ENVIRONMENTAL LIFE CYCLE ASSESSMENT OF LINOLEUM 
Marieke Gorree

Jeroen Guinée

Gjalt Huppes

Lauran van Oers

Centre of Environmental Science (CML)

Leiden University

P.O. Box 9518

2300 RA Leiden

The Netherlands

CML report 151 - Section Substances \& Products 


\title{
ENVIRONMENTAL LIFE CYCLE ASSESSMENT OF LINOLEUM
}

Research commissioned by Forbo-Krommenie B.V.

\author{
Marieke Gorree \\ Jeroen Guinée \\ Gjalt Huppes \\ Lauran van Oers \\ Centre of Environmental Science (CML) \\ Leiden University \\ P.O. Box 9518 \\ 2300 RA Leiden \\ The Netherlands
}

CML report 151 - Section Substances \& Products 
Copies can be ordered as follows:

- by telephone: (+31) 715277485

- by writing to: CML Library, P.O. Box 9518, 2300 RA Leiden, The Netherlands

- by fax: (+31) 715275587

- by e mail: eroos@cml.leidenuniv.nl

Please mention report number, and name and address to whom the report is to be sent

ISBN: 90-5191-129-7

Printed by: Universitair Grafisch Bedrijf, Leiden

${ }^{\circ}$ Centre of Environmental Science (CML), Leiden 2000 


\section{Content}

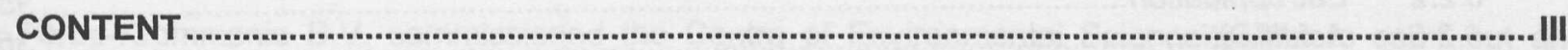

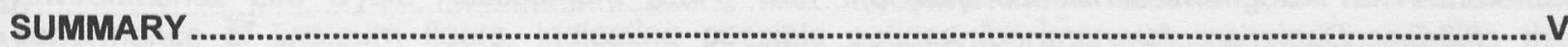

CRITICAL REVIEW STATEMENT OF LCA OF LINOLEUM FLOORS.................................................X

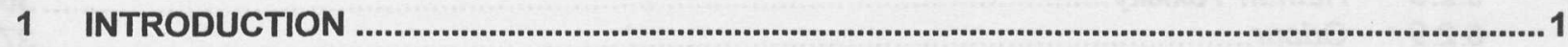

1.1 GENERAL

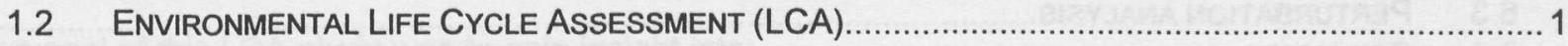

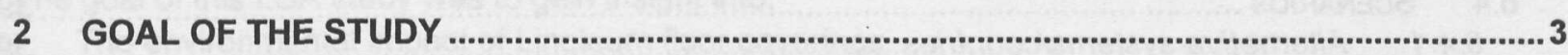

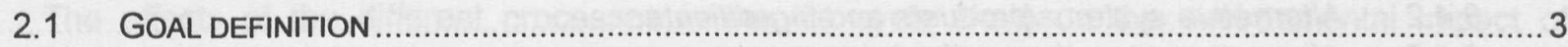

2.2 INTENDED APPLICATION OF THE STUDY RESULTS.

2.3 INITIATOR

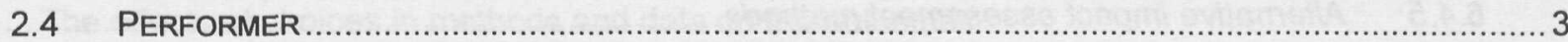

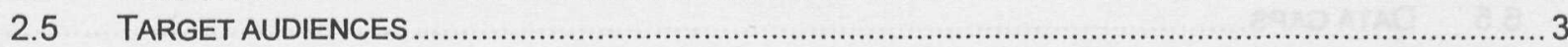

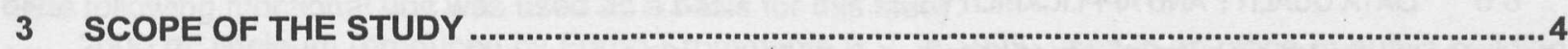

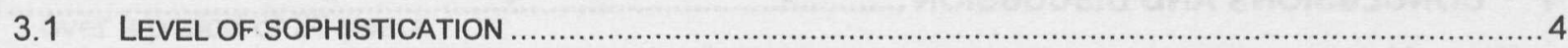

3.2 TYPE OF ANALYSIS: DESCRIPTIVE AND CHANGE ORIENTED

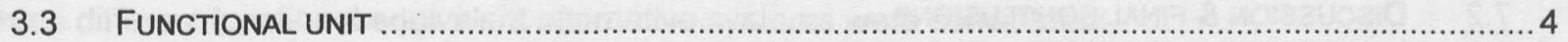

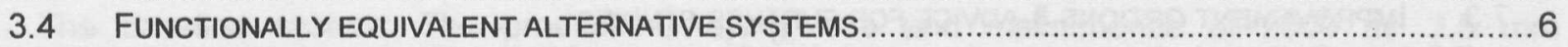

$3.5 \quad$ INITIAL CHOICES ON METHODS AND DATA

3.6 ISO GUIDELINES CONCERNING THIRD PARTY REPORTING AND PEER REVIEW...............................

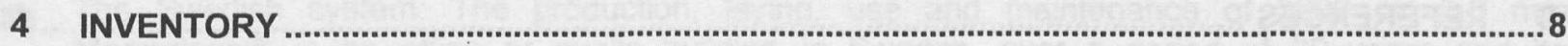

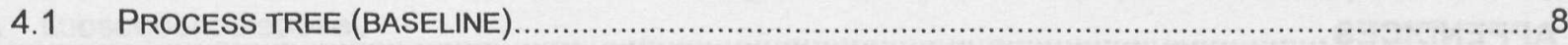

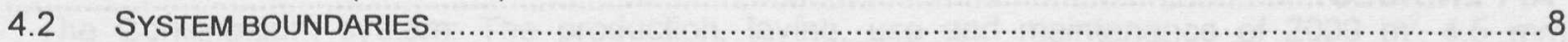

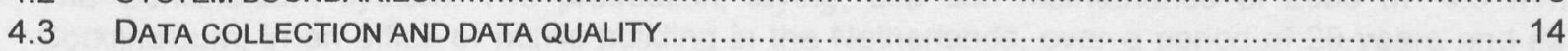

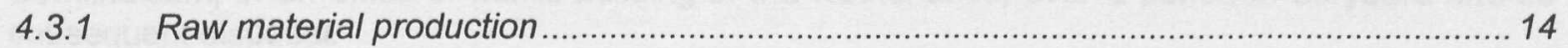

4.3.2 Processes which take place at Forbo-Krommenie B.V. ............................................ 15

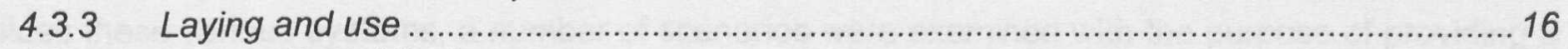

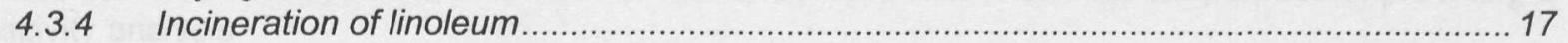

4.3.5 Other processes (transport, energy, landfill) ............................................................. 17

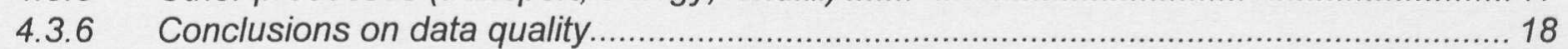

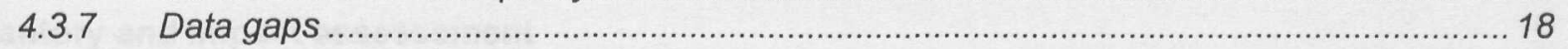

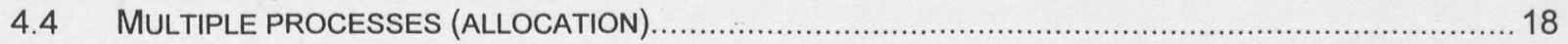

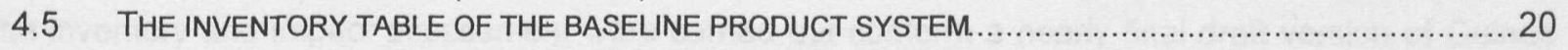

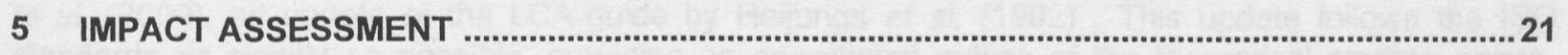

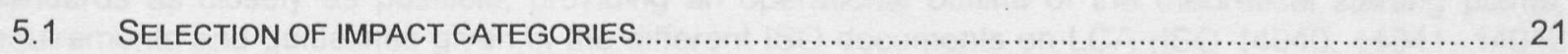

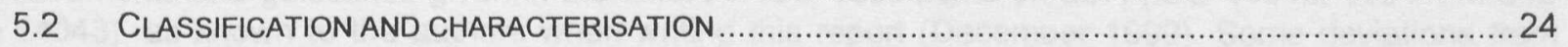

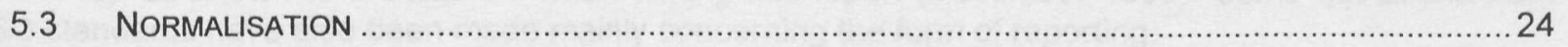

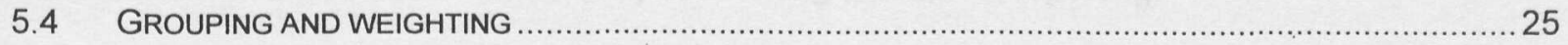

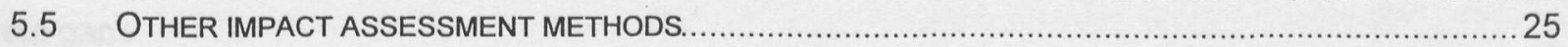

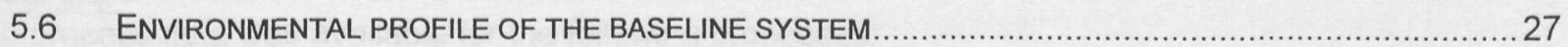

5.7 INPUTS AND OUTPUTS NOT ASSIGNED TO AN IMPACT CATEGORY _.............................................. 27

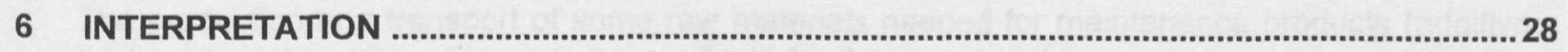

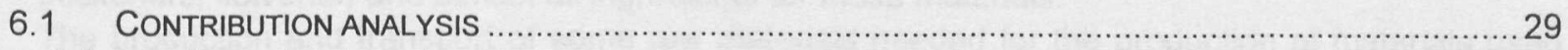

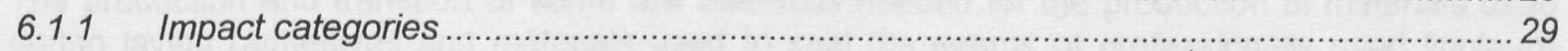

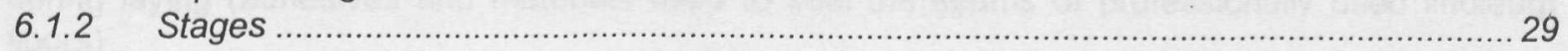

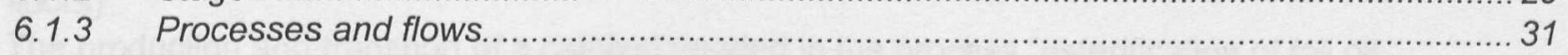




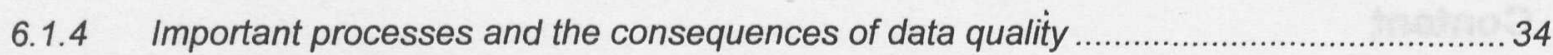

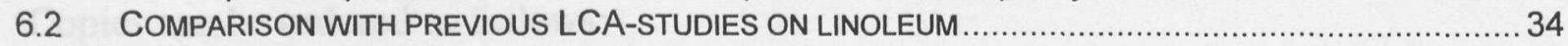

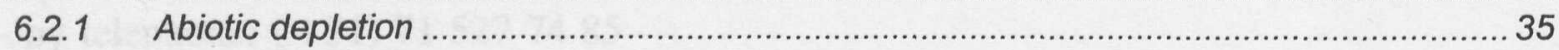

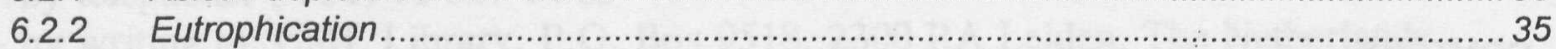

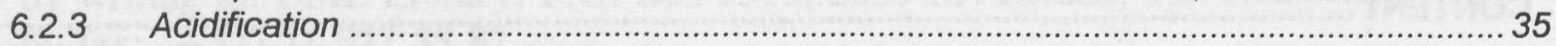

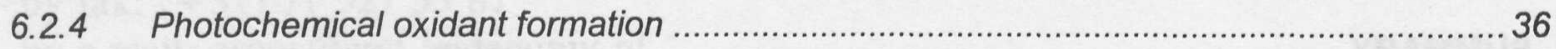

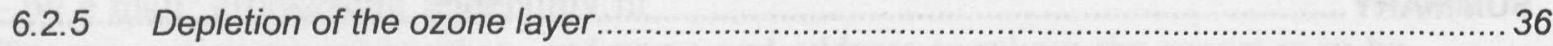

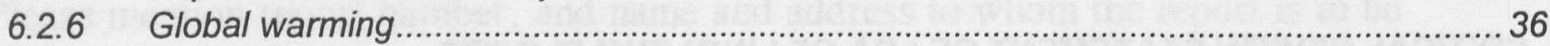

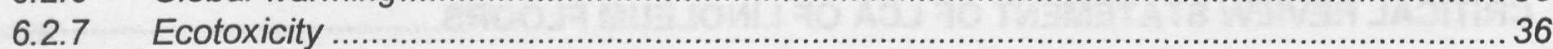

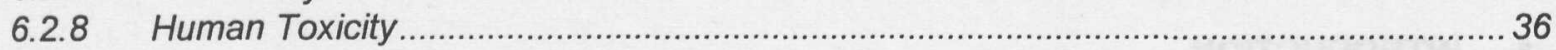

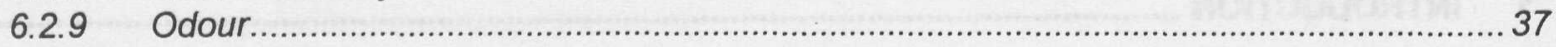

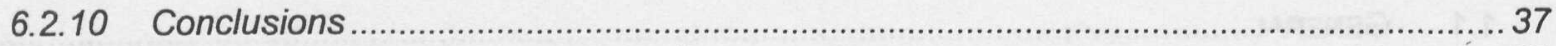

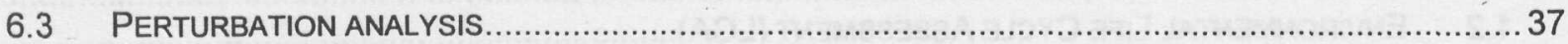

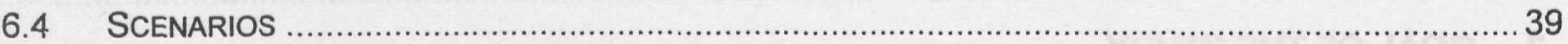

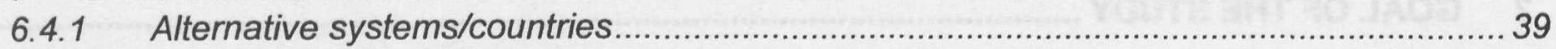

6.4.2 Alternative systems/products and ingredients ..................................................... 40

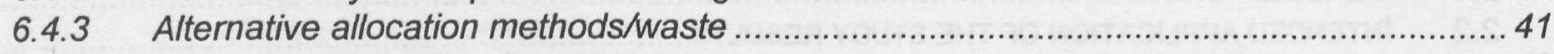

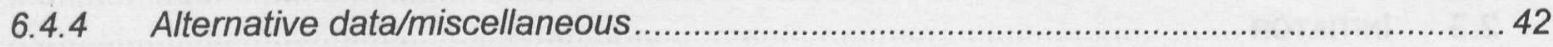

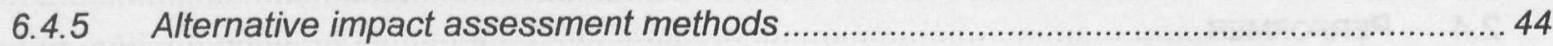

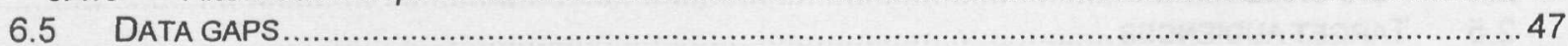

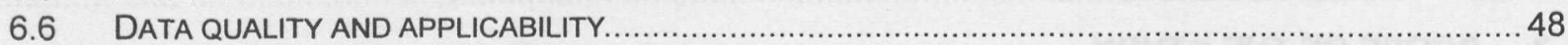

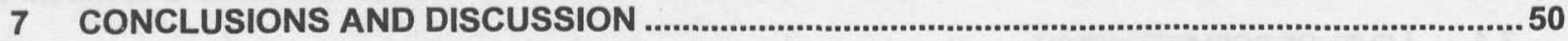

7.1 MAIN CONCLUSIONS FROM INVENTORY, IMPACT ASSESSMENT AND INTERPRETATION SUMMARISED50

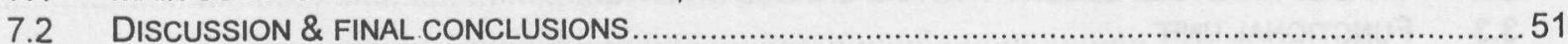

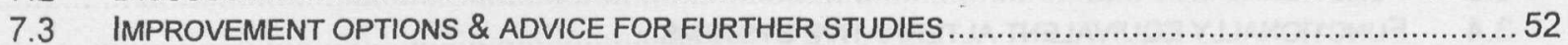

7.3.1 Options to improve environmental performance of Forbo-Krommenie B. V. ................52

7.3.2 Advice for future studies on linoleum ......................................................... 53

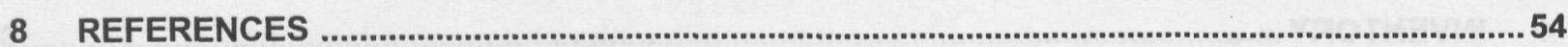

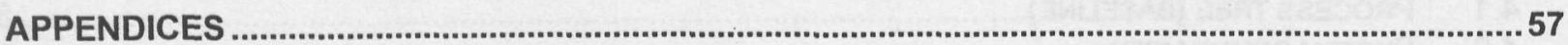




\section{Summary}

\section{Introduction}

Forbo-Krommenie B.V. commissioned the Centre of Environmental Science (CML) to carry out an Environmental Life Cycle Assessment (LCA) with the purpose of assessing the environmental performance of linoleum floors, indicating possible options for improvement, and assessing the sensitivity of the results to methodical choices. The method followed in this study is based on Guinee et al. (2000) an update of the CML guide on LCA from Heijungs et al. (1992).

\section{Goal and siope}

The goal of this LCA study was to gain insight into:

- The environmental impact of Linoleum floor coverings.

- The effects of the different processes in the life cycle chain on the environmental impact of linoleum.

- Identifying possible improvements,

- The effects of choices in methods and data on the outcomes.

The following functional unit was used as a basis for this study: $2000 \mathrm{~m}^{2}$ linoleum (produced by Forbo-Krommenie B.V. in 1998) used in an office or public building over a period of 20 years.

Three different functional equivalent alternative systems were considered:

- The baseline system: The production, laying, use and maintenance of $2000 \mathrm{~m}^{2} 2.5 \mathrm{~mm}$ Marmoleum $^{\otimes}$, in an office or public building in the Netherlands, over a period of 20 years and its subsequent disposal.

- The Swedish system: The production, laying, use and maintenance of $2000 \mathrm{~m}^{2} 2.0 \mathrm{~mm}$ Marmoleum $^{\Theta_{1}}$ in an office or public building in Sweden, over a period of 20 years and its subsequent disposal.

- The Corklinoleum system: The production, laying, use and maintenance of $2000 \mathrm{~m}^{2} 4.5 \mathrm{~mm}$ Corklinoleum, in an office or public building in the Netherlands, over a period of 20 years and its subsequent disposal.

Besides these various systems, a number of scenarios were examined with the purpose of providing a sensitivity analysis.

\section{Inventory and impact assessement}

The inventory and impact assessment were carried out conform a nearly final draft version of Guineé et al. (2000), an update of the LCA-guide by Heijungs et al. (1992). This update follows the ISO standards as closely as possible, providing an operational outline of the theoretical starting points, requirements and guidelines given in the different ISO documents on LCA (ISO 14040, 14041, 14042 \& 14043), as known to the authors when writing this report (December 1999). Some deviations from ISO standards have also been made mainly concerning the form of reporting.

\section{Data gaps}

No process data was available for the following processes:

- The production and transport of pesticides (use and emission of pesticides is included).

- The production and transport of some raw materials needed for maintenance products (additives, thickeners, solvents) and almost all ingredients for those materials.

- The production and transport of some raw materials needed for the production of materials used during laying (adhesives and materials used to seal the seams of professionally used linoleum floors)

- The production and transport of a catalyst needed in the process "esterification of tall oil ". 
- The production and transport of the fertiliser S needed in the process "growing linseed ".

- The production and transport of the maintenance product used for removing the polymer dressing of linoleum floor covering needed in the process "the use and maintenance of $1 \mathrm{~m} 2$ linoleum for 20 years".

\section{Impact categories}

The following impact categories are included in this study:

- Extraction of abiotic resources

- Climate change

- Depletion of the ozone layer

- Human toxicity

- Eco-toxicity

- subcategory: aquatic eco-toxicity

- subcategory: terrestrial eco-toxicity

- subcategory: sediment eco-toxicity

- Photo-oxidant formation

- Acidification

- Eutrophication

- odour

Besides the impact assessment method described in Guinée et al. (2000), a few other impact assessment methods were applied to determine the extent to which the results of the study are influenced by the method of impact assessment used.

\section{Flows not assigned to an impact category}

In total there were 264 inputs/outputs that could not be assigned to an impact category. These are mainly from the ETH database. A large portion of the emissions (132 emissions) are radioactive emissions. Since radiation is not included in this study (because no consensus has yet been reached concerning the impact assessment method to be used) it could not be included in the impact assessment results.

\section{Interpretation and conclusions}

\section{Main contributing processes}

The processes or groups of processes that contribute largely to more than one impact category are:

- The growing of linseed (emissions of $\mathrm{NH}_{3}, \mathrm{~N}_{2} \mathrm{O}$, pesticides).

- Gas and electricity used at Forbo-Krommenie B.V.

- Oil used for the production of maintenance products.

- The transport of raw materials.

- The incineration of linoleum.

- Coal used for the production of detergents and acrylic dispersions/emulsions.

\section{Scenarios}

The following conclusions were drawn from the scenarios:

- Extra transport by freighter to the USA has less impact than was expected.

- The influence of maintenance in the "use " phase is not negligible as is often thought.

- The influence of other pigments than $\mathrm{TiO} 2$ can be considerable. $\mathrm{TiO} 2$ is not representative for other pigments. The impact of other pigments may be high even though their mass share in the product is much lower.

- Using tall oil in linoleum is better for the environment than using only linseed oil.

- Using $2.0 \mathrm{~mm}$ linoleum is better for the environment. However, this is only the case if the life span of the $2.0 \mathrm{~mm}$ linoleum is more or less the same as for the $2.5 \mathrm{~mm}$ version.

- Substitution of useful heat produced during the incinerating of linoleum with avoided electricity use is the best of the studied alternatives. 
- Reduction in the use of pesticide only effects terrestrial ecotoxicity, and not the other toxicity categories.

- Reduction in the use of $\mathrm{N}$-fertiliser affects the results for global warming, eutrophication and acidification substantially.

- Reduction of the transportation distance for raw materials has some effect on aquatic and sediment ecotoxicity.

- Changes in the composition of the VOC emissions at Forbo-Krommenie B.V. have a considerable influence on the category "odour ". A better estimate of the composition of these emissions is necessary in order to assess the effects on "odour " and "depletion of the ozone layer " properly in an LCA on linoleum products.

- The influence of capital goods is certainly not negligible. According to a very rough scenario leaving out capital goods may lead to an underestimation of $1-10 \%$. The influence of the missing chemicals could be even more substantial. According to a very rough scenario these data gaps may lead to an underestimation of $5-40 \%$.

Data quality and applicability

The main conclusions following from the analysis of data quality are:

- The results of the study are applicable for analysis of the described systems only. This means that:

- The results should not be used to compare the environmental performance of linoleum produced by Forbo-Krommenie B.V. to other products.

- The results should not be used to compare systems with different capital goods because capital goods are not included.

- The results should not be used to compare different maintenance systems because too much data on chemicals in maintenance products is missing.

- The results for "odour " and "depletion of the ozone layer " should be presented with some care as the unreliable VOC estimations at Forbo-Krommenie B.V. play an important role in these impact categories.

\section{Discussion \& final conclusions}

Main contributing processes

In the stage "production of raw materials" the processes "growing of linseed" and "transport" are important processes. The data for this process depend largely on assumptions. If these assumptions are not realistic, this may have large influences on results. Therefore, it is advisable to try to ground these assumptions with better data. Still, Forbo-Krommenie B.V. could improve their environmental performance on many impact categories by using linseed that is cultivated with less fertiliser and less pesticides.

In the stage "production of linoleum " the energy used at Forbo-Krommenie B.V. is most important. We expect that this data is valid and reliable. Saving on the use of electricity and gas is therefore an opportunity for improvement.

In the stage "laying and use ", the coal and oil used during the production of maintenance products is important. Data on the production of maintenance products is not complete. The results of the comparison of the Dutch and Swedish scenario showed a heavy influence of the lack of data on the production of Swedish maintenance products. Therefore, no conclusions can be based on the comparison of these maintenance systems, other than that maintenance is not negligible. Better data on maintenance products is needed before more conclusions can be drawn on the influence of maintenance.

In the stage "disposal " the emissions produced during incineration are important. However, the figure of $100 \%$ incineration of used linoleum and the linoleum waste from Forbo-Krommenie B.V. is only an assumption. From the scenario analysis followed that substitution of useful heat produced during the incinerating of linoleum with avoided electricity use shows the best environmental profile of all studied alternatives for handling waste. Therefore incineration seems a better alternative than landfill: 
However, this result should be considered with some care, because for landfills average ETH-data was used. These are probably not very realistic for the landfill of linoleum.

\section{Composition of linoleum}

In the scenarios a number of variation in linoleum composition have been studied. This showed that:

- The sensitivity analysis showed that the contribution of pigments other than $\mathrm{TiO}_{2}$ to all impact categories can be considerable. However the data on pigments could not be checked for quality. Moreover, these data were not provided by the suppliers of the pigments and the representativeness of these data might be disputed. There is a great variety of possible pigments, but little available information concerning their environmental performance. A more detailed analysis on this point focusing on the pigments that are actually used by Forbo-Krommenie B.V., aimed at finding pigments that are the most environment-friendly could be valuable.

- The $2.0 \mathrm{~mm}$ gauge has a considerably better environmental performance than the $2.5 \mathrm{~mm}$ gauge. On average, the results for this gauge are $15 \%$ lower. If the life span of both floors is comparable, Forbo-Krommenie B.V. might consider producing relatively more $2.0 \mathrm{~mm}$ products as a means of improving their environmental performance.

- Linoleum with tall oil has a better environmental profile than linoleum without tall oil, because the use of linseed is lower.

- Compared to the baseline $2.5 \mathrm{~mm}$ linoleum cork linoleum produces better results in most categories, but performs considerably worse in the categories "abiotic depletion " and "odour ". Were the gas use during drying and milling of cork-granulate reduced, it would improve the results for these categories.

\section{Data gaps}

The influence of capital goods is certainly not negligible. According to a very rough scenario leaving out capital goods may lead to an underestimation of $1-10 \%)$. The influence of the missing chemicals could be even more substantial. According to a very rough scenario these data gaps may lead to an underestimation of $5-40 \%$ ). Therefore, the results should not be used to compare systems with different capital goods or different maintenance systems. The results of the study are applicable for analysis of the described systems only.

\section{VOC}

The result for "odour " and "depletion of the ozone layer " should be considered with some care, since the emissions of VOC from Forbo-Krommenie B.V., which play an important role in these impact categories, were not specified.

\section{Improvement options \& advice for further studies}

\section{Improvement options}

- Forbo-Krommenie B.V. could improve their environmental performance on many impact categories by using linseed that is cultivated with less fertilisers and less pesticides. This seems a more promising option than reducing transportation distances for raw materials.

- Saving on the use of electricity and gas at Forbo-Krommenie B.V. is also an area for improvement.

- The pigments used can have a large influence on environmental performance. A more detailed analysis on this point, aimed at finding pigments, which are safest for the environment, could be valuable.

- The $2.0 \mathrm{~mm}$ gauge has a considerably better environmental performance than the $2.5 \mathrm{~mm}$ gauge. On an average, the results for this gauge are $15 \%$ lower. If the lifetime of both floors is comparable, Forbo-Krommenie B.V. might consider producing relatively more $2.0 \mathrm{~mm}$ products in order to improve their environmental performance.

- Linoleum with tall oil shows a better environmental profile than linoleum without tall oil. Therefore, a reduction in the amount of tall oil, in favour of linseed oil, is not advisable.

- Reducing gas use during the drying and milling of cork-granulate would improve the results for "abiotic depletion" and "odour " for cork linoleum. For other categories cork linoleum already produces better results compared to baseline $2.5 \mathrm{~mm}$ linoleum. 
Advice for future studies on linoleum

It is advisable to give the following topics extra attention in future studies on linoleum as the data on these topics is fragmented and its influence on the environmental profile of linoleum could be considerable:

- The production and use of maintenance products, especially the Swedish type.

- The production and use of pigments other than TiO2.

- Emissions of individual VOC at the site of Forbo-Krommenie B.V. 


\section{Critical review statement of LCA of linoleum floors}

CML has performed a Life Cycle Assessment (LCA) of linoleum floors, commissioned by Forbo Krommenie B.V. The critical review has been performed in three phases, and two meetings have been held. The last review was to confirm that the comments in the second phase where taken into account.

The goal of the study has been to learn about the environmental impact of the Linoleum floor coverings and the different contributing processes, to identify possible improvements and to learn about effects of choices in methods and data on the outcome. The first part of the goal is definitely met, since the results show which processes that contribute significantly to the different environmental impacts. Out of the identified significant issues, improvement options are suggested. These can be studied further in improvement assessments. The effects of choices in methods and data are partly studied and concluded on.

The critical review has been performed in order to:

- check that the methodology is in compliance with international standards on LCA, ISO 14040 14043 , and scientifically and technically valid

- give advice on the data sources used

- check that the report is transparent and consistent

- check that the interpretation and the conclusions drawn reflect the limitations identified and the goal of the study

\section{Compliance}

The study has been performed according to all main requirements of the ISO 14040, 14041, 14042 and 14043 standards. Requirements such as sensitivity analysis in order to check the chosen criteria for inclusion of inputs and outputs is rather detailed, and hardly ever possible to fulfil for each input and output! The impact assessment give often a result that is incomplete (e.g. for photo oxidant formation, toxicity and odour) more because of lack of data than on the deficiencies of the characterisation methods. Since conclusions are based on more robust impact categories, the uncertainty within these impact categories does not effect the conclusions.

\section{Data}

Site specific data have been used when available. There are data gaps in the study, but many of the gaps have by a sensitivity check been shown not to be of large importance for the main results. Important data gaps may be the pigments used, the maintenance products used (the production of these products) and the VOCs from the linoleum production and storage. Other data gaps are emissions from landfill and incineration of Linoleum.

Essential assumptions for data and data sources are documented in a transparent way in an annex. However, the inputs and outputs for each process is only documented in the LCA tool, which is not so easy to use, why the inventory calculations have not been checked.

\section{Methods}

The methodology used is presented in a clear way. Some deviations from ISO recommendations exist, e.g. for incineration and landfill, where the allocation used in the ETH database has been used instead of a methodology recommended by the ISO 14041. This seems to have a small contribution to the total results, however, why this probably does not influence the main results.

\section{Interpretation}

The interpretation has been done in accordance with the requirements of 14043 , and in line with the goal and scope. The sensitivity analysis of e.g. the pesticide use show in an illustrative way how the uncertainty in amounts of pesticide and fertiliser effects the different impact categories. The conclusions are drawn in line with the goal and scope.

The conclusion is that the report can contribute to the increased knowledge of Forbo about the environmental impact of Linoleum floor covering, and that it is a good basis for further studies. The results can be communicated externally, since there is a third party report that shows how the study 
has been performed. It should, however, be clear when results are communicated that the results can not be compared to results of other studies, since the choice of methodology, assumptions, system boundaries, data for e.g. electricity production etc often differ to a large extent.

At last the critical reviewer would like to thank for a good and constructive co-operation.

Elin Eriksson

CIT Ekologik, Stiftelsen CHALMERS INDUSTRITEKNIK

Göteborg, 2000-06-20 



\section{Introduction}

\subsection{General}

Linoleum is a floor covering consisting of a binder made from linseed oil and/or vegetable drying oils and rosin mixed with wood flour and/or cork, inorganic filler and pigments, on a carrier of jute. ForboKrommenie B.V. is the world's largest producer of linoleum floor covering (Marmoleum ${ }^{\circledast}$, Artoleum ${ }^{\circledast}$, Corklinoleum and Walton) and of other linoleum products (Desk Top ${ }^{\circledR}$ and Bulletin Board ${ }^{\circledR}$ ). The products are sold all over the world but principally in Western Europe and the USA. Forbo-Krommenie B.V. commissioned the Centre of Environmental Science (CML) to carry out an Environmental Life Cycle Assessment (LCA) in order to assess the environmental performance of linoleum floors, to indicate possible options for improvement, and to assess the sensitivity of the results in methodical choices.

Elin Eriksson, of Chalmers Industriteknik in Sweden carried out a peer review.

\subsection{Environmental Life Cycle Assessment (LCA)}

The LCA is defined by ISO as a "compilation and evaluation of the inputs and outputs and the potential environmental impacts of a product system throughout its life cycle " (ISO 14040, 1997). It is a method that is meant, based on a 'cradle-to-grave' approach, to systematically evaluate the environmental impacts of products and activities. This approach is based on the identification and quantification of the flows of substances and materials (or other interventions) to and from the economy and the environment, during the entire life cycle of the product or activity. Economy is defined here as all human activities that take place more or less isolated from the environment. In other publication, sometimes the word 'technosphere' is used to describe this concept. In Figure 1 the main methodological framewc, $k$ according to ISO is given.

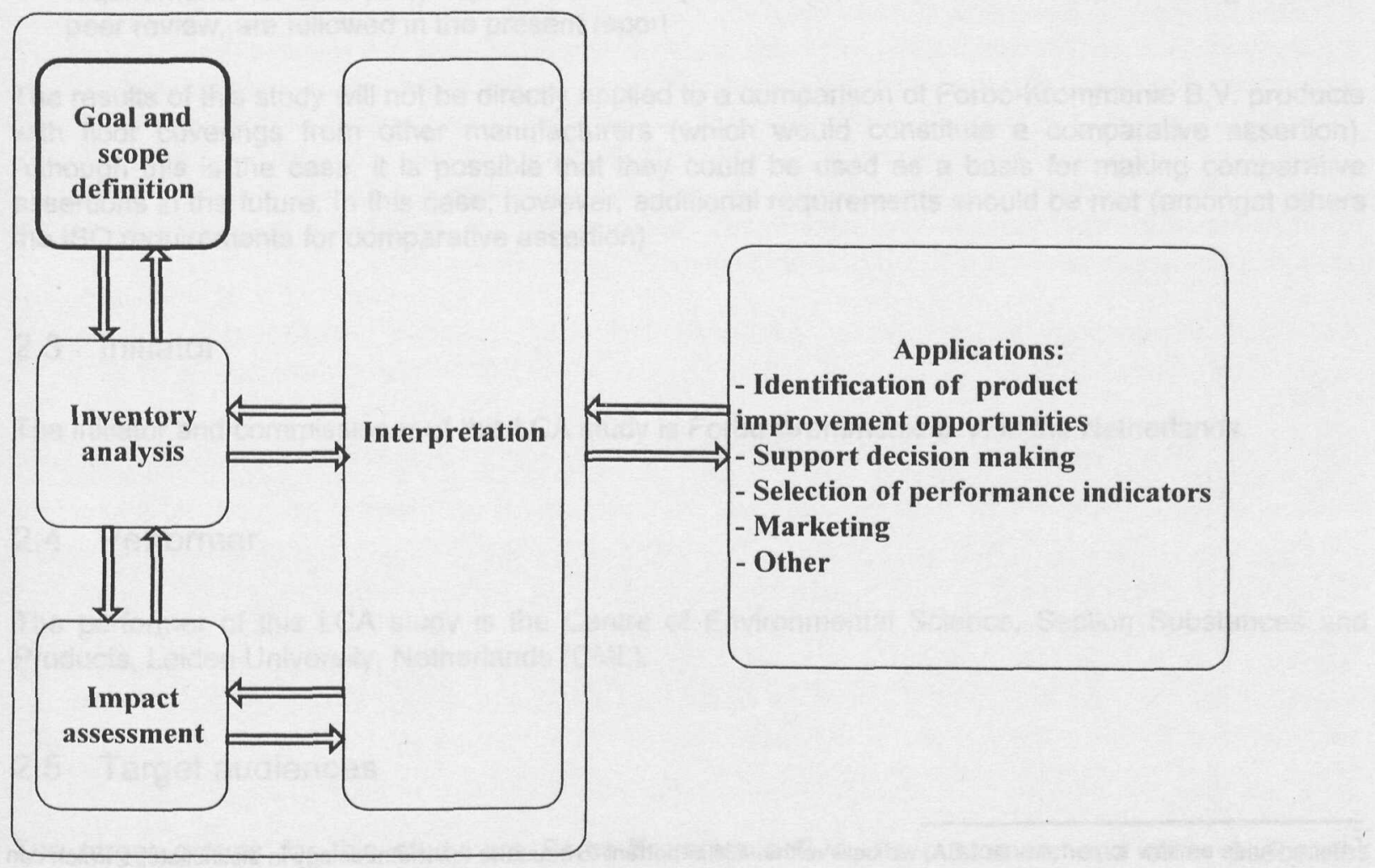

Figure 1: The general methodological framework for LCA (ISO 14040, 1997). 
In ISO $14040,14041,14042$ \& 14043 requirements are stated for every phase of the LCA and even more technical reports and standards are in development. These ISO standards and guidelines are very important in providing an international reference on principles, framework and terminology for conducting and reporting LCA studies. The ISO standards, however, do not supply the reader with a "cookbook " outlining step-by-step operational guidelines for conducting a LCA study. ISO standards contain elements that shall or should be considered when conducting an LCA, and when communicating the results.

Currently, Guinée et al. (2000) are writing an update of the LCA-guide by Heijungs et al., (1992). This update follows the ISO standards and guidelines as closely as possible, providing an operational outline of the theoretical starting points, requirements and guidelines given in the different. ISO documents on LCA (ISO 14040, 14041, 14042 \& 14043), as known to the authors at the moment of writing (December 1999). Guinée et al. (2000) take the Guide by Heijungs et al. (1992) as a starting point, and then further update this guide ${ }^{1}$ by including all relevant developments which have taken place since its publication, with particular reference to the work taking place within the SETAC community. Some additions to ISO standards have been made in order to achieve operationalisation. Some deviations from ISO standards have also been made, but only if there were really significant arguments to do so. The main deviations concern the form of reporting. In Guinee et al. (2000) the guidelines for reporting are different for separate goal and scope studies (which is in line with ISO), and for final reports, in which the description and discussion of several topics is not placed in the goal and scope, but rather in later sections of the report closer to the topic itself (e.g. data quality, system boundaries etc.). Additions and deviations have all been documented comprehensively in the guide. The present study is based on the nearly final draft version of the updated guide (Guinee et al., 2000).

\footnotetext{
${ }^{1}$ For a Guide on how to perform an LCA, we believe that it is important to structure the methodology in distinct steps which can be documented and reported separately in an LCA-report, and which follow a logic working plan. Iterations between steps are always possible and necessary, as LCA is an iterative technique, but iteration as such is not an individual step.
} 


\section{Goal of the study}

In the goal definition of an LCA, besides the goal also the intended application, initiator, performer and intended audience should be stated according to ISO (ISO, 14040). In this chapter, these topics are described sequentially.

\subsection{Goal definition}

The goal of this LCA study is to gain insignt into:

- The environmental impact of linoleum floor coverings.

- The effects of the different processes in the life cycle chain on the environmental impact of linoleum floor covering.

- Identifying possible improvements

- The effects of choices in methods and data on the outcomes.

\subsection{Intended application of the study results}

The results of this LCA will be used:

- For product and process development:

Forbo-Krommenie B.V. wants to be able to analyse (with the aid of the delivered software) the effects of process changes in terms of technology, input and product composition on the total environmental impact. This information can, in turn, be used to prioritise different measures that may be taken to improve Forbo-Krommenie B.V.'s environmental performance.

- As a basis for answering questions received from business relations

- Possibly as basis for a brochure that serves marketing needs by communicating the environmental performance of Forbo-Krommenie B.V.'s products to external relations. Therefore, the requirements for third-party reports from ISO (ISO 14040, 14041 and 14042), including a critical peer review, are followed in the present report

The results of this study will not be directly applied to a comparison of Forbo-Krommenie B.V. products with floor coverings from other manufacturers (which would constitute a comparative assertion). Although this is the case, it is possible that they could be used as a basis for making comparative assertions in the future. In this case, however, additional requirements should be met (amongst others the ISO requirements for comparative assertion).

\subsection{Initiator}

The initiator and commissioner of this LCA study is Forbo-Krommenie B.V. in the Netherlands.

\subsection{Performer}

The performer of this LCA study is the Centre of Environmental Science, Section Substances and Products, Leiden University, Netherlands (CML).

\subsection{Target audiences}

The target groups for this study are Forbo-Krommenie B.V., its customers and other associates interested in the environmental performance of linoleum products. 


\section{Scope of the study}

In this chapter, principal choices concerning the scope of the study are outlined. These include the breadth and depth of the study, functional units and functionally equivalent systems. These are described in $\S 3.1$ to 3.4 .

According to ISO, main choices and assumptions on the next phases of the LCA should be described under scope. These include choices and assumptions on system boundaries, data collection, applicability etc. However, Guinée et al. (2000) advise doing so only in the initial goal and scope description. In the description of a fully elaborated LCA, such assumptions should be treated, in as far as possible, where the topic itself is described in the report, since assumptions and choices often change during typically iterative LCA processes. In order to adhere as closely as possible to the ISO guidelines, we summarise the main assumptions and choices in $\S 3.5$.

In § 3.6 finally the ISO guidelines concerning third party reporting and the need for a peer review are described.

\subsection{Level of sophistication}

An LCA can be performed so as to articulate a range of levels of sophistication. These levels are described in Guinée et al. (2000). For this linoleum LCA, a detailed LCA is carried out using default methods and sensitivity analysis on non-default methods. This is the operative level for a professional study, for a vast but not overly complex application. The ISO guidelines are followed as closely as possible (ISO 14040, 14042, 14042 \& 14043), including the recommended sensitivity analysis.

\subsection{Type of analysis: descriptive and change oriented}

This LCA describes environmental burdens that may be attributed to (the use of) a certain amount of a product or service (= descriptive) but also focuses on arguments to improve technologies and production processes (change oriented). This LCA should, therefore, be considered as a change oriented LCA rather than as a descriptive one. One of the main requirements of a change oriented LCA is that the processes included should be up to date so, that a fair comparison may be made between a system with "old " processes and a changed system with improved processes, both based on recent data. In this study we used the most recent data available, mainly from 1998. The "marginal average " discussions currently taking place in LCA fora are not included in this study. ${ }^{2}$

\subsection{Functional unit}

The functional unit describes the main function(s) fulfilled by a product system and indicates how much of this function is considered.

This study is carried out in the form of an LCA of a baseline product system which is combined with a sensitivity analysis in order to gain insight into such topics as the effects of differences in production methods, data quality, use, and countries to which the product is sold. In this sensitivity analysis data, methods and choices are varied and, in some scenarios even, the product system has been changed substantially. In order to avoid the necessity of defining a different functional unit for every scenario, it was decided to keep the functional unit applicable to all scenarios by making the definition rather broad. This is most useful in the present study in which a number of scenarios play an important role.

\footnotetext{
${ }^{2}$ All inventory data is average data. In the impact assessment, both average and marginal methods are often used as the best
} available methods per impact category, leaving no choice here. This method is either average or marginal. 
For example, it makes it possible to use the same functional unit for a linoleum floor used in the Netherlands and in Sweden. However should the result of this study be used in other applications, (e.g. in a comparative assertion concerning different floor coverings ) the functional unit should be defined more rigidly, in order to avoid large differences in functionality between the alternative systems.

For the definition of the functional unit, the following elements were considered:

- The function.

- The spatial context.

- The temporal context.

- The users behaviour.

- The unit.

- The quantity.

These elements are hereafter discussed in relation to the LCA on linoleum.

\section{Function}

Linoleum's function is floor covering. Linoleum floor covering can be used in different situations, e.g. in private homes, public buildings or offices. The situation and the intensity of use influence the life span of the floor. In this study we focused on linoleum floor covering in public and commercial use areas as the baseline LCA. Linoleum is sold as a floor covering mainly to hospitals, schools, offices, etc.

\section{Spatial context}

While all linoleum is produced in a factory in Assendelft ${ }^{3}$, in the Netherlands, linoleum is used all over the world. This means that there are differences in the distance and method of transport of the linoleum floor covering as well as possible differences in the use and disposal phases. The differences between countries in the use of floor covering may include differences in maintenance (frequency of cleaning, use of cleaning products) and differences in life span (as a function of the maintenance, frequency of moving, fashion, etc.). The differences in the disposal of floor covering may include differences in recycling and differences in the ratio between combustion and dump. There may also be variance in how electricity is produced in different countries.

Because the linoleum floor covering produced by Forbo-Krommenie B.V. is used in many countries, a choice had to be made for the baseline system and the scenarios. The baseline LCA is determined as a function of the country in which most linoleum is sold, namely the Netherlands. Sweden is also a very important market for linoleum. One of the scenarios in the sensitivity analysis will, therefore, be based on Sweden. Linoleum is also sold to the USA because this has large consequences for transport distance, transport to the USA will also be included in the sensitivity analysis. To ensure the functional unit's applicability in all scenarios, no definition of the country of use is included in the functional unit.

\section{Temporal context}

1998 isthe most recent year for which Forbo-Krommenie B.V. process data is available. This year will, therefore, be the reference year for the study.

The life span of the linoleum floor can be interpreted as the technical life span or the economic life span. The technical life span is based on wear as a result of use. However, the economic life span of a floor is shorter because a floor may be removed for aesthetic reasons, for instance, when new users move into a building or when a building is renovated. This is generally referred to as the "econiomic life span ". The economic life span may also be influenced by the country in which the floor is used (see also spatial context). For this study, the economic life span of linoleum floors in public buildings is estimated to be 20 years (source: Forbo-Krommenie B.V.). The life span in private homes is probably shorter because people frequently replace old floor covering when moving into a new home.

\section{User behaviour}

As mentioned above consumer behaviour may differ between countries. This may influence life span and the use of maintenance products. Actual use and maintenance in a public building in the

\footnotetext{
${ }^{3}$ There also is a Forbo-Krommenie B.V. factory in Scotland. This is not included in this study.
} 
Netherlands is included in the baseline system. In the Swedish scenario, we included the actual use and maintenance in a public building in Sweden, however, there was less information on Swedish maintenance than on Dutch maintenance (see Table 2 for the maintenance frequencies in public buildings in both countries).

Unit

The unit not only depends on the function, but also on the spatial and temporal context. The unit should preferably be expressed in SI units. For this study we have defined the unit as: $\mathrm{m}^{2}$ year.

Quantity

The quantity in the baseline LCA is taken as: $40000 \mathrm{~m}^{2}$.year. This is the use of $2000 \mathrm{~m}^{2}$ linoleum over a period of 20 years. $2000 \mathrm{~m}^{2}$ is representative of the floor area in an average-sized office or public building and 20 year is the economic life span of the floor in the Netherlands according to ForboKrommenie B.V.

This leads to the following functional unit:

The use of $2000 \mathrm{~m}^{2}$ linoleum (produced by Forbo-Krommenie B.V. in 1998) in an office or public building over a period of 20 years.

\subsection{Functionally equivalent alternative systems}

After establishing the functional unit, one or more product systems capable of producing the functional unit defined above are selected.

Linoleum floor covering from Forbo-Krommenie B.V. is available in three types:

- Plain and decorative linoleum

- Corklinoleum

- Plain and decorative linoleum on a corkment backing

These are available in different gauges. For the baseline system we focused on the types of linoleum floor covering most sold, namely plain and decorative. Forbo-Krommenie B.V. sells this type of linoleum under the brand names Marmoleum ${ }^{\circledast}$, Artoleum ${ }^{\circledast}$ and Walton. Although patterns and colours vary, the overall composition of these products is the same, with the exception of the pigments. 2.5 $\mathrm{mm}$ is the best-selling gauge ( $70 \%$ of the total return). Marmoleum $2.5 \mathrm{~mm}$ is, therefore, used in the baseline system. In Sweden, there is a large market for $2.0 \mathrm{~mm}$ Marmoleum ${ }^{\circledast}$ hence this type will be used in the "Swedish scenario ". Corklinoleum differs substantially from Marmoleum ${ }^{\oplus}$ as it is thicker $(4.5 \mathrm{~mm})$ and cork is used as filler instead of wood flour.

In conclusion, three different functional equivalent alternative systems are considered:

- The baseline system: This consists of the production, laying, use and maintenance of $2000 \mathrm{~m}^{2} 2.5$ $\mathrm{mm}$ Marmoleum ${ }^{\circledast}$, in an office or public building in the Netherlands, over a period of 20 years and its subsequent disposal.

- The Swedish system: This consists of the production, laying, use and maintenance of $2000 \mathrm{~m}^{2} 2.0$ $\mathrm{mm}$ Marmoleum $^{\circledR}$, in an office or public building in Sweden, over a period of 20 years and its subsequent disposal.

- The Corklinoleum system: This consists of the production, laying, use and maintenance of $2000 \mathrm{~m}^{2}$ $4.5 \mathrm{~mm}$ Corklinoleum, in an office or public building in the Netherlands, over a period of 20 years and its subsequent disposal.

Forbo-Krommenie B.V. manufactures other products (e.g. Artoleum ${ }^{\circledast}$, Walton) and gauges which sometimes means using slightly different production processes. However, the focus of this study was not to discuss all products manufactured by Forbo-Krommenie B.V., but rather to discuss a few relevant alternatives. Forbo-Krommenie B.V. has indicated that the three different systems described above are the most relevant. 


\subsection{Initial choices on methods and data}

ISO requires that a number of choices on methods data and on the applicability of the study be described and discussed within the scope of the study (See ISO 14040, 140401). In accordance with Guinée et al. (2000) we decided to write up most of these discussions in the final report, closer to the point were these choices were made. In order to adhere closely to ISO standards, we summarised these topics below and included a reference as to where the full description/discussion on this topic may be found in the report.

- System boundaries

In defining the system boundaries we followed the guidelines given in Guinée et al. (2000) which are described in the Inventory in § 4.2. The most significant choice made was to leave out the capital gciods for producing linoleum.

- Criteria for inclusion of data

Where possible in this study, we used case-specific data on processes provided by the companies were these processes take place. If this information was not complete or did not seem reliable, we used available data from other inventory studies. If case-specific information was not available, we also used other sources such as the ETH database $(E T H, 1996)$ or data from other inventory studies. More detailed choices on the inclusion of data are described in $\S 4.3$ and appendix A.

- Data quality

The quality of data is discussed in the inventory section where a description of the data used in the study is given: $\S 4.3$.

- Allocation procedures

These are described in the section of the inventory where the allocation is carried out: $\S 4.4$.

- Main assumptions

The main assumptions influencing the outcome of the study are made in the inventory phase (see also above). They are, therefore, described in chapter 4 (Inventory). These assumptions concern waste treatment (incineration or landfill, substitution of useful heat when incinerating linoleum), life span, maintenance systems etc.. Other assumptions are outlined in appendix A.

- Applicability of the study

The results of this study can be used for the application mentioned in $\S 2.2$. The study is designed to describe the functional, alternative systems mentioned above and to analyse the not too large variations on these systems. In this form, the results cannot be used for comparative assertion or for radical changes to the system (e.g. changes leading to variance in capital goods). A more thorough discussion on data quality and the resulting applicability of the results of this study may be found in $\S 6.6$.

\subsection{ISO guidelines concerning third party reporting and peer review}

Because the results of this study will probably be made public. Therefore, it has been compiled is in accordance with the ISO guidelines with respect to third party reporting. Although at present there is no comparative assertion at stake, the results may be used for this at a later stage. The study, therefore, also accords with the ISO guidelines with respect to comparative assertion and includes a peer review by an independent LCA-expert (see ISO 14040, ISO 14041, ISO 14042 and ISO 14043). This review is included in this report. 


\section{Inventory}

In the inventory analysis, data was collected and in- and outputs were attributed to the processes. Aggregating the data ultimately resulted in a list of all environmental in- and outputs in the product system namely, the inventory table. In this chapter, all basic choices made during the construction and quantification of the product system are described. The process tree and system boundaries are described in $\S 4.1$ and 4.2 ., data gathering in $\S 4.3$ allocation of data in $\S 4.4$ and the resulting inventory table in $\S 4.5$ and appendix B.

For this study, we used a new software tool currently being developed at the CML: CMLCA 2.0 (Heijungs, 2000). This software is better adapted to producing results using different inventory allocation and impact assessment methods than current commercial packages. This affords the user greater freedom in defining scenarios. Unfortunately, however, the CML package is not commercially available, hence no user-friendly guide has been developed and no helpdesk exists. While this is the case, the software is available free of charge on the CML-internet site:

http://www.leidenuniv.nl/interfac/cml/ssp/cmlca.html

\subsection{Process tree (baseline)}

In Figure 2 the process tree for the baseline scenario is depicted.

\section{Validity}

This product system is representative for a large share of the linoleum production at Forbo-Krommenie B.V. There is, however, considerable variability in product systems for different linoleum products made by Forbo-Krommenie B.V. Therefore, in $\S 6.4$ some alternative systems are analysed and discussed (see also § 3.4).

\section{Reliability}

The system is not complete because capital goods are not included. All other relevant processes were included in the system. Processes for which no data was available and for which it was not possible to make a sound estime of the process data, were included in the system as "data gaps ". In

Figure 2 these are shown by a grey fill. From some processes, only part of the data could be gathered. These processes are marked with one or more asterisks. A description appears below the figure describing what is and what is not included for those processes. More detailed information may be found in appendix $A$.

\subsection{System boundaries}

In an ideal LCA situation one would be able to trace all streams up to the level of natural resources (with no human effort yet invested in the material)emissions without any further future human interventions. This, however, commonly gives rise to a problematic, infinite historical regression. Given this then, boundaries for the system must be established.

Three types of boundaries are distinguished between:

1. the product system and the environment,

2. included and disregarded processes,

3. the product system under investigation and other product systems. 


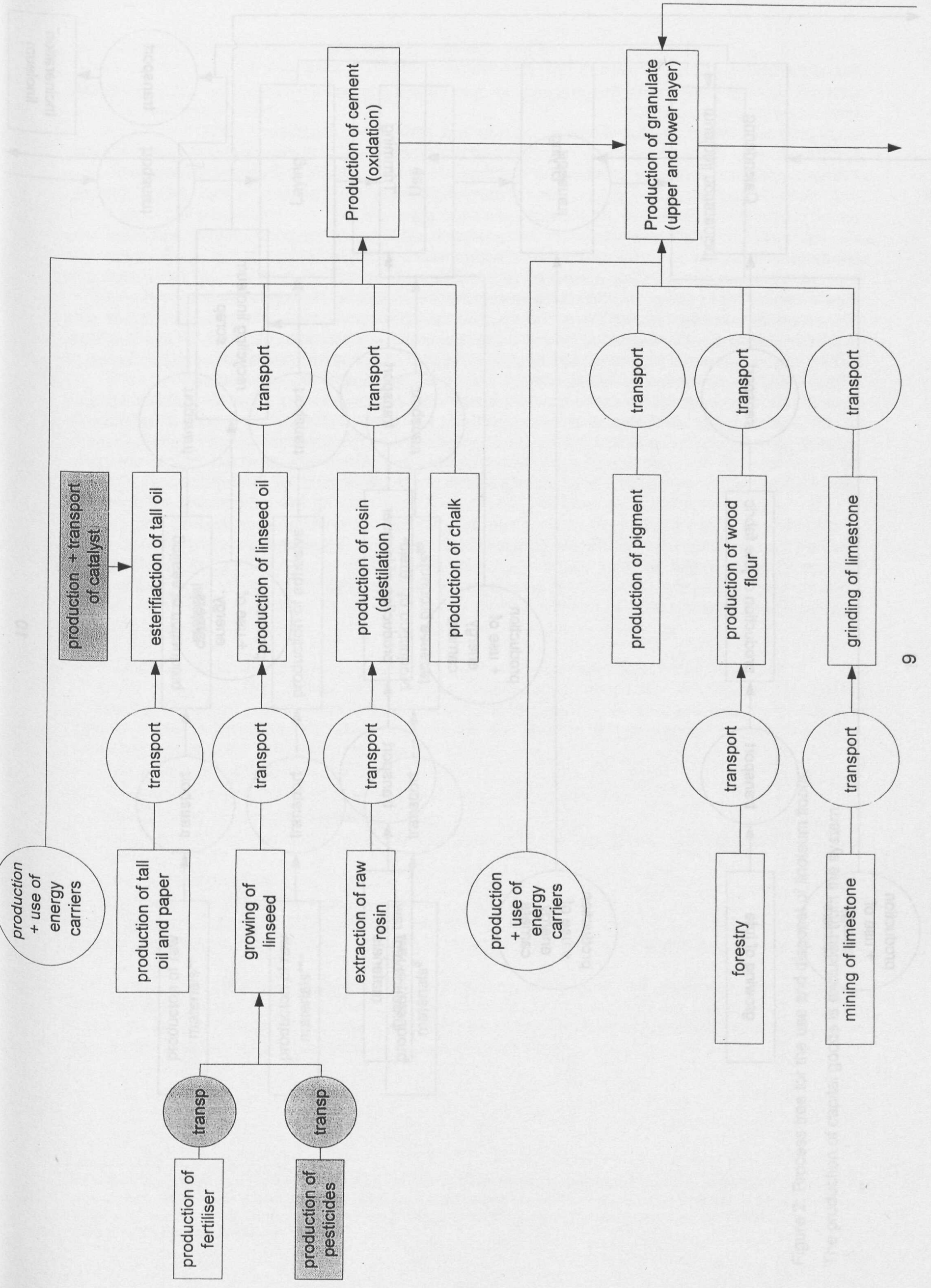




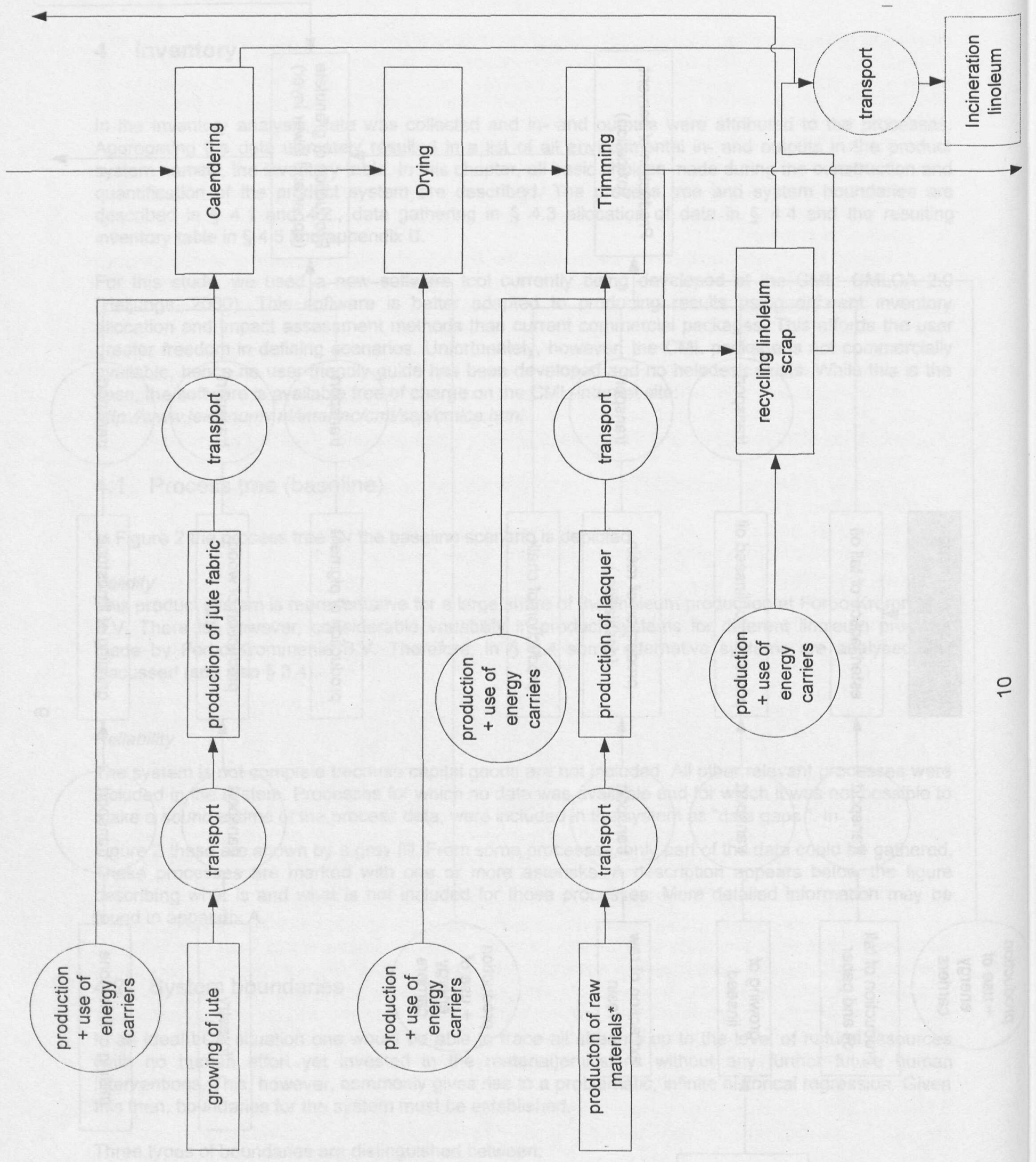



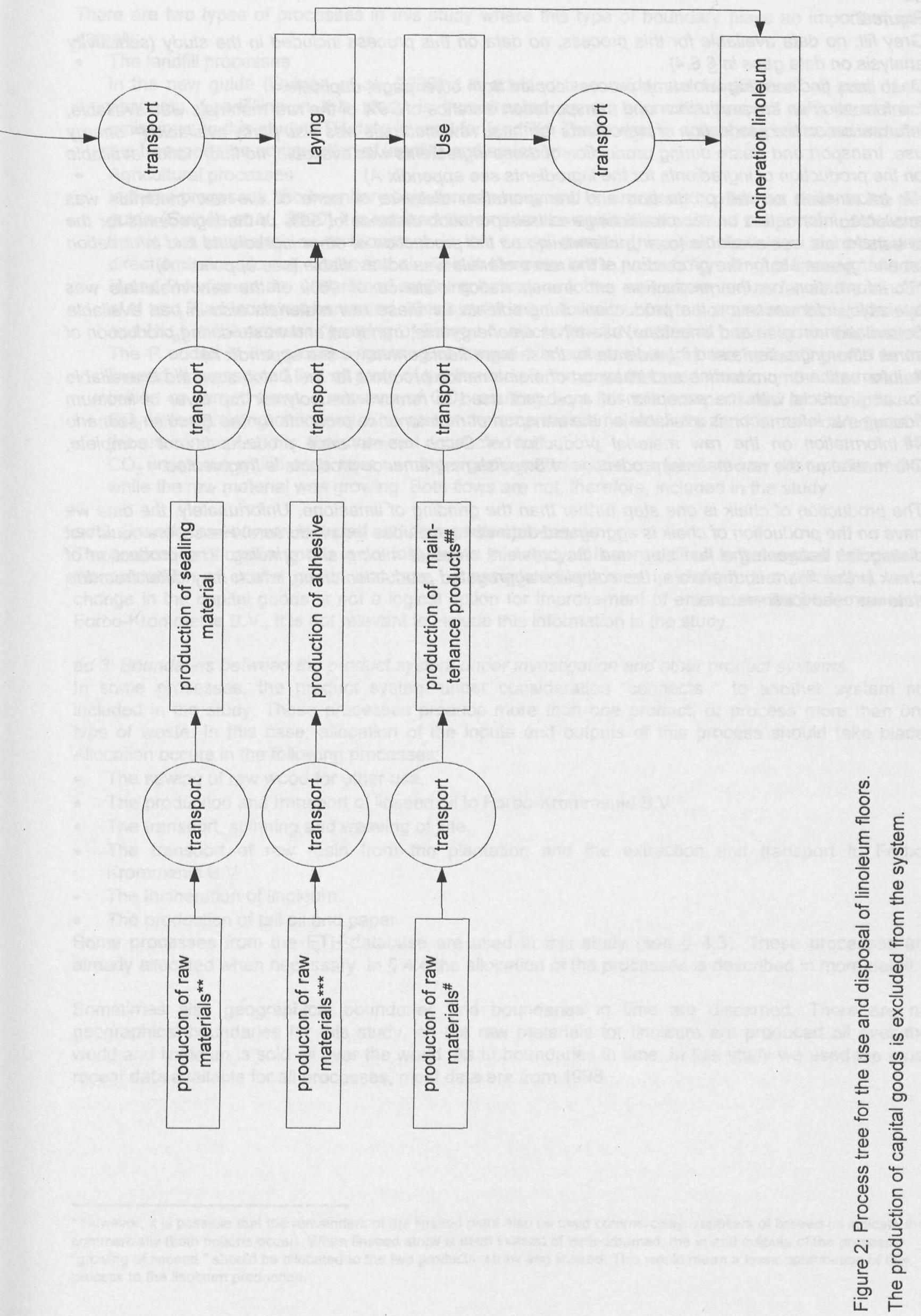
ad

Figure 2:

Grey fill: no data available for this process, no data on this process included in the study (sensitivity analysis on data gaps in §6.4).

Dash lines (incinerating linoleum): process copied from other page, duplicate.

*: information on the production and transportation distance of $96 \%$ of the raw materials was available, information on the production of ingredients for these raw materials was also partly available (= energy use, transport and waste during production of these ingredients was available, no information available on the production of ingredients for the ingredients see appendix A)

**: Information on the production and transportation distance of none of the raw materials was available, information on the production and transportation distance of $50 \%$ of the ingredients for the raw materials was available (rosin), information on the production of other ingredients and information on energy use etc for the production of the raw materials was not available (see appendix A).

***: information on the production and transportation distance of $98 \%$ of the raw materials was available, information on the production of ingredients for these raw materials also in part available (information on gum and limestone, as well as on energy use, transport and waste during production of some other ingredients and ingredients for these ingredients unknown see appendix A).

\#: information on production and transport of maintenance products for the Dutch scenario is available for all products with the exception of a product used to remove the polymer top layer of linoleum flooring. No information is available on the transport of maintenance products for the Swedish scenario \#\#:Information on the raw material production of Dutch maintenance products is not complete, information on the raw material production of Swedish maintenance products is fragmented.

The production of chalk is one step further than the grinding of limestone. Unfortunately, the data we have on the production of chalk is aggregated data which includes the precursors. Hence, we could not distinguish between the last step and the previous steps of mining and grinding. The production of chalk in this figure is, therefore, the complete aggregated production chain, which may differ from the data we used for limestone. 
ad 1: Boundaries between product system and environment

There are two types of processes in this study where this type of boundary plays an important role, namely:

- The landfill processes

In the new guide (Guinée et al., 2000) it is advised to consider the landfill itself as part of the economy. Landfilling in this study is thus treated as an economic process with inputs and emissions. In this study ETH-data is used for landfills. These data is aggregated data and includes the transport, the construction of landfills and emissions.

- Agricultural processes

In these processes, the boundary between economy and environment is often not clear. In the new guide (Guinée et al., 2000) it is advised to consider the agricultural soil and the portion of the crop that is not harvested, as environment. In this study. therefore, applied pesticides are treated as direct emissions to soil, water and air. This is the case in the process "growing of linseed ". Added $\mathrm{N}$ and $\mathrm{P}$ fertilisers are understood as being emissions to the environment in as far as the amount of $\mathrm{N}$ and $\mathrm{P}$ added is not harvested. This results in an emission of $\mathrm{N}$ to soil and into the air (as $\mathrm{N}_{2} \mathrm{O}$ and $\mathrm{NH}_{3}$ ) in the process "growing of linseed ", since not all $\mathrm{N}$ added is harvested in the linseed. The $\mathrm{P}$ added in the process "growing of linseed " is compensated by the harvesting of $\mathrm{P}$ in the linseed. It is assumed that all parts of the linseed plant except the seed remain on the land and are buried or burned there. Emissions of $\mathrm{CO}_{2}$ resulting from this are compensated by the uptake of $\mathrm{CO}_{2}$ in the growing of linseed. The part of the crop that remains on the land is considered part of the environment and is, therefore, not accounted for ${ }^{4}$.

$\mathrm{CO}_{2}$ emissions which arise from burning organic material, are compensated by the uptake of $\mathrm{CO}_{2}$ while the raw material was growing. Both flows are not, therefore, included in the study.

\section{ad 2: Boundaries between included and disregarded processes}

The production of capital goods is not included in this study. It is very difficult to get a good estimate of the production of capital goods that may be associated with one unit of product (linoleum). As a change in the capital goods is not a logical option for improvement of environmental performance at Forbo-Krommenie B.V., it is not relevant to include this information in the study.

\section{ad 3: Boundaries between the product system under investigation and other product systems}

In some processes, the product system under consideration "connects " to another system not included in the study. These processes produce more than one product, or process more than one type of waste. In this case, allocation of the inputs and outputs of this process should take place. Allocation occurs in the following processes:

- The sawing of raw wood for other use.

- The production and transport of linseed oil to Forbo-Krommenie B.V.

- The transport, spinning and weaving of jute.

- The transport of raw rosin from the plantation and the extraction and transport to ForboKrommenie B.V

- The Incineration of linoleum.

- The production of tall oil and paper

Some processes from the ETH-database are used in this study (see $\S 4.3$ ). These processes are already allocated when necessary. In $\S 4.4$ the allocation of the processes is described in more detail.

Sometimes also geographical boundaries and boundaries in time are discerned. There are no geographical boundaries for this study, as the raw materials for linoleum are produced all over the world and linoleum is sold all over the world. As to boundaries in time: In this study we used the most recent data available for all processes, most data are from 1998.

\footnotetext{
${ }^{4}$ However, it is possible that the remainders of the linseed plant also be used commercially. suppliers of linseed oil indicate that commercially (both options occur). When linseed straw is used instead of buried/burned, the in-and outputs of the process "growing of linseed " should be allocated to the two products: straw and linseed. This would mean a lower contribution of this process to the linoleum production.
} 


\subsection{Data collection and data quality}

All the economical and environmental in- and outputs are described and quantified per process. The validity (representativeness) and reliability (completeness, variability and uncertainty) of the process data are described in so far as this is possible. In the interpretation phase of the LCA, possible effects of the quality of data are analysed (see Wrisberg et al., 1999).

Data was collected by Forbo-Krommenie B.V. and CML. A description is given below of the data collected, as well as of its validity and reliability.

\subsubsection{Raw material production}

General data for the production of raw materials

Data concerning production and transport (means and distance) of most raw materials used in the Forbo-Krommenie B.V. processes was provided by Forbo-Krommenie B.V.

\section{Vvalidity}

This data was provided by Forbo-Krommenie B.V. 's suppliers and should be, therefore, representative per definition for the processes concerned. Wood flour is supplied mainly by English and German suppliers, cork by Portuguese suppliers, (all cork is produced in Portugal), jute is supplied by Indian, Dutch and French suppliers(all jute is grown in India and Bangladesh), linseed oil is supplied by Dutch and German suppliers ( all linseed is grown in Canada), gum rosin is supplied by Dutch and German suppliers(all raw rosin is produced in Indonesia).

\section{Reliability}

The data provided is not always complete. Figures on emissions resulting from the use of fossil fuels were often not provided. Therefore, we used aggregated ETH-data on the use of these fuels instead of data on the supply of these fuels. Data on some processes consists of rough estimates. Important assumptions are made for linseed: every year an insecticide and a pesticide are used on $20 \%$ of the linseed fields in the prescribed amount(based on personal communications from suppliers of linseed) $1 \%$ of the $\mathrm{N}$ applied evaporates as $\mathrm{NH}_{3}$ and $1 \%$ as $\mathrm{N}_{2} \mathrm{O}$ (based on Välimaa \& Stadig, 1998). Data on some processes is lacking. The lacking data is mentioned in $\S 4.2 \mathrm{ad} 2$, and marked in the process tree. The variability may be considerable. Transportation distance in particular can vary widely because the transport is delivered by different suppliers. Data on the amount of product produced per unit of raw material varies less. We took average values for all data on raw materials where information from more than one supplier was available. Where possible, we used weighted averages based on the percentage of the raw material that Forbo-Krommenie B.V. bought from different suppliers. The uncertainty of this data is not known.

\section{Pigments}

The aggregated data on the production of $\mathrm{TiO}_{2}$ used in the baseline study, and the yellow and red pigments used in the sensitivity analysis, are provided by PE Product Engineering, Germany (PE, 1999, pers. comm. K. Saur).

\section{Validity and reliability}

The validity and reliability of this data is unknown although it was more complete than the data provided by Forbo-Krommenie B.V 's suppliers. As this is aggregated data, all upstream processes such as energy use, production and the mining of Ti-ore etc. are included. Data on the transportation of the pigment to Forbo-Krommenie B.V. was based on the information provided by Forbo-Krommenie B.V.'s suppliers.

\section{Fertilisers}

The aggregated data on the production and transportation of fertilisers is taken from Chalmers (Davis \& Haglund, 1999). An estimate of the percentage of $\mathrm{N}$ applied as fertiliser emitted into the air is from Välimaa \& Stadig (1998). 


\section{Validity}

This data is representative for the European situation, while linseed production takes place outside Europe, in Canada. While the fertilisers used in Canada are probably comparable to those used in Europe, transportation distances might differ.

\section{Reliability}

Concerning the completeness: it is not certain that the emissions resulting form the production of energy carriers are included in the data. We assumed that this was not the case and, therefore, added the ETH-data on supplied energy carriers (oil, energy etc.). We have no information as to the variability and uncertainty of the data.

Other products used during raw material production

The aggregated data on a few specific products (chalk, paper) was derived from the ETH database (ETH, 1996).

\section{Validity}

This data is mainly representative for European situations.

\section{Reliability}

There is no exact information on the reliability of this data, however, the list of emissions and inputs per process is very complete. ETH is high-status data and is often used in LCA-studies. However, one should keep in mind that this database is primary intended as an inventory of energy production systems. The processes closely related to the production of energy are more reliable than the more remote processes, such as production of materials.

\subsubsection{Processes which take place at Forbo-Krommenie B.V.}

\section{General}

Data on processes occurring at the production site in Assendelft is provided by Forbo-Krommenie B.V. The receipt of linoleum from the three alternative product systems is indicated in Table 1.

Table 1. The receipt of linoleum 2,5 mm (Dutch system) $2.0 \mathrm{~mm}$ (Swedish system) and cork-linoleum in $\mathrm{g} / \mathrm{m}^{2}$.

\begin{tabular}{llll}
\hline & $2.5 \mathrm{~mm}$ & $2.0 \mathrm{~mm}$ & cork \\
\hline tall oil & 398 & 316 & 380 \\
gum rosin & 76 & 55 & 3 \\
linseed oil & 588 & 452 & 842 \\
wood flour & 901 & 688 & - \\
cork granulate & - & - & 1329 \\
limestone & 592 & 441 & 143 \\
pigment & 101 & 76 & 263 \\
jute & 233 & 260 & 230 \\
lacquer & 12 & 12 & 10 \\
TOTAL & 2900 & 2300 & 3200 \\
\hline
\end{tabular}

\section{Validity}

This data is per definition representative for the processes concerned.

\section{Reliability}

It is expected that the data is as complete as possible, because the authors of this study and ForboKrommenie B.V. had frequent contact. However, emissions resulting from the use of gasare probably not complete as only a limited list of substances was given. Therefore, we used aggregated ETH-data on the use of gas (including the production of this gas). The variability and uncertainty of the data given by Forbo-Krommenie B.V. is unknown. 
VOC emissions from Forbo-Krommenie B.V.

Data on the total emissions of VOCs (not methane volatile organic compounds) from the production site are provided by Forbo-Krommenie B.V. Unfortunately, the individual VOC emissions at ForboKrommenie B.V. are not measured. Only the total VOC-emission is measured. Therefore, the emission of individual substances had to be estimated, based on data from Hauschild \& Wenzel (1998) on VOC mixtures for solvent use. For other unspecified VOC (e.g. from ETH-processes) emissions, the VOC mixtures for stationary combustion from Hauschild and Wenzel (1998) are used.

\section{Valididty}

The data from Haschild \& Wenzel (1998) is probably not very representative for the specific emissions from Forbo-Krommenie B.V.'s site.

\section{Reliability}

The completeness, variability and uncertainty of the data from Hauschild \& Wenzel (1998) are unknown. However, the data seems very complete, and includes a list of great variety of individual VOCs.

\subsubsection{Laying and use}

Data on use and laying as well as on the products used during laying and use of the linoleum, is provided by Forbo-Krommenie B.V.

The maintenance sequences for the Dutch and Swedish systems are given in table 2.

Table 2. Maintenance sequences for the Dutch and Swedish system per $20 \mathrm{~m}^{2}$ year (for linoleum with a life span of 20 years).

\begin{tabular}{|c|c|c|c|}
\hline unit of operation & material used per $\mathrm{m}^{2}$ & $\begin{array}{l}\text { Dutch } \\
\text { system } \\
\text { frequency in }\end{array}$ & $\begin{array}{l}\text { Swedish } \\
\text { system } \\
\text { years use }\end{array}$ \\
\hline first cleaning & $\begin{array}{r}0.16 \text { I water } \\
1.3 e-3 \text { I cleaner }\end{array}$ & 1 & \\
\hline & 0.02 I sealer & & \\
\hline dust wiping & 0.005 wiping cloths & 5200 & 240 \\
\hline spot cleaning & $\begin{array}{r}1.7 \mathrm{e}-4 \text { I cleaner } \\
0.02 \text { I water }\end{array}$ & 5200 & \\
\hline spot s & 0.001 & 260 & \\
\hline stripping + resealing & $\begin{array}{r}0.02 \text { I stripper } \\
0.04 \text { I sealer }\end{array}$ & 6 & \\
\hline initial & 3e-3 I cleaner with wax & & \\
\hline humid moping, wax & $3 e-5$ I cleaner with wax & & 3120 \\
\hline humid mopping, detergent & $3 e-4$ I cleaner & & 1040 \\
\hline
\end{tabular}

Validity

Data on the laying and maintenance of linoleum is mainly provided by Forbo-Krommenie B.V. They are representative for laying and maintenance in public and commercial buildings.

Data on adhesives and maintenance products is provided by Forbo-Krommenie B.V.'s suppliers and should be, therefore, per definition representative for the processes concerned.

Reliability

Data on the production of laying and maintenance products is including emissions, material use and energy use. Information on the production and transportation distance of $100 \%$ of the raw materials used for sealing material is available, information on the production of ingredients for these raw materials is also available in part. Information on the production and transportation distance of $98 \%$ of 
the raw materials used for adhesives is available, information on the production of ingredients for these raw materials is also available in part. Information on production and transport of maintenance products for the Dutch scenario is available for almost all products (except for a product used to remove the old seals of a linoleum floor). Information on the production and transport of maintenance products for the Swedish scenario is not complete. Information on the raw material production of Dutch maintenance products is not complete. Information on the raw material production of Swedish maintenance products is fragmented (see appendix A). No information is available on the variability and uncertainty of the data.

\subsubsection{Incineration of linoleum}

Data on the incineration of linoleum is from TNO and provided by Forbo-Krommenie B.V. (van Loo \& de Koning, 1994). All linoleum waste from the calenders, the trimming department and final disposals is assumed to be incinerated. It is assumed that the waste that remains during laying goes to landfill. The $\mathrm{CO}_{2}$ emissions during the incineration of linoleum are not included in the study. They are compensated by the uptake of $\mathrm{CO}_{2}$ by the linseed and wood, which serve as the raw material for linoleum.

\section{Validity and reliability}

The data concerning which part of the linoleum is burned and which part is land filled is based on assumptions. Other assumptions are also possible. The data on the emissions and energy content wile incinerating linoleum is, per definition, representative for the processes concerned because the analysis and calculations carried out by TNO (well known Dutch independent research institute) are carried out on linoleum manufactured by Forbo-Krommenie B.V. The assumption made by Van Loo \& De Coning that the efficiency of energy production while incinerating linoleum can be $75 \%$, seems too high. In this study, we used a lower but still high value of $40 \%$. However, this high value is not unrealistic when we look only at the marginal change in electricity production when adding a certain amount of linoleum to an already working waste incineration.

\subsubsection{Other processes (transport, energy, landfill)}

Data on transport, by truck, boat and train, production and use of energy carriers, and landfilling is derived from the ETH database (ETH, 1996).

\section{Validity}

Electricity:

Electricity data is very detailed in the ETH database. Given this, the electricity needed for those processes which take place on Forbo-Krommenie B.V.'s site, Dutch electricity production could be taken to be representative. For other electricity use, the European average was taken. This data is representative for the European situation but perhaps not for other countries.

Other energy carriers, transport and landfill:

Data on the use of other energy carriers, and transport is representative for European situations. Their representativeness for non-European countries (India, Indonesia, and Canada) is not known. This data is mostly representative for European situations. Emissions from transport by truck in Asian countries such as India and Indonesia are possibly higher Landfill: because the trucks that are used are older and the fuel used might be different.

For all types of waste in this study, data for landfill sites from the ETH-database are used. These are aggregated data from landfill sites for inert waste, hazardous waste, chemical waste and radioactive waste. The data include the infrastructure and the long term emissions from the different types of landfill sites. These emissions are probably not very representative for emissions resulting from linoleum, or other specific wastes. However, this was the only date available. 
Reliability

Landfill:

All the data we used came from the ETH-database and is aggregated data, meaning that all processes are followed from the cradle. This means that, in the case of landfill, also the infrastructure (building of the landfill site etc.) necessary for landfilling is included. Also, longterm emissions from landfill are included.

Energy and transportation:

This cradle approach was also applied in the case of transportation processes and energy carriers. There is no exact information on the reliability of data although the list of emissions and inputs per process is very complete. ETH is high-status data and often used in LCA-studies. However, one should keep in mind that this database was created primarily as an inventory of energy production systems. The processes closely related to the production of energy are more reliable than more remote processes, such as landfilling and transport.

\subsubsection{Conclusions on data quality}

Most data on the production of raw materials is representative for the specific processes, but reliability is not known. They differ largely in completeness. Data on the processes which take place at ForboKrommenie B.V. is representative and probably more reliable (because more complete) than that on the production of raw materials. Data on the composition of the VOC emissions is lacking. The data on the incineration of linoleum is representative and seems complete. However, the assumption that electricity is produced with $40 \%$ efficiency might be somewhat high. The ETH data is probably representative and reliable for the energy data. The data on transportation processes, landfill processes and other processes is probably less representative and reliable.

In $\S 6.4$ sensitivity analysis in the form of scenarios is carried out to study the effects of changes in data for processes with a high influence on the results for which the data is less reliable.

\subsubsection{Data gaps}

No process data was available for the following processes:

- The production and transport of pesticides (the use and emission of pesticides is included).

- The production and transport of some raw materials needed for maintenance products (additives, thickeners, solvents) and virtually all of the ingredients for those materials.

- The production and transport of some raw materials needed for the production of materials used during laying (adhesives and material used to seal the seams of professionally used linoleum floors).

- The production and transport of a catalyst needed in the process "esterification of tall oil ".

- The production and transport of the fertiliser S needed in the process "growing of linseed ".

- The production and transport of the maintenance product used for removing the top layer of linoleum floor covering needed in the process "the use and maintenance of $1 \mathrm{~m} 2$ linoleum for 20 years".

In $\S 0$ a sensitivity analysis is carried out to study the effects of these datagaps.

\subsection{Multiple processes (allocation)}

There are three kinds of multiple processes, namely:

- Co-production: simultaneous production of economically valuable products, goods or services.

- Combined waste disposal: simultaneous processing of more than one stream of waste, with a negative value

- Open-loop recycling: processing a waste stream of one production process so that it can be reused in another. 
Furthermore, a combination of these multiple processes may be found, such as the production of electricity (valuable product) through a waste incinerator.

For these multiple processes, a solution has to be found by either avoiding multiple processes by dividing the process into sub-processes or by enlarging the system under investigation so that the coproducts are also involved, or by allocation (dividing the in- and outputs of the processes over the products). According to ISO 14041 (1998), the following steps have to be taken when multiple processes occur:

\section{Step 1}

If it is possible, one should try to avoid allocation by dividing the multiple processes into sub-processes or by enlarging the system under investigation so that the co-products are also included.

\section{Step 2}

If it is not possible to do so, an allocation based upon causal physical relations is preferred; e.g. the amount of mercury $(\mathrm{Hg})$ in the emission of a waste combustion laying may be attributed to every product containing mercury to be burned according to its content.

\section{Step 3}

If it is not possible to make an assessment based on a causal physical relationship, then other relations should be used such as an allocation proportional to the economical value of the products.

In $\S 4.2$ ad 3 the multiple processes for the product system under investigation have been mentioned. In this study, we followed the three ISO 14041 steps as closely as possible. In only one case an ISO step-one procedure (substitution) could be applied (see ISO 14041, 1998). Expansion of function was not applied because it could result in a very large and complex system, producing many products. It would then be difficult to identify possible improvement options for Forbo-Krommenie B.V. ISO steptwo procedures (allocation based on causal relations) were not possible (see ISO 14041, 1998). Most processes that had to be allocated involved co-production. In these cases, allocation based on causal physical relations is almost never possible. In most cases, therefore, an ISO step-three procedure was followed (see ISO 14041, 1998). Where possible, we used the economic value of the products because we feel the trigger for a production process will be the product with the highest economic value. Where this was not possible, we carried out a mass-based allocation (which is less desirable because the product with the highest mass is not automatically the one which triggers the process) or a proxy.

Allocation occurred for the following processes:

- The sawing of raw wood for other uses.

$>$ This process produces wood remainders and production wood.

$>$ In this case, the inputs and outputs of the process are partitioned between the two products, based on their economic value (step 3 procedure). The remainders form $45 \%$ of the total mass produced. The value of production wood is a factor 4.5 higher than that of the wood remainders. Therefore, $15 \%$ of the total value produced is from wood remainders.

- The production and transport of linseed oil to Forbo-Krommenie B.V.

$>$ This process produces linseed oil and linseed expellers (used as cattle fodder)

$>$ In this case, the inputs and outputs of the process are partitioned between the two products based on economic value (step 3 procedure). The value of the produced linseed expellers is not negligible. It is $43 \%$ of the total value produced.

- The transport, spinning and weaving of jute.

$>$ This process produces jute and re-usable jute waste.

$>$ In this case, all in- and outputs are allocated to the jute (step 3 procedure) because the value of the amount of jute-waste produced is almost negligible compared to the jute.

- The transport raw rosin from the plantation and the extraction and transport to Forbo-Krommenie B.V.

$>$ This process produces gum rosin and turpentine.

$>$ In this case, the inputs and outputs of the process are partitioned between the two products based on economic value (step 3 procedure). The value of the produced gum rosin is $91 \%$ of the total value produced 
- The production of tall oil and paper.

$>$ This process produces tall oil and paper

$>$ The inputs and outputs of the process are partitioned between the two products based on economic value (step 3 procedure). The value of the amount of tall oil produced is only $1 \%$ of the total produced value.

- The incineration of linoleum.

$>$ This process treats waste and produces energy.

$>$ The produced energy has to be allocated. This is done by seeing the energy as a substitute for another process, that being the production of electricity in the Netherlands (ETH-process for Dutch average electricity), with an efficiency of 0.4 (step 1 procedure).

Conclusion for six processes the in- and outputs had to be allocated, namely

- The sawing of raw wood for other use.

- The production and transport of linseed oil to Forbo-Krommenie B.V.

- The transport, spinning and weaving of jute.

- The transport of raw rosin from the plantation and the extraction and transport to ForboKrommenie B.V.

- The production of tall oil and paper .

- The incineration of linoleum.

In five cases, economic allocation was applied and in one case -the incineration of linoleum- a substitution has taken place.

\subsection{The inventory table of the baseline product system}

Data collection has resulted in a database with processes and accompanying in- and outputs. These processes are coupled based on the functional unit. An inventory table is then the result of aggregation and of scaling all data for the process tree.

In this LCA study, calculations were made using CML software. The CML package uses the matrix method to calculate the inventory table which belongs to the functional unit (ILV,1991; Heijungs, 1992).

The inventory table of the baseline product system and the two alternative equivalent systems is given in appendix B.

\footnotetext{
${ }^{5}$ In this study we did not separate the production of pulp and paper. Often these processes take place in the same factory. Therefore, the in- and-outputs in the production of paper and tall oil are allocated over the products tall-oil and paper. If pulpproduction were seen as a separate process, the allocation should take place over the products tall oil and pulp. This would mean a different allocation as the economic value of the pulp is probably much lower than that of the resulting paper leading to a higher contribution of this process to the linoleum production.
} 


\section{Impact assessment}

In the impact assessment, the potential effects of the in- and outputs are identified and characterised. For the baseline and most scenarios, the impact categories as well as characterisation and normalisation are based on Guinée et al. (2000). This is briefly described in $\S 5.1$ to 5.4 .

There are, however, other impact assessment approaches which are also applied to the baseline system as sensitivity analysis scenarios. These methods are briefly described in $\S 5.5$. Not all of these methods are in accordance with ISO standards. Methods and scenarios that do not follow the ISO standards are described in separate textboxes, so that any third party reading the report can recognise them as such. When comparing the different impact assessment methods, the approach of Guinée et al. (2000) is referred to as CML-2000. In $\S 5.6$ the environrnental profile of the baseline system is described and in $\S 5.7$ the inputs and outputs not assigned to an impact category are listed.

\subsection{Selection of impact categories}

It was first established which of the environmental problems and impact categories are taken into account. In this study, the impact categories are based on Guinée et al (2000), as presented in Table 3. Depending on the expected emissions and extractions taking place during the life cycle of linoleum floor coverings, the relevant categories were selected from this list.

Table 3. Default list of impact categories

\begin{tabular}{ll}
\hline Impact category & Spatial scope \\
\hline A. Input related categories & global \\
\hline Extraction of abiotic resources & global \\
$\begin{array}{l}\text { Extraction of biotic resources } \\
\text { Land use }\end{array}$ & local \\
subcategory: Increase of land competition & Cont/reg/local \\
subcategory: Degradation of life support functions & Cont/reg/local \\
subcategory: Degradation of biodiversity & \\
B. Output related categories & \\
\hline Climate change & Global \\
Depletion of the ozone ayer & Global \\
Human toxicity & Glob/cont/reg/loc \\
Eco-toxicity & \\
subcategory: aquatic eco-toxicity fresh water & Glob/cont/reg/loc \\
subcategory: aquatic eco-toxicity sea water & Glob/cont/reg/loc \\
subcategory: terrestrial eco-toxicity & Glob/cont/reg/loc \\
subcategory: sediment eco-toxicity fresh water & Glob/cont/reg/loc \\
subcategory: sediment eco-toxicity sea water & Glob/cont/reg/loc \\
Photo-oxidant formation & Cont/reg/loc \\
Acidification & Cont/reg/loc \\
Eutrophication & Cont/reg/loc \\
Odour & Local \\
Noise & Local \\
Radiation & Regional/local \\
Casualties & Local \\
Waste heat & Local \\
\hline
\end{tabular}

In Table 4 the impact categories considered in this study are listed. The characterisation factors for these 11 impact categories are listed in appendix $\mathrm{C}$. Below, these categories and the corresponding indicators are explained briefly. For a more detailed description and justification of the chosen 
indicators we refer to Guinée et al. (2000), in which the indicators are described according to ISO standards.

Table 4. Impact categories, methods and normalisation data for the impact assessment method according to Guinée et al. (2000)

\begin{tabular}{|c|c|c|c|}
\hline Impact category & characterisation method & unit & $\begin{array}{l}\text { Normalisation } \\
\text { data }\end{array}$ \\
\hline $\begin{array}{l}\text { A. INPUT } \\
\text { CATEGORIES }\end{array}$ & & & \\
\hline Extraction of abiotic resources & Guinée (1995) & $\mathrm{kg}$ antimony eq. & $2.20 \mathrm{E} 12$ \\
\hline \multicolumn{4}{|l|}{ B. Output related categories } \\
\hline Climate change & Houghton et al (1994 \& 1995) & $\mathrm{kg} \mathrm{CO} 2$ eq. & 2.27E13 \\
\hline Depletion of the ozone layer & WMO $(1991,1995,1998)$ & kg CFC-11 eq. & 3.61E8 \\
\hline Human toxicity & Huijbregts (1999a) & $\begin{array}{l}\mathrm{kg} \quad 1,4 \text {-dichloro- } \\
\text { benzene eq. }\end{array}$ & 1.45E13 \\
\hline Eco-toxicity & & & \\
\hline subcategory: aquatic eco-toxicity 6 & Huijbregts (1999a) & $\begin{array}{l}\mathrm{kg} \quad 1,4 \text {-dichloro- } \\
\text { benzene eq. }\end{array}$ & 7.61E13 \\
\hline subcategory: terrestrial eco-toxicity & Huijbregts (1999a) & $\begin{array}{l}\mathrm{kg} \quad 1,4 \text {-dichloro- } \\
\text { benzene eq.. }\end{array}$ & 1.13E11 \\
\hline $\begin{array}{l}\text { subcategory: sediment eco- } \\
\text { toxicity6 }\end{array}$ & Huijbregts (1999a) & $\begin{array}{l}\mathrm{kg} \quad 1,4 \text {-dichloro- } \\
\text { benzene eq. }\end{array}$ & 9.19E12 \\
\hline Photo-oxidant formation & $\begin{array}{l}\text { Derwent et al. (1998) and } \\
\text { Jenkin \& Hayman (1999) }\end{array}$ & $\mathrm{kg}$ ethylene eq. & 6.26E9 \\
\hline Acidification & Huijbregts (1999b) & $\mathrm{kg} \mathrm{SO} 2$ eq. & $6.41 \mathrm{e} 10$ \\
\hline Eutrophication & Heijungs et al. (1992) & $\mathrm{kg} \mathrm{PO}_{4}$ eq. & $1.08 \mathrm{e} 11$ \\
\hline Odour & Heijungs et al. (1992) & $\mathrm{m}^{3}$ & $4.96 \mathrm{e} 17$ \\
\hline
\end{tabular}

\section{Extraction of abiotic resources}

Problem definition:

The decrease of abiotic resources, the "dead " material resources such as iron ore, fossil fuels etc. that occur as inflows in LCA.

Indicator:

The method based on the current reserves and the rate of deaccumulation of these reserves developed by Guinée (1995).

\section{Climate change}

Problem definition:

The effect of emissions as a result of human activities on the radiative forcing (=heat radiation absorption) of the atmosphere. This, in turn, can result in adverse effects on ecosystem health, human health and material welfare. Most of these emissions enhance the radiative forcing, resulting in a rise in the earth's temperature.. This is popularly referred to as the "Greenhouse effect".

Indicator:

The Global Warming Potentials (GWPs) of the IPCC. The GWP of a substance is the ratio between the contribution to the heat radiation absorption resulting from the instantaneous release of $1 \mathrm{~kg}$ of a greenhouse gas and an equal emission of carbon dioxide $\left(\mathrm{CO}_{2}\right)$ integrated over time. (Houghton et al., 1994 \& 1995)

\footnotetext{
${ }^{6}$ In a recent update of Huibregts (1999a) that became available during the writing of this study, these subcategories are split into two fresh water and salt water. For this study we decided to use the old list of only three subcategories because using the new list would imply changing all water-emission figures in the database, which was not possible in the time left. We did use the new characterisation factors, but we added the characterisation results for fresh water and marine systems weighted in the way the old factors were weighted, based on the respective volumes of salt water and fresh water for the aquatic factors and weight of the salt water and fresh water sediment. This basically meant that the salt water compartments prevailed, because of their high factors and large volume/weight.
} 


\section{Depletion of the ozone layer}

\section{Problem definition:}

The problem of the breakdown of stratospheric ozone as a result of human emissions. Because of the thinning of the ozone layer, a larger fraction of the sun's UV-B radiation reaches the earth's surface. This can have harmful effects on human health, animal heath, terrestrial and aquatic ecosystems, biochemical cycles, as well as on materials.

Indicator:

The Ozone Depletion Potentials of the WMO. The ODP is defined as the ratio between ozone breakdown in a state of equilibrium due to annual emissions (flux in $\mathrm{kg} \cdot \mathrm{yr}^{-1}$ ) of a quantity of a substance released into the atmosphere and the breakdown of ozone in a state of equilibrium due to an equal quantity of CFC-11 (WMO, 1991, 1995, 1998).

\section{Human Toxicity:}

\section{Problem definition:}

This impact category contains the effects of toxic substances in the environment on humans.

Indicator:

The characterisation factors from Huijbregts (1999a) based on modelling the fate of toxic substances, along with exposure and risk for humans with the fate model USES-LCA.

\section{Ecotoxicity}

\section{Problem definition:}

Eco-toxicological impacts are the effects of toxic substances on aquatic, terrestrial and sediment ecosystems.

Indicator:

The characterisation factors from Huijbregts (1999a) based on modelling the fate of toxic substances, along with risk for ecosystems with the fate model USES-LCA.

\section{Photo-oxidant formation}

Problem definition:

Photo-oxidant formation is the formation of reactive substances (mainly ozone), which are injurious to human health and ecosystems, and which may damage crops.

Indicator:

The Photochemical Ozone Creation Potentials (POCPs). A POCP of a VOC is the ratio between the change in ozone concentration due to a change in the emission of that VOC and the change in ozone concentration due to a change in the emission of ethylene (Derwent et al., 1998 andJenkin \& Hayman, 1999).

\section{Acidification}

Problem definition:

Acidifying substances cause a large number of diverse impacts on soil, groundwater, surface water, organisms, ecosystems and materials (buildings). Examples are fish dying in Scandinavian lakes, forest decline and the crumbling of building materials.

Indicator:

The average European factors from Huijbregts (1999b). This method accounts for fate and regional sensitivity from ecosystems.

\section{Eutrophication}

\section{Problem definition:}

Eutrophication includes all impacts due to a too high level of macro-nutrients in the environment. Nitrogen $(N)$ and phosphorus $(P)$ are the most important eutrophicating elements. This enrichment may cause an undesirable shift in the composition of a species and an increased production of biomass within aquatic and terrestrial ecosystems. In addition, high nutrient concentrations can also make surface waters unacceptable for drinking water supply. An increased production of biomass in aquatic ecosystems may lead to low oxygen concentrations because the decomposition of this biomass needs oxygen (measured as BOD). This is also placed in the same category. 
Indicator:

The factors from Heijungs et al. (1992) based on the contribution of $\mathrm{N}$ and $\mathrm{P}$ to the average composition of aquatic organisms: $\mathrm{C}_{106} \mathrm{H}_{263} \mathrm{O}_{110} \mathrm{~N}_{16} \mathrm{P}$, which is assumed to be representative of the average composition of biomass.

\section{Odour}

Problem definition:

Odour is a problem when a given concentration of odorous substances is experienced as unpleasant. Whether or not an odour will be experienced as stench will depend on the individual exposed to it. However, above a certain emission level all individuals will experience this odour as stench.

Indicator:

The factors in Heijungs et al. (1992), based on Odour Threshold Values (OTV). The OTI of a substance is the concentration of a given substance, under defined standard conditions, at which $50 \%$ of a representative sample of the population can just detect the difference between a sample of air mixed with that substance and a sample of clean air.

\section{Other impact categories}

For most of the other categories mentioned in Table 3 the characterisation methods are not yet operational (extraction of biotic resources, land use, noise and radiation). The category casualties, noise, and waste heat are not taken into account in most LCA-studies, unless there is reason to believe that they are especially important for the study. This is not the case for the present study. These categories are very local and the results are difficult to interpret in relation to the other categories. Moreover, for these categories there is no normalisation data, so it is impossible to compare these categories to the others, because they are not in the same dimensions.

The reasoning above also holds for the impact category "odour ". However, "odour " is an important category for Forbo-Krommenie B.V. because of the typical linoleum "odour ". Therefore "odour " was included in the study. However the emissions of the VOCs which give rise to the "odour " at ForboKrommenie B.V. are not known individually. It would be necessary to have this information for each individual case in order to properly include this category in an LCA. In the present case we had to make an estimate, which makes the results for this category somewhat more uncertain than those for the other categories. In $\S 6.4 .4$ we carried out a sensitivity analysis to study the effects of this estimated VOC-composition on "odour".

\subsection{Classification and characterisation}

In this step, the in- and outputs are attributed to all relevant impact categories and the contribution to the different impact categories iscalculated based on the characterisation factors/methods chosen. All in- and outputs are taken to contribute to these categories to their potential full amount, which means that we did not account for the possible occurence of parallel impacts ( $=$ the contribution of the substance to one impact category diminishes the contribution to another) to occur. The characterisation factors used in this study are listed in appendix $\mathrm{C}$, based on the preferred methods listed in Guinée et al. (2000). The results are aggregated per impact category. This then yields in one indicator result per impact category.

\subsection{Normalisation}

In this step, the indicator result per impact category is given as a fraction of the reference contribution of a certain region or person, over a particular interval of time, to this impact category. Among these reference contributions (normalisation data) one may find the annual contribution to the impact categories under consideration per the entire world, or the Netherlands, or a world citizen. The normalisation step makes it possible to compare the contributions of the different impact categories, since they are now in the same dimensions: e.g. a fraction of the annual worldwide (or Dutch) contribution to this category. As a step towards grouping and weighting, normalisation often is indispensable. 
In this study, the results are normalised based on the contribution of the world in 1989 to abiotic depletion and the contribution of the world in 1992/1993 to the output-related categories. The last figures are based on Dutch emissions of pesticides in 1992 and other substances in 1993, extrapolated to the world level. This is the most recent normalisation data available (source: Blonk et al., 1997). Normalisation data is given in Table 4.

\subsection{Grouping and weighting}

Following normalisation, the results per impact category may be grouped or even weighted.

Grouping means that the categories are being formed, sorted and, if desired, classified. One might, for example think of classifications based upon the spatial scale of a certain environmental problem (local, regional, global), or of a classification based on a scale of relative importance. In this way, results are presented in an orderly fashion.

Weighting on the other hand, goes somewhat further. The results for a certain category are multiplied by a weighting factor. Such a factor is based on the relative importance of the particular impact category. This makes it possible to compare different categories and even add them up to get one one final LCA-result. The weighting factors may be based on different approaches. Guinée et al (2000) recommend basing these weighting factors on some form of panel method. A number of methods are available based upon the 'panel-method' through which a representative panel attributes weighting factors to the different environmental problems. However, these methods only concentrate on a part of the effect categories.

ISO 14042 (1999) does not permit weighting in cases where the results of an LCA will be used for a comparative assertion, that is, comparison of products for which the results will be used externally. Yet while this is the case for weighting, grouping is allowed in comparative assertion. Unfortunately no practical methods for grouping are yet known to the authors of this study.

Because no complete weighting set covering all relevant impact categories is available at the present time, we did not carry out a weighting for the three equivalent alternatives studied. However in order to compare the results of the method described by Guinée et al. (2000), which will, for convenience sake, be referred to as the CML 2000 with methods which combine impact assessment and weighting in one factor, we had to apply a weighting to the CML-2000 results (see further $\S 5.5$ ). We chose to weight all impact categories equally with a factor 1 with the exception of the ecotoxicological impact categories which are weighted together as one (so that each of the three ecotoxicological categories was weighted with a factor $1 / 3$ ).

\subsection{Other impact assessment methods}

To check to what extent the results of the study are influenced by the method of impact assessment that is used, 5 different impact assessment methods were compared to the method used in this study in the scenario analysis

- The Ecoindicator 95 (Goedkoop, 1995) ${ }^{7}$

- The ExternE method (EC, 1995)

- The EPS system (Steen, 1993; Steen 1996)

Impact assessment method not following the ISO standards:

- The Ecoscarcity method, two versions (Ahbe et al, 1990; Braunschweig et al, 1994)

\footnotetext{
${ }^{7}$ Recently, a new version of the ecoindicator (the ecoindicator 99) and EPS were published. However, these methods were not yet available at the moment that the database CMLCA was being set up.
} 
These methods are described briefly below. The method used in this study is referred to, for convenience, as the CML 2000 method. For a more extended description we refer the reader to the literature cited.

In the Ecoindicator 95 (Goedkoop, 1995), the impact categories are more or less similar to those in the CML 2000 method. The main difference is that, in the characterisation phase, no inter-effect weighting takes place for toxic substances (metals, pesticides and carcinogenic substances are treated separately). They are weighted against each other using a distance-to-target method. The targets are based upon a defined no-effect level, assumed at safeguard subject level.

In the ExternE method (ExternE, 1995) or the Impact Pathway Analysis, the damage of an emission is calculated using fate models and exposure-damage functions. In principle, the damages are valuated on the basis of willingness-to-pay. Although the damages could probability be calculated and presented separately from the valuation step, this is seldom done. Mostly, one set of factors is applied directly to the emission and extraction data, which is not in accordance with ISO standards.

The EPS (Steen, 1993; Steen, 1996) method is a valuation method based on safeguard subjects and a valuation based on willingness-to-pay. Although the contribution to the safeguard subjects could be calculated and presented separately from the valuation step, this is seldom done. Moreover the list of safeguard subjects is rather limited. Mostly, one set of factors is applied directly to the emission and extraction data, which is not in accordance with ISO standards. The method and the derivation of factors are not transparent.

Impact assessment methods which do not follow the ISO standards:

In the Ecoscarcity method (Ahbe et al. 1990; Braunschweig et al. 1994; Baumann, 1992) interventions are weighted against each other directly at the level of emissions and extractions, using a distance-totarget method based on (Swiss or Norwegian ) policy targets. All goals are considered equally important. One important drawback to the method is its dependence on the goals in time and country. 


\subsection{Environmental profile of the baseline system}

In Table 5 the results of the characterisation and normalisation phase are shown.

Table 5. Environmental profile of the baseline system

\begin{tabular}{|c|c|c|}
\hline categories & characterisation results & normalisation results \\
\hline $\begin{array}{l}\text { Depletion of abiotic } \\
\text { resources }\end{array}$ & $1.12 \mathrm{E}+03 \mathrm{~kg}$ antimony eq. & $5.09 \mathrm{E}-10 \mathrm{yr}$. \\
\hline $\begin{array}{l}\text { Photochemical oxidant } \\
\text { formation }\end{array}$ & $1.06 \mathrm{E}+01 \mathrm{~kg}$ ethylene eq. & 1.69E-09 yr. \\
\hline $\begin{array}{l}\text { Depletion of the ozone } \\
\text { layer }\end{array}$ & $2.06 \mathrm{E}-02^{*} \mathrm{~kg}$ CFC-11 eq. & $5.69 E-11^{*}$ yr. \\
\hline Global warming & $1.72 \mathrm{E}+04 \mathrm{~kg} \mathrm{CO} 2 \mathrm{eq}$. & $7.58 \mathrm{E}-10 \mathrm{yr}$. \\
\hline Human toxicity & 8.30E+03 kg 1,4-dichlorobenzene eq. & $5.71 E-10$ yr. \\
\hline Aquatic ecotoxicity & $8.40 \mathrm{E}+05 \mathrm{~kg}$ 1,4-dichlorobenzene eq. & $1.10 \mathrm{E}-08$ yr. \\
\hline Sediment ecotoxicity & 7.38E+05 kg 1,4-dichlorobenzene eq. & 8.07E-08 yr. \\
\hline Terrestrial ecotoxicity & $2.36 \mathrm{E}+02 \mathrm{~kg}$ 1,4-dichlorobenzene eq. & 2.09E-09 yr. \\
\hline Acidification & $8.44 E+01 \mathrm{~kg} \mathrm{SO} 2 \mathrm{eq}$ & 1.32E-09 yr. \\
\hline Eutrophication & 1.29E+01 kg PO4- eq. & 1.19E-10 yr. \\
\hline Odour & $1.59 \mathrm{E}+08 \mathrm{~m} 3$ & $3.20 \mathrm{E}-10 \mathrm{yr}$. \\
\hline
\end{tabular}

Ex $=\cdot 10^{x}$

*: These numbers would be $8 \%$ higher if the leak of HCFC-22 at the calenders at Forbo-Krommenie B.V. in 1998 were included. However, this leak is regarded in this study as an incident.

**: average of the results for the three ecotoxicity subcategories

\subsection{Inputs and outputs not assigned to an impact category}

In total 264 inputs/outputs are not assigned to an impact category. These are listed in appendix D. These are mainly from the ETH database. A large share (132 emissions) of the emissions are radioactive emissions. Since the impact category "radiation " is not included in this study (because no consensus has yet been reached on which impact assessment method to use for this category), these radioactive emissions could not be included in the impact assessment results. Also, some inputs are also not assigned to an impact category. The input of land is not included, because a method for the impact category "land-use " is still in development. For other inputs no characterisation factors have as yet been developed. 


\section{Interpretation}

In the interpretation, the results of the life cycle inventory and the life cycle impact assessment (LCIA), are summarised, analysed and discussed as a basis for conclusions, recommendations and decision making, in accordance with the goal and scope.

In ISO document 14043 (1998) it is proposed that interpretation be carried out in three steps:

- Identification of the significant issues.

- Evaluation.

- Conclusions, recommendations and reporting.

These steps were followed in the present study on linoleum floors. The first two steps are followed in a recursive process. Conclusions are based on the combined results of the two preceding steps.

The aim of the identification of significant issues is to get answers to the questions defined in the goal and scope of the study. In this study, two types of analysis were carried out:

- Contribution analysis: Significant contributions to the total result are mapped by expressing, for example, the contribution of substances, processes, life cycle stages and/or impact categories in $\%$ of the total results. The results of the contribution analysis for the baseline system are reported in $\S 6.1$.

- Anomaly Assessment: On the basis of experience, for example, unusual or remarkable deviations from expected or normal results are determined. This is a method of checking for possible errors. These errors can then be repaired by adjusting the "incorrect "input data or model choices etc. Unusual results remaining at the end of the study (ergo, which are not the result of a repairable error) which cannot be explained are reported.

After the presentation and analysis of the results for the baseline system in $\S 6.1$, the reliability and validity of the data is further checked in the evaluation which is described in the following sections. "The objectives of the evaluation are to establish and enhance the confidence in and the reliability of the result of the study " (ISO 14043, 1998). The ISO 14043 guidelines are not yet worked out in detail. ISO mentions a completeness check, a sensitivity check, a consistency check, an uncertainty analysis and an assessment of data quality as elements of the evaluation. Van den Berg et al. (1998) give a framework for quality assessment in LCA. They distinguish between validity and reliability of input (process) data and models. Validity refers to representativeness, consistency, relevancy, as well as to whether or not data and models match the scope of the study. Reliability refers to reproducibility, uncertainty, completeness, etc. The validity and reliability of the product system, data and methods is considered throughout the entire study (see, for example, the description of the product system, process data and allocation methods in $\S 4.1$ to 4.4 and the consequences of data quality in $\S 6.1 .4$ ), but conclusions on the effects of data quality can only be drawn after such an evaluation.

In $\S 6.2$ a first validity check of the total results for the baseline system is carried out by comparing the results of this study with results from other studies on linoleum floor coverings. In $\S 6.3$ and $\S 6.4$ the reliability of product system, data and methods is further checked through various forms of sensitivity analysis. In $\S 6.3$ a perturbation analysis is carried out. In this analysis, the influence of small variations on the results in each individual economic and environmental flow is analysed. The result can be used to answer the question for which flows the result is the most sensitive. These are the flows for which the ratio between change in result and change in flow are highest. In $\S 6.4$ the sensitivity of product system, data and methods is checked by means of different scenarios. Data quality is one of the reasons for carrying out some scenarios. Finally in $\S 0$. the consequences of the datagaps (completeness check) are evaluated.

Conclusions and recommendations are treated in chapter 7. 


\subsection{Contribution analysis}

In the contribution analysis, the contribution of the different stages in the production chain, processes, emissions and extractions is analysed. This analysis provides insight into the most important stages, processes and flows contributing to a certain impact category. This insight may be used in several ways. First, the knowledge may be combined with the knowledge on data quality. Clearly, the most important stages, processes and flows should be based on valid and reliable data. Important processes or flows that are based on low quality data might be analysed further in a sensitivity analysis. Second, the processes that contribute much to the impact categories are the first processes to focus on, when looking for opportunities for improvement.

\subsubsection{Impact categories}

In the last column of Table 5 , the characterisation and normalisation results are presented for all categories. The one result for ecotoxicity is the average for all 3 ecotoxicity categories. The result for ecotoxicity is very high, approximately a factor 20 higher than the result for the next "important " categories namely, acidification and oxidant formation. In most weighting methods, the weighting factors differ no more than a factor 10 . Therefore, aquatic ecotoxicity is probably the most important impact category when a combined result would be calculated based on some sort of weighting. This is not done here as no encompassing weighting method is available and ISO does not permit weighting for comparative assertion.

The result for "odour " should be considered with some care, as the emissions of VOC from ForboKrommenie B.V. were not specified. The total amount of VOC was known, but not the individual substances. To calculate the contribution of the VOC-emission to the different categories, the individual substances in this VOC-emission had to be known. These are estimated based on solvent use (source: Hauschild \& Wenzel,1998). However, it is not known if this mixture of individual VOC is representative for Forbo-Krommenie B.V.

\subsubsection{Stages}

In Figure 3, the contribution of the different life cycle stages to the category results is presented. The total of all positive contributions is set at $100 \%$.

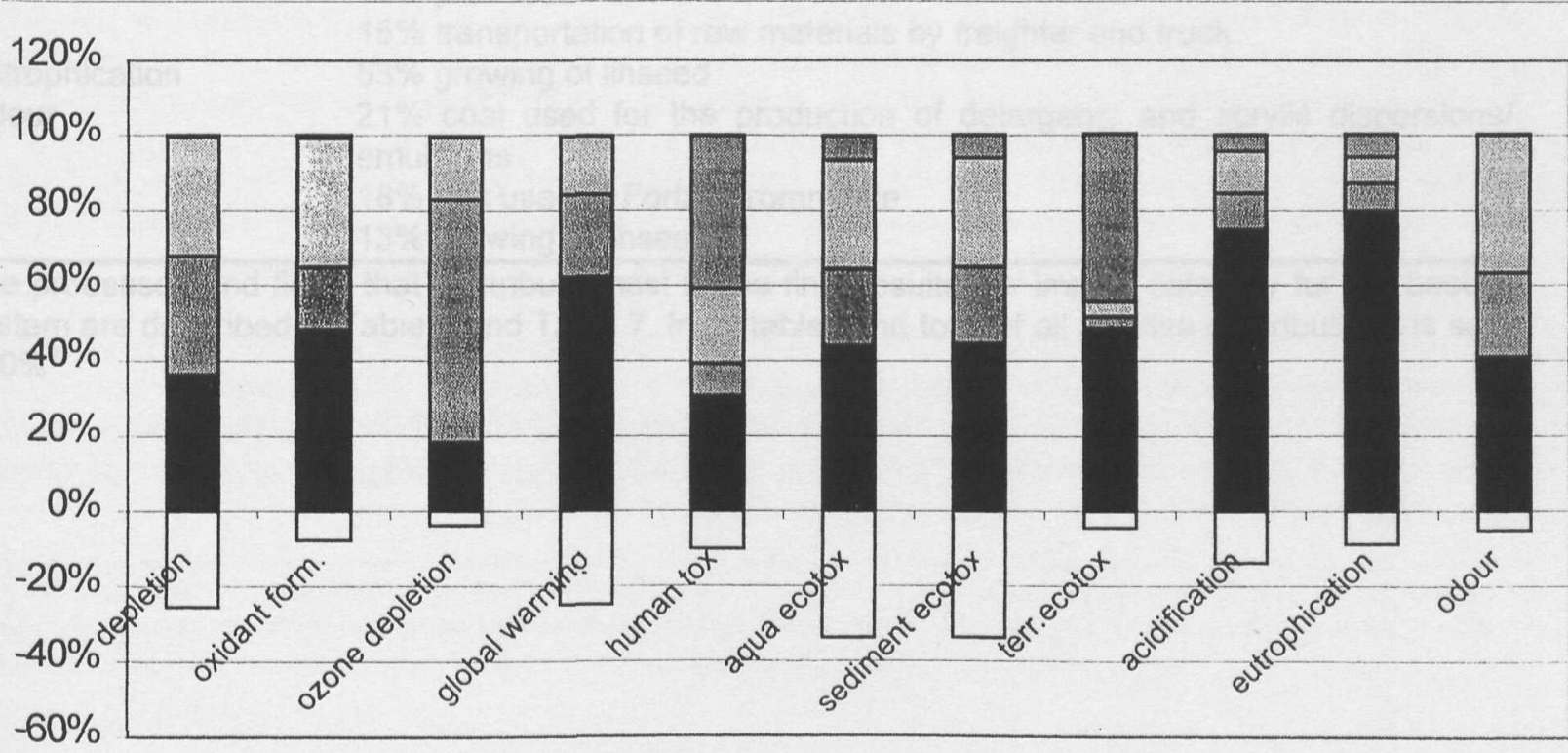

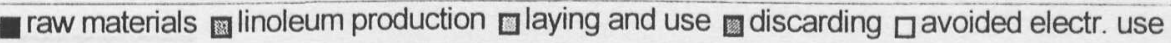

Figure 3. The contribution of life cycle stages to the category results 
- The stage "raw materials " includes all of the processes which take place for the production of raw materials (including transport) needed for the production of linoleum. These are all inputs of ForboKrommenie B.V. (materials and half-products), with the exception of energy carriers.

- The stage "linoleum production " includes all of the processes taking place at Forbo-Krommenie B.V., including processes such as steam production and seaving of cork (cork-linoleum only).

- The stage "laying and use "includes all of the processes which take place during the laying and use of the floor including transport from Forbo-Krommenie B.V. and maintenance of the floor.

- The stage "disposal "includes transport to the waste incinerator as well as the incineration of linoleum.

It is apparent that the production of raw materials is the main contributor for most categories. Exceptions to this are the category "depletion of the ozone layer ", where linoleum production is the main contributing stage $(64 \%)$ and the category "human toxicity ", where the contribution of the disposal stage is the main contributing stage $(41 \%)$. For abiotic depletion, the contribution of raw materials, linoleum production, laying and use is almost equal (resp. $36 \%, 32 \%$ and $32 \%$ ) while for "odour ", the contribution of the laying and use stages almost equals that of the raw materials stage (41\% resp. $37 \%)$.

The contribution of the disposal phase is negative for most impact categories, except for human toxicity and terrestrial ecotoxicity. This is due to the "avoided emissions ", caused by the production of useful heat when the linoleum is incinerated which is then used for electricity production. 


\subsubsection{Processes and flows}

Table 6. Main processes contributing to the environmental profile of the baseline system

\begin{tabular}{|c|c|}
\hline impact category & rocesses \\
\hline abiotic depletion & $\begin{array}{l}17 \% \text { gas used by Forbo-Krommenie } \\
15 \% \text { electricity used by Forbo-Krommenie } \\
13 \% \text { oil used for the production of maintenance products (detergents and } \\
\text { acrylic dispersions/emulsions) }\end{array}$ \\
\hline $\begin{array}{l}\text { Photochemical } \\
\text { oxidant formation }\end{array}$ & $\begin{array}{l}25 \% \text { VOC emissions due to transportation of raw material (total } 44 \% \text { VOC } \\
\text { emissions during raw material production } \\
27 \% \text { VOC emissions caused by oil use during the production of } \\
\text { maintenance products (total } 32 \% \text { VOC emissions during laying and use) }\end{array}$ \\
\hline $\begin{array}{l}\text { Depletion of the ozor } \\
\text { layer }\end{array}$ & $62 \%$ VOC emissions by the processes taking place at $F$ \\
\hline Global warming & $\begin{array}{l}37 \% \text { growing of linseed (mainly } \mathrm{N} 2 \mathrm{O} \text { emissions as a result of fertiliser use) } \\
14 \% \text { electricity used by Forbo-Krommenie B.V. } \\
7 \% \text { gas used by Forbo-Krommenie }\end{array}$ \\
\hline Human toxicity & $\begin{array}{l}40 \% \text { incineration of linoleum } \\
17 \% \text { VOC emissions during raw material production (10\% from } \\
\text { transportation by truck and freighter } \\
11 \% \text { VOC emissions caused by oil use during the production of } \\
\text { maintenance products (total } 13 \% \text { VOC emissions during laying and use) }\end{array}$ \\
\hline Aquat & $\begin{array}{l}20 \% \text { electricity used by Forbo-Krommenie B.V. } \\
12 \% \text { coal used for the production of detergents and acrylic dispersions/ } \\
\text { emulsions } \\
9 \% \text { transportation of raw materials by sea freighter }\end{array}$ \\
\hline Sediment ecotoxicity & $\begin{array}{l}19 \% \text { electricity used by Forbo-Krommenie B.V. } \\
12 \% \text { coal used for the production of detergents and acrylic dispersions/ } \\
\text { emulsions } \\
10 \% \text { transportation of raw materials by sea freighter }\end{array}$ \\
\hline $\begin{array}{l}\text { Ecotoxicity } \\
\text { terrestrial } \\
\text { Acidification }\end{array}$ & $\begin{array}{l}44 \% \text { incineration of linoleum } \\
37 \% \text { growing of linseed } \\
28 \% \text { growing of linseed }\left(\mathrm{NH}_{3}\right) \\
15 \% \text { production of } \mathrm{TiO} 2 \\
15 \% \text { transportation of raw materials by freighter and truck }\end{array}$ \\
\hline $\begin{array}{l}\text { Eutrophication } \\
\text { Odour }\end{array}$ & $\begin{array}{l}53 \% \text { growing of linseed } \\
21 \% \text { coal used for the production of detergents and acrylic dispersions/ } \\
\text { emulsions } \\
18 \% \text { gas used at Forbo-Krommenie } \\
13 \% \text { growing of linseed }\end{array}$ \\
\hline
\end{tabular}

The processes and flows that contribute most to the final results per impact category for the baseline system are described in Table 6 and Table 7 . In all tables, the total of all positive contributions is set at $100 \%{ }^{8}$.

\footnotetext{
${ }^{8}$. This then excludes the negative contributions of avoided energy use through the production of useful heat during the incineration of linoleum.
} 
Table 7. Main flows contributing to the environmental profile of the baseline system

\begin{tabular}{ll}
\hline impact category & flows \\
\hline abiotic depletion & $50 \%$ crude oil \\
Photochemical & $45 \%$ natural gas \\
oxidant formation & $32 \%$ ethylene to air \\
Depletion of the ozone layer & $18 \%$ formaldehyde to air \\
& $62 \% 1,1,1$-trichloroethane to air \\
Global warming & $35 \% \mathrm{HALON}-1301$ to air \\
& $56 \% \mathrm{~N} 2 \mathrm{O}$ to air \\
Human toxicity & $41 \% \mathrm{CO} 2$ to air \\
& $34 \%$ benzene to air \\
Aquatic Ecotoxicity & $30 \%$ arsenic to air \\
& $10 \%$ cadmium to air \\
Sediment ecotoxicity & $34 \%$ vanadium to air \\
Ecotoxicity terrestrial & $32 \%$ barium to water \\
Acidification & $36 \%$ vanadium to air \\
& $31 \%$ barium to water \\
Eutrophication & $38 \%$ mercury to air \\
Odour & $27 \%$ trichlorfon to soil \\
\hline
\end{tabular}

Table 6 shows that a limited number of processes is responsible for the highest contributions to most impact categories. In Table 8, these main contributing processes are viewed in detail. Again, the total of all positive contributions is set at $100 \%$. From the close resemblance between the percentages in both columns it can be seen that, for all these processes, a limited set of emissions is responsible for the high score of an impact category.

The results for "odour " should be considered with care. The special "odour " related to linoleum , perceptible at Forbo-Krommenie B.V. 's site, is not included in this study. Emissions of VOC during the oxidation of cement and the drying of linoleum cause this "odour ". Unfortunately, the individual VOC emissions at Forbo-Krommenie B.V. are not measured. Only the total VOC-emission is measured. Therefore, the emission of individual substances had to be estimated based on a standard emission profile which itself was based on solvent use. Apparently, this estimate does not match the real VOC mix very closely, as $\mathrm{H} 2 \mathrm{~S}$ emitted during the production of gas is cited as the main cause of "odour " for the life cycle stage "production of linoleum ". The emissions of VOC at Forbo-Krommenie B.V. are responsible for only $2 \%$ of the total result for "odour ", which seems unlikely. Therefore, we may conclude that "odour " is probably underestimated by using this standard emission profile.

The results for "depletion of the ozone layer " are probably overestimated because of the use of this standard emission profile. $62 \%$ of the ozone depletion is caused by VOC-emissions from ForboKrommenie B.V. (see Table 6). This is entirely the result of the emission of 1,1,1-trichlorethane (see Table 7). This is a solvent which is part of the standard emission profile, but which is not used at Forbo-Krommenie B.V. 
Table 8. Processes contributing more than $10 \%$ to more than one category.

\begin{tabular}{|c|c|c|c|c|}
\hline process & $\begin{array}{l}\text { categories the process } \\
\text { contributes to }\end{array}$ & $\%$ & mainly caused by & $\%$ \\
\hline \multicolumn{5}{|c|}{ The growing of linseed } \\
\hline & eutrophication & 53 & $\mathrm{NH} 3$ & 53 \\
\hline & terrestrial ecotoxicity & 37 & pesticides & 37 \\
\hline & global warming & 37 & $\mathrm{~N} 20$ & 37 \\
\hline & acidification & 28 & $\mathrm{NH} 3$ & 28 \\
\hline & odour & 13 & NH3 & 13 \\
\hline \multicolumn{5}{|c|}{ gas and electricity use at Forbo-Krommenie B.V. } \\
\hline & abiotic depletion & 32 & natural gas & 28 \\
\hline & global warming & 21 & $\mathrm{CO} 2$ & 21 \\
\hline & aquatic ecotoxicity (only electr.) & 20 & $\begin{array}{l}\text { heavy metals } \\
\text { (mainly barium } \\
\text { and vanadium) }\end{array}$ & 18 \\
\hline & sediment ecotoxicity (only electr.) & 19 & $\begin{array}{l}\text { heavy metals } \\
\text { (mainly barium } \\
\text { and vanadium) }\end{array}$ & 19 \\
\hline & odour (only gas) & 18 & $\mathrm{H} 2 \mathrm{~S}$ & 17 \\
\hline \multicolumn{5}{|c|}{ Oil used for the production of maintenance products } \\
\hline & photo. oxidant formation & 27 & VOC-mix* & 27 \\
\hline & abiotic depletion & 13 & crude oil & 13 \\
\hline & human toxicity & 11 & VOC-mix* & 11 \\
\hline \multicolumn{5}{|c|}{ Transportation of raw materials } \\
\hline & oxidant formation & 25 & VOC-mix* & 25 \\
\hline & aquatic ecotoxicity (only freighter) & 9 & $\begin{array}{l}\text { heavy metals } \\
\text { (mainly vanadium) }\end{array}$ & 9 \\
\hline & sediment ecotoxicity (only freighter) & 10 & $\begin{array}{l}\text { heavy metals } \\
\text { (mainly vanadium) }\end{array}$ & 10 \\
\hline & human & 10 & VOC-mix* & 10 \\
\hline & acidification & 15 & $\mathrm{SO} 2$ & 9 \\
\hline \multicolumn{5}{|c|}{ Incineration of linoleum } \\
\hline & terrestrial ecotoxicity & 44 & $\begin{array}{l}\text { heavy metals } \\
\text { (mainly mercurv) }\end{array}$ & 44 \\
\hline & human toxicity & 40 & $\begin{array}{l}\text { heavy metals } \\
\text { (mainly cadmium } \\
\text { and arsenic }\end{array}$ & 39 \\
\hline \multicolumn{5}{|c|}{$\begin{array}{l}\text { coal used for the production of detergents and acrylic } \\
\text { dispersions/ emulsions }\end{array}$} \\
\hline & odour & 21 & $\mathrm{H} 2 \mathrm{~S}$ & 21 \\
\hline & aquatic ecotoxicity & 12 & $\begin{array}{l}\text { heavy metals } \\
\text { (mainly barium } \\
\text { and vanadium) }\end{array}$ & 12 \\
\hline & sediment ecotoxicity & 12 & $\begin{array}{l}\text { heavy metals } \\
\text { (mainly barium } \\
\text { and vanadium) }\end{array}$ & 12 \\
\hline
\end{tabular}

\footnotetext{
*: VOC emissions are usually given as a total the VOC-mix. However, for the impact assessment, individual substances are needed. A standard emission profile representative for stationary combustion (Hauschild \& Wenzel, 1998) is used to estimate which individual substances are present in this mix (see also § 4.3.2).
}

Some remarks are also in order as to the high contribution of heavy metals to the categories "human toxicity " and "ecotoxicity". The high contribution of persistent metals is not always in accordance with the expectations based on knowledge of present toxic risk effects. This is not due to faults in the 
characterisation factors for heavy metals. The multimedia model used to calculate toxicity factors is based on state-of-the-art knowledge concerning the fate of substances. It is a result of a general starting point in Guinée et al. (2000), that being that in an LCA, all effects now and in the future are taken into account. In other words: all methods used for effect assessment, are where possible, based on a time horizon of eternity. Because heavy metals are very persistent in the environment, since they do not break down as organic substances do, their contribution to toxicity themes, especially aquatic and sediment ecotoxicity, is very high. Therefore, the results should be considered with some care. It should be kept in mind that the high score for metals is a result of taking future toxic risks into account. If these future risks are evaluated as being lower than the present risks, the contribution of persistent substances would also be lower in the future. For this study, this would mean that the influence of the more degradable but very toxic pesticides would increase relatively.

\subsubsection{Important processes and the consequences of data quality}

If the most important processes are known, the consequences of data quality should be considered. The main question is: Is the data which underlies these processes valid and reliable?

In the stage "production of raw materials ", the growing of linseed and transport are important processes. The growing of linseed is overall a very important process contributing greatly to more than 5 impact categories. The data for this process is valid because it is representative. Its reliability, however, may be disputed. The emissions of $\mathrm{NH}_{3}, \mathrm{~N}_{2} \mathrm{O}$ and pesticides both depend largely on assumptions. The emission of $\mathrm{NH}_{3}$ and $\mathrm{N}_{2} \mathrm{O}$ are based on the assumption that $1 \%$ of applied fertiliser evaporates as $\mathrm{NH}_{3}$ and that $1 \%$ as $\mathrm{N}_{2} \mathrm{O}$ goes into the air (Välimaa \& Stadig, 1998). The emission of pesticides is based on the assumption that on $20 \%$ of the fields, one herbicide and one pesticide is used. This information is based on communications with a supplier. If these assumptions are not realistic, influences on the result may be great. Therefore, a sensitivity analysis of fertiliser and pesticide use is carried out in $\S 6.4 .4$.

In the stage "production of linoleum ", the energy used at Forbo-Krommenie B.V. is most important. We expect that this data is valid and reliable. Therefore, these processes are not analysed further in the sensitivity analysis.

In the stage "laying and use ", the coal and oil used during the production of maintenance products is important. Data on the production of maintenance products is not complete. Therefore, in the scenario analysis, maintenance options are analysed in greater depth (see $§ 6.4 .1$ ).

In the stage "disposal ", the emissions produced during incineration are important. However, the figure of $100 \%$ incineration of used linoleum and the linoleum waste from Forbo-Krommenie B.V., is only an assumption. Therefore, a sensitivity analysis has been carried out in which the waste is not incinerated but landfilled (see 6.4.3)

\subsection{Comparison with previous LCA-studies on linoleum}

The comparison of the results from this study with those of other studies on linoleum floor coverings, serves as a first validity check of the total results for the baseline system.

Three other LCA-studies on linoleum floorings were known at the time this report was being written:

- Potting \& Blok carried out an LCA-study, in which four different types of floor covering including linoleum were compared (Potting \& Blok, 1993, 1995).

- Jönsson et al. (1995) carried out an LCA-study in which three different types of floor covering including linoleum were compared. However, they did not calculate the contributions to impact categories but, rather compared the alternatives chiefly on the basis of inventory data. A large part of the data they used was based on Potting and Blok (1993).

- Günther and Langowski (1997) carried out an LCA-study, in which seven different types of floor covering, including linoleum, were compared. However, from this most recent study, only very few 
results for linoleum are presented. No information concerning the contribution of processes or stages is presented, only the total results of the whole life cycle of linoleum are presented and compared to other floor coverings. Also, only a small selection of impact assessment categories is presented (no human toxicity or ecotoxicity, no oxidant formation, no "odour "). This makes the results of this study less useful for comparison.

Below, the results of our study are, where possible, compared to results from other LCA-studies. If a study is not mentioned, no data were available on the subject in question.

\subsubsection{Abiotic depletion}

In this study, the contribution of raw material production is the highest $(36 \%)$, followed by the contribution of linoleum laying and use (32\%), and production of linoleum (32\%). However, the main contributing processes are the use of electricity and gas at Forbo-Krommenie B.V.

Potting \& Blok $(1993,1995)$ state that of the use of primal energy carriers ( $\approx$ abiotic depletion) $66 \%$ is required for linoleum production and $20 \%$ for the production of pigment (TiO2). In the study of Jöhnsson et al. (1995), linoleum production is also responsible for the major portion of electricity use of and for the use of fossil fuels (respectively $44 \%$ and $67 \%$ ). Also, in their study, the electricity used for the production of $\mathrm{TiO} 2$ is high: $30 \%$.

The higher contribution of the other stages - raw material production and laying and use - in our study can be explained by two factors:

- The estimate of the energy used at Forbo-Krommenie B.V. in this study is lower than that which appears in other studies. In this study, the electricity used at Forbo-Krommenie per $1 \mathrm{~m}^{2}$ laid linoleum is approximately $8 \mathrm{MJ}$. Potting and Blok give a value of $15 \mathrm{MJ}$ and Jöhnsson et al.16 MJ.

- In our study, we gathered more data, especially on the laying and use phase.

Likewise, the contribution of the production and transport of $\mathrm{TiO} 2$ is much lower (only $5 \%$ ) in our study. We used other data on the production of TiO2 than Potting \& Blok and Jöhnsson. According to the supplier of our data on $\mathrm{TiO} 2$, the production of $\mathrm{TiO} 2$ has a relatively low energy need (pers. comm. Dr. Saur, PE).

\subsubsection{Eutrophication}

In this study, the growing of linseed is responsible for $53 \%$ of eutrophication. This is in accordance with Potting and Blok, who state that the eutrophication result is almost completely caused by the growing of linseed.

\subsubsection{Acidification}

In this study, $28 \%$ of the acidification is caused by the growing of linseed, $15 \%$ by the production of $\mathrm{TiO} 2$ and $15 \%$ by the transport of raw materials. Potting and Blok state that $44 \%$ is caused by transport (a figure which includes the transport of raw materials only) and $30 \%$ by the production of linoleum. Jöhnsson et al. state that the emissions of $\mathrm{SO}$ and $\mathrm{NO}_{x}$ are caused for a great part by transport (62\% and $31 \%)$.

One explanation for the high contribution of linseed growing in our study is the assumption that $1 \%$ of the applied $\mathrm{N}$-fertiliser evaporates as $\mathrm{NH}_{3}$. In the other studies, no emissions of $\mathrm{NH}_{3}$ are produced as no emission of $\mathrm{NH}_{3}$ is found in the inventory tables. 


\subsubsection{Photochemical oxidant formation}

In this study $27 \%$ of the photochemical oxidant formation is caused by VOC emissions resulting from oil use when producing maintenance products, and $25 \%$ is caused by VOC emissions resulting from transport by truck and freighter.

Potting and Blok found that $70 \%$ was caused by the VOC emission during the production of linoleum, and $15 \%$ by the incineration of linoleum. Jöhnsson et al. also found that $87 \%$ of the VOC emissions are a result of linoleum production, however, they based their data mainly on Potting and Blok.

In our study, the contribution of linoleum production to total VOC emissions is only $22 \%$. The total amount of VOC emitted per $\mathrm{m}^{2}$ laid linoleum by the factory at Krommenie issomewhat lower $(2 \mathrm{~g})$ in our study than in those of Potting and Blok, and Jöhnsson (both $5 \mathrm{~g}$ ). However, the total emitted amount is higher in our study: $19 \mathrm{~g}$ in our study, $7 \mathrm{~g}$ in Potting and Blok and $6 \mathrm{~g}$ in Jöhnsson respectively.

Since our data on VOC emissions comes directly from Forbo-Krommenie B.V., while the data presented in other studies comes mainly from the environmental permits, our data is more realistic. Also, the data on the other stages in our study appears to be more complete.

\subsubsection{Depletion of the ozone layer}

In this study, we recorded a relatively low score for ozone depletion compared to the other impact categories. Potting and Blok, and Jöhnsson et al. assumed no ozone depleting emissions.

\subsubsection{Global warming}

In this study, we found that $37 \%$ of the global warming is caused by the growing of linseed and $21 \%$ by the use of electricity and gas during linoleum production.

Potting and Blok found that $66 \%$ was caused by the production of linoleum and $20 \%$ by the production of pigment (TiO2). Also in Jöhnsson et al. linoleum production was responsible for $58 \%$ of $\mathrm{CO}_{2}$ emissions.

One explanation for the high contribution of linseed growing in our study is the assumption that $1 \%$ of the applied $\mathrm{N}$-fertiliser evaporates as $\mathrm{N}_{2} \mathrm{O}$. In the other studies, no emissions of $\mathrm{N}_{2} \mathrm{O}$ take place (no emission of $\mathrm{N}_{2} \mathrm{O}$ are found in the inventory tables). The contribution of the production of $\mathrm{TiO}_{2}$ in our study is only $4 \%$.

\subsubsection{Ecotoxicity}

In this study, we found a relatively high contribution for ecotoxicity. Potting and Blok, and Jöhnsson et al. found almost no emissions to water. Potting and Blok mention the use of pesticides for the growing of linseed, but were not able to quantify the emissions.

\subsubsection{Human Toxicity}

In this study, human toxicity is mainly caused by emissions of heavy metals and benzene as a result of the incineration of linoleum as well as of VOC emissions which occur during the production of raw materials. Potting and Blok present an impact category "spoiled air " ( $\approx$ human toxicity by air emission). The result for this category is mainly caused by the emission of dust during production of limestone. They also mention that the contribution of pesticides could not be quantified properly, but is probably considerable. In our study, however, the influence of pesticide use on human toxicity is relatively low $(<1 \%)$. 


\subsubsection{Odour}

Odour is not mentioned in other studies, so no comparison could be made.

\subsubsection{Conclusions}

When compared with the other studies, more data was compiled here on life cycle stages, such as raw material production, use and maintenance (e.g. emissions which ocuur during the production of linseed or in the production of maintenance products). This resulted in a relatively large contribution of these life cycle stages to the final environmental profile. However, it should be kept in mind that these data were not validated or made public.

Furthermore, we used emission data directly provide by Forbo-Krommenie B.V. These values were lower than the values from the environmental permit for the factory in Assendelft which were used in the other studies. The lower values are the result of improvements made by Forbo-Krommenie B.V. after the submission of the permit. For instance, lacquers with less solvents are now used and more VOCs are removed by the incinerators. The data provided by Forbo-Krommenie B.V. is more realistic and, therefore, the lower contribution of the lifecycle stage "production of linoleum " is also more realistic than that offered in the other studies. This data is amongst others reported in environmental reports and in reports by Central Statistics Netherlands.

\subsection{Perturbation analysis}

In the perturbation analysis, the influence of a variation in each economic flow on the final result is calculated using matrix techniques. This may provide insight into which processes have a more than proportional effect on the end result. These processes could then become the focal point for further sensitivity analysis. In table 2, the results of the perturbation analysis are presented. The factor is the ratio between the change in the flow and the change in the result. If this factor is higher than 1 for a given flow, this means that a change in the flow concerned results in a more than proportional change in the result.

Table 9 shows that "laid linoleum " is the most important economic flow for all impact categories. For global warming, and aquatic and sediment ecotoxicity, a change in this flow results in a more than $10 \%$ higher change in the final category result. However, since this economic flow $\left(2000 \mathrm{~m}^{2}\right)$ is directly coupled to the functional unit $\left(2000 \mathrm{~m}^{2} \cdot 20\right.$ years), there is no uncertainty concerning this flow. Therefore, the flow is not evaluated in the uncertainty analysis based on scenarios in $\S 6.4$. It is concluded that no environmental flow has more than a proportional effect on the final results per impact category. 
Table 9. perturbation analysis

\begin{tabular}{|c|c|c|c|c|}
\hline category & economic flow & $\begin{array}{l}\text { ratio } \\
\text { change } \\
\text { flowl } \\
\text { change } \\
\text { result }\end{array}$ & environmental flow & $\begin{array}{l}\text { ratio } \\
\text { change } \\
\text { flow/ } \\
\text { change } \\
\text { result }\end{array}$ \\
\hline abiotic depletion & laid linoleum & 1.03 & none above 1 & - \\
\hline photo. oxidant formation & none above 1 & - & none above 1 & - \\
\hline $\begin{array}{l}\text { depletion of the ozone } \\
\text { layer }\end{array}$ & none above 1 & - & none above 1 & - \\
\hline \multirow{4}{*}{ global warming } & laid linoleum & 1.18 & none above 1 & - \\
\hline & $2.5 \mathrm{~mm}$ linoleum packed & 1.09 & & \\
\hline & $2.5 \mathrm{~mm}$ linoleum trimmed & 1.06 & & \\
\hline & $2.5 \mathrm{~mm}$ linoleum dried & 1.03 & & \\
\hline human toxicity & none above 1 & & none above 1 & - \\
\hline aquatic ecotoxicity & laid linoleum & 1.12 & none above 1 & - \\
\hline sediment ecotoxicity & laid linoleum & 1.12 & none above 1 & - \\
\hline terrestrial ecotoxicity & none above 1 & & none above 1 & - \\
\hline acidification & laid linoleum & 1.02 & none above 1 & - \\
\hline eutrophication & none above 1 & & none above 1 & \\
\hline odour & none above 1 & - & none above 1 & - \\
\hline
\end{tabular}




\subsection{Scenarios}

Scenario analysis is used to verify what percentage of the results for the baseline system is influenced by differences in system, processes or process data, or by differences in methods.

\subsubsection{Alternative systems/countries}

In $\S 3.4$, we defined two functionally equivalent alternatives along with the baseline system. One of these systems is the Swedish system. Analysing this system provides insight into the effects of different maintenance systems, different gauges and different transportation distances for the linoleum. To evaluate the influence of transportation distances, the USA scenario is introduced.

In Figure 4, the results for three alternative scenarios related to the country of use are presented.

- The Swedish scenario: in Sweden, a thinner gauge of $2.0 \mathrm{~mm}$ is frequently used in public buildings. Because of the different climate in Sweden, a different maintenance system is also used. This system is based on wax rather than on stripping and on adding a new polymer top layer as is the case in the Netherlands.

- The $2.0 \mathrm{~mm}$ system NL is the $2.0 \mathrm{~mm}$ gauge used in the Netherlands, using the Dutch maintenance system.

- The scenario for the US is the same as the baseline system, with an additional transport distance (transport to and in the USA).

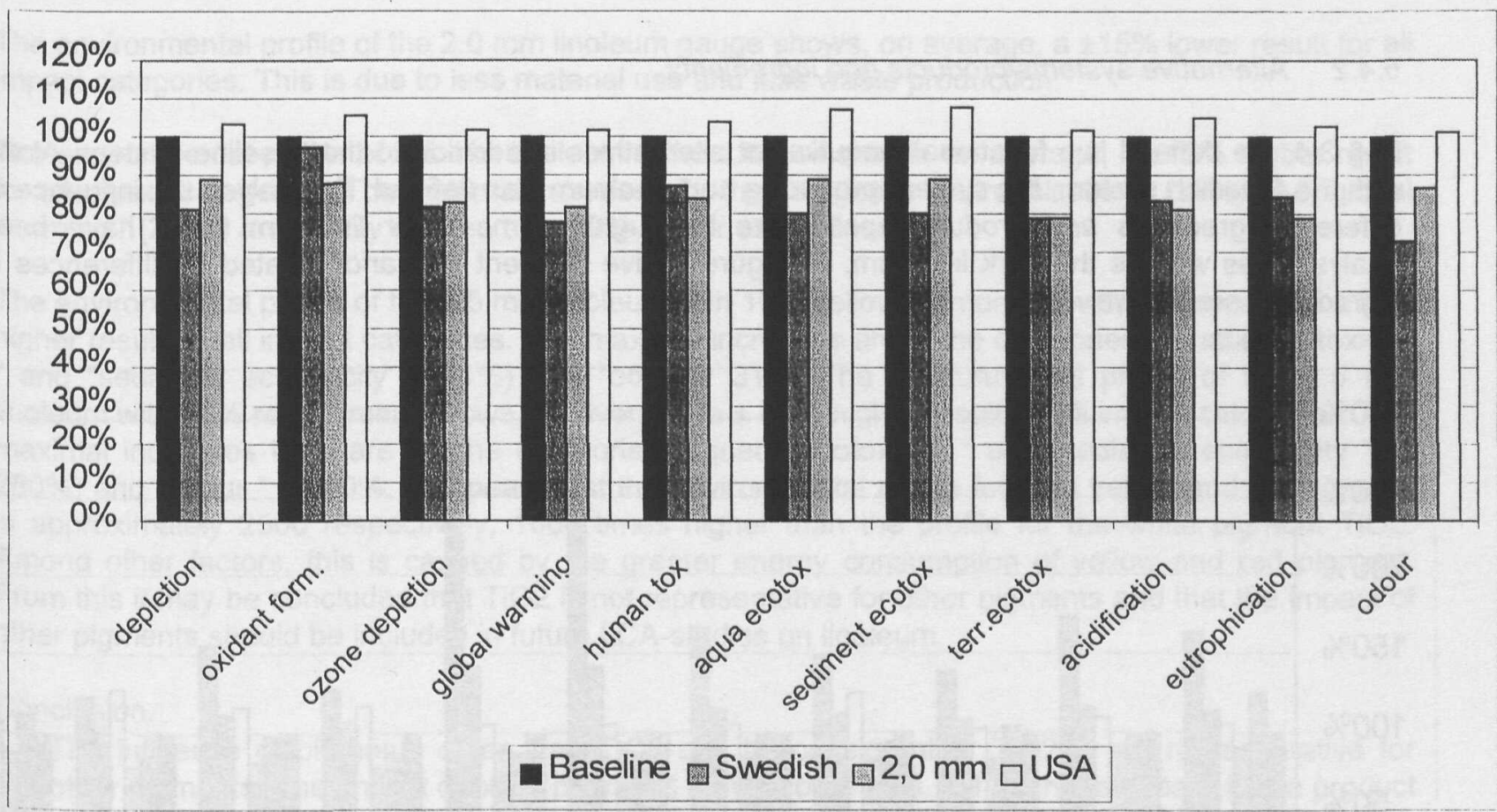

Figure 4 . Environmental profile for the baseline system and three alternative scenarios related to the country of use.

This figure would indicate that despite the longer transportation distances, the Swedish system yields lower results $(3-27 \%)$ than the baseline. This is caused by the lower contribution of the Swedish maintenance system and the fact that less material is needed for the gauge $2.0 \mathrm{~mm}$. However, the lower contribution of the Swedish maintenance system is at least partly the result of lack of data. Data on the emissions and use of energy during the production of maintenance products is less complete than for the Dutch system. 
The $2.0 \mathrm{~mm}$ gauge used in the Netherlands scores lower for oxidant formation, human toxicity, terrestrial ecotoxicity, acidification and eutrophication. For the other categories, however, it scores higher than the Swedish scenario. This means that maintenance has more influence than transportation distance for those categories. It appears that the environmental profile (the list of the results for all impact categories) for the Swedish maintenance system is indeed $80-90 \%$ lower than for the Dutch maintenance system.

This is at least partly a result of data gaps for the production of maintenance products for the Swedish method. It followed from the contribution analysis that the oil and coal consumed during the production of maintenance products contributes considerably to human toxicity, ecotoxicity, abiotic depletion and photo oxidant formation. Although the validity and reliability of the ETH data used for these processes can be questioned, it at least indicates that the contribution of the production processes for maintenance products are not negligible. Based on this study, no other conclusions can be drawn concerning the difference between Dutch and Swedish maintenance systems.

The results for the USA scenario are higher for every impact category, however, not much higher. The maximal increase in ecotoxicity was found in sediment ecotoxicity, which was $8 \%$ higher for the USA scenario as compared to the baseline scenario.

Conclusion :

- The extra transport by freighter to the USA has less impact than was expected.

- The influence of maintenance is not negligible, as is often thought.

\subsubsection{Alternative systems/products and ingredients}

In $\S 3.4$, we defined two functionally equivalent alternatives in addition to the baseline system. Along with the Swedish system, the system producing cork linoleum was defined. To analyse the influence of different ingredients and product types more thoroughly some other linoleum types have been analysed, as well as the cork linoleum. In Figure 5, five different scenarios related to differences in ingredients are shown.

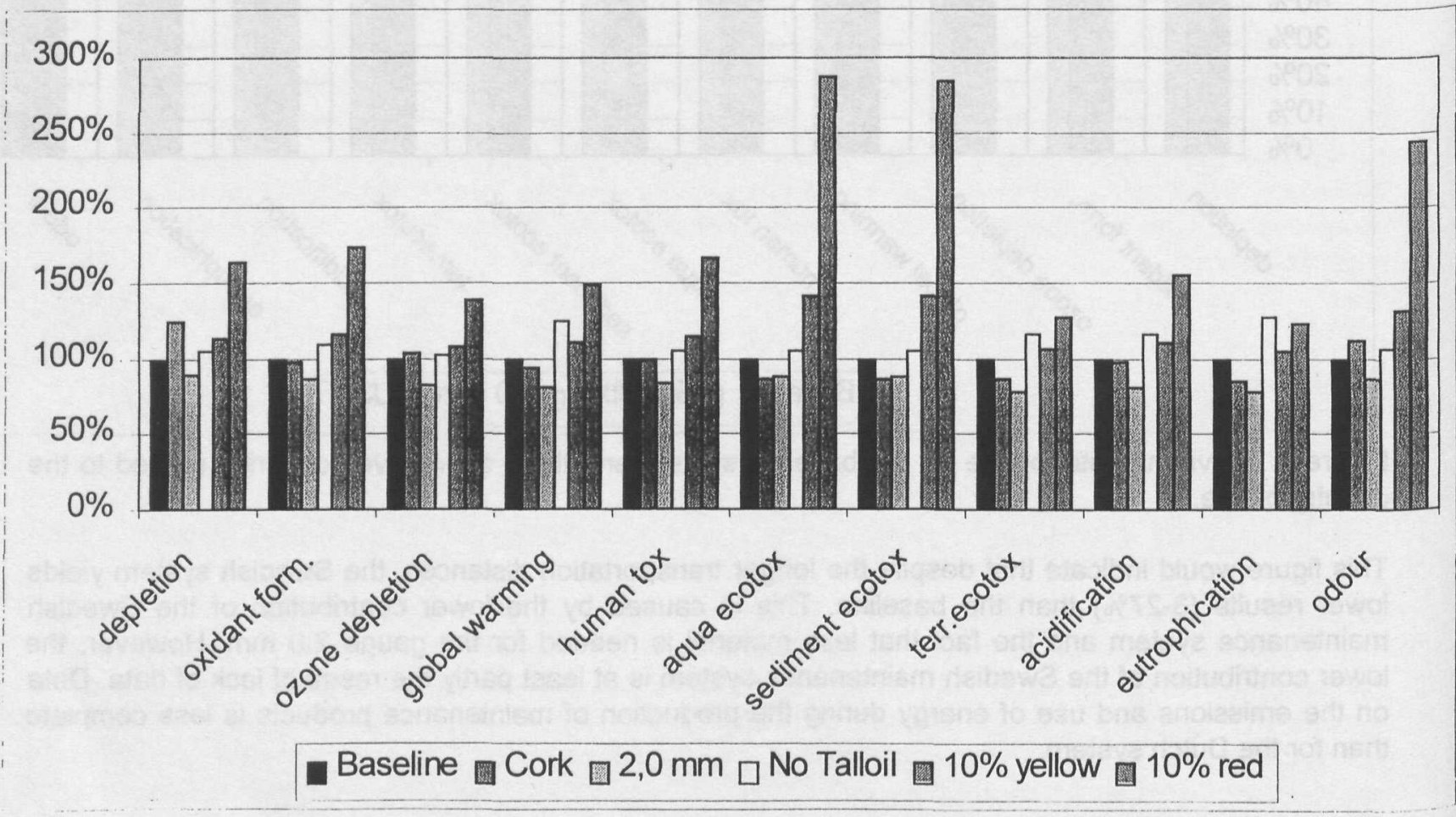


Figure 5. Environmental profile for the baseline system and five alternative scenarios related to differences in ingredients.

- The cork-linoleum is based on $4.5 \mathrm{~mm}$ cork linoleum. In the production of cork linoleum, cork powder is used instead of wood flour. This cork powder is first sieved at Forbo-Krommenie B.V. The composition of this linoleum also differs from normal Marmoleum.

- The $2.0 \mathrm{~mm}$ gauge used in the Netherlands was already described above.

- "No tall oil " linoleum receipt is made without tall oil.

- " $10 \%$ yellow and $10 \%$ red " are scenarios in which $10 \%$ of the $\mathrm{TiO} 2$ is replaced by respectively yellow and red organic pigments. In the linoleum produced by Forbo-Krommenie B.V., an average $16 \%$ of the pigment consists of pigment other than $\mathrm{TiO} 2.12 \%$ consists of iron oxides and $4 \%$ of organic pigment (source: Forbo-Krommenie B.V.). thus, $10 \%$ organic pigments is approximately twice as high as the actual amount of organic pigments, but lower than the total amount of "alternative " pigments.

The environmental profile of cork-linoleum shows lower results for most impact categories than the baseline scenario. This decrease varies from 1 to $13 \%$ (for the ecotoxicity categories). This decrease is mainly the result of using fewer materials in addition to cork (relatively more cork is used than wood powder) which also results in a reduction in the use of electricity during raw material production, and of linseed. However, the results for the impact categories "abiotic depletion ", "odour " and "depletion of the ozone layer " are respectively $25 \%, 13 \%$ and $3 \%$ higher than those in the baseline scenario. For "abiotic depletion " and "odour " this is mainly the result of a high use of gas during the milling and drying of cork, and for "depletion of the ozone layer "of an increase in the VOC emissions from the production site in Assendelft ( $9 \%$ higher).

The environmental profile of the $2.0 \mathrm{~mm}$ linoleum gauge shows, on average, $a \pm 15 \%$ lower result for all impact categories. This is due to less material use and less waste production.

The environmental profile of $2.5 \mathrm{~mm}$ linoleum without tall oil shows, on average, a $\pm 15 \%$ higher result for all impact categories. The maximal increases for the categories "eutrophication " $(28 \%)$ and "global warming " $(25 \%)$, are mainly caused by a $40 \%$ higher use of linseed.

The environmental profile of the $2.5 \mathrm{~mm}$ linoleum with $10 \%$ yellow pigment shows, on average, $a \pm 15 \%$ higher result for all impact categories. The maximal increases are in the categories "aquatic ecotoxicity "and "sediment ecotoxicity " $(41 \%)$ and "odour " $31 \%$. The environmental profile of the $2.5 \mathrm{~mm}$ linoleum with $10 \%$ red pigment shows, on average, $a \pm 80 \%$ higher result for all impact categories. The maximal increases here are for the categories "aquatic ecotoxicity " and "sediment ecotoxicity " ( \pm $280 \%$ ) and "odour " $\pm 240 \%$. It appears that the environmental profile for $1 \mathrm{~kg}$ yellow and red pigment is approximately 2500 respectively, 1000 times higher than the profile for the white pigment TiO2. Among other factors, this is caused by the greater energy consumption of yellow and red pigment. From this it may be concluded that $\mathrm{TiO}_{2}$ is not representative for other pigments and that the impact of other pigments should be included in future LCA-studies on linoleum.

\section{Conclusion:}

- The influence of pigments other than $\mathrm{TiO} 2$ can be considerable. $\mathrm{TiO} 2$ is not representative for other pigments. The impact of other pigments may also be high, although their share in the product is much lower.

- Using tall oil in the linoleum is better for the environment than using only linseed oil and gum rosin.

- Using $2.0 \mathrm{~mm}$ linoleum is better for the environment. However, this is only the case if the life span of the $2.0 \mathrm{~mm}$ linoleum is more or less the same as that of the $2.5 \mathrm{~mm}$ gauge.

\subsubsection{Alternative allocation methods/waste}

Data on the treatment of waste depends for a large part, on assumptions. It is assumed, for example, that all old linoleum and all linoleum waste from Forbo-Krommenie B.V. is incinerated. It is also 
assumed that electricity is produced just as it is assumed that the efficiency is $40 \%$. To analyse the effect of these assumptions, we used a few scenarios in which waste treatment is varied.

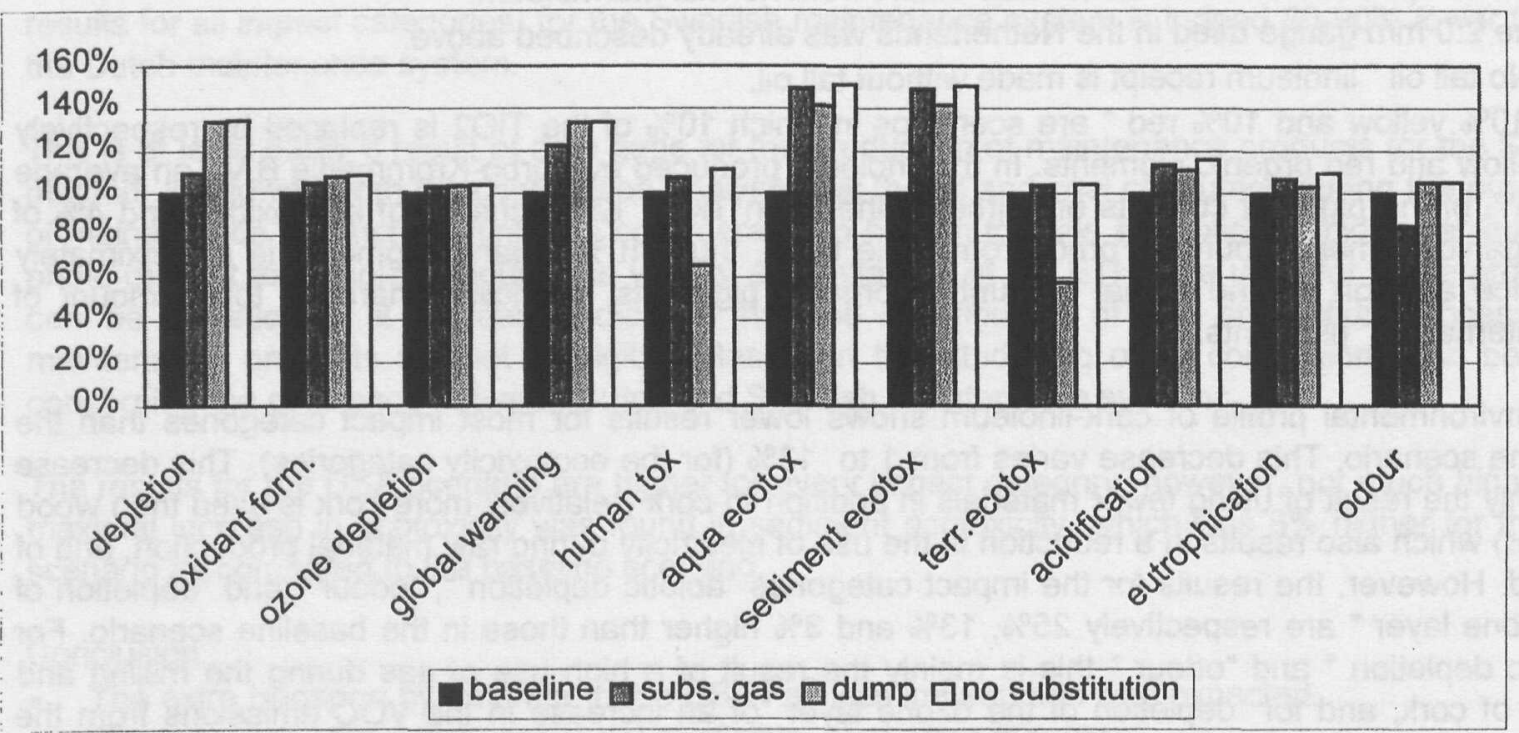

Figure 6. Environmental profile for the baseline system and three different scenarios related to handling linoleum waste

In Figure 6, three different scenarios related to handling linoleum waste are shown.

- The first scenario is an alternative allocation method for the heat produced by theincineration of linoleum. It is assumed in this scenario that the heat is used for heating (e.g. city heating) Heat is, therefore, replaced with avoided gas use.

- The second scenario is based on dumping the linoleum in a landfill site, rather than incinerating it.

- The third scenario is an alternative allocation method for the heat produced by the incineration of linoleum. Instead of the substitution method the surplus method is used, which means that the produced is not used and that no inputs or outputs are allocated to this flow.

Figure 6 shows that the allocation method chosen for the baseline received the lowest score for virtually every impact category. Substitution of gas use for electricity use results in a $15 \%$ lower score for "odour " and a higher score for all other impact categories. Dumping the waste on a landfill site results in a substantial lower score for human toxicity (34\% lower) and terrestrial ecotoxicity ( $42 \%$ lower), this, however, is not surprising since in both categories, emissions resulting from the incineration of linoleum cause the highest score. For all other categories, the results are higher. Not surprisingly, the scenario in which the heat produced is not used, shows the highest results for all impact categories.

\subsubsection{Alternative data/miscellaneous}

In Table 10, some scenarios related to input data are presented.

- Because the growing of linseed is an important process and because data is partially based on assumptions (see discussions on data quality in $\S$ 4.3.1and 6.1.4), three different scenarios concerning pesticide emissions and $\mathrm{N}$-emission were evaluated.

- The scenario is based on the assumption that all pesticides that could be used according to the supplier are indeed used on every hectare, in the prescribed dose. Since the suppliers also 
indicated that on only approx. $20 \%$ of the fields a pesticide is used, and that at no time are all pesticides used at the same field, this represents the worse possible scenario.

- The scenario based on the absence of pesticide use.

- The scenario based on a lower use of $\mathrm{N}$-fertiliser $(0.04 \mathrm{~kg} \mathrm{~N}$ per kg linseed instead of $0.068 \approx$ the amount taken up yearly by the linseed)

- Given the low score of the VOC-emissions at Forbo-Krommenie B.V. in relation to the H2S score on the impact category "odour " an alternative scenario was evaluated assuming all emitted VOC were butanal ("that is, having a relatively high "odour " factor).

- In order to ascertain whether shorter transportation distances for the raw materials would yield a substantially lower environmental profile, a scenario was analysed based on a $50 \%$ lower transportation distance per barge, for all raw materials.

Table 10. Environmental profiles for various scenarios with alternative input data, relative to the baseline system (in \%).

\begin{tabular}{|c|c|c|c|c|c|}
\hline & $\begin{array}{l}\text { high } \\
\text { pesticide }\end{array}$ & $\begin{array}{l}\text { no } \\
\text { pesticide }\end{array}$ & $\begin{array}{l}\text { lower use } \\
\mathrm{N} \text {-fertiliser }\end{array}$ & $\begin{array}{l}\text { of all VOC a } \\
\text { Forbo- } \\
\text { Krommenie } \\
\text { B.V. } \\
\text { butanal }\end{array}$ & $\begin{array}{l}\text { at } 50 \% \text { shorter } \\
\text { transportation by } \\
\text { e freighter } \\
=\end{array}$ \\
\hline Depletion of abiotic resources & 102 & 100 & 97 & $\mathrm{nr}$ & 98 \\
\hline Photochemical oxidant formation & 104 & 100 & 98 & $\mathrm{nr}$ & 98 \\
\hline Depletion of the ozone layer & 101 & 100 & 99 & $\mathrm{nr}$ & 99 \\
\hline Global warming & 101 & 100 & 76 & $\mathrm{nr}$ & 99 \\
\hline Human toxicity & 105 & 100 & 98 & $\mathrm{nr}$ & 98 \\
\hline Aquatic ecotoxicity & 102 & 100 & 95 & $\mathrm{nr}$ & 93 \\
\hline Sediment ecotoxicity & 102 & 100 & 95 & $\mathrm{nr}$ & 93 \\
\hline Terrestrial ecotoxicity & 162 & 61 & 99 & $\mathrm{nr}$ & 99 \\
\hline Acidification & 101 & 100 & 85 & $\mathrm{nr}$ & 96 \\
\hline Eutrophication & 102 & 100 & 74 & $\mathrm{nr}$ & 98 \\
\hline Odour & 101 & 100 & 91 & 2100 & 99 \\
\hline
\end{tabular}

$\mathrm{nr}=$ not relevant

The "high pesticide use "scenario shows mainly a higher score in the category "terrestrial ecotoxicity". The effect on human toxicity is relatively low (only a $5 \%$ increase), which is in accordance with the observation made above concerning the relatively low contribution of pesticide emissions to human toxicity. The effect on aquatic and sediment toxicity is still not very high. The effect of heavy metals continues to dominate these impact categories. Not surprisingly, the "no pesticide use "scenario shows the opposite effect. The impact category "terrestrial toxicity " shows a decrease of $39 \%$ while the other categories show no decrease.

The "lower use of $\mathrm{N}$-fertiliser " scenario shows lower results for every impact category. Global warming, acidification and eutrophication show a decrease of $15-26 \%$. The influence on the other categories is lower.

The replacement of all VOC-emissions by butanal (hence a worse case estimate for "odour ") leads to an increase in the result for the impact category "odour " by a factor of 21 . In this scenario, $95 \%$ of the score for "odour "is caused by the VOC-emissions at Forbo-Krommenie B.V. Thus, assumptions concerning the composition of the VOC emissions at Forbo-Krommenie B.V.have a considerable influence on the category "odour".

The scenario in which transport by sea-freighter is halved shows slightly lower results for all impact categories. The greatest decrease is seen in the category "ecotoxicity " for water and sediment $(7 \%)$. 
Conclusions:

- Reduction of pesticides only effects terrestrial ecotoxicity, and not the other toxicity categories.

- Reduction in the use of $\mathrm{N}$-fertiliser mostly effects the results for global warming, eutrophication and acidification.

- If the assumption that $1 \%$ of the applied $\mathrm{N}$ evaporates as $\mathrm{NH}_{3}$ and $1 \%$ as $\mathrm{N}_{2} \mathrm{O}$ is not realistic, but rather too high, the results for global warming, eutrophication and acidification are overestimated.

- Reduction in the transportation distance for raw materials has some effect on aquatic and sediment ecotoxicity.

- Assumptions concerning the composition of VOC emissions at Forbo-Krommenie B.V. have a considerable influence on the category "odour ". A better estimate of the composition of these emissions is necessary in order to assess the effects on "odour " properly in an LCA on linoleum products.

\subsubsection{Alternative impact assessment methods}

NB! The methods used in this section are not in accordance with ISO standards and gujdelines, as weighting is performed, and methods combining characterisation and weighting as one step are used.

In addition to the CML-2000 method (Guinée et al., 2000) which is followed in this study, there are a number of other methods for impact assessment in LCA. Some of these methods follow more or less the same structure as the CML-method in that they first calculate results per impact category by multiplying the emissions/extractions with characterisation factors. These results per impact category can then possibly be weighted and summed to such an indicator as Ecoindicator 95: Goedkoop, 1995. Yet other methods use factors which combine the characterisation and weighting step in one, resulting directly in one final value (e.g. EPS: Steen, 1996; Ecoscarcity: Ahbe et al., 1990, Baumann, 1992; ExternE: EC, 1995).

A few of those alternative methods are applied to the baseline scenario in order to determine how dependent the results are on the method used. The additional methods we used are:

- The Ecoindicator 95 (Goedkoop, 1995).

- ExternE (EC, 1995).

- $\operatorname{EPS}$ (Steen, 1996).

- Swiss Ecoscarcity (Ahbe et al., 1990).

- Norwegian Ecoscarcity (Baumann, 1992).

The ecoindicator is comparable to CML-2000, in that characterisation, normalisation and weighting are done in separate steps. Therefore, the contribution of the different life cycle stages to the individual impact categories could be visualised and compared to the unweighted results for the CML-2000 method. For the other methods, characterisation and weighting are combined in one step to yield one weighted total result. Therefore, a comparison of these methods with the CML-2000 method was only possible when the results per impact category for the CML-2000 method were also weighted. Hence, we applied an equal weighting to all categories (counting ecotoxicity as one category), resulting in Figure 8 and Table 11.

In

Figure 7 the results calculated with the Ecoindicator 95 are presented. When this figure is compared with Figure 3 , it becomes apparent that the results for impact categories, which are part of both methods, are very similar. However, the Ecoindicator has a different way of dealing with toxic substances. Their categories i.e. "heavy metals ", "pesticides ", "carcinogenic substances ", are grouped according to substance characteristic/effect type, while the CML-method is more focused on such endpoints as risk to ecosystems and risk to humans ${ }^{9}$. When all categories are weighted and

${ }^{9}$ in the new version of the ecoindicator, the ecoindicator 99 the impact categories are defined even more at endpoint level than in the CML-method: human health, ecosystem quality and resources. However, this method was not available yet at the moment the database of CMLCA was set up. 
summed, the most important impact category is heavy metals, which accounts for $75 \%$ of the total positive score (excl. negative value of linoleum incineration). If one were to use equal weights for every impact category, "sediment ecotoxicity " would be the most important impact category for the CMLmethod $(67 \%)$, attributable largely to heavy metals. However, the high contribution of heavy metals to ecotoxicity in the CML-2000 method should be considered with some care, as was discussed above see $(\S 6.1 .3)$.

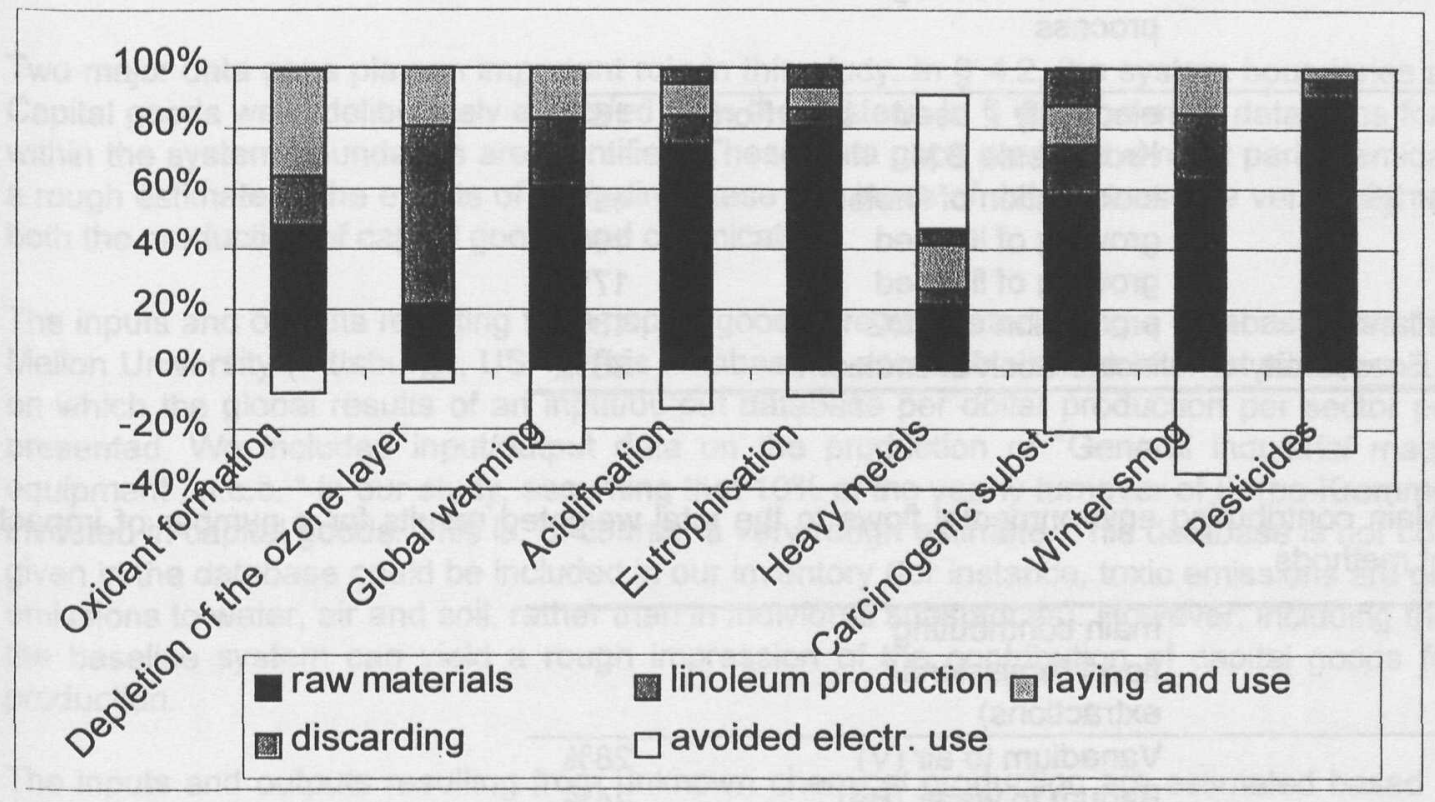

Figure 7. Environmental profile of the baseline system calculated with the Ecoindicator 95, split-up into life cycle stages.

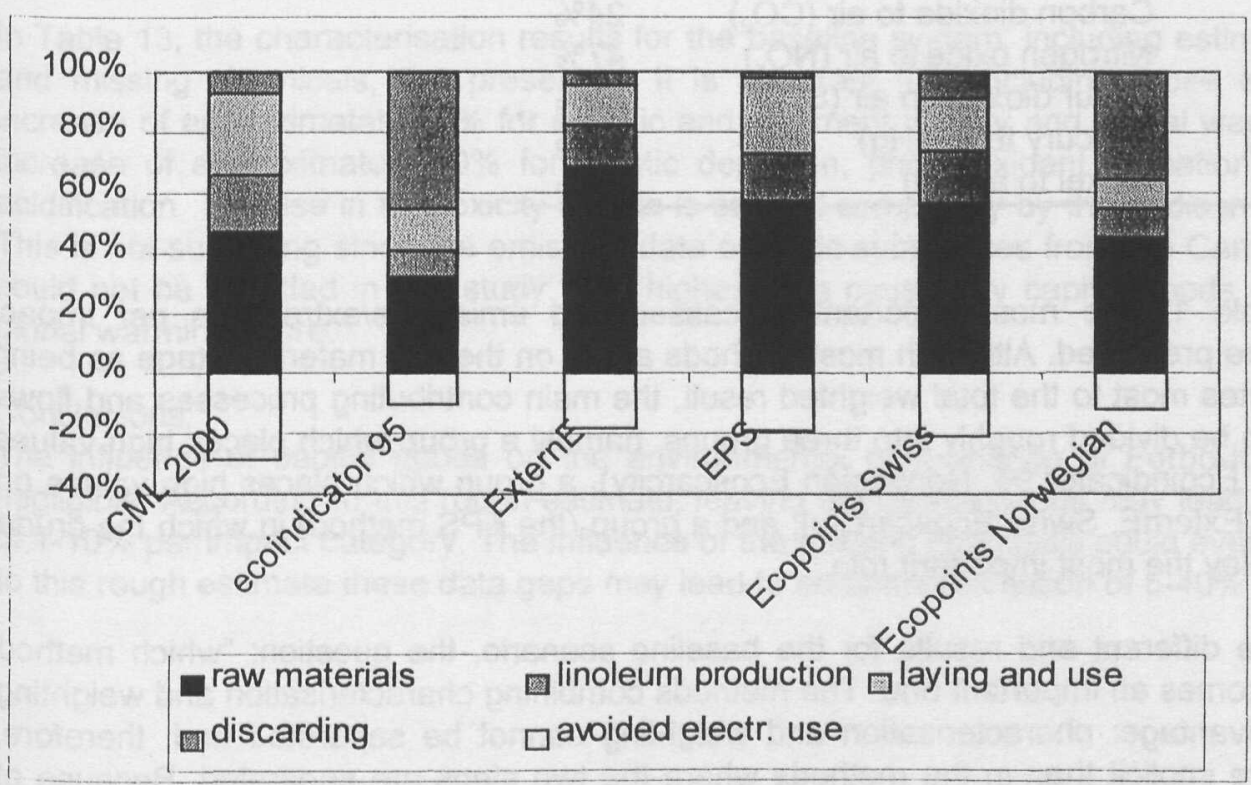

Figure 8. Total weighted results for the baseline system calculated with different methods.

In Figure 8 , the results calculated with the methods combining characterisation and weighting in one step are compared with the weighted results for the Ecoindicator 95 and the CML 2000 method, using the arbitrary weighting factor of 1 for each impact category (except the ecotoxicity categories, which were first averaged and the average result was then weighted with a factor of 1 ). All methods indicate 
the raw material stage as the most important, with the exception of the Ecoindicator 95 which rates the disposal stage the highest.

Table 11. Main contributing processes to the total positive weighted results for a number of impact assessment methods (excluding the negative result of incinerating linoleum).

\begin{tabular}{lll}
\hline IA method & $\begin{array}{l}\text { main contributing } \\
\text { process }\end{array}$ \\
\hline CML 2000 & $\begin{array}{l}\text { electricity used by } \\
\text { Krommenie B.V. }\end{array}$ & \\
Ecoindicator 95 & incineration of linoleum & $16 \%$ \\
ExternE & growing of linseed & $45 \%$ \\
EPS & growing of linseed & $19 \%$ \\
Swiss Ecoscarcity & production of TiO2 & $17 \%$ \\
Norwegian Ecoscarcity & incineration of linoleum & $36 \%$ \\
\hline
\end{tabular}

Table 12. Main contributing environmental flows to the total weighted results for a number of impact assessment methods

\begin{tabular}{lll}
\hline IA method & $\begin{array}{l}\text { main contributing } \\
\text { flows (emissions/ } \\
\text { extractions) }\end{array}$ \\
\hline CML 2000 & Vanadium to air (V) & $28 \%$ \\
& Barium to water (Ba) & $24 \%$ \\
Ecoindicator 95 & Cadmium to air (Cd) & $25 \%$ \\
ExternE & Lead to air (Pb) & $13 \%$ \\
& Sulfur dioxide to air $\left(\mathrm{SO}_{2}\right)$ & $38 \%$ \\
EPS & Nitrogen oxide to air $\left(\mathrm{NO}_{x}\right)$ & $24 \%$ \\
& Crude oil (resource) & $26 \%$ \\
Swiss Ecoscarcity & Carbon dioxide to air $\left(\mathrm{CO}_{2}\right)$ & $24 \%$ \\
& Nitrogen oxide to air $\left(\mathrm{NO}_{x}\right)$ & $47 \%$ \\
Norwegian Ecoscarcity & Sulfur dioxide to air $\left(\mathrm{SO}_{2}\right)$ & $29 \%$ \\
& Mercury to air $(\mathrm{Hg})$ & $25 \%$ \\
& Nickel to air (Ni) & $24 \%$ \\
\hline
\end{tabular}

In Table 11 and Table 12 the most important processes and emissions/extractions per impact assessment method are presented. Although most methods agree on the raw materials stage as being the stage that contributes most to the total weighted result, the main contributing processes and flows vary. The methods can be divided roughly into three groups, namely a group which places high values on metals (CML 2000, Ecoindicator 95, Norwegian Ecoscarcity), a group which places high values on acidifying substances (ExternE, Swiss Ecoscarcity), and a group (the EPS method) in which the crude oil resource and $\mathrm{CO}_{2}$ play the most important role.

Since all methods give different end results for the baseline scenario, the question: "which method should be used? ", becomes an important one. The methods combining characterisation and weighting have one major disadvantage: characterisation and weighting cannot be separated and, therefore, value choices are more implicit than in the methods where the two steps are separated. Because of this combined step, these methods are likewise not compatible with the ISO framework (ISO 14042, 1999). In the ISO framework, the environmental profile resulting from classification and characterisation is an important result on its own, and the more value-based weighting procedures are clearly a separate optional element (ISO 14042, 1999). Doing the analysis in two steps leads to greater transparency. It makes it possible to analyse the data more thoroughly by providing more information on the types of environmental impacts that can be expected. Moreover, combined methods often work with much smaller sets of factors. The EPS, ExternE and Ecoscarcity methods used in this study, use 
sets of 10 to $<70$ different factors, whereas both the CML 2000 method and the Ecoindicator 95 have more than 1000 factors. Therefore, we advise using a two step method and because the factors from the CML 2000 method are more up to date than those of the Ecoindicator 95, we advise using the results from the CML 2000 method $^{10}$

\subsection{Data gaps}

Two major data gaps play an important role in this study. In $\S 4.2$, the system boundaries are defined. Capital goods were deliberately excluded from the system. In $\S 4.3$, the main data gaps for processes within the system boundaries are identified. These data gaps are for the most part chemicals. To give a rough estimate of the effects of excluding these two types of data, we used a very rough estimate for both the production of capital goods and chemicals.

The inputs and outputs resulting from capital goods are estimated using a database from the Carnegie Mellon University (Pittsburgh, USA). This database is accessible at the internet site (www.eiolca.net), on which the global results of an input/output database per dollar production per sector per year are presented. We included input/output data on the production of "General industrial machinery and equipment, n.e.c. " in our study, assuming that $10 \%$ of the yearly turnover of Forbo-Krommenie B.V. is invested in capital goods. This is, of course, a very rough estimate. This database is not complete and given in the database could be included in our inventory (for instance, toxic emissions are given in total emissions to water, air and soil, rather than in individual substances). However, including these data in the baseline system can yield a rough impression of the contribution of capital goods for linoleum production.

The inputs and outputs resulting from unknown chemical production are estimated based on data on the production of organic chemicals in the ETH-database. This is also a very rough estimate. This data is probably not entirely representative for the missing chemicals and the reliability of the data on this general organic chemical will not be very high. Not all chemicals for which data was unavailable will be organic chemicals. However, the emissions for organic chemicals in the ETH-database are higher than for the inorganic chemicals, hence choosing the organic chemical yields a sort of worse case estimate.

In Table 13, the characterisation results for the baseline system, including estimates for capital goods and missing chemicals, are presented. It is apparent that including those estimates leads to an increase of approximately $40 \%$ for aquatic and sediment toxicity and global warming as well as to an increase of approximately $20 \%$ for abiotic depletion, photo oxidant formation, global warming and acidification. The rise in the toxicity results is caused completely by the addition of data on chemicals. This is not surprising since the emission data on toxic substances from the Carnegie Mellon database could not be included in this study. The highest rise caused by capital goods is the $10 \%$ rise of the global warming score.

\section{Conclusions:}

The influence of capital goods on the environmental performance of Forbo-Krommenie B.V. is not negligible. According to this rough estimate, leaving out capital goods may lead to an underestimation of $1-10 \%$ per impact category. The influence of the missing chemicals could even be higher. According to this rough estimate these data gaps may lead to an underestimation of $5-40 \%$ per impact category.

\footnotetext{
${ }^{10}$ In future LCA studies, the Ecoindicator 99 might well be a valuable addition to the CML 2000 method for Forbo-Krommie BV.
} 
Table 13. Characterisation results for the baseline system including estimates for capital goods and missing chemicals, relative to the baseline system (in \%).

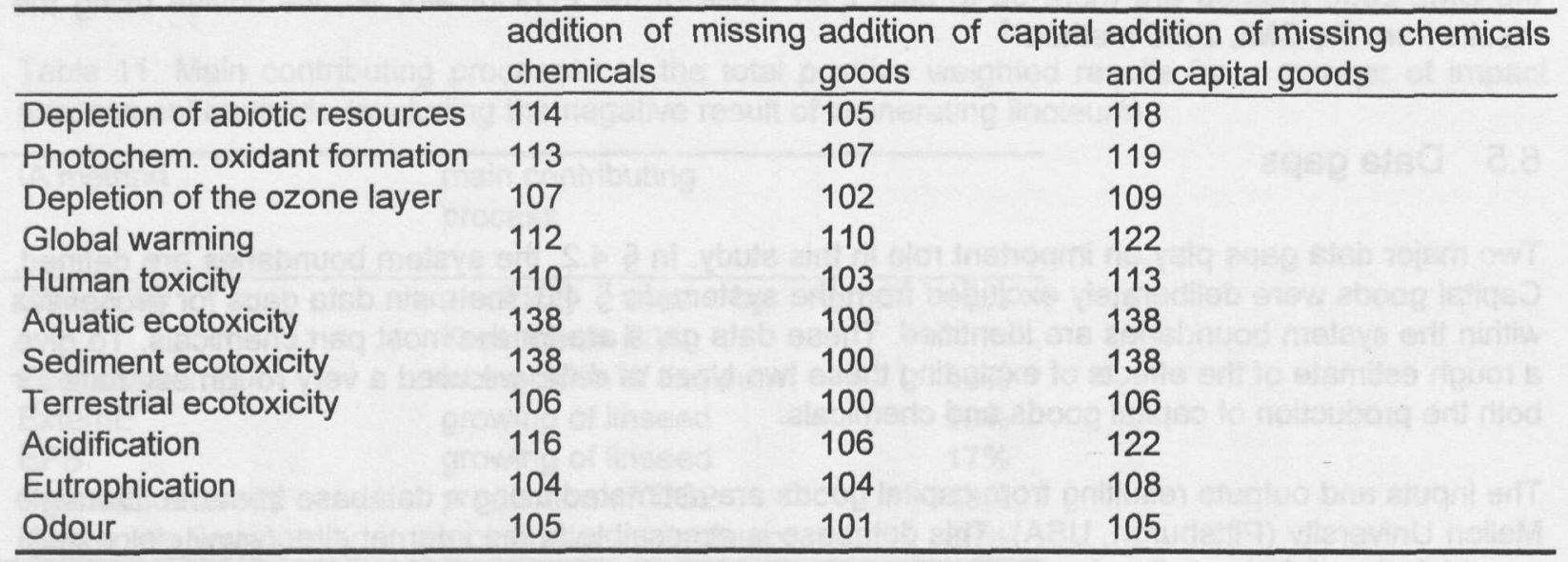

\subsection{Data quality and applicability}

Combining the results from the contribution analysis with the knowledge on the quality of the data the following conclusions may be drawn :

- Data on the growing of linseed is partially based on assumptions. Because this process has a large influence on the outcome of the study (see $\S 6.1 .3$ and the scenarios in $\S 6.4 .4$ ), it is advisable to try to ground these assumptions with better data.

- Better data on maintenance products is needed. The results of the comparison of the Dutch and Swedish scenario are influenced by a lack of data on the production of Swedish maintenance products (see scenarios in $\S 6.4 .1$ ). Therefore, no conclusions can be based on the comparison of these maintenance systems, other than that maintenance is not negligible.

- Because the data on red an yellow pigments comes from personal communications only, a quality check was not possible. However, inclusion of this data did show that its influence can be considerable (see scenarios in § 6.4.2).

- The estimate of the composition of VOC emissions at Forbo-Krommenie B.V. is not very realistic. As a consequence, the result for "depletion of the ozone layer " is overestimated and the result for "odour " is underestimated (see scenario in $\S 6.4 .4$ and the discussion on "depletion of the ozone layer " in § 6.1.3, Table 6 and Table 8). These results are therefore, less reliable.

- The use of average data for the landfilling process is not very representative for the effects of dumped linoleum. However only a small fraction of the linoleum is supposed to be dumped. Moreover the contribution of the process landfilling is not important for any of the impact categories. So the use of these average data probably does not have a high influence on the result of this study.

- Because the linseed straw was not included in the study (see $\S 4.2$ ), the emissions of the burning of this straw other than $\mathrm{CO}_{2}$ are not included in this study. This might result in an underestimation of the contribution of the process " growing of linseed " which is already a very important contributing process.

- According to a very rough estimate leaving out capital goods may lead to an underestimation of 1$10 \%$ per impact category (see scenario in $\S 6.5$ ).

- The influence of the missing chemicals (mainly in maintenance products, lacquers and adhesives) on the environmental profile could be even more substantial. According to a very rough estimate, these data gaps may lead to an underestimation of $5-40 \%$ per impact category (see scenario in $\S$ 6.5).

Based on these conclusions we would state that:

- The results of the study are applicable for analysis of the described systems only. This means that:

- The results should not be used to compare the environmental performance of linoleum produced by Forbo-Krommenie B.V. to other products. 
- The results should not be used to compare systems with different capital goods because capital goods are not included.

- The results should not be used to compare different maintenance systems because too much data on chemicals in maintenance products is missing.

- The results for "odour " and "depletion of the ozone layer " should be presented with some care as the unreliable VOC estimations at Forbo-Krommenie B.V. play an important role in these impact categories. 


\section{Conclusions and discussion}

In this chapter, the main conclusions following from the study are summarised, and the consequences for Forbo-Krommenie B.V. are outlined.

\subsection{Main conclusions from inventory, impact assessment and interpretation summarised}

\section{Data gaps}

No process data was available for the following processes:

- The production and transport of pesticides (use and emission of pesticides is included).

- The production and transport of the fertiliser S needed in the process "growing linseed ".

- The production and transport of some raw materials needed for maintenance products (additives, thickeners, solvents) and almost all ingredients for those materials.

- The production and transport of some raw materials needed for the production of materials used during laying (adhesives and materials used to seal the seams for professionally used linoleum floors).

- The production and transport of a catalyst needed in the process "esterification of tall oil ".

- The production and transport of the maintenance product used for removing the polymer dressing on linoleum floor covering needed in the process "the use and maintenance of $1 \mathrm{~m} 2$ linoleum for 20 years".

\section{Flows not assigned to an impact category}

In total there are 264 inputs/outputs that could not be assigned to an impact category. These are mainly from the ETH database. A large portion of the emissions (132 emissions) are radioactive emissions. Since radiation is not included in this study (because no consensus has yet been reached concerning the impact assessment method to be used) it could not be included in the impact assessment results.

\section{Main contributing processes}

The processes or groups of processes that contribute largely to more than one impact category are:

- The growing of linseed (emissions of $\mathrm{NH}_{3}, \mathrm{~N}_{2} \mathrm{O}$, pesticides).

- Gas and electricity used at Forbo-Krommenie B.V.

- Oil used for the production of maintenance products.

- The transport of raw materials.

- The incineration of linoleum.

- Coal used for the production of detergents and acrylic dispersions/emulsions.

\section{Scenarios}

The following conclusions were drawn from the scenarios:

- Extra transport by freighter to the USA has less impact than was expected.

- The influence of maintenance in the "use "phase is not negligible as is often thought.

- The influence of other pigments than $\mathrm{TiO}_{2}$ can be considerable. $\mathrm{TiO} 2$ is not representative for other pigments. The impact of other pigments may be high even though their mass share in the product is much lower.

- Using tall oil in linoleum is better for the environment than using only linseed oil.

- Using $2.0 \mathrm{~mm}$ linoleum is better for the environment. However, this is only the case if the life span of the $2.0 \mathrm{~mm}$ linoleum is more or less the same as for the $2.5 \mathrm{~mm}$ version.

- Substitution of useful heat produced during the incinerating of linoleum with avoided electricity use is the best of the studied alternatives.

- Reduction in the use of pesticide only effects terrestrial ecotoxicity, and not the other toxicity categories. 
- Reduction in the use of $\mathrm{N}$-fertiliser affects the results for global warming, eutrophication and acidification substantially.

- Reduction of the transportation distance for raw materials has some effect on aquatic and sediment ecotoxicity.

- Changes in the composition of the VOC emissions at Forbo-Krommenie B.V. have a considerable influence on the category "odour ". A better estimate of the composition of these emissions is necessary in order to assess the effects on "odour " and "depletion of the ozone layer " properly in an LCA on linoleum products.

- The influence of capital goods is certainly not negligible. According to a very rough scenario leaving out capital goods may lead to an underestimation of $1-10 \%$. The influence of the missing chemicals could be even more substantial. According to a very rough scenario these data gaps may lead to an underestimation of $5-40 \%$.

\subsection{Discussion \& final conclusions}

\section{Main contributing processes}

In the stage "production of raw materials " the growing of linseed and transport are important processes. The growing of linseed is overall a very important process contributing greatly to more than 5 impact categories (see $\S 6.1 .3$ ), mainly caused by the emissions of $\mathrm{NH}_{3}$. The data for this process is valid because it is representative. However, its reliability may be disputed. The emissions of both $\mathrm{NH}_{3}$, $\mathrm{N}_{2} \mathrm{O}$ and pesticides depend largely on assumptions. If these assumptions are not realistic, this may have large influences on results (see the scenarios in 6.4.4). Therefore it is advisable to try to ground these assumptions with better data. Still, Forbo-Krommenie B.V. could improve their environmental performance on many impact categories by using linseed that is cultivated with less fertiliser and less pesticides.

This high impact of the process "growing of linseed" also is responsible for the difference between linoleum with and without tall oil (see scenario in 6.4.2). Linoleum with tall oil has a better environmental profile than linoleum without tall oil because the use of linseed is lower. Therefore, reduction of the amount of tall oil in favour of linseed oil does not improve environmental performance.

In the stage "production of linoleum " the energy used at Forbo-Krommenie B.V. is most important. We expect that this data is valid and reliable. Saving on the use of electricity and gas is therefore an opportunity for improvement. This is an option or which Forbo-Krommenie B.V. is not dependent on its suppliers.

In the stage "laying and use ", the coal and oil used during the production of maintenance products is important. Data on the production of maintenance products is not complete. Therefore, in the scenario analysis, maintenance options were analysed in greater detail (see $\S 6.4 .1$ ) The results of the comparison of the Dutch and Swedish scenario showed a heavy influence of the lack of data on the production of Swedish maintenance products (see scenarios in $\S 6.4 .1$ ). Therefore, no conclusions can be based on the comparison of these maintenance systems, other than that maintenance is not negligible. Better data on maintenance products is needed before more conclusions can be drawn on the influence of maintenance.

In the stage "disposal " the emissions produced during incineration are important. However the figure of $100 \%$ incineration of used linoleum and the linoleum waste from Forbo-Krommenie B.V., is only an assumption. Therefore, a sensitivity analysis has been carried out in which the waste is not incinerated but landfilled (see 6.4.3). From this scenario followed that substitution of useful heat produced during the incinerating of linoleum with avoided electricity use shows the best environmental profile of the studied alternatives. Therefore incineration seems a better alternative than landfilling. However, this result should be considered with some care, because for landfilling average ETH-data was used. These are probably not very realistic for the landfill of linoleum. 


\section{Composition of linoleum}

In the scenarios a number of variation in linoleum composition have been studied (see $\S$ 6.4.2). This showed that:

- The pigments used can have a large influence on environmental performance. However the data on pigments could not be checked for quality. Moreover, these data were not provided by the suppliers of the pigments and the representativeness of these data might be disputed. A more detailed analysis on this point focusing on the pigments which are actually used by ForboKrommenie B.V., aimed at finding pigments which are the most environment-friendly could be valuable.

- The $2.0 \mathrm{~mm}$ gauge has a considerably better environmental performance than the $2.5 \mathrm{~mm}$ gauge. On average, the results for this gauge are $15 \%$ lower. If the life span of both floors is comparable, Forbo-Krommenie B.V. might consider producing relatively more $2.0 \mathrm{~mm}$ products and advising their use on well-smoothed surfaces as a means of improving their environmental performance.

- Linoleum with tall oil has a better environmental profile than linoleum without tall oil because the use of linseed is lower (see also discussing before on linseed).

- Compared to the baseline $2.5 \mathrm{~mm}$ linoleum cork linoleum produces better results in most categories, but performs considerably worse in the categories "abiotic depletion "and "odour ". Were the gas use during drying and milling of cork-granulate reduced, it would improve the results for these categories.

\section{Datagaps}

The influence of capital goods is certainly not negligible. According to a very rough scenario leaving out capital goods may lead to an underestimation of $1-10 \%$ (see $\S 6.5$ ). The influence of the missing chemicals could be even more substantial. According to a very rough scenario these data gaps may lead to an underestimation of $5-40 \%$ (see $\S 6.5$ ). Therefore, the results should not be used to compare systems with different capital goods or different maintenance systems. The results of the study are applicable for analysis of the described systems only.

VOC

The result for "odour " and "depletion of the ozone layer " should be considered with some care, since the emissions of VOC from Forbo-Krommenie B.V., which play an important role in these impact categories, were not specified. From the scenario in $\S 6.4 .4$ followed that the individual VOC composition of the emission from Frobo-Kromenie B.V. can have a large influence the results for "odour".

\subsection{Improvement options \& advice for further studies}

\subsubsection{Options to improve environmental performance of Forbo-Krommenie B.V.}

Raw material use

Forbo-Krommenie B.V. could improve their environmental performance on many impact categories by using linseed that is cultivated with less fertiliser and less pesticides. This seems a more promising option than reducing transportation distances for raw materials.

\section{Energy use}

Not surprisingly, saving on the use of electricity and gas is also an opportunity for improvement. This is an option or which Forbo-Krommenie B.V. is not dependent on its suppliers.

\section{Composition of linoleum}

The pigments used can have a large influence on environmental performance. A more detailed analysis on this point, aimed at finding pigments which are the most environment-friendly could be valuable.

The $2.0 \mathrm{~mm}$ gauge has a considerably better environmental performance than the $2.5 \mathrm{~mm}$ gauge. On average, the results for this gauge are $15 \%$ lower. If the life span of both floors is comparable, Forbo- 
Krommenie B.V. might consider producing relatively more $2.0 \mathrm{~mm}$ products and advising their use on well-smoothed surfaces as a means of improving their environmental performance.

Linoleum with tall oil has a better environmental profile than linoleum without tall oil because the use of linseed is lower. Therefore, reduction of the amount of tall oil in favour of linseed oil does not improve environmental performance.

Compared to the baseline $2.5 \mathrm{~mm}$ linoleum cork linoleum produces better results in most categories, but performs considerably worse in the categories "abiotic depletion " and "odour ". Were the gas use during drying and milling of cork-granulate reduced, it would improve the results for these categories.

\subsubsection{Advice for future studies on linoleum}

We advice giving the following topics extra attention in future studies on linoleum, as the data on these topics is fragmented and their influence on the environmental profile of linoleum could be considerable:

- The production and use of maintenance products, especially the Swedish type

- The production and use of pigments other than TiO2. There is a great variety of possible pigments, but little information is available concerning their environmental performance. Our sensitivity analysis showed that the contribution of these pigments can be considerable.

- Emissions of individual VOC at the site of Forbo-Krommenie B.V.

Given the normative nature of evaluation and weighting, this step should be separated from the characterisation step (conform ISO), for transparency reasons and to show the effects of various assumptions. Therefore, we advise using a two step method for future LCA-studies on linoleum products. As no independent nationally or internationally authorised weighting sets are currently available, we advise using no weighting, if this not necessary. 


\section{References}

Ahbe, S., A. Braunschweig, \& R. Müller-Wenk, 1990: Methodik für Oekobilanzen, auf der Basis ökologischer Optimierung. Schriftenreihe Umwelt Nr 133, Bundesamt für Umwelt, Wald und Landshaft (BUWAL), Bern, Switzerland

Baumann, H., 1992: LCA: Utvärdering med index. Beräkning av tva uppsättningar norska index. CITekologik 1992:2. Chalmers, Göteborg. Sweden.

Berg, N, W. van den, G. Huppes, E.W. Lindeijer, B.L. van der Ven \& M.N. Wrisberg, 1998: Quality assessment in LCA. Framework and survey, draft.

Blonk, T.J., M. Lafleur, R. Spriensma, S. Stevens, M. Goedkoop, A. Agterberg, B. van Engelenburg \& K. Blok, 1997: Drie referentieniveaus voor normalisatie in LCA: Nederlands grondgebied 1993/1994; Nederlandse eindconsumptie 1993/1994; West-Europees grondgebied begin jaren 1990. RIZAwerkdocument $97.110 x$.

Braunschweig, A., R. Förster, P. Hofstetter, R. Müller-Wenk, 1994: Evaluation und Weiterentwicklung von Bewertungsmethoden für Ökobilanzen - Erste Ergebnisse. IWÖ-Diskussionsbeitrag $\mathrm{Nr} 19$, Zwischenbericht des Nationalprojects $\mathrm{Nr}$ 5001-35066, SSP Umwelt, Institut für Wirtschaft und Ökologie (IWÖ) St. Gallen, Switzerland

Davis, J \& C. Haglund, 1999: Life Cycle Inventory (LCI) of fertiliser production. SIK-report No. 654. Chalmers University of Technology, Sweden.

Derwent, R.G., M.E. Jenkin, S.M. Saunders \& M.J. Pilling, 1998: Photochemical ozone creation potentials for organic compounds in Northwest Europe calculated with a master chemical mechanism. Atmospheric Environment, 32. p 2429-2441.

EC (European Commission); 1995: ExternE: Externalities of Energy. Volume 1-5. European Commission, Directorate-General XII, Science, Research and Development, Luxembourg

ETH, 1996: Ökoinventare von Energiesystemen. Eidgenössische Technische Hochschule, Zürich. Database.

Goedkoop, M., 1995: The Ecoindicator 95, Weighting method for environmental effects that damage ecosystems or human health on a European scale. Final report, NOH report 9523, Pre Consultants, Amersfoort, Netherlands.

Guinée, J.B., 1995. Development of a methodology for the environmental life-cycle assessment of products; with a case study on margarines. Thesis, Leiden University.

Guinée, J.B., M. Gorree, R. Heijungs, G. Huppes, R. Kleijn, H.A. Udo de Haes, E. van der Voet \& M.N. Wrisberg, 2000: Environmental Life Cycle Assessment. Backgrounds. Centre of Environmental Science (CML), Leiden University, Leiden. In prep. draft version October 1998 available on the website: http://www.leidenuniv.nl/interfac/cml/lca2/index.html

Günther, A. \& H.C. Langowski, 1997: Life Cycle Assessment study on resilient floor coverings. Int. J. LCA 2 (2). pp. $73-80$.

Hauschild, M \& H. Wenzel, 1998: Environmental Assessment of products. Volume 2: Scientific background. Chapman \& Hall, London.

Heijungs, R. 2000. CMLCA 2.0. on website: http.//www.leidenuniv.nl/interfac/cml/ssp/cmlca.html. 
Heijungs, R., J.B. Guinée, G. Huppes, R.M. Lankreijer, H.A. Udo de Haes, A. Wegener Sleeswijk, A.M.M. Ansems, P.G. Eggels, R. van Duin \& H.P. de Goede, 1992: Environmental Life Cycle Assessment of products. Guide and Backgrounds. Centre of Environmental Science (CML), Leiden University, Leiden.

Houghton, J.T., L.G. Meira Filho, J. Bruce, H. Lee, B.A. Callander, E. Haites, N. Harris \& K. Maskell (eds), 1994: Climate change 1994. Radiative forcing of climate change and an evaluation of the IPCC IS92 Emissions scenarios. Cambridge University Press, Cambridge.

Houghton, J.T., L.G. Meira Filho, B.A. Callander, N. Harris, A. Kattenberg \& K. Maskell, 1995: Climate change 1995. The science of climate change; contribution of WGI to the second assessment report of the intergovernmental panel on climate change. Cambridge University Press, Cambridge.

Huijbregts, M.A.J., 1999a: Priority assessment of toxic substances in LCA. Development and application of the multi-media fate, exposure and effect model USES-LCA. Interfaculty Department of Environmental Science, Faculty of Environmental Science, University of Amsterdam, Amsterdam.

Huijbregts, M.A.J., 1999b: Life-cycle impact assessment of acidifying and eutrophying air pollutants. I: Calculation of equivalency factors with RAINS-LCA. Interfaculty Department of Environmental Science, Faculty of Environmental Science, University of Amsterdam, Amsterdam.

ILV, 1991: Umweltprofile von Packstoffen und Packmitteln, Methode (Entwurf). Fraunhofer-Institut für Lebensmitteltechnologie und Verpackung, Gesellschaft für Verpackungsmarktforschung, Institut für Energy- und Umweltforschung, München.

ISO 14040, 1997: ISO 14040: 1997, Environmental management - Life cycle assessment - Principles and framework. International Organisation for Standardisation (ISO).

ISO 14041, 1998: Final draft ISO/FDIS 14041. Environmental management - Life cycle assessment Goal and scope definition and inventory analysis. International Organisation for Standardisation (ISO).

ISO 14042, 1999: Final draft ISO/FDIS 14042. Environmental management - Life cycle assessment - Life cycle impact assessment. International Organisation for Standardisation (ISO).

ISO 14043, 1998: Committee draft ISO/DIS 14043. Environmental management - Life cycle assessment - Life cycle interpretation. International Organisation for Standardisation (ISO).

Jöhnsson, A., A.M. Tillman \& T. Svensson, 1995: Life-cycle assessment of flooring materials. Report A5: 1995. Swedish Council for building research.

Jenkin, M.E. \& G.D. Hayman, 1999. Photochemical ozone creation potentials for oxygenated volatile organic compounds: sensitivity to variations in kinetic and mechanistic parameters. Atmospheric Environment, 33. p 1275-21293.

Loo, S van \& de Koning, J, 1994: Oriëntatie op de thermische verwerking van Marmoleum (In Dutch). TNO, MEP, Apeldoorn, The Netherlands

PE, 1999: Product Engeneering, Dettingen, Germany: Personal communications Dr. K. Saur.

Potting, J \& K. Blok, 1993: The environmental life-cycle assessment of four types of floor covering. Science shop co-ordinating centre of Utrecht University, Utrecht.

Steen, B. \& S.O.Ryding, 1993: The EPS Enviro-Accounting Method, An Application of Environmental Accounting Principles for Evaluation and Valuation of Environmental Impact in Product Design. AFRreport 11, Avfallsforskningsradet (AFR), Swedish Environmental Protection Agency, Stockholm, Sweden 
Steen, B., 1996: EPS-Default Valuation of Environmental Impacts from Emission and Use of Resources. Version 1996, AFR report 111, Avfallsforskningsradet (AFR), Swedish Environmental Protection Agency, Stockholm, Sweden

Välimaa, C. \& M. Stadig, 1998: Växtnäring / livscykelanalys. Swedish Institute for food and biotechnology. SIK-rapport nr. 6371998.

WMO (world Meteorological Organisation), 1992: Scientific assessment of ozone depletion: 1991. Report no. 25. Geneva.

WMO (world Meteorological Organisation), 1995: Scientific assessment of ozone depletion: 1994. Report no. 37. Geneva.

WMO (world Meteorological Organisation), 1998: Scientific assessment of ozone depletion: 1998. Report no. 44. Geneva.

Wrisberg, M.N. N.W. an den Berg, G. Huppes, E. Lindeijer \& B.L. van der Ven, 1999: Operational quality assessment in LCA: A semi quantitative method, draft. 
Appendices 
Below the data sources per process are described. Information on suppliers is not published in this list.

P1: Production of 2,5 mm Marmoleum/Artoleum at FORBO-KROMMENIE B.V. (in M2)

Conversion of $\mathrm{kg}$ linoleum in $\mathrm{m}^{2}$ linoleum and adding packaging and the additional "general" energy use and waste production

- data sources: annex 1: flowsheet linoleum production, 2 and 4 Forbo-Krommenie B.V.

- $\quad$ waste $=$ not recycled packaging and domestic waste (annex 1: flowsheet linoleum production) $\Rightarrow>$ ETH-848: abfall in inertstoffdeponie

P2: Other yearly use of steam and electricity at Forbo-Krommenie B.V.

General energy use not related to one specific process in Forbo-Krommenie B.V.

- data electricity: annex 5 Forbo-Krommenie B.V. $=14 \% ;-4 \%$ to recycling (Keuken \& de Koning, 1997) $=10 \%$

- data steam: Keuken \& de Koning, 1997 (=soda inst. + heating buildings)

\section{P3: Recycling scrap linoleum}

Converting scrap linoleum from trimming at Forbo-Krommenie B.V. into reusable scrap:

- data electricity: Keuken \& de Koning, $1997=4 \%$, total use annex 5 Forbo-Krommenie B.V., see also P2

- data emission dust/particles: annex 6 Forbo-Krommenie B.V.

- remaining data: annex 1: flowsheet linoleum production, Forbo-Krommenie B.V.

- remaining linoleum waste is incinerated

P4 \& P5: Production linoleum granulate lower \& upper layer

Process taking place at Forbo-Krommenie B.V.-Krommenie. The reference amount is de total granulate production by Forbo-Krommenie B.V. in 1998

- data emission particles/dust and NMVOC: annex 6 Forbo-Krommenie B.V.

- data electricity: annex 5 Forbo-Krommenie B.V.

- remaining data: annex 1: flowsheet linoleum production, and 4 Forbo-Krommenie B.V.; see for calculations exel-file "berekening benodigde hoev grondstof"; In this file, the amounts needed for a certain type of linoleum are calculated based on the total inflow in 1998 (annex 1: flowsheet linoleum production) and the ratio between the materials in the final product (annex 4)

\section{P6: Esterification of tall oil}

Process taking place at Forbo-Krommenie B.V.-Krommenie. The reference amount is de total tall oil production by Forbo-Krommenie B.V. in 1998

- data electricity and use of gas: annex 5 Forbo-Krommenie B.V.

- data emission dust/particles: annex 6 Forbo-Krommenie B.V.

- remaining data: annex 1 Forbo-Krommenie B.V.

\section{P7: Production of linoleum cement (oxidation)}

Process taking place at Forbo-Krommenie B.V.-Krommenie. The reference amount is de total cement production by Forbo-Krommenie B.V. in 1998

- data electricity and use of gas: annex 5 Forbo-Krommenie B.V.

- data emission dust/particles and NMVOC: annex 6 Forbo-Krommenie B.V.

- data steam use: Keuken \& de Koning, 1997

- remaining data: annex 1 Forbo-Krommenie B.V.

- waste $($ annex 1) $\Rightarrow$ ETH-848: abfall in inertstoffdeponie

P8: Kalandering linoleum

Process taking place at Forbo-Krommenie B.V.-Krommenie. The reference amount is de total flow of linoleum from the kalanders at Forbo-Krommenie B.V. in 1998

- data electricity use: annex 5 Forbo-Krommenie B.V. 
- data emission NMVOC: annex 6 Forbo-Krommenie B.V.

- data steam use: Keuken \& de Koning, 1997

- remaining: annex 1 and 4 Forbo-Krommenie B.V.; see for calculations exel-file "berekening benodigde hoev grondstof"; In this file, the amounts needed for a certain type of linoleum are calculated based on the total inflow in 1998 (annex 1) and the ratio between the materials in the final product (annex 4)

- Granulate waste and jute waste are treated together as linoleum waste. This waste is burned and treated as "linoleum waste"(although the ration between jute and granulate is somewhat higher than the ratio jute/cement in the final product (annex 4)).

\section{P9: Production of steam at Forbo-Krommenie B.V.}

Proces taking place at Forbo-Krommenie B.V.-Krommenie. Reference amount is all steam produced and used by Forbo-Krommenie B.V. (100\%) in 1998. Gas input is the gas use of the steam kettles

- data gas use:

- ammount: annex 5 Forbo-Krommenie B.V.

- process: ETH-427: Erdgas in Industriefeuerung >100kW Euro (aggregated process production and use of gas)

- data emission $\mathrm{NO}_{\mathrm{x}}$ : annex 6 Forbo-Krommenie B.V.

P10: Electricity use at Forbo-Krommenie B.V.

Reference amount is all electricity used by Forbo-Krommenie B.V. (100\%) in 1998. Input is electricity from the Dutch net.

- data electricity use:

- amount: annex 5 Forbo-Krommenie B.V.

- ETH-99: Strom Mittelspannung - Bezug in NL Import

\section{P11: Drying of 2,5 mm Marmoleum/Artoleum}

Process taking place at Forbo-Krommenie B.V.-Krommenie. The reference amount is de total amount of linoleum from the drying chambers at Forbo-Krommenie B.V. in 1998

- data electricity and use of gas: annex 5 Forbo-Krommenie B.V.

- data steam use: Keuken \& de Koning, 1997

- data emission NMVOC: annex 6 Forbo-Krommenie B.V.

- remaining data: annex 1 Forbo-Krommenie B.V.

\section{P12: Trimming of $2,5 \mathrm{~mm}$ Marmoleum/Artoleum}

Process taking place at Forbo-Krommenie B.V.-Krommenie. The reference amount is de total amount of trimmed linoleum at Forbo-Krommenie B.V. in 1998

- data electricity use: annex 5 Forbo-Krommenie B.V.

- data steam use: Keuken \& de Koning, 1997

- data emission NMVOC: annex 6 Forbo-Krommenie B.V. databronnen:

- data lacquer: annex 1 and 4 Forbo-Krommenie B.V.; see for calculations exel-file "berekening benodigde hoev grondstof"; In this file, the amounts needed for a certain type of linoleum are calculated based on the total inflow in 1998 (annex 1) and the ratio between the materials in the final product (annex 4)

- remaining data: annex 1 Forbo-Krommenie B.V.

- $\quad$ waste $=$ remaining lacquer $=>$ ETH-849: abfall in reststoffdeponie

\section{P13: Production + transport to Forbo-Krommenie B.V. of wood flour (milling)}

Milling and transportation from the mill to Forbo-Krommenie B.V.. Reference amount is $1 \mathrm{~kg}$ wood flower

- ratio between suppliers to Forbo-Krommenie B.V. known

- all data received from suppliers via Forbo-Krommenie B.V.

- data use of electricity:

- $\quad$ amount: weighted average suppliers

- process: ETH-102: Strom Mittelspannung - Bezug in UCPTE 
- data transportation distance: weighted average distance from plant suppliers to Forbo-Krommenie B.V.

- ratio raw wood/wood reminders: information from one supplier only

\section{P14 \& P15: Sawing of raw wood}

- ratio between suppliers to Forbo-Krommenie B.V. known

- allocation between wood remainders and production wood is based on data on economic value provided by Forbo-Krommenie B.V. ( value produced wood remainders $=15 \%$ )

- all data received from suppliers via Forbo-Krommenie B.V.

- data electricity use: data form one supplier only

- ratio use production wood/wood remainders: data from one supplier only

\section{P16: Forestry (production of wood)+ transport to mill}

Production of wood + transportation to the sawmill

- ratio between suppliers to Forbo-Krommenie B.V. known

- all data received from suppliers via Forbo-Krommenie B.V.

- no fertiliser use (although one supplier mentions the use of $P$ )

- data for use of diesel: date from one supplier only

- transportation distance forest to mill: weighted average suppliers

\section{P17: Production of linseed oil and transport to Forbo-Krommenie B.V.}

- ratio between suppliers to Forbo-Krommenie B.V. known

- allocation between linseed oil and linseed expellers is based on data on economic value provided by one supplier (price) and all suppliers (amount produced, all give the same produced amount per $\mathrm{kg}$ linseed; value produced inseed oil $=57 \%$ )

- all data received from suppliers via Forbo-Krommenie B.V.

- data use of gas:

- amount: data from the largest provider for Forbo-Krommenie B.V.. In this case not the weighted average is chosen because the energy type of other supplier(s) is not known.

- process: ETH-427: Erdgas in Industriefeuerung $>100 \mathrm{~kW}$ Euro (aggregated process production and use of gas)

- the amount linseed oil produced from $1 \mathrm{~kg}$ linseed: weighted average suppliers. All give the same amounts for linseed oil and expellers

- transport by inland freighter is based on a weighted average of suppliers of the transportation distance from plant to Forbo-Krommenie B.V. $(=184.5 \mathrm{e}-3 \mathrm{tkm} \times 0.33)+$ the transportation distance from the harbour to the plant (= 154e-3 tkm).

\section{P18: Growing of linseed}

Worse case scenario for pesticide emissions: assumption all mentioned pesticides are used and all in the maximal dose.

- suppliers to Forbo-Krommenie B.V. known, ratio not known

- all data received from suppliers via Forbo-Krommenie B.V.

- transportation of seed by truck in India, by sea freighter and by inland freighter in Europa is based on the average distances of suppliers; transport by truck can also partly be done by train (Linora has a transportation distance in Europe of $0 \mathrm{~km}$ by inland freighter because they are situated in the harbour)

- use of diesel: average suppliers (0.429 MJ \& $1.74 \mathrm{MJ})$.

- use of $\mathrm{N}$ - and P-fertiliser: average suppliersI

- use of fertiliser S: one supplier (not accounted for, treated as datagap ( $\mathrm{S}$ is not mentioned by other supplier(s)

- use of pesticides: herbicides: one supplier, insecticides: another supplier

The amount used is the maximal amount in the worse case scenario (in P113 the best estimate for pesticide use is used, based on the assumption that on $20 \%$ of the fields one herbicide and one pesticide is used (based on e-mail supplier). The total amount used is seen as an emission. Division of the emissions over water/air/soil based on the MJP-G emission evaluation. 
- emissions of $\mathrm{NH} 3$ and $\mathrm{N} 2 \mathrm{O}$ to air are based on the assumption that $1 \%$ of the applied fertiliser evaporates as $\mathrm{NH} 3$ and $1 \%$ as $\mathrm{N} 2 \mathrm{O}$ to air (Välimaa \& Stadig, 1998). The weights are adapted to the molecular weight of the substances. The emission of $\mathrm{N}$ to soil is based on the difference between the dose of $\mathrm{N}$-fertiliser and the estimated amount harvested in the linseed minus the amount emitted to air.

\section{P19: Transport, spinning and weaving of jute}

Including all transportation of yarn and jute in India and Europe

- ratio between suppliers to Forbo-Krommenie B.V. known

- all data received from suppliers via Forbo-Krommenie B.V.

- data use of electricity for spinning: one supplier (these are the only data on spinning): $1.44 \mathrm{MJ}$

- data use of electricity for weaving: weighted average all suppliers who gave realistic information on this: $1 \mathrm{MJ}$, unrealistic high value for electricity use was not used

- data use of gas for weaving: one supplier

- data use of oil for weaving: not known, therefore not included (some suppliers mention the use of oil but give no, or unrealistic high values)

- Transportation per sea freighter India-Rotterdam: one supplier (other companies give no data for transportation from India to Europe)

- Transportation in India per truck: one supplier $425 \mathrm{~km}$ (other companies give no data for transportation in India)

- Transportation in Europe per truck: $375 \mathrm{~km}$ (based on a weighted average of the transportation distances given by all suppliers

- re-usable jute waste $2 \%$ : two suppliers (both give the same value)

- other waste $1,5 \%$ : one supplier (based on a total waste of $3,5 \%$ of which $2 \%$ is re-usable, see before) $\Rightarrow$ ETH-848: abfall in inertstoffdeponie

- use of starch, oil and salt: one supplier (this supplier gave besides answer to our questions also their environmental report, therefore there data are more complete than those of the other companies)

P20: Transport raw rosin from plantation, extraction and transport to Forbo-Krommenie B.V.

- all data received from suppliers via Forbo-Krommenie B.V.

- Ratio for suppliers not known, therefore unweighted averages are used

- allocation between linseed oil and linseed expellers is based on data on economic value provided by suppliers (both give approx. the same economic value: value produced gum rosin $=91 \%$ )

- transportation in Indonesia by truck: average of the figures of suppliers (very rough estimation

- transportation Amsterdam - Krommenie: $20 \mathrm{~km}$

- average distance Indonesia - Amsterdam by sea freighter: summary data Forbo-Krommenie B.V. 1997 (1999 data not reliable)

- production of rosin and terpentine, input of raw rosin: twol suppliers 1999 (both give the same figures)

- use middle fuel oil: one supplier

\section{P21: Mining of limestone}

- data from only one supplier available (via Forbo-Krommenie B.V.)

- data sources: only use diesel:

- alternative possibility: ETH, not tried because of lack of time

\section{P22: Grinding + transport of limestone}

- data source: all data from one supplier (via Forbo-Krommenie B.V.)

- assumption: emission from particles are due to grinding

- waste $=>E T H-848$ : abfall in inertstoffdeponie

- $\quad$ assumption oil $=$ middle fuel oil

\section{P23 \& 24: Transport of tall oil and paper}

Based on the location of the only supplier for tall oil

- transport by truck, 125 km Rotterdam- Krommenie: estimation 
- transport by sea freighter: supplier, 1999 (via Forbo-Krommenie B.V.)

- transport by train, supplier - harbour: supplier, 1999 (via Forbo-Krommenie B.V.)

- data on the production process of paper (and tall oil) from ETH (ETH-184), data from supplier seem not complete

\section{P25, P26 \& P27: Production of $\mathrm{TiO}_{2}$}

- data sources:

- P25: PE Germany, these data are most complete because they are aggregated data including mining, transportation, energy use etc.

- P26: sulphate process:

- all data received from suppliers via Forbo-Krommenie B.V. (ratio known)

- sulfuric acid and Ti-ore: weighted average suppliers

- use of gas, emissions $\mathrm{NO}_{x}$, heavy metals $\& \mathrm{TiO}_{2}$ : main supplier

- transport by truck \& solid waste: one supplier

- transport Ti-ore very rough estimation (based on ore from Norway)

- P27, chloride process: data from one supplier (via Forbo-Krommenie B.V.)

- waste $=>E T H-848$ : abfall in inertstoffdeponie

\section{P28: Burning Linoleum}

- the production of usefull energy is substituted by electricity (ETH-99: Strom Mittelspannung Bezug in NL Import).

- The exchange rate is assumed $0.4 \mathrm{MJ} / \mathrm{MJ}$

- $\quad$ waste $=>E T H-848$ : abfall in inertstoffdeponie

- assumption: transportation distance to incinerator: $100 \mathrm{~km}$.

\section{P29: production of starch}

- data source: Spin 1994

- data not complete: date concerning the use of raw materials (potatos cereals) are lacking

- note the amounts of water emissions (sulphate as S, salt as CL; etc.)

- data concerning waste are a rough estimation (mostly terra and slug)

\section{P30, 31, 120, 121 \& 122: production lacquer, adhesives and maintenance products}

- data sources: one supplier (via Forbo-Krommenie B.V.)

- data concerning the production of raw materials (additives, thickeners, solvents and waxdispersions) are not available.

- assumtion 11 product $=1 \mathrm{~kg}$

chemical waste $=>E T H-849$ : abfall in reststoffdeponie

\section{P32, 33, 34 \& 37: Production of fertiliser}

- aggregated data including transport

- data: Davis \& Hagland, 1999

- assumption: emissions resulting from the use of energy carriers are included in Davis \& Hagland, interventions related to the production of these energy carriers are not included, Therefor the related ETH-processes are added.

energy content of diesel, oil and cole are from BINAS, weight oil and diesel from ETH.

- waste:

- radioactive waste $=>E T H-631$ :radioactiver abfall in depot $\mathrm{C}$

- hazardous waste $=>$ abfall in reactor deponie $(50 \%$ ETH-884: Kunststoffe in Reaktordeponie \& $50 \%$ ETH-870: Holz in Reaktordeponie)

- industrial waste $=>E T H-848$ : Abfaelle in Inertstoffdeponie

- $\quad$ solid waste $=>$ ETH-848: Abfaelle in Inertstoffdeponie

\section{P35: Linoleum installation $(2,5 \mathrm{~mm})$}

Inputs and outputs related to installation of linoleum

- assumption: $6 \%$ of the linoleum is wasted during installation (see Potting \& Blok, 1993) 
- waste $=6 \%$ linoleum + packaging + marmoweld $($ small fraction $) \Rightarrow$ not burned but landfilled: ETH 848: Abfaelle in Inertstoffdeponie

- assumption: transportation distance $=100 \mathrm{~km}$ in NL $(=0.29 \mathrm{tkm})$

- use of adhesives and other product from suppliers (via Forbo-Krommenie B.V.)

P36: The use and maintenance of $1 \mathrm{~m} 2$ linoleum for 20 years

- Maintenance and use of products is based on information from Forbo-Krommenie B.V.

- all linoleum is considered to be burned after 20 years

P38 -83 \& 85: ETH-processes

datasource: ETH 1996

Transportation by truck is assumed to take place with $40 \mathrm{t}$ trucks

P84: Production of raw gum resin

Empty process, according to Forbo-Krommenie B.V., the harvesting of raw gum rosin is done mainly by hand.

P86 \& P87: production of tall oil (and paper)

- allocation between those two products based on data on economic value provided by ForboKrommenie B.V.

- value produced tall oil $=1 \%$

P88 -P90: Production of DouPol, InterClean \& DuoClean

- data sources: one supplier (via Forbo-Krommenie B.V.)

- chemical waste $=\mathrm{ETH}-849$ : Abfaelle in Reststoffdeponie

P91: Translation NMVOC based on the emissions during stationary combustion

- data source: Hauschild \& Wenzel (1998)

P92: growing of linseed (best estimate)

- See P18 for data

- assumption $20 \%$ of the fields one herbicide and one pesticide is used.

P93 -P96: Production of detergents

- Selection of three detergents

- Data from Stalmans et al. (1995)

P97-P103, P60, P137 \& P141: Maintenance options for linoleum

- data for P97-P103: Forbo-Krommenie B.V.

- data for P137-P141: Forbo-Krommenie B.V.-Forshaga, Fax 9 July 1999.

P104: Production of Marmoweld

- data source: one supplier (via Forbo-Krommenie B.V.)

- chemical waste $=>$ ETH-849: Abfaelle in Reststoffdeponie

P105-P108: Production of ingredients for maintenance products, adhesive etc.

- data source:one supplier (via Forbo-Krommenie B.V.)

- chemical waste $=$ ETH-849: Abfaelle in Reststoffdeponie

- solid waste $=\mathrm{ETH}-848$ : Abfaelle in Inertstoffdeponie

P109: Production of yarn

Empty process, according to Forbo-Krommenie B.V., the growing and harvesting of yarn is done mainly by hand 
P110-P129: copies of ETH processes and NMVOC translation

These are needed to split the total system into four different stages: production of raw materials, production of linoleum at Forbo-Krommenie B.V., use and maintenance, disposal

P130: Translation NMVOC based on the emissions during solvent use

- data source: Hauschild \& Wenzel (1998)

- used as an estimation for the emission of NMVOC at Forbo-Krommenie B.V.

P131: SWEDISH SCENARIO: The use and maintenance of $1 \mathrm{~m} 22,0 \mathrm{~mm}$ linoleum for 20 years and the disposal

Differences with P36 based on the Swedisch maintenance (information Forbo-Krommenie B.V.-

Forshaga, Fax 9 July 1999) and the difference in weight between 2, $5 \mathrm{~mm}$ and 2,0 mm linoleum

P132: SWEDISH SCENARIO: linoleum installation $(2,0 \mathrm{~mm})$

Difference with P35 are due to the transportation distance and the difference in weight between 2, 5 $\mathrm{mm}$ and 2,0 $\mathrm{mm}$ linoleum

- assumption transportation to Sweden: $1000 \mathrm{~km}$ by freighter and $1000 \mathrm{~km}$ by truck

P133-P136: SWEDISH SCENARIO:

Difference with $\mathrm{P} 1, \mathrm{P} 8, \mathrm{P} 11$ and $\mathrm{P} 12$ are due to the difference in weight and relative composition between 2, $5 \mathrm{~mm}$ and 2,0 $\mathrm{mm}$ linoleum see annex 4 and see for calculations exel-file "berekening benodigde hoèv grondstof"

P142-P148: Scenario Cork- linoleum

Difference with P4, P5, P8, P11, P12, P35 \& P36 are due to the difference in weight and relative composition between 2, $5 \mathrm{~mm}$ and Cork-linoleum see annex 4 and see for calculations exelfile."berekening benodigde hoev grondstof"

\section{P149: Seaving cork}

Reference flow is total amount of cork sieved in 1998 at Forbo-Krommenie B.V.

- data electricity use: annex 5 Forbo-Krommenie B.V.

- data emission dust: annex 6 Forbo-Krommenie B.V.

P150: transportation of cork to mill, milling and transportation to Forbo-Krommenie B.V. of cork granulate

- data from the main supplier

- Note: cork granulate is a by-product of corks used for bottles etc. However nothing is known of the production of this main product.

- Data on energy use from supplier (via Forbo-Krommenie B.V.)

It is assumed that the energy mentioned by supplier used for drying is all used for the production of the granulate and not for corks, as these are not mentioned by the suppliers and the cork granulate is explicitly mentioned as the product by supplier.

- The transportation of the cork granulate to the Portuguese harbour and to Forbo-Krommenie B.V. is based on the average values from all suppliers (via Forbo-Krommenie B.V.). $+60 \mathrm{~km}$ for transportation in the Netherlands (estimation)

- The transportation from the woods to the mill is "allocated" based on mass (assuming that $1 \mathrm{~kg}$ cork from the woods results in corks and cork granulate without much waste), as economic values of corks and cork-granulate is not known. Distance between the woods and the mill is based on the average data from all suppliers (via Forbo-Krommenie B.V.).

P151-P153: SCENARIO USA

- an assumption is made about the extra transportation distance per boat and truck 


\section{P154-P162: SCENARIO NO TALL OIL}

Production of linoleum cement (oxidation) without tall oil. Process taking place at Forbo-Krommenie B.V.-Krommenie. The reference amount is de total cement production by Forbo-Krommenie B.V. in 1998

- data on ratio linseed oil / resin: annex 4 and 4a Forbo-Krommenie B.V.

- data electricity and use of gas: annex 5 Forbo-Krommenie B.V.

- data emission dust/particles and NMVOC: annex 6 Forbo-Krommenie B.V.

- data steam use: Keuken \& de Koning, 1997

- remaining data: annex 1 Forbo-Krommenie B.V.

\section{P163-P164: Dutch $2.0 \mathrm{~mm}$}

- data from the production of $2.0 \mathrm{~mm}$ (Swedish scenario) combined with installation, use and, maintenance in the Netherlands

P164-P166: Production of Yellow and Red pigment

- Aggregated data on the production of two pigments, data from CE

\section{P167: Production of organic chemical}

- Transportation by truck based on the assumption that the distance from production site to user is $100 \mathrm{~km}$

- other data from ETH: production of organic chemical

\section{P168: Production of capital goods}

- estimation of the yearly investments in capital goods of Forbo-Krommenie B.V. of the yearly production in fl.

- emissions and environmental inputs per $1 \mathrm{e} 6$ \$ from Carnegie Mellon. internet-site

- hazardous waste: => abfall in reactor deponie (50\% ETH-884: Kunststoffe in Reaktordeponie \& 50\% ETH-870: Holz in Reaktordeponie)

- conversion $\$=>\mathrm{fl}$. based on the values of $25 / 4 / 2000$

Annex 1 Forbo-Krommenie B.V.: Flowsheet linoleum production 1998

Annex 2 Forbo-Krommenie B.V.: mass balance linoleum production

Annex 4 Forbo-Krommenie B.V.: composition linoleum $2.0 \mathrm{~mm}, 2.5 \mathrm{~mm}$, linoleum without tall oil, corklinoleum and bulletin board

Annex 5 Forbo-Krommenie B.V.: Energy use Forbo-Krommenie B.V.: 1998

Annex 6 Forbo-Krommenie B.V.: Emissions to air Forbo-Krommenie B.V. 1998 
Appendix B. Intervention tables

\begin{tabular}{|c|c|c|c|}
\hline Environmental flow & $\begin{array}{l}\text { BAseline } \\
\text { (Dutch) }\end{array}$ & Swedish & Cork \\
\hline [E16] cobalt (Co) from earth resources & $-3.16 \mathrm{E}-06$ & $-2.67 \mathrm{E}-06$ & $-3.06 \mathrm{E}-06 \mathrm{~kg}$ \\
\hline [E17] copper $(\mathrm{Cu})$ from earth resources & -0.7435 & -0.74809 & $-0.66445 \mathrm{~kg}$ \\
\hline [E18] chromium $(\mathrm{Cr})$ from earth resources & -0.26267 & -0.35548 & $-0.23928 \mathrm{~kg}$ \\
\hline [E33] iron (Fe) from earth resources & -150.33 & -149.98 & $-129.96 \mathrm{~kg}$ \\
\hline [E38] lead $(\mathrm{Pb})$ from earth resources & -0.8873 & -1.3594 & $-0.83331 \mathrm{~kg}$ \\
\hline [E41] manganese $(\mathrm{Mn})$ from earth resources & -0.092737 & -0.12201 & $-0.083536 \mathrm{~kg}$ \\
\hline [E43] molybdenum (Mo) from earth resources & $-2.90 \mathrm{E}-06$ & $-1.95 E-06$ & $-2.85 \mathrm{E}-06 \mathrm{~kg}$ \\
\hline [E46] nickel (Ni) from earth resources & -0.15328 & -0.20956 & $-0.14015 \mathrm{~kg}$ \\
\hline [E49] palladium ( $P d)$ from earth resources & $-5.53 E-07$ & $-7.91 E-07$ & $-5.19 \mathrm{E}-07 \mathrm{~kg}$ \\
\hline [E50] phosphorus $(P)$ from earth resources & -16.445 & -12.495 & $-11.692 \mathrm{~kg}$ \\
\hline [E51] platinum (Pt) from earth resources & $-6.59 E-07$ & $-9.49 E-07$ & $-6.20 \mathrm{E}-07 \mathrm{~kg}$ \\
\hline [E57] rhenium (Re) from earth resources & $-4.93 E-07$ & $-6.90 \mathrm{E}-07$ & $-4.63 \mathrm{E}-07 \mathrm{~kg}$ \\
\hline [E58] rhodium ( $R h$ ) from earth resources & $-5.94 \mathrm{E}-07$ & $-8.52 E-07$ & $-5.59 \mathrm{E}-07 \mathrm{~kg}$ \\
\hline [E65] silver (Ag) from earth resources & -0.0046019 & -0.0040948 & $-0.0043543 \mathrm{~kg}$ \\
\hline [E68] sulfur (S) from earth resources & -27.031 & -9.5491 & $-27.031 \mathrm{~kg}$ \\
\hline$[E 75]$ tin $(\mathrm{Sn})$ from earth resources & -0.0025542 & -0.002271 & $-0.0024167 \mathrm{~kg}$ \\
\hline [E78] uranium $(U)$ from earth resources & -0.051527 & -0.038311 & $-0.0394 \mathrm{~kg}$ \\
\hline [E83] zinc $(\mathrm{Zn})$ from earth resources & -0.023439 & -0.03648 & $-0.021899 \mathrm{~kg}$ \\
\hline [E85] oil crude from earth resources & -1602 & -1427.7 & $-1610.1 \mathrm{~kg}$ \\
\hline [E86] natural gas from earth resources & -1174.8 & -784.1 & $-2040.2 \mathrm{~m} 3$ \\
\hline [E87] coal soft from earth resources & -1148.5 & -713.99 & $-1181.9 \mathrm{~kg}$ \\
\hline [E88] coal hard from earth resources & -529.61 & -391.07 & $-371.26 \mathrm{~kg}$ \\
\hline [E90] 1,1,1-trichloroethane (methyl chloroform; $\mathrm{HC}-140 \mathrm{a}$ ) to air & 0.11022 & 0.085923 & $0.11954 \mathrm{~kg}$ \\
\hline [E97] 1,2,3-Trimethyl Benzene to air & 0.19434 & 0.18674 & $0.18891 \mathrm{~kg}$ \\
\hline [E100] 1,2,4-trimethylbenzene to air & 0.23057 & 0.21499 & $0.22821 \mathrm{~kg}$ \\
\hline [E109] 1,3,5-trimethylbenzene (mesitylene) to air & 0.20222 & 0.19288 & $0.19746 \mathrm{~kg}$ \\
\hline [E123] 1-Butyl Acetate to air & 0.025271 & 0.0197 & $0.027408 \mathrm{~kg}$ \\
\hline [E130] 1-Decane to air & 0.069229 & 0.053968 & $0.075081 \mathrm{~kg}$ \\
\hline [E131] 1-Dodecane to air & 0.086402 & 0.067355 & $0.093707 \mathrm{~kg}$ \\
\hline [E134] 1-Heptane to air & 0.037392 & 0.029149 & $0.040553 \mathrm{~kg}$ \\
\hline [E137] 1-Hexane to air & 0.057614 & 0.044913 & $0.062485 \mathrm{~kg}$ \\
\hline [E141] 1-Nonane to air & 0.080836 & 0.063016 & $0.08767 \mathrm{~kg}$ \\
\hline [E143] 1-Octane to air & 0.028805 & 0.022455 & $0.031241 \mathrm{~kg}$ \\
\hline [E147] 1-Pentane to air & 0.014401 & 0.011226 & $0.015618 \mathrm{~kg}$ \\
\hline [E150] 1-Propyl Benzene to air & 0.19025 & 0.18339 & $0.18458 \mathrm{~kg}$ \\
\hline [E154] 1-Undecane to air & 0.086402 & 0.067355 & $0.093707 \mathrm{~kg}$ \\
\hline [E168] 2,3,7,8-TCDD (tetrachloride-dibenzo-dioxin) to air & $7.49 \mathrm{E}-10$ & $7.09 \mathrm{E}-10$ & $6.12 \mathrm{E}-10 \mathrm{~kg}$ \\
\hline [E182] 2.3- Dimethylbutane to air & 0.020225 & 0.015767 & $0.021935 \mathrm{~kg}$ \\
\hline [E199] 2-Methylheptane to air & 0.063408 & 0.04943 & $0.068768 \mathrm{~kg}$ \\
\hline [E200] 2-Methylhexane to air & 0.017201 & 0.013409 & $0.018655 \mathrm{~kg}$ \\
\hline [E201] 2-Methylnonane to air & 0.040416 & 0.031507 & $0.043833 \mathrm{~kg}$ \\
\hline [E202] 2-Methyloctane to air & 0.026012 & 0.020278 & $0.028211 \mathrm{~kg}$ \\
\hline [E203] 2-Methylpentane to air & 0.051797 & 0.040378 & $0.056175 \mathrm{~kg}$ \\
\hline [E223] 3-Methylhexane to air & 0.014408 & 0.011232 & $0.015626 \mathrm{~kg}$ \\
\hline [E224] 3-Methylpentane to air & 0.037392 & 0.029149 & $0.040553 \mathrm{~kg}$ \\
\hline [E232] Acetaldehyde (ethanal) to air & 0.001233 & 0.0009408 & $0.0009588 \mathrm{~kg}$ \\
\hline [E233] Acetic acid to air & 0.0096433 & 0.0069185 & $0.012838 \mathrm{~kg}$ \\
\hline [E234] Acetone (2-propanon) to air & 0.11139 & 0.086818 & $0.1204 \mathrm{~kg}$ \\
\hline
\end{tabular}


[E235] Acetylene to air

[E236] Acrolein (2-propenal) to air [E239] aldehydes (unspec.) to air [E242] alkanes (unspec.) to air [E243] alkenes (unspec.) to air [E249] ammonia, ammonium to air [E252] antimony to air [E253] aromatics (unspecified) to air

[E254] arsenic to air

[E258] barium to air

[E261] Benzaldehyde to air

[E262] Benzene to air

[E264] benzo[a]pyrene to air

[E269] beryllium to air

[E286] butane (unspec.) to air

[E384] cadmium to air

[E392] Carbon dioxide to air

[E394] Carbon Monoxide to air

[E397] CFC-11, to air

[E399] CFC-114 to air

[E401] CFC-12 to air

[E402] CFC-13 to air

[E411] chlorpyriphos to air

[E412] chromium (unspecified) to air

[E420] cobalt to air

[E421] copper to air

[E425] Cyanides to air

[E440] deltamethrin to air

[E448] Dichloromethane (Methylene Chloride) to air

[E460] dimethoate to air

[E468] Dinitrogen oxide (nitrous oxide) to air

[E482] Ethane to air

[E484] Ethanol to air

[E487] Ethyl Acetate to air

[E495] Ethylbenzene to air

[E498] Ethylene (ethene) to air

[E500] Ethylene Dichloride to air

[E510] Formaldehyde (methanal) to air

[E515] glyphosate to air

[E520] HALON-1301 to air

[E528] HCFC-22 (Chlorodifluormethane) to air

[E533] heptane to air

[E536] hexachlorobenzene to air

[E544] HFC-134a (1,1,1,2-tetrafluoroethane) to air

[E559] hydrogen chloride to air

[E560] hydrogen fluoride to air

[E561] hydrogen sulfide to air

[E570] isobutyl acetate to air

[E573] isopentane to air

[E577] isopropyl acetate to air

[E578] isopropyl benzene (cumene) to air

[E581] lead to air

\begin{tabular}{rrr}
0.72736 & 0.70768 & $0.70128 \mathrm{~kg}$ \\
$1.06 \mathrm{E}-06$ & $1.05 \mathrm{E}-06$ & $9.63 \mathrm{E}-07 \mathrm{~kg}$ \\
$4.00 \mathrm{E}-05$ & $2.96 \mathrm{E}-05$ & $2.84 \mathrm{E}-05 \mathrm{~kg}$ \\
0.047882 & 0.039719 & $0.043909 \mathrm{~kg}$ \\
0.0022927 & 0.0017444 & $0.00137 \mathrm{~kg}$ \\
21.408 & 16.266 & $15.22 \mathrm{~kg}$ \\
0.0029855 & 0.002347 & $0.002986 \mathrm{~kg}$ \\
0.0015145 & 0.0010898 & $0.0013002 \mathrm{~kg}$ \\
0.0078946 & 0.0064217 & $0.0078653 \mathrm{~kg}$ \\
0.000547 & 0.0003929 & $0.0004062 \mathrm{~kg}$ \\
$5.53 \mathrm{E}-07$ & $5.50 \mathrm{E}-07$ & $5.03 \mathrm{E}-07 \mathrm{~kg}$ \\
1.5063 & 1.4696 & $1.4633 \mathrm{~kg}$ \\
$3.54 \mathrm{E}-05$ & $2.64 \mathrm{E}-05$ & $3.33 \mathrm{E}-05 \mathrm{~kg}$ \\
$1.11 \mathrm{E}-05$ & $8.22 \mathrm{E}-06$ & $8.20 \mathrm{E}-06 \mathrm{~kg}$ \\
0.1652 & 0.13447 & $0.18827 \mathrm{~kg}$ \\
0.0059176 & 0.0047136 & $0.0059012 \mathrm{~kg}$ \\
7254.8 & 5839.8 & $8938.5 \mathrm{~kg}$ \\
57.156 & 47.533 & $90.837 \mathrm{~kg}$ \\
$1.25 \mathrm{E}-05$ & $9.09 \mathrm{E}-06$ & $8.19 \mathrm{E}-06 \mathrm{~kg}$ \\
0.000423 & 0.0003144 & $0.0003058 \mathrm{~kg}$ \\
$2.69 \mathrm{E}-06$ & $1.96 \mathrm{E}-06$ & $1.76 \mathrm{E}-06 \mathrm{~kg}$ \\
$1.68 \mathrm{E}-06$ & $1.22 \mathrm{E}-06$ & $1.10 \mathrm{E}-06 \mathrm{~kg}$ \\
0.013647 & 0.010369 & $0.0097026 \mathrm{~kg}$ \\
0.0033873 & 0.0026978 & $0.0032314 \mathrm{~kg}$ \\
0.0011762 & 0.0009416 & $0.0010649 \mathrm{~kg}$ \\
0.040295 & 0.02905 & $0.039852 \mathrm{~kg}$ \\
\hline $8.48 \mathrm{E}-05$ & $6.68 \mathrm{E}-05$ & $7.55 \mathrm{E}-05 \mathrm{~kg}$ \\
$3.70 \mathrm{E}-05$ & $2.81 \mathrm{E}-05$ & $2.63 \mathrm{E}-05 \mathrm{~kg}$ \\
0.087648 & 0.068326 & $0.095056 \mathrm{~kg}$ \\
0.0056904 & 0.0043235 & $0.0040457 \mathrm{~kg}$ \\
\hline 30.384 & 23.159 & $21.622 \mathrm{~kg}$ \\
3.2256 & 3.056 & $3.2881 \mathrm{~kg}$ \\
0.40033 & 0.31205 & $0.43337 \mathrm{~kg}$ \\
0.043323 & 0.033773 & $0.046986 \mathrm{~kg}$ \\
0.22002 & 0.20704 & $0.21555 \mathrm{~kg}$ \\
3.3703 & 3.5362 & $3.24 \mathrm{~kg}$ \\
0.0001221 & $8.44 \mathrm{E}-05$ & $0.0001518 \mathrm{~kg}$ \\
3.6656 & 3.5582 & $3.5377 \mathrm{~kg}$ \\
0.0017776 & 0.0013506 & $0.0012638 \mathrm{~kg}$ \\
0.0005758 & 0.0005186 & $0.0005448 \mathrm{~kg}$ \\
$2.98 \mathrm{E}-06$ & $2.17 \mathrm{E}-06$ & $1.99 \mathrm{E}-06 \mathrm{~kg}$ \\
0.028313 & 0.024539 & $0.026862 \mathrm{~kg}$ \\
\hline $.56 \mathrm{E}-09$ & $5.10 \mathrm{E}-09$ & $4.13 \mathrm{E}-09 \mathrm{~kg}$ \\
$-7.32 \mathrm{E}-17$ & $-4.97 \mathrm{E}-17$ & $-1.33 \mathrm{E}-16 \mathrm{~kg}$ \\
1.3237 & 1.0317 & $1.3006 \mathrm{~kg}$ \\
0.15508 & 0.12061 & $0.14523 \mathrm{~kg}$ \\
0.049989 & 0.034578 & $0.06183 \mathrm{~kg}$ \\
0.031468 & 0.024531 & $0.034128 \mathrm{~kg}$ \\
0.18729 & 0.0066939 & $0.0093128 \mathrm{~kg}$ \\
0.15153 & 0.15187 & $0.021128 \mathrm{~kg}$ \\
& 0.12166 & $0.18117 \mathrm{~kg}$ \\
\hline & & $0.15189 \mathrm{~kg}$ \\
\hline
\end{tabular}


[E584] malathion to air

0.018935

[E585] Mangane to air

[E586] MCPA to air

[E588] mercury to air

[E590] meta-Ethyltoluene to air

[E592] meta-Xylene (1,3-dimethylbenzene) to air

[E596] Methane to air

[E598] Methanol to air

[E599] methomyl to air

[E600] Methyl Acetate to air

[E608] Methyl Ethyl Ketone to air

[E611] Methyl Isobutyl Ketone (4-methylpentanon-2) to air

[E619] Methyl trans-Butyl Ether to air

[E627] molybdenum to air

[E630] nickel to air

[E634] nitrogen to air

[E637] nitrogen oxides to air

[E642] ortho-Ethyltoluene to air

[E643] ortho-Xylene (1,2-dimethylbenzene) to air

[E647] para-Ethyltoluene to air

[E651] para-Xylene (1,4-dimethylbenzene) to air

[E653] pentachlorobenzene to air

[E655] pentachlorophenol to air

[E660] Perfluoroethane (CFC-116) to air

[E662] Perfluoromethane (CFC-14) to air

[E665] permethrin to air

[E668] Phenol to air

[E671] Phosphorus to air

[E676] PM10 to air

[E677] Polycyclic Aromatic Hydrocarbons (PAH) (unspecified) to air [E680] Propane to air

[E681] Propanoic acid (propionic acid) to air

[E682] Propionaldehyde (propanal) to air

[E686] Propylene to air

[E697] selenium to air

[E702] sulphur dioxide to air

[E703] Sulphur hexafluoride to air

[E711] tetrachloroethylene (PER) (tetrachloroethene) to air

[E712] Tetrachloromethane (carbon tetrachloride) (HC-10) to air

[E715] thallium to air

[E717] tin to air

[E720] Toluene to air

[E729] trichlorfon to air

[E730] Trichloroethylene (tri) to air

[E731] Trichloromethane (chloroform) to air

[E736] vanadium to air

[E738] Vinyl Chloride (chloroethene) to air

[E741] zinc to air

[E743] 1,1,1-trichloroethane to air

[E772] ammonia, ammonium to fresh water

[E775] antimony to fresh water

[E776] arsenic to fresh water $\begin{array}{lll}0.045489 & 0.037225 & 0.044606 \mathrm{~kg}\end{array}$

$\begin{array}{lll}0.0037265 & 0.0028313 & 0.0026494 \mathrm{~kg}\end{array}$

$\begin{array}{lll}0.0031749 & 0.0024938 & 0.0031691 \mathrm{~kg}\end{array}$

$0.19987 \quad 0.19105 \quad 0.19492 \mathrm{~kg}$

$\begin{array}{lll}0.24148 & 0.22349 & 0.24004 \mathrm{~kg}\end{array}$

$24.552 \quad 17.966 \quad 26.609 \mathrm{~kg}$

$\begin{array}{lll}0.062396 & 0.048629 & 0.066711 \mathrm{~kg}\end{array}$

$\begin{array}{lll}0.0065717 & 0.0049931 & 0.0046723 \\ k g\end{array}$

$0.011298 \quad 0.0088071 \quad 0.012253 \mathrm{~kg}$

$0.19977 \quad 0.15573 \quad 0.21666 \mathrm{~kg}$

$\begin{array}{lll}0.068888 & 0.053702 & 0.074711 \mathrm{~kg}\end{array}$

$\begin{array}{lll}7.41 \mathrm{E}-05 & 0.0001159 & 6.96 \mathrm{E}-05 \mathrm{~kg}\end{array}$

$\begin{array}{llll}0.0002665 & 0.0002209 & 0.0002134 \\ k g\end{array}$

$\begin{array}{lll}0.010368 & 0.0091329 & 0.0090703 \mathrm{~kg}\end{array}$

$\begin{array}{lll}0.33587 & 0.22525 & 0.57431 \mathrm{~kg}\end{array}$

$\begin{array}{lll}37.382 & 37.059 & 39.359 \mathrm{~kg}\end{array}$

$0.20072 \quad 0.19171 \quad 0.19583 \mathrm{~kg}$

$\begin{array}{lll}0.22227 & 0.20852 & 0.21921 \mathrm{~kg}\end{array}$

$0.19988 \quad 0.19106 \quad 0.19492 \mathrm{~kg}$

$\begin{array}{lll}0.24061 & 0.22264 & 0.2392 \mathrm{~kg}\end{array}$

$1.22 \mathrm{E}-08 \quad 1.36 \mathrm{E}-08 \quad 1.10 \mathrm{E}-08 \mathrm{~kg}$

$1.97 \mathrm{E}-09 \quad 2.20 \mathrm{E}-09 \quad 1.78 \mathrm{E}-09 \mathrm{~kg}$

5.71E-05 8.26E-05 5.02E-05 kg

$\begin{array}{lll}0.0005136 & 0.0007435 & 0.0004517 \mathrm{~kg}\end{array}$

$0.0005539 \quad 0.0004209 \quad 0.0003938 \mathrm{~kg}$

$5.29 \mathrm{E}-06 \quad 2.78 \mathrm{E}-06 \quad 4.19 \mathrm{E}-06 \mathrm{~kg}$

$\begin{array}{llll}0.0006957 & 0.0005796 & 0.0005622 & k g\end{array}$

$\begin{array}{lll}6.8834 & 5.9896 & 7.8811 \mathrm{~kg}\end{array}$

$\begin{array}{lll}0.0005312 & 0.0004486 & 0.0007896 \mathrm{~kg}\end{array}$

$\begin{array}{lll}0.20094 & 0.15927 & 0.24263 \mathrm{~kg}\end{array}$

$0.0006523 \quad 0.0004381 \quad 0.0012385 \mathrm{~kg}$

$5.53 \mathrm{E}-07 \quad 5.50 \mathrm{E}-07 \quad 5.03 \mathrm{E}-07 \mathrm{~kg}$

$\begin{array}{lll}0.73645 & 0.71683 & 0.70988 \mathrm{~kg}\end{array}$

$\begin{array}{lll}0.0015874 & 0.0013327 & 0.0014367 \mathrm{~kg}\end{array}$

$\begin{array}{lll}41.229 & 34.083 & 46.494 \mathrm{~kg}\end{array}$

3.38E-06 2.56E-06 2.40E-06 kg

$0.095065 \quad 0.074108 \quad 0.1031 \mathrm{~kg}$

3.03E-05 2.10E-05 $3.71 \mathrm{E}-05 \mathrm{~kg}$

4.17E-06 3.07E-06 $3.17 \mathrm{E}-06 \mathrm{~kg}$

$\begin{array}{lll}0.017938 & 0.014104 & 0.018044 \mathrm{~kg}\end{array}$

$\begin{array}{lll}0.67142 & 0.59426 & 0.68648 \mathrm{~kg}\end{array}$

$\begin{array}{lll}0.0088882 & 0.0067531 & 0.0063192 \mathrm{~kg}\end{array}$

$\begin{array}{llll}0.10667 & 0.083157 & 0.11569 & \mathrm{~kg}\end{array}$

3.22E-06 2.23E-06 $4.01 \mathrm{E}-06 \mathrm{~kg}$

$0.028781 \quad 0.02507 \quad 0.024776 \mathrm{~kg}$

$1.99 \mathrm{E}-05 \quad 1.37 \mathrm{E}-05 \quad 2.47 \mathrm{E}-05 \mathrm{~kg}$

$\begin{array}{lll}0.049842 & 0.063005 & 0.046987 \\ \mathrm{~kg}\end{array}$

$9.90 \mathrm{E}-07 \quad 1.55 \mathrm{E}-06 \quad 9.31 \mathrm{E}-07 \mathrm{~kg}$

$\begin{array}{lll}0.12186 & 0.10656 & 0.11161 \mathrm{~kg}\end{array}$

$\begin{array}{lll}0.045258 & 0.040636 & 0.044124 \mathrm{~kg}\end{array}$

$\begin{array}{lll}0.00292 & 0.0016916 & 0.0023658\end{array} \mathrm{~kg}$ 
[E780] barium to fresh water

[E783] benzene to fresh water

[E791] Biological Oxygen Demand (BOD) to fresh water

[E792] Borium to fresh water

[E794] cadmium to fresh water

[E802] Chemical oxigen demand (COD) to fresh water

[E806] Chlorine to fresh water

[E807] chlorobenzene to fresh water

[E810] chlorpyriphos to fresh water

[E811] chromium III to fresh water

[E812] chromium VI to fresh water

[E814] cobalt to fresh water

[E815] copper to fresh water

[E821] deltamethrin to fresh water

[E824] Di(2-ethylhexyl)phtalate to fresh water

[E826] Dibutylphtalate to fresh water

[E835] dimethoate to fresh water

[E836] Dimethylphtalate to fresh water

[E846] ethylbęnzene to fresh water

[E855] Formaldehyde (methanal) to fresh water

[E856] glyphosate to fresh water

[E861] hydrogen sulfide to fresh water

[E865] lead to fresh water

[E868] malathion to fresh water

[E869] mangane to fresh water

[E870] MCPA to fresh water

[E872] mercury to fresh water

[E876] methomyl to fresh water

[E882] molybdenum to fresh water

[E885] nickel to fresh water

[E886] Nitrate to fresh water

[E887] Nitrites to fresh water

[E888] Nitrogen to fresh water

[E898] permethrin to fresh water

[E900] phenol to fresh water

[E901] Phosphate to fresh water

[E902] Phosphorus to fresh water

[E914] sulphates to fresh water

[E915] sulphur dioxide to fresh water

[E916] tetrachloroethylene (PER) to fresh water

[E917] Tetrachloromethane (carbon tetrachloride) to fresh water

[E920] tin to fresh water

[E922] toluene to fresh water

[E925] tributyltinoxide to fresh water

[E926] trichlorfon to fresh water

[E927] Trichloroethylene (tri) to fresh water

[E928] Trichloromethane $=$ chloroform to fresh water

[E930] vanadium to fresh water

[E931] Vinyl Chloride (chloroethene) to fresh water

[E932] zinc to fresh water

[E1185] chlorpyriphos to agricultural soil

[E1196] deltamethrin to agricultural soil

\begin{tabular}{|c|c|c|}
\hline 0.29769 & 0.23175 & $0.26625 \mathrm{~kg}$ \\
\hline 0.0098211 & 0.0089104 & $99 \mathrm{~kg}$ \\
\hline 0.0020506 & 0.0016212 & $0.0034845 \mathrm{~kg}$ \\
\hline 0.0019806 & 0.0017887 & $0.0015409 \mathrm{~kg}$ \\
\hline 0.0054054 & 0.0048521 & $0.0043737 \mathrm{~kg}$ \\
\hline 1.3879 & 1.1939 & $1.2867 \mathrm{~kg}$ \\
\hline 127.88 & 114.33 & $166.99 \mathrm{~kg}$ \\
\hline 3.84E-09 & 6.07E-09 & $3.63 \mathrm{E}-09 \mathrm{~kg}$ \\
\hline $6.50 \mathrm{E}-05$ & 4.94E-05 & 4.62E-05 kg \\
\hline 0.015471 & 0.009258 & $0.012726 \mathrm{~kg}$ \\
\hline 8.06E-06 & $6.10 \mathrm{E}-06$ & $5.68 \mathrm{E}-06 \mathrm{~kg}$ \\
\hline 0.0028391 & 0.0016195 & $0.0022961 \mathrm{~kg}$ \\
\hline 0.008216 & 0.0050571 & $0.0066519 \mathrm{~kg}$ \\
\hline 9.64E-07 & 7.33E-07 & $6.86 \mathrm{E}-07 \mathrm{~kg}$ \\
\hline $1.31 \mathrm{E}-08$ & $1.56 \mathrm{E}-08$ & $1.40 \mathrm{E}-08 \mathrm{~kg}$ \\
\hline $1.72 E-08$ & 7.69E-09 & $3.78 \mathrm{E}-08 \mathrm{~kg}$ \\
\hline 2.69E-05 & 2.05E-05 & $1.92 \mathrm{E}-05 \mathrm{~kg}$ \\
\hline $1.08 \mathrm{E}-07$ & 4.81E-08 & $2.37 \mathrm{E}-07 \mathrm{~kg}$ \\
\hline 0.0017412 & 0.0015808 & $0.0016458 \mathrm{~kg}$ \\
\hline $1.42 E-06$ & 2.09E-06 & $1.31 \mathrm{E}-06 \mathrm{~kg}$ \\
\hline 0.0001516 & 0.0001152 & $0.0001078 \mathrm{~kg}$ \\
\hline 0.0010726 & 0.000638 & $0.0010335 \mathrm{~kg}$ \\
\hline 0.011849 & 0.0085415 & $0.010118 \mathrm{~kg}$ \\
\hline 7.77E-05 & $5.90 \mathrm{E}-05$ & $5.52 \mathrm{E}-05 \mathrm{~kg}$ \\
\hline 0.03632 & 0.022695 & $0.029637 \mathrm{~kg}$ \\
\hline 0.0001624 & 0.0001234 & $0.0001155 \mathrm{~kg}$ \\
\hline 1.23E-05 & 1.09E-05 & $1.35 \mathrm{E}-05 \mathrm{~kg}$ \\
\hline $5.01 \mathrm{E}-05$ & 3.81E-05 & $3.56 \mathrm{E}-05 \mathrm{~kg}$ \\
\hline 0.0046464 & 0.0028464 & $0.0036547 \mathrm{~kg}$ \\
\hline 0.0075558 & 0.0044645 & $0.0061394 \mathrm{~kg}$ \\
\hline 0.17294 & 0.21881 & $0.15077 \mathrm{~kg}$ \\
\hline 0.0023119 & 0.0017155 & $0.0016562 \mathrm{~kg}$ \\
\hline 0.13044 & 0.11989 & $0.12352 \mathrm{~kg}$ \\
\hline 1.45E-05 & 1.10E-05 & 1.03E-05 kg \\
\hline 0.010866 & 0.009833 & $0.01097 \mathrm{~kg}$ \\
\hline 0.090854 & 0.053482 & $0.073737 \mathrm{~kg}$ \\
\hline 0.054532 & 0.041434 & $0.038792 \mathrm{~kg}$ \\
\hline 27.741 & 20.561 & $35.938 \mathrm{~kg}$ \\
\hline 0.003465 & 0.0030096 & $0.0032144 \mathrm{~kg}$ \\
\hline $1.66 \mathrm{E}-07$ & 1.14E-07 & 2.06E-07 kg \\
\hline 2.53E-07 & $1.75 \mathrm{E}-07$ & $3.14 \mathrm{E}-07 \mathrm{~kg}$ \\
\hline 0.000594 & 0.0004506 & $0.0004186 \mathrm{~kg}$ \\
\hline 0.0081126 & 0.0073558 & $0.0078049 \mathrm{~kg}$ \\
\hline 0.0003989 & 0.0003822 & $0.0003806 \mathrm{~kg}$ \\
\hline 0.0001163 & 8.84E-05 & $8.27 \mathrm{E}-05 \mathrm{~kg}$ \\
\hline 1.05E-05 & 7.23E-06 & $1.30 \mathrm{E}-05 \mathrm{~kg}$ \\
\hline 3.84E-05 & 2.65E-05 & 4.77E-05 kg \\
\hline 0.0075229 & 0.004374 & $0.0060531 \mathrm{~kg}$ \\
\hline $4.70 \mathrm{E}-08$ & $3.25 E-08$ & $5.84 \mathrm{E}-08 \mathrm{~kg}$ \\
\hline 0.028126 & 0.020642 & $0.023242 \mathrm{~kg}$ \\
\hline 0.011104 & 0.0084366 & $0.0078945 \mathrm{~kg}$ \\
\hline 0.0003298 & 0.0002506 & $0.0002345 \mathrm{~kg}$ \\
\hline
\end{tabular}


[E1210] dimethoate to agricultural soil [E1231] glyphosate to agricultural soil [E1242] malathion to agricultural soil [E1243] MCPA to agricultural soil [E1249] methomyl to agricultural soil [E1268] permethrin to agricultural soil [E1292] trichlorfon to agricultural soil [E1333] arsenic to agricultural soil [E1349] cadmium to industrial soil [E1364] chromium III to industrial soil [E1367] cobalt to industrial soil [E1368] copper to industrial soil [E1417] lead to industrial soil [E1423] mercury to industrial soil [E1436] nickel to industrial soil [E1476] zinc to industrial soil

[E1478] Barit ab Erz to industrial soil [E1479] Bauxit to industrial soil [E1480] Bentonit ab Erz to industrial soil [E1481] dolomite to industrial soil [E1482] Erdoelgas to industrial soil [E1483] flussspat to industrial soil [E1484] Grubengas (Methan) to industrial soil [E1485] Holz to industrial soil

[E1486] Kalkstein vor Abbau to industrial soil [E1488] Kies vor Abbau to industrial soil [E1489] quarzsand to industrial soil [E1490] Sand vor Abbau to industrial soil [E1491] Space Benthos II-III to industrial soil [E1492] Space Benthos II-IV to industrial soil [E1493] Space II-III to industrial soil [E1494] Space III-IV to industrial soil [E1495] Space II-IV to industrial soil [E1496] Space IV-IV to industrial soil [E1497] Steinsalz vor Abbau to industrial soil [E1498] titanium ore to industrial soil [E1499] Ton to industrial soil [E1500] Wasser to industrial soil [E1501] heat to industrial soil [E1502] Aluminium from earth resources [E1503] B from earth resources [E1504] Br from earth resources [E1505] Butene from earth resources [E1506] Ca from earth resources

[E1507] Dichloromonofluormethane from earth resources [E1508] Iron from earth resources [E1509] He from earth resources [E1510] Hexane from earth resources [E1511] I (lodium) from earth resources [E1512] K (kalium) from earth resources [E1513] La (Lanthane) from earth resources [E1514] Mg from earth resources

\begin{tabular}{rrr}
\hline 0.0046329 & 0.0035201 & $0.0032939 \mathrm{~kg}$ \\
0.027697 & 0.021044 & $0.019692 \mathrm{~kg}$ \\
0.01541 & 0.011708 & $0.010956 \mathrm{~kg}$ \\
0.058163 & 0.044192 & $0.041352 \mathrm{~kg}$ \\
\hline .0053631 & 0.0040748 & $0.003813 \mathrm{~kg}$ \\
\hline 0.0049602 & 0.0037688 & $0.0035266 \mathrm{~kg}$ \\
0.035502 & 0.026974 & $0.025241 \mathrm{~kg}$ \\
\hline $4.53 \mathrm{E}-05$ & $3.82 \mathrm{E}-05$ & $5.01 \mathrm{E}-05 \mathrm{~kg}$ \\
\hline $1.38 \mathrm{E}-05$ & $1.20 \mathrm{E}-05$ & $1.12 \mathrm{E}-05 \mathrm{~kg}$ \\
\hline .0005669 & 0.0004785 & $0.0006268 \mathrm{~kg}$ \\
\hline $1.95 \mathrm{E}-06$ & $1.75 \mathrm{E}-06$ & $1.84 \mathrm{E}-06 \mathrm{~kg}$ \\
\hline $9.74 \mathrm{E}-06$ & $8.73 \mathrm{E}-06$ & $9.21 \mathrm{E}-06 \mathrm{~kg}$ \\
\hline $.64 \mathrm{E}-05$ & $4.14 \mathrm{E}-05$ & $4.35 \mathrm{E}-05 \mathrm{~kg}$ \\
\hline $3.23 \mathrm{E}-07$ & $2.71 \mathrm{E}-07$ & $3.06 \mathrm{E}-07 \mathrm{~kg}$ \\
\hline $1.46 \mathrm{E}-05$ & $1.31 \mathrm{E}-05$ & $1.38 \mathrm{E}-05 \mathrm{~kg}$ \\
0.001797 & 0.0015212 & $0.0019713 \mathrm{~kg}$ \\
-8.5448 & -7.245 & $-9.5006 \mathrm{~kg}$ \\
\hline-11.922 & -12.876 & $-16.103 \mathrm{~kg}$ \\
\hline-694.45 & -528.02 & $-494.17 \mathrm{~kg}$ \\
\hline 0.059254 & 0.051494 & $0.056212 \mathrm{~kg}$ \\
\hline 0.008399 & 0.0006165 & $0.0005532 \mathrm{~kg}$ \\
\hline 0.03008 & 0.028755 & $0.025389 \mathrm{~kg}$ \\
\hline $.03 \mathrm{E}-05$ & $1.30 \mathrm{E}-05$ & $1.43 \mathrm{E}-05 \mathrm{~kg}$ \\
0.016229 & -119.29 & $-111.62 \mathrm{~kg}$ \\
\hline 0.012889 & 0.0092554 & $0.0096966 \mathrm{~kg}$
\end{tabular}


[E1515] LT Radio. Rn222 from earth resources [E1516] $\mathrm{Na}$ from earth resources [E1517] NMVOC from earth resources [E1518] Pentane from earth resources [E1519] Pt (Platina) from earth resources [E1520] Radio. Aerosole from earth resources [E1521] Radio. Ag110m from earth resources [E1522] Radio. Am241 from earth resources [E1523] Radio. other beta from earth resources [E1524] Radio. Ar4 from earth resources [E1525] Radio. Ba140 to air [E1526] Radio. C14 to air [E1527] Radio. Ce141 to air [E1528] Radio. Ce144 to air [E1529] Radio. Cm alpha to air [E1530] Radio. Cm242 to air [E1531] Radio. Cm244 to air [E1532] Radio. Co57 to air [E1533] Radio. Co58 to air [E1534] Radio. Co60 to air [E1535] Radio. Cr51 to air [E1536] Radio. Cs134 to air [E1537] Radio. Cs137 to air [E1538] Radio. nobel gasses to air [E1539] Radio. Fe59 to air [E1540] Radio. H3 to air [E1541] Radio. I129 to air [E1542] Radio. 1131 air [E1543] Radio. I133 air [E1544] Radio. I135 air [E1545] Radio. K40 air [E1546] Radio. Kr85 air [E1547] Radio. Kr85m air [E1548] Radio. Kr87 air [E1549] Radio. Kr88 air [E1550] Radio. Kr89 air [E1551] Radio. La140 air [E1552] Radio. Mn54 air [E1553] Radio. Nb95 air [E1554] Radio. Np237 air [E1555] Radio. Pa234m air [E1556] Radio. Pb210 air [E1557] Radio. Pm147 air [E1558] Radio. Po210 air [E1559] Radio. Pu alpha air [E1560] Radio. Pu238 air [E1561] Radio. Pu241 Beta air [E1562] Radio. Ra226 air [E1563] Radio. Ra228 air [E1564] Radio. Rn220 air [E1565] Radio. Rn222 air [E1566] Radio. Ru103 air

\begin{tabular}{|c|c|c|}
\hline $65 E+06$ & $1.97 E+06$ & 1.89E+06 kBq \\
\hline 0.011814 & 0.0098601 & $0.0096186 \mathrm{~kg}$ \\
\hline 17.312 & 16.389 & $17.008 \mathrm{~kg}$ \\
\hline 0.19573 & 0.16048 & $0.22067 \mathrm{~kg}$ \\
\hline $4.20 E-06$ & 6.66E-06 & $3.95 \mathrm{E}-06 \mathrm{~kg}$ \\
\hline $9.92 E+05$ & $7.54 \mathrm{E}+05$ & $7.06 \mathrm{E}+05 \mathrm{kBq}$ \\
\hline 9.33E-06 & $6.49 E-06$ & $4.68 \mathrm{E}-06 \mathrm{kBq}$ \\
\hline 0.0003799 & 0.0002815 & $0.0002709 \mathrm{kBq}$ \\
\hline $1.48 \mathrm{E}-05$ & 1.10E-05 & 1.09E-05 kBq \\
\hline 19.056 & 13.184 & $9.2345 \mathrm{kBq}$ \\
\hline 0.0001509 & 0.0001116 & $0.0001078 \mathrm{kBq}$ \\
\hline 34.305 & 25.412 & $24.493 \mathrm{kBq}$ \\
\hline 0.0010366 & 0.0007875 & $0.0007369 \mathrm{kBq}$ \\
\hline 0.0040299 & 0.0029868 & $0.0028739 \mathrm{kBq}$ \\
\hline 0.000602 & 0.0004462 & $0.0004292 \mathrm{kBq}$ \\
\hline 1.22E-09 & $8.89 E-10$ & $7.84 \mathrm{E}-10 \mathrm{kBq}$ \\
\hline $1.11 \mathrm{E}-08$ & 8.03E-09 & 7.08E-09 kBq \\
\hline 1.47E-08 & $1.02 E-08$ & 7.05E-09 kBq \\
\hline 0.0004868 & 0.0003639 & $0.0003585 \mathrm{kBq}$ \\
\hline 0.000794 & 0.0005895 & $0.0005691 \mathrm{kBq}$ \\
\hline $5.74 \mathrm{E}-05$ & 4.19E-05 & $3.81 \mathrm{E}-05 \mathrm{kBq}$ \\
\hline 0.014332 & 0.010623 & $0.010232 \mathrm{kBq}$ \\
\hline 0.027794 & 0.020604 & $0.019827 \mathrm{kBq}$ \\
\hline 19.928 & 14.821 & $14.761 \mathrm{kBq}$ \\
\hline $4.60 \mathrm{E}-07$ & 3.33E-07 & $2.86 \mathrm{E}-07 \mathrm{kBq}$ \\
\hline 273.09 & 202.91 & $196.13 \mathrm{kBq}$ \\
\hline 0.10834 & 0.080301 & $0.077249 \mathrm{kBq}$ \\
\hline 0.038408 & 0.028519 & $0.028123 \mathrm{kBq}$ \\
\hline 0.0059792 & 0.0044723 & $0.004417 \mathrm{kBq}$ \\
\hline 0.0085463 & 0.0063941 & $0.0063138 \mathrm{kBq}$ \\
\hline 0.040251 & 0.029833 & $0.026833 \mathrm{kBq}$ \\
\hline $1.86 E+06$ & $1.38 E+06$ & $1.33 \mathrm{E}+06 \mathrm{kBq}$ \\
\hline 10.868 & 8.0681 & $7.9729 \mathrm{kBq}$ \\
\hline 3.4243 & 2.5372 & $2.4874 \mathrm{kBq}$ \\
\hline 77.319 & 57.827 & $57.065 \mathrm{kBq}$ \\
\hline 3.4543 & 2.5649 & $2.5374 \mathrm{kBq}$ \\
\hline $4.85 E-05$ & $3.56 \mathrm{E}-05$ & $3.31 \mathrm{E}-05 \mathrm{kBq}$ \\
\hline 1.43E-05 & $1.04 \mathrm{E}-05$ & 9.37E-06 kBq \\
\hline 2.17E-06 & $1.55 \mathrm{E}-06$ & 1.26E-06 kBq \\
\hline $1.99 E-08$ & 1.47E-08 & $1.42 \mathrm{E}-08 \mathrm{kBq}$ \\
\hline 0.011927 & 0.00884 & $0.0084895 \mathrm{kBq}$ \\
\hline 0.27468 & 0.20356 & $0.18929 \mathrm{kBq}$ \\
\hline 0.010237 & 0.0075874 & $0.0072982 \mathrm{kBq}$ \\
\hline 0.39136 & 0.29006 & $0.26702 \mathrm{kBq}$ \\
\hline 0.001203 & 0.000892 & $0.0008578 \mathrm{kBq}$ \\
\hline 2.67E-08 & 1.93E-08 & 1.67E-08 kBq \\
\hline 0.033142 & 0.02456 & $0.023628 \mathrm{kBq}$ \\
\hline 0.41101 & 0.30445 & $0.29084 \mathrm{kBc}$ \\
\hline 0.0198 & 0.014681 & $0.01322 \mathrm{kBq}$ \\
\hline 0.28035 & 0.10169 & $-0.32118 \mathrm{kBq}$ \\
\hline 28817 & 21346 & $20519 \mathrm{kBq}$ \\
\hline $2.46 \mathrm{E}-07$ & 1.79E-07 & $1.62 \mathrm{E}-07 \mathrm{kBq}$ \\
\hline
\end{tabular}


[E1567] Radio. Ru106 air [E1568] Radio. Sb124 air [E1569] Radio. Sb125 air [E1570] Radio. Sr89 air [E1571] Radio. Sr90 air [E1572] Radio. Tc99 air [E1573] Radio. Te123m air [E1574] Radio. Th228 air [E1575] Radio. Th230 air [E1576] Radio. Th232 air [E1577] Radio. Th234 air [E1578] Radio. U alpha air [E1579] Radio. U234 air [E1580] Radio. U235 air [E1581] Radio. U238 air [E1582] Radio. Xe131m air [E1583] Radio. Xe133 air [E1584] Radio. Xe133m air [E1585] Radiq. Xe135 air [E1586] Radio. Xe135m air [E1587] Radio. Xe137 air [E1588] Radio. Xe138 air [E1589] Radio. Zn65 air [E1590] Radio. Zr95 air [E1591] Sc (Scandium) air [E1592] Si air [E1593] Sr air [E1594] Th (Thorium) air [E1595] Ti (Titanium) air [E1596] U (Uranium) air [E1597] Zr (Zirkonium) air [E1598] heat air [E1599] Xylene air [E1600] Acenaphthylene air [E1601] Alkane air [E1602] Alkene air [E1603] AOX air [E1604] Barite air [E1605] BSB5 air [E1606] Ethylene Dichloride air [E1607] acid air [E1608] aluminium air [E1609] berilium air [E1610] calcium air [E1611] Cs (Cesium) air [E1612] Cyanide (CN) air [E1613] DOC air

[E1614] fat and oil air [E1615] fatty acids as $C$ air [E1616] Glutaraldehyde air [E1617] Hexachloroethane air [E1618] HOCl air

\begin{tabular}{rrr}
0.1203 & 0.089198 & $0.08578 \mathrm{kBq}$ \\
$3.20 \mathrm{E}-06$ & $2.27 \mathrm{E}-06$ & $1.80 \mathrm{E}-06 \mathrm{kBq}$ \\
$2.76 \mathrm{E}-06$ & $2.05 \mathrm{E}-06$ & $2.01 \mathrm{E}-06 \mathrm{kBq}$ \\
$2.60 \mathrm{E}-05$ & $1.90 \mathrm{E}-05$ & $1.72 \mathrm{E}-05 \mathrm{kBq}$ \\
0.019893 & 0.014742 & $0.014185 \mathrm{kBq}$ \\
$8.43 \mathrm{E}-07$ & $6.25 \mathrm{E}-07$ & $6.01 \mathrm{E}-07 \mathrm{kBq}$ \\
$3.86 \mathrm{E}-05$ & $2.67 \mathrm{E}-05$ & $1.85 \mathrm{E}-05 \mathrm{kBq}$ \\
0.01681 & 0.012463 & $0.011222 \mathrm{kBq}$ \\
0.13255 & 0.098175 & $0.09432 \mathrm{kBq}$ \\
0.010646 & 0.0078946 & $0.0071069 \mathrm{kBq}$ \\
0.011927 & 0.00884 & $0.0084895 \mathrm{kBq}$ \\
\hline 0.42686 & 0.31621 & $0.30379 \mathrm{kBq}$ \\
0.14307 & 0.10604 & $0.10186 \mathrm{kBq}$ \\
0.0069344 & 0.0051375 & $0.0049379 \mathrm{kBq}$ \\
0.17194 & 0.12735 & $0.12103 \mathrm{kBq}$ \\
15.69 & 11.623 & $11.382 \mathrm{kBq}$ \\
1382.9 & 1031.4 & $1014.6 \mathrm{kBq}$ \\
0.31314 & 0.2196 & $0.16467 \mathrm{kBq}$ \\
\hline 376.59 & 280.39 & $276.54 \mathrm{kBq}$ \\
\hline 103.03 & 76.487 & $75.55 \mathrm{kBq}$ \\
\hline 0.37108 & 0.33606 & $0.35378 \mathrm{~kg}$ \\
\hline .0002058 & 0.0001755 & $0.0002302 \mathrm{~kg}$ \\
$1.40 \mathrm{E}-09$ & $9.64 \mathrm{E}-10$ & $1.73 \mathrm{E}-09 \mathrm{~kg}$ \\
0.0026837 & 0.0017771 & $0.0009119 \mathrm{~kg}$
\end{tabular}


[E1619] hydrocarbons air

[E1620] iod air

[E1621] K air

[E1622] metals water

[E1623] Methylene chloride water

[E1624] Mg water

[E1625] MTBE water

[E1626] Fe water

[E1627] Fluoride water

[E1628] silver water

[E1629] Na water

[E1630] OCl water

[E1631] PAH Polycyclic. Aromatic Hydrocarbons water

[E1632] Radio. Ag110m water

[E1633] Radio. Alpha-radiator water

[E1634] Radio. Am241 water

[E1635] Radio. Ba140 water

[E1636] Radio. C14 water

[E1637] Radio., Cd109 water

[E1638] Radio. Ce141 water

[E1639] Radio. Ce144 water

[E1640] Radio. Cm alpha water

[E1641] Radio. Co57 water

[E1642] Radio. Co58 water

[E1643] Radio. Co60 water

[E1644] Radio. Cr51 water

[E1645] Radio. Cs134 water

[E1646] Radio. Cs136 water

[E1647] Radio. Cs137 water

[E1648] Radio. Fe59 water

[E1649] Radio. H3 water

[E1650] Radio. I129 water

[E1651] Radio. I131 water

[E1652] Radio. I133 water

[E1653] Radio. K 40 water

[E1654] Radio. La140 water

[E1655] Radio. Mn54 water

[E1656] Radio. Mo99 water

[E1657] Radio. Na24 water

[E1658] Radio. Nb95 water

[E1659] Radio. Np237 water

[E1660] Radio. Nucl. mixed water

[E1661] Radio. Pa234m water

[E1662] Radio. $\mathrm{Pb} 210$ water

[E1663] Radio. Po 210 water

[E1664] Radio. Pu alpha water

[E1665] Radio. Pu241 beta water

[E1666] Radio. Ra 224 water

[E1667] Radio. Ra 226 water

[E1668] Radio. Ra 228 water

[E1669] Radio. Ru103 water

[E1670] Radio. Ru106 water

\begin{tabular}{|c|c|c|}
\hline 0.057618 & 0.045586 & $0.098622 \mathrm{~kg}$ \\
\hline 0.0072367 & 0.006564 & $0.0068406 \mathrm{~kg}$ \\
\hline 0.78508 & 0.56897 & $0.68592 \mathrm{~kg}$ \\
\hline 0.21239 & 0.16805 & $0.36548 \mathrm{~kg}$ \\
\hline 0.0007879 & 0.0006643 & $0.0008714 \mathrm{~kg}$ \\
\hline 1.2327 & 0.73724 & $1.0021 \mathrm{~kg}$ \\
\hline $6.14 \mathrm{E}-06$ & 9.54E-06 & $5.76 \mathrm{E}-06 \mathrm{~kg}$ \\
\hline 1.583 & 1.116 & $1.4166 \mathrm{~kg}$ \\
\hline 0.041717 & 0.038489 & $0.040781 \mathrm{~kg}$ \\
\hline 5.34E-05 & 4.93E-05 & $4.95 \mathrm{E}-05 \mathrm{~kg}$ \\
\hline 33.808 & 36.107 & $31.754 \mathrm{~kg}$ \\
\hline 0.0026838 & 0.0017772 & $0.000912 \mathrm{~kg}$ \\
\hline 0.0009813 & 0.0009035 & $0.0009259 \mathrm{~kg}$ \\
\hline 0.13338 & 0.10046 & $0.10174 \mathrm{kBq}$ \\
\hline 9.95E-06 & 7.22E-06 & $6.33 \mathrm{E}-06 \mathrm{kBq}$ \\
\hline 0.04997 & 0.037035 & $0.035631 \mathrm{kBq}$ \\
\hline 0.0018854 & 0.0014023 & $0.0013965 \mathrm{kBq}$ \\
\hline 2.5249 & 1.8719 & $1.8003 \mathrm{kBq}$ \\
\hline 1.09E-05 & 8.10E-06 & 8.06E-06 kBq \\
\hline 0.000281 & 0.000209 & $0.0002082 \mathrm{kBq}$ \\
\hline 1.1441 & 0.84783 & $0.81592 \mathrm{kBq}$ \\
\hline 0.066147 & 0.049024 & $0.047166 \mathrm{kBq}$ \\
\hline 0.0019274 & 0.0014334 & $0.0014279 \mathrm{kBq}$ \\
\hline 0.80412 & 0.59925 & $0.59832 \mathrm{kBq}$ \\
\hline 12.005 & 8.9017 & $8.5967 \mathrm{kBq}$ \\
\hline 0.041348 & 0.030751 & $0.030629 \mathrm{kBq}$ \\
\hline 2.5684 & 1.9046 & $1.833 \mathrm{kBq}$ \\
\hline 1.01E-05 & 7.51E-06 & 7.48E-06 kBq \\
\hline 23.629 & 17.522 & $16.862 \mathrm{kBq}$ \\
\hline 3.33E-05 & $2.48 \mathrm{E}-05$ & 2.47E-05 kBq \\
\hline 74827 & 55454 & $53362 \mathrm{kBq}$ \\
\hline 7.2324 & 5.3602 & $5.1569 \mathrm{kBq}$ \\
\hline 0.011341 & 0.0084699 & $0.0084802 \mathrm{kBq}$ \\
\hline 0.0086151 & 0.0064071 & $0.0063817 \mathrm{kBq}$ \\
\hline-0.10853 & -0.095951 & $-0.14957 \mathrm{kBq}$ \\
\hline 0.0003905 & 0.0002904 & $0.0002892 \mathrm{kBq}$ \\
\hline 1.7279 & 1.2809 & $1.2334 \mathrm{kBq}$ \\
\hline 0.0001317 & $9.79 \mathrm{E}-05$ & $9.75 \mathrm{E}-05 \mathrm{kBq}$ \\
\hline 0.057934 & 0.043087 & $0.042918 \mathrm{kBq}$ \\
\hline 0.0010686 & 0.0007947 & $0.0007915 \mathrm{kBq}$ \\
\hline 0.0031877 & 0.0023631 & $0.0022732 \mathrm{kBq}$ \\
\hline 8663.6 & 6582.5 & $6159.6 \mathrm{kBq}$ \\
\hline 0.22079 & 0.16361 & $0.15715 \mathrm{kBq}$ \\
\hline-0.08698 & -0.076886 & $-0.11975 \mathrm{kBq}$ \\
\hline-0.08698 & -0.076886 & $-0.11975 \mathrm{kBq}$ \\
\hline 0.19893 & 0.14742 & $0.14185 \mathrm{kBq}$ \\
\hline 4.9382 & 3.6605 & $3.5212 \mathrm{kBq}$ \\
\hline 3.6161 & 3.2814 & $3.4178 \mathrm{kBq}$ \\
\hline 917.09 & 680.65 & $654.35 \mathrm{kBq}$ \\
\hline 7.2369 & 6.5642 & $6.8408 \mathrm{kBq}$ \\
\hline 0.0006312 & 0.0004695 & $0.0004676 \mathrm{kBq}$ \\
\hline 12.03 & 8.9198 & $8.578 \mathrm{kBq}$ \\
\hline
\end{tabular}


[E1671] Radio. Sb122 water [E1672] Radio. Sb124 water [E1673] Radio. Sb125 water [E1674] Radio. Spalt- u. Aktiv. prod. water [E1675] Radio. Sr89 water [E1676] Radio. Sr90 water [E1677] Radio. Tc99 water [E1678] Radio. Tc99m water [E1679] Radio. Te123m water [E1680] Radio. Te132 water [E1681] Radio. Th 228 water [E1682] Radio. Th 232 water [E1683] Radio. Th230 water [E1684] Radio. Th234 water [E1685] Radio. U 238 water [E1686] Radio. $U$ alpha water [E1687] Radio. U234 water [E1688] Radio. U235 water [E1689] Radio: Y90 water [E1690] Radio. Zn65 water [E1691] Radio. Zr95 water [E1692] Rb (Rubidium) water [E1693] salt water [E1694] Sb water [E1695] Se water [E1696] Si water [E1697] solved substances water [E1698] Solvents (Cl) water [E1699] Sr water [E1700] Sulfite water [E1701] suspended substanses water [E1702] Ti water [E1703] TOC water [E1704] Triethylene Glykol water [E1705] volatile organic comp. as C water [E1706] W (wolfram) water [E1707] Xylene water [E1708] heat water [E1709] aluminium water [E1710] C water [E1711] calcium water [E1712] iron water [E1713] mangane water [E1714] N water [E1715] oil water [E1716] oil biol. water [E1717] $P$ water [E1718] S water [E1719] chlorine water [E1720] fluor water [E1722] Te water [E1723] heavy metals water

\begin{tabular}{|c|c|c|}
\hline 0018854 & 0.0014023 & $0.0013965 \mathrm{kBq}$ \\
\hline 0.056806 & 0.042554 & $0.042771 \mathrm{kBq}$ \\
\hline 0.015382 & 0.011441 & $0.011394 \mathrm{kBq}$ \\
\hline 0.066298 & 0.046154 & $0.033236 \mathrm{kBq}$ \\
\hline 0.0042549 & 0.0031644 & $0.0031522 \mathrm{kBq}$ \\
\hline 2.4117 & 1.7873 & $1.7198 \mathrm{kBq}$ \\
\hline 1.2649 & 0.93755 & $0.90198 \mathrm{kBq}$ \\
\hline 0.0008871 & 0.0006598 & $0.0006571 \mathrm{kBq}$ \\
\hline 7.95E-05 & $5.91 \mathrm{E}-05$ & $5.89 \mathrm{E}-05 \mathrm{kBq}$ \\
\hline 3.25E-05 & 2.42E-05 & $2.41 \mathrm{E}-05 \mathrm{kBq}$ \\
\hline 14.472 & 13.14 & $13.679 \mathrm{kBq}$ \\
\hline-0.020261 & -0.017916 & $-0.027917 \mathrm{kBq}$ \\
\hline 34.544 & 25.593 & $24.585 \mathrm{kBq}$ \\
\hline 0.22203 & 0.16448 & $0.15794 \mathrm{kBq}$ \\
\hline 0.63372 & 0.46337 & $0.42257 \mathrm{kBq}$ \\
\hline 14.416 & 10.674 & $10.253 \mathrm{kBq}$ \\
\hline 0.2948 & 0.21842 & $0.20985 \mathrm{kBq}$ \\
\hline 0.43957 & 0.32561 & $0.31281 \mathrm{kBq}$ \\
\hline 0.0002179 & 0.0001621 & $0.0001614 \mathrm{kBq}$ \\
\hline 0.12238 & 0.091018 & $0.090654 \mathrm{kBq}$ \\
\hline 0.1026 & 0.076042 & $0.073147 \mathrm{kBq}$ \\
\hline 0.0007254 & 0.0006583 & $0.0006854 \mathrm{~kg}$ \\
\hline 2.1405 & 1.5307 & $1.5652 \mathrm{~kg}$ \\
\hline 6.13E-06 & 5.89E-06 & $1.74 \mathrm{E}-06 \mathrm{~kg}$ \\
\hline 0.0073071 & 0.0042149 & $0.0059036 \mathrm{~kg}$ \\
\hline 0.0010827 & 0.0008914 & $0.0011027 \mathrm{~kg}$ \\
\hline 0.82706 & 0.62933 & $0.70293 \mathrm{~kg}$ \\
\hline 1.65E-05 & 1.67E-05 & $1.42 \mathrm{E}-05 \mathrm{~kg}$ \\
\hline 0.45548 & 0.40803 & $0.42822 \mathrm{~kg}$ \\
\hline 0.0027271 & 0.0040448 & $0.0024617 \mathrm{~kg}$ \\
\hline 5.3479 & 4.5812 & $5.916 \mathrm{~kg}$ \\
\hline 0.085895 & 0.049627 & $0.069535 \mathrm{~kg}$ \\
\hline 30.201 & 26.114 & $24.335 \mathrm{~kg}$ \\
\hline 0.016669 & 0.011046 & $0.02897 \mathrm{~kg}$ \\
\hline 0.025337 & 0.023 & $0.023946 \mathrm{~kg}$ \\
\hline $3.10 \mathrm{E}-05$ & 2.30E-05 & $2.08 \mathrm{E}-05 \mathrm{~kg}$ \\
\hline 0.007042 & 0.0063974 & $0.0067654 \mathrm{~kg}$ \\
\hline-0.0006908 & -0.0005762 & $-0.0006933 \mathrm{TJ}$ \\
\hline 0.11333 & 0.095586 & $0.12529 \mathrm{~kg}$ \\
\hline 0.38271 & 0.32062 & $0.40813 \mathrm{~kg}$ \\
\hline 0.45319 & 0.38233 & $0.501 \mathrm{~kg}$ \\
\hline 0.22665 & 0.19118 & $0.25068 \mathrm{~kg}$ \\
\hline 0.0045319 & 0.0038233 & $0.00501 \mathrm{~kg}$ \\
\hline 55.395 & 42.088 & $39.384 \mathrm{~kg}$ \\
\hline 0.070075 & 0.062012 & $0.065932 \mathrm{~kg}$ \\
\hline 0.0048416 & 0.0037557 & $0.0043744 \mathrm{~kg}$ \\
\hline 0.0065833 & 0.0054251 & $0.0070187 \mathrm{~kg}$ \\
\hline 0.068008 & 0.057367 & $0.075192 \mathrm{~kg}$ \\
\hline 0.029266 & 0.023157 & $0.050361 \mathrm{~kg}$ \\
\hline 0.01087 & 0.008601 & $0.018706 \mathrm{~kg}$ \\
\hline 0.0001157 & $9.09 E-05$ & $0.0001164 \mathrm{~kg}$ \\
\hline 8.79E-19 & $6.68 \mathrm{E}-19$ & $6.25 \mathrm{E}-19 \mathrm{~kg}$ \\
\hline
\end{tabular}


[E1724] metals water

[E1725] oil water

[E1726] HC water

[E1727] bromoxynil water

[E1728] clopyralid water

[E1729] lambda-cyhalothrin water

[E1730] sethoxydim water

[E1731] bromoxynil water

[E1732] clopyralid water

[E1733] lambda-cyhalothrin water

[E1734] sethoxidim soil

[E1735] bromoxynil soil

[E1736] clopyralid soil

[E1737] lambda-cyhalothrin soil

[E1738] sethoxidim soil

\begin{tabular}{rrr}
$7.35 \mathrm{E}-07$ & $5.59 \mathrm{E}-07$ & $5.23 \mathrm{E}-07 \mathrm{~kg}$ \\
0.03575 & 0.027162 & $0.025417 \mathrm{~kg}$ \\
$1.77 \mathrm{E}-05$ & $1.34 \mathrm{E}-05$ & $1.26 \mathrm{E}-05 \mathrm{~kg}$ \\
0.0042804 & 0.0032522 & $0.0030432 \mathrm{~kg}$ \\
0.0043056 & 0.0032714 & $0.0030611 \mathrm{~kg}$ \\
$2.67 \mathrm{E}-05$ & $2.03 \mathrm{E}-05$ & $1.90 \mathrm{E}-05 \mathrm{~kg}$ \\
0.0029208 & 0.0022192 & $0.0020766 \mathrm{~kg}$ \\
0.016971 & 0.012894 & $0.012066 \mathrm{~kg}$ \\
0.0034747 & 0.00264 & $0.0024704 \mathrm{~kg}$ \\
0.0004155 & 0.0003157 & $0.0002954 \mathrm{~kg}$ \\
0.026186 & 0.019896 & $0.018618 \mathrm{~kg}$ \\
0.0001093 & $8.30 \mathrm{E}-05$ & $7.77 \mathrm{E}-05 \mathrm{~kg}$ \\
$4.00 \mathrm{E}-05$ & $3.04 \mathrm{E}-05$ & $2.85 \mathrm{E}-05 \mathrm{~kg}$ \\
$1.16 \mathrm{E}-06$ & $8.80 \mathrm{E}-07$ & $8.23 \mathrm{E}-07 \mathrm{~kg}$ \\
0.0001498 & 0.0001138 & $0.0001065 \mathrm{~kg}$ \\
\hline
\end{tabular}


Category $=[C 1]$ Depletion of abiotic resources Description $=$ Problem oriented approach, manual 1999

Author $=$ Guinee, 1995

Date $=11 / 1 / 99$

\section{Environmental resources}

Environmental resource

[E1] actinium (AC) from earth resources

[E2] aluminium (AI) from earth resources

[E3] antimony (Sb) from earth resources

[E4] argon (Ar) from earth resources

[E5] arsenic (As) from earth resources

[E6] barium (Ba) from earth resources

[E7] beryllium $(\mathrm{Be})$ from earth resources

[E8] bismuth (Bi) from earth resources

[E9] boron (B) from earth resources

[E10] bromine $(\mathrm{Br})$ from earth resources

[E11] cadmium (Cd) from earth resources

[E12] calcium (Ca) from earth resources

[E13] cerium (Ce) from earth resources

[E14] cesium (Cs) from earth resources

[E15] chlorine (Cl) from earth resources

[E16] cobalt (Co) from earth resources

[E17] copper (Cu) from earth resources

[E18] chromium ( $\mathrm{Cr}$ ) from earth resources

[E19] dysprosium (Dy) from earth resources

[E20] erbium ( $E r)$ from earth resources

[E21] europium (Eu) from earth resources

[E22] fluorine $(F)$ from earth resources

[E23] gadolinium (Gd) from earth resources

[E24] gallium $(\mathrm{Ga})$ from earth resources

[E25] germanium $(\mathrm{Ge})$ from earth resources

[E26] gold $(\mathrm{Au})$ from earth resources

[E27] hafnium $(\mathrm{Hf})$ from earth resources

[E28] helium (He) from earth resources

[E29] holmium $(\mathrm{Ho})$ from earth resources

[E30] indium (In) from earth resources

[E31] iodine (I) from earth resources

[E32] iridium (Ir) from earth resources

[E33] iron ( $\mathrm{Fe})$ from earth resources

[E34] kalium (K;potassium) from earth resources

[E35] krypton (Kr) from earth resources

[E36] lanthanum (La) from earth resources

[E37] lithium ( $\mathrm{Li}$ ) from earth resources

[E38] lead (Pb) from earth resources

[E39] lutetium (Lu) from earth resources

[E40] magnesium $(\mathrm{Mg}$ ) from earth resources

[E41] manganese $(\mathrm{Mn})$ from earth resources

[E42] mercury $(\mathrm{Hg})$ from earth resources

[E43] molybdenum (Mo) from earth resources

[E44] neodymium (Nd) from earth resources

[E45] neon ( $\mathrm{Ne})$ from earth resources

[E46] nickel (Ni) from earth resources

[E47] niobium $(\mathrm{Nb})$ from earth resources

[E48] osmium (Os) from earth resources

[E49] palladium $(\mathrm{Pd})$ from earth resources

[E50] phosphorus (P) from earth resources

[E51] platinum $(\mathrm{Pt})$ from earth resources

[E52] polonium ( $\mathrm{Po}$ ) from earth resources

[E53] praseodymium (Pr) from earth resources

[E54] protactinium $(\mathrm{Pa})$ from earth resources

[E55] radium $(\mathrm{Ra})$ from earth resources

[E56] radon $(\mathrm{Rn})$ from earth resources

[E57] rhenium (Re) from earth resources

[E58] rhodium $(\mathrm{Rh})$ from earth resources

Value

Unit

$6.33 \mathrm{E}+13 \mathrm{~kg}$ antimony eq. $/ \mathrm{kg}$

$1.00 \mathrm{E}-08 \mathrm{~kg}$ antimony eq. $/ \mathrm{kg}$

$1 \mathrm{~kg}$ antimony eq. $/ \mathrm{kg}$

$4.71 \mathrm{E}-07 \mathrm{~kg}$ antimony eq. $/ \mathrm{kg}$

$0.00917 \mathrm{~kg}$ antimony eq. $/ \mathrm{kg}$

$1.06 \mathrm{E}-10 \mathrm{~kg}$ antimony eq. $/ \mathrm{kg}$

$3.19 \mathrm{E}-05 \mathrm{~kg}$ antimony eq. $/ \mathrm{kg}$

$0.0731 \mathrm{~kg}$ antimony eq. $/ \mathrm{kg}$

$0.00467 \mathrm{~kg}$ antimony eq. $/ \mathrm{kg}$

$0.00667 \mathrm{~kg}$ antimony eq. $/ \mathrm{kg}$

$0.33 \mathrm{~kg}$ antimony eq. $/ \mathrm{kg}$

$7.08 \mathrm{E}-10 \mathrm{~kg}$ antimony eq. $/ \mathrm{kg}$

$5.32 \mathrm{E}-09 \mathrm{~kg}$ antimony eq. $/ \mathrm{kg}$

$1.91 \mathrm{E}-05 \mathrm{~kg}$ antimony eq. $/ \mathrm{kg}$

$4.86 \mathrm{E}-08 \mathrm{~kg}$ antimony eq. $/ \mathrm{kg}$

$2.62 \mathrm{E}-05 \mathrm{~kg}$ antimony eq. $/ \mathrm{kg}$

$0.00194 \mathrm{~kg}$ antimony eq. $/ \mathrm{kg}$

$0.000858 \mathrm{~kg}$ antimony eq. $/ \mathrm{kg}$

$2.13 \mathrm{E}-06 \mathrm{~kg}$ antimony eq. $/ \mathrm{kg}$

$2.44 \mathrm{E}-06 \mathrm{~kg}$ antimony eq. $/ \mathrm{kg}$

$1.33 \mathrm{E}-05 \mathrm{~kg}$ antimony eq. $/ \mathrm{kg}$

$2.96 \mathrm{E}-06 \mathrm{~kg}$ antimony eq. $/ \mathrm{kg}$

$6.57 \mathrm{E}-07 \mathrm{~kg}$ antimony eq. $/ \mathrm{kg}$

$1.03 \mathrm{E}-07 \mathrm{~kg}$ antimony eq. $/ \mathrm{kg}$

$1.47 \mathrm{E}-06 \mathrm{~kg}$ antimony eq. $/ \mathrm{kg}$

$89.5 \mathrm{~kg}$ antimony eq. $/ \mathrm{kg}$

$8.67 \mathrm{E}-07 \mathrm{~kg}$ antimony eq. $/ \mathrm{kg}$

$148 \mathrm{~kg}$ antimony eq. $/ \mathrm{kg}$

$1.33 \mathrm{E}-05 \mathrm{~kg}$ antimony eq. $/ \mathrm{kg}$

$0.00903 \mathrm{~kg}$ antimony eq. $/ \mathrm{kg}$

$0.0427 \mathrm{~kg}$ antimony eq. $/ \mathrm{kg}$

$32.3 \mathrm{~kg}$ antimony eq. $/ \mathrm{kg}$

$8.43 E-08 \mathrm{~kg}$ antimony eq. $/ \mathrm{kg}$

$3.13 \mathrm{E}-08 \mathrm{~kg}$ antimony eq. $/ \mathrm{kg}$

$20.9 \mathrm{~kg}$ antimony eq. $/ \mathrm{kg}$

2.13E-08 kg antimony eq. $/ \mathrm{kg}$

9.23E-06 kg antimony eq. $/ \mathrm{kg}$

$0.0135 \mathrm{~kg}$ antimony eq. $/ \mathrm{kg}$

$7.66 \mathrm{E}-05 \mathrm{~kg}$ antimony eq. $/ \mathrm{kg}$

$3.73 \mathrm{E}-09 \mathrm{~kg}$ antimony eq. $/ \mathrm{kg}$

$1.38 \mathrm{E}-05 \mathrm{~kg}$ antimony eq. $/ \mathrm{kg}$

$0.495 \mathrm{~kg}$ antimony eq. $/ \mathrm{kg}$

$0.0317 \mathrm{~kg}$ antimony eq. $/ \mathrm{kg}$

$1.94 \mathrm{E}-17 \mathrm{~kg}$ antimony eq. $/ \mathrm{kg}$

$0.325 \mathrm{~kg}$ antimony eq. $/ \mathrm{kg}$

$0.000108 \mathrm{~kg}$ antimony eq. $/ \mathrm{kg}$

$2.31 \mathrm{E}-05 \mathrm{~kg}$ antimony eq. $/ \mathrm{kg}$

$14.4 \mathrm{~kg}$ antimony eq. $/ \mathrm{kg}$

$0.323 \mathrm{~kg}$ antimony eq. $/ \mathrm{kg}$

$8.44 \mathrm{E}-05 \mathrm{~kg}$ antimony eq. $/ \mathrm{kg}$

$1.29 \mathrm{~kg}$ antimony eq. $/ \mathrm{kg}$

$4.79 \mathrm{E}+14 \mathrm{~kg}$ antimony eq. $/ \mathrm{kg}$

$2.85 \mathrm{E}-07 \mathrm{~kg}$ antimony eq. $/ \mathrm{kg}$

$9.77 \mathrm{E}+06 \mathrm{~kg}$ antimony eq. $/ \mathrm{kg}$

$2.36 \mathrm{E}+07 \mathrm{~kg}$ antimony eq. $/ \mathrm{kg}$

$1.20 \mathrm{E}+20 \mathrm{~kg}$ antimony eq. $/ \mathrm{kg}$

$0.766 \mathrm{~kg}$ antimony eq. $/ \mathrm{kg}$

$32.3 \mathrm{~kg}$ antimony eq. $/ \mathrm{kg}$ 
[E59] rubidium $(\mathrm{Rb})$ from earth resources

[E60] ruthenium $(\mathrm{Ru})$ from earth resources

[E61] samarium $(\mathrm{Sm})$ from earth resources

[E62] scandium (Sc) from earth resources

[E63] selenium (Se) from earth resources

[E64] silicium ( $\mathrm{Si}$; silicon) from earth resources

[E65] silver $(\mathrm{Ag})$ from earth resources

[E66] Sodium ( $\mathrm{Na}$ ) from earth resources

[E67] strontium $(\mathrm{Sr})$ from earth resources

[E68] sulfur (S) from earth resources

[E69] tantalum ( $\mathrm{Ta}$ ) from earth resources

$[E 70]$ tellurium $(\mathrm{Te})$ from earth resources

[E71] terbium (Tb) from earth resources

[E72] thallium (TI) from earth resources

[E73] thorium (Th) from earth resources

[E74] thulium ( $\mathrm{Tm})$ from earth resources

$[\mathrm{E} 75]$ tin $(\mathrm{Sn})$ from earth resources

[E76] titanium (Ti) from earth resources

[E77] tungsten (W); wolfraam from earth resources

[E78] uranium $(U)$ from earth resources

$[E 79]$ vanadium $(V)$ from earth resources

[E80] xenon (Xe) from earth resources

[E81] ytterbium (Yb) from earth resources

[E82] yttrium $(Y)$ from earth resources

[E83] zinc $(\mathrm{Zn})$ from earth resources

[E84] Zirconium $(\mathrm{Zr})$ from earth resources

[E85] oil crude from earth resources

[E86] natural gas from earth resources

[E87] coal soft from earth resources

[E88] coal hard from earth resources

[E89] coal total from earth resources

[E1506] Erdoelgas from earth resources

Environmental emissions

Environmental emission

Category $=[\mathrm{C} 2]$ Photochemical oxidant formation (Derwent)

Description = Problem oriented approach, manual 1999; high NOx

Author $=$ Derwent et al., 1998

Date $=27-09-1999$

Environmental resources

Environmental resource

Environmental emissions

Environmental emission

[E90] 1,1,1-trichloroethane (methyl chloroform; HC-140a) to air

[E97] 1,2,3-Trimethyl Benzene to air

[E100] 1,2,4-trimethylbenzene to air

[E109] 1,3,5-trimethylbenzene (mesitylene) to air

[E111] 1,3-Butadiene to air

[E119] 1-Butane to air

[E120] 1-Butanol to air

[E121] 1-Butene to air

[E122] 1-Butoxy Propanol to air

[E123] 1-Butyl Acetate to air

[E130] 1-Decane to air

[E131] 1-Dodecane to air

[E134] 1-Heptane to air

[E137] 1-Hexane to air

[E138] 1-Hexene to air

[E139] 1-Methoxy-2-Propanol to air

[E141] 1-Nonane to air

[E143] 1-Octane to air

[E147] 1-Pentane to air
2.36E-09 kg antimony eq. $/ \mathrm{kg}$

$32.3 \mathrm{~kg}$ antimony eq. $/ \mathrm{kg}$

$5.32 \mathrm{E}-07 \mathrm{~kg}$ antimony eq. $/ \mathrm{kg}$

$3.96 \mathrm{E}-08 \mathrm{~kg}$ antimony eq. $/ \mathrm{kg}$

$0.475 \mathrm{~kg}$ antimony eq. $/ \mathrm{kg}$

$2.99 \mathrm{E}-11 \mathrm{~kg}$ antimony eq. $/ \mathrm{kg}$

$1.84 \mathrm{~kg}$ antimony eq. $/ \mathrm{kg}$

$8.24 \mathrm{E}-11 \mathrm{~kg}$ antimony eq. $/ \mathrm{kg}$

$1.12 \mathrm{E}-06 \mathrm{~kg}$ antimony eq. $/ \mathrm{kg}$

$0.000358 \mathrm{~kg}$ antimony eq. $/ \mathrm{kg}$

$6.77 \mathrm{E}-05 \mathrm{~kg}$ antimony eq. $/ \mathrm{kg}$

$52.8 \mathrm{~kg}$ antimony eq. $/ \mathrm{kg}$

2.36E-05 kg antimony eq. $/ \mathrm{kg}$

$5.05 \mathrm{E}-05 \mathrm{~kg}$ antimony eq. $/ \mathrm{kg}$

$2.08 \mathrm{E}-07 \mathrm{~kg}$ antimony eq. $/ \mathrm{kg}$

$8.31 \mathrm{E}-05 \mathrm{~kg}$ antimony eq. $/ \mathrm{kg}$

$0.033 \mathrm{~kg}$ antimony eq. $/ \mathrm{kg}$

$4.40 \mathrm{E}-08 \mathrm{~kg}$ antimony eq. $/ \mathrm{kg}$

$0.0117 \mathrm{~kg}$ antimony eq. $/ \mathrm{kg}$

$0.00287 \mathrm{~kg}$ antimony eq. $/ \mathrm{kg}$

$1.16 \mathrm{E}-06 \mathrm{~kg}$ antimony eq. $/ \mathrm{kg}$

$17500 \mathrm{~kg}$ antimony eq. $/ \mathrm{kg}$

2.13E-06 kg antimony eq. $/ \mathrm{kg}$

$3.34 \mathrm{E}-07 \mathrm{~kg}$ antimony eq. $/ \mathrm{kg}$

$0.000992 \mathrm{~kg}$ antimony eq. $/ \mathrm{kg}$

$1.86 \mathrm{E}-05 \mathrm{~kg}$ antimony eq. $/ \mathrm{kg}$

$0.436 \mathrm{~kg}$ antimony eq. $/ \mathrm{kg}$

$0.32 \mathrm{~kg}$ antimony eq. $/ \mathrm{m} 3$

$0.00851 \mathrm{~kg}$ antimony eq. $/ \mathrm{kg}$

$0.006 \mathrm{~kg}$ antimony eq. $/ \mathrm{kg}$

$0.00363 \mathrm{~kg}$ antimony eq. $/ \mathrm{kg}$

$0.32 \mathrm{~kg}$ antimony eq. $/ \mathrm{Nm} 3$

Value

Unit

Value

Unit

Value

Unit

$0.009 \mathrm{~kg}$ ethylene eq. $/ \mathrm{kg}$

$1.27 \mathrm{~kg}$ ethylene eq. $/ \mathrm{kg}$

$1.28 \mathrm{~kg}$ ethylene eq. $/ \mathrm{kg}$

$1.38 \mathrm{~kg}$ ethylene eq. $/ \mathrm{kg}$

$0.851 \mathrm{~kg}$ ethylene eq. $/ \mathrm{kg}$

$0.352 \mathrm{~kg}$ ethylene eq. $/ \mathrm{kg}$

$0.612 \mathrm{~kg}$ ethylene eq. $/ \mathrm{kg}$

$1.08 \mathrm{~kg}$ ethylene eq. $/ \mathrm{kg}$

$0.436 \mathrm{~kg}$ ethylene eq. $/ \mathrm{kg}$

$0.241 \mathrm{~kg}$ ethylene eq. $/ \mathrm{kg}$

$0.384 \mathrm{~kg}$ ethylene eq. $/ \mathrm{kg}$

$0.357 \mathrm{~kg}$ ethylene eq. $/ \mathrm{kg}$

$0.494 \mathrm{~kg}$ ethylene eq. $/ \mathrm{kg}$

$0.482 \mathrm{~kg}$ ethylene eq. $/ \mathrm{kg}$

$0.874 \mathrm{~kg}$ ethylene eq. $/ \mathrm{kg}$

$0.368 \mathrm{~kg}$ ethylene eq. $/ \mathrm{kg}$

$0.414 \mathrm{~kg}$ ethylene eq. $/ \mathrm{kg}$

$0.453 \mathrm{~kg}$ ethylene eq. $/ \mathrm{kg}$

$0.395 \mathrm{~kg}$ ethylene eq. $/ \mathrm{kg}$ 
$0.842 \mathrm{~kg}$ ethylene eq. $/ \mathrm{kg}$

$0.407 \mathrm{~kg}$ ethylene eq. $/ \mathrm{kg}$

$0.142 \mathrm{~kg}$ ethylene eq. $/ \mathrm{kg}$

[E200] 2-Methylhexane to air

$0.411 \mathrm{~kg}$ ethylene eq. $/ \mathrm{kg}$

$0.42 \mathrm{~kg}$ ethylene eq. $/ \mathrm{kg}$

[E203] 2-Methylpentane to air

[E211] 3,5-Diethyltoluene to air

$1.3 \mathrm{~kg}$ ethylene eq. $/ \mathrm{kg}$

[E212] 3,5-Dimethylethylbenzene to air

$1.32 \mathrm{~kg}$ ethylene eq. $/ \mathrm{kg}$

[E218] 3-Methyl-1-Butene to air

[E219] 3-Methylbutan-1-ol to air

[E220] 3-Methylbutan-2-ol to air

[E223] 3-Methylhexane to air

[E224] 3-Methylpentane to air

[E227] 3-Pentanol to air

[E232] Acetaldehyde (ethanal) to air

[E233] Acetic acid to air

[E234] Acetone (2-propanon) to air

[E235] Acetylene to air

[E262] Benzaldehyde to air

[E263] Benzene to air

[E291] Butyraldehyde (butanal) to air

[E417] cis-2-Butene to air

[E418] cis-2-Hexene to air

[E419] cis-2-Pentene to air

[E420] cis-Dichloroethene to air

[E429] Cyclohexane to air

[E430] Cyclohexanol to air

[E431] Cyclohexanone to air

[E445] Diacetone alcohol to air

[E449] Dichloromethane (Methylene Chloride) to air

[E453] Diethyl Ether to air

[E455] Diethylketone to air

[E460] Diisopropylether to air

[E463] Dimethyl Ether to air

[E483] Ethane to air

[E485] Ethanol to air

[E488] Ethyl Acetate to air

[E495] Ethyl- trans-Butyl Ether to air

[E496] Ethylbenzene to air

[E499] Ethylene (ethene) to air

[E502] Ethylene Glycol to air

[E511] Formaldehyde (methanal) to air

[E512] Formic acid to air

[E539] Hexan-2-one to air

[E540] Hexan-3-one to air

[E570] isobutane to air

[E571] isobutanol (2-methylpropanol-1) to air

[E572] isobutene (2-methylpropene) to air

[E575] isobutyraldehyde to air

[E576] isopentane to air

[E578] isoprene to air

[E579] isopropanol to air

[E580] isopropyl acetate to air

[E581] isopropyl benzene (cumene) to air

[E594] meta-Ethyltoluene to air

[E596] meta-Xylene (1,3-dimethylbenzene) to air

[E600] Methane to air

[E602] Methanol to air

$0.671 \mathrm{~kg}$ ethylene eq. $/ \mathrm{kg}$

$0.412 \mathrm{~kg}$ ethylene eq. $/ \mathrm{kg}$

$0.366 \mathrm{~kg}$ ethylene eq. $/ \mathrm{kg}$.

$0.364 \mathrm{~kg}$ ethylene eq. $/ \mathrm{kg}$

$0.479 \mathrm{~kg}$ ethylene eq. $/ \mathrm{kg}$

$0.422 \mathrm{~kg}$ ethylene eq. $/ \mathrm{kg}$

$0.641 \mathrm{~kg}$ ethylene eq. $/ \mathrm{kg}$

$0.097 \mathrm{~kg}$ ethylene eq. $/ \mathrm{kg}$

$0.094 \mathrm{~kg}$ ethylene eq. $/ \mathrm{kg}$

$0.085 \mathrm{~kg}$ ethylene eq. $/ \mathrm{kg}$

$-0.092 \mathrm{~kg}$ ethylene eq. $/ \mathrm{kg}$

$0.218 \mathrm{~kg}$ ethylene eq. $/ \mathrm{kg}$

$0.795 \mathrm{~kg}$ ethylene eq. $/ \mathrm{kg}$

$1.15 \mathrm{~kg}$ ethylene eq. $/ \mathrm{kg}$

$1.07 \mathrm{~kg}$ ethylene eq. $/ \mathrm{kg}$

$1.12 \mathrm{~kg}$ ethylene eq. $/ \mathrm{kg}$

$0.447 \mathrm{~kg}$ ethylene eq. $/ \mathrm{kg}$

$0.29 \mathrm{~kg}$ ethylene eq. $/ \mathrm{kg}$

$0.446 \mathrm{~kg}$ ethylene eq. $/ \mathrm{kg}$

$0.299 \mathrm{~kg}$ ethylene eq. $/ \mathrm{kg}$

$0.262 \mathrm{~kg}$ ethylene eq. $/ \mathrm{kg}$

$0.068 \mathrm{~kg}$ ethylene eq. $/ \mathrm{kg}$

$0.467 \mathrm{~kg}$ ethylene eq. $/ \mathrm{kg}$

$0.414 \mathrm{~kg}$ ethylene eq. $/ \mathrm{kg}$

$0.476 \mathrm{~kg}$ ethylene eq. $/ \mathrm{kg}$

$0.174 \mathrm{~kg}$ ethylene eq. $/ \mathrm{kg}$

$0.123 \mathrm{~kg}$ ethylene eq. $/ \mathrm{kg}$

$0.386 \mathrm{~kg}$ ethylene eq. $/ \mathrm{kg}$

$0.213 \mathrm{~kg}$ ethylene eq. $/ \mathrm{kg}$

$0.214 \mathrm{~kg}$ ethylene eq. $/ \mathrm{kg}$

$0.73 \mathrm{~kg}$ ethylene eq. $/ \mathrm{kg}$

$1 \mathrm{~kg}$ ethylene eq. $/ \mathrm{kg}$

$0.382 \mathrm{~kg}$ ethylene eq. $/ \mathrm{kg}$

$0.519 \mathrm{~kg}$ ethylene eq. $/ \mathrm{kg}$

$0.032 \mathrm{~kg}$ ethylene eq. $/ \mathrm{kg}$

$0.572 \mathrm{~kg}$ ethylene eq. $/ \mathrm{kg}$

$0.599 \mathrm{~kg}$ ethylene eq. $/ \mathrm{kg}$

$0.307 \mathrm{~kg}$ ethylene eq. $/ \mathrm{kg}$

$0.375 \mathrm{~kg}$ ethylene eq. $/ \mathrm{kg}$

$0.627 \mathrm{~kg}$ ethylene eq. $/ \mathrm{kg}$

$0.514 \mathrm{~kg}$ ethylene eq. $/ \mathrm{kg}$

$0.405 \mathrm{~kg}$ ethylene eq. $/ \mathrm{kg}$

$1.09 \mathrm{~kg}$ ethylene eq. $/ \mathrm{kg}$

$0.14 \mathrm{~kg}$ ethylene eq. $/ \mathrm{kg}$

$0.213 \mathrm{~kg}$ ethylene eq. $/ \mathrm{kg}$

$0.5 \mathrm{~kg}$ ethylene eq. $/ \mathrm{kg}$

$1.02 \mathrm{~kg}$ ethylene eq. $/ \mathrm{kg}$

$1.11 \mathrm{~kg}$ ethylene eq. $/ \mathrm{kg}$

$0.006 \mathrm{~kg}$ ethylene eq. $/ \mathrm{kg}$

$0.131 \mathrm{~kg}$ ethylene eq. $/ \mathrm{kg}$ 
[E604] Methyl Acetate to air [E609] Methyl Choride to air

[E613] Methyl Ethyl Ketone to air

[E614] Methyl Formate to air

[E616] Methyl Isobutyl Ketone (4-methylpentanon-2) to air

[E622] Methyl propyl Ketone to air

[E624] Methyl trans-Butyl Ether to air

[E625] Methyl-Isopropylketone to air

[E627] Methyl-transbutylketone to air

[E634] Neopentane to air

[E649] ortho-Ethyltoluene to air

[E650] ortho-Xylene (1,2-dimethylbenzene) to air

[E654] para-Ethyltoluene to air

[E658] para-Xylene (1,4-dimethylbenzene) to air

[E663] Pentanaldehyde to air

[E687] Propane to air

[E688] Propanoic acid (propionic acid) to air

[E689] Propionaldehyde (propanal) to air

[E691] Propyl Acetate to air

[E693] Propylene to air

[E695] Propylene Glycol to air

[E701] sec-Butanol to air

[E703] sec-Buytl Acetate to air

[E706] styrene (vinylbenzene) to air

[E716] tertiary-Butanol to air

[E717] tertiary-Butyl Acetate to air

[E718] tetrachloroethylene (PER) (tetrachloroethene) to air

[E727] Toluene to air

[E729] trans-2-Butene to air

[E730] trans-2-Hexene to air

[E731] trans-2-Pentene to air

[E732] trans-dichloroethene to air

[E737] Trichloroethylene (tri) to air

[E738] Trichloromethane (chloroform) to air
$0.046 \mathrm{~kg}$ ethylene eq. $/ \mathrm{kg}$

$0.005 \mathrm{~kg}$ ethylene eq. $/ \mathrm{kg}$

$0.373 \mathrm{~kg}$ ethylene eq. $/ \mathrm{kg}$

$0.033 \mathrm{~kg}$ ethylene eq. $/ \mathrm{kg}$

$0.49 \mathrm{~kg}$ ethylene eq. $/ \mathrm{kg}$

$0.548 \mathrm{~kg}$ ethylene eq. $/ \mathrm{kg}$

$0.152 \mathrm{~kg}$ ethylene eq. $/ \mathrm{kg}$

$0.364 \mathrm{~kg}$ ethylene eq. $/ \mathrm{kg}$

$0.323 \mathrm{~kg}$ ethylene eq. $/ \mathrm{kg}$

$0.173 \mathrm{~kg}$ ethylene eq. $/ \mathrm{kg}$

$0.898 \mathrm{~kg}$ ethylene eq. $/ \mathrm{kg}$

$1.05 \mathrm{~kg}$ ethylene eq. $/ \mathrm{kg}$

$0.906 \mathrm{~kg}$ ethylene eq. $/ \mathrm{kg}$

$1.01 \mathrm{~kg}$ ethylene eq. $/ \mathrm{kg}$

$0.765 \mathrm{~kg}$ ethylene eq. $/ \mathrm{kg}$

$0.176 \mathrm{~kg}$ ethylene eq. $/ \mathrm{kg}$

$0.15 \mathrm{~kg}$ ethylene eq. $/ \mathrm{kg}$

$0.798 \mathrm{~kg}$ ethylene eq. $/ \mathrm{kg}$

$0.29 \mathrm{~kg}$ ethylene eq. $/ \mathrm{kg}$

$1.12 \mathrm{~kg}$ ethylene eq. $/ \mathrm{kg}$

$0.457 \mathrm{~kg}$ ethylene eq. $/ \mathrm{kg}$

$0.4 \mathrm{~kg}$ ethylene eq. $/ \mathrm{kg}$

$0.267 \mathrm{~kg}$ ethylene eq. $/ \mathrm{kg}$

$0.142 \mathrm{~kg}$ ethylene eq. $/ \mathrm{kg}$

$0.123 \mathrm{~kg}$ ethylene eq. $/ \mathrm{kg}$ $0.065 \mathrm{~kg}$ ethylene eq. $/ \mathrm{kg}$ $0.029 \mathrm{~kg}$ ethylene eq. $/ \mathrm{kg}$ $0.637 \mathrm{~kg}$ ethylene eq. $/ \mathrm{kg}$ $1.13 \mathrm{~kg}$ ethylene eq. $/ \mathrm{kg}$ $1.07 \mathrm{~kg}$ ethylene eq. $/ \mathrm{kg}$

$1.12 \mathrm{~kg}$ ethylene eq. $/ \mathrm{kg}$ $0.392 \mathrm{~kg}$ ethylene eq. $/ \mathrm{kg}$ $0.325 \mathrm{~kg}$ ethylene $\mathrm{eq} . / \mathrm{kg}$ $0.023 \mathrm{~kg}$ ethylene eq. $/ \mathrm{kg}$

Category $=[\mathrm{C} 3]$ Depletion of the ozone layer

Description = Problem oriented approach, manual 1999; ozone depletion steady state Author $=$ WMO, 1991, 1994, 1998

Date $=11 / 1 / 99$

Environmental resources

Environmental resource

Environmental emissions

Environmental emission

[E90] 1,1,1-trichloroethane (methyl chloroform; HC-140a) to air

[E398] CFC-11 to air

[E399] CFC-113 to air

[E400] CFC-114 to air

[E401] CFC-115 to air

[E402] CFC-12 to air

[E517] HALON (HBFC-2401) to air

[E518] HALON-1201 (HBFC-1201) to air

[E519] HALON-1202 to air

[E520] HALON-1211 to air

[E521] HALON-1301 to air

[E522] HALON-2311 (HBFC-2311) to air

[E523] HALON-2402 (Di-Broom-tetra-Fluor-ethane) to air

[E525] HCFC-123 (1,1-Di-Chloro-222-Tri-Fluorethaan) to air

[E526] HCFC-124 (1-Chloro-1222-Tetra-Fluor-Ethane) to air

[E527] HCFC-141b (Dichlorofluorethane) to air

[E528] HCFC-142b (1-Chloro-1,1-Difluorethane) to air

[E529] HCFC-22 (Chlorodifluormethane) to air

[E530] HCFC-225ca to air

[E531] HCFC-225cb to air

[E608] Methyl Bromide to air
Value

Value

$0.12 \mathrm{~kg} \mathrm{CFC}-11$ eq. $/ \mathrm{kg}$

$1 \mathrm{~kg}$ CFC-11 eq. $/ \mathrm{kg}$

$0.9 \mathrm{~kg} \mathrm{CFC}-11$ eq. $/ \mathrm{kg}$

$0.85 \mathrm{~kg}$ CFC-11 eq. $/ \mathrm{kg}$

$0.4 \mathrm{~kg} \mathrm{CFC}-11$ eq. $/ \mathrm{kg}$

$0.82 \mathrm{~kg}$ CFC-11 eq. $/ \mathrm{kg}$

$0.25 \mathrm{~kg}$ CFC-11 eq. $/ \mathrm{kg}$

$1.4 \mathrm{~kg} \mathrm{CFC}-11 \mathrm{eq} . / \mathrm{kg}$

$1.25 \mathrm{~kg} \mathrm{CFC}-11$ eq. $/ \mathrm{kg}$

$5.1 \mathrm{~kg} \mathrm{CFC}-11$ eq. $/ \mathrm{kg}$

$12 \mathrm{~kg} \mathrm{CFC}-11 \mathrm{eq} . / \mathrm{kg}$

$0.14 \mathrm{~kg}$ CFC-11 eq. $/ \mathrm{kg}$

$7 \mathrm{~kg} \mathrm{CFC}-11$ eq. $/ \mathrm{kg}$

$0.014 \mathrm{~kg}$ CFC-11 eq. $/ \mathrm{kg}$

$0.03 \mathrm{~kg}$ CFC-11 eq. $/ \mathrm{kg}$

$0.1 \mathrm{~kg} \mathrm{CFC}-11 \mathrm{eq} . / \mathrm{kg}$

$0.05 \mathrm{~kg} \mathrm{CFC}-11$ eq. $/ \mathrm{kg}$

$0.04 \mathrm{~kg}$ CFC-11 eq. $/ \mathrm{kg}$

$0.02 \mathrm{~kg} \mathrm{CFC}-11$ eq. $/ \mathrm{kg}$

$0.02 \mathrm{~kg}$ CFC-11 eq. $/ \mathrm{kg}$

$0.4 \mathrm{~kg} \mathrm{CFC}-11$ eq. $/ \mathrm{kg}$ 
Category $=[C 4]$ Global warming (100 years)

Description = Problem oriented approach, manual 1999; GWP100 Author $=$ Houghton et al., $1994 \& 1995$

Date $=27-09-1999$

Environmental resources

Environmental resource

Environmental emissions

Environmental emission

[E90] 1,1,1-trichloroethane (methyl chloroform; HC-140a) to air

[E393] Carbon dioxide to air

[E398] CFC-11 to air

[E399] CFC-113 to air

[E400] CFC-114 to air

[E401] CFC-115 to air

[E402] CFC-12 to air

[E403] CFC-13 to air

[E449] Dichloromethane (Methylene Chloride) to air

[E469] Dinitrogen oxide (nitrous oxide) to air

[E521] HALON-1301 to air

[E525] HCFC-123 (1,1-Di-Chloro-222-Tri-Fluorethaan) to air

[E526] HCFC-124 (1-Chloro-1222-Tetra-Fluor-Ethane) to air

[E527] HCFC-141b (Dichlorofluorethane) to air

[E528] HCFC-142b (1-Chloro-1,1-Difluorethane) to air

[E529] HCFC-22 (Chlorodifluormethane) to air

[E530] HCFC-225ca to air

[E531] HCFC-225cb to air

[E543] HFC-125 (Pentafluoroethane) to air

[E544] HFC-134 (1,1,1,2-tetra-fluor-ethane) to air

[E545] HFC-134a (1,1,1,2-tetrafluoroethane) to air

[E546] HFC-143 to air

[E547] HFC-143a (1,1,1-trifluoroethane) to air

[E548] HFC-152a (1,1-difluoroethane) to air

[E549] HFC-227ea to air

[E550] HFC-23 to air

[E551] HFC-236fa to air

[E552] HFC-245ca to air

[E553] HFC-32 to air

[E554] HFC-41 to air

[E555] HFC-43-10mee to air

[E600] Methane to air

[E609] Methyl Choride to air

[E665] Perfluorobutane to air

[E666] Perfluorocyclobutane to air

[E667] Perfluoroethane (CFC-116) to air

[E668] Perfluorohexane to air

[E669] Perfluoromethane (CFC-14) to air

[E670] Perfluoropentane to air

[E671] Perfluoropropane to air

[E710] Sulphur hexafluoride to air

[E719] Tetrachloromethane (carbon tetrachloride) ( $\mathrm{HC}-10)$ to air

[E738] Trichloromethane (chloroform) to air
Value

Unit

Value

Unit

$110 \mathrm{~kg} \mathrm{CO} 2 \mathrm{eq} . / \mathrm{kg}$

$1 \mathrm{~kg} \mathrm{CO} 2 \mathrm{eq} . / \mathrm{kg}$

$4000 \mathrm{~kg} \mathrm{CO} 2 \mathrm{eq} . / \mathrm{kg}$

$5000 \mathrm{~kg} \mathrm{CO} 2 \mathrm{eq} . / \mathrm{kg}$

$9300 \mathrm{~kg} \mathrm{CO} 2 \mathrm{eq} . / \mathrm{kg}$

$9300 \mathrm{~kg} \mathrm{CO} 2 \mathrm{eq} . / \mathrm{kg}$

$8500 \mathrm{~kg} \mathrm{CO} 2 \mathrm{eq} . / \mathrm{kg}$

$11700 \mathrm{~kg} \mathrm{CO} 2 \mathrm{eq} . / \mathrm{kg}$

$9 \mathrm{~kg} \mathrm{CO} 2 \mathrm{eq} . / \mathrm{kg}$

$310 \mathrm{~kg} \mathrm{CO} 2 \mathrm{eq} . / \mathrm{kg}$

$5600 \mathrm{~kg} \mathrm{CO} 2 \mathrm{eq} . / \mathrm{kg}$

$93 \mathrm{~kg} \mathrm{CO} 2 \mathrm{eq} . / \mathrm{kg}$

$480 \mathrm{~kg} \mathrm{CO} 2 \mathrm{eq} . / \mathrm{kg}$

$630 \mathrm{~kg} \mathrm{CO} 2 \mathrm{eq} . / \mathrm{kg}$

$2000 \mathrm{~kg} \mathrm{CO} 2 \mathrm{eq} . / \mathrm{kg}$

$1700 \mathrm{~kg} \mathrm{CO} 2 \mathrm{eq} . / \mathrm{kg}$

$170 \mathrm{~kg} \mathrm{CO} 2 \mathrm{eq} . / \mathrm{kg}$

$530 \mathrm{~kg} \mathrm{CO} 2 \mathrm{eq} . / \mathrm{kg}$

$2800 \mathrm{~kg} \mathrm{CO} 2 \mathrm{eq} . / \mathrm{kg}$

$1000 \mathrm{~kg} \mathrm{CO} 2 \mathrm{eq} . / \mathrm{kg}$

$1300 \mathrm{~kg} \mathrm{CO} 2 \mathrm{eq} . / \mathrm{kg}$

$300 \mathrm{~kg} \mathrm{CO} 2 \mathrm{eq} . / \mathrm{kg}$

$3800 \mathrm{~kg} \mathrm{CO} 2$ eq. $/ \mathrm{kg}$

$140 \mathrm{~kg} \mathrm{CO} 2 \mathrm{eq} . / \mathrm{kg}$

$2900 \mathrm{~kg} \mathrm{CO} 2 \mathrm{eq} . / \mathrm{kg}$

$11700 \mathrm{~kg} \mathrm{CO} 2 \mathrm{eq} . / \mathrm{kg}$

$6300 \mathrm{~kg} \mathrm{CO} 2 \mathrm{eq} . / \mathrm{kg}$

$560 \mathrm{~kg} \mathrm{CO} 2 \mathrm{eq} . / \mathrm{kg}$

$650 \mathrm{~kg} \mathrm{CO} 2 \mathrm{eq} . / \mathrm{kg}$

$13000 \mathrm{~kg} \mathrm{CO} 2 \mathrm{eq} . / \mathrm{kg}$

$1300 \mathrm{~kg} \mathrm{CO} 2 \mathrm{eq} . / \mathrm{kg}$

$21 \mathrm{~kg} \mathrm{CO} 2 \mathrm{eq} . / \mathrm{kg}$

$25 \mathrm{~kg} \mathrm{CO} 2 \mathrm{eq} . / \mathrm{kg}$

$7000 \mathrm{~kg} \mathrm{CO} 2 \mathrm{eq} . / \mathrm{kg}$

$8700 \mathrm{~kg} \mathrm{CO} 2 \mathrm{eq} . / \mathrm{kg}$

$9200 \mathrm{~kg} \mathrm{CO} 2 \mathrm{eq} . / \mathrm{kg}$

$7400 \mathrm{~kg} \mathrm{CO} 2 \mathrm{eq} . / \mathrm{kg}$

$6500 \mathrm{~kg} \mathrm{CO} 2 \mathrm{eq} . / \mathrm{kg}$

$7500 \mathrm{~kg} \mathrm{CO} 2 \mathrm{eq} . / \mathrm{kg}$

$7000 \mathrm{~kg} \mathrm{CO} 2 \mathrm{eq} . / \mathrm{kg}$

$23900 \mathrm{~kg} \mathrm{CO} 2 \mathrm{eq} . / \mathrm{kg}$

$1400 \mathrm{~kg} \mathrm{CO} 2 \mathrm{eq} . / \mathrm{kg}$

$5 \mathrm{~kg} \mathrm{CO} 2 \mathrm{eq} . / \mathrm{kg}$

Category $=[\mathrm{C} 5]$ Human toxicity

Description = Problem oriented approach, manual 1999; HTP

Author = Huijbregts, 1999

Date $=27-09-1999$

Environmental resources

Environmental resource 
Environmental emissions

Environmental emission

[E90] 1,1,1-trichloroethane (methyl chloroform; HC-140a) to air

[E94] 1,2,3,4-tetrachlorobenzene to air

[E95] 1,2,3,5-tetrachlorobenzene to air

[E96] 1,2,3-trichlorobenzene to air

[E98] 1,2,4,5-tetrachlorobenzene to air

[E99] 1,2,4-trichlorobenzene to air

[E102] 1,2-dichlorobenzene to air

[E103] 1,2-dichloroethane to air

[E107] 1,3,5-trichlorobenzene to air

[E111] 1,3-Butadiene to air

[E112] 1,3-dichlorobenzene to air

[E116] 1,4-dichlorobenzene to air

[E128] 1-chloro-4-nitrobenzene to air

[E166] 2,3,4,6-tetrachlorophenol to air

[E168] 2,3,7,8-TCDD (tetrachloride-dibenzo-dioxin) to air

[E172] 2,4,5-T to air

[E173] 2,4,5-trichlorophenol to air

[E174] 2,4,6-trichlorophenol to air

[E175] 2,4-D to air

[E176] 2,4-dichlorophenol to air

[E185] 2-chlorophenol to air

[E208] 3,4-dichloroaniline to air

[E217] 3-chloroaniline to air

[E228] 4-chloroaniline to air

[E231] acephate to air

[E236] Acrolein (2-propenal) to air

[E237] Acrylonitrile to air

[E241] aldicarb to air

[E242] aldrin to air

[E250] ammonia, ammonium to air

[E251] anilazine to air

[E252] anthracene to air

[E253] antimony to air

[E255] arsenic to air

[E256] atrazine to air

[E257] azinphos-ethyl to air

[E258] azinphos-methyl to air

[E259] barium to air

[E260] benomyl to air

[E261] bentazone to air

[E263] Benzene to air

[E269] benzylchloride to air

[E270] beryllium to air

[E272] bifenthrin to air

[E290] Butylbenzylphtalate to air

[E385] cadmium to air

[E387] captafol to air

[E388] captan to air

[E389] carbaryl to air

[E390] carbendazim to air

[E391] carbofuran to air

[E394] carbon disulfide to air

[E404] chlordane to air

[E405] chlorfenvinphos to air

[E406] chloridazon to air

[E407] chlorobenzene to air

[E410] chlorothalonil to air

[E411] chlorpropham to air

[E412] chlorpyriphos to air

[E413] chromium (unspecified) to air

[E414] chromium III to air

[E415] chromium VI to air

[E421] cobalt to air

[E422] copper to air

[E423] coumaphos to air
Value

Unit

17 kg 1,4-dichlorobenzene eq." $/ \mathrm{kg}$

$50 \mathrm{~kg}$ 1,4-dichlorobenzene eq." $/ \mathrm{kg}$

$46 \mathrm{~kg}$ 1,4-dichlorobenzene eq." $/ \mathrm{kg}$

$130 \mathrm{~kg}$ 1,4-dichlorobenzene eq." $/ \mathrm{kg}$

35 kg 1,4-dichlorobenzene eq." $/ \mathrm{kg}$

$120 \mathrm{~kg}$ 1,4-dichlorobenzene eq." $/ \mathrm{kg}$

$9.1 \mathrm{~kg}$ 1,4-dichlorobenzene eq." $/ \mathrm{kg}$

$6.8 \mathrm{~kg}$ 1,4-dichlorobenzene eq." $/ \mathrm{kg}$

$120 \mathrm{~kg}$ 1,4-dichlorobenzene eq." $/ \mathrm{kg}$

2200 kg 1,4-dichlorobenzene eq." $/ \mathrm{kg}$

62 kg 1,4-dichlorobenzene eq."/kg

$1 \mathrm{~kg}$ 1,4-dichlorobenzene eq." $/ \mathrm{kg}$

$1200 \mathrm{~kg}$ 1,4-dichlorobenzene eq." $/ \mathrm{kg}$

290 kg 1,4-dichlorobenzene eq." $/ \mathrm{kg}$

$1.90 \mathrm{E}+09 \mathrm{~kg}$ 1,4-dichlorobenzene eq."/kg

$0.89 \mathrm{~kg}$ 1,4-dichlorobenzene eq." $/ \mathrm{kg}$

$8.3 \mathrm{~kg}$ 1,4-dichlorobenzene eq."/kg

$14000 \mathrm{~kg}$ 1,4-dichlorobenzene eq." $/ \mathrm{kg}$

$6.6 \mathrm{~kg}$ 1,4-dichlorobenzene eq." $/ \mathrm{kg}$

$95 \mathrm{~kg}$ 1,4-dichlorobenzene eq." $/ \mathrm{kg}$

22 kg 1,4-dichlorobenzene eq." $/ \mathrm{kg}$

$220 \mathrm{~kg}$ 1,4-dichlorobenzene eq."/kg

17000 kg 1,4-dichlorobenzene eq."/kg

$260 \mathrm{~kg}$ 1,4-dichlorobenzene eq." $/ \mathrm{kg}$

$3.1 \mathrm{~kg}$ 1,4-dichlorobenzene eq." $/ \mathrm{kg}$

$57 \mathrm{~kg}$ 1,4-dichlorobenzene eq." $/ \mathrm{kg}$

$3400 \mathrm{~kg}$ 1,4-dichlorobenzene eq."/kg

$72 \mathrm{~kg}$ 1,4-dichlorobenzene eq." $/ \mathrm{kg}$

$19 \mathrm{~kg}$ 1,4-dichlorobenzene eq." $/ \mathrm{kg}$

$0.1 \mathrm{~kg}$ 1,4-dichlorobenzene eq." $/ \mathrm{kg}$

$0.072 \mathrm{~kg}$ 1,4-dichlorobenzene eq." $/ \mathrm{kg}$

$0.52 \mathrm{~kg}$ 1,4-dichlorobenzene eq." $/ \mathrm{kg}$

$6700 \mathrm{~kg}$ 1,4-dichlorobenzene eq." $/ \mathrm{kg}$

$3.50 \mathrm{E}+05 \mathrm{~kg}$ 1,4-dichlorobenzene eq." $/ \mathrm{kg}$

$4.5 \mathrm{~kg}$ 1,4-dichlorobenzene eq." $/ \mathrm{kg}$

$200 \mathrm{~kg}$ 1,4-dichlorobenzene eq." $/ \mathrm{kg}$

$14 \mathrm{~kg}$ 1,4-dichlorobenzene eq." $/ \mathrm{kg}$

$760 \mathrm{~kg}$ 1,4-dichlorobenzene eq." $/ \mathrm{kg}$

$0.021 \mathrm{~kg} 1,4$-dichlorobenzene eq." $/ \mathrm{kg}$

$2.1 \mathrm{~kg}$ 1,4-dichlorobenzene eq." $/ \mathrm{kg}$

1900 kg 1,4-dichlorobenzene eq."/kg

$3500 \mathrm{~kg}$ 1,4-dichlorobenzene eq." $/ \mathrm{kg}$

$2.30 E+05 \mathrm{~kg}$ 1,4-dichlorobenzene eq." $/ \mathrm{kg}$

$19 \mathrm{~kg}$ 1,4-dichlorobenzene eq." $/ \mathrm{kg}$

$10 \mathrm{~kg}$ 1,4-dichlorobenzene eq."/kg

1.50E+05 kg 1,4-dichlorobenzene eq." $/ \mathrm{kg}$

87 kg 1,4-dichlorobenzene eq."/kg

$0.59 \mathrm{~kg}$ 1,4-dichlorobenzene eq." $/ \mathrm{kg}$

$3.2 \mathrm{~kg}$ 1,4-dichlorobenzene eq." $/ \mathrm{kg}$

19 kg 1,4-dichlorobenzene eq."/kg

200 kg 1,4-dichlorobenzene eq." $/ \mathrm{kg}$

$2.4 \mathrm{~kg}$ 1,4-dichlorobenzene eq." $/ \mathrm{kg}$

$6700 \mathrm{~kg}$ 1,4-dichlorobenzene eq." $/ \mathrm{kg}$

$270 \mathrm{~kg}$ 1,4-dichlorobenzene eq." $/ \mathrm{kg}$

$0.013 \mathrm{~kg} \mathrm{1,4-dichlorobenzene} \mathrm{eq."} / \mathrm{kg}$

$9.2 \mathrm{~kg}$ 1,4-dichlorobenzene eq." $/ \mathrm{kg}$

$8.4 \mathrm{~kg}$ 1,4-dichlorobenzene eq." $/ \mathrm{kg}$

$0.34 \mathrm{~kg} 1,4$-dichlorobenzene eq." $/ \mathrm{kg}$

$21 \mathrm{~kg}$ 1,4-dichlorobenzene eq." $/ \mathrm{kg}$

$650 \mathrm{~kg}$ 1,4-dichlorobenzene eq." $/ \mathrm{kg}$

$650 \mathrm{~kg}$ 1,4-dichlorobenzene eq." $/ \mathrm{kg}$

3.40E+06 kg 1,4-dichlorobenzene eq." $/ \mathrm{kg}$

17000 kg 1,4-dichlorobenzene eq." $/ \mathrm{kg}$

$4300 \mathrm{~kg}$ 1,4-dichlorobenzene eq."/kg

780 kg 1,4-dichlorobenzene eq." $/ \mathrm{kg}$ 
[E425] cyanazine to air

[E437] cypermethrin to air

[E438] cyromazine to air

[E439] DDT to air

[E441] deltamethrin to air

[E442] demeton to air

[E443] desmetryn to air

[E444] Di(2-ethylhexyl)phtalate to air

[E446] diazinon to air

[E448] Dibutylphtalate to air

[E449] Dichloromethane (Methylene Chloride) to air

[E450] dichlorprop to air

[E451] dichlorvos to air

[E452] dieldrin to air

[E456] Diethylphtalate to air

[E457] Dihexylphtalate to air

[E458] Diisodecylphtalate to air

[E459] Diisooctylphtalate to air

[E461] dimethoate to air

[E468] Dimethylphtalate to air

[E470] dinoseb to air

[E471] dinoterb to air

[E472] Dioctylphtalate to air

[E475] disulfothon to air

[E476] diuron to air

[E478] DNOC to air

[E480] endosulfan to air

[E481] endrin to air

[E487] ethoprophos to air

[E496] Ethylbenzene to air

[E499] Ethylene (ethene) to air

[E504] fenitrothion to air

[E505] fenthion to air

[E506] fentin acetate to air

[E507] fentin chloride to air

[E508] fentin hydroxide to air

[E510] folpet to air

[E511] Formaldehyde (methanal) to air

[E516] glyphosate to air

[E533] heptachlor to air

[E535] heptenophos to air

[E536] hexachloro-1,3-butadiene to air

[E537] hexachlorobenzene to air

[E561] hydrogen chloride to air

[E563] hydrogen sulfide to air

[E568] iprodione to air

[E583] isoproturon to air

[E584] lead to air

[E585] lindane to air

[E586] linuron to air

[E587] malathion to air

[E589] MCPA to air

[E590] mecoprop to air

[E592] mercury to air

[E595] metamitron to air

[E596] meta-Xylene (1,3-dimethylbenzene) to air

[E597] metazachlor to air

[E598] methabenzthiazuron to air

[E603] methomyl to air

[E626] methyl-mercury to air

[E628] metobromuron to air

[E629] metolachlor to air

[E630] mevinphos to air

[E632] molybdenum to air

[E633] Naphtalene to air

[E635] nickel to air

[E641] nitrogen dioxide to air
$3.5 \mathrm{~kg}$ 1,4-dichlorobenzene eq." $/ \mathrm{kg}$

$170 \mathrm{~kg}$ 1,4-dichlorobenzene eq." $/ \mathrm{kg}$

38 kg 1,4-dichlorobenzene eq."/kg

$110 \mathrm{~kg}$ 1,4-dichlorobenzene eq." $/ \mathrm{kg}$

$1.6 \mathrm{~kg}$ 1,4-dichlorobenzene eq." $/ \mathrm{kg}$

$71 \mathrm{~kg} \mathrm{1,4-dichlorobenzene} \mathrm{eq."} / \mathrm{kg}$

$95 \mathrm{~kg}$ 1,4-dichlorobenzene eq." $/ \mathrm{kg}$

$2.6 \mathrm{~kg} \mathrm{1,4-dichlorobenzene} \mathrm{eq."} / \mathrm{kg}$

$59 \mathrm{~kg}$ 1,4-dichlorobenzene eq." $/ \mathrm{kg}$

25 kg 1,4-dichlorobenzene eq."/kg

$2 \mathrm{~kg}$ 1,4-dichlorobenzene eq." $/ \mathrm{kg}$

$1.1 \mathrm{~kg}$ 1,4-dichlorobenzene eq." $/ \mathrm{kg}$

$100 \mathrm{~kg}$ 1,4-dichlorobenzene eq."/kg

$13000 \mathrm{~kg}$ 1,4-dichlorobenzene eq." $/ \mathrm{kg}$

$0.32 \mathrm{~kg}$ 1,4-dichlorobenzene eq." $/ \mathrm{kg}$

7000 kg 1,4-dichlorobenzene eq." $/ \mathrm{kg}$

$46 \mathrm{~kg}$ 1,4-dichlorobenzene eq."/kg

$310 \mathrm{~kg}$ 1,4-dichlorobenzene eq."/kg

$44 \mathrm{~kg}$ 1,4-dichlorobenzene eq."/kg

$210 \mathrm{~kg}$ 1,4-dichlorobenzene eq." $/ \mathrm{kg}$

$3600 \mathrm{~kg}$ 1,4-dichlorobenzene eq."/kg

$170 \mathrm{~kg}$ 1,4-dichlorobenzene eq." $/ \mathrm{kg}$

$19 \mathrm{~kg}$ 1,4-dichlorobenzene eq." $/ \mathrm{kg}$

290 kg 1,4-dichlorobenzene eq."/kg

$290 \mathrm{~kg}$ 1,4-dichlorobenzene eq." $/ \mathrm{kg}$

$160 \mathrm{~kg}$ 1,4-dichlorobenzene eq."/kg

$6.7 \mathrm{~kg}$ 1,4-dichlorobenzene eq."//kg

1200 kg 1,4-dichlorobenzene eq." $/ \mathrm{kg}$

$1100 \mathrm{~kg}$ 1,4-dichlorobenzene eq."/kg

$0.97 \mathrm{~kg}$ 1,4-dichlorobenzene eq."/kg

$0.64 \mathrm{~kg}$ 1,4-dichlorobenzene eq." $/ \mathrm{kg}$

$5.9 \mathrm{~kg}$ 1,4-dichlorobenzene eq." $/ \mathrm{kg}$

$63 \mathrm{~kg}$ 1,4-dichlorobenzene eq." $/ \mathrm{kg}$

2200 kg 1,4-dichlorobenzene eq." $/ \mathrm{kg}$

$840 \mathrm{~kg}$ 1,4-dichlorobenzene eq."/kg

$850 \mathrm{~kg}$ 1,4-dichlorobenzene eq." $/ \mathrm{kg}$

$2 \mathrm{~kg}$ 1,4-dichlorobenzene eq." $/ \mathrm{kg}$

$0.83 \mathrm{~kg}$ 1,4-dichlorobenzene eq." $/ \mathrm{kg}$

$0.0031 \mathrm{~kg}$ 1,4-dichlorobenzene eq."/kg

$40 \mathrm{~kg}$ 1,4-dichlorobenzene eq." $/ \mathrm{kg}$

$23 \mathrm{~kg}$ 1,4-dichlorobenzene eq." $/ \mathrm{kg}$

79000 kg 1,4-dichlorobenzene eq." $/ \mathrm{kg}$

$3.20 \mathrm{E}+06 \mathrm{~kg}$ 1,4-dichlorobenzene eq." $/ \mathrm{kg}$

$0.5 \mathrm{~kg}$ 1,4-dichlorobenzene eq." $/ \mathrm{kg}$

$0.71 \mathrm{~kg}$ 1,4-dichlorobenzene eq." $/ \mathrm{kg}$

$0.28 \mathrm{~kg}$ 1,4-dichlorobenzene eq." $/ \mathrm{kg}$

$130 \mathrm{~kg}$ 1,4-dichlorobenzene eq." $/ \mathrm{kg}$

$470 \mathrm{~kg}$ 1,4-dichlorobenzene eq." $/ \mathrm{kg}$

$610 \mathrm{~kg}$ 1,4-dichlorobenzene eq." $/ \mathrm{kg}$

$14 \mathrm{~kg}$ 1,4-dichlorobenzene eq." $/ \mathrm{kg}$

$0.035 \mathrm{~kg}$ 1,4-dichlorobenzene eq." $/ \mathrm{kg}$

$15 \mathrm{~kg}$ 1,4-dichlorobenzene eq. "/kg

$120 \mathrm{~kg}$ 1,4-dichlorobenzene eq." $/ \mathrm{kg}$

$6000 \mathrm{~kg} \mathrm{1,4-dichlorobenzene} \mathrm{eq."} / \mathrm{kg}$

$0.88 \mathrm{~kg} 1,4$-dichlorobenzene eq." $/ \mathrm{kg}$

0.027 kg 1,4-dichlorobenzene eq." $/ \mathrm{kg}$

$6.8 \mathrm{~kg}$ 1,4-dichlorobenzene eq." $/ \mathrm{kg}$

7.1 kg 1,4-dichlorobenzene eq." $/ \mathrm{kg}$

$6.2 \mathrm{~kg}$ 1,4-dichlorobenzene eq." $/ \mathrm{kg}$

$58000 \mathrm{~kg} \mathrm{1,4-dichlorobenzene} \mathrm{eq."} / \mathrm{kg}$

55 kg 1,4-dichlorobenzene eq." $/ \mathrm{kg}$

$2.6 \mathrm{~kg}$ 1,4-dichlorobenzene eq." $/ \mathrm{kg}$

$1 \mathrm{~kg}$ 1,4-dichlorobenzene eq." $/ \mathrm{kg}$

$5400 \mathrm{~kg}$ 1,4-dichlorobenzene eq." $/ \mathrm{kg}$

$8.1 \mathrm{~kg}$ 1,4-dichlorobenzene eq." $/ \mathrm{kg}$

$35000 \mathrm{~kg}$ 1,4-dichlorobenzene eq."/kg

$1.3 \mathrm{~kg}$ 1,4-dichlorobenzene eq." $/ \mathrm{kg}$ 
[E650] ortho-Xylene (1,2-dimethylbenzene) to air

[E651] oxamyl to air

[E652] oxydemethon-methyl to air

[E655] parathion-ethyl to air

[E656] parathion-methyl to air

[E658] para-Xylene $(1,4$-dimethylbenzene) to air

[E660] pentachlorobenzene to air

[E661] pentachloronitrobenzene to air

[E662] pentachlorophenol to air

[E672] permethrin to air

[E675] Phenol to air

[E679] phoxim to air

[E680] Phtalic anhydride to air

[E682] pirimicarb to air

[E683] PM10 to air

[E684] Polycyclic Aromatic Hydrocarbons (PAH) (unspecified) to air

[E685] Polycyclic Aromatic Hydrocarbons Carcinogenic- (carcinogenic-PAH) to air

[E686] propachlor to air

[E690] propoxur to air

[E697] Propylene Oxide to air

[E698] pyrazophos to air

[E704] selenium to air

[E705] simazine to air

[E706] styrene (vinylbenzene) to air

[E709] sulphur dioxide to air

[E718] tetrachloroethylene (PER) (tetrachloroethene) to air

[E719] Tetrachloromethane (carbon tetrachloride) $(\mathrm{HC}-10)$ to air

[E722] thallium to air

[E723] Thiram to air

[E724] tin to air

[E725] tolclophos-methyl to air

[E727] Toluene to air

[E733] tri-allate to air

[E734] triazophos to air

[E735] tributyltinoxide to air

[E736] trichlorfon to air

[E737] Trichloroethylene (tri) to air

[E738] Trichloromethane (chloroform) to air

[E739] trifluarin to air

[E743] vanadium to air

[E745] Vinyl Chloride (chloroethene) to air

[E748] zinc to air

[E749] zineb to air

[E750] 1,1,1-trichloroethane to air

[E751] 1,2,3,4-tetrachlorobenzene to air

[E752] 1,2,3,5-tetrachlorobenzene to air

[E753] 1,2,3-trichlorobenzene to air

[E754] 1,2,4,5-tetrachlorobenzene to air

[E755] 1,2,4-trichlorobenzene to air

[E756] 1,2-dichlorobenzene to air

[E757] 1,2-dichloroethane to air

[E758] 1,3,5-trichlorobenzene to air

[E759] 1,3-butadiene to air

[E760] 1,3-dichlorobenzene to air

[E761] 1,4-dichlorobenzene to air

[E762] 1-chloro-4-nitrobenzene to air

[E763] 2,3,4,6-tetrachlorophenol to air

[E764] 2,3,7,8-TCDD to air

[E765] 2,4,5-T to air

[E766] 2,4,5-trichlorophenol to air

[E767] 2,4,6-trichlorophenol to fresh water

[E768] 2,4-D to fresh water

[E769] 2,4-dichlorophenol to fresh water

[E770] 2-chlorophenol to fresh water

[E771] 3,4-dichloroaniline to fresh water

[E772] 3-chloroaniline to fresh water

[E773] 4-chloroaniline to fresh water
$0.12 \mathrm{~kg}$ 1,4-dichlorobenzene eq." $/ \mathrm{kg}$

$1.4 \mathrm{~kg}$ 1,4-dichlorobenzene eq." $/ \mathrm{kg}$

$120 \mathrm{~kg}$ 1,4-dichlorobenzene eq." $/ \mathrm{kg}$

$3.3 \mathrm{~kg}$ 1,4-dichlorobenzene eq." $/ \mathrm{kg}$

$53 \mathrm{~kg}$ 1,4-dichlorobenzene eq." $/ \mathrm{kg}$

$0.043 \mathrm{~kg}$ 1,4-dichlorobenzene eq." $/ \mathrm{kg}$

$410 \mathrm{~kg}$ 1,4-dichlorobenzene eq." $/ \mathrm{kg}$

$190 \mathrm{~kg}$ 1,4-dichlorobenzene eq." $/ \mathrm{kg}$

$5.1 \mathrm{~kg}$ 1,4-dichlorobenzene eq." $/ \mathrm{kg}$

$0.85 \mathrm{~kg}$ 1,4-dichlorobenzene eq. "/kg

$0.52 \mathrm{~kg}$ 1,4-dichlorobenzene eq." $/ \mathrm{kg}$

$0.97 \mathrm{~kg}$ 1,4-dichlorobenzene eq." $/ \mathrm{kg}$

$0.41 \mathrm{~kg}$ 1,4-dichlorobenzene eq." $/ \mathrm{kg}$

$3.4 \mathrm{~kg}$ 1,4-dichlorobenzene eq." $/ \mathrm{kg}$

$0.82 \mathrm{~kg} 1,4$-dichlorobenzene eq. "/kg

$5.70 \mathrm{E}+05 \mathrm{~kg}$ 1,4-dichlorobenzene eq. "/kg

$5.70 \mathrm{E}+05 \mathrm{~kg}$ 1,4-dichlorobenzene eq." $/ \mathrm{kg}$

12 kg 1,4-dichlorobenzene eq." $/ \mathrm{kg}$

$37 \mathrm{~kg}$ 1,4-dichlorobenzene eq." $/ \mathrm{kg}$

1300 kg 1,4-dichlorobenzene eq." $/ \mathrm{kg}$

$25 \mathrm{~kg}$ 1,4-dichlorobenzene eq." $/ \mathrm{kg}$

48000 kg 1,4-dichlorobenzene eq."/kg

$33 \mathrm{~kg}$ 1,4-dichlorobenzene eq." $/ \mathrm{kg}$

$0.047 \mathrm{~kg}$ 1,4-dichlorobenzene eq." $/ \mathrm{kg}$

$0.096 \mathrm{~kg}$ 1,4-dichlorobenzene eq." $/ \mathrm{kg}$

$5.5 \mathrm{~kg}$ 1,4-dichlorobenzene eq." $/ \mathrm{kg}$

$220 \mathrm{~kg}$ 1,4-dichlorobenzene eq." $/ \mathrm{kg}$

4.30E+05 kg 1,4-dichlorobenzene eq." $/ \mathrm{kg}$

19 kg 1,4-dichlorobenzene eq." $/ \mathrm{kg}$

$1.7 \mathrm{~kg}$ 1,4-dichlorobenzene eq." $/ \mathrm{kg}$

$0.06 \mathrm{~kg}$ 1,4-dichlorobenzene eq." $/ \mathrm{kg}$

$0.33 \mathrm{~kg}$ 1,4-dichlorobenzene eq." $/ \mathrm{kg}$

$9.7 \mathrm{~kg}$ 1,4-dichlorobenzene eq." $/ \mathrm{kg}$

$210 \mathrm{~kg}$ 1,4-dichlorobenzene eq." $/ \mathrm{kg}$

$7500 \mathrm{~kg}$ 1,4-dichlorobenzene eq." $/ \mathrm{kg}$

$4.4 \mathrm{~kg}$ 1,4-dichlorobenzene eq." $/ \mathrm{kg}$

$34 \mathrm{~kg}$ 1,4-dichlorobenzene eq." $/ \mathrm{kg}$

$13 \mathrm{~kg}$ 1,4-dichlorobenzene eq." $/ \mathrm{kg}$

$1.7 \mathrm{~kg}$ 1,4-dichlorobenzene eq." $/ \mathrm{kg}$

6200 kg 1,4-dichlorobenzene eq." $/ \mathrm{kg}$

$84 \mathrm{~kg}$ 1,4-dichlorobenzene eq." $/ \mathrm{kg}$

$100 \mathrm{~kg}$ 1,4-dichlorobenzene eq." $/ \mathrm{kg}$

$4.8 \mathrm{~kg}$ 1,4-dichlorobenzene eq." $/ \mathrm{kg}$

$17 \mathrm{~kg}$ 1,4-dichlorobenzene eq." $/ \mathrm{kg}$

$160 \mathrm{~kg}$ 1,4-dichlorobenzene eq." $/ \mathrm{kg}$

$92 \mathrm{~kg}$ 1,4-dichlorobenzene eq." $/ \mathrm{kg}$

$130 \mathrm{~kg}$ 1,4-dichlorobenzene eq." $/ \mathrm{kg}$

$180 \mathrm{~kg}$ 1,4-dichlorobenzene eq." $/ \mathrm{kg}$

$120 \mathrm{~kg}$ 1,4-dichlorobenzene eq." $/ \mathrm{kg}$

$8.9 \mathrm{~kg}$ 1,4-dichlorobenzene eq." $/ \mathrm{kg}$

$28 \mathrm{~kg}$ 1,4-dichlorobenzene eq." $/ \mathrm{kg}$

$120 \mathrm{~kg}$ 1,4-dichlorobenzene eq." $/ \mathrm{kg}$

7000 kg 1,4-dichlorobenzene eq." $/ \mathrm{kg}$

$74 \mathrm{~kg}$ 1,4-dichlorobenzene eq. "/kg

$1.1 \mathrm{~kg}$ 1,4-dichlorobenzene eq." $/ \mathrm{kg}$

$1700 \mathrm{~kg}$ 1,4-dichlorobenzene eq." $/ \mathrm{kg}$

$35 \mathrm{~kg}$ 1,4-dichlorobenzene eq." $/ \mathrm{kg}$

$8.60 \mathrm{E}+08 \mathrm{~kg}$ 1,4-dichlorobenzene eq." $/ \mathrm{kg}$

$1.9 \mathrm{~kg}$ 1,4-dichlorobenzene eq." $/ \mathrm{kg}$

$45 \mathrm{~kg}$ 1,4-dichlorobenzene eq." $/ \mathrm{kg}$

$9100 \mathrm{~kg}$ 1,4-dichlorobenzene eq." $/ \mathrm{kg}$

$3.5 \mathrm{~kg}$ 1,4-dichlorobenzene eq." $/ \mathrm{kg}$

$16 \mathrm{~kg} \mathrm{1,4-dichlorobenzene} \mathrm{eq."} / \mathrm{kg}$

$70 \mathrm{~kg}$ 1,4-dichlorobenzene eq." $/ \mathrm{kg}$

$130 \mathrm{~kg}$ 1,4-dichlorobenzene eq." $/ \mathrm{kg}$

3500 kg 1,4-dichlorobenzene eq." $/ \mathrm{kg}$

2900 kg 1,4-dichlorobenzene eq."/kg 
[E774] acephate to fresh water [E775] Acrolein (2-propenal) to fresh water [E776] acrylonitrile to fresh water [E777] aldicarb to fresh water [E778] aldrin to fresh water [E780] anilazine to fresh water [E781] anthracene to fresh water [E782] antimony to fresh water [E783] arsenic to fresh water [E784] atrazine to fresh water [E785] azinphos-ethyl to fresh water [E786] azinphos-methyl to fresh water [E787] barium to fresh water [E788] benomyl to fresh water [E789] bentazone to fresh water [E790] benzene to fresh water [E795] benzylchloride to fresh water [E796] beryllium to fresh water [E797] bifenthrin to fresh water [E800] Butylbenzylphtalate to fresh water [E801] cadmium to fresh water [E802] captafol to fresh water [E803] captan to fresh water [E804] carbaryl to fresh water [E805] carbendazim to fresh water [E806] carbofuran to fresh water [E807] carbon disulfide to fresh water [E808] Carcinogenic PAHs to fresh water [E810] chlordane to fresh water [E811] chlorfenvinphos to fresh water [E812] chloridazon to fresh water [E814] chlorobenzene to fresh water [E815] chlorothalonil to fresh water [E816] chlorpropham to fresh water [E817] chlorpyriphos to fresh water [E818] chromium III to fresh water [E819] chromium VI to fresh water [E821] cobalt to fresh water [E822] copper to fresh water [E823] coumaphos to fresh water [E824] cyanazine to fresh water [E825] cypermethrin to fresh water [E826] cyromazine to fresh water [E827] DDT to fresh water [E828] deltamethrin to fresh water [E829] demeton to fresh water [E830] desmetryn to fresh water [E831] Di(2-ethylhexyl)phtalate to fresh water [E832] diazinon to fresh water

[E833] Dibutylphtalate to fresh water

[E834] Dichloromethane (Methylene Chloride) to fresh water

[E835] dichlorprop to fresh water

[E836] dichlorvos to fresh water

[E837] dieldrin to fresh water

[E838] Diethylphtalate to fresh water

[E839] Dihexylphtalate to fresh water

[E840] Diisodecylphtalate to fresh water

[E841] Diisooctylphtalate to fresh water

[E842] dimethoate to fresh water

[E843] Dimethylphtalate to fresh water

[E844] dinoseb to fresh water

[E845] dinoterb to fresh water

[E846] Dioctylphtalate to fresh water

[E847] disulfothon to fresh water

[E848] diuron to fresh water

[E849] DNOC to fresh water

[E850] endosulfan to fresh water
$2.1 \mathrm{~kg}$ 1,4-dichlorobenzene eq. "/kg

59 kg 1,4-dichlorobenzene eq." $/ \mathrm{kg}$

$7100 \mathrm{~kg}$ 1,4-dichlorobenzene eq."/kg

$61 \mathrm{~kg}$ 1,4-dichlorobenzene eq." $/ \mathrm{kg}$

$6000 \mathrm{~kg}$ 1,4-dichlorobenzene eq." $/ \mathrm{kg}$

$0.24 \mathrm{~kg}$ 1,4-dichlorobenzene eq." $/ \mathrm{kg}$

$2.1 \mathrm{~kg}$ 1,4-dichlorobenzene eq." $/ \mathrm{kg}$

5100 kg 1,4-dichlorobenzene eq." $/ \mathrm{kg}$

950 kg 1,4-dichlorobenzene eq."//kg

$4.6 \mathrm{~kg}$ 1,4-dichlorobenzene eq." $/ \mathrm{kg}$

$460 \mathrm{~kg}$ 1,4-dichlorobenzene eq." $/ \mathrm{kg}$

$2.5 \mathrm{~kg}$ 1,4-dichlorobenzene eq." $/ \mathrm{kg}$

$630 \mathrm{~kg}$ 1,4-dichlorobenzene eq." $/ \mathrm{kg}$

$0.14 \mathrm{~kg}$ 1,4-dichlorobenzene eq." $/ \mathrm{kg}$

$0.73 \mathrm{~kg}$ 1,4-dichlorobenzene eq." $/ \mathrm{kg}$

$1800 \mathrm{~kg}$ 1,4-dichlorobenzene eq." $/ \mathrm{kg}$

2400 kg 1,4-dichlorobenzene eq." $/ \mathrm{kg}$

14000 kg 1,4-dichlorobenzene eq."/kg

$98 \mathrm{~kg}$ 1,4-dichlorobenzene eq." $/ \mathrm{kg}$

$0.086 \mathrm{~kg}$ 1,4-dichlorobenzene eq." $/ \mathrm{kg}$

$23 \mathrm{~kg}$ 1,4-dichlorobenzene eq." $/ \mathrm{kg}$

$500 \mathrm{~kg} \mathrm{1,4-dichlorobenzene} \mathrm{eq."} / \mathrm{kg}$

$0.0053 \mathrm{~kg}$ 1,4-dichlorobenzene eq." $/ \mathrm{kg}$

$4.7 \mathrm{~kg}$ 1,4-dichlorobenzene eq." $/ \mathrm{kg}$

$2.5 \mathrm{~kg}$ 1,4-dichlorobenzene eq." $/ \mathrm{kg}$

56 kg 1,4-dichlorobenzene eq." $/ \mathrm{kg}$

$2.4 \mathrm{~kg}$ 1,4-dichlorobenzene eq." $/ \mathrm{kg}$

2.80E+05 kg 1,4-dichlorobenzene eq." $/ \mathrm{kg}$

$740 \mathrm{~kg}$ 1,4-dichlorobenzene eq." $/ \mathrm{kg}$

$810 \mathrm{~kg}$ 1,4-dichlorobenzene eq." $/ \mathrm{kg}$

$0.14 \mathrm{~kg}$ 1,4-dichlorobenzene eq." $/ \mathrm{kg}$

$9.1 \mathrm{~kg}$ 1,4-dichlorobenzene eq." $/ \mathrm{kg}$

$6.7 \mathrm{~kg}$ 1,4-dichlorobenzene eq." $/ \mathrm{kg}$

$1 \mathrm{~kg} 1,4$-dichlorobenzene eq." $/ \mathrm{kg}$

$44 \mathrm{~kg}$ 1,4-dichlorobenzene eq." $/ \mathrm{kg}$

$2.1 \mathrm{~kg}$ 1,4-dichlorobenzene eq. "/kg

$3.4 \mathrm{~kg}$ 1,4-dichlorobenzene eq." $/ \mathrm{kg}$

$97 \mathrm{~kg}$ 1,4-dichlorobenzene eq." $/ \mathrm{kg}$

$1.3 \mathrm{~kg}$ 1,4-dichlorobenzene eq." $/ \mathrm{kg}$

$10000 \mathrm{~kg}$ 1,4-dichlorobenzene eq." $/ \mathrm{kg}$

$6 \mathrm{~kg}$ 1,4-dichlorobenzene eq." $/ \mathrm{kg}$

$5.5 \mathrm{~kg}$ 1,4-dichlorobenzene eq." $/ \mathrm{kg}$

$5.4 \mathrm{~kg}$ 1,4-dichlorobenzene eq." $/ \mathrm{kg}$

$37 \mathrm{~kg}$ 1,4-dichlorobenzene eq." $/ \mathrm{kg}$

$2.8 \mathrm{~kg} \mathrm{1,4-dichlorobenzene} \mathrm{eq."} / \mathrm{kg}$

$720 \mathrm{~kg}$ 1,4-dichlorobenzene eq." $/ \mathrm{kg}$

$50 \mathrm{~kg}$ 1,4-dichlorobenzene eq." $/ \mathrm{kg}$

$0.91 \mathrm{~kg}$ 1,4-dichlorobenzene eq." $/ \mathrm{kg}$

$66 \mathrm{~kg}$ 1,4-dichlorobenzene eq." $/ \mathrm{kg}$

$0.54 \mathrm{~kg}$ 1,4-dichlorobenzene eq." $/ \mathrm{kg}$

$1.8 \mathrm{~kg}$ 1,4-dichlorobenzene eq." $/ \mathrm{kg}$

$24 \mathrm{~kg}$ 1,4-dichlorobenzene eq. "/kg

$0.34 \mathrm{~kg} 1,4$-dichlorobenzene eq. $/ / \mathrm{kg}$

$45000 \mathrm{~kg}$ 1,4-dichlorobenzene eq." $/ \mathrm{kg}$

$0.14 \mathrm{~kg}$ 1,4-dichlorobenzene eq." $/ \mathrm{kg}$

14000 kg 1,4-dichlorobenzene eq." $/ \mathrm{kg}$

$19 \mathrm{~kg}$ 1,4-dichlorobenzene eq. "/kg

$18 \mathrm{~kg}$ 1,4-dichlorobenzene eq. "/ $/ \mathrm{kg}$

$18 \mathrm{~kg}$ 1,4-dichlorobenzene eq." $/ \mathrm{kg}$

$7.2 \mathrm{~kg}$ 1,4-dichlorobenzene eq." $/ \mathrm{kg}$

$160 \mathrm{~kg}$ 1,4-dichlorobenzene eq." $/ \mathrm{kg}$

$2.5 \mathrm{~kg}$ 1,4-dichlorobenzene eq. "/kg

$6.3 \mathrm{~kg}$ 1,4-dichlorobenzene eq." $/ \mathrm{kg}$ $340 \mathrm{~kg}$ 1,4-dichlorobenzene eq." $/ \mathrm{kg}$ $340 \mathrm{~kg}$ 1,4-dichlorobenzene eq." $/ \mathrm{kg}$

$59 \mathrm{~kg}$ 1,4-dichlorobenzene eq. $/ \mathrm{kg}$

17 kg 1,4-dichlorobenzene eq." $/ \mathrm{kg}$ 
[E851] endrin to fresh water

[E852] ethoprophos to fresh water

[E853] ethylbenzene to fresh water

[E854] ethylene to fresh water

[E855] fenitrothion to fresh water

[E856] fenthion to fresh water

[E857] fentin acetate to fresh water

[E858] fentin chloride to fresh water

[E859] fentin hydroxide to fresh water

[E861] folpet to fresh water

[E862] Formaldehyde (methanal) to fresh water

[E863] glyphosate to fresh water

[E864] heptachlor to fresh water

[E865] heptenophos to fresh water

[E866] hexachloro-1,3-butadiene to fresh water

[E867] hexachlorobenzene to fresh water

[E871] iprodione to fresh water

[E872] isoproturon to fresh water

[E873] lead to fresh water

[E874] lindane to fresh water

[E875] linuron to fresh water

[E876] malathion to fresh water

[E878] MCPA to fresh water

[E879] mecoprop to fresh water

[E880] mercury to fresh water

[E881] metamitron to fresh water

[E882] metazachlor to fresh water

[E883] methabenzthiazuron to fresh water

[E884] methomyl to fresh water

[E885] methylbromide to fresh water

[E886] methyl-mercury to fresh water

[E887] metobromuron to fresh water

[E888] metolachlor to fresh water

[E889] mevinphos to fresh water

[E890] molybdenum to fresh water

[E891] $\mathrm{m}$-xylene to fresh water

[E892] naphtalene to fresh water

[E893] nickel to fresh water

[E899] oxamyl to fresh water

[E900] oxydemethon-methyl to fresh water

[E901] o-xylene to fresh water

[E902] parathion-ethyl to fresh water

[E903] parathion-methyl to fresh water

[E904] pentachlorobenzene to fresh water

[E905] pentachloronitrobenzene to fresh water

[E906] pentachlorophenol to fresh water

[E907] permethrin to fresh water

[E909] phenol to fresh water

[E912] phoxim to fresh water

[E913] Phtalic anhydride to fresh water

[E914] pirimicarb to fresh water

[E916] propachlor to fresh water

[E917] propoxur to fresh water

[E918] propylene oxide to fresh water

[E919] p-xylene to fresh water

[E920] pyrazophos to fresh water

[E921] selenium to fresh water

[E922] simazine to fresh water

[E923] styrene (vinylbenzene) to fresh water

[E926] tetrachloroethylene (PER) to fresh water

[E927] Tetrachloromethane (carbon tetrachloride) $(\mathrm{HC}-10)$ to fresh water

[E928] thallium to fresh water

[E929] Thiram to fresh water

[E930] tin to fresh water

[E931] tolclophos-methyl to fresh water

[E932] toluene to fresh water

[E933] tri-allate to fresh water
$6000 \mathrm{~kg}$ 1,4-dichlorobenzene eq." $/ \mathrm{kg}$ $1800 \mathrm{~kg}$ 1,4-dichlorobenzene eq. "/kg $0.83 \mathrm{~kg}$ 1,4-dichlorobenzene eq." $/ \mathrm{kg}$ $0.65 \mathrm{~kg}$ 1,4-dichlorobenzene eq." $/ \mathrm{kg}$

$22 \mathrm{~kg} \mathrm{1,4-dichlorobenzene} \mathrm{eq."} / \mathrm{kg}$

$93 \mathrm{~kg}$ 1,4-dichlorobenzene eq." $/ \mathrm{kg}$ $880 \mathrm{~kg}$ 1,4-dichlorobenzene eq." $/ \mathrm{kg}$ $860 \mathrm{~kg}$ 1,4-dichlorobenzene eq." $/ \mathrm{kg}$ $870 \mathrm{~kg}$ 1,4-dichlorobenzene eq." $/ \mathrm{kg}$ $8.6 \mathrm{~kg}$ 1,4-dichlorobenzene eq." $/ \mathrm{kg}$ $0.037 \mathrm{~kg}$ 1,4-dichlorobenzene eq." $/ \mathrm{kg}$ $0.066 \mathrm{~kg}$ 1,4-dichlorobenzene eq." $/ \mathrm{kg}$ $3400 \mathrm{~kg}$ 1,4-dichlorobenzene eq." $/ \mathrm{kg}$

$1.3 \mathrm{~kg}$ 1,4-dichlorobenzene eq." $/ \mathrm{kg}$ $80000 \mathrm{~kg}$ 1,4-dichlorobenzene eq. "//kg 5.60E+06 kg 1,4-dichlorobenzene eq." $/ \mathrm{kg}$

$0.18 \mathrm{~kg}$ 1,4-dichlorobenzene eq." $/ \mathrm{kg}$

$13 \mathrm{~kg}$ 1,4-dichlorobenzene eq." $/ \mathrm{kg}$

$12 \mathrm{~kg}$ 1,4-dichlorobenzene eq." $/ \mathrm{kg}$ $830 \mathrm{~kg}$ 1,4-dichlorobenzene eq." $/ \mathrm{kg}$ $110 \mathrm{~kg}$ 1,4-dichlorobenzene eq." $/ \mathrm{kg}$ $0.24 \mathrm{~kg}$ 1,4-dichlorobenzene eq." $/ \mathrm{kg}$ $15 \mathrm{~kg}$ 1,4-dichlorobenzene eq." $/ \mathrm{kg}$ $200 \mathrm{~kg}$ 1,4-dichlorobenzene eq." $/ \mathrm{kg}$ $1400 \mathrm{~kg}$ 1,4-dichlorobenzene eq." $/ \mathrm{kg}$ $0.16 \mathrm{~kg}$ 1,4-dichlorobenzene eq." $/ \mathrm{kg}$

$1.7 \mathrm{~kg}$ 1,4-dichlorobenzene eq." $/ \mathrm{kg}$

$2.6 \mathrm{~kg}$ 1,4-dichlorobenzene eq."/kg

$3.3 \mathrm{~kg}$ 1,4-dichlorobenzene eq." $/ \mathrm{kg}$ $300 \mathrm{~kg}$ 1,4-dichlorobenzene eq." $/ \mathrm{kg}$

15000 kg 1,4-dichlorobenzene eq."/kg

$8 \mathrm{~kg}$ 1,4-dichlorobenzene eq." $/ \mathrm{kg}$

$0.55 \mathrm{~kg}$ 1,4-dichlorobenzene eq. "/kg

$11 \mathrm{~kg}$ 1,4-dichlorobenzene eq." $/ \mathrm{kg}$

$5500 \mathrm{~kg}$ 1,4-dichlorobenzene eq." $/ \mathrm{kg}$

$0.34 \mathrm{~kg}$ 1,4-dichlorobenzene eq." $/ \mathrm{kg}$

$5.6 \mathrm{~kg}$ 1,4-dichlorobenzene eq." $/ \mathrm{kg}$ $330 \mathrm{~kg}$ 1,4-dichlorobenzene eq." $/ \mathrm{kg}$

$0.36 \mathrm{~kg}$ 1,4-dichlorobenzene eq." $/ \mathrm{kg}$

$74 \mathrm{~kg}$ 1,4-dichlorobenzene eq." $/ \mathrm{kg}$

$0.42 \mathrm{~kg}$ 1,4-dichlorobenzene eq." $/ \mathrm{kg}$

$31 \mathrm{~kg}$ 1,4-dichlorobenzene eq." $/ \mathrm{kg}$

$100 \mathrm{~kg}$ 1,4-dichlorobenzene eq." $/ \mathrm{kg}$

1200 kg 1,4-dichlorobenzene eq."//kg

$91 \mathrm{~kg}$ 1,4-dichlorobenzene eq. " $/ \mathrm{kg}$

$7.2 \mathrm{~kg}$ 1,4-dichlorobenzene eq." $/ \mathrm{kg}$

$23 \mathrm{~kg}$ 1,4-dichlorobenzene eq." $/ \mathrm{kg}$

$0.049 \mathrm{~kg}$ 1,4-dichlorobenzene eq. $/ \mathrm{kg}$ $120 \mathrm{~kg}$ 1,4-dichlorobenzene eq." $/ \mathrm{kg}$

$0.00011 \mathrm{~kg} 1,4$-dichlorobenzene eq." $/ \mathrm{kg}$

$1.7 \mathrm{~kg}$ 1,4-dichlorobenzene eq." $/ \mathrm{kg}$

$1.6 \mathrm{~kg}$ 1,4-dichlorobenzene eq." $/ \mathrm{kg}$

$1.3 \mathrm{~kg}$ 1,4-dichlorobenzene eq." $/ \mathrm{kg}$ $2600 \mathrm{~kg}$ 1,4-dichlorobenzene eq."//kg $0.35 \mathrm{~kg}$ 1,4-dichlorobenzene eq." $/ \mathrm{kg}$

$53 \mathrm{~kg}$ 1,4-dichlorobenzene eq." $/ \mathrm{kg}$

$56000 \mathrm{~kg}$ 1,4-dichlorobenzene eq." $/ \mathrm{kg}$

$9.7 \mathrm{~kg}$ 1,4-dichlorobenzene eq." $/ \mathrm{kg}$

$0.085 \mathrm{~kg}$ 1,4-dichlorobenzene eq." $/ \mathrm{kg}$

$5.7 \mathrm{~kg}$ 1,4-dichlorobenzene eq." $/ \mathrm{kg}$

$220 \mathrm{~kg}$ 1,4-dichlorobenzene eq." $/ \mathrm{kg}$

$2.30 \mathrm{E}+05 \mathrm{~kg}$ 1,4-dichlorobenzene eq." $/ \mathrm{kg}$

$3.3 \mathrm{~kg}$ 1,4-dichlorobenzene eq." $/ \mathrm{kg}$

$0.017 \mathrm{~kg}$ 1,4-dichlorobenzene eq." $/ \mathrm{kg}$

$1 \mathrm{~kg}$ 1,4-dichlorobenzene eq." $/ \mathrm{kg}$

$0.3 \mathrm{~kg}$ 1,4-dichlorobenzene eq. " $/ \mathrm{kg}$

$83 \mathrm{~kg}$ 1,4-dichlorobenzene eq." $/ \mathrm{kg}$ 
[E934] triazophos to fresh water [E935] tributyltinoxide to fresh water [E936] trichlorfon to fresh water [E937] Trichloroethylene (tri) to fresh water [E938] Trichloromethane $=$ chloroform to fresh water [E939] trifluarin to fresh water

[E940] vanadium to fresh water

[E941] Vinyl Chloride (chloroethene) to fresh water

[E942] zinc to fresh water

[E943] zineb to fresh water

[E944] 1,1,1-trichloroethane to fresh water

[E945] 1,2,3,4-tetrachlorobenzene to fresh water

[E946] 1,2,3,5-tetrachlorobenzene to fresh water

[E947] 1,2,3-trichlorobenzene to fresh water

[E948] 1,2,4,5-tetrachlorobenzene to fresh water

[E949] 1,2,4-trichlorobenzene to fresh water

[E950] 1,2-dichlorobenzene to fresh water

[E951] 1,2-dichloroethane to fresh water

[E952] 1,3,5-trichlorobenzene to fresh water

[E953] 1,3-butadiene to fresh water

[E954] 1,3-dichlorobenzene to fresh water

[E955] 1,4-dichlorobenzene to fresh water

[E956] 1-chloro-4-nitrobenzene to fresh water

[E957] 2,3,4,6-tetrachlorophenol to fresh water

[E958] 2,3,7,8-TCDD to fresh water

[E959] 2,4,5-T to fresh water

[E960] 2,4,5-trichlorophenol to fresh water

[E961] 2,4,6-trichlorophenol to sea water

[E962] 2,4-D to sea water

[E963] 2,4-dichlorophenol to sea water

[E964] 2-chlorophenol to sea water

[E965] 3,4-dichloroaniline to sea water

[E966] 3-chloroaniline to sea water

[E967] 4-chloroaniline to sea water

[E968] acephate to sea water

[E969] Acrolein (2-propenal) to sea water

[E970] acrylonitrile to sea water

[E971] aldicarb to sea water

[E972] aldrin to sea water

[E974] anilazine to sea water

[E975] anthracene to sea water

[E976] antimony to sea water

[E977] arsenic to sea water

[E978] atrazine to sea water

[E979] azinphos-ethyl to sea water

[E980] azinphos-methyl to sea water

[E981] barium to sea water

[E982] benomyl to sea water

[E983] bentazone to sea water

[E984] benzene to sea water

[E989] benzylchloride to sea water

[E990] beryllium to sea water

[E991] bifenthrin to sea water

[E994] Butylbenzylphtalate to sea water

[E995] cadmium to sea water

[E996] captafol to sea water

[E997] captan to sea water

[E998] carbaryl to sea water

[E999] carbendazim to sea water

[E1000] carbofuran to sea water

[E1001] carbon disulfide to sea water

[E1002] Carcinogenic PAHs to sea water

[E1004] chlordane to sea water

[E1005] chlorfenvinphos to sea water

[E1006] chloridazon to sea water

[E1008] chlorobenzene to sea water

[E1009] chlorothalonil to sea water
$320 \mathrm{~kg}$ 1,4-dichlorobenzene eq. "/kg $3400 \mathrm{~kg}$ 1,4-dichlorobenzene eq."/kg

$0.37 \mathrm{~kg}$ 1,4-dichlorobenzene eq." $/ \mathrm{kg}$

$33 \mathrm{~kg}$ 1,4-dichlorobenzene eq." $/ \mathrm{kg}$

$13 \mathrm{~kg}$ 1,4-dichlorobenzene eq." $/ \mathrm{kg}$

$97 \mathrm{~kg}$ 1,4-dichlorobenzene eq." $/ \mathrm{kg}$

$3200 \mathrm{~kg}$ 1,4-dichlorobenzene eq." / $/ \mathrm{kg}$

$140 \mathrm{~kg}$ 1,4-dichlorobenzene eq." $/ \mathrm{kg}$

$0.58 \mathrm{~kg}$ 1,4-dichlorobenzene eq." $/ \mathrm{kg}$

$1.7 \mathrm{~kg}$ 1,4-dichlorobenzene eq." $/ \mathrm{kg}$

$9.9 \mathrm{~kg}$ 1,4-dichlorobenzene eq." $/ \mathrm{kg}$

$30 \mathrm{~kg}$ 1,4-dichlorobenzene eq."/kg

$25 \mathrm{~kg}$ 1,4-dichlorobenzene eq." $/ \mathrm{kg}$

$62 \mathrm{~kg}$ 1,4-dichlorobenzene eq."//kg

$30 \mathrm{~kg}$ 1,4-dichlorobenzene eq." $/ \mathrm{kg}$

$56 \mathrm{~kg}$ 1,4-dichlorobenzene eq." $/ \mathrm{kg}$

$4.1 \mathrm{~kg}$ 1,4-dichlorobenzene eq." $/ \mathrm{kg}$

$5.5 \mathrm{~kg}$ 1,4-dichlorobenzene eq." $/ \mathrm{kg}$

$54 \mathrm{~kg}$ 1,4-dichlorobenzene eq." $/ \mathrm{kg}$

$450 \mathrm{~kg}$ 1,4-dichlorobenzene eq." $/ \mathrm{kg}$

$30 \mathrm{~kg}$ 1,4-dichlorobenzene eq."/ $/ \mathrm{kg}$

$0.47 \mathrm{~kg}$ 1,4-dichlorobenzene eq." $/ \mathrm{kg}$

$220 \mathrm{~kg} 1,4$-dichlorobenzene eq." $/ \mathrm{kg}$

$0.26 \mathrm{~kg}$ 1,4-dichlorobenzene eq." $/ \mathrm{kg}$

4.20E+08 kg 1,4-dichlorobenzene eq." $/ \mathrm{kg}$

$0.0054 \mathrm{~kg} 1,4$-dichlorobenzene eq." $/ \mathrm{kg}$

$0.61 \mathrm{~kg}$ 1,4-dichlorobenzene eq." $/ \mathrm{kg}$

$47 \mathrm{~kg} \mathrm{1,4-dichlorobenzene} \mathrm{eq."} / \mathrm{kg}$

6.70E-05 kg 1,4-dichlorobenzene eq." $/ \mathrm{kg}$

$0.065 \mathrm{~kg}$ 1,4-dichlorobenzene eq." $/ \mathrm{kg}$

$0.35 \mathrm{~kg}$ 1,4-dichlorobenzene eq." $/ \mathrm{kg}$

$1.5 \mathrm{~kg}$ 1,4-dichlorobenzene eq." $/ \mathrm{kg}$

$2.1 \mathrm{~kg}$ 1,4-dichlorobenzene eq. "/kg

$4 \mathrm{~kg}$ 1,4-dichlorobenzene eq." $/ \mathrm{kg}$

$0.00051 \mathrm{~kg} 1,4$-dichlorobenzene eq." $/ \mathrm{kg}$

$0.8 \mathrm{~kg} \mathrm{1,4-dichlorobenzene} \mathrm{eq."} / \mathrm{kg}$

$51 \mathrm{~kg} \mathrm{1,4-dichlorobenzene} \mathrm{eq."} / \mathrm{kg}$

$0.24 \mathrm{~kg}$ 1,4-dichlorobenzene eq." $/ \mathrm{kg}$

780 kg 1,4-dichlorobenzene eq." $/ \mathrm{kg}$

$0.00082 \mathrm{~kg}$ 1,4-dichlorobenzene eq." $/ \mathrm{kg}$

$0.16 \mathrm{~kg}$ 1,4-dichlorobenzene eq." $/ \mathrm{kg}$

$8600 \mathrm{~kg}$ 1,4-dichlorobenzene eq." $/ \mathrm{kg}$

$2400 \mathrm{~kg}$ 1,4-dichlorobenzene eq."//kg

$0.018 \mathrm{~kg}$ 1,4-dichlorobenzene eq." $/ \mathrm{kg}$

$1.6 \mathrm{~kg}$ 1,4-dichlorobenzene eq." $/ \mathrm{kg}$

$0.0057 \mathrm{~kg}$ 1,4-dichlorobenzene eq." $/ \mathrm{kg}$

$800 \mathrm{~kg}$ 1,4-dichlorobenzene eq." $/ \mathrm{kg}$

$0.00024 \mathrm{~kg}$ 1,4-dichlorobenzene eq." $/ \mathrm{kg}$

$0.0022 \mathrm{~kg} 1,4$-dichlorobenzene eq." $/ \mathrm{kg}$

$210 \mathrm{~kg}$ 1,4-dichlorobenzene eq. "/ $/ \mathrm{kg}$

$55 \mathrm{~kg}$ 1,4-dichlorobenzene eq. "/kg

$16000 \mathrm{~kg}$ 1,4-dichlorobenzene eq." $/ \mathrm{kg}$

$0.75 \mathrm{~kg}$ 1,4-dichlorobenzene eq." $/ \mathrm{kg}$

$0.00085 \mathrm{~kg}$ 1,4-dichlorobenzene eq." $/ \mathrm{kg}$

$100 \mathrm{~kg}$ 1,4-dichlorobenzene eq." $/ \mathrm{kg}$

$9.7 \mathrm{~kg}$ 1,4-dichlorobenzene eq." $/ \mathrm{kg}$

5.40E-06 kg 1,4-dichlorobenzene eq. " $/ \mathrm{kg}$

$0.0019 \mathrm{~kg} 1,4$-dichlorobenzene eq." $/ \mathrm{kg}$

$0.002 \mathrm{~kg}$ 1,4-dichlorobenzene eq."/kg

$0.21 \mathrm{~kg}$ 1,4-dichlorobenzene eq." $/ \mathrm{kg}$

$0.48 \mathrm{~kg}$ 1,4-dichlorobenzene eq." $/ \mathrm{kg}$

$29000 \mathrm{~kg}$ 1,4-dichlorobenzene eq." $/ \mathrm{kg}$

$1200 \mathrm{~kg}$ 1,4-dichlorobenzene eq." $/ \mathrm{kg}$

$3.8 \mathrm{~kg}$ 1,4-dichlorobenzene eq." $/ \mathrm{kg}$

$0.0021 \mathrm{~kg} \mathrm{1,4-dichlorobenzene} \mathrm{eq."} / \mathrm{kg}$

$5.2 \mathrm{~kg}$ 1,4-dichlorobenzene eq." $/ \mathrm{kg}$

$0.45 \mathrm{~kg}$ 1,4-dichlorobenzene eq." $/ \mathrm{kg}$ 
[E1010] chlorpropham to sea water [E1011] chlorpyriphos to sea water [E1012] chromium III to sea water [E1013] chromium VI to sea water [E1015] cobalt to sea water [E1016] copper to sea water [E1017] coumaphos to sea water [E1018] cyanazine to sea water [E1019] cypermethrin to sea water [E1020] cyromazine to sea water [E1021] DDT to sea water [E1022] deltamethrin to sea water [E1023] demeton to sea water [E1024] desmetryn to sea water [E1025] Di(2-ethylhexyl)phtalate to sea water [E1026] diazinon to sea water [E1027] Dibutylphtalate to sea water

[E1028] Dichloromethane (Methylene Chloride) to sea water [E1029] dichlorprop to sea water

[E1030] dichlorvos to sea water

[E1031] dieldrin to sea water

[E1032] Diethylphtalate to sea water

[E1033] Dihexylphtalate to sea water

[E1034] Diisodecylphtalate to sea water

[E1035] Diisooctylphtalate to sea water

[E1036] dimethoate to sea water

[E1037] Dimethylphtalate to sea water

[E1038] dinoseb to sea water

[E1039] dinoterb to sea water

[E1040] Dioctylphtalate to sea water

[E1041] disulfothon to sea water

[E1042] diuron to sea water

[E1043] DNOC to sea water

[E1044] endosulfan to sea water

[E1045] endrin to sea water

[E1046] ethoprophos to sea water

[E1047] ethylbenzene to sea water

[E1048] ethylene to sea water

[E1049] fenitrothion to sea water

[E1050] fenthion to sea water

[E1051] fentin acetate to sea water

[E1052] fentin chloride to sea water

[E1053] fentin hydroxide to sea water

[E1055] folpet to sea water

[E1056] Formaldehyde (methanal) to sea water

[E1057] glyphosate to sea water

[E1058] heptachlor to sea water

[E1059] heptenophos to sea water

[E1060] hexachloro-1,3-butadiene to sea water

[E1061] hexachlorobenzene to sea water

[E10.65] iprodione to sea water

[E1066] isoproturon to sea water

[E1067] lead to sea water

[E1068] lindane to sea water

[E1069] linuron to sea water

[E1070] malathion to sea water

[E1072] MCPA to sea water

[E1073] mecoprop to sea water

[E1074] mercury to sea water

[E1075] metamitron to sea water

[E1076] metazachlor to sea water

[E1077] methabenzthiazuron to sea water

[E1078] methomyl to sea water

[E1079] methylbromide to sea water

[E1080] methyl-mercury to sea water

[E1081] metobromuron to sea water

[E1082] metolachlor to sea water
$0.0043 \mathrm{~kg}$ 1,4-dichlorobenzene eq." $/ \mathrm{kg}$

$0.038 \mathrm{~kg}$ 1,4-dichlorobenzene eq." $/ \mathrm{kg}$

$10 \mathrm{~kg}$ 1,4-dichlorobenzene eq."/kg

$17 \mathrm{~kg}$ 1,4-dichlorobenzene eq." $/ \mathrm{kg}$

$60 \mathrm{~kg}$ 1,4-dichlorobenzene eq." $/ \mathrm{kg}$

$5.9 \mathrm{~kg}$ 1,4-dichlorobenzene eq." $/ \mathrm{kg}$

$220 \mathrm{~kg}$ 1,4-dichlorobenzene eq." $/ \mathrm{kg}$

0.0096 kg 1,4-dichlorobenzene eq." $/ \mathrm{kg}$

$0.026 \mathrm{~kg}$ 1,4-dichlorobenzene eq." $/ \mathrm{kg}$

$0.0026 \mathrm{~kg}$ 1,4-dichlorobenzene eq." $/ \mathrm{kg}$

34 kg 1,4-dichlorobenzene eq." $/ \mathrm{kg}$

$0.033 \mathrm{~kg}$ 1,4-dichlorobenzene eq." $/ \mathrm{kg}$

$0.3 \mathrm{~kg}$ 1,4-dichlorobenzene eq." $/ \mathrm{kg}$

$0.12 \mathrm{~kg}$ 1,4-dichlorobenzene eq." $/ \mathrm{kg}$

0.04 kg 1,4-dichlorobenzene eq." $/ \mathrm{kg}$

$0.27 \mathrm{~kg}$ 1,4-dichlorobenzene eq."/ $/ \mathrm{kg}$

$0.003 \mathrm{~kg}$ 1,4-dichlorobenzene eq." $/ \mathrm{kg}$

$0.3 \mathrm{~kg}$ 1,4-dichlorobenzene eq." $/ \mathrm{kg}$

$0.097 \mathrm{~kg}$ 1,4-dichlorobenzene eq." $/ \mathrm{kg}$

$0.0023 \mathrm{~kg}$ 1,4-dichlorobenzene eq." $/ \mathrm{kg}$

5500 kg 1,4-dichlorobenzene eq." $/ \mathrm{kg}$

$0.00057 \mathrm{~kg}$ 1,4-dichlorobenzene eq." $/ \mathrm{kg}$ $370 \mathrm{~kg}$ 1,4-dichlorobenzene eq." $/ \mathrm{kg}$

$3.2 \mathrm{~kg}$ 1,4-dichlorobenzene eq." $/ \mathrm{kg}$

$9.7 \mathrm{~kg}$ 1,4-dichlorobenzene eq." $/ \mathrm{kg}$

$0.0033 \mathrm{~kg}$ 1,4-dichlorobenzene eq." $/ \mathrm{kg}$

$0.0084 \mathrm{~kg}$ 1,4-dichlorobenzene eq." $/ \mathrm{kg}$

$0.63 \mathrm{~kg}$ 1,4-dichlorobenzene eq." $/ \mathrm{kg}$

$0.0029 \mathrm{~kg}$ 1,4-dichlorobenzene eq." $/ \mathrm{kg}$

$1.3 \mathrm{~kg}$ 1,4-dichlorobenzene eq." $/ \mathrm{kg}$

$1.5 \mathrm{~kg}$ 1,4-dichlorobenzene eq." $/ \mathrm{kg}$

$1.5 \mathrm{~kg}$ 1,4-dichlorobenzene eq." $/ \mathrm{kg}$

0.0015 kg 1,4-dichlorobenzene eq." $/ \mathrm{kg}$

$0.042 \mathrm{~kg}$ 1,4-dichlorobenzene eq." $/ \mathrm{kg}$ 1600 kg 1,4-dichlorobenzene eq." $/ \mathrm{kg}$

$13 \mathrm{~kg}$ 1,4-dichlorobenzene eq." $/ \mathrm{kg}$

$0.07 \mathrm{~kg}$ 1,4-dichlorobenzene eq." $/ \mathrm{kg}$

$0.047 \mathrm{~kg}$ 1,4-dichlorobenzene eq." $/ \mathrm{kg}$ $0.09 \mathrm{~kg}$ 1,4-dichlorobenzene eq." $/ \mathrm{kg}$ $0.46 \mathrm{~kg}$ 1,4-dichlorobenzene eq." $/ \mathrm{kg}$

$4.1 \mathrm{~kg}$ 1,4-dichlorobenzene eq." $/ \mathrm{kg}$

$12 \mathrm{~kg}$ 1,4-dichlorobenzene eq." $/ \mathrm{kg}$

$4.1 \mathrm{~kg}$ 1,4-dichlorobenzene eq." $/ \mathrm{kg}$

$0.31 \mathrm{~kg} 1,4$-dichlorobenzene eq." $/ \mathrm{kg}$

2.80E-05 kg 1,4-dichlorobenzene eq." $/ \mathrm{kg}$

$1.50 \mathrm{E}-05 \mathrm{~kg}$ 1,4-dichlorobenzene eq." $/ \mathrm{kg}$

$43 \mathrm{~kg}$ 1,4-dichlorobenzene eq." $/ \mathrm{kg}$

$0.0023 \mathrm{~kg}$ 1,4-dichlorobenzene eq." $/ \mathrm{kg}$

$39000 \mathrm{~kg}$ 1,4-dichlorobenzene eq." $/ \mathrm{kg}$

$3.40 E+06$ kg 1,4-dichlorobenzene eq."/kg

$0.00012 \mathrm{~kg} \mathrm{1,4-dichlorobenzene} \mathrm{eq."} / \mathrm{kg}$

$0.029 \mathrm{~kg}$ 1,4-dichlorobenzene eq." $/ \mathrm{kg}$

$79 \mathrm{~kg}$ 1,4-dichlorobenzene eq." $/ \mathrm{kg}$

$6.1 \mathrm{~kg}$ 1,4-dichlorobenzene eq." $/ \mathrm{kg}$

$0.65 \mathrm{~kg}$ 1,4-dichlorobenzene eq." $/ \mathrm{kg}$

$0.00084 \mathrm{~kg}$ 1,4-dichlorobenzene eq." $/ \mathrm{kg}$

$0.037 \mathrm{~kg} \mathrm{1,4-dichlorobenzene} \mathrm{eq."} / \mathrm{kg}$

$0.84 \mathrm{~kg} 1,4$-dichlorobenzene eq." $/ \mathrm{kg}$

$8200 \mathrm{~kg}$ 1,4-dichlorobenzene eq. "/kg

3.20E-05 kg 1,4-dichlorobenzene eq."/kg 0.0024 kg 1,4-dichlorobenzene eq." $/ \mathrm{kg}$ $0.0082 \mathrm{~kg}$ 1,4-dichlorobenzene eq." $/ \mathrm{kg}$ 0.0014 kg 1,4-dichlorobenzene eq." $/ \mathrm{kg}$

$25 \mathrm{~kg}$ 1,4-dichlorobenzene eq." $/ \mathrm{kg}$ $88000 \mathrm{~kg}$ 1,4-dichlorobenzene eq."/kg

$0.076 \mathrm{~kg} \mathrm{1,4-dichlorobenzene} \mathrm{eq.} \mathrm{"/kg}$ $0.00085 \mathrm{~kg}$ 1,4-dichlorobenzene eq." $/ \mathrm{kg}$ 
[E1083] mevinphos to sea water [E1084] molybdenum to sea water [E1085] m-xylene to sea water [E1086] naphtalene to sea water [E1087] nickel to sea water [E1093] oxamyl to sea water [E1094] oxydemethon-methyl to sea water [E1095] o-xylene to sea water [E1096] parathion-ethyl to sea water [E1097] parathion-methyl to sea water [E1098] pentachlorobenzene to sea water [E1099] pentachloronitrobenzene to sea water [E1100] pentachlorophenol to sea water

[E1101] permethrin to sea water

[E1103] phenol to sea water

[E1106] phoxim to sea water

[E1107] Phtalic anhydride to sea water

[E1108] pirimicarb to sea water

[E1110] propachlor to sea water

[E1111] propoxur to sea water

[E1112] propylene oxide to sea water

[E1113] p-xylene to sea water

[E1114] pyrazophos to sea water

[E1115] selenium to sea water

[E1116] simazine to sea water

[E1117] styrene (vinylbenzene) to sea water

[E1120] tetrachloroethylene (PER) to sea water

[E1121] Tetrachloromethane (carbon tetrachloride) (HC-10) to sea water

[E1122] thallium to sea water

[E1123] Thiram to sea water

[E1124] tin to sea water

[E1125] tolclophos-methyl to sea water

[E1126] toluene to sea water

[E1127] tri-allate to sea water

[E1128] triazophos to sea water

[E1129] tributyltinoxide to sea water

[E1130] trichlorfon to sea water

[E1131] Trichloroethylene (tri) to sea water

[E1132] Trichloromethane $=$ chloroform to sea water

[E1133] trifluarin to sea water

[E1134] vanadium to sea water

[E1135] Vinyl Chloride (chloroethene) to sea water

[E1136] zinc to sea water

[E1137] zineb to sea water

[E1138] 1,1,1-trichloroethane to sea water

[E1139] 1,2,3,4-tetrachlorobenzene to sea water

[E1140] 1,2,3,5-tetrachlorobenzene to sea water

[E1141] 1,2,3-trichlorobenzene to sea water

[E1142] 1,2,4,5-tetrachlorobenzene to sea water

[E1143] 1,2,4-trichlorobenzene to sea water

[E1144] 1,2-dichlorobenzene to sea water

[E1145] 1,2-dichloroethane to sea water

[E1146] 1,3,5-trichlorobenzene to sea water

[E1147] 1,3-butadiene to sea water

[E1148] 1,3-dichlorobenzene to sea water

[E1149] 1,4-dichlorobenzene to sea water

[E1150] 1-chloro-4-nitrobenzene to sea water

[E1151] 2,3,4,6-tetrachlorophenol to sea water

[E1152] 2,3,7,8-TCDD to sea water

[E1153] 2,4,5-T to sea water

[E1154] 2,4,5-trichlorophenol to sea water

[E1155] 2,4,6-trichlorophenol to agricultural soil

[E1156] 2,4-D to agricultural soil

[E1157] 2,4-dichlorophenol to agricultural soil

[E1158] 2-chlorophenol to agricultural soil

[E1159] 3,4-dichloroaniline to agricultural soil

[E1160] 3-chloroaniline to agricultural soil
$0.0018 \mathrm{~kg} \mathrm{1,4-dichlorobenzene} \mathrm{eq."} / \mathrm{kg}$ $6800 \mathrm{~kg}$ 1,4-dichlorobenzene eq." $/ \mathrm{kg}$ $0.01 \mathrm{~kg}$ 1,4-dichlorobenzene eq." $/ \mathrm{kg}$ $0.19 \mathrm{~kg} \mathrm{1,4-dichlorobenzene} \mathrm{eq."} / \mathrm{kg}$

$750 \mathrm{~kg}$ 1,4-dichlorobenzene eq." $/ \mathrm{kg}$

1.40E-05 kg 1,4-dichlorobenzene eq." $/ \mathrm{kg}$

$0.01 \mathrm{~kg}$ 1,4-dichlorobenzene eq." $/ \mathrm{kg}$

0.026 kg 1,4-dichlorobenzene eq." $/ \mathrm{kg}$

$0.18 \mathrm{~kg} \mathrm{1,4-dichlorobenzene} \mathrm{eq."} / \mathrm{kg}$

$0.54 \mathrm{~kg} \mathrm{1,4-dichlorobenzene} \mathrm{eq."} / \mathrm{kg}$

$410 \mathrm{~kg}$ 1,4-dichlorobenzene eq." $/ \mathrm{kg}$

$46 \mathrm{~kg}$ 1,4-dichlorobenzene eq." $/ \mathrm{kg}$

$0.14 \mathrm{~kg}$ 1,4-dichlorobenzene eq." $/ \mathrm{kg}$

$0.26 \mathrm{~kg}$ 1,4-dichlorobenzene eq." $/ \mathrm{kg}$

8.00E-05 kg 1,4-dichlorobenzene eq." $/ \mathrm{kg}$

$0.29 \mathrm{~kg}$ 1,4-dichlorobenzene eq." $/ \mathrm{kg}$

1.00E-07 kg 1,4-dichlorobenzene eq." $/ \mathrm{kg}$

$0.0013 \mathrm{~kg}$ 1,4-dichlorobenzene eq." $/ \mathrm{kg}$

0.0026 kg 1,4-dichlorobenzene eq."/kg

0.00039 kg 1,4-dichlorobenzene eq." $/ \mathrm{kg}$

$16 \mathrm{~kg}$ 1,4-dichlorobenzene eq." $/ \mathrm{kg}$

$0.013 \mathrm{~kg}$ 1,4-dichlorobenzene eq." $/ \mathrm{kg}$

$0.23 \mathrm{~kg} 1,4$-dichlorobenzene eq." $/ \mathrm{kg}$

63000 kg 1,4-dichlorobenzene eq." $/ \mathrm{kg}$

$0.016 \mathrm{~kg}$ 1,4-dichlorobenzene eq." $/ \mathrm{kg}$

$0.01 \mathrm{~kg}$ 1,4-dichlorobenzene eq." $/ \mathrm{kg}$

$2.8 \mathrm{~kg}$ 1,4-dichlorobenzene eq. "/kg

$170 \mathrm{~kg} \mathrm{1,4-dichlorobenzene} \mathrm{eq."} / \mathrm{kg}$

2.90E+05 kg 1,4-dichlorobenzene eq." $/ \mathrm{kg}$

0.00066 kg 1,4-dichlorobenzene eq." $/ \mathrm{kg}$

$0.11 \mathrm{~kg} \mathrm{1,4-dichlorobenzene} \mathrm{eq."} / \mathrm{kg}$

$0.065 \mathrm{~kg}$ 1,4-dichlorobenzene eq." $/ \mathrm{kg}$

0.039 kg 1,4-dichlorobenzene eq." $/ \mathrm{kg}$

$1.2 \mathrm{~kg}$ 1,4-dichlorobenzene eq." $/ \mathrm{kg}$

$1.6 \mathrm{~kg} \mathrm{1,4-dichlorobenzene} \mathrm{eq."} / \mathrm{kg}$

$55 \mathrm{~kg}$ 1,4-dichlorobenzene eq." $/ \mathrm{kg}$

3.10E-05 kg 1,4-dichlorobenzene eq." $/ \mathrm{kg}$

$14 \mathrm{~kg}$ 1,4-dichlorobenzene eq." $/ \mathrm{kg}$

$6 \mathrm{~kg}$ 1,4-dichlorobenzene eq." $/ \mathrm{kg}$

$6 \mathrm{~kg}$ 1,4-dichlorobenzene eq." $/ \mathrm{kg}$

$6200 \mathrm{~kg}$ 1,4-dichlorobenzene eq." $/ \mathrm{kg}$

$43 \mathrm{~kg}$ 1,4-dichlorobenzene eq." $/ \mathrm{kg}$

$3.2 \mathrm{~kg}$ 1,4-dichlorobenzene eq. "/ $/ \mathrm{kg}$

0.00082 kg 1,4-dichlorobenzene eq." $/ \mathrm{kg}$

$16 \mathrm{~kg}$ 1,4-dichlorobenzene eq." $/ \mathrm{kg}$

$80 \mathrm{~kg}$ 1,4-dichlorobenzene eq." $/ \mathrm{kg}$

$180 \mathrm{~kg}$ 1,4-dichlorobenzene eq. "/kg

$56 \mathrm{~kg}$ 1,4-dichlorobenzene eq." $/ \mathrm{kg}$

$84 \mathrm{~kg}$ 1,4-dichlorobenzene eq." $/ \mathrm{kg}$

$42 \mathrm{~kg}$ 1,4-dichlorobenzene eq." $/ \mathrm{kg}$

$7.3 \mathrm{~kg}$ 1,4-dichlorobenzene eq."//kg

$1300 \mathrm{~kg}$ 1,4-dichlorobenzene eq." $/ \mathrm{kg}$

$69 \mathrm{~kg}$ 1,4-dichlorobenzene eq." $/ \mathrm{kg}$

$3100 \mathrm{~kg} \mathrm{1,4-dichlorobenzene} \mathrm{eq."} / \mathrm{kg}$

$250 \mathrm{~kg}$ 1,4-dichlorobenzene eq." $/ \mathrm{kg}$

$2.9 \mathrm{~kg}$ 1,4-dichlorobenzene eq." $/ \mathrm{kg}$

22000 kg 1,4-dichloroberizene eq." $/ \mathrm{kg}$

$31 \mathrm{~kg}$ 1,4-dichlorobenzene eq." $/ \mathrm{kg}$

1.30E+09 kg 1,4-dichlorobenzene eq."/kg

$5.8 \mathrm{~kg}$ 1,4-dichlorobenzene eq." $/ \mathrm{kg}$

$5.3 \mathrm{~kg}$ 1,4-dichlorobenzene eq." $/ \mathrm{kg}$

1800 kg 1,4-dichlorobenzene eq." $/ / \mathrm{kg}$

$47 \mathrm{~kg}$ 1,4-dichlorobenzene eq." $/ \mathrm{kg}$

$740 \mathrm{~kg}$ 1,4-dichlorobenzene eq." $/ \mathrm{kg}$

$8.3 \mathrm{~kg}$ 1,4-dichlorobenzene eq." $/ \mathrm{kg}$

$1700 \mathrm{~kg}$ 1,4-dichlorobenzene eq." $/ \mathrm{kg}$

$30000 \mathrm{~kg}$ 1,4-dichlorobenzene eq." $/ \mathrm{kg}$ 
[E1161] 4-chloroaniline to agricultural soil

[E1162] acephate to agricultural soil

[E1163] Acrolein (2-propenal) to agricultural soil

[E1164] acrylonitrile to agricultural soil

[E1165] aldicarb to agricultural soil

[E1166] aldrin to agricultural soil

[E1168] anilazine to agricultural soil

[E1169] anthracene to agricultural soil

[E1170] antimony to agricultural soil

[E1171] arsenic to agricultural soil

[E1172] atrazine to agricultural soil

[E1173] azinphos-ethyl to agricultural soil

[E1174] azinphos-methyl to agricultural soil

[E1175] barium to agricultural soil

[E1176] benomyl to agricultural soil

[E1177] bentazone to agricultural soil

[E1178] benzene to agricultural soil

[E1183] benzylchloride to agricultural soil

[E1184] beryllium to agricultural soil

[E1185] bifenthrin to agricultural soil

[E1186] Butylbenzylphtalate to agricultural soil

[E1187] cadmium to agricultural soil

[E1188] captafol to agricultural soil

[E1189] captan to agricultural soil

[E1190] carbaryl to agricultural soil

[E1191] carbendazim to agricultural soil

[E1192] carbofuran to agricultural soil

[E1193] carbon disulfide to agricultural soil

[E1194] Carcinogenic PAHs to agricultural soil

[E1195] chlordane to agricultural soil

[E1196] chlorfenvinphos to agricultural soil

[E1197] chloridazon to agricultural soil

[E1198] chlorobenzene to agricultural soil

[E1199] chlorothalonil to agricultural soil

[E1200] chlorpropham to agricultural soil

[E1201] chlorpyriphos to agricultural soil

[E1202] chromium III to agricultural soil

[E1203] chromium VI to agricultural soil

[E1205] cobalt to agricultural soil

[E1206] copper to agricultural soil

[E1207] coumaphos to agricultural soil

[E1208] cyanazine to agricultural soil

[E1209] cypermethrin to agricultural soil

[E1210] cyromazine to agricultural soil

[E1211] DDT to agricultural soil

[E1212] deltamethrin to agricultural soil

[E1213] demeton to agricultural soil

[E1214] desmetryn to agricultural soil

[E1215] Di(2-ethylhexyl)phtalate to agricultural soil

[E1216] diazinon to agricultural soil

[E1217] Dibuty/phtalate to agricultural soil

[E1218] Dichloromethane (Methylene Chloride) to agricultural soil

[E1219] dichlorprop to agricultural soil

[E1220] dichlorvos to agricultural soil

[E1221] dieldrin to agricultural soil

[E1222] Diethylphtalate to agricultural soil

[E1223] Dihexylphtalate to agricultural soil

[E1224] Diisodecylphtalate to agricultural soil

[E1225] Diisooctylphtalate to agricultural soil

[E1226] dimethoate to agricultural soil

[E1227] Dimethylphtalate to agricultural soil

[E1228] dinoseb to agricultural soil

[E1229] dinoterb to agricultural soil

[E1230] Dioctylphtalate to agricultural soil

[E1231] disulfothon to agricultural soil

[E1232] diuron to agricultural soil

[E1233] DNOC to agricultural soil
$35000 \mathrm{~kg}$ 1,4-dichlorobenzene eq." $/ \mathrm{kg}$

22 kg 1,4-dichlorobenzene eq." $/ \mathrm{kg}$

$230 \mathrm{~kg}$ 1,4-dichlorobenzene eq." $/ \mathrm{kg}$

4.90E+05 kg 1,4-dichlorobenzene eq." $/ \mathrm{kg}$

$510 \mathrm{~kg}$ 1,4-dichlorobenzene eq." $/ \mathrm{kg}$

$4700 \mathrm{~kg}$ 1,4-dichlorobenzene eq." $/ \mathrm{kg}$

$0.08 \mathrm{~kg}$ 1,4-dichlorobenzene eq." $/ \mathrm{kg}$

$0.51 \mathrm{~kg}$ 1,4-dichlorobenzene eq." $/ \mathrm{kg}$

8900 kg 1,4-dichlorobenzene eq."/kg

32000 kg 1,4-dichlorobenzene eq." $/ \mathrm{kg}$

$21 \mathrm{~kg}$ 1,4-dichlorobenzene eq." $/ \mathrm{kg}$

$760 \mathrm{~kg}$ 1,4-dichlorobenzene eq." $/ \mathrm{kg}$

$39 \mathrm{~kg}$ 1,4-dichlorobenzene eq." $/ \mathrm{kg}$

$360 \mathrm{~kg}$ 1,4-dichlorobenzene eq." $/ \mathrm{kg}$

$0.43 \mathrm{~kg}$ 1,4-dichlorobenzene eq." $/ \mathrm{kg}$

$15 \mathrm{~kg}$ 1,4-dichlorobenzene eq." $/ \mathrm{kg}$

$15000 \mathrm{~kg}$ 1,4-dichlorobenzene eq." $/ \mathrm{kg}$

$5500 \mathrm{~kg}$ 1,4-dichlorobenzene eq." $/ \mathrm{kg}$

13000 kg 1,4-dichlorobenzene eq." $/ \mathrm{kg}$

$29 \mathrm{~kg}$ 1,4-dichlorobenzene eq." $/ \mathrm{kg}$

$0.31 \mathrm{~kg}$ 1,4-dichlorobenzene eq." $/ \mathrm{kg}$

20000 kg 1,4-dichlorobenzene eq."/kg

$960 \mathrm{~kg}$ 1,4-dichlorobenzene eq." $/ \mathrm{kg}$

$0.097 \mathrm{~kg}$ 1,4-dichlorobenzene eq." $/ \mathrm{kg}$

$21 \mathrm{~kg}$ 1,4-dichlorobenzene eq." $/ \mathrm{kg}$

$140 \mathrm{~kg}$ 1,4-dichlorobenzene eq." $/ \mathrm{kg}$

$1400 \mathrm{~kg}$ 1,4-dichlorobenzene eq."/kg

$3.6 \mathrm{~kg}$ 1,4-dichlorobenzene eq." $/ \mathrm{kg}$

$71000 \mathrm{~kg}$ 1,4-dichlorobenzene eq." $/ \mathrm{kg}$

$2800 \mathrm{~kg}$ 1,4-dichlorobenzene eq."/kg

$1200 \mathrm{~kg}$ 1,4-dichlorobenzene eq." $/ \mathrm{kg}$

$2.2 \mathrm{~kg}$ 1,4-dichlorobenzene eq." $/ \mathrm{kg}$

$7.1 \mathrm{~kg}$ 1,4-dichlorobenzene eq." $/ \mathrm{kg}$

$0.94 \mathrm{~kg}$ 1,4-dichlorobenzene eq." $/ \mathrm{kg}$

$2.1 \mathrm{~kg}$ 1,4-dichlorobenzene eq." $/ \mathrm{kg}$

$14 \mathrm{~kg}$ 1,4-dichlorobenzene eq." $/ \mathrm{kg}$

$5100 \mathrm{~kg}$ 1,4-dichlorobenzene eq." $/ \mathrm{kg}$

8500 kg 1,4-dichlorobenzene eq."/kg

$2400 \mathrm{~kg}$ 1,4-dichlorobenzene eq." $/ \mathrm{kg}$

$94 \mathrm{~kg}$ 1,4-dichlorobenzene eq." $/ \mathrm{kg}$

$11000 \mathrm{~kg}$ 1,4-dichlorobenzene eq."/kg

$24 \mathrm{~kg}$ 1,4-dichlorobenzene eq." $/ \mathrm{kg}$

$5200 \mathrm{~kg}$ 1,4-dichlorobenzene eq." $/ \mathrm{kg}$

$280 \mathrm{~kg}$ 1,4-dichlorobenzene eq." $/ \mathrm{kg}$

$270 \mathrm{~kg}$ 1,4-dichlorobenzene eq." $/ \mathrm{kg}$

0.16 kg 1,4-dichlorobenzene eq."/kg

5700 kg 1,4-dichlorobenzene eq."/kg

$650 \mathrm{~kg}$ 1,4-dichlorobenzene eq." $/ \mathrm{kg}$

$1.8 \mathrm{~kg}$ 1,4-dichlorobenzene eq." $/ \mathrm{kg}$

$120 \mathrm{~kg}$ 1,4-dichlorobenzene eq." $/ \mathrm{kg}$

$1.3 \mathrm{~kg}$ 1,4-dichlorobenzene eq." $/ \mathrm{kg}$

$2.4 \mathrm{~kg}$ 1,4-dichlorobenzene eq." $/ \mathrm{kg}$

$4.5 \mathrm{~kg}$ 1,4-dichlorobenzene eq." $/ \mathrm{kg}$

$0.97 \mathrm{~kg}$ 1,4-dichlorobenzene eq." $/ \mathrm{kg}$

$7600 \mathrm{~kg}$ 1,4-dichlorobenzene eq." $/ \mathrm{kg}$

$0.057 \mathrm{~kg}$ 1,4-dichlorobenzene eq." $/ \mathrm{kg}$

1200 kg 1,4-dichlorobenzene eq."/kg

$110 \mathrm{~kg}$ 1,4-dichlorobenzene eq." $/ \mathrm{kg}$

$32 \mathrm{~kg}$ 1,4-dichlorobenzene eq."/kg

$320 \mathrm{~kg}$ 1,4-dichlorobenzene eq." $/ \mathrm{kg}$

$28 \mathrm{~kg}$ 1,4-dichlorobenzene eq." $/ \mathrm{kg}$

$560 \mathrm{~kg}$ 1,4-dichlorobenzene eq." $/ \mathrm{kg}$

0.36 kg 1,4-dichlorobenzene eq."/kg

$8.6 \mathrm{~kg}$ 1,4-dichlorobenzene eq." $/ \mathrm{kg}$

$170 \mathrm{~kg}$ 1,4-dichlorobenzene eq." $/ \mathrm{kg}$

$170 \mathrm{~kg}$ 1,4-dichlorobenzene eq." $/ \mathrm{kg}$ $280 \mathrm{~kg}$ 1,4-dichlorobenzene eq." $/ \mathrm{kg}$ 
[E1234] endosulfan to agricultural soil

[E1235] endrin to agricultural soil

[E1236] ethoprophos to agricultural soil

[E1237] ethylbenzene to agricultural soil

[E1238] ethylene to agricultural soil

[E1239] fenitrothion to agricultural soil

[E1240] fenthion to agricultural soil

[E1241] fentin acetate to agricultural soil

[E1242] fentin chloride to agricultural soil

[E1243] fentin hydroxide to agricultural soil

[E1245] folpet to agricultural soil

[E1246] Formaldehyde (methanal) to agricultural soil

[E1247] glyphosate to agricultural soil

[E1248] heptachlor to agricultural soil

[E1249] heptenophos to agricultural soil

[E1250] hexachloro-1,3-butadiene to agricultural soil

[E1251] hexachlorobenzene to agricultural soil

[E1255] iprodione to agricultural soil

[E1256] isoproturon to agricultural soil

[E1257] lead to agricultural soil

[E1258] lindane to agricultural soil

[E1259] linuron to agricultural soil

[E1260] malathion to agricultural soil

[E1261] MCPA to agricultural soil

[E1262] mecoprop to agricultural soil

[E1263] mercury to agricultural soil

[E1264] metamitron to agricultural soil

[E1265] metazachlor to agricultural soil

[E1266] methabenzthiazuron to agricultural soil

[E1267] methomyl to agricultural soil

[E1268] methylbromide to agricultural soil

[E1269] methyl-mercury to agricultural soil

[E1270] metobromuron to agricultural soil

[E1271] metolachlor to agricultural soil

[E1272] mevinphos to agricultural soil

[E1273] molybdenum to agricultural soil

[E1274] $m$-xylene to agricultural soil

[E1275] naphtalene to agricultural soil

[E1276] nickel to agricultural soil

[E1278] oxamyl to agricultural soil

[E1279] oxydemethon-methyl to agricultural soil

[E1280] o-xylene to agricultural soil

[E1281] parathion-ethyl to agricultural soil

[E1282] parathion-methyl to agricultural soil

[E1283] pentachlorobenzene to agricultural soil

[E1284] pentachloronitrobenzene to agricultural soil

[E1285] pentachlorophenol to agricultural soil

[E1286] permethrin to agricultural soil

[E1288] phenol to agricultural soil

[E1289] phoxim to agricultural soil

[E1290] Phtalic anhydride to agricultural soil

[E1291] pirimicarb to agricultural soil

[E1293] propachlor to agricultural soil

[E1294] propoxur to agricultural soil

[E1295] propylene oxide to agricultural soil

[E1296] $p$-xylene to agricultural soil

[E1297] pyrazophos to agricultural soil

[E1298] selenium to agricultural soil

[E1299] simazine to agricultural soil

[E1300] styrene (vinylbenzene) to agricultural soil

[E1302] tetrachloroethylene (PER) to agricultural soil

[E1303] Tetrachloromethane (carbon tetrachloride) (HC-10) to agricultural soil

[E1304] thallium to agricultural soil

[E1305] Thiram to agricultural soil

[E1306] tin to agricultural soil

[E1307] tolclophos-methyl to agricultural soil

[E1308] toluene to agricultural soil
$0.26 \mathrm{~kg} \mathrm{1,4-dichlorobenzene} \mathrm{eq."} / \mathrm{kg}$ $8400 \mathrm{~kg} 1,4$-dichlorobenzene eq." $/ \mathrm{kg}$ $5700 \mathrm{~kg}$ 1,4-dichlorobenzene eq." $/ \mathrm{kg}$ $0.75 \mathrm{~kg}$ 1,4-dichlorobenzene eq." $/ \mathrm{kg}$

$0.78 \mathrm{~kg}$ 1,4-dichlorobenzene eq." $/ \mathrm{kg}$

$12 \mathrm{~kg}$ 1,4-dichlorobenzene eq." $/ \mathrm{kg}$

$30 \mathrm{~kg}$ 1,4-dichlorobenzene eq." $/ \mathrm{kg}$

$72 \mathrm{~kg}$ 1,4-dichlorobenzene eq."//kg

$130 \mathrm{~kg}$ 1,4-dichlorobenzene eq." $/ \mathrm{kg}$

$88 \mathrm{~kg} 1,4$-dichlorobenzene eq." $/ \mathrm{kg}$

$13 \mathrm{~kg}$ 1,4-dichlorobenzene eq."//kg

$2.3 \mathrm{~kg}$ 1,4-dichlorobenzene eq." $/ \mathrm{kg}$

$0.015 \mathrm{~kg}$ 1,4-dichlorobenzene eq." $/ \mathrm{kg}$

$670 \mathrm{~kg}$ 1,4-dichlorobenzene eq." $/ \mathrm{kg}$

$3.4 \mathrm{~kg}$ 1,4-dichlorobenzene eq." $/ \mathrm{kg}$

$30000 \mathrm{~kg}$ 1,4-dichlorobenzene eq." $/ \mathrm{kg}$

$3.30 \mathrm{E}+07 \mathrm{~kg}$ 1,4-dichlorobenzene eq." $/ \mathrm{kg}$

$1.8 \mathrm{~kg}$ 1,4-dichlorobenzene eq." $/ \mathrm{kg}$

$960 \mathrm{~kg}$ 1,4-dichlorobenzene eq." $/ \mathrm{kg}$

$3300 \mathrm{~kg}$ 1,4-dichlorobenzene eq." $/ \mathrm{kg}$

$490 \mathrm{~kg}$ 1,4-dichlorobenzene eq." $/ \mathrm{kg}$

$170 \mathrm{~kg}$ 1,4-dichlorobenzene eq." $/ \mathrm{kg}$

$0.026 \mathrm{~kg} 1,4$-dichlorobenzene eq." $/ \mathrm{kg}$

100 kg 1,4-dichlorobenzene eq." $/ \mathrm{kg}$

$740 \mathrm{~kg}$ 1,4-dichlorobenzene eq." $/ \mathrm{kg}$

5900 kg 1,4-dichlorobenzene eq." $/ \mathrm{kg}$

$6.5 \mathrm{~kg}$ 1,4-dichlorobenzene eq. "/ $/ \mathrm{kg}$

49 kg 1,4-dichlorobenzene eq." $/ \mathrm{kg}$

$51 \mathrm{~kg}$ 1,4-dichlorobenzene eq." $/ \mathrm{kg}$

$43 \mathrm{~kg} 1,4$-dichlorobenzene eq." $/ \mathrm{kg}$

$260 \mathrm{~kg}$ 1,4-dichlorobenzene eq." $/ \mathrm{kg}$

20000 kg 1,4-dichlorobenzene eq."/kg

$410 \mathrm{~kg}$ 1,4-dichlorobenzene eq." $/ \mathrm{kg}$

$11 \mathrm{~kg}$ 1,4-dichlorobenzene eq."//kg

$5.7 \mathrm{~kg}$ 1,4-dichlorobenzene eq." $/ \mathrm{kg}$

$6200 \mathrm{~kg}$ 1,4-dichlorobenzene eq." $/ \mathrm{kg}$

$3.8 \mathrm{~kg}$ 1,4-dichlorobenzene eq. "/kg

$4.8 \mathrm{~kg}$ 1,4-dichlorobenzene eq." $/ \mathrm{kg}$

2700 kg 1,4-dichlorobenzene eq." $/ \mathrm{kg}$

$10 \mathrm{~kg}$ 1,4-dichlorobenzene eq." $/ \mathrm{kg}$

$610 \mathrm{~kg}$ 1,4-dichlorobenzene eq. "/ $/ \mathrm{kg}$

$5 \mathrm{~kg}$ 1,4-dichlorobenzene eq." $/ \mathrm{kg}$

$2.9 \mathrm{~kg}$ 1,4-dichlorobenzene eq." $/ \mathrm{kg}$

$24 \mathrm{~kg}$ 1,4-dichlorobenzene eq." $/ \mathrm{kg}$

$4500 \mathrm{~kg} \mathrm{1,4-dichlorobenzene} \mathrm{eq."} / \mathrm{kg}$

$72 \mathrm{~kg}$ 1,4-dichlorobenzene eq." $/ \mathrm{kg}$

$0.15 \mathrm{~kg}$ 1,4-dichlorobenzene eq." $/ \mathrm{kg}$

$11 \mathrm{~kg}$ 1,4-dichlorobenzene eq." $/ \mathrm{kg}$

$1.9 \mathrm{~kg}$ 1,4-dichlorobenzene eq." $/ \mathrm{kg}$

$25 \mathrm{~kg}$ 1,4-dichlorobenzene eq." $/ \mathrm{kg}$

$0.01 \mathrm{~kg}$ 1,4-dichlorobenzene eq." $/ \mathrm{kg}$

$26 \mathrm{~kg}$ 1,4-dichlorobenzene eq." $/ \mathrm{kg}$

$15 \mathrm{~kg}$ 1,4-dichlorobenzene eq." $/ \mathrm{kg}$

$270 \mathrm{~kg}$ 1,4-dichlorobenzene eq." $/ \mathrm{kg}$

2.20E+05 kg 1,4-dichlorobenzene eq." $/ \mathrm{kg}$

$3 \mathrm{~kg}$ 1,4-dichlorobenzene eq. " $/ \mathrm{kg}$

$51 \mathrm{~kg}$ 1,4-dichloroberizene eq."//kg

$29000 \mathrm{~kg}$ 1,4-dichlorobenzene eq." $/ \mathrm{kg}$

$210 \mathrm{~kg}$ 1,4-dichlorobenzene eq." $/ \mathrm{kg}$

$0.48 \mathrm{~kg}$ 1,4-dichlorobenzene eq." $/ \mathrm{kg}$

$6.4 \mathrm{~kg}$ 1,4-dichlorobenzene eq." $/ \mathrm{kg}$

$220 \mathrm{~kg}$ 1,4-dichlorobenzene eq." $/ \mathrm{kg}$

2.00E+06 kg 1,4-dichlorobenzene eq." $/ \mathrm{kg}$

$7.9 \mathrm{~kg}$ 1,4-dichlorobenzene eq." $/ \mathrm{kg}$

$13 \mathrm{~kg}$ 1,4-dichlorobenzene eq." $/ \mathrm{kg}$

$11 \mathrm{~kg}$ 1,4-dichlorobenzene eq." $/ \mathrm{kg}$

$0.35 \mathrm{~kg}$ 1,4-dichlorobenzene eq." $/ \mathrm{kg}$ 
[E1309] tri-allate to agricultural soil

[E1310] triazophos to agricultural soil

[E1311] tributyltinoxide to agricultural soil

[E1312] trichlorfon to agricultural soil

[E1313] Trichloroethylene (tri) to agricultural soil

[E1314] Trichloromethane $=$ chloroform to agricultural soil

[E1315] trifluarin to agricultural soil

[E1316] vanadium to agricultural soil

[E1317] Vinyl Chloride (chloroethene) to agricultural soil

[E1318] zinc to agricultural soil

[E1319] zineb to agricultural soil

[E1320] 1,1,1-trichloroethane to agricultural soil

[E1321] 1,2,3,4-tetrachlorobenzene to agricultural soil

[E1322] 1,2,3,5-tetrachlorobenzene to agricultural soil

[E1323] 1,2,3-trichlorobenzene to agricultural soil

[E1324] 1,2,4,5-tetrachlorobenzene to agricultural soil

[E1325] 1,2,4-trichlorobenzene to agricultural soil

[E1326] 1,2-dichlorobenzene to agricultural soil

[E1327] 1,2-dichloroethane to agricultural soil

[E1328] 1,3,5-trichlorobenzene to agricultural soil

[E1329] 1,3-butadiene to agricultural soil

[E1330] 1,3-dichlorobenzene to agricultural soil

[E1331] 1,4-dichlorobenzene to agricultural soil

[E1332] 1-chloro-4-nitrobenzene to agricultural soil

[E1333] 2,3,4,6-tetrachlorophenol to agricultural soil

[E1334] 2,3,7,8-TCDD to agricultural soil

[E1335] 2,4,5-T to agricultural soil

[E1336] 2,4,5-trichlorophenol to agricultural soil

[E1337] 2,4,6-trichlorophenol to industrial soil

[E1338] 2,4-D to industrial soil

[E1339] 2,4-dichlorophenol to industrial soil

[E1340] 2-chlorophenol to industrial soil

[E1341] 3,4-dichloroaniline to industrial soil

[E1342] 3-chloroaniline to industrial soil

[E1343] 4-chloroaniline to industrial soil

[E1344] acephate to industrial soil

[E1345] Acrolein (2-propenal) to industrial soil

[E1346] acrylonitrile to industrial soil

[E1347] aldicarb to industrial soil

[E1348] aldrin to industrial soil

[E1350] anilazine to industrial soil

[E1351] anthracene to industrial soil

[E1352] antimony to industrial soil

[E1353] arsenic to industrial soil

[E1354] atrazine to industrial soil

[E1355] azinphos-ethyl to industrial soil

[E1356] azinphos-methyl to industrial soil

[E1357] barium to industrial soil

[E1358] benomyl to industrial soil

[E1359] bentazone to industrial soil

[E1360] benzene to industrial soil

[E1365] benzylchloride to industrial soil

[E1366] beryllium to industrial soil

[E1367] bifenthrin to industrial soil

[E1368] Butylbenzylphtalate to industrial soil

[E1369] cadmium to industrial soil

[E1370] captafol to industrial soil

[E1371] captan to industrial soil

[E1372] carbaryl to industrial soil

[E1373] carbendazim to industrial soil

[E1374] carbofuran to industrial soil

[E1375] carbon disulfide to industrial soil

[E1376] Carcinogenic PAHs to industrial soil

[E1377] chlordane to industrial soil

[E1378] chlorfenvinphos to industrial soil

[E1379] chloridazon to industrial soil

[E1380] chlorobenzene to industrial soil
$5.8 \mathrm{~kg}$ 1,4-dichlorobenzene eq. " $/ \mathrm{kg}$ 1200 kg 1,4-dichlorobenzene eq."/kg $290 \mathrm{~kg}$ 1,4-dichlorobenzene eq." $/ \mathrm{kg}$

$33 \mathrm{~kg}$ 1,4-dichlorobenzene eq." $/ \mathrm{kg}$

32 kg 1,4-dichlorobenzene eq." $/ \mathrm{kg}$

$14 \mathrm{~kg}$ 1,4-dichlorobenzene eq." $/ \mathrm{kg}$

$120 \mathrm{~kg}$ 1,4-dichlorobenzene eq." $/ \mathrm{kg}$

$19000 \mathrm{~kg}$ 1,4-dichlorobenzene eq." $/ \mathrm{kg}$

$520 \mathrm{~kg} \mathrm{1,4-dichlorobenzene} \mathrm{eq."} / \mathrm{kg}$

64 kg 1,4-dichlorobenzene eq." $/ \mathrm{kg}$

20 kg 1,4-dichlorobenzene eq." $/ \mathrm{kg}$

$16 \mathrm{~kg}$ 1,4-dichlorobenzene eq." $/ \mathrm{kg}$

$5.2 \mathrm{~kg}$ 1,4-dichlorobenzene eq." $/ \mathrm{kg}$

$14 \mathrm{~kg}$ 1,4-dichlorobenzene eq. " $/ \mathrm{kg}$

54 kg 1,4-dichlorabenzene eq." $/ \mathrm{kg}$

$5.4 \mathrm{~kg}$ 1,4-dichlorobenzene eq." $/ \mathrm{kg}$

$43 \mathrm{~kg}$ 1,4-dichlorobenzene eq." $/ \mathrm{kg}$

$6.9 \mathrm{~kg}$ 1,4-dichlorobenzene eq." $/ \mathrm{kg}$

$5.7 \mathrm{~kg}$ 1,4-dichlorobenzene eq. " $/ \mathrm{kg}$

$52 \mathrm{~kg}$ 1,4-dichlorobenzene eq." $/ \mathrm{kg}$

2200 kg 1,4-dichlorobenzene eq." $/ \mathrm{kg}$

$50 \mathrm{~kg}$ 1,4-dichlorobenzene eq." $/ \mathrm{kg}$

$0.74 \mathrm{~kg}$ 1,4-dichlorobenzene eq." $/ \mathrm{kg}$

460 kg 1,4-dichlorobenzene eq." $/ \mathrm{kg}$

$1.6 \mathrm{~kg}$ 1,4-dichlorobenzene eq." $/ \mathrm{kg}$

$1.00 \mathrm{E}+07 \mathrm{~kg}$ 1,4-dichlorobenzene eq." $/ \mathrm{kg}$

$0.18 \mathrm{~kg}$ 1,4-dichlorobenzene eq." $/ \mathrm{kg}$

$2.9 \mathrm{~kg}$ 1,4-dichlorobenzene eq." $/ \mathrm{kg}$

$170 \mathrm{~kg}$ 1,4-dichlorobenzene eq." $/ \mathrm{kg}$

$0.72 \mathrm{~kg}$ 1,4-dichlorobenzene eq." $/ \mathrm{kg}$

$1.9 \mathrm{~kg}$ 1,4-dichlorobenzene eq." $/ \mathrm{kg}$

$1.4 \mathrm{~kg}$ 1,4-dichlorobenzene eq." $/ \mathrm{kg}$

$31 \mathrm{~kg}$ 1,4-dichlorobenzene eq." $/ \mathrm{kg}$

$460 \mathrm{~kg}$ 1,4-dichlorobenzene eq." $/ \mathrm{kg}$

$510 \mathrm{~kg}$ 1,4-dichlorobenzene eq. "/kg

$0.31 \mathrm{~kg} 1,4$-dichlorobenzene eq." $/ \mathrm{kg}$

$17 \mathrm{~kg}$ 1,4-dichlorobenzene eq." $/ \mathrm{kg}$

$1500 \mathrm{~kg}$ 1,4-dichlorobenzene eq."//kg

$13 \mathrm{~kg}$ 1,4-dichlorobenzene eq." $/ \mathrm{kg}$

$160 \mathrm{~kg}$ 1,4-dichlorobenzene eq." $/ \mathrm{kg}$

$0.0003 \mathrm{~kg}$ 1,4-dichlorobenzene eq." $/ \mathrm{kg}$

0.02 kg 1,4-dichlorobenzene eq."/kg

$2600 \mathrm{~kg}$ 1,4-dichlorobenzene eq."/kg

$1000 \mathrm{~kg}$ 1,4-dichlorobenzene eq." $/ \mathrm{kg}$

$0.88 \mathrm{~kg}$ 1,4-dichlorobenzene eq." $/ \mathrm{kg}$

$6.9 \mathrm{~kg}$ 1,4-dichlorobenzene eq." $/ \mathrm{kg}$

0.099 kg 1,4-dichlorobenzene eq." $/ \mathrm{kg}$

$320 \mathrm{~kg}$ 1,4-dichlorobenzene eq." $/ \mathrm{kg}$

$0.0011 \mathrm{~kg}$ 1,4-dichlorobenzene eq." $/ \mathrm{kg}$

$0.16 \mathrm{~kg} \mathrm{1,4-dichlorobenzene} \mathrm{eq."} / \mathrm{kg}$

1600 kg 1,4-dichlorobenzene eq." $/ \mathrm{kg}$

$490 \mathrm{~kg} \mathrm{1,4-dichlorobenzene} \mathrm{eq."} / \mathrm{kg}$

7000 kg 1,4-dichlorobenzene eq." $/ \mathrm{kg}$

$0.3 \mathrm{~kg}$ 1,4-dichlorobenzene eq." $/ \mathrm{kg}$

$0.0018 \mathrm{~kg} 1,4$-dichlorobenzene eq." $/ \mathrm{kg}$

$67 \mathrm{~kg}$ 1,4-dichlorobenzene eq." $/ \mathrm{kg}$

$79 \mathrm{~kg}$ 1,4-dichlorobenzene eq." $/ \mathrm{kg}$

$0.00011 \mathrm{~kg}$ 1,4-dichlorobenzene eq. "/kg

$0.15 \mathrm{~kg}$ 1,4-dichlorobenzene eq." $/ \mathrm{kg}$

$0.43 \mathrm{~kg}$ 1,4-dichlorobenzene eq." $/ \mathrm{kg}$

$8 \mathrm{~kg}$ 1,4-dichlorobenzene eq." $/ \mathrm{kg}$

$2.2 \mathrm{~kg}$ 1,4-dichlorobenzene eq." $/ \mathrm{kg}$

$2700 \mathrm{~kg}$ 1,4-dichlorobenzene eq." $/ \mathrm{kg}$

$27 \mathrm{~kg}$ 1,4-dichlorobenzene eq." $/ \mathrm{kg}$

$44 \mathrm{~kg}$ 1,4-dichlorobenzene eq." $/ \mathrm{kg}$

$0.02 \mathrm{~kg}$ 1,4-dichlorobenzene eq. "/ $/ \mathrm{kg}$

$6.8 \mathrm{~kg}$ 1,4-dichlorobenzene eq." $/ \mathrm{kg}$ 
[E1381] chlorothalonil to industrial soil [E1382] chlorpropham to industrial soil [E1383] chlorpyriphos to industrial soil [E1384] chromium III to industrial soil [E1385] chromium VI to industrial soil [E1387] cobalt to industrial soil [E1388] copper to industrial soil [E1389] coumaphos to industrial soil [E1390] cyanazine to industrial soil [E1391] cypermethrin to industrial soil [E1392] cyromazine to industrial soil [E1393] DDT to industrial soil [E1394] deltamethrin to industrial soil [E1395] demeton to industrial soil [E1396] desmetryn to industrial soil [E1397] Di(2-ethylhexyl)phtalate to industrial soil [E1398] diazinon to industrial soil

[E1399] Dibutylphtalate to industrial soil

[E1400] Dichloromethane (Methylene Chloride) to industrial soil [E1401] dichlorprop to industrial soil

[E1402] dichlorvos to industrial soil

[E1403] dieldrin to industrial soil

[E1404] Diethylphtalate to industrial soil

[E1405] Dihexylphtalate to industrial soil

[E1406] Diisodecylphtalate to industrial soil

[E1407] Diisooctylphtalate to industrial soil

[E1408] dimethoate to industrial soil

[E1409] Dimethylphtalate to industrial soil

[E1410] dinoseb to industrial soil

[E1411] dinoterb to industrial soil

[E1412] Dioctylphtalate to industrial soil

[E1413] disulfothon to industrial soil

[E1414] diuron to industrial soil

[E1415] DNOC to industrial soil

[E1416] endosulfan to industrial soil

[E1417] endrin to industrial soil

[E1418] ethoprophos to industrial soil

[E1419] ethylbenzene to industrial soil

[E1420] ethylene to industrial soil

[E1421] fenitrothion to industrial soil

[E1422] fenthion to industrial soil

[E1423] fentin acetate to industrial soil

[E1424] fentin chloride to industrial soil

[E1425] fentin hydroxide to industrial soil

[E1427] folpet to industrial soil

[E1428] Formaldehyde (methanal) to industrial soil

[E1429] glyphosate to industrial soil

[E1430] heptachlor to industrial soil

[E1431] heptenophos to industrial soil

[E1432] hexachloro-1,3-butadiene to industrial soil

[E1433] hexachlorobenzene to industrial soil

[E1437] iprodione to industrial soil

[E1438] isoproturon to industrial soil

[E1439] lead to industrial soil

[E1440] lindane to industrial soil

[E1441] linuron to industrial soil

[E1442] malathion to industrial soil

[E1443] MCPA to industrial soil

[E1444] mecoprop to industrial soil

[E1445] mercury to industrial soil

[E1446] metamitron to industrial soil

[E1447] metazachlor to industrial soil

[E1448] methabenzthiazuron to industrial soil

[E1449] methomyl to industrial soil

[E1450] methylbromide to industrial soil

[E1451] methyl-mercury to industrial soil

[E1452] metobromuron to industrial soil
$1 \mathrm{~kg}$ 1,4-dichlorobenzene eq. " $/ \mathrm{kg}$ $0.081 \mathrm{~kg}$ 1,4-dichlorobenzene eq." $/ \mathrm{kg}$ $0.14 \mathrm{~kg}$ 1,4-dichlorobenzene eq." $/ \mathrm{kg}$ $300 \mathrm{~kg}$ 1,4-dichlorobenzene eq." $/ \mathrm{kg}$ 500 kg 1,4-dichlorobenzene eq." $/ \mathrm{kg}$ $59 \mathrm{~kg}$ 1,4-dichlorobenzene eq." $/ \mathrm{kg}$ $1.3 \mathrm{~kg}$ 1,4-dichlorobenzene eq."//kg $1600 \mathrm{~kg}$ 1,4-dichlorobenzene eq." $/ \mathrm{kg}$ $0.35 \mathrm{~kg}$ 1,4-dichlorobenzene eq." $/ \mathrm{kg}$

$1.8 \mathrm{~kg}$ 1,4-dichlorobenzene eq." $/ \mathrm{kg}$

$1.3 \mathrm{~kg}$ 1,4-dichlorobenzene eq." $/ \mathrm{kg}$

$1.8 \mathrm{~kg}$ 1,4-dichlorobenzene eq." $/ \mathrm{kg}$

$0.03 \mathrm{~kg} \mathrm{1,4-dichlorobenzene} \mathrm{eq."} / \mathrm{kg}$

$89 \mathrm{~kg} \mathrm{1,4-dichlorobenzene} \mathrm{eq."} / \mathrm{kg}$

$2.9 \mathrm{~kg}$ 1,4-dichlorobenzene eq." $/ \mathrm{kg}$

$0.0052 \mathrm{~kg} 1,4$-dichlorobenzene eq." $/ \mathrm{kg}$

$3.2 \mathrm{~kg}$ 1,4-dichlorobenzene eq." $/ \mathrm{kg}$

$0.013 \mathrm{~kg} 1,4$-dichlorobenzene eq." $/ \mathrm{kg}$

$1.3 \mathrm{~kg}$ 1,4-dichlorobenzene eq." $/ \mathrm{kg}$

$0.26 \mathrm{~kg}$ 1,4-dichlorobenzene eq." $/ \mathrm{kg}$

$0.036 \mathrm{~kg}$ 1,4-dichlorobenzene eq." $/ \mathrm{kg}$

$1500 \mathrm{~kg}$ 1,4-dichlorobenzene eq." $/ \mathrm{kg}$

$0.0033 \mathrm{~kg}$ 1,4-dichlorobenzene eq."/ $/ \mathrm{kg}$

$14 \mathrm{~kg}$ 1,4-dichlorobenzene eq." $/ \mathrm{kg}$

$0.038 \mathrm{~kg}$ 1,4-dichlorobenzene eq."/kg

$0.052 \mathrm{~kg}$ 1,4-dichlorobenzene eq." $/ \mathrm{kg}$

$3 \mathrm{~kg}$ 1,4-dichlorobenzene eq." $/ \mathrm{kg}$

$0.27 \mathrm{~kg}$ 1,4-dichlorobenzene eq." $/ \mathrm{kg}$

$97 \mathrm{~kg}$ 1,4-dichlorobenzene eq." $/ \mathrm{kg}$

$0.12 \mathrm{~kg}$ 1,4-dichlorobenzene eq." $/ \mathrm{kg}$

$0.0088 \mathrm{~kg}$ 1,4-dichlorobenzene eq." $/ \mathrm{kg}$

$2 \mathrm{~kg}$ 1,4-dichlorobenzene eq." $/ \mathrm{kg}$

2 kg 1,4-dichlorobenzene eq." $/ \mathrm{kg}$

$2.8 \mathrm{~kg}$ 1,4-dichlorobenzene eq." $/ \mathrm{kg}$

$0.016 \mathrm{~kg}$ 1,4-dichlorobenzene eq." $/ \mathrm{kg}$

$750 \mathrm{~kg}$ 1,4-dichlorobenzene eq."/ $/ \mathrm{kg}$

$380 \mathrm{~kg}$ 1,4-dichlorobenzene eq. "/kg

$0.5 \mathrm{~kg} 1,4$-dichlorobenzene eq. $/ \mathrm{kg}$

$0.62 \mathrm{~kg} \mathrm{1,4-dichlorobenzene} \mathrm{eq."} / \mathrm{kg}$

$0.32 \mathrm{~kg} 1,4$-dichlorobenzene eq." $/ \mathrm{kg}$

$1.5 \mathrm{~kg}$ 1,4-dichlorobenzene eq." $/ \mathrm{kg}$

$9.2 \mathrm{~kg}$ 1,4-dichlorobenzene eq." $/ \mathrm{kg}$

$13 \mathrm{~kg}$ 1,4-dichlorobenzene eq." $/ \mathrm{kg}$

$8.5 \mathrm{~kg}$ 1,4-dichlorobenzene eq." $/ \mathrm{kg}$

$1.5 \mathrm{~kg}$ 1,4-dichlorobenzene eq." $/ \mathrm{kg}$

$0.019 \mathrm{~kg}$ 1,4-dichlorobenzene eq." $/ \mathrm{kg}$

$0.00065 \mathrm{~kg}$ 1,4-dichlorobenzene eq." $/ \mathrm{kg}$

$4.4 \mathrm{~kg}$ 1,4-dichlorobenzene eq." $/ \mathrm{kg}$

$0.02 \mathrm{~kg}$ 1,4-dichlorobenzene eq." $/ \mathrm{kg}$

$35000 \mathrm{~kg}$ 1,4-dichlorobenzene eq." $/ \mathrm{kg}$

1.30E+06 kg 1,4-dichlorobenzene eq." $/ \mathrm{kg}$

$0.0032 \mathrm{~kg}$ 1,4-dichlorobenzene eq." $/ \mathrm{kg}$

$2.8 \mathrm{~kg}$ 1,4-dichlorobenzene eq." $/ \mathrm{kg}$

$290 \mathrm{~kg}$ 1,4-dichlorobenzene eq." $/ \mathrm{kg}$

$52 \mathrm{~kg}$ 1,4-dichlorobenzene eq. $/ \mathrm{kg}$

$9.4 \mathrm{~kg}$ 1,4-dichlorobenzene eq." $/ \mathrm{kg}$

$0.00095 \mathrm{~kg}$ 1,4-dichlorobenzene eq." $/ \mathrm{kg}$

$0.97 \mathrm{~kg}$ 1,4-dichlorobenzene eq." $/ \mathrm{kg}$

$42 \mathrm{~kg}$ 1,4-dichlorobenzene eq." $/ \mathrm{kg}$

$1100 \mathrm{~kg}$ 1,4-dichlorobenzene eq." $/ \mathrm{kg}$

$0.012 \mathrm{~kg}$ 1,4-dichlorobenzene eq." $/ \mathrm{kg}$

$0.16 \mathrm{~kg} \mathrm{1,4-dichlorobenzene} \mathrm{eq."} / \mathrm{kg}$

$0.36 \mathrm{~kg}$ 1,4-dichlorobenzene eq." $/ \mathrm{kg}$

$0.69 \mathrm{~kg}$ 1,4-dichlorobenzene eq. " $/ \mathrm{kg}$

$260 \mathrm{~kg}$ 1,4-dichlorobenzene eq." $/ \mathrm{kg}$

$11000 \mathrm{~kg}$ 1,4-dichlorobenzene eq." $/ \mathrm{kg}$

$1.9 \mathrm{~kg}$ 1,4-dichlorobenzene eq." $/ \mathrm{kg}$ 
[E1453] metolachlor to industrial soil

[E1454] mevinphos to industrial soil

[E1455] molybdenum to industrial soil

[E1456] $m$-xylene to industrial soil

[E1457] naphtalene to industrial soil

[E1458] nickel to industrial soil

[E1460] oxamyl to industrial soil

[E1461] oxydemethon-methyl to industrial soil

[E1462] o-xylene to industrial soil

[E1463] parathion-ethyl to industrial soil

[E1464] parathion-methyl to industrial soil

[E1465] pentachlorobenzene to industrial soil

[E1466] pentachloronitrobenzene to industrial soil

[E1467] pentachlorophenol to industrial soil

[E1468] permethrin to industrial soil

[E1470] phenol to industrial soil

[E1471] phoxim to industrial soil

[E1472] Phtalic anhydride to industrial soil

[E1473] pirimicarb to industrial soil

[E1475] propachlor to industrial soil

[E1476] propoxur to industrial soil

[E1477] propylene oxide to industrial soil

[E1478] p-xylene to industrial soil

[E1479] pyrazophos to industrial soil

[E1480] selenium to industrial soil

[E1481] simazine to industrial soil

[E1482] styrene (vinylbenzene) to industrial soil

[E1484] tetrachloroethylene (PER) to industrial soil

[E1485] Tetrachloromethane (carbon tetrachloride) $(\mathrm{HC}-10)$ to industrial soil

[E1486] thallium to industrial soil

[E1487] Thiram to industrial soil

[E1488] tin to industrial soil

[E1489] tolclophos-methyl to industrial soil

[E1490] toluene to industrial soil

[E1491] tri-allate to industrial soil

[E1492] triazophos to industrial soil

[E1493] tributyltinoxide to industrial soil

[E1494] trichlorfon to industrial soil

[E1495] Trichloroethylene (tri) to industrial soil

[E1496] Trichloromethane $=$ chloroform to industrial soil

[E1497] trifluarin to industrial soil

[E1498] vanadium to industrial soil

[E1499] Vinyl Chloride (chloroethene) to industrial soil

[E1500] zinc to industrial soil

[E1501] zineb to industrial soil

Category $=[$ [C6] Aquatic ecotoxicity fresh water

Description = Problem oriented approach, manual 1999; AETP

Author = Huijbregts, 1999

Date $=10 / 11 / 99$

Environmental resources

Environmental resource

Value

Environmental emissions

Environmental emission

[E90] 1,1,1-trichloroethane (methyl chloroform; HC-140a) to air

[E94] 1,2,3,4-tetrachlorobenzene to air

[E95] 1,2,3,5-tetrachlorobenzene to air

[E96] 1,2,3-trichlorobenzene to air

[E98] 1,2,4,5-tetrachlorobenzene to air

[E99] 1,2,4-trichlorobenzene to air

[E102] 1,2-dichlorobenzene to air

[E103] 1,2-dichloroethane to air

[E107] 1,3,5-trichlorobenzene to air

[E111] 1,3-Butadiene to air
$0.11 \mathrm{~kg}$ 1,4-dichlorobenzene eq. "/ $/ \mathrm{kg}$ $0.055 \mathrm{~kg}$ 1,4-dichlorobenzene eq." $/ \mathrm{kg}$

$3100 \mathrm{~kg}$ 1,4-dichlorobenzene eq." $/ \mathrm{kg}$

$0.019 \mathrm{~kg}$ 1,4-dichlorobenzene eq." $/ \mathrm{kg}$

$1.6 \mathrm{~kg}$ 1,4-dichlorobenzene eq." $/ \mathrm{kg}$

$200 \mathrm{~kg}$ 1,4-dichlorobenzene eq." $/ \mathrm{kg}$

$0.068 \mathrm{~kg}$ 1,4-dichlorobenzene eq." $/ \mathrm{kg}$

$3.8 \mathrm{~kg}$ 1,4-dichlorobenzene eq." $/ \mathrm{kg}$

0.076 kg 1,4-dichlorobenzene eq." $/ \mathrm{kg}$

$0.11 \mathrm{~kg}$ 1,4-dichlorobenzene eq." $/ \mathrm{kg}$

$1.7 \mathrm{~kg}$ 1,4-dichlorobenzene eq." $/ \mathrm{kg}$

$140 \mathrm{~kg}$ 1,4-dichlorobenzene eq." $/ \mathrm{kg}$

$4.3 \mathrm{~kg}$ 1,4-dichlorobenzene eq." $/ \mathrm{kg}$

0.039 kg 1,4-dichlorobenzene eq." $/ \mathrm{kg}$

$0.021 \mathrm{~kg} \mathrm{1,4-dichlorobenzene} \mathrm{eq."} / \mathrm{kg}$

0.006 kg 1,4-dichlorobenzene eq." $/ \mathrm{kg}$

$0.38 \mathrm{~kg}$ 1,4-dichlorobenzene eq." $/ \mathrm{kg}$

6.60E-07 kg 1,4-dichlorobenzene eq." $/ \mathrm{kg}$

$0.29 \mathrm{~kg}$ 1,4-dichlorobenzene eq. "/kg

$0.14 \mathrm{~kg}$ 1,4-dichlorobenzene eq."/kg

$0.27 \mathrm{~kg}$ 1,4-dichlorobenzene eq." $/ \mathrm{kg}$

$590 \mathrm{~kg}$ 1,4-dichlorobenzene eq." $/ \mathrm{kg}$

$0.025 \mathrm{~kg}$ 1,4-dichlorobenzene eq." $/ \mathrm{kg}$

$1.2 \mathrm{~kg}$ 1,4-dichlorobenzene eq. "/kg

28000 kg 1,4-dichlorobenzene eq." $/ \mathrm{kg}$

$2.2 \mathrm{~kg}$ 1,4-dichlorobenzene eq." $/ \mathrm{kg}$

$0.018 \mathrm{~kg}$ 1,4-dichlorobenzene eq." $/ \mathrm{kg}$

$5.2 \mathrm{~kg}$ 1,4-dichlorobenzene eq." $/ \mathrm{kg}$

220 kg 1,4-dichlorobenzene eq." $/ \mathrm{kg}$

1.20E+05 kg 1,4-dichlorobenzene eq." $/ \mathrm{kg}$

$0.25 \mathrm{~kg}$ 1,4-dichlorobenzene eq." $/ \mathrm{kg}$

$0.52 \mathrm{~kg}$ 1,4-dichlorobenzene eq." $/ \mathrm{kg}$

$0.04 \mathrm{~kg} 1,4$-dichlorobenzene eq. $/ \mathrm{kg}$

$0.21 \mathrm{~kg}$ 1,4-dichlorobenzene eq." $/ \mathrm{kg}$

$0.36 \mathrm{~kg}$ 1,4-dichlorobenzene eq." $/ \mathrm{kg}$

$37 \mathrm{~kg}$ 1,4-dichlorobenzene eq." $/ \mathrm{kg}$

$43 \mathrm{~kg}$ 1,4-dichlorobenzene eq." $/ \mathrm{kg}$

$0.02 \mathrm{~kg}$ 1,4-dichlorobenzene eq." $/ \mathrm{kg}$

$32 \mathrm{~kg}$ 1,4-dichlorobenzene eq." $/ \mathrm{kg}$

$10 \mathrm{~kg}$ 1,4-dichlorobenzene eq." $/ \mathrm{kg}$

$0.68 \mathrm{~kg}$ 1,4-dichlorobenzene eq." $/ \mathrm{kg}$

$1700 \mathrm{~kg}$ 1,4-dichlorobenzene eq." $/ \mathrm{kg}$

$83 \mathrm{~kg}$ 1,4-dichlorobenzene eq." $/ \mathrm{kg}$

$0.42 \mathrm{~kg}$ 1,4-dichlorobenzene eq."/kg

$0.1 \mathrm{~kg}$ 1,4-dichlorobenzene eq."/kg
Value 0.00012 kg 1,4-dichlorobenzene eq." $/ \mathrm{kg}$

$0.1 \mathrm{~kg}$ 1,4-dichlorobenzene eq." $/ \mathrm{kg}$

$0.073 \mathrm{~kg}$ 1,4-dichlorobenzene eq." $/ \mathrm{kg}$

0.0085 kg 1,4-dichlorobenzene eq." $/ \mathrm{kg}$

$0.073 \mathrm{~kg}$ 1,4-dichlorobenzene eq." $/ \mathrm{kg}$

$0.0099 \mathrm{~kg}$ 1,4-dichlorobenzene eq." $/ \mathrm{kg}$

$0.0029 \mathrm{~kg}$ 1,4-dichlorobenzene eq." $/ \mathrm{kg}$

0.00012 kg 1,4-dichlorobenzene eq." $/ \mathrm{kg}$

$0.016 \mathrm{~kg}$ 1,4-dichlorobenzene eq." $/ \mathrm{kg}$

3.30E-07 kg 1,4-dichlorobenzene eq."/kg 
[E112] 1,3-dichlorobenzene to air [E116] 1,4-dichlorobenzene to air [E128] 1-chloro-4-nitrobenzene to air [E166] 2,3,4,6-tetrachlorophenol to air [E168] 2,3,7,8-TCDD (tetrachloride-dibenzo-dioxin) to air [E172] 2,4,5-T to air

[E173] 2,4,5-trichlorophenol to air [E174] 2,4,6-trichlorophenol to air [E175] 2,4-D to air

[E176] 2,4-dichlorophenol to air [E185] 2-chlorophenol to air [E208] 3,4-dichloroaniline to air [E217] 3-chloroaniline to air [E228] 4-chloroaniline to air [E231] acephate to air [E236] Acrolein (2-propenal) to air [E237] Acrylonitrile to air [E241] aldicarb to air [E242] aldrin to air [E251] anilazine to air [E252] anthracene to air [E253] antimony to air [E255] arsenic to air [E256] atrazine to air [E257] azinphos-ethyl to air [E258] azinphos-methyl to air [E259] barium to air [E260] benomyl to air [E261] bentazone to air [E263] Benzene to air [E264] benzo[a]anthracene to air [E265] benzo[a]pyrene to air [E266] benzo[ghi]perylene to air [E267] benzo[k]fluoranthrene to air [E269] benzylchloride to air [E270] beryllium to air [E272] bifenthrin to air [E290] Butylbenzylphtalate to air [E385] cadmium to air [E387] captafol to air [E388] captan to air [E389] carbaryl to air [E390] carbendazim to air [E391] carbofuran to air [E394] carbon disulfide to air [E404] chlordane to air [E405] chlorfenvinphos to air [E406] chloridazon to air [E407] chlorobenzene to air [E410] chlorothalonil to air [E411] chlorpropham to air [E412] chlorpyriphos to air [E413] chromium (unspecified) to air [E414] chromium III to air [E415] chromium VI to air [E416] chrysene to air [E421] cobalt to air [E422] copper to air [E423] coumaphos to air [E425] cyanazine to air [E437] cypermethrin to air [E438] cyromazine to air [E439] DDT to air

[E441] deltamethrin to air [E442] demeton to air [E443] desmetryn to air [E444] Di(2-ethylhexyl)phtalate to air
0.0024 kg 1,4-dichlorobenzene eq."/kg 0.0024 kg 1,4-dichlorobenzene eq." $/ \mathrm{kg}$

$11 \mathrm{~kg}$ 1,4-dichlorobenzene eq." $/ \mathrm{kg}$

80 kg 1,4-dichlorobenzene eq." $/ \mathrm{kg}$

2.10E+06 kg 1,4-dichlorobenzene eq."/kg 0.85 kg 1,4-dichlorobenzene eq." $/ \mathrm{kg}$ 15 kg 1,4-dichlorobenzene eq."/kg 5.9 kg 1,4-dichlorobenzene eq."/kg 39 kg 1,4-dichlorobenzene eq."/kg $1.4 \mathrm{~kg} \mathrm{1,4-dichlorobenzene} \mathrm{eq."} / \mathrm{kg}$ $13 \mathrm{~kg}$ 1,4-dichlorobenzene eq."/kg 1700 kg 1,4-dichlorobenzene eq. "/kg 100 kg 1,4-dichlorobenzene eq." $/ \mathrm{kg}$

2 kg 1,4-dichlorobenzene eq."/kg 79 kg 1,4-dichlorobenzene eq."/kg $520 \mathrm{~kg} \mathrm{1,4-dichlorobenzene} \mathrm{eq."} / \mathrm{kg}$ $0.41 \mathrm{~kg}$ 1,4-dichlorobenzene eq."/kg 51000 kg 1,4-dichlorobenzene eq."/kg $2.7 \mathrm{~kg}$ 1,4-dichlorobenzene eq." $/ \mathrm{kg}$ 14 kg 1,4-dichlorobenzene eq."/kg 140 kg 1,4-dichlorobenzene eq." $/ \mathrm{kg}$ $3.7 \mathrm{~kg}$ 1,4-dichlorobenzene eq." $/ \mathrm{kg}$ $50 \mathrm{~kg} \mathrm{1,4-dichlorobenzene} \mathrm{eq.} \mathrm{"/kg}$ $360 \mathrm{~kg} \mathrm{1,4-dichlorobenzene} \mathrm{eq."} / \mathrm{kg}$ 290 kg 1,4-dichlorobenzene eq."/kg 420 kg 1,4-dichlorobenzene eq." $/ \mathrm{kg}$

$43 \mathrm{~kg} \mathrm{1,4-dichlorobenzene} \mathrm{eq."/kg}$

$30 \mathrm{~kg} \mathrm{1,4-dichlorobenzene} \mathrm{eq."} / \mathrm{kg}$ $5.6 \mathrm{~kg} \mathrm{1,4-dichlorobenzene} \mathrm{eq."} / \mathrm{kg}$ 8.40E-05 kg 1,4-dichlorobenzene eq." $/ \mathrm{kg}$ 42 kg 1,4-dichlorobenzene eq." $/ \mathrm{kg}$ $88 \mathrm{~kg} \mathrm{1,4-dichlorobenzene} \mathrm{eq.} \mathrm{"/kg}$ 44 kg 1,4-dichlorobenzene eq."/kg 3900 kg 1,4-dichlorobenzene eq."/kg 0.76 kg 1,4-dichlorobenzene eq."/kg 17000 kg 1,4-dichlorobenzene eq." $/ \mathrm{kg}$ $820 \mathrm{~kg} \mathrm{1,4-dichlorobenzene} \mathrm{eq.} \mathrm{"/kg}$ $0.4 \mathrm{~kg} 1,4$-dichlorobenzene eq." $/ \mathrm{kg}$ $290 \mathrm{~kg} \mathrm{1,4-dichlorobenzene} \mathrm{eq."/kg}$ 20000 kg 1,4-dichlorobenzene eq."/kg 16 kg 1,4-dichlorobenzene eq." $/ \mathrm{kg}$ $110 \mathrm{~kg}$ 1,4-dichlorobenzene eq." $/ \mathrm{kg}$ 3000 kg 1,4-dichlorobenzene eq." $/ \mathrm{kg}$ 900 kg 1,4-dichlorobenzene eq."/kg $0.033 \mathrm{~kg} \mathrm{1,4-dichlorobenzene} \mathrm{eq.} \mathrm{"/kg}$ 270 kg 1,4-dichlorobenzene eq."/kg

32 kg 1,4-dichlorobenzene eq." $/ \mathrm{kg}$ 0.026 kg 1,4-dichlorobenzene eq." $/ \mathrm{kg}$ 0.00047 kg 1,4-dichlorobenzene eq."/kg 2.5 kg 1,4-dichlorobenzene eq." $/ \mathrm{kg}$ $2.3 \mathrm{~kg} \mathrm{1,4-dichlorobenzene} \mathrm{eq."} / \mathrm{kg}$ 520 kg 1,4-dichlorobenzene eq." $/ \mathrm{kg}$ $1.9 \mathrm{~kg}$ 1,4-dichlorobenzene eq." $/ \mathrm{kg}$ $1.9 \mathrm{~kg} \mathrm{1,4-dichlorobenzene} \mathrm{eq."} / \mathrm{kg}$ 7.7 kg 1,4-dichlorobenzene eq."/kg $39 \mathrm{~kg}$ 1,4-dichlorobenzene eq." $/ \mathrm{kg}$ 640 kg 1,4-dichlorobenzene eq."/kg 220 kg 1,4-dichlorobenzene eq." $/ \mathrm{kg}$ 2.40E+05 kg 1,4-dichlorobenzene eq." $/ \mathrm{kg}$ 1900 kg 1,4-dichlorobenzene eq."/kg 84000 kg 1,4-dichlorobenzene eq."/kg 3500 kg 1,4-dichlorobenzene eq." $/ \mathrm{kg}$ $320 \mathrm{~kg} \mathrm{1,4-dichlorobenzene} \mathrm{eq."/kg}$ 1800 kg 1,4-dichlorobenzene eq."/kg

$23 \mathrm{~kg}$ 1,4-dichlorobenzene eq. "/kg $6.8 \mathrm{~kg} \mathrm{1,4-dichlorobenzene} \mathrm{eq."/kg}$ 0.35 kg 1,4-dichlorobenzene eq." $/ \mathrm{kg}$ 
[E446] diazinon to air

[E448] Dibutylphtalate to air

[E449] Dichloromethane (Methylene Chloride) to air

[E450] dichlorprop to air

[E451] dichlorvos to air

[E452] dieldrin to air

[E456] Diethylphtalate to air

[E457] Dihexylphtalate to air

[E458] Diisodecylphtalate to air

[E459] Diisooctylphtalate to air

[E461] dimethoate to air

[E468] Dimethylphtalate to air

[E470] dinoseb to air

[E471] dinoterb to air

[E472] Dioctylphtalate to air

[E475] disulfothon to air

[E476] diuron to air

[E478] DNOC to air

[E480] endosulfan to air

[E481] endrin to air

[E487] ethoprophos to air

[E496] Ethylbenzene to air

[E499] Ethylene (ethene) to air

[E504] fenitrothion to air

[E505] fenthion to air

[E506] fentin acetate to air

[E507] fentin chloride to air

[E508] fentin hydroxide to air

[E509] fluoranthrene to air

[E510] folpet to air

[E511] Formaldehyde (methanal) to air

[E516] glyphosate to air

[E533] heptachlor to air

[E535] heptenophos to air

[E536] hexachloro-1,3-butadiene to air

[E537] hexachlorobenzene to air

[E566] indeno[1,2,3-cd]pyrene to air

[E568] iprodione to air

[E583] isoproturon to air

[E584] lead to air

[E585] lindane to air

[E586] linuron to air

[E587] malathion to air

[E589] MCPA to air

[E590] mecoprop to air

[E592] mercury to air

[E595] metamitron to air

[E596] meta-Xylene (1,3-dimethylbenzene) to air

[E597] metazachlor to air

[E598] methabenzthiazuron to air

[E603] methomyl to air

[E626] methyl-mercury to air

[E628] metobromuron to air

[E629] metolachlor to air

[E630] mevinphos to air

[E632] molybdenum to air

[E633] Naphtalene to air

[E635] nickel to air

[E650] ortho-Xylene (1,2-dimethylbenzene) to air

[E651] oxamyl to air

[E652] oxydemethon-methyl to air

[E655] parathion-ethyl to air

[E656] parathion-methyl to air

[E658] para-Xylene (1,4-dimethylbenzene) to air

[E660] pentachlorobenzene to air

[E661] pentachloronitrobenzene to air

[E662] pentachlorophenol to air
$230 \mathrm{~kg}$ 1,4-dichlorobenzene eq." $/ \mathrm{kg}$

$0.56 \mathrm{~kg}$ 1,4-dichlorobenzene eq." $/ \mathrm{kg}$

3.30E-05 kg 1,4-dichlorobenzene eq."/kg

$0.099 \mathrm{~kg}$ 1,4-dichlorobenzene eq." $/ \mathrm{kg}$

$510 \mathrm{~kg}$ 1,4-dichlorobenzene eq." $/ \mathrm{kg}$

$200 \mathrm{~kg}$ 1,4-dichlorobenzene eq." $/ \mathrm{kg}$

$0.42 \mathrm{~kg} \mathrm{1,4-dichlorobenzene} \mathrm{eq."} / \mathrm{kg}$

$0.5 \mathrm{~kg}$ 1,4-dichlorobenzene eq." $/ \mathrm{kg}$

$0.56 \mathrm{~kg}$ 1,4-dichlorobenzene eq." $/ \mathrm{kg}$

$0.12 \mathrm{~kg}$ 1,4-dichlorobenzene eq." $/ \mathrm{kg}$

$13 \mathrm{~kg}$ 1,4-dichlorobenzene eq." $/ \mathrm{kg}$

$0.052 \mathrm{~kg}$ 1,4-dichlorobenzene eq."/kg

$10000 \mathrm{~kg}$ 1,4-dichlorobenzene eq." $/ \mathrm{kg}$

$2900 \mathrm{~kg}$ 1,4-dichlorobenzene eq." $/ \mathrm{kg}$

$0.016 \mathrm{~kg}$ 1,4-dichlorobenzene eq." $/ \mathrm{kg}$

27 kg 1,4-dichlorobenzene eq."/kg

$27 \mathrm{~kg}$ 1,4-dichlorobenzene eq." $/ \mathrm{kg}$

$3.4 \mathrm{~kg}$ 1,4-dichlorobenzene eq." $/ \mathrm{kg}$

$45 \mathrm{~kg}$ 1,4-dichlorobenzene eq." $/ \mathrm{kg}$

$1100 \mathrm{~kg}$ 1,4-dichlorobenzene eq." $/ \mathrm{kg}$

$2400 \mathrm{~kg}$ 1,4-dichlorobenzene eq."/ $/ \mathrm{kg}$

$0.00013 \mathrm{~kg}$ 1,4-dichlorobenzene eq."//kg

$1.40 \mathrm{E}-11 \mathrm{~kg}$ 1,4-dichlorobenzene eq."/kg

$2500 \mathrm{~kg}$ 1,4-dichlorobenzene eq." $/ \mathrm{kg}$

$2500 \mathrm{~kg} 1,4$-dichlorobenzene eq." $/ \mathrm{kg}$

$4300 \mathrm{~kg}$ 1,4-dichlorobenzene eq." $/ \mathrm{kg}$

1800 kg 1,4-dichlorobenzene eq."/kg

4200 kg 1,4-dichlorobenzene eq." $/ \mathrm{kg}$

$18 \mathrm{~kg}$ 1,4-dichlorobenzene eq." $/ \mathrm{kg}$

$410 \mathrm{~kg}$ 1,4-dichlorobenzene eq." $/ \mathrm{kg}$

$8.3 \mathrm{~kg}$ 1,4-dichlorobenzene eq." $/ \mathrm{kg}$

$22 \mathrm{~kg}$ 1,4-dichlorobenzene eq." $/ \mathrm{kg}$

$1.4 \mathrm{~kg}$ 1,4-dichlorobenzene eq." $/ \mathrm{kg}$

$120 \mathrm{~kg} \mathrm{1,4-dichlorobenzene} \mathrm{eq."} / \mathrm{kg}$

$46 \mathrm{~kg}$ 1,4-dichlorobenzene eq." $/ \mathrm{kg}$

$1.3 \mathrm{~kg} 1,4$-dichlorobenzene eq." $/ \mathrm{kg}$

170 kg 1,4-dichlorobenzene eq." $/ \mathrm{kg}$

$2.8 \mathrm{~kg}$ 1,4-dichlorobenzene eq." $/ \mathrm{kg}$

190 kg 1,4-dichlorobenzene eq." $/ \mathrm{kg}$

$2.4 \mathrm{~kg}$ 1,4-dichlorobenzene eq." $/ \mathrm{kg}$

$52 \mathrm{~kg}$ 1,4-dichlorobenzene eq." $/ \mathrm{kg}$

$40 \mathrm{~kg}$ 1,4-dichlorobenzene eq." $/ \mathrm{kg}$

$1800 \mathrm{~kg}$ 1,4-dichlorobenzene eq." $/ \mathrm{kg}$

$1.1 \mathrm{~kg}$ 1,4-dichlorobenzene eq." $/ \mathrm{kg}$

37 kg 1,4-dichlorobenzene eq." /kg

$320 \mathrm{~kg}$ 1,4-dichlorobenzene eq." $/ \mathrm{kg}$

$0.93 \mathrm{~kg} 1,4$-dichlorobenzene eq." $/ \mathrm{kg}$

4.40E-05 kg 1,4-dichlorobenzene eq."/kg

$7.4 \mathrm{~kg}$ 1,4-dichlorobenzene eq."/kg

$70 \mathrm{~kg}$ 1,4-dichlorobenzene eq." $/ \mathrm{kg}$

$14000 \mathrm{~kg}$ 1,4-dichlorobenzene eq." $/ \mathrm{kg}$

7300 kg 1,4-dichlorobenzene eq." $/ \mathrm{kg}$

$49 \mathrm{~kg}$ 1,4-dichlorobenzene eq." $/ \mathrm{kg}$

1500 kg 1,4-dichlorobenzene eq." $/ \mathrm{kg}$

$9300 \mathrm{~kg}$ 1,4-dichlorobenzene eq." $/ \mathrm{kg}$

$97 \mathrm{~kg}$ 1,4-dichlorobenzene eq." $/ \mathrm{kg}$

$0.5 \mathrm{~kg}$ 1,4-dichlorobenzene eq." $/ \mathrm{kg}$

$630 \mathrm{~kg}$ 1,4-dichlorobenzene eq." $/ \mathrm{kg}$

9.30E-05 kg 1,4-dichlorobenzene eq."/kg

56 kg 1,4-dichlorobenzene eq." $/ \mathrm{kg}$

2400 kg 1,4-dichlorobenzene eq."/kg

2800 kg 1,4-dichlorobenzene eq." $/ \mathrm{kg}$

$990 \mathrm{~kg}$ 1,4-dichlorobenzene eq." $/ \mathrm{kg}$

6.10E-05 kg 1,4-dichlorobenzene eq." $/ \mathrm{kg}$

$0.37 \mathrm{~kg}$ 1,4-dichlorobenzene eq." $/ \mathrm{kg}$

$47 \mathrm{~kg}$ 1,4-dichlorobenzene eq." $/ \mathrm{kg}$

$11 \mathrm{~kg}$ 1,4-dichlorobenzene eq. "/kg 
[E672] permethrin to air

[E674] phenanthrene to air

[E675] Phenol to air

[E679] phoxim to air

[E680] Phtalic anhydride to air

[E682] pirimicarb to air

[E685] Polycyclic Aromatic Hydrocarbons Carcinogenic- (carcinogenic-PAH) to air

[E686] propachlor to air

[E690] propoxur to air

[E697] Propylene Oxide to air

[E698] pyrazophos to air

[E704] selenium to air

[E705] simazine to air

[E706] styrene (vinylbenzene) to air

[E718] tetrachloroethylene (PER) (tetrachloroethene) to air

[E719] Tetrachloromethane (carbon tetrachloride) (HC-10) to air

[E722] thallium to air

[E723] Thiram to air

[E724] tin to air

[E725] tolclophos-methyl to air

[E727] Toluene to air

[E733] tri-allate to air

[E734] triazophos to air

[E735] tributyltinoxide to air

[E736] trichlorfon to air

[E737] Trichloroethylene (tri) to air

[E738] Trichloromethane (chloroform) to air

[E739] trifluarin to air

[E743] vanadium to air

[E745] Vinyl Chloride (chloroethene) to air

[E748] zinc to air

[E749] zineb to air

[E750] 1,1,1-trichloroethane to air

[E751] 1,2,3,4-tetrachlorobenzene to air

[E752] 1,2,3,5-tetrachlorobenzene to air

[E753] 1,2,3-trichlorobenzene to air

[E754] 1,2,4,5-tetrachlorobenzene to air

[E755] 1,2,4-trichlorobenzene to air

[E756] 1,2-dichlorobenzene to air

[E757] 1,2-dichloroethane to air

[E758] 1,3,5-trichlorobenzene to air

[E759] 1,3-butadiene to air

[E760] 1,3-dichlorobenzene to air

[E761] 1,4-dichlorobenzene to air

[E762] 1-chloro-4-nitrobenzene to air

[E763] 2,3,4,6-tetrachlorophenol to air

[E764] 2,3,7,8-TCDD to air

[E765] 2,4,5-T to air

[E766] 2,4,5-trichlorophenol to air

[E767] 2,4,6-trichlorophenol to fresh water

[E768] 2,4-D to fresh water

[E769] 2,4-dichlorophenol to fresh wate

[E770] 2-chlorophenol to fresh water

[E771] 3,4-dichloroaniline to fresh water

[E772] 3-chloroaniline to fresh water

[E773] 4-chloroaniline to fresh water

[E774] acephate to fresh water

[E775] Acrolein (2-propenal) to fresh water

[E776] acrylonitrile to fresh water

[E777] aldicarb to fresh water

[E778] aldrin to fresh water

[E780] anilazine to fresh water

[E781] anthracene to fresh water

[E782] antimony to fresh water

[E783] arsenic to fresh water

[E784] atrazine to fresh water

[E785] azinphos-ethyl to fresh water
$16000 \mathrm{~kg} \mathrm{1,4-dichlorobenzene} \mathrm{eq."} / \mathrm{kg}$

$1.3 \mathrm{~kg}$ 1,4-dichlorobenzene eq." $/ \mathrm{kg}$

$1.5 \mathrm{~kg}$ 1,4-dichlorobenzene eq." $/ \mathrm{kg}$

$0.44 \mathrm{~kg} 1,4$-dichlorobenzene eq." $/ \mathrm{kg}$

$0.0082 \mathrm{~kg}$ 1,4-dichlorobenzene eq." $/ \mathrm{kg}$

2400 kg 1,4-dichlorobenzene eq." $/ \mathrm{kg}$

$170 \mathrm{~kg}$ 1,4-dichlorobenzene eq." $/ \mathrm{kg}$

$20 \mathrm{~kg}$ 1,4-dichlorobenzene eq." $/ \mathrm{kg}$

25000 kg 1,4-dichlorobenzene eq."/kg

$0.037 \mathrm{~kg}$ 1,4-dichlorobenzene eq." $/ \mathrm{kg}$

$180 \mathrm{~kg}$ 1,4-dichlorobenzene eq." $/ \mathrm{kg}$

$550 \mathrm{~kg}$ 1,4-dichlorobenzene eq." $/ \mathrm{kg}$

$2100 \mathrm{~kg} \mathrm{1,4-dichlorobenzene} \mathrm{eq."} / \mathrm{kg}$

$5.10 \mathrm{E}-05 \mathrm{~kg}$ 1,4-dichlorobenzene eq." $/ \mathrm{kg}$

$0.00041 \mathrm{~kg} 1,4$-dichlorobenzene eq. "/kg

$0.00025 \mathrm{~kg}$ 1,4-dichlorobenzene eq." $/ \mathrm{kg}$

$1600 \mathrm{~kg}$ 1,4-dichlorobenzene eq." $/ \mathrm{kg}$

2700 kg 1,4-dichlorobenzene eq." $/ \mathrm{kg}$

$2.5 \mathrm{~kg}$ 1,4-dichlorobenzene eq. "/kg

$0.15 \mathrm{~kg}$ 1,4-dichlorobenzene eq." $/ \mathrm{kg}$

7.00E-05 kg 1,4-dichlorobenzene eq." $/ \mathrm{kg}$

$61 \mathrm{~kg}$ 1,4-dichlorobenzene eq." $/ \mathrm{kg}$

$3300 \mathrm{~kg}$ 1,4-dichlorobenzene eq."/kg

$7700 \mathrm{~kg}$ 1,4-dichlorobenzene eq."/kg

13000 kg 1,4-dichlorobenzene eq." $/ \mathrm{kg}$

3.80E-05 kg 1,4-dichlorobenzene eq." $/ \mathrm{kg}$

9.50E-05 kg 1,4-dichlorobenzene eq." $/ \mathrm{kg}$

$9.9 \mathrm{~kg}$ 1,4-dichlorobenzene eq." $/ \mathrm{kg}$

$1700 \mathrm{~kg}$ 1,4-dichlorobenzene eq." $/ \mathrm{kg}$

2.90E-06 kg 1,4-dichlorobenzene eq." $/ \mathrm{kg}$

$18 \mathrm{~kg}$ 1,4-dichlorobenzene eq." $/ \mathrm{kg}$

$940 \mathrm{~kg}$ 1,4-dichlorobenzene eq. $/ / \mathrm{kg}$

$0.11 \mathrm{~kg}$ 1,4-dichlorobenzene eq." $/ \mathrm{kg}$

$16 \mathrm{~kg}$ 1,4-dichlorobenzene eq." $/ \mathrm{kg}$

$14 \mathrm{~kg}$ 1,4-dichlorobenzene eq." $/ \mathrm{kg}$

$4 \mathrm{~kg}$ 1,4-dichlorobenzene eq." $/ \mathrm{kg}$

$13 \mathrm{~kg}$ 1,4-dichlorobenzene eq." $/ \mathrm{kg}$

$3.5 \mathrm{~kg}$ 1,4-dichlorobenzene eq." $/ \mathrm{kg}$

$1 \mathrm{~kg}$ 1,4-dichlorobenzene eq." $/ \mathrm{kg}$

$0.023 \mathrm{~kg} \mathrm{1,4-dichlorobenzene} \mathrm{eq."/kg}$

$5 \mathrm{~kg}$ 1,4-dichlorobenzene eq." $/ \mathrm{kg}$

$3 \mathrm{~kg}$ 1,4-dichlorobenzene eq." $/ \mathrm{kg}$

$1.2 \mathrm{~kg}$ 1,4-dichlorobenzene eq." $/ \mathrm{kg}$

$1 \mathrm{~kg}$ 1,4-dichlorobenzene eq." $/ \mathrm{kg}$

860 kg 1,4-dichlorobenzene eq." $/ \mathrm{kg}$

$5200 \mathrm{~kg}$ 1,4-dichlorobenzene eq." $/ \mathrm{kg}$

$1.70 \mathrm{E}+08 \mathrm{~kg}$ 1,4-dichlorobenzene eq. "/kg

17 kg 1,4-dichlorobenzene eq." $/ \mathrm{kg}$

1600 kg 1,4-dichlorobenzene eq." $/ \mathrm{kg}$

$290 \mathrm{~kg}$ 1,4-dichlorobenzene eq." $/ \mathrm{kg}$

$400 \mathrm{~kg}$ 1,4-dichlorobenzene eq." $/ \mathrm{kg}$

$170 \mathrm{~kg}$ 1,4-dichlorobenzene eq." $/ \mathrm{kg}$

1600 kg 1,4-dichlorobenzene eq." $/ \mathrm{kg}$

19000 kg 1,4-dichlorobenzene eq."/kg

2500 kg 1,4-dichlorobenzene eq." $/ \mathrm{kg}$

$3100 \mathrm{~kg}$ 1,4-dichlorobenzene eq."/kg

1100 kg 1,4-dichlorobenzene eq." $/ \mathrm{kg}$

2.50E+05 kg 1,4-dichlorobenzene eq." $/ \mathrm{kg}$

$79 \mathrm{~kg}$ 1,4-dichlorobenzene eq." $/ \mathrm{kg}$

$4.40 \mathrm{E}+05 \mathrm{~kg} 1.4$-dichlorobenzene eq." $/ \mathrm{kg}$

12000 kg 1,4-dichlorobenzene eq." $/ \mathrm{kg}$

$1100 \mathrm{~kg}$ 1,4-dichlorobenzene eq." $/ \mathrm{kg}$

57000 kg 1,4-dichlorobenzene eq." $/ \mathrm{kg}$

$20 \mathrm{~kg}$ 1,4-dichlorobenzene eq." $/ \mathrm{kg}$

$210 \mathrm{~kg}$ 1,4-dichlorobenzene eq." $/ \mathrm{kg}$

$5000 \mathrm{~kg}$ 1,4-dichlorobenzene eq." $/ \mathrm{kg}$

2.70E+05 kg 1,4-dichlorobenzene eq." $/ \mathrm{kg}$ 
[E786] azinphos-methyl to fresh water

[E787] barium to fresh water

[E788] benomyl to fresh water

[E789] bentazone to fresh water

[E790] benzene to fresh water

[E791] benzo[a]anthracene to fresh water

[E792] benzo[a]pyrene to fresh water

[E793] benzo[ghi]perylene to fresh water

[E794] benzo[k]fluoranthrene to fresh water

[E795] benzylchloride to fresh water

[E796] beryllium to fresh water

[E797] bifenthrin to fresh water

[E800] Butylbenzylphtalate to fresh water

[E801] cadmium to fresh water

[E802] captafol to fresh water

[E803] captan to fresh water

[E804] carbaryl to fresh water

[E805] carbendazim to fresh water

[E806] carbofuran to fresh water

[E807] carbon disulfide to fresh water

[E808] Carcinogenic PAHs to fresh water

[E810] chlordane to fresh water

[E811] chlorfenvinphos to fresh water

[E812] chloridazon to fresh water

[E814] chlorobenzene to fresh water

[E815] chlorothalonil to fresh water

[E816] chlorpropham to fresh water

[E817] chlorpyriphos to fresh water

[E818] chromium III to fresh water

[E819] chromium VI to fresh water

[E820] chrysene to fresh water

[E821] cobalt to fresh water

[E822] copper to fresh water

[E823] coumaphos to fresh water

[E824] cyanazine to fresh water

[E825] cypermethrin to fresh water

[E826] cyromazine to fresh water

[E827] DDT to fresh water

[E828] deltamethrin to fresh water

[E829] demeton to fresh water

[E830] desmetryn to fresh water

[E831] Di(2-ethylhexyl)phtalate to fresh water

[E832] diazinon to fresh water

[E833] Dibutylphtalate to fresh water

[E834] Dichloromethane (Methylene Chloride) to fresh water

[E835] dichlorprop to fresh water

[E836] dichlorvos to fresh water

[E837] dieldrin to fresh water

[E838] Diethylphtalate to fresh water

[E839] Dihexylphtalate to fresh water

[E840] Diisodecylphtalate to fresh water

[E841] Diisooctylphtalate to fresh water

[E842] dimethoate to fresh water

[E843] Dimethylphtalate to fresh water

[E844] dinoseb to fresh water

[E845] dinoterb to fresh water

[E846] Dioctylphtalate to fresh water

[E847] disulfothon to fresh water

[E848] diuron to fresh water

[E849] DNOC to fresh water

[E850] endosulfan to fresh water

[E851] endrin to fresh water

[E852] ethoprophos to fresh water

[E853] ethylbenzene to fresh water

[E854] ethylene to fresh water

[E855] fenitrothion to fresh water

[E856] fenthion to fresh water
52000 kg 1,4-dichlorobenzene eq." $/ \mathrm{kg}$

$230 \mathrm{~kg}$ 1,4-dichlorobenzene eq." $/ \mathrm{kg}$

$6800 \mathrm{~kg} \mathrm{1,4-dichlorobenzene} \mathrm{eq."} / \mathrm{kg}$

$51 \mathrm{~kg}$ 1,4-dichlorobenzene eq." $/ \mathrm{kg}$

$0.091 \mathrm{~kg}$ 1,4-dichlorobenzene eq." $/ \mathrm{kg}$

1.10E+05 kg 1,4-dichlorobenzene eq."/kg

$2.50 \mathrm{E}+05 \mathrm{~kg}$ 1,4-dichlorobenzene eq." $/ \mathrm{kg}$

$52000 \mathrm{~kg}$ 1,4-dichlorobenzene eq."/kg

1.20E+06 kg 1,4-dichlorobenzene eq." $/ \mathrm{kg}$

200 kg 1,4-dichlorobenzene eq."/kg

$91000 \mathrm{~kg}$ 1,4-dichlorobenzene eq."/kg

2.40E+05 kg 1,4-dichlorobenzene eq." $/ \mathrm{kg}$

$76 \mathrm{~kg}$ 1,4-dichlorobenzene eq." $/ \mathrm{kg}$

$1500 \mathrm{~kg}$ 1,4-dichlorobenzene eq." $/ \mathrm{kg}$

5.40E+05 kg 1,4-dichlorobenzene eq." $/ \mathrm{kg}$

$2100 \mathrm{~kg}$ 1,4-dichlorobenzene eq." $/ \mathrm{kg}$

$4500 \mathrm{~kg}$ 1,4-dichlorobenzene eq." $/ \mathrm{kg}$

38000 kg 1,4-dichlorobenzene eq." $/ \mathrm{kg}$

$13000 \mathrm{~kg}$ 1,4-dichlorobenzene eq."/kg

$110 \mathrm{~kg}$ 1,4-dichlorobenzene eq." $/ \mathrm{kg}$

28000 kg 1,4-dichlorobenzene eq." /kg

$90000 \mathrm{~kg}$ 1,4-dichlorobenzene eq."/kg

$1100 \mathrm{~kg}$ 1,4-dichlorobenzene eq." $/ \mathrm{kg}$

$31 \mathrm{~kg}$ 1,4-dichlorobenzene eq." $/ \mathrm{kg}$

$0.36 \mathrm{~kg}$ 1,4-dichlorobenzene eq." $/ \mathrm{kg}$

370 kg 1,4-dichlorobenzene eq."/kg

$83 \mathrm{~kg}$ 1,4-dichlorobenzene eq." $/ \mathrm{kg}$

6.40E+05 kg 1,4-dichlorobenzene eq." $/ \mathrm{kg}$

$6.9 \mathrm{~kg}$ 1,4-dichlorobenzene eq." $/ \mathrm{kg}$

$28 \mathrm{~kg}$ 1,4-dichlorobenzene eq." $/ \mathrm{kg}$

19000 kg 1,4-dichlorobenzene eq." $/ \mathrm{kg}$

$3400 \mathrm{~kg}$ 1,4-dichlorobenzene eq." $/ \mathrm{kg}$

1200 kg 1,4-dichlorobenzene eq."/kg

$2.00 \mathrm{E}+07 \mathrm{~kg}$ 1,4-dichlorobenzene eq." $/ \mathrm{kg}$

$54000 \mathrm{~kg}$ 1,4-dichlorobenzene eq." $/ \mathrm{kg}$

7.90E+06 kg 1,4-dichlorobenzene eq." $/ \mathrm{kg}$

26000 kg 1,4-dichlorobenzene eq." $/ \mathrm{kg}$

$29000 \mathrm{~kg}$ 1,4-dichlorobenzene eq."/kg

6.50E+05 kg 1,4-dichlorobenzene eq." $/ \mathrm{kg}$

22000 kg 1,4-dichlorobenzene eq."/kg

$190 \mathrm{~kg}$ 1,4-dichlorobenzene eq."/kg

79 kg 1,4-dichlorobenzene eq." $/ \mathrm{kg}$

1.10E+05 kg 1,4-dichlorobenzene eq." $/ \mathrm{kg}$

$79 \mathrm{~kg}$ 1,4-dichlorobenzene eq." $/ \mathrm{kg}$

$0.012 \mathrm{~kg}$ 1,4-dichlorobenzene eq." $/ \mathrm{kg}$

$5.3 \mathrm{~kg}$ 1,4-dichlorobenzene eq." $/ \mathrm{kg}$

1.20E+05 kg 1,4-dichlorobenzene eq." $/ \mathrm{kg}$

79000 kg 1,4-dichlorobenzene eq."/kg

$34 \mathrm{~kg}$ 1,4-dichlorobenzene eq." $/ \mathrm{kg}$

$110 \mathrm{~kg}$ 1,4-dichlorobenzene eq." $/ \mathrm{kg}$

86 kg 1,4-dichlorobenzene eq." $/ \mathrm{kg}$

$21 \mathrm{~kg}$ 1,4-dichlorobenzene eq." $/ \mathrm{kg}$

$170 \mathrm{~kg}$ 1,4-dichlorobenzene eq."//kg

$3.1 \mathrm{~kg}$ 1,4-dichlorobenzene eq." $/ \mathrm{kg}$

3.20E+05 kg 1,4-dichlorobenzene eq." $/ \mathrm{kg}$

$2.30 \mathrm{E}+05 \mathrm{~kg}$ 1,4-dichlorobenzene eq."/kg

$2.8 \mathrm{~kg}$ 1,4-dichlorobenzene eq." $/ \mathrm{kg}$

$64000 \mathrm{~kg}$ 1,4-dichlorobenzene eq."/kg

64000 kg 1,4-dichlorobenzene eq."/kg

$110 \mathrm{~kg}$ 1,4-dichlorobenzene eq." $/ \mathrm{kg}$

$28000 \mathrm{~kg}$ 1,4-dichlorobenzene eq."/kg

7.00E+05 kg 1,4-dichlorobenzene eq."/kg

$1.50 \mathrm{E}+05 \mathrm{~kg}$ 1,4-dichlorobenzene eq." $/ \mathrm{kg}$

$0.55 \mathrm{~kg}$ 1,4-dichlorobenzene eq." $/ \mathrm{kg}$

$0.022 \mathrm{~kg}$ 1,4-dichlorobenzene eq. "/kg

$2.40 \mathrm{E}+05 \mathrm{~kg}$ 1,4-dichlorobenzene eq." $/ \mathrm{kg}$

$9.10 \mathrm{E}+05 \mathrm{~kg}$ 1,4-dichlorobenzene eq." $/ \mathrm{kg}$ 
[E857] fentin acetate to fresh water [E858] fentin chloride to fresh water [E859] fentin hydroxide to fresh water [E860] fluoranthrene to fresh water [E861] folpet to fresh water

[E862] Formaldehyde (methanal) to fresh water

[E863] glyphosate to fresh water

[E864] heptachlor to fresh water

[E865] heptenophos to fresh water

[E866] hexachloro-1,3-butadiene to fresh water

[E867] hexachlorobenzene to fresh water

[E870] indeno[1,2,3-cd]pyrene to fresh water

[E871] iprodione to fresh water

[E872] isoproturon to fresh water

[E873] lead to fresh water

[E874] lindane to fresh water

[E875] linuron to fresh water

[E876] malathion to fresh water

[E878] MCPA to fresh water

[E879] mecoprop to fresh water

[E880] mercury to fresh water

[E881] metamitron to fresh water

[E882] metazachlor to fresh water

[E883] methabenzthiazuron to fresh water

[E884] methomyl to fresh water

[E885] methylbromide to fresh water

[E886] methyl-mercury to fresh water

[E887] metobromuron to fresh water

[E888] metolachlor to fresh water

[E889] mevinphos to fresh water

[E890] molybdenum to fresh water

[E891] m-xylene to fresh water

[E892] naphtalene to fresh water

[E893] nickel to fresh water

[E899] oxamyl to fresh water

[E900] oxydemethon-methyl to fresh water

[E901] o-xylene to fresh water

[E902] parathion-ethyl to fresh water

[E903] parathion-methyl to fresh water

[E904] pentachlorobenzene to fresh water

[E905] pentachloronitrobenzene to fresh water

[E906] pentachlorophenol to fresh water

[E907] permethrin to fresh water

[E908] phenanthrene to fresh water

[E909] phenol to fresh water

[E912] phoxim to fresh water

[E913] Phtalic anhydride to fresh water

[E914] pirimicarb to fresh water

[E916] propachlor to fresh water

[E917] propoxur to fresh water

[E918] propylene oxide to fresh water

[E919] p-xylene to fresh water

[E920] pyrazophos to fresh water

[E921] selenium to fresh water

[E922] simazine to fresh water

[E923] styrene (vinylbenzene) to fresh water

[E926] tetrachloroethylene (PER) to fresh water

[E927] Tetrachloromethane (carbon tetrachloride) $(\mathrm{HC}-10)$ to fresh water

[E928] thallium to fresh water

[E929] Thiram to fresh water

[E930] tin to fresh water

[E931] tolclophos-methyl to fresh water

[E932] toluene to fresh water

[E933] tri-allate to fresh water

[E934] triazophos to fresh water

[E935] tributyltinoxide to fresh water

[E936] trichlorfon to fresh water
2.70E+05 kg 1,4-dichlorobenzene eq." $/ \mathrm{kg}$ 1.70E+05 kg 1,4-dichlorobenzene eq."/kg

2.70E+05 kg 1,4-dichlorobenzene eq."/kg

13000 kg 1,4-dichlorobenzene eq."/kg

82000 kg 1,4-dichlorobenzene eq."/kg

280 kg 1,4-dichlorobenzene eq."/kg

1400 kg 1,4-dichlorobenzene eq."/kg

18000 kg 1,4-dichlorobenzene eq."/kg

22000 kg 1,4-dichlorobenzene eq."/kg

45000 kg 1,4-dichlorobenzene eq."/kg

$150 \mathrm{~kg} \mathrm{1,4-dichlorobenzene} \mathrm{eq."/kg}$

77000 kg 1,4-dichlorobenzene eq."/kg

160 kg 1,4-dichlorobenzene eq."/kg

1900 kg 1,4-dichlorobenzene eq." $/ \mathrm{kg}$

$9.6 \mathrm{~kg}$ 1,4-dichlorobenzene eq. "/kg

6500 kg 1,4-dichlorobenzene eq."/kg

31000 kg 1,4-dichlorobenzene eq."/kg

2.10E+05 kg 1,4-dichlorobenzene eq."/kg

27 kg 1,4-dichlorobenzene eq." $/ \mathrm{kg}$

380 kg 1,4-dichlorobenzene eq." $/ \mathrm{kg}$

1700 kg 1,4-dichlorobenzene eq." $/ \mathrm{kg}$

23 kg 1,4-dichlorobenzene eq."/kg

150 kg 1,4-dichlorobenzene eq. "/kg

$1100 \mathrm{~kg} \mathrm{1,4-dichlorobenzene} \mathrm{eq."/kg}$

1.40E+05 kg 1,4-dichlorobenzene eq."/kg

19 kg 1,4-dichlorobenzene eq."/kg

39000 kg 1,4-dichlorobenzene eq."/kg

430 kg 1,4-dichlorobenzene eq."/kg

38000 kg 1,4-dichlorobenzene eq." $/ \mathrm{kg}$

5.90E+05 kg 1,4-dichlorobenzene eq."/kg

480 kg 1,4-dichlorobenzene eq." $/ \mathrm{kg}$

0.06 kg 1,4-dichlorobenzene eq." $/ \mathrm{kg}$

660 kg 1,4-dichlorobenzene eq."/kg

3200 kg 1,4-dichlorobenzene eq." $/ \mathrm{kg}$

650 kg 1,4-dichlorobenzene eq."/kg

70000 kg 1,4-dichlorobenzene eq."/kg

$0.56 \mathrm{~kg}$ 1,4-dichlorobenzene eq. " $/ \mathrm{kg}$

1.20E+06 kg 1,4-dichlorobenzene eq."/kg

2.90E+05 kg 1,4-dichlorobenzene eq." $/ \mathrm{kg}$

$51 \mathrm{~kg}$ 1,4-dichlorobenzene eq." $/ \mathrm{kg}$

4000 kg 1,4-dichlorobenzene eq."/kg

$710 \mathrm{~kg} \mathrm{1,4-dichlorobenzene} \mathrm{eq."} / \mathrm{kg}$

5.00E+06 kg 1,4-dichlorobenzene eq." $/ \mathrm{kg}$

520 kg 1,4-dichlorobenzene eq. "/kg

240 kg 1,4-dichlorobenzene eq." $/ \mathrm{kg}$

$2600 \mathrm{~kg} \mathrm{1,4-dichlorobenzene} \mathrm{eq.} \mathrm{"/kg}$

$0.55 \mathrm{~kg} \mathrm{1,4-dichlorobenzene} \mathrm{eq."} / \mathrm{kg}$

36000 kg 1,4-dichlorobenzene eq."/kg

1200 kg 1,4-dichlorobenzene eq." $/ \mathrm{kg}$

2.60E+05 kg 1,4-dichlorobenzene eq." $/ \mathrm{kg}$

4 kg 1,4-dichlorobenzene eq."/kg

0.55 kg 1,4-dichlorobenzene eq. " $/ \mathrm{kg}$

49000 kg 1,4-dichlorobenzene eq."/kg

2900 kg 1,4-dichlorobenzene eq."/kg

27000 kg 1,4-dichlorobenzene eq."/kg

0.44 kg 1,4-dichlorobenzene eq." $/ \mathrm{kg}$

$0.7 \mathrm{~kg} \mathrm{1,4-dichlorobenzene} \mathrm{eq."} / \mathrm{kg}$

$0.21 \mathrm{~kg} \mathrm{1,4-dichlorobenzene} \mathrm{eq."} / \mathrm{kg}$

8000 kg 1,4-dichlorobenzene eq." $/ \mathrm{kg}$

98000 kg 1,4-dichlorobenzene eq." $/ \mathrm{kg}$

$10 \mathrm{~kg} \mathrm{1,4-dichlorobenzene} \mathrm{eq."} / \mathrm{kg}$

$500 \mathrm{~kg} \mathrm{1,4-dichlorobenzene} \mathrm{eq."} / \mathrm{kg}$

0.29 kg 1,4-dichlorobenzene eq." $/ \mathrm{kg}$

49000 kg 1,4-dichlorobenzene eq."/kg

1.70E+05 kg 1,4-dichlorobenzene eq." $/ \mathrm{kg}$

4.50E+05 kg 1,4-dichlorobenzene eq." $/ \mathrm{kg}$

4.10E+05 kg 1,4-dichlorobenzene eq."/kg 
[E937] Trichloroethylene (tri) to fresh water

[E938] Trichloromethane $=$ chloroform to fresh water

[E939] trifluarin to fresh water

[E940] vanadium to fresh water

[E941] Vinyl Chloride (chloroethene) to fresh water

[E942] zinc to fresh water

[E943] zineb to fresh water

[E944] 1,1,1-trichloroethane to fresh water

[E945] 1,2,3,4-tetrachlorobenzene to fresh water

[E946] 1,2,3,5-tetrachlorobenzene to fresh water

[E947] 1,2,3-trichlorobenzene to fresh water

[E948] 1,2,4,5-tetrachlorobenzene to fresh water

[E949] 1,2,4-trichlorobenzene to fresh water

[E950] 1,2-dichlorobenzene to fresh water

[E951] 1,2-dichloroethane to fresh water

[E952] 1,3,5-trichlorobenzene to fresh water

[E953] 1,3-butadiene to fresh water

[E954] 1,3-dichlorobenzene to fresh water

[E955] 1,4-dichlorobenzene to fresh water

[E956] 1-chloro-4-nitrobenzene to fresh water

[E957] 2,3,4,6-tetrachlorophenol to fresh water

[E958] 2,3,7,8-TCDD to fresh water

[E959] 2,4,5-T to fresh water

[E960] 2,4,5-trichlorophenol to fresh water

[E961] 2,4,6-trichlorophenol to sea water

[E962] 2,4-D to sea water

[E963] 2,4-dichlorophenol to sea water

[E964] 2-chlorophenol to sea water

[E965] 3,4-dichloroaniline to sea water

[E966] 3-chloroaniline to sea water

[E967] 4-chloroaniline to sea water

[E968] acephate to sea water

[E969] Acrolein (2-propenal) to sea water

[E970] acrylonitrile to sea water

[E971] aldicarb to sea water

[E972] aldrin to sea water

[E974] anilazine to sea water

[E975] anthracene to sea water

[E976] antimony to sea water

[E977] arsenic to sea water

[E978] atrazine to sea water

[E979] azinphos-ethyl to sea water

[E980] azinphos-methyl to sea water

[E981] barium to sea water

[E982] benomyl to sea water

[E983] bentazone to sea water

[E984] benzene to sea water

[E985] benzo[a]anthracene to sea water

[E986] benzo[a]pyrene to sea water

[E987] benzo[ghi]perylene to sea water

[E988] benzo[k]fluoranthrene to sea water

[E989] benzylchloride to sea water

[E990] beryllium to sea water

[E991] bifenthrin to sea water

[E994] Butylbenzylphtalate to sea water

[E995] cadmium to sea water

[E996] captafol to sea water

[E997] captan to sea water

[E998] carbaryl to sea water

[E999] carbendazim to sea water

[E1000] carbofuran to sea water

[E1001] carbon disulfide to sea water

[E1002] Carcinogenic PAHs to sea water

[E1004] chlordane to sea water

[E1005] chlorfenvinphos to sea water

[E1006] chloridazon to sea water

[E1008] chlorobenzene to sea water
$0.097 \mathrm{~kg}$ 1,4-dichlorobenzene eq." $/ \mathrm{kg}$

$0.042 \mathrm{~kg}$ 1,4-dichlorobenzene eq."/kg

$27000 \mathrm{~kg}$ 1,4-dichlorobenzene eq." $/ \mathrm{kg}$

$9000 \mathrm{~kg}$ 1,4-dichlorobenzene eq." $/ \mathrm{kg}$

$0.028 \mathrm{~kg}$ 1,4-dichlorobenzene eq." $/ \mathrm{kg}$

$92 \mathrm{~kg}$ 1,4-dichlorobenzene eq." $/ \mathrm{kg}$

28000 kg 1,4-dichlorobenzene eq."/kg

7.20E-05 kg 1,4-dichlorobenzene eq."/kg

$0.038 \mathrm{~kg}$ 1,4-dichlorobenzene eq."/kg

$0.03 \mathrm{~kg}$ 1,4-dichlorobenzene eq." $/ \mathrm{kg}$

$0.0039 \mathrm{~kg} 1,4$-dichlorobenzene eq." $/ \mathrm{kg}$

$0.029 \mathrm{~kg}$ 1,4-dichlorobenzene eq." $/ \mathrm{kg}$

$0.0044 \mathrm{~kg} 1,4$-dichlorobenzene eq." $/ \mathrm{kg}$

$0.0013 \mathrm{~kg}$ 1,4-dichlorobenzene eq." $/ \mathrm{kg}$

8.80E-05 kg 1,4-dichlorobenzene eq."/kg

$0.007 \mathrm{~kg} 1,4$-dichlorobenzene eq. "/kg

5.60E-08 kg 1,4-dichlorobenzene eq. "/kg

$0.0011 \mathrm{~kg} 1,4$-dichlorobenzene eq." $/ \mathrm{kg}$

$0.0011 \mathrm{~kg}$ 1,4-dichlorobenzene eq." $/ \mathrm{kg}$

$1.9 \mathrm{~kg}$ 1,4-dichlorobenzene eq."/kg

$0.0013 \mathrm{~kg}$ 1,4-dichlorobenzene eq." $/ \mathrm{kg}$

$1.30 \mathrm{E}+05 \mathrm{~kg}$ 1,4-dichlorobenzene eq." $/ \mathrm{kg}$

1.70E-10 kg 1,4-dichlorobenzene eq."/kg

$0.054 \mathrm{~kg}$ 1,4-dichlorobenzene eq." $/ \mathrm{kg}$

$0.00024 \mathrm{~kg}$ 1,4-dichlorobenzene eq." $/ \mathrm{kg}$

1.10E-10 kg 1,4-dichlorobenzene eq."/kg

0.00029 kg 1,4-dichlorobenzene eq." $/ \mathrm{kg}$

$0.0067 \mathrm{~kg}$ 1,4-dichlorobenzene eq." $/ \mathrm{kg}$

$0.0012 \mathrm{~kg}$ 1,4-dichlorobenzene eq." $/ \mathrm{kg}$

3.70E-06 kg 1,4-dichlorobenzene eq."/kg

$0.011 \mathrm{~kg}$ 1,4-dichlorobenzene eq." $/ \mathrm{kg}$

6.00E-08 kg 1,4-dichlorobenzene eq." $/ \mathrm{kg}$

$5 \mathrm{~kg}$ 1,4-dichlorobenzene eq." $/ \mathrm{kg}$

$0.006 \mathrm{~kg}$ 1,4-dichlorobenzene eq." $/ \mathrm{kg}$

$0.12 \mathrm{~kg}$ 1,4-dichlorobenzene eq." $/ \mathrm{kg}$

$1.3 \mathrm{~kg}$ 1,4-dichlorobenzene eq." $/ \mathrm{kg}$

1.10E-07 kg 1,4-dichlorobenzene eq." $/ \mathrm{kg}$

$17 \mathrm{~kg}$ 1,4-dichlorobenzene eq." $/ \mathrm{kg}$

7.60E-21 kg 1,4-dichlorobenzene eq."/kg

3.80E-20 kg 1,4-dichlorobenzene eq." $/ \mathrm{kg}$

$0.0083 \mathrm{~kg}$ 1,4-dichlorobenzene eq." $/ \mathrm{kg}$

$0.041 \mathrm{~kg}$ 1,4-dichlorobenzene eq."/ $/ \mathrm{kg}$

$0.00011 \mathrm{~kg}$ 1,4-dichlorobenzene eq." $/ \mathrm{kg}$

2.40E-19 kg 1,4-dichlorobenzene eq."/kg

8.90E-08 kg 1,4-dichlorobenzene eq." $/ \mathrm{kg}$

7.40E-09 kg 1,4-dichlorobenzene eq." $/ \mathrm{kg}$

9.20E-06 kg 1,4-dichlorobenzene eq." $/ \mathrm{kg}$

$1.1 \mathrm{~kg}$ 1,4-dichlorobenzene eq." $/ \mathrm{kg}$

$0.28 \mathrm{~kg}$ 1,4-dichlorobenzene eq." $/ \mathrm{kg}$

$0.049 \mathrm{~kg} 1,4$-dichlorobenzene eq." $/ \mathrm{kg}$

$9.1 \mathrm{~kg}$ 1,4-dichlorobenzene eq." $/ \mathrm{kg}$

$0.011 \mathrm{~kg}$ 1,4-dichlorobenzene eq." $/ \mathrm{kg}$

1.60E-16 kg 1,4-dichlorobenzene eq." $/ \mathrm{kg}$

$0.055 \mathrm{~kg} 1,4$-dichlorobenzene eq." $/ \mathrm{kg}$

3.20E-05 kg 1,4-dichlorobenzene eq." $/ \mathrm{kg}$

$2.50 \mathrm{E}-20 \mathrm{~kg}$ 1,4-dichlorobenzene eq." $/ \mathrm{kg}$

5.00E-05 kg 1,4-dichlorobenzene eq."/kg

6.50E-07 kg 1,4-dichlorobenzene eq." $/ \mathrm{kg}$

$1.90 \mathrm{E}-06 \mathrm{~kg}$ 1,4-dichlorobenzene eq." $/ \mathrm{kg}$

2.40E-08 kg 1,4-dichlorobenzene eq."/kg

$0.00018 \mathrm{~kg} 1,4$-dichlorobenzene eq." $/ \mathrm{kg}$

$0.0065 \mathrm{~kg} \mathrm{1,4-dichlorobenzene} \mathrm{eq."} / \mathrm{kg}$

$0.12 \mathrm{~kg}$ 1,4-dichlorobenzene eq." $/ \mathrm{kg}$

$31 \mathrm{~kg}$ 1,4-dichlorobenzene eq." $/ \mathrm{kg}$

5.60E-05 kg 1,4-dichlorobenzene eq." $/ \mathrm{kg}$

$0.0035 \mathrm{~kg}$ 1,4-dichlorobenzene eq." $/ \mathrm{kg}$

$0.00026 \mathrm{~kg}$ 1,4-dichlorobenzene eq."/kg 
[E1009] chlorothalonil to sea water [E1010] chlorpropham to sea water [E1011] chlorpyriphos to sea water [E1012] chromium III to sea water [E1013] chromium VI to sea water [E1014] chrysene to sea water [E1015] cobalt to sea water [E1016] copper to sea water [E1017] coumaphos to sea water [E1018] cyanazine to sea water [E1019] cypermethrin to sea water [E1020] cyromazine to sea water [E1021] DDT to sea water

[E1022] deltamethrin to sea water

[E1023] demeton to sea water

[E1024] desmetryn to sea water

[E1025] Di(2-ethylhexyl)phtalate to sea water

[E1026] diazinon to sea water

[E1027] Dibutylphtalate to sea water

[E1028] Dichloromethane (Methylene Chloride) to sea water

[E1029] dichlorprop to sea water

[E1030] dichlorvos to sea water

[E1031] dieldrin to sea water

[E1032] Diethylphtalate to sea water

[E1033] Dihexylphtalate to sea water

[E1034] Diisodecylphtalate to sea water

[E1035] Diisooctylphtalate to sea water

[E1036] dimethoate to sea water

[E1037] Dimethylphtalate to sea water

[E1038] dinoseb to sea water

[E1039] dinoterb to sea water

[E1040] Dioctylphtalate to sea water

[E1041] disulfothon to sea water

[E1042] diuron to sea water

[E1043] DNOC to sea water

[E1044] endosulfan to sea water

[E1045] endrin to sea water

[E1046] ethoprophos to sea water

[E1047] ethylbenzene to sea water

[E1048] ethylene to sea water

[E1049] fenitrothion to sea water

[E1050] fenthion to sea water

[E1051] fentin acetate to sea water

[E1052] fentin chloride to sea water

[E1053] fentin hydroxide to sea water

[E1054] fluoranthrene to sea water

[E1055] folpet to sea water

[E1056] Formaldehyde (methanal) to sea water

[E1057] glyphosate to sea water

[E1058] heptachlor to sea water

[E1059] heptenophos to sea water

[E1060] hexachloro-1,3-butadiene to sea water

[E1061] hexachlorobenzene to sea water

[E1064] indeno[1,2,3-cd]pyrene to sea water

[E1065] iprodione to sea water

[E1066] isoproturon to sea water

[E1067] lead to sea water

[E1068] lindane to sea water

[E1069] linuron to sea water

[E1070] malathion to sea water

[E1072] MCPA to sea water

[E1073] mecoprop to sea water

[E1074] mercury to sea water

[E1075] metamitron to sea water

[E1076] metazachlor to sea water

[E1077] methabenzthiazuron to sea water

[E1078] methomyl to sea water
$0.14 \mathrm{~kg}$ 1,4-dichlorobenzene eq. "/kg 2.80E-05 kg 1,4-dichlorobenzene eq." $/ \mathrm{kg}$

$0.23 \mathrm{~kg}$ 1,4-dichlorobenzene eq." $/ \mathrm{kg}$

8.80E-23 kg 1,4-dichlorobenzene eq." $/ \mathrm{kg}$ 3.50E-22 kg 1,4-dichlorobenzene eq." $/ \mathrm{kg}$

$0.26 \mathrm{~kg}$ 1,4-dichlorobenzene eq." $/ \mathrm{kg}$

1.20E-18 kg 1,4-dichlorobenzene eq." $/ \mathrm{kg}$

4.10E-20 kg 1,4-dichlorobenzene eq."/kg

$110 \mathrm{~kg}$ 1,4-dichlorobenzene eq. "/kg

2.50E-06 kg 1,4-dichlorobenzene eq." $/ \mathrm{kg}$

$2.4 \mathrm{~kg}$ 1,4-dichlorobenzene eq." $/ \mathrm{kg}$

8.10E-07 kg 1,4-dichlorobenzene eq." $/ \mathrm{kg}$

15 kg 1,4-dichlorobenzene eq." $/ \mathrm{kg}$

$3.2 \mathrm{~kg}$ 1,4-dichlorobenzene eq. $" / \mathrm{kg}$

$0.017 \mathrm{~kg} 1,4$-dichlorobenzene eq." $/ \mathrm{kg}$

4.10E-06 kg 1,4-dichlorobenzene eq." $/ \mathrm{kg}$

0.0016 kg 1,4-dichlorobenzene eq." $/ \mathrm{kg}$

$0.064 \mathrm{~kg} 1,4$-dichlorobenzene eq." $/ \mathrm{kg}$

2.90E-05 kg 1,4-dichlorobenzene eq." $/ \mathrm{kg}$

5.00E-06 kg 1,4-dichlorobenzene eq." $/ \mathrm{kg}$

1.60E-12 kg 1,4-dichlorobenzene eq." $/ \mathrm{kg}$

$0.011 \mathrm{~kg} 1,4$-dichlorobenzene eq."/ $/ \mathrm{kg}$

16 kg 1,4-dichlorobenzene eq." $/ \mathrm{kg}$

7.90E-05 kg 1,4-dichlorobenzene eq." $/ \mathrm{kg}$

$0.011 \mathrm{~kg} 1,4$-dichlorobenzene eq." $/ \mathrm{kg}$

$0.038 \mathrm{~kg} 1,4$-dichlorobenzene eq." $/ \mathrm{kg}$

$0.0039 \mathrm{~kg}$ 1,4-dichlorobenzene eq." $/ \mathrm{kg}$

7.40E-06 kg 1,4-dichlorobenzene eq."/kg

3.80E-07 kg 1,4-dichlorobenzene eq." $/ \mathrm{kg}$

$0.11 \mathrm{~kg}$ 1,4-dichlorobenzene eq." $/ \mathrm{kg}$

$0.042 \mathrm{~kg}$ 1,4-dichlorobenzene eq." $/ \mathrm{kg}$

$0.00014 \mathrm{~kg}$ 1,4-dichlorobenzene eq." $/ \mathrm{kg}$

$0.013 \mathrm{~kg}$ 1,4-dichlorobenzene eq." $/ \mathrm{kg}$

$0.013 \mathrm{~kg}$ 1,4-dichlorobenzene eq." $/ \mathrm{kg}$

2.10E-08 kg 1,4-dichlorobenzene eq." $/ \mathrm{kg}$

$0.021 \mathrm{~kg} \mathrm{1,4-dichlorobenzene} \mathrm{eq."} / \mathrm{kg}$

$6.1 \mathrm{~kg}$ 1,4-dichlorobenzene eq." $/ \mathrm{kg}$

$1 \mathrm{~kg}$ 1,4-dichlorobenzene eq." $/ \mathrm{kg}$

9.40E-06 kg 1,4-dichlorobenzene eq." $/ \mathrm{kg}$

$1.00 \mathrm{E}-12 \mathrm{~kg}$ 1,4-dichlorobenzene eq." $/ \mathrm{kg}$

0.0099 kg 1,4-dichlorobenzene eq." $/ \mathrm{kg}$

$0.26 \mathrm{~kg}$ 1,4-dichlorobenzene eq." $/ \mathrm{kg}$

$0.087 \mathrm{~kg}$ 1,4-dichlorobenzene eq." $/ \mathrm{kg}$

$18 \mathrm{~kg}$ 1,4-dichlorobenzene eq." $/ \mathrm{kg}$

$0.029 \mathrm{~kg} 1,4$-dichlorobenzene eq." $/ \mathrm{kg}$

$0.87 \mathrm{~kg} 1,4$-dichlorobenzene eq. "/ $/ \mathrm{kg}$

$16 \mathrm{~kg}$ 1,4-dichlorobenzene eq." $/ \mathrm{kg}$

$0.00021 \mathrm{~kg} 1,4$-dichlorobenzene eq." $/ \mathrm{kg}$

2.10E-11 kg 1,4-dichlorobenzene eq." $/ \mathrm{kg}$

$0.039 \mathrm{~kg} 1,4$-dichlorobenzene eq." $/ \mathrm{kg}$

$0.0013 \mathrm{~kg}$ 1,4-dichlorobenzene eq." $/ \mathrm{kg}$

$23 \mathrm{~kg}$ 1,4-dichlorobenzene eq. "/ $/ \mathrm{kg}$

$1.1 \mathrm{~kg}$ 1,4-dichlorobenzene eq." $/ \mathrm{kg}$

$0.00074 \mathrm{~kg}$ 1,4-dichlorobenzene eq." $/ \mathrm{kg}$

3.80E-09 kg 1,4-dichlorobenzene eq." $/ \mathrm{kg}$

2.90E-05 kg 1,4-dichlorobenzene eq." $/ \mathrm{kg}$

5.60E-23 kg 1,4-dichlorobenzene eq." $/ \mathrm{kg}$

$0.11 \mathrm{~kg}$ 1,4-dichlorobenzene eq." $/ \mathrm{kg}$

$0.06 \mathrm{~kg} 1,4$-dichlorobenzene eq. $/ / \mathrm{kg}$

$0.018 \mathrm{~kg}$ 1,4-dichlorobenzene eq." $/ \mathrm{kg}$

5.30E-13 kg 1,4-dichlorobenzene eq." $/ \mathrm{kg}$

3.80E-10 kg 1,4-dichlorobenzene eq." $/ \mathrm{kg}$

$6.8 \mathrm{~kg} 1,4$-dichlorobenzene eq." $/ \mathrm{kg}$

6.80E-10 kg 1,4-dichlorobenzene eq." $/ \mathrm{kg}$

$3.00 \mathrm{E}-06 \mathrm{~kg}$ 1,4-dichlorobenzene eq." $/ \mathrm{kg}$

9.20E-05 kg 1,4-dichlorobenzene eq." $/ \mathrm{kg}$

0.0085 kg 1,4-dichlorobenzene eq." $/ \mathrm{kg}$ 
[E1079] methylbromide to sea water [E1080] methyl-mercury to sea water [E1081] metobromuron to sea water [E1082] metolachlor to sea water [E1083] mevinphos to sea water [E1084] molybdenum to sea water [E1085] m-xylene to sea water [E1086] naphtalene to sea water [E1087] nickel to sea water [E1093] oxamyl to sea water [E1094] oxydemethon-methyl to sea water [E1095] o-xylene to sea water [E1096] parathion-ethyl to sea water [E1097] parathion-methyl to sea water [E1098] pentachlorobenzene to sea water

[E1099] pentachloronitrobenzene to sea water

[E1100] pentachlorophenol to sea water

[E1101] permethrin to sea water

[E1102] phenanthrene to sea water

[E1103] phenol to sea water

[E1106] phoxim to sea water

[E1107] Phtalic anhydride to sea water

[E1108] pirimicarb to sea water

[E1110] propachlor to sea water

[E1111] propoxur to sea water

[E1112] propylene oxide to sea water

[E1113] p-xylene to sea water

[E1114] pyrazophos to sea water

[E1115] selenium to sea water

[E1116] simazine to sea water

[E1117] styrene (vinylbenzene) to sea water

[E1120] tetrachloroethylene (PER) to sea water

[E1121] Tetrachloromethane (carbon tetrachloride) (HC-10) to sea water

[E1122] thallium to sea water

[E1123] Thiram to sea water

[E1124] tin to sea water

[E1125] tolclophos-methyl to sea water

[E1126] toluene to sea water

[E1127] tri-allate to sea water

[E1128] triazophos to sea water

[E1129] tributyltinoxide to sea water

[E1130] trichlorfon to sea water

[E1131] Trichloroethylene (tri) to sea water

[E1132] Trichloromethane $=$ chloroform to sea water

[E1133] trifluarin to sea water

[E1134] vanadium to sea water

[E1135] Vinyl Chloride (chloroethene) to sea water

[E1136] zinc to sea water

[E1137] zineb to sea water

[E1138] 1,1,1-trichloroethane to sea water

[E1139] 1,2,3,4-tetrachlorobenzene to sea water

[E1140] 1,2,3,5-tetrachlorobenzene to sea water

[E1141] 1,2,3-trichlorobenzene to sea water

[E1142] 1,2,4,5-tetrachlorobenzene to sea water

[E1143] 1,2,4-trichlorobenzene to sea water

[E1144] 1,2-dichlorobenzene to sea water

[E1145] 1,2-dichloroethane to sea water

[E1146] 1,3,5-trichlorobenzene to sea water

[E1147] 1,3-butadiene to sea water

[E1148] 1,3-dichlorobenzene to sea water

[E1149] 1,4-dichlorobenzene to sea water

[E1150] 1-chloro-4-nitrobenzene to sea water

[E1151] 2,3,4,6-tetrachlorophenol to sea water

[E1152] 2,3,7,8-TCDD to sea water

[E1153] 2,4,5-T to sea water

[E1154] 2,4,5-trichlorophenol to sea water

[E1155] 2,4,6-trichlorophenol to agricultural soil
0.0023 kg 1,4-dichlorobenzene eq." $/ \mathrm{kg}$ $160 \mathrm{~kg}$ 1,4-dichlorobenzene eq." $/ \mathrm{kg}$ $0.0016 \mathrm{~kg}$ 1,4-dichlorobenzene eq."//kg

$0.07 \mathrm{~kg} \mathrm{1,4-dichlorobenzene} \mathrm{eq."} / \mathrm{kg}$ 6.90E-05 kg 1,4-dichlorobenzene eq."//kg $6.60 \mathrm{E}-19 \mathrm{~kg}$ 1,4-dichlorobenzene eq." $/ \mathrm{kg}$ 7.20E-06 kg 1,4-dichlorobenzene eq."/kg $0.011 \mathrm{~kg}$ 1,4-dichlorobenzene eq." $/ \mathrm{kg}$ $6.10 \mathrm{E}-19 \mathrm{~kg}$ 1,4-dichlorobenzene eq." $/ \mathrm{kg}$ 4.50E-07 kg 1,4-dichlorobenzene eq." $/ \mathrm{kg}$ $0.0003 \mathrm{~kg}$ 1,4-dichlorobenzene eq." $/ \mathrm{kg}$ 1.50E-05 kg 1,4-dichlorobenzene eq."//kg $0.2 \mathrm{~kg}$ 1,4-dichlorobenzene eq." $/ \mathrm{kg}$ $0.12 \mathrm{~kg} \mathrm{1,4-dichlorobenzene} \mathrm{eq."} / \mathrm{kg}$ $0.24 \mathrm{~kg} 1,4$-dichlorobenzene eq." $/ \mathrm{kg}$

$11 \mathrm{~kg}$ 1,4-dichlorobenzene eq." $/ \mathrm{kg}$

1.20E-05 kg 1,4-dichlorobenzene eq."//kg

$10 \mathrm{~kg}$ 1,4-dichlorobenzene eq." $/ \mathrm{kg}$ $0.058 \mathrm{~kg} 1,4$-dichlorobenzene eq." $/ \mathrm{kg}$ 1.70E-05 kg 1,4-dichlorobenzene eq."/kg $0.033 \mathrm{~kg} \mathrm{1,4-dichlorobenzene} \mathrm{eq."} / \mathrm{kg}$ 4.60E-11 kg 1,4-dichlorobenzene eq." $/ \mathrm{kg}$ $0.00089 \mathrm{~kg} \mathrm{1,4-dichlorobenzene} \mathrm{eq.} / / \mathrm{kg}$ 0.0005 kg 1,4-dichlorobenzene eq." $/ \mathrm{kg}$ 0.00012 kg 1,4-dichlorobenzene eq." $/ \mathrm{kg}$ 0.00044 kg 1,4-dichlorobenzene eq." $/ \mathrm{kg}$ 1.00E-05 kg 1,4-dichlorobenzene eq."/kg $0.0023 \mathrm{~kg} \mathrm{1,4-dichlorobenzene} \mathrm{eq."} / \mathrm{kg}$

7.40E-18 kg 1,4-dichlorobenzene eq." $/ \mathrm{kg}$ $0.0045 \mathrm{~kg}$ 1,4-dichlorobenzene eq." $/ \mathrm{kg}$ 1.00E-05 kg 1,4-dichlorobenzene eq." $/ \mathrm{kg}$ $0.0002 \mathrm{~kg} 1,4$-dichlorobenzene eq." $/ \mathrm{kg}$ 0.00019 kg 1,4-dichlorobenzene eq." $/ \mathrm{kg}$

7.90E-18 kg 1,4-dichlorobenzene eq." $/ \mathrm{kg}$ $0.026 \mathrm{~kg}$ 1,4-dichlorobenzene eq." $/ \mathrm{kg}$

$9.50 \mathrm{E}-23 \mathrm{~kg}$ 1,4-dichlorobenzene eq. "/ $/ \mathrm{kg}$ $0.029 \mathrm{~kg}$ 1,4-dichlorobenzene eq." $/ \mathrm{kg}$ 8.30E-06 kg 1,4-dichlorobenzene eq."/kg

$1.1 \mathrm{~kg}$ 1,4-dichlorobenzene eq." $/ \mathrm{kg}$ $0.079 \mathrm{~kg} \mathrm{1,4-dichlorobenzene} \mathrm{eq."} / \mathrm{kg}$

$3 \mathrm{~kg}$ 1,4-dichlorobenzene eq." $/ \mathrm{kg}$ 5.30E-06 kg 1,4-dichlorobenzene eq."/kg 1.60E-05 kg 1,4-dichlorobenzene eq." $/ \mathrm{kg}$ 4.50E-05 kg 1,4-dichlorobenzene eq."/kg

$1.8 \mathrm{~kg}$ 1,4-dichlorobenzene eq." $/ \mathrm{kg}$

2.40E-18 kg 1,4-dichlorobenzene eq."/kg

1.40E-06 kg 1,4-dichlorobenzene eq."/kg

1.80E-21 kg 1,4-dichlorobenzene eq."/kg

$0.0036 \mathrm{~kg} 1,4$-dichlorobenzene eq." $/ \mathrm{kg}$

$0.00037 \mathrm{~kg}$ 1,4-dichlorobenzene eq." $/ \mathrm{kg}$ $0.028 \mathrm{~kg} \mathrm{1,4-dichlorobenzene} \mathrm{eq."} / \mathrm{kg}$ $0.083 \mathrm{~kg}$ 1,4-dichlorobenzene eq." $/ \mathrm{kg}$ $0.023 \mathrm{~kg} 1,4$-dichlorobenzene eq." $/ \mathrm{kg}$ $0.025 \mathrm{~kg}$ 1,4-dichlorobenzene eq." $/ \mathrm{kg}$ $0.02 \mathrm{~kg} 1,4$-dichlorobenzene eq. " $/ \mathrm{kg}$ $0.019 \mathrm{~kg}$ 1,4-dichlorobenzene eq." $/ \mathrm{kg}$

$0.00075 \mathrm{~kg}$ 1,4-dichlorobenzene eq." $/ \mathrm{kg}$ $0.054 \mathrm{~kg}$ 1,4-dichlorobenzene eq." $/ \mathrm{kg}$

5.70E-05 kg 1,4-dichlorobenzene eq." $/ \mathrm{kg}$ $0.018 \mathrm{~kg}$ 1,4-dichlorobenzene eq." $/ \mathrm{kg}$ $0.014 \mathrm{~kg}$ 1,4-dichlorobenzene eq."/kg $150 \mathrm{~kg}$ 1,4-dichlorobenzene eq." $/ \mathrm{kg}$

$32 \mathrm{~kg}$ 1,4-dichlorobenzene eq." $/ \mathrm{kg}$

1.20E+05 kg 1,4-dichlorobenzene eq." $/ \mathrm{kg}$ $0.44 \mathrm{~kg}$ 1,4-dichlorobenzene eq." $/ \mathrm{kg}$

$28 \mathrm{~kg}$ 1,4-dichlorobenzene eq." $/ \mathrm{kg}$

$1.2 \mathrm{~kg}$ 1,4-dichlorobenzene eq." $/ \mathrm{kg}$ 
[E1156] 2,4-D to agricultural soil [E1157] 2,4-dichlorophenol to agricultural soil [E1158] 2-chlorophenol to agricultural soil [E1159] 3,4-dichloroaniline to agricultural soil [E1160] 3-chloroaniline to agricultural soil [E1161] 4-chloroaniline to agricultural soil [E1162] acephate to agricultural soil

[E1163] Acrolein (2-propenal) to agricultural soil [E1164] acrylonitrile to agricultural soil

[E1165] aldicarb to agricultural soil

[E1166] aldrin to agricultural soil

[E1168] anilazine to agricultural soil

[E1169] anthracene to agricultural soil

[E1170] antimony to agricultural soil

[E1171] arsenic to agricultural soil

[E1172] atrazine to agricultural soil

[E1173] azinphos-ethyl to agricultural soil

[E1174] azinphos-methyl to agricultural soil

[E1175] barium to agricultural soil

[E1176] benomyl to agricultural soil

[E1177] bentazone to agricultural soil

[E1178] benzene to agricultural soil

[E1179] benzo[a]anthracene to agricultural soil

[E1180] benzo[a]pyrene to agricultural soil

[E1181] benzo[ghi]perylene to agricultural soil

[E1182] benzo[k]fluoranthrene to agricultural soil

[E1183] benzylchloride to agricultural soil

[E1184] beryllium to agricultural soil

[E1185] bifenthrin to agricultural soil

[E1186] Butylbenzylphtalate to agricultural soil

[E1187] cadmium to agricultural soil

[E1188] captafol to agricultural soil

[E1189] captan to agricultural soil

[E1190] carbaryl to agricultural soil

[E1191] carbendazim to agricultural soil

[E1192] carbofuran to agricultural soil

[E1193] carbon disulfide to agricultural soil

[E1194] Carcinogenic PAHs to agricultural soil

[E1195] chlordane to agricultural soil

[E1196] chlorfenvinphos to agricultural soil

[E1197] chloridazon to agricultural soil

[E1198] chlorobenzene to agricultural soil

[E1199] chlorothalonil to agricultural soil

[E1200] chlorpropham to agricultural soil

[E1201] chlorpyriphos to agricultural soil

[E1202] chromium III to agricultural soil

[E1203] chromium VI to agricultural soil

[E1204] chrysene to agricultural soil

[E1205] cobalt to agricultural soil

[E1206] copper to agricultural soil

[E1207] coumaphos to agricultural soil

[E1208] cyanazine to agricultural soil

[E1209] cypermethrin to agricultural soil

[E1210] cyromazine to agricultural soil

[E1211] DDT to agricultural soil

[E1212] deltamethrin to agricultural soil

[E1213] demeton to agricultural soil

[E1214] desmetryn to agricultural soil

[E1215] Di(2-ethylhexyl)phtalate to agricultural soil

[E1216] diazinon to agricultural soil

[E1217] Dibutylphtalate to agricultural soil

[E1218] Dichloromethane (Methylene Chloride) to agricultural soil

[E1219] dichlorprop to agricultural soil

[E1220] dichlorvos to agricultural soil

[E1221] dieldrin to agricultural soil

[E1222] Diethylphtalate to agricultural soil

[E1223] Dihexylphtalate to agricultural soil
$29 \mathrm{~kg}$ 1,4-dichlorobenzene eq." $/ \mathrm{kg}$

$2.5 \mathrm{~kg}$ 1,4-dichlorobenzene eq." $/ \mathrm{kg}$

$7.9 \mathrm{~kg}$ 1,4-dichlorobenzene eq." $/ \mathrm{kg}$

$1800 \mathrm{~kg}$ 1,4-dichlorobenzene eq." $/ \mathrm{kg}$

$74 \mathrm{~kg}$ 1,4-dichlorobenzene eq. $/ \mathrm{kg}$

$170 \mathrm{~kg}$ 1,4-dichlorobenzene eq." $/ \mathrm{kg}$

$51 \mathrm{~kg} \mathrm{1,4-dichlorobenzene} \mathrm{eq."} / \mathrm{kg}$

$45000 \mathrm{~kg}$ 1,4-dichlorobenzene eq." $/ \mathrm{kg}$

$6.5 \mathrm{~kg}$ 1,4-dichlorobenzene eq." $/ \mathrm{kg}$

$96000 \mathrm{~kg}$ 1,4-dichlorobenzene eq." $/ \mathrm{kg}$

$280 \mathrm{~kg}$ 1,4-dichlorobenzene eq." $/ \mathrm{kg}$

$0.21 \mathrm{~kg}$ 1,4-dichlorobenzene eq." $/ \mathrm{kg}$

$82 \mathrm{~kg}$ 1,4-dichlorobenzene eq."//kg

$10 \mathrm{~kg}$ 1,4-dichlorobenzene eq." $/ \mathrm{kg}$

$130 \mathrm{~kg}$ 1,4-dichlorobenzene eq." $/ \mathrm{kg}$

$340 \mathrm{~kg}$ 1,4-dichlorobenzene eq." $/ \mathrm{kg}$

$2800 \mathrm{~kg}$ 1,4-dichlorobenzene eq." $/ \mathrm{kg}$

$190 \mathrm{~kg}$ 1,4-dichlorobenzene eq." $/ \mathrm{kg}$

$110 \mathrm{~kg}$ 1,4-dichlorobenzene eq." $/ \mathrm{kg}$

$4.6 \mathrm{~kg}$ 1,4-dichlorobenzene eq."//kg

$8.3 \mathrm{~kg}$ 1,4-dichlorobenzene eq." $/ \mathrm{kg}$

$0.00072 \mathrm{~kg}$ 1,4-dichlorobenzene eq." $/ \mathrm{kg}$

$62 \mathrm{~kg}$ 1,4-dichlorobenzene eq." $/ \mathrm{kg}$

$130 \mathrm{~kg}$ 1,4-dichlorobenzene eq." $/ \mathrm{kg}$

$61 \mathrm{~kg}$ 1,4-dichlorobenzene eq." $/ \mathrm{kg}$

5200 kg 1,4-dichlorobenzene eq." $/ \mathrm{kg}$

$0.92 \mathrm{~kg}$ 1,4-dichlorobenzene eq." $/ \mathrm{kg}$

$46000 \mathrm{~kg}$ 1,4-dichlorobenzene eq." $/ \mathrm{kg}$

$100 \mathrm{~kg}$ 1,4-dichlorobenzene eq." $/ \mathrm{kg}$

$0.025 \mathrm{~kg}$ 1,4-dichlorobenzene eq." $/ \mathrm{kg}$

$780 \mathrm{~kg}$ 1,4-dichlorobenzene eq." $/ \mathrm{kg}$

27000 kg 1,4-dichlorobenzene eq." $/ \mathrm{kg}$

$0.4 \mathrm{~kg}$ 1,4-dichlorobenzene eq." $/ \mathrm{kg}$

$23 \mathrm{~kg}$ 1,4-dichlorobenzene eq." $/ \mathrm{kg}$

2000 kg 1,4-dichlorobenzene eq." $/ \mathrm{kg}$

$580 \mathrm{~kg}$ 1,4-dichlorobenzene eq." $/ \mathrm{kg}$

$0.34 \mathrm{~kg}$ 1,4-dichlorobenzene eq." $/ \mathrm{kg}$

$58 \mathrm{~kg}$ 1,4-dichlorobenzene eq." $/ \mathrm{kg}$

94 kg 1,4-dichlorobenzene eq." $/ \mathrm{kg}$

$16 \mathrm{~kg}$ 1,4-dichlorobenzene eq." $/ \mathrm{kg}$

$1.8 \mathrm{~kg}$ 1,4-dichlorobenzene eq." $/ \mathrm{kg}$

$0.0032 \mathrm{~kg} 1,4$-dichlorobenzene eq." $/ \mathrm{kg}$

$1 \mathrm{~kg}$ 1,4-dichlorobenzene eq." $/ \mathrm{kg}$

$1.8 \mathrm{~kg}$ 1,4-dichlorobenzene eq." $/ \mathrm{kg}$ $360 \mathrm{~kg}$ 1,4-dichlorobenzene eq." $/ \mathrm{kg}$

$5.3 \mathrm{~kg}$ 1,4-dichlorobenzene eq." $/ \mathrm{kg}$

$21 \mathrm{~kg} \mathrm{1,4-dichlorobenzene} \mathrm{eq."} / \mathrm{kg}$

74 kg 1,4-dichlorobenzene eq." $/ \mathrm{kg}$

17000 kg 1,4-dichlorobenzene eq." $/ \mathrm{kg}$

$590 \mathrm{~kg}$ 1,4-dichlorobenzene eq." $/ \mathrm{kg}$

$1.00 \mathrm{E}+06 \mathrm{~kg}$ 1,4-dichlorobenzene eq." $/ \mathrm{kg}$ $810 \mathrm{~kg}$ 1,4-dichlorobenzene eq." $/ \mathrm{kg}$

$2.00 E+05 \mathrm{~kg}$ 1,4-dichlorobenzene eq." $/ \mathrm{kg}$

$6500 \mathrm{~kg} \mathrm{1,4-dichlorobenzene} \mathrm{eq."} / \mathrm{kg}$

87 kg 1,4-dichlorobenzene eq."/kg

24 kg 1,4-dichlorobenzene eq." $/ \mathrm{kg}$

$800 \mathrm{~kg}$ 1,4-dichlorobenzene eq." $/ \mathrm{kg}$

$3 \mathrm{~kg}$ 1,4-dichlorobenzene eq. " $/ \mathrm{kg}$

0.0015 kg 1,4-dichlorobenzene eq." //kg $1300 \mathrm{~kg}$ 1,4-dichlorobenzene eq."/kg

$0.079 \mathrm{~kg}$ 1,4-dichlorobenzene eq." $/ \mathrm{kg}$

0.00016 kg 1,4-dichlorobenzene eq." $/ \mathrm{kg}$

$0.013 \mathrm{~kg}$ 1,4-dichlorobenzene eq." $/ \mathrm{kg}$

$74 \mathrm{~kg}$ 1,4-dichlorobenzene eq." $/ \mathrm{kg}$

$600 \mathrm{~kg}$ 1,4-dichlorobenzene eq." $/ \mathrm{kg}$

$0.16 \mathrm{~kg}$ 1,4-dichlorobenzene eq." $/ \mathrm{kg}$

$0.018 \mathrm{~kg}$ 1,4-dichlorobenzene eq." $/ \mathrm{kg}$ 
[E1224] Diisodecylphtalate to agricultural soil

[E1225] Diisooctylphtalate to agricultural soil

[E1226] dimethoate to agricultural soil

[E1227] Dimethylphtalate to agricultural soil

[E1228] dinoseb to agricultural soil

[E1229] dinoterb to agricultural soil

[E1230] Dioctylphtalate to agricultural soil

[E1231] disulfothon to agricultural soil

[E1232] diuron to agricultural soil

[E1233] DNOC to agricultural soil

[E1234] endosulfan to agricultural soil

[E1235] endrin to agricultural soil

[E1236] ethoprophos to agricultural soil

[E1237] ethylbenzene to agricultural soil

[E1238] ethylene to agricultural soil

[E1239] fenitrothion to agricultural soil

[E1240] fenthion to agricultural soil

[E1241] fentin acetate to agricultural soil

[E1242] fentin chloride to agricultural soil

[E1243] fentin hydroxide to agricultural soil

[E1244] fluoranthrene to agricultural soil

[E1245] folpet to agricultural soil

[E1246] Formaldehyde (methanal) to agricultural soil

[E1247] glyphosate to agricultural soil

[E1248] heptachlor to agricultural soil

[E1249] heptenophos to agricultural soil

[E1250] hexachloro-1,3-butadiene to agricultural soil

[E1251] hexachlorobenzene to agricultural soil

[E1254] indeno[1,2,3-cd]pyrene to agricultural soil

[E1255] iprodione to agricultural soil

[E1256] isoproturon to agricultural soil

[E1257] lead to agricultural soil

[E1258] lindane to agricultural soil

[E1259] linuron to agricultural soil

[E1260] malathion to agricultural soil

[E1261] MCPA to agricultural soil

[E1262] mecoprop to agricultural soil

[E1263] mercury to agricultural soil

[E1264] metamitron to agricultural soil

[E1265] metazachlor to agricultural soil

[E1266] methabenzthiazuron to agricultural soil

[E1267] methomyl to agricultural soil

[E1268] methylbromide to agricultural soil

[E1269] methyl-mercury to agricultural soil

[E1270] metobromuron to agricultural soil

[E1271] metolachlor to agricultural soil

[E1272] mevinphos to agricultural soil

[E1273] molybdenum to agricultural soi

[E1274] m-xylene to agricultural soil

[E1275] naphtalene to agricultural soil

[E1276] nickel to agricultural soil

[E1278] oxamyl to agricultural soil

[E1279] oxydemethon-methyl to agricultural soil

[E1280] o-xylene to agricultural soil

[E1281] parathion-ethyl to agricultural soil

[E1282] parathion-methyl to agricultural soil

[E1283] pentachlorobenzene to agricultural soil

[E1284] pentachloronitrobenzene to agricultural soil

[E1285] pentachlorophenol to agricultural soil

[E1286] permethrin to agricultural soil

[E1287] phenanthrene to agricultural soil

[E1288] phenol to agricultural soil

[E1289] phoxim to agricultural soil

[E1290] Phtalic anhydride to agricultural soil

[E1291] pirimicarb to agricultural soil

[E1293] propachlor to agricultural soil

[E1294] propoxur to agricultural soil
$0.0046 \mathrm{~kg}$ 1,4-dichlorobenzene eq." $/ \mathrm{kg}$

0.00062 kg 1,4-dichlorobenzene eq." $/ \mathrm{kg}$

$8.9 \mathrm{~kg}$ 1,4-dichlorobenzene eq." $/ \mathrm{kg}$

$0.0074 \mathrm{~kg}$ 1,4-dichlorobenzene eq." $/ \mathrm{kg}$

20000 kg 1,4-dichlorobenzene eq."/kg

$330 \mathrm{~kg}$ 1,4-dichlorobenzene eq."/kg

4.20E-05 kg 1,4-dichlorobenzene eq." $/ \mathrm{kg}$

72 kg 1,4-dichlorobenzene eq." $/ \mathrm{kg}$

72 kg 1,4-dichlorobenzene eq." $/ \mathrm{kg}$

$1.2 \mathrm{~kg}$ 1,4-dichlorobenzene eq." $/ \mathrm{kg}$

$2.2 \mathrm{~kg}$ 1,4-dichlorobenzene eq." $/ \mathrm{kg}$

$21000 \mathrm{~kg}$ 1,4-dichlorobenzene eq." $/ \mathrm{kg}$

$11000 \mathrm{~kg}$ 1,4-dichlorobenzene eq."/kg

$0.0018 \mathrm{~kg}$ 1,4-dichlorobenzene eq." $/ \mathrm{kg}$

1.10E-09 kg 1,4-dichlorobenzene eq."/kg

760 kg 1,4-dichlorobenzene eq." $/ \mathrm{kg}$

$3500 \mathrm{~kg}$ 1,4-dichlorobenzene eq."/kg

$380 \mathrm{~kg}$ 1,4-dichlorobenzene eq." $/ \mathrm{kg}$

$250 \mathrm{~kg}$ 1,4-dichlorobenzene eq."/kg

$380 \mathrm{~kg}$ 1,4-dichlorobenzene eq." $/ \mathrm{kg}$

$19 \mathrm{~kg}$ 1,4-dichlorobenzene eq." $/ \mathrm{kg}$

$4500 \mathrm{~kg}$ 1,4-dichlorobenzene eq."/kg

$15 \mathrm{~kg}$ 1,4-dichlorobenzene eq." $/ \mathrm{kg}$

$0.92 \mathrm{~kg} \mathrm{1,4-dichlorobenzene} \mathrm{eq."} / \mathrm{kg}$

$2.3 \mathrm{~kg}$ 1,4-dichlorobenzene eq. "/kg

$31 \mathrm{~kg}$ 1,4-dichlorobenzene eq. "/kg

$70 \mathrm{~kg}$ 1,4-dichlorobenzene eq." $/ \mathrm{kg}$

$3.2 \mathrm{~kg}$ 1,4-dichlorobenzene eq." $/ \mathrm{kg}$

90 kg 1,4-dichlorobenzene eq." $/ \mathrm{kg}$

$0.23 \mathrm{~kg}$ 1,4-dichlorobenzene eq." $/ \mathrm{kg}$

$170 \mathrm{~kg}$ 1,4-dichlorobenzene eq." $/ \mathrm{kg}$

$6.5 \mathrm{~kg}$ 1,4-dichlorobenzene eq." $/ \mathrm{kg}$

97 kg 1,4-dichlorobenzene eq."/kg

$690 \mathrm{~kg}$ 1,4-dichlorobenzene eq." $/ \mathrm{kg}$

$160 \mathrm{~kg}$ 1,4-dichlorobenzene eq." $/ \mathrm{kg}$

$0.46 \mathrm{~kg}$ 1,4-dichlorobenzene eq." $/ \mathrm{kg}$

$30 \mathrm{~kg}$ 1,4-dichlorobenzene eq." $/ \mathrm{kg}$

$850 \mathrm{~kg}$ 1,4-dichlorobenzene eq." $/ \mathrm{kg}$

$0.41 \mathrm{~kg}$ 1,4-dichlorobenzene eq." $/ \mathrm{kg}$

$3.9 \mathrm{~kg}$ 1,4-dichlorobenzene eq." $/ \mathrm{kg}$

$44 \mathrm{~kg}$ 1,4-dichlorobenzene eq." $/ \mathrm{kg}$

$14000 \mathrm{~kg}$ 1,4-dichlorobenzene eq." $/ \mathrm{kg}$

$0.14 \mathrm{~kg}$ 1,4-dichlorobenzene eq." $/ \mathrm{kg}$

19000 kg 1,4-dichlorobenzene eq." $/ \mathrm{kg}$

$95 \mathrm{~kg}$ 1,4-dichlorobenzene eq." $/ \mathrm{kg}$

1900 kg 1,4-dichlorobenzene eq." $/ \mathrm{kg}$

$350 \mathrm{~kg}$ 1,4-dichlorobenzene eq."//kg

$260 \mathrm{~kg}$ 1,4-dichlorobenzene eq." $/ \mathrm{kg}$

$0.0019 \mathrm{~kg} \mathrm{1,4-dichlorobenzene} \mathrm{eq."/kg}$

$3.8 \mathrm{~kg}$ 1,4-dichlorobenzene eq." $/ \mathrm{kg}$

1700 kg 1,4-dichlorobenzene eq." $/ \mathrm{kg}$

30 kg 1,4-dichlorobenzene eq." $/ \mathrm{kg}$

$970 \mathrm{~kg}$ 1,4-dichlorobenzene eq." $/ \mathrm{kg}$

0.0025 kg 1,4-dichlorobenzene eq."/kg

$500 \mathrm{~kg}$ 1,4-dichlorobenzene eq." $/ \mathrm{kg}$

$1100 \mathrm{~kg}$ 1,4-dichlorobenzene eq." $/ \mathrm{kg}$

$0.59 \mathrm{~kg}$ 1,4-dichlorobenzene eq." $/ \mathrm{kg}$

$15 \mathrm{~kg}$ 1,4-dichlorobenzene eq."//kg

$0.33 \mathrm{~kg}$ 1,4-dichlorobenzene eq." $/ \mathrm{kg}$

$920 \mathrm{~kg}$ 1,4-dichlorobenzene eq." $/ \mathrm{kg}$

$0.29 \mathrm{~kg}$ 1,4-dichlorobenzene eq."/kg

$3.5 \mathrm{~kg}$ 1,4-dichlorobenzene eq." $/ \mathrm{kg}$

$4.4 \mathrm{~kg}$ 1,4-dichlorobenzene eq." $/ \mathrm{kg}$

4.80E-05 kg 1,4-dichlorobenzene eq." $/ \mathrm{kg}$

$1700 \mathrm{~kg}$ 1,4-dichlorobenzene eq."/kg

$17 \mathrm{~kg}$ 1,4-dichlorobenzene eq."/kg

20000 kg 1,4-dichlorobenzene eq."/kg 
[E1295] propylene oxide to agricultural soil [E1296] p-xylene to agricultural soil

[E1297] pyrazophos to agricultural soil

[E1298] selenium to agricultural soil

[E1299] simazine to agricultural soil

[E1300] styrene (vinylbenzene) to agricultural soil

[E1302] tetrachloroethylene (PER) to agricultural soil

[E1303] Tetrachloromethane (carbon tetrachloride) (HC-10) to agricultural soil

[E1304] thallium to agricultural soil

[E1305] Thiram to agricultural soil

[E1306] tin to agricultural soil

[E1307] tolclophos-methyl to agricultural soil

[E1308] toluene to agricultural soil

[E1309] tri-allate to agricultural soil

[E1310] triazophos to agricultural soil

[E1311] tributyltinoxide to agricultural soil

[E1312] trichlorfon to agricultural soil

[E1313] Trichloroethylene (tri) to agricultural soil

[E1314] Trichloromethane $=$ chloroform to agricultural soil

[E1315] trifluarin to agricultural soil

[E1316] vanadium to agricultural soil

[E1317] Vinyl Chloride (chloroethene) to agricultural soil

[E1318] zinc to agricultural soil

[E1319] zineb to agricultural soil

[E1320] 1,1,1-trichloroethane to agricultural soil

[E1321] 1,2,3,4-tetrachlorobenzene to agricultural soil

[E1322] 1,2,3,5-tetrachlorobenzene to agricultural soil

[E1323] 1,2,3-trichlorobenzene to agricultural soil

[E1324] 1,2,4,5-tetrachlorobenzene to agricultural soil

[E1325] 1,2,4-trichlorobenzene to agricultural soil

[E1326] 1,2-dichlorobenzene to agricultural soil

[E1327] 1,2-dichloroethane to agricultural soil

[E1328] 1,3,5-trichlorobenzene to agricultural soil

[E1329] 1,3-butadiene to agricultural soil

[E1330] 1,3-dichlorobenzene to agricultural soil

[E1331] 1,4-dichlorobenzene to agricultural soil

[E1332] 1-chloro-4-nitrobenzene to agricultural soil

[E1333] 2,3,4,6-tetrachlorophenol to agricultural soil

[E1334] 2,3,7,8-TCDD to agricultural soil

[E1335] 2,4,5-T to agricultural soil

[E1336] 2,4,5-trichlorophenol to agricultural soil

[E1337] 2,4,6-trichlorophenol to industrial soil

[E1338] 2,4-D to industrial soil

[E1339] 2,4-dichlorophenol to industrial soil

[E1340] 2-chlorophenol to industrial soil

[E1341] 3,4-dichloroaniline to industrial soil

[E1342] 3-chloroaniline to industrial soil

[E1343] 4-chloroaniline to industrial soil

[E1344] acephate to industrial soil

[E1345] Acrolein (2-propenal) to industrial soil

[E1346] acrylonitrile to industrial soil

[E1347] aldicarb to industrial soil

[E1348] aldrin to industrial soil

[E1350] anilazine to industrial soil

[E1351] anthracene to industrial soil

[E1352] antimony to industrial soil

[E1353] arsenic to industrial soil

[E1354] atrazine to industrial soil

[E1355] azinphos-ethyl to industrial soil

[E1356] azinphos-methyl to industrial soil

[E1357] barium to industrial soil

[E1358] benomyl to industrial soil

[E1359] bentazone to industrial soil

[E1360] benzene to industrial soil

[E1361] benzo[a]anthracene to industrial soil

[E1362] benzo[a]pyrene to industrial soil

[E1363] benzo[ghi]perylene to industrial soil
$0.42 \mathrm{~kg}$ 1,4-dichlorobenzene eq." $/ \mathrm{kg}$

$0.0014 \mathrm{~kg} 1,4$-dichlorobenzene eq." $/ \mathrm{kg}$

$250 \mathrm{~kg}$ 1,4-dichlorobenzene eq." $/ \mathrm{kg}$

1500 kg 1,4-dichlorobenzene eq." $/ \mathrm{kg}$

$2300 \mathrm{~kg}$ 1,4-dichlorobenzene eq." $/ \mathrm{kg}$

$0.0015 \mathrm{~kg}$ 1,4-dichlorobenzene eq."//kg

$0.0022 \mathrm{~kg}$ 1,4-dichlorobenzene eq." $/ \mathrm{kg}$

$0.00056 \mathrm{~kg}$ 1,4-dichlorobenzene eq." $/ \mathrm{kg}$

4200 kg 1,4-dichlorobenzene eq." $/ \mathrm{kg}$

$690 \mathrm{~kg}$ 1,4-dichlorobenzene eq." $/ \mathrm{kg}$

$6.9 \mathrm{~kg}$ 1,4-dichlorobenzene eq." $/ \mathrm{kg}$

$3.1 \mathrm{~kg}$ 1,4-dichlorobenzene eq."//kg

$0.0011 \mathrm{~kg}$ 1,4-dichlorobenzene eq." $/ \mathrm{kg}$

$50 \mathrm{~kg} \mathrm{1,4-dichlorobenzene} \mathrm{eq."} / \mathrm{kg}$

5800 kg 1,4-dichlorobenzene eq."/kg

$1100 \mathrm{~kg}$ 1,4-dichlorobenzene eq." $/ \mathrm{kg}$

$3300 \mathrm{~kg}$ 1,4-dichlorobenzene eq." $/ \mathrm{kg}$

$0.00046 \mathrm{~kg}$ 1,4-dichlorobenzene eq."/kg

$0.00047 \mathrm{~kg}$ 1,4-dichlorobenzene eq." $/ \mathrm{kg}$

$40 \mathrm{~kg}$ 1,4-dichlorobenzene eq." $/ \mathrm{kg}$

4700 kg 1,4-dichlorobenzene eq."//kg

6.40E-05 kg 1,4-dichlorobenzene eq." $/ \mathrm{kg}$

$48 \mathrm{~kg}$ 1,4-dichlorobenzene eq. " $/ \mathrm{kg}$

$370 \mathrm{~kg}$ 1,4-dichlorobenzene eq." $/ \mathrm{kg}$

$0.00037 \mathrm{~kg}$ 1,4-dichlorobenzene eq." $/ \mathrm{kg}$

$0.1 \mathrm{~kg}$ 1,4-dichlorobenzene eq." $/ \mathrm{kg}$

$0.19 \mathrm{~kg}$ 1,4-dichlorobenzene eq."/kg

$0.03 \mathrm{~kg} \mathrm{1,4-dichlorobenzene} \mathrm{eq."} / \mathrm{kg}$

$0.09 \mathrm{~kg}$ 1,4-dichlorobenzene eq." $/ \mathrm{kg}$

$0.032 \mathrm{~kg}$ 1,4-dichlorobenzene eq." $/ \mathrm{kg}$

0.019 kg 1,4-dichlorobenzene eq." $/ \mathrm{kg}$

$0.00075 \mathrm{~kg}$ 1,4-dichlorobenzene eq." $/ \mathrm{kg}$

$0.066 \mathrm{~kg}$ 1,4-dichlorobenzene eq." $/ \mathrm{kg}$

5.70E-05 kg 1,4-dichlorobenzene eq."/kg

$0.018 \mathrm{~kg}$ 1,4-dichlorobenzene eq." $/ \mathrm{kg}$

$0.014 \mathrm{~kg}$ 1,4-dichlorobenzene eq." $/ \mathrm{kg}$

$150 \mathrm{~kg}$ 1,4-dichlorobenzene eq." $/ \mathrm{kg}$

$120 \mathrm{~kg}$ 1,4-dichlorobenzene eq."//kg

4.90E+05 kg 1,4-dichlorobenzene eq."/kg

$1.5 \mathrm{~kg}$ 1,4-dichlorobenzene eq." $/ \mathrm{kg}$

99 kg 1,4-dichlorobenzene eq." $/ \mathrm{kg}$

$4.8 \mathrm{~kg}$ 1,4-dichlorobenzene eq." $/ \mathrm{kg}$

$82 \mathrm{~kg}$ 1,4-dichlorobenzene eq." $/ \mathrm{kg}$

$9.2 \mathrm{~kg}$ 1,4-dichlorobenzene eq." $/ \mathrm{kg}$

$31 \mathrm{~kg}$ 1,4-dichlorobenzene eq." $/ \mathrm{kg}$

4000 kg 1,4-dichlorobenzene eq." $/ \mathrm{kg}$

$250 \mathrm{~kg}$ 1,4-dichlorobenzene eq." $/ \mathrm{kg}$

$490 \mathrm{~kg}$ 1,4-dichlorobenzene eq." $/ \mathrm{kg}$

$160 \mathrm{~kg}$ 1,4-dichlorobenzene eq." $/ \mathrm{kg}$

45000 kg 1,4-dichlorobenzene eq." $/ \mathrm{kg}$

$8.1 \mathrm{~kg}$ 1,4-dichlorobenzene eq." $/ \mathrm{kg}$

96000 kg 1,4-dichlorobenzene eq." $/ \mathrm{kg}$

$290 \mathrm{~kg}$ 1,4-dichlorobenzene eq."/kg

$0.86 \mathrm{~kg}$ 1,4-dichlorobenzene eq." $/ \mathrm{kg}$

$320 \mathrm{~kg}$ 1,4-dichlorobenzene eq." $/ \mathrm{kg}$

$10 \mathrm{~kg}$ 1,4-dichlorobenzene eq." $/ \mathrm{kg}$

$130 \mathrm{~kg}$ 1,4-dichlorobenzene eq."/kg

$930 \mathrm{~kg}$ 1,4-dichlorobenzene eq." $/ \mathrm{kg}$

3700 kg 1,4-dichlorobenzene eq." $/ \mathrm{kg}$

$800 \mathrm{~kg}$ 1,4-dichlorobenzene eq." $/ \mathrm{kg}$

$110 \mathrm{~kg}$ 1,4-dichlorobenzene eq." $/ \mathrm{kg}$

$18 \mathrm{~kg}$ 1,4-dichlorobenzene eq." $/ \mathrm{kg}$

11 kg 1,4-dichlorobenzene eq." $/ \mathrm{kg}$

$0.00072 \mathrm{~kg} 1,4$-dichlorobenzene eq." $/ \mathrm{kg}$

$250 \mathrm{~kg}$ 1,4-dichlorobenzene eq." $/ \mathrm{kg}$

$530 \mathrm{~kg}$ 1,4-dichlorobenzene eq." $/ \mathrm{kg}$

$240 \mathrm{~kg}$ 1,4-dichlorobenzene eq. "/kg 
[E1364] benzo[k]fluoranthrene to industrial soil

[E1365] benzylchloride to industrial soil

[E1366] beryllium to industrial soil

[E1367] bifenthrin to industrial soil

[E1368] Butylbenzylphtalate to industrial soil

[E1369] cadmium to industrial soil

[E1370] captafol to industrial soil

[E1371] captan to industrial soil

[E1372] carbaryl to industrial soil

[E1373] carbendazim to industrial soil

[E1374] carbofuran to industrial soil

[E1375] carbon disulfide to industrial soil

[E1376] Carcinogenic PAHs to industrial soil

[E1377] chlordane to industrial soil

[E1378] chlorfenvinphos to industrial soil

[E1379] chloridazon to industrial soil

[E1380] chlorobenzene to industrial soil

[E1381] chlorothalonil to industrial soil

[E1382] chlorpropham to industrial soil

[E1383] chlorpyriphos to industrial soil

[E1384] chromium III to industrial soil

[E1385] chromium VI to industrial soil

[E1386] chrysene to industrial soil

[E1387] cobalt to industrial soil

[E1388] copper to industrial soil

[E1389] coumaphos to industrial soil

[E1390] cyanazine to industrial soil

[E1391] cypermethrin to industrial soil

[E1392] cyromazine to industrial soil

[E1393] DDT to industrial soil

[E1394] deltamethrin to industrial soil

[E1395] demeton to industrial soil

[E1396] desmetryn to industrial soil

[E1397] Di(2-ethylhexyl)phtalate to industrial soil

[E1398] diazinon to industrial soil

[E1399] Dibutylphtalate to industrial soil

[E1400] Dichloromethane (Methylene Chloride) to industrial soil

[E1401] dichlorprop to industrial soil

[E1402] dichlorvos to industrial soil

[E1403] dieldrin to industrial soil

[E1404] Diethylphtalate to industrial soil

[E1405] Dihexylphtalate to industrial soil

[E1406] Diisodecylphtalate to industrial soil

[E1407] Diisooctylphtalate to industrial soil

[E1408] dimethoate to industrial soil

[E1409] Dimethylphtalate to industrial soil

[E1410] dinoseb to industrial soil

[E1411] dinoterb to industrial soil

[E1412] Dioctylphtalate to industrial soil

[E1413] disulfothon to industrial soil

[E1414] diuron to industrial soil

[E1415] DNOC to industrial soil

[E1416] endosulfan to industrial soil

[E1417] endrin to industrial soil

[E1418] ethoprophos to industrial soil

[E1419] ethylbenzene to industrial soil

[E1420] ethylene to industrial soil

[E1421] fenitrothion to industrial soil

[E1422] fenthion to industrial soil

[E1423] fentin acetate to industrial soil

[E1424] fentin chloride to industrial soil

[E1425] fentin hydroxide to industrial soil

[E1426] fluoranthrene to industrial soil

[E1427] folpet to industrial soil

[E1428] Formaldehyde (methanal) to industrial soil

[E1429] glyphosate to industrial soil

[E1430] heptachlor to industrial soil
20000 kg 1,4-dichlorobenzene eq." $/ \mathrm{kg}$

$3.2 \mathrm{~kg}$ 1,4-dichlorobenzene eq." $/ \mathrm{kg}$

46000 kg 1,4-dichlorobenzene eq." $/ \mathrm{kg}$

$410 \mathrm{~kg}$ 1,4-dichlorobenzene eq." $/ \mathrm{kg}$

$0.1 \mathrm{~kg}$ 1,4-dichlorobenzene eq." $/ \mathrm{kg}$

$780 \mathrm{~kg}$ 1,4-dichlorobenzene eq." $/ \mathrm{kg}$

83000 kg 1,4-dichlorobenzene eq." $/ \mathrm{kg}$

$4.7 \mathrm{~kg}$ 1,4-dichlorobenzene eq." $/ \mathrm{kg}$

$120 \mathrm{~kg}$ 1,4-dichlorobenzene eq." $/ \mathrm{kg}$

$6100 \mathrm{~kg}$ 1,4-dichlorobenzene eq." $/ \mathrm{kg}$

1800 kg 1,4-dichlorobenzene eq."/kg

$0.34 \mathrm{~kg}$ 1,4-dichlorobenzene eq." $/ \mathrm{kg}$

$230 \mathrm{~kg}$ 1,4-dichlorobenzene eq." $/ \mathrm{kg}$

$370 \mathrm{~kg}$ 1,4-dichlorobenzene eq." $/ \mathrm{kg}$

$59 \mathrm{~kg}$ 1,4-dichlorobenzene eq." $/ \mathrm{kg}$

$3.9 \mathrm{~kg}$ 1,4-dichlorobenzene eq." $/ \mathrm{kg}$

$0.0032 \mathrm{~kg}$ 1,4-dichlorobenzene eq." $/ \mathrm{kg}$

$3.7 \mathrm{~kg} \mathrm{1,4-dichlorobenzene} \mathrm{eq."} / \mathrm{kg}$

$6.4 \mathrm{~kg}$ 1,4-dichlorobenzene eq." $/ \mathrm{kg}$

1400 kg 1,4-dichlorobenzene eq."/kg

$5.3 \mathrm{~kg}$ 1,4-dichlorobenzene eq." $/ \mathrm{kg}$

$21 \mathrm{~kg}$ 1,4-dichlorobenzene eq." $/ \mathrm{kg}$ 290 kg 1,4-dichlorobenzene eq." $/ \mathrm{kg}$ $1700 \mathrm{~kg}$ 1,4-dichlorobenzene eq." $/ \mathrm{kg}$ $590 \mathrm{~kg}$ 1,4-dichlorobenzene eq." $/ \mathrm{kg}$

3.10E+06 kg 1,4-dichlorobenzene eq."/kg $3000 \mathrm{~kg}$ 1,4-dichlorobenzene eq."/kg

6.90E+05 kg 1,4-dichlorobenzene eq." $/ \mathrm{kg}$ $6500 \mathrm{~kg}$ 1,4-dichlorobenzene eq." $/ \mathrm{kg}$ $340 \mathrm{~kg}$ 1,4-dichlorobenzene eq. "/kg

$96 \mathrm{~kg}$ 1,4-dichlorobenzene eq." $/ \mathrm{kg}$ 2600 kg 1,4-dichlorobenzene eq." $/ \mathrm{kg}$

$11 \mathrm{~kg} \mathrm{1,4-dichlorobenzene} \mathrm{eq."} / \mathrm{kg}$

0.006 kg 1,4-dichlorobenzene eq."/kg $4600 \mathrm{~kg}$ 1,4-dichlorobenzene eq." $/ \mathrm{kg}$ $0.31 \mathrm{~kg}$ 1,4-dichlorobenzene eq." $/ \mathrm{kg}$

$0.00016 \mathrm{~kg}$ 1,4-dichlorobenzene eq." $/ \mathrm{kg}$

$0.051 \mathrm{~kg}$ 1,4-dichlorobenzene eq." $/ \mathrm{kg}$

$300 \mathrm{~kg}$ 1,4-dichlorobenzene eq." $/ \mathrm{kg}$

2300 kg 1,4-dichlorobenzene eq." $/ \mathrm{kg}$

$0.63 \mathrm{~kg} \mathrm{1,4-dichlorobenzene} \mathrm{eq."} / \mathrm{kg}$

$0.074 \mathrm{~kg}$ 1,4-dichlorobenzene eq." $/ \mathrm{kg}$

$0.018 \mathrm{~kg}$ 1,4-dichlorobenzene eq." $/ \mathrm{kg}$

0.0025 kg 1,4-dichlorobenzene eq." $/ \mathrm{kg}$

$28 \mathrm{~kg}$ 1,4-dichlorobenzene eq." $/ \mathrm{kg}$

0.029 kg 1,4-dichlorobenzene eq." $/ \mathrm{kg}$

58000 kg 1,4-dichlorobenzene eq."/kg

1300 kg 1,4-dichlorobenzene eq."/kg

$0.00017 \mathrm{~kg}$ 1,4-dichlorobenzene eq." $/ \mathrm{kg}$ $290 \mathrm{~kg}$ 1,4-dichlorobenzene eq."/kg $290 \mathrm{~kg}$ 1,4-dichlorobenzene eq." $/ \mathrm{kg}$

$4.5 \mathrm{~kg} \mathrm{1,4-dichlorobenzene} \mathrm{eq."} / \mathrm{kg}$

9 kg 1,4-dichlorobenzene eq." $/ \mathrm{kg}$

$71000 \mathrm{~kg}$ 1,4-dichlorobenzene eq." $/ \mathrm{kg}$ 30000 kg 1,4-dichlorobenzene eq." $/ \mathrm{kg}$ $0.0018 \mathrm{~kg}$ 1,4-dichlorobenzene eq." $/ \mathrm{kg}$

1.10E-09 kg 1,4-dichlorobenzene eq."//kg $3000 \mathrm{~kg} \mathrm{1,4-dichlorobenzene} \mathrm{eq."} / \mathrm{kg}$ 14000 kg 1,4-dichlorobenzene eq."/kg $1500 \mathrm{~kg}$ 1,4-dichlorobenzene eq." $/ \mathrm{kg}$ $990 \mathrm{~kg}$ 1,4-dichlorobenzene eq." $/ \mathrm{kg}$ $1500 \mathrm{~kg}$ 1,4-dichlorobenzene eq."/kg

$76 \mathrm{~kg}$ 1,4-dichlorobenzene eq." $/ \mathrm{kg}$ 13000 kg 1,4-dichlorobenzene eq."/kg

$44 \mathrm{~kg}$ 1,4-dichlorobenzene eq." $/ \mathrm{kg}$

$3.7 \mathrm{~kg}$ 1,4-dichlorobenzene eq."/kg

$8.9 \mathrm{~kg}$ 1,4-dichlorobenzene eq." $/ \mathrm{kg}$ 
[E1431] heptenophos to industrial soil

[E1432] hexachloro-1,3-butadiene to industrial soil

[E1433] hexachlorobenzene to industrial soil

[E1436] indeno[1,2,3-cd]pyrene to industrial soil

[E1437] iprodione to industrial soil

[E1438] isoproturon to industrial soil

[E1439] lead to industrial soil

[E1440] lindane to industrial soil

[E1441] linuron to industrial soil

[E1442] malathion to industrial soil

[E1443] MCPA to industrial soil

[E1444] mecoprop to industrial soil

[E1445] mercury to industrial soil

[E1446] metamitron to industrial soil

[E1447] metazachlor to industrial soil

[E1448] methabenzthiazuron to industrial soil

[E1449] methomyl to industrial soil

[E1450] methylbromide to industrial soil

[E1451] methyl-mercury to industrial soil

[E1452] metobromuron to industrial soil

[E1453] metolachlor to industrial soil

[E1454] mevinphos to industrial soil

[E1455] molybdenum to industrial soil

[E1456] $m$-xylene to industrial soil

[E1457] naphtalene to industrial soil

[E1458] nickel to industrial soil

[E1460] oxamyl to industrial soil

[E1461] oxydemethon-methyl to industrial soil

[E1462] o-xylene to industrial soil

[E1463] parathion-ethyl to industrial soil

[E1464] parathion-methyl to industrial soil

[E1465] pentachlorobenzene to industrial soil

[E1466] pentachloronitrobenzene to industrial soil

[E1467] pentachlorophenol to industrial soil

[E1468] permethrin to industrial soil

[E1469] phenanthrene to industrial soil

[E1470] phenol to industrial soil

[E1471] phoxim to industrial soil

[E1472] Phtalic anhydride to industrial soil

[E1473] pirimicarb to industrial soil

[E1475] propachlor to industrial soil

[E1476] propoxur to industrial soil

[E1477] propylene oxide to industrial soil

[E1478] p-xylene to industrial soil

[E1479] pyrazophos to industrial soil

[E1480] selenium to industrial soil

[E1481] simazine to industrial soil

[E1482] styrene (vinylbenzene) to industrial soil

[E1484] tetrachloroethylene (PER) to industrial soil

[E1485] Tetrachloromethane (carbon tetrachloride) $(\mathrm{HC}-10)$ to industrial soil

[E1486] thallium to industrial soil

[E1487] Thiram to industrial soil

[E1488] tin to industrial soil

[E1489] tolclophos-methyl to industrial soil

[E1490] toluene to industrial soil

[E1491] tri-allate to industrial soil

[E1492] triazophos to industrial soil

[E1493] tributyltinoxide to industrial soil

[E1494] trichlorfon to industrial soil

[E1495] Trichloroethylene (tri) to industrial soil

[E1496] Trichloromethane=chloroform to industrial soil

[E1497] trifluarin to industrial soil

[E1498] vanadium to industrial soil

[E1499] Vinyl Chloride (chloroethene) to industrial soil

[E1500] zinc to industrial soil

[E1501] zineb to industrial soil
120 kg 1,4-dichlorobenzene eq." $/ \mathrm{kg}$

$84 \mathrm{~kg}$ 1,4-dichlorobenzene eq." $/ \mathrm{kg}$

$4.3 \mathrm{~kg}$ 1,4-dichlorobenzene eq." $/ \mathrm{kg}$

$360 \mathrm{~kg}$ 1,4-dichlorobenzene eq." $/ \mathrm{kg}$

$1.9 \mathrm{~kg}$ 1,4-dichlorobenzene eq." $/ \mathrm{kg}$

$400 \mathrm{~kg}$ 1,4-dichlorobenzene eq. "/kg

$6.5 \mathrm{~kg}$ 1,4-dichlorobenzene eq." $/ \mathrm{kg}$

370 kg 1,4-dichlorobenzene eq." $/ \mathrm{kg}$

$2400 \mathrm{~kg}$ 1,4-dichlorobenzene eq." $/ \mathrm{kg}$

$650 \mathrm{~kg}$ 1,4-dichlorobenzene eq." $/ \mathrm{kg}$

$1.7 \mathrm{~kg}$ 1,4-dichlorobenzene eq." $/ \mathrm{kg}$

$78 \mathrm{~kg}$ 1,4-dichlorobenzene eq." $/ \mathrm{kg}$

$850 \mathrm{~kg}$ 1,4-dichlorobenzene eq." $/ \mathrm{kg}$

$1.5 \mathrm{~kg}$ 1,4-dichlorobenzene eq." $/ \mathrm{kg}$

$14 \mathrm{~kg}$ 1,4-dichlorobenzene eq." $/ \mathrm{kg}$

$140 \mathrm{~kg}$ 1,4-dichlorobenzene eq." $/ \mathrm{kg}$

28000 kg 1,4-dichlorobenzene eq." $/ \mathrm{kg}$

$0.14 \mathrm{~kg}$ 1,4-dichlorobenzene eq." $/ \mathrm{kg}$

19000 kg 1,4-dichlorobenzene eq."/kg

$95 \mathrm{~kg}$ 1,4-dichlorobenzene eq." $/ \mathrm{kg}$

$5800 \mathrm{~kg}$ 1,4-dichlorobenzene eq." $/ \mathrm{kg}$

$1500 \mathrm{~kg}$ 1,4-dichlorobenzene eq." $/ \mathrm{kg}$

260 kg 1,4-dichlorobenzene eq."/kg

$0.0019 \mathrm{~kg} 1,4$-dichlorobenzene eq." $/ \mathrm{kg}$

$12 \mathrm{~kg}$ 1,4-dichlorobenzene eq." $/ \mathrm{kg}$

$1700 \mathrm{~kg} \mathrm{1,4-dichlorobenzene} \mathrm{eq."/kg}$

$120 \mathrm{~kg}$ 1,4-dichlorobenzene eq." $/ \mathrm{kg}$

$3600 \mathrm{~kg}$ 1,4-dichlorobenzene eq." $/ \mathrm{kg}$

$0.0025 \mathrm{~kg}$ 1,4-dichlorobenzene eq." $/ \mathrm{kg}$

1900 kg 1,4-dichlorobenzene eq." $/ \mathrm{kg}$

$4400 \mathrm{~kg} \mathrm{1,4-dichlorobenzene} \mathrm{eq."} / \mathrm{kg}$

$1.1 \mathrm{~kg}$ 1,4-dichlorobenzene eq."/kg

$58 \mathrm{~kg}$ 1,4-dichlorobenzene eq." $/ \mathrm{kg}$

$1.3 \mathrm{~kg}$ 1,4-dichlorobenzene eq." $/ \mathrm{kg}$

$3700 \mathrm{~kg}$ 1,4-dichlorobenzene eq." $/ \mathrm{kg}$

$1.2 \mathrm{~kg}$ 1,4-dichlorobenzene eq." $/ \mathrm{kg}$

$13 \mathrm{~kg}$ 1,4-dichlorobenzene eq." $/ \mathrm{kg}$

$7.9 \mathrm{~kg} 1,4$-dichlorobenzene eq." $/ \mathrm{kg}$

3.10E-05 kg 1,4-dichlorobenzene eq." $/ \mathrm{kg}$

$5200 \mathrm{~kg}$ 1,4-dichlorobenzene eq." $/ \mathrm{kg}$

64 kg 1,4-dichlorobenzene eq." $/ \mathrm{kg}$

$54000 \mathrm{~kg}$ 1,4-dichlorobenzene eq." $/ \mathrm{kg}$

$0.48 \mathrm{~kg} 1,4$-dichlorobenzene eq." $/ \mathrm{kg}$

0.0014 kg 1,4-dichlorobenzene eq." $/ \mathrm{kg}$

$990 \mathrm{~kg}$ 1,4-dichlorobenzene eq." $/ \mathrm{kg}$

1500 kg 1,4-dichlorobenzene eq." $/ \mathrm{kg}$

$5600 \mathrm{~kg} \mathrm{1,4-dichlorobenzene} \mathrm{eq."} / \mathrm{kg}$

0.0026 kg 1,4-dichlorobenzene eq." $/ \mathrm{kg}$

$0.00056 \mathrm{~kg}$ 1,4-dichlorobenzene eq."//kg

$0.00056 \mathrm{~kg}$ 1,4-dichlorobenzene eq." $/ \mathrm{kg}$ $4200 \mathrm{~kg}$ 1,4-dichlorobenzene eq." $/ \mathrm{kg}$ $4400 \mathrm{~kg}$ 1,4-dichlorobenzene eq." $/ \mathrm{kg}$

$6.9 \mathrm{~kg}$ 1,4-dichlorobenzene eq." $/ \mathrm{kg}$

$9.2 \mathrm{~kg}$ 1,4-dichlorobenzene eq." $/ \mathrm{kg}$

$0.0011 \mathrm{~kg} 1,4$-dichlorobenzene eq." $/ \mathrm{kg}$

$200 \mathrm{~kg}$ 1,4-dichlorobenzene eq." $/ \mathrm{kg}$

19000 kg 1,4-dichlorobenzene eq." $/ \mathrm{kg}$ $4200 \mathrm{~kg}$ 1,4-dichlorobenzene eq." $/ \mathrm{kg}$

$18000 \mathrm{~kg}$ 1,4-dichlorobenzene eq." $/ \mathrm{kg}$

$0.00046 \mathrm{~kg}$ 1,4-dichlorobenzene eq." $/ \mathrm{kg}$

$0.00047 \mathrm{~kg} 1,4$-dichlorobenzene eq." $/ \mathrm{kg}$

$160 \mathrm{~kg}$ 1,4-dichlorobenzene eq." $/ \mathrm{kg}$

$4700 \mathrm{~kg}$ 1,4-dichlorobenzene eq." $/ \mathrm{kg}$

6.40E-05 kg 1,4-dichlorobenzene eq." $/ \mathrm{kg}$

$48 \mathrm{~kg}$ 1,4-dichlorobenzene eq." $/ \mathrm{kg}$

1400 kg 1,4-dichlorobenzene eq." $/ \mathrm{kg}$ 
Category $=[C 7]$ Aquatic ecotoxicity salt water

Description = Problem oriented approach, manual 1999; AETP

Author $=$ anonymous

Date $=1 / 18 / 00$

Environmental resources

Environmental resource

Environmental emissions

Environmental emission

[E90] 1,1,1-trichloroethane (methyl chloroform; $\mathrm{HC}-140 \mathrm{a}$ ) to air

[E94] 1,2,3,4-tetrachlorobenzene to air

[E95] 1,2,3,5-tetrachlorobenzene to air

[E96] 1,2,3-trichlorobenzene to air

[E98] 1,2,4,5-tetrachlorobenzene to air

[E99] 1,2,4-trichlorobenzene to air

[E102] 1,2-dichlorobenzene to air

[E103] 1,2-dichloroethane to air

[E107] 1,3,5-trichlorobenzene to air

[E111] 1,3-Butadiene to air

[E112] 1,3-dichlorobenzene to air

[E116] 1,4-dichlorobenzene to air

[E128] 1-chloro-4-nitrobenzene to air

[E166] 2,3,4,6-tetrachlorophenol to air

[E168] 2,3,7,8-TCDD (tetrachloride-dibenzo-dioxin) to air

[E172] 2,4,5-T to air

[E173] 2,4,5-trichlorophenol to air

[E174] 2,4,6-trichlorophenol to air

[E175] 2,4-D to air

[E176] 2,4-dichlorophenol to air

[E185] 2-chlorophenol to air

[E208] 3,4-dichloroaniline to air

[E217] 3-chloroaniline to air

[E228] 4-chloroaniline to air

[E231] acephate to air

[E236] Acrolein (2-propenal) to air

[E237] Acrylonitrile to air

[E241] aldicarb to air

[E242] aldrin to air

[E251] anilazine to air

[E252] anthracene to air

[E253] antimony to air

[E255] arsenic to air

[E256] atrazine to air

[E257] azinphos-ethyl to air

[E258] azinphos-methyl to air

[E259] barium to air

[E260] benomyl to air

[E261] bentazone to air

[E263] Benzene to air

[E264] benzo[a]anthracene to air

[E265] benzo[a]pyrene to air

[E266] benzo[ghi]perylene to air

[E267] benzo[k]fluoranthrene to air

[E269] benzylchloride to air

[E270] beryllium to air

[E272] bifenthrin to air

[E290] Butylbenzylphtalate to air

[E385] cadmium to air

[E387] captafol to air

[E388] captan to air

[E389] carbaryl to air

[E390] carbendazim to air

[E391] carbofuran to air

[E394] carbon disulfide to air

[E404] chlordane to air
Value

Value

Unit

$0.33 \mathrm{~kg}$ 1,4-dichlorobenzene eq." $/ \mathrm{kg}$

$17 \mathrm{~kg}$ 1,4-dichlorobenzene eq." $/ \mathrm{kg}$

$18 \mathrm{~kg}$ 1,4-dichlorobenzene eq." $/ \mathrm{kg}$

$2.1 \mathrm{~kg}$ 1,4-dichlorobenzene eq." $/ \mathrm{kg}$

$15 \mathrm{~kg}$ 1,4-dichlorobenzene eq." $/ \mathrm{kg}$

$2 \mathrm{~kg}$ 1,4-dichlorobenzene eq." $/ \mathrm{kg}$

$0.67 \mathrm{~kg}$ 1,4-dichlorobenzene eq." $/ \mathrm{kg}$

$0.082 \mathrm{~kg} 1,4$-dichlorobenzene eq." $/ \mathrm{kg}$

$3 \mathrm{~kg}$ 1,4-dichlorobenzene eq. $" / \mathrm{kg}$

2.70E-06 kg 1,4-dichlorobenzene eq." /kg

$0.46 \mathrm{~kg}$ 1,4-dichlorobenzene eq." $/ \mathrm{kg}$

$0.74 \mathrm{~kg}$ 1,4-dichlorobenzene eq." $/ \mathrm{kg}$

$390 \mathrm{~kg}$ 1,4-dichlorobenzene eq." $/ \mathrm{kg}$

$130 \mathrm{~kg}$ 1,4-dichlorobenzene eq." $/ \mathrm{kg}$

$3.00 E+08$ kg 1,4-dichlorobenzene eq." $/ \mathrm{kg}$

$0.2 \mathrm{~kg}$ 1,4-dichlorobenzene eq." $/ \mathrm{kg}$

$53 \mathrm{~kg}$ 1,4-dichlorobenzene eq." $/ \mathrm{kg}$

$3.9 \mathrm{~kg}$ 1,4-dichlorobenzene eq." $/ \mathrm{kg}$

$5.3 \mathrm{~kg}$ 1,4-dichlorobenzene eq." $/ \mathrm{kg}$

$1.3 \mathrm{~kg}$ 1,4-dichlorobenzene eq." $/ \mathrm{kg}$

$12 \mathrm{~kg}$ 1,4-dichlorobenzene eq."/kg

1700 kg 1,4-dichlorobenzene eq." $/ \mathrm{kg}$

$23 \mathrm{~kg}$ 1,4-dichlorobenzene eq." $/ \mathrm{kg}$

$1.7 \mathrm{~kg}$ 1,4-dichlorobenzene eq." $/ \mathrm{kg}$

19 kg 1,4-dichlorobenzene eq." $/ \mathrm{kg}$

$570 \mathrm{~kg}$ 1,4-dichlorobenzene eq." $/ \mathrm{kg}$

$0.91 \mathrm{~kg}$ 1,4-dichlorobenzene eq." $/ \mathrm{kg}$

8200 kg 1,4-dichlorobenzene eq."/kg

$61 \mathrm{~kg} \mathrm{1,4-dichlorobenzene} \mathrm{eq."} / \mathrm{kg}$

$8.3 \mathrm{~kg} \mathrm{1,4-dichlorobenzene} \mathrm{eq."} / \mathrm{kg}$

1700 kg 1,4-dichlorobenzene eq."/kg

$33000 \mathrm{~kg}$ 1,4-dichlorobenzene eq." $/ \mathrm{kg}$

2.30E+05 kg 1,4-dichlorobenzene eq." $/ \mathrm{kg}$ $280 \mathrm{~kg}$ 1,4-dichlorobenzene eq." $/ \mathrm{kg}$ 160 kg 1,4-dichlorobenzene eq." $/ \mathrm{kg}$ $200 \mathrm{~kg}$ 1,4-dichlorobenzene eq." $/ \mathrm{kg}$

7.80E+05 kg 1,4-dichlorobenzene eq." $/ \mathrm{kg}$

$21 \mathrm{~kg}$ 1,4-dichlorobenzene eq." $/ \mathrm{kg}$

$0.62 \mathrm{~kg}$ 1,4-dichlorobenzene eq." $/ \mathrm{kg}$

$0.0028 \mathrm{~kg}$ 1,4-dichlorobenzene eq." $/ \mathrm{kg}$

$1000 \mathrm{~kg} \mathrm{1,4-dichlorobenzene} \mathrm{eq."/kg}$

$1400 \mathrm{~kg}$ 1,4-dichlorobenzene eq." $/ \mathrm{kg}$

$1700 \mathrm{~kg}$ 1,4-dichlorobenzene eq." $/ \mathrm{kg}$

1.20E+05 kg 1,4-dichlorobenzene eq."/kg

$2.1 \mathrm{~kg}$ 1,4-dichlorobenzene eq." $/ \mathrm{kg}$

4.70E+08 kg 1,4-dichlorobenzene eq." $/ \mathrm{kg}$

$1000 \mathrm{~kg}$ 1,4-dichlorobenzene eq." $/ \mathrm{kg}$

$0.32 \mathrm{~kg}$ 1,4-dichlorobenzene eq." $/ \mathrm{kg}$

$1.10 \mathrm{E}+06 \mathrm{~kg}$ 1,4-dichlorobenzene eq." $/ \mathrm{kg}$

27000 kg 1,4-dichlorobenzene eq." $/ \mathrm{kg}$

$10 \mathrm{~kg}$ 1,4-dichlorobenzene eq." $/ \mathrm{kg}$

$120 \mathrm{~kg}$ 1,4-dichlorobenzene eq." $/ \mathrm{kg}$

720 kg 1,4-dichlorobenzene eq."/kg

$150 \mathrm{~kg}$ 1,4-dichlorobenzene eq."/kg

$1.5 \mathrm{~kg}$ 1,4-dichlorobenzene eq." $/ \mathrm{kg}$

61000 kg 1,4-dichlorobenzene eq."/kg 
[E405] chlorfenvinphos to air

[E406] chloridazon to air

[E407] chlorobenzene to air

[E410] chlorothalonil to air

[E411] chlorpropham to air

[E412] chlorpyriphos to air

[E413] chromium (unspecified) to air

[E414] chromium III to air

[E415] chromium VI to air

[E416] chrysene to air

[E421] cobalt to air

[E422] copper to air

[E423] coumaphos to air

[E425] cyanazine to air

[E437] cypermethrin to air

[E438] cyromazine to air

[E439] DDT to air

[E441] deltamethrin to air

[E442] demeton to air

[E443] desmetryn to air

[E444] Di(2-ethylhexyl)phtalate to air

[E446] diazinon to air

[E448] Dibutylphtalate to air

[E449] Dichloromethane (Methylene Chloride) to air

[E450] dichlorprop to air

[E451] dichlorvos to air

[E452] dieldrin to air

[E456] Diethylphtalate to air

[E457] Dihexylphtalate to air

[E458] Diisodecylphtalate to air

[E459] Diisooctylphtalate to air

[E461] dimethoate to air

[E468] Dimethylphtalate to air

[E470] dinoseb to air

[E471] dinoterb to air

[E472] Dioctylphtalate to air

[E475] disulfothon to air

[E476] diuron to air

[E478] DNOC to air

[E480] endosulfan to air

[E481] endrin to air

[E487] ethoprophos to air

[E496] Ethylbenzene to air

[E499] Ethylene (ethene) to air

[E504] fenitrothion to air

[E505] fenthion to air

[E506] fentin acetate to air

[E507] fentin chloride to air

[E508] fentin hydroxide to air

[E509] fluoranthrene to air

[E510] folpet to air

[E511] Formaldehyde (methanal) to air

[E516] glyphosate to air

[E533] heptachlor to air

[E535] heptenophos to air

[E536] hexachloro-1,3-butadiene to air

[E537] hexachlorobenzene to air

[E566] indeno[1,2,3-cd]pyrene to air

[E568] iprodione to air

[E583] isoproturon to air

[E584] lead to air

[E585] lindane to air

[E586] linuron to air

[E587] malathion to air

[E589] MCPA to air

[E590] mecoprop to air

[E592] mercury to air
$11 \mathrm{~kg}$ 1,4-dichlorobenzene eq." $/ \mathrm{kg}$ $0.22 \mathrm{~kg}$ 1,4-dichlorobenzene eq." $/ \mathrm{kg}$

$0.11 \mathrm{~kg}$ 1,4-dichlorobenzene eq." $/ \mathrm{kg}$

$51 \mathrm{~kg}$ 1,4-dichlorobenzene eq." $/ \mathrm{kg}$

$0.64 \mathrm{~kg}$ 1,4-dichlorobenzene eq." $/ \mathrm{kg}$

$62 \mathrm{~kg}$ 1,4-dichlorobenzene eq." $/ \mathrm{kg}$

$5200 \mathrm{~kg}$ 1,4-dichlorobenzene eq." $/ \mathrm{kg}$

$5200 \mathrm{~kg}$ 1,4-dichlorobenzene eq." $/ \mathrm{kg}$

21000 kg 1,4-dichlorobenzene eq." $/ \mathrm{kg}$

$410 \mathrm{~kg}$ 1,4-dichlorobenzene eq. "/ $/ \mathrm{kg}$

$5.40 \mathrm{E}+06$ kg 1,4-dichlorobenzene eq." $/ \mathrm{kg}$

$8.90 \mathrm{E}+05 \mathrm{~kg}$ 1,4-dichlorobenzene eq." $/ \mathrm{kg}$

$3.40 \mathrm{E}+05 \mathrm{~kg}$ 1,4-dichlorobenzene eq. "/kg

$630 \mathrm{~kg}$ 1,4-dichlorobenzene eq." $/ \mathrm{kg}$

19000 kg 1,4-dichlorobenzene eq." $/ \mathrm{kg}$

920 kg 1,4-dichlorobenzene eq."/kg

$86000 \mathrm{~kg}$ 1,4-dichlorobenzene eq." $/ \mathrm{kg}$

$3500 \mathrm{~kg}$ 1,4-dichlorobenzene eq."/kg

$9.1 \mathrm{~kg}$ 1,4-dichlorobenzene eq." $/ \mathrm{kg}$

$2.6 \mathrm{~kg}$ 1,4-dichlorobenzene eq." $/ \mathrm{kg}$

$2.4 \mathrm{~kg}$ 1,4-dichlorobenzene eq." $/ \mathrm{kg}$

$120 \mathrm{~kg}$ 1,4-dichlorobenzene eq." $/ \mathrm{kg}$

$0.44 \mathrm{~kg} 1,4$-dichlorobenzene eq." $/ \mathrm{kg}$

$0.0038 \mathrm{~kg}$ 1,4-dichlorobenzene eq." $/ \mathrm{kg}$

$0.062 \mathrm{~kg} 1,4$-dichlorobenzene eq." $/ \mathrm{kg}$

$410 \mathrm{~kg}$ 1,4-dichlorobenzene eq." $/ \mathrm{kg}$

$5200 \mathrm{~kg}$ 1,4-dichlorobenzene eq."/kg

$0.34 \mathrm{~kg}$ 1,4-dichlorobenzene eq." $/ \mathrm{kg}$

$1.7 \mathrm{~kg}$ 1,4-dichlorobenzene eq." $/ \mathrm{kg}$

$4.7 \mathrm{~kg}$ 1,4-dichlorobenzene eq." $/ \mathrm{kg}$

$3.6 \mathrm{~kg}$ 1,4-dichlorobenzene eq." $/ \mathrm{kg}$

$1.6 \mathrm{~kg}$ 1,4-dichlorobenzene eq." $/ \mathrm{kg}$

$0.027 \mathrm{~kg}$ 1,4-dichlorobenzene eq." $/ \mathrm{kg}$

$4600 \mathrm{~kg}$ 1,4-dichlorobenzene eq."/kg

$7300 \mathrm{~kg}$ 1,4-dichlorobenzene eq." $/ \mathrm{kg}$

$0.54 \mathrm{~kg}$ 1,4-dichlorobenzene eq." $/ \mathrm{kg}$

$20 \mathrm{~kg}$ 1,4-dichlorobenzene eq." $/ \mathrm{kg}$

20 kg 1,4-dichlorobenzene eq." $/ \mathrm{kg}$

$1.3 \mathrm{~kg}$ 1,4-dichlorobenzene eq." $/ \mathrm{kg}$

$19 \mathrm{~kg}$ 1,4-dichlorobenzene eq."//kg

49000 kg 1,4-dichlorobenzene eq." $/ \mathrm{kg}$

$710 \mathrm{~kg}$ 1,4-dichlorobenzene eq." $/ \mathrm{kg}$

$0.0008 \mathrm{~kg}$ 1,4-dichlorobenzene eq." $/ \mathrm{kg}$

7.90E-11 kg 1,4-dichlorobenzene eq." $/ \mathrm{kg}$

1500 kg 1,4-dichlorobenzene eq." $/ \mathrm{kg}$

1600 kg 1,4-dichlorobenzene eq."/kg

21000 kg 1,4-dichlorobenzene eq."/kg

47000 kg 1,4-dichlorobenzene eq."/kg

20000 kg 1,4-dichlorobenzene eq."/kg

200 kg 1,4-dichlorobenzene eq." $/ \mathrm{kg}$

$2300 \mathrm{~kg}$ 1,4-dichlorobenzene eq." $/ \mathrm{kg}$

$1.6 \mathrm{~kg}$ 1,4-dichlorobenzene eq." $/ \mathrm{kg}$

17 kg 1,4-dichlorobenzene eq." $/ \mathrm{kg}$

2.9 kg 1,4-dichlorobenzene eq. "/kg

$78 \mathrm{~kg} 1,4$-dichlorobenzene eq. "/kg

77000 kg 1,4-dichlorobenzene eq." $/ \mathrm{kg}$

2400 kg 1,4-dichlorobenzene eq."/kg

$7300 \mathrm{~kg}$ 1,4-dichlorobenzene eq." $/ \mathrm{kg}$

$0.32 \mathrm{~kg}$ 1,4-dichlorobenzene eq." $/ \mathrm{kg}$

$32 \mathrm{~kg} \mathrm{1,4-dichlorobenzene} \mathrm{eq."} / \mathrm{kg}$

7000 kg 1,4-dichlorobenzene eq." $/ \mathrm{kg}$

$52 \mathrm{~kg}$ 1,4-dichlorobenzene eq." $/ \mathrm{kg}$

27 kg 1,4-dichlorobenzene eq." $/ \mathrm{kg}$

1400 kg 1,4-dichlorobenzene eq."/kg

$0.28 \mathrm{~kg}$ 1,4-dichlorobenzene eq." $/ \mathrm{kg}$

$4.1 \mathrm{~kg}$ 1,4-dichlorobenzene eq."//kg

$1.20 \mathrm{E}+06 \mathrm{~kg}$ 1,4-dichlorobenzene eq." $/ \mathrm{kg}$ 
[E595] metamitron to air

[E596] meta-Xylene (1,3-dimethylbenzene) to air

[E597] metazachlor to air

[E598] methabenzthiazuron to air

[E603] methomyl to air

[E626] methyl-mercury to air

[E628] metobromuron to air

[E629] metolachlor to air

[E630] mevinphos to air

[E632] molybdenum to air

[E633] Naphtalene to air

[E635] nickel to air

[E650] ortho-Xylene (1,2-dimethylbenzene) to air

[E651] oxamyl to air

[E652] oxydemethon-methyl to air

[E655] parathion-ethyl to air

[E656] parathion-methyl to air

[E658] para-Xylene (1,4-dimethylbenzene) to air

[E660] pentachlorobenzene to air

[E661] pentachloronitrobenzene to air

[E662] pentachlorophenol to air

[E672] permethrin to air

[E674] phenanthrene to air

[E675] Phenol to air

[E679] phoxim to air

[E680] Phtalic anhydride to air

[E682] pirimicarb to air

[E685] Polycyclic Aromatic Hydrocarbons Carcinogenic- (carcinogenic-PAH) to air

[E686] propachlor to air

[E690] propoxur to air

[E697] Propylene Oxide to air

[E698] pyrazophos to air

[E704] selenium to air

[E705] simazine to air

[E706] styrene (vinylbenzene) to air

[E718] tetrachloroethylene (PER) (tetrachloroethene) to air

[E719] Tetrachloromethane (carbon tetrachloride) $(\mathrm{HC}-10)$ to air

[E722] thallium to air

[E723] Thiram to air

[E724] tin to air

[E725] tolclophos-methyl to air

[E727] Toluene to air

[E733] tri-allate to air

[E734] triazophos to air

[E735] tributyltinoxide to air

[E736] trichlorfon to air

[E737] Trichloroethylene (tri) to air

[E738] Trichloromethane (chloroform) to air

[E739] trifluarin to air

[E743] vanadium to air

[E745] Vinyl Chloride (chloroethene) to air

[E748] zinc to air

[E749] zineb to air

[E750] 1,1,1-trichloroethane to air

[E751] 1,2,3,4-tetrachlorobenzene to air

[E752] 1,2,3,5-tetrachlorobenzene to air

[E753] 1,2,3-trichlorobenzene to air

[E754] 1,2,4,5-tetrachlorobenzene to air

[E755] 1,2,4-trichlorobenzene to air

[E756] 1,2-dichlorobenzene to air

[E757] 1,2-dichloroethane to air

[E758] 1,3,5-trichlorobenzene to air

[E759] 1,3-butadiene to air

[E760] 1,3-dichlorobenzene to air

[E761] 1,4-dichlorobenzene to air

[E762] 1-chloro-4-nitrobenzene to air

[E763] 2,3,4,6-tetrachlorophenol to air
$0.25 \mathrm{~kg}$ 1,4-dichlorobenzene eq." $/ \mathrm{kg}$

$0.00039 \mathrm{~kg}$ 1,4-dichlorobenzene eq." $/ \mathrm{kg}$

$2.2 \mathrm{~kg}$ 1,4-dichlorobenzene eq." $/ \mathrm{kg}$

$25 \mathrm{~kg}$ 1,4-dichlorobenzene eq." $/ \mathrm{kg}$

$3900 \mathrm{~kg}$ 1,4-dichlorobenzene eq." $/ \mathrm{kg}$

$2.80 \mathrm{E}+07 \mathrm{~kg}$ 1,4-dichlorobenzene eq."/kg

$42 \mathrm{~kg}$ 1,4-dichlorobenzene eq." $/ \mathrm{kg}$

$380 \mathrm{~kg}$ 1,4-dichlorobenzene eq." $/ \mathrm{kg}$

$5400 \mathrm{~kg}$ 1,4-dichlorobenzene eq." $/ \mathrm{kg}$

$1.90 \mathrm{E}+06 \mathrm{~kg}$ 1,4-dichlorobenzene eq."/kg

$0.91 \mathrm{~kg}$ 1,4-dichlorobenzene eq." $/ \mathrm{kg}$

$3.80 \mathrm{E}+06 \mathrm{~kg}$ 1,4-dichlorobenzene eq. "/kg

$0.00091 \mathrm{~kg} \mathrm{1,4-dichlorobenzene} \mathrm{eq."} / \mathrm{kg}$

$1.4 \mathrm{~kg}$ 1,4-dichlorobenzene eq." $/ \mathrm{kg}$

$500 \mathrm{~kg}$ 1,4-dichlorobenzene eq." $/ \mathrm{kg}$

$3100 \mathrm{~kg}$ 1,4-dichlorobenzene eq."/kg

720 kg 1,4-dichlorobenzene eq."//kg

$0.00061 \mathrm{~kg}$ 1,4-dichlorobenzene eq." $/ \mathrm{kg}$

$170 \mathrm{~kg}$ 1,4-dichlorobenzene eq."/kg

$6000 \mathrm{~kg}$ 1,4-dichlorobenzene eq." $/ \mathrm{kg}$

$40 \mathrm{~kg}$ 1,4-dichlorobenzene eq." $/ \mathrm{kg}$

$31000 \mathrm{~kg}$ 1,4-dichlorobenzene eq." $/ \mathrm{kg}$

$7.3 \mathrm{~kg}$ 1,4-dichlorobenzene eq." $/ \mathrm{kg}$

$0.55 \mathrm{~kg}$ 1,4-dichlorobenzene eq." $/ \mathrm{kg}$

$1.6 \mathrm{~kg}$ 1,4-dichlorobenzene eq." $/ \mathrm{kg}$

$0.0085 \mathrm{~kg}$ 1,4-dichlorobenzene eq." $/ \mathrm{kg}$

$410 \mathrm{~kg}$ 1,4-dichlorobenzene eq." $/ \mathrm{kg}$

$4300 \mathrm{~kg}$ 1,4-dichlorobenzene eq."/kg

$7.1 \mathrm{~kg}$ 1,4-dichlorobenzene eq." $/ \mathrm{kg}$

$1800 \mathrm{~kg}$ 1,4-dichlorobenzene eq." $/ \mathrm{kg}$

$0.14 \mathrm{~kg}$ 1,4-dichlorobenzene eq." $/ \mathrm{kg}$

$94 \mathrm{~kg}$ 1,4-dichlorobenzene eq. "/kg

2.10E+07 kg 1,4-dichlorobenzene eq." $/ \mathrm{kg}$

$280 \mathrm{~kg}$ 1,4-dichlorobenzene eq." $/ \mathrm{kg}$

$0.00051 \mathrm{~kg}$ 1,4-dichlorobenzene eq." $/ \mathrm{kg}$

$0.34 \mathrm{~kg}$ 1,4-dichlorobenzene eq." $/ \mathrm{kg}$

$1.2 \mathrm{~kg}$ 1,4-dichlorobenzene eq." $/ \mathrm{kg}$

2.60E+07 kg 1,4-dichlorobenzene eq." $/ \mathrm{kg}$

310 kg 1,4-dichlorobenzene eq." $/ \mathrm{kg}$

$7500 \mathrm{~kg} \mathrm{1,4-dichlorobenzene} \mathrm{eq."} / \mathrm{kg}$

$1.4 \mathrm{~kg}$ 1,4-dichlorobenzene eq." $/ \mathrm{kg}$

$0.0007 \mathrm{~kg}$ 1,4-dichlorobenzene eq." $/ \mathrm{kg}$

$150 \mathrm{~kg}$ 1,4-dichlorobenzene eq." $/ \mathrm{kg}$

850 kg 1,4-dichlorobenzene eq." $/ \mathrm{kg}$

3.10E+05 kg 1,4-dichlorobenzene eq." $/ \mathrm{kg}$

$1800 \mathrm{~kg}$ 1,4-dichlorobenzene eq." $/ \mathrm{kg}$

0.0027 kg 1,4-dichlorobenzene eq." $/ \mathrm{kg}$

$0.059 \mathrm{~kg}$ 1,4-dichlorobenzene eq." $/ \mathrm{kg}$

$100 \mathrm{~kg}$ 1,4-dichlorobenzene eq." $/ \mathrm{kg}$

1.20E+07 kg 1,4-dichlorobenzene eq." $/ \mathrm{kg}$

0.00013 kg 1,4-dichlorobenzene eq." $/ \mathrm{kg}$

67000 kg 1,4-dichlorobenzene eq."/kg

$410 \mathrm{~kg}$ 1,4-dichlorobenzene eq." $/ \mathrm{kg}$

$0.32 \mathrm{~kg}$ 1,4-dichlorobenzene eq." $/ \mathrm{kg}$

$16 \mathrm{~kg}$ 1,4-dichlorobenzene eq." $/ \mathrm{kg}$

$17 \mathrm{~kg}$ 1,4-dichlorobenzene eq." $/ \mathrm{kg}$

$2.1 \mathrm{~kg}$ 1,4-dichlorobenzene eq." $/ \mathrm{kg}$

$14 \mathrm{~kg}$ 1,4-dichlorobenzene eq." $/ \mathrm{kg}$

$2 \mathrm{~kg}$ 1,4-dichlorobenzene eq." $/ \mathrm{kg}$

$0.66 \mathrm{~kg} 1,4$-dichlorobenzene eq." $/ \mathrm{kg}$

$0.081 \mathrm{~kg}$ 1,4-dichlorobenzene eq." $/ \mathrm{kg}$

$3 \mathrm{~kg}$ 1,4-dichlorobenzene eq."/kg

$0.0087 \mathrm{~kg} 1,4$-dichlorobenzene eq." $/ \mathrm{kg}$

$0.46 \mathrm{~kg}$ 1,4-dichlorobenzene eq." $/ \mathrm{kg}$

$0.73 \mathrm{~kg}$ 1,4-dichlorobenzene eq." $/ \mathrm{kg}$

$370 \mathrm{~kg}$ 1,4-dichlorobenzene eq."/kg

$91 \mathrm{~kg}$ 1,4-dichlorobenzene eq." $/ \mathrm{kg}$ 
[E787] barium to fresh water

[E788] benomyl to fresh water

[E789] bentazone to fresh water

[E790] benzene to fresh water

[E791] benzo[a]anthracene to fresh water

[E792] benzo[a]pyrene to fresh water

[E793] benzo[ghi]perylene to fresh water

[E794] benzo[k]fluoranthrene to fresh water

[E795] benzylchloride to fresh water

[E796] beryllium to fresh water

[E797] bifenthrin to fresh water

[E800] Butylbenzylphtalate to fresh water

[E801] cadmium to fresh water

[E802] captafol to fresh water

[E803] captan to fresh water

[E804] carbaryl to fresh water

[E805] carbendazim to fresh water

[E806] carbofuran to fresh water

[E807] carbon disulfide to fresh water

[E808] Carcinogenic PAHs to fresh water

[E810] chlordane to fresh water

[E811] chlorfenvinphos to fresh water

[E812] chloridazon to fresh water

[E814] chlorobenzene to fresh water

[E815] chlorothalonil to fresh water

[E816] chlorpropham to fresh water

[E817] chlorpyriphos to fresh water

[E818] chromium III to fresh water

[E819] chromium VI to fresh water

[E820] chrysene to fresh water

[E821] cobalt to fresh water

[E822] copper to fresh water

[E823] coumaphos to fresh water

[E824] cyanazine to fresh water

[E825] cypermethrin to fresh water

[E826] cyromazine to fresh water

[E827] DDT to fresh water

[E828] deltamethrin to fresh water

[E829] demeton to fresh water

[E830] desmetryn to fresh water

[E831] Di(2-ethylhexyl)phtalate to fresh water

[E832] diazinon to fresh water

[E833] Dibutylphtalate to fresh water

[E834] Dichloromethane (Methylene Chloride) to fresh water

[E835] dichlorprop to fresh water

4.50E+07 kg 1,4-dichlorobenzene eq." $/ \mathrm{kg}$

$0.061 \mathrm{~kg} 1,4$-dichlorobenzene eq." $/ \mathrm{kg}$

$64 \mathrm{~kg} \mathrm{1,4-dichlorobenzene} \mathrm{eq."} / \mathrm{kg}$

$1.6 \mathrm{~kg}$ 1,4-dichlorobenzene eq." $/ \mathrm{kg}$

$2.3 \mathrm{~kg}$ 1,4-dichlorobenzene eq." $/ \mathrm{kg}$

$0.25 \mathrm{~kg}$ 1,4-dichlorobenzene eq." $/ \mathrm{kg}$

$13 \mathrm{~kg}$ 1,4-dichlorobenzene eq." $/ \mathrm{kg}$

$2800 \mathrm{~kg}$ 1,4-dichlorobenzene eq." $/ \mathrm{kg}$

$11 \mathrm{~kg} \mathrm{1,4-dichlorobenzene} \mathrm{eq."/kg}$

$14 \mathrm{~kg}$ 1,4-dichlorobenzene eq." $/ \mathrm{kg}$

$15 \mathrm{~kg}$ 1,4-dichlorobenzene eq." $/ \mathrm{kg}$

$1100 \mathrm{~kg}$ 1,4-dichlorobenzene eq." $/ \mathrm{kg}$

$0.54 \mathrm{~kg}$ 1,4-dichlorobenzene eq." $/ \mathrm{kg}$

$7400 \mathrm{~kg}$ 1,4-dichlorobenzene eq."/kg

$210 \mathrm{~kg}$ 1,4-dichlorobenzene eq." $/ \mathrm{kg}$

$0.25 \mathrm{~kg}$ 1,4-dichlorobenzene eq." $/ \mathrm{kg}$

$3000 \mathrm{~kg}$ 1,4-dichlorobenzene eq." $/ \mathrm{kg}$

$27000 \mathrm{~kg}$ 1,4-dichlorobenzene eq." $/ \mathrm{kg}$

1.20E+05 kg 1,4-dichlorobenzene eq." $/ \mathrm{kg}$

490 kg 1,4-dichlorobenzene eq." $/ \mathrm{kg}$

$1000 \mathrm{~kg}$ 1,4-dichlorobenzene eq."/kg

$35 \mathrm{~kg}$ 1,4-dichlorobenzene eq."/kg

8.30E+05 kg 1,4-dichlorobenzene eq." $/ \mathrm{kg}$

$8.6 \mathrm{~kg}$ 1,4-dichlorobenzene eq." $/ \mathrm{kg}$

$0.22 \mathrm{~kg}$ 1,4-dichlorobenzene eq." $/ \mathrm{kg}$

$0.0027 \mathrm{~kg}$ 1,4-dichlorobenzene eq." $/ \mathrm{kg}$

$8300 \mathrm{~kg} \mathrm{1,4-dichlorobenzene} \mathrm{eq."/kg}$

12000 kg 1,4-dichlorobenzene eq." $/ \mathrm{kg}$

$9100 \mathrm{~kg} \mathrm{1,4-dichlorobenzene} \mathrm{eq."} / \mathrm{kg}$

4.40E+05 kg 1,4-dichlorobenzene eq." $/ \mathrm{kg}$

$1.2 \mathrm{~kg}$ 1,4-dichlorobenzene eq." $/ \mathrm{kg}$

5.40E+08 kg 1,4-dichlorobenzene eq." $/ \mathrm{kg}$

$210 \mathrm{~kg}$ 1,4-dichlorobenzene eq." $/ \mathrm{kg}$

$0.053 \mathrm{~kg}$ 1,4-dichlorobenzene eq." $/ \mathrm{kg}$

$2.20 \mathrm{E}+05 \mathrm{~kg}$ 1,4-dichlorobenzene eq." $/ \mathrm{kg}$ 80000 kg 1,4-dichlorobenzene eq. "/kg

$0.1 \mathrm{~kg}$ 1,4-dichlorobenzene eq." $/ \mathrm{kg}$

$1.4 \mathrm{~kg}$ 1,4-dichlorobenzene eq." $/ \mathrm{kg}$

$580 \mathrm{~kg}$ 1,4-dichlorobenzene eq." $/ \mathrm{kg}$

$44 \mathrm{~kg}$ 1,4-dichlorobenzene eq." $/ \mathrm{kg}$

$1.8 \mathrm{~kg}$ 1,4-dichlorobenzene eq." $/ \mathrm{kg}$

5500 kg 1,4-dichlorobenzene eq." $/ \mathrm{kg}$ 8900 kg 1,4-dichlorobenzene eq." $/ \mathrm{kg}$

5.7 kg 1,4-dichlorobenzene eq." $/ \mathrm{kg}$

$1.2 \mathrm{~kg}$ 1,4-dichlorobenzene eq." $/ \mathrm{kg}$

$0.11 \mathrm{~kg} \mathrm{1,4-dichlorobenzene} \mathrm{eq."} / \mathrm{kg}$

$40 \mathrm{~kg}$ 1,4-dichlorobenzene eq."/kg

$0.35 \mathrm{~kg}$ 1,4-dichlorobenzene eq." $/ \mathrm{kg}$

$240 \mathrm{~kg}$ 1,4-dichlorobenzene eq." $/ \mathrm{kg}$

860 kg 1,4-dichlorobenzene eq." $/ \mathrm{kg}$

$3400 \mathrm{~kg}$ 1,4-dichlorobenzene eq." $/ \mathrm{kg}$

$3000 \mathrm{~kg}$ 1,4-dichlorobenzene eq." $/ \mathrm{kg}$

4.40E+06 kg 1,4-dichlorobenzene eq." $/ \mathrm{kg}$

$2.30 \mathrm{E}+05 \mathrm{~kg}$ 1,4-dichlorobenzene eq." $/ \mathrm{kg}$

$3.00 \mathrm{E}+06 \mathrm{~kg}$ 1,4-dichlorobenzene eq." $/ \mathrm{kg}$

$190 \mathrm{~kg}$ 1,4-dichlorobenzene eq." $/ \mathrm{kg}$

10000 kg 1,4-dichlorobenzene eq." $/ \mathrm{kg}$

$1000 \mathrm{~kg}$ 1,4-dichlorobenzene eq." $/ \mathrm{kg}$

$4400 \mathrm{~kg}$ 1,4-dichlorobenzene eq." $/ \mathrm{kg}$

980 kg 1,4-dichlorobenzene eq." $/ \mathrm{kg}$

96 kg 1,4-dichlorobenzene eq." $/ \mathrm{kg}$

$1.5 \mathrm{~kg}$ 1,4-dichlorobenzene eq." $/ \mathrm{kg}$

$0.37 \mathrm{~kg}$ 1,4-dichlorobenzene eq." $/ \mathrm{kg}$

$640 \mathrm{~kg}$ 1,4-dichlorobenzene eq." $/ \mathrm{kg}$

$0.077 \mathrm{~kg}$ 1,4-dichlorobenzene eq." $/ \mathrm{kg}$

$0.0035 \mathrm{~kg} 1,4$-dichlorobenzene eq." $/ \mathrm{kg}$

0.015 kg 1,4-dichlorobenzene eq."/kg 
[E855] fenitrothion to fresh water

[E856] fenthion to fresh water

[E857] fentin acetate to fresh water

[E858] fentin chloride to fresh water

[E859] fentin hydroxide to fresh water

[E860] fluoranthrene to fresh water

[E861] folpet to fresh water

[E862] Formaldehyde (methanal) to fresh water

[E863] glyphosate to fresh water

[E864] heptachlor to fresh water

[E865] heptenophos to fresh water

[E866] hexachloro-1,3-butadiene to fresh water

[E867] hexachlorobenzene to fresh water

[E870] indeno[1,2,3-cd]pyrene to fresh water

[E871] iprodione to fresh water

[E872] isoproturon to fresh water

[E873] lead to fresh water

[E874] lindane to fresh water

[E875] linuron to fresh water

[E876] malathion to fresh water

[E878] MCPA to fresh water

[E879] mecoprop to fresh water

[E880] mercury to fresh water

[E881] metamitron to fresh water

[E882] metazachlor to fresh water

[E883] methabenzthiazuron to fresh water

[E884] methomyl to fresh water

[E885] methylbromide to fresh water

[E886] methyl-mercury to fresh water

[E887] metobromuron to fresh water

[E888] metolachlor to fresh water

[E889] mevinphos to fresh water

[E890] molybdenum to fresh water

[E891] m-xylene to fresh water

[E892] naphtalene to fresh water

[E893] nickel to fresh water

[E899] oxamyl to fresh water

[E900] oxydemethon-methyl to fresh water

[E901] o-xylene to fresh water

[E902] parathion-ethyl to fresh water

[E903] parathion-methyl to fresh water

[E904] pentachlorobenzene to fresh water

[E905] pentachloronitrobenzene to fresh water

[E906] pentachlorophenol to fresh water

[E907] permethrin to fresh water

[E908] phenanthrene to fresh water

[E909] phenol to fresh water

[E912] phoxim to fresh water

12 kg 1,4-dichlorobenzene eq."/kg 9000 kg 1,4-dichlorobenzene eq."/kg $0.11 \mathrm{~kg} \mathrm{1,4-dichlorobenzene} \mathrm{eq."/kg}$

1.2 kg 1,4-dichlorobenzene eq."/kg

$2.3 \mathrm{~kg}$ 1,4-dichlorobenzene eq."/kg

0.43 kg 1,4-dichlorobenzene eq."/kg

0.75 kg 1,4-dichlorobenzene eq." $/ \mathrm{kg}$

0.0017 kg 1,4-dichlorobenzene eq." $/ \mathrm{kg}$ 5900 kg 1,4-dichlorobenzene eq."/kg 5400 kg 1,4-dichlorobenzene eq." $/ \mathrm{kg}$

0.035 kg 1,4-dichlorobenzene eq." /kg

120 kg 1,4-dichlorobenzene eq."/kg

120 kg 1,4-dichlorobenzene eq."/kg

0.34 kg 1,4-dichlorobenzene eq."/kg

$11 \mathrm{~kg}$ 1,4-dichlorobenzene eq."/kg

3.40E+05 kg 1,4-dichlorobenzene eq." $/ \mathrm{kg}$ 3500 kg 1,4-dichlorobenzene eq."/kg 0.0014 kg 1,4-dichlorobenzene eq." $/ \mathrm{kg}$

2.80E-05 kg 1,4-dichlorobenzene eq."/kg 670 kg 1,4-dichlorobenzene eq."/kg 3600 kg 1,4-dichlorobenzene eq." $/ \mathrm{kg}$ 3200 kg 1,4-dichlorobenzene eq." $/ \mathrm{kg}$ 19000 kg 1,4-dichlorobenzene eq."/kg 3100 kg 1,4-dichlorobenzene eq."/kg 870 kg 1,4-dichlorobenzene eq."/kg 12000 kg 1,4-dichlorobenzene eq."/kg 0.19 kg 1,4-dichlorobenzene eq."/kg 4.2 kg 1,4-dichlorobenzene eq." $/ \mathrm{kg}$

12 kg 1,4-dichlorobenzene eq."/kg

11 kg 1,4-dichlorobenzene eq."/kg 75000 kg 1,4-dichlorobenzene eq." $/ \mathrm{kg}$ 2400 kg 1,4-dichlorobenzene eq."/kg 15000 kg 1,4-dichlorobenzene eq."/kg 0.015 kg 1,4-dichlorobenzene eq."/kg

$20 \mathrm{~kg}$ 1,4-dichlorobenzene eq." $/ \mathrm{kg}$

1100 kg 1,4-dichlorobenzene eq."/kg

88 kg 1,4-dichlorobenzene eq." $/ \mathrm{kg}$ 560 kg 1,4-dichlorobenzene eq."/kg 770 kg 1,4-dichlorobenzene eq."/kg 0.036 kg 1,4-dichlorobenzene eq." $/ \mathrm{kg}$

$0.67 \mathrm{~kg} \mathrm{1,4-dichlorobenzene} \mathrm{eq."} / \mathrm{kg}$ 2.10E+05 kg 1,4-dichlorobenzene eq." $/ \mathrm{kg}$ 0.063 kg 1,4-dichlorobenzene eq." $/ \mathrm{kg}$ $1.3 \mathrm{~kg} \mathrm{1,4-dichlorobenzene} \mathrm{eq."/kg}$ 25 kg 1,4-dichlorobenzene eq."/kg 4200 kg 1,4-dichlorobenzene eq." $/ \mathrm{kg}$

$3.5 \mathrm{~kg} \mathrm{1,4-dichlorobenzene} \mathrm{eq."/kg}$

4.90E+06 kg 1,4-dichlorobenzene eq."/kg

64 kg 1,4-dichlorobenzene eq."/kg 580 kg 1,4-dichlorobenzene eq." $/ \mathrm{kg}$ 570 kg 1,4-dichlorobenzene eq." $/ \mathrm{kg}$

2.10E+06 kg 1,4-dichlorobenzene eq."/kg 0.0021 kg 1,4-dichlorobenzene eq." $/ \mathrm{kg}$

$1.1 \mathrm{~kg} \mathrm{1,4-dichlorobenzene} \mathrm{eq."} / \mathrm{kg}$

2.20E+06 kg 1,4-dichlorobenzene eq." $/ \mathrm{kg}$ $0.18 \mathrm{~kg}$ 1,4-dichlorobenzene eq." $/ \mathrm{kg}$ 140 kg 1,4-dichlorobenzene eq."/kg 0.0025 kg 1,4-dichlorobenzene eq."/kg 5300 kg 1,4-dichlorobenzene eq."/kg 1500 kg 1,4-dichlorobenzene eq."/kg 170 kg 1,4-dichlorobenzene eq."/kg 2800 kg 1,4-dichlorobenzene eq."/kg 12 kg 1,4-dichlorobenzene eq."/kg 27000 kg 1,4-dichlorobenzene eq."/kg

$10 \mathrm{~kg}$ 1,4-dichlorobenzene eq." $/ \mathrm{kg}$ 0.056 kg 1,4-dichlorobenzene eq."/kg 5 kg 1,4-dichlorobenzene eq."/kg 
[E913] Phtalic anhydride to fresh water [E914] pirimicarb to fresh water

[E916] propachlor to fresh water

[E917] propoxur to fresh water

[E918] propylene oxide to fresh water

[E919] p-xylene to fresh water

[E920] pyrazophos to fresh water

[E921] selenium to fresh water

[E922] simazine to fresh water

[E923] styrene (vinylbenzene) to fresh water

[E926] tetrachloroethylene (PER) to fresh water

[E927] Tetrachloromethane (carbon tetrachloride) (HC-10) to fresh water

[E928] thallium to fresh water

[E929] Thiram to fresh water

[E930] tin to fresh water

[E931] tolclophos-methyl to fresh water

[E932] toluene to fresh water

[E933] tri-allate to fresh water

[E934] triazophos to fresh water

[E935] tributyltinoxide to fresh water

[E936] trichlorfon to fresh water

[E937] Trichloroethylene (tri) to fresh water

[E938] Trichloromethane $=$ chloroform to fresh water

[E939] trifluarin to fresh water

[E940] vanadium to fresh water

[E941] Vinyl Chloride (chloroethene) to fresh water

[E942] zinc to fresh water

[E943] zineb to fresh water

[E944] 1,1,1-trichloroethane to fresh water

[E945] 1,2,3,4-tetrachlorobenzene to fresh water

[E946] 1,2,3,5-tetrachlorobenzene to fresh water

[E947] 1,2,3-trichlorobenzene to fresh water

[E948] 1,2,4,5-tetrachlorobenzene to fresh water

[E949] 1,2,4-trichlorobenzene to fresh water

[E950] 1,2-dichlorobenzene to fresh water

[E951] 1,2-dichloroethane to fresh water

[E952] 1,3,5-trichlorobenzene to fresh water

[E953] 1,3-butadiene to fresh water

[E954] 1,3-dichlorobenzene to fresh water

[E955] 1,4-dichlorobenzene to fresh water

[E956] 1-chloro-4-nitrobenzene to fresh water

[E957] 2,3,4,6-tetrachlorophenol to fresh water

[E958] 2,3,7,8-TCDD to fresh water

[E959] 2,4,5-T to fresh water

[E960] 2,4,5-trichlorophenol to fresh water

[E961] 2,4,6-trichlorophenol to sea water

[E962] 2,4-D to sea water

[E963] 2,4-dichlorophenol to sea water

[E964] 2-chlorophenol to sea water

[E965] 3,4-dichloroaniline to sea water

[E966] 3-chloroaniline to sea water

[E967] 4-chloroaniline to sea water

[E968] acephate to sea water

[E969] Acrolein (2-propenal) to sea water

[E970] acrylonitrile to sea water

[E971] aldicarb to sea water

[E972] aldrin to sea water

[E974] anilazine to sea water

[E975] anthracene to sea water

[E976] antimony to sea water

[E977] arsenic to sea water

[E978] atrazine to sea water

[E979] azinphos-ethyl to sea water

[E980] azinphos-methyl to sea water

[E981] barium to sea water

[E982] benomyl to sea water

[E983] bentazone to sea water
4.10E-06 kg 1,4-dichlorobenzene eq."//kg $160 \mathrm{~kg}$ 1,4-dichlorobenzene eq." $/ \mathrm{kg}$ $2.4 \mathrm{~kg}$ 1,4-dichlorobenzene eq." $/ \mathrm{kg}$ $500 \mathrm{~kg}$ 1,4-dichlorobenzene eq." $/ \mathrm{kg}$ 0.064 kg 1,4-dichlorobenzene eq." $/ \mathrm{kg}$

$0.0022 \mathrm{~kg}$ 1,4-dichlorobenzene eq." $/ \mathrm{kg}$

$120 \mathrm{~kg}$ 1,4-dichlorobenzene eq." $/ \mathrm{kg}$

$2.50 \mathrm{E}+07 \mathrm{~kg}$ 1,4-dichlorobenzene eq."/kg

$140 \mathrm{~kg}$ 1,4-dichlorobenzene eq." $/ \mathrm{kg}$

$0.0022 \mathrm{~kg}$ 1,4-dichlorobenzene eq." $/ \mathrm{kg}$

$0.34 \mathrm{~kg}$ 1,4-dichlorobenzene eq." $/ \mathrm{kg}$

$1.1 \mathrm{~kg}$ 1,4-dichlorobenzene eq." $/ \mathrm{kg}$

2.70E+07 kg 1,4-dichlorobenzene eq." $/ \mathrm{kg}$

$75 \mathrm{~kg}$ 1,4-dichlorobenzene eq." $/ \mathrm{kg}$

$1200 \mathrm{~kg}$ 1,4-dichlorobenzene eq." $/ \mathrm{kg}$

$4.4 \mathrm{~kg}$ 1,4-dichlorobenzene eq." $/ \mathrm{kg}$

$0.0012 \mathrm{~kg} \mathrm{1,4-dichlorobenzene} \mathrm{eq."} / \mathrm{kg}$

$780 \mathrm{~kg}$ 1,4-dichlorobenzene eq." $/ \mathrm{kg}$

$1500 \mathrm{~kg}$ 1,4-dichlorobenzene eq."//kg

2.10E+05 kg 1,4-dichlorobenzene eq." $/ \mathrm{kg}$

$83 \mathrm{~kg}$ 1,4-dichlorobenzene eq." $/ \mathrm{kg}$

$0.0033 \mathrm{~kg}$ 1,4-dichlorobenzene eq." $/ \mathrm{kg}$

$0.058 \mathrm{~kg}$ 1,4-dichlorobenzene eq."/kg

$420 \mathrm{~kg}$ 1,4-dichlorobenzene eq." $/ \mathrm{kg}$

$8.60 \mathrm{E}+06 \mathrm{~kg}$ 1,4-dichlorobenzene eq." $/ \mathrm{kg}$

$0.00038 \mathrm{~kg}$ 1,4-dichlorobenzene eq." $/ \mathrm{kg}$

$14000 \mathrm{~kg} \mathrm{1,4-dichlorobenzene} \mathrm{eq."/kg}$

$250 \mathrm{~kg}$ 1,4-dichlorobenzene eq." $/ \mathrm{kg}$

$0.28 \mathrm{~kg}$ 1,4-dichlorobenzene eq." $/ \mathrm{kg}$

$15 \mathrm{~kg}$ 1,4-dichlorobenzene eq." $/ \mathrm{kg}$

$16 \mathrm{~kg}$ 1,4-dichlorobenzene eq." $/ \mathrm{kg}$

$3.6 \mathrm{~kg}$ 1,4-dichlorobenzene eq." $/ \mathrm{kg}$

$13 \mathrm{~kg}$ 1,4-dichlorobenzene eq." $/ \mathrm{kg}$

$3.1 \mathrm{~kg}$ 1,4-dichlorobenzene eq." $/ \mathrm{kg}$

$0.95 \mathrm{~kg}$ 1,4-dichlorobenzene eq." $/ \mathrm{kg}$

$0.091 \mathrm{~kg}$ 1,4-dichlorobenzene eq." $/ \mathrm{kg}$

$4.5 \mathrm{~kg} \mathrm{1,4-dichlorobenzene} \mathrm{eq."} / \mathrm{kg}$

$0.73 \mathrm{~kg}$ 1,4-dichlorobenzene eq." $/ \mathrm{kg}$

$1 \mathrm{~kg}$ 1,4-dichlorobenzene eq." $/ \mathrm{kg}$

$1 \mathrm{~kg}$ 1,4-dichlorobenzene eq." $/ \mathrm{kg}$

$370 \mathrm{~kg}$ 1,4-dichlorobenzene eq." $/ \mathrm{kg}$

$220 \mathrm{~kg}$ 1,4-dichlorobenzene eq." $/ \mathrm{kg}$

5.00E+08 kg 1,4-dichlorobenzene eq." $/ \mathrm{kg}$

$0.4 \mathrm{~kg}$ 1,4-dichlorobenzene eq." $/ \mathrm{kg}$

120 kg 1,4-dichlorobenzene eq." $/ \mathrm{kg}$

$7.6 \mathrm{~kg}$ 1,4-dichlorobenzene eq." $/ \mathrm{kg}$

$10 \mathrm{~kg}$ 1,4-dichlorobenzene eq." $/ \mathrm{kg}$

$3.7 \mathrm{~kg}$ 1,4-dichlorobenzene eq." $/ \mathrm{kg}$

$46 \mathrm{~kg}$ 1,4-dichlorobenzene eq." $/ \mathrm{kg}$

$3300 \mathrm{~kg}$ 1,4-dichlorobenzene eq." $/ \mathrm{kg}$

$59 \mathrm{~kg}$ 1,4-dichlorobenzene eq." $/ \mathrm{kg}$

$96 \mathrm{~kg}$ 1,4-dichlorobenzene eq." $/ \mathrm{kg}$

$37 \mathrm{~kg}$ 1,4-dichlorobenzene eq." $/ \mathrm{kg}$

$8900 \mathrm{~kg}$ 1,4-dichlorobenzene eq." $/ \mathrm{kg}$

$3.1 \mathrm{~kg}$ 1,4-dichlorobenzene eq." $/ \mathrm{kg}$

$15000 \mathrm{~kg}$ 1,4-dichlorobenzene eq." $/ \mathrm{kg}$

8000 kg 1,4-dichlorobenzene eq." $/ \mathrm{kg}$

$20 \mathrm{~kg}$ 1,4-dichlorobenzene eq." $/ \mathrm{kg}$

$18000 \mathrm{~kg} \mathrm{1,4-dichlorobenzene} \mathrm{eq."} / \mathrm{kg}$

49000 kg 1,4-dichlorobenzene eq."/kg

$3.40 \mathrm{E}+05 \mathrm{~kg}$ 1,4-dichlorobenzene eq." $/ \mathrm{kg}$

$610 \mathrm{~kg}$ 1,4-dichlorobenzene eq." $/ \mathrm{kg}$

$5900 \mathrm{~kg}$ 1,4-dichlorobenzene eq." $/ \mathrm{kg}$

$1000 \mathrm{~kg}$ 1,4-dichlorobenzene eq." $/ \mathrm{kg}$

1.10E+06 kg 1,4-dichlorobenzene eq." $/ \mathrm{kg}$

$150 \mathrm{~kg}$ 1,4-dichlorobenzene eq." $/ \mathrm{kg}$

$1.2 \mathrm{~kg}$ 1,4-dichlorobenzene eq." $/ \mathrm{kg}$ 
[E984] benzene to sea water

[E985] benzo[a]anthracene to sea water [E986] benzo[a]pyrene to sea water

[E987] benzo[ghi]perylene to sea water

[E988] benzo[k]fluoranthrene to sea water

[E989] benzylchloride to sea water

[E990] beryllium to sea water

[E991] bifenthrin to sea water

[E994] Butylbenzylphtalate to sea water

[E995] cadmium to sea water

[E996] captafol to sea water

[E997] captan to sea water

[E998] carbaryl to sea water

[E999] carbendazim to sea water

[E1000] carbofuran to sea water

[E1001] carbon disulfide to sea water

[E1002] Carcinogenic PAHs to sea water

[E1004] chlordane to sea water

[E1005] chlorfenvinphos to sea water

[E1006] chloridazon to sea water

[E1008] chlorobenzene to sea water

[E1009] chlorothalonil to sea water

[E1010] chlorpropham to sea water

[E1011] chlorpyriphos to sea water

[E1012] chromium III to sea water

[E1013] chromium VI to sea water

[E1014] chrysene to sea water

[E1015] cobalt to sea water

[E1016] copper to sea water

[E1017] coumaphos to sea water

[E1018] cyanazine to sea water

[E1019] cypermethrin to sea water

[E1020] cyromazine to sea water

[E1021] DDT to sea water

[E1022] deltamethrin to sea water

[E1023] demeton to sea water

[E1024] desmetryn to sea water

[E1025] Di(2-ethylhexyl)phtalate to sea water

[E1026] diazinon to sea water

[E1027] Dibutylphtalate to sea water

[E1028] Dichloromethane (Methylene Chloride) to sea water

[E1029] dichlorprop to sea water

[E1030] dichlorvos to sea water

[E1031] dieldrin to sea water

[E1032] Diethylphtalate to sea water

[E1033] Dihexylphtalate to sea water

[E1034] Diisodecylphtalate to sea water

[E1035] Diisooctylphtalate to sea water

[E1036] dimethoate to sea water

[E1037] Dimethylphtalate to sea water

[E1038] dinoseb to sea water

[E1039] dinoterb to sea water

[E1040] Dioctylphtalate to sea water

[E1041] disulfothon to sea water

[E1042] diuron to sea water

[E1043] DNOC to sea water

[E1044] endosulfan to sea water

[E1045] endrin to sea water

[E1046] ethoprophos to sea water

[E1047] ethylbenzene to sea water

[E1048] ethylene to sea water

[E1049] fenitrothion to sea water

[E1050] fenthion to sea water

[E1051] fentin acetate to sea water

[E1052] fentin chloride to sea water

[E1053] fentin hydroxide to sea water

[E1054] fluoranthrene to sea water
0.015 kg 1,4-dichlorobenzene eq."/kg

85000 kg 1,4-dichlorobenzene eq."/kg

1.20E+05 kg 1,4-dichlorobenzene eq."/kg

65000 kg 1,4-dichlorobenzene eq."/kg

1.50E+06 kg 1,4-dichlorobenzene eq."/kg

$7.8 \mathrm{~kg} \mathrm{1,4-dichlorobenzene} \mathrm{eq."} / \mathrm{kg}$

6.40E+08 kg 1,4-dichlorobenzene eq."/kg

8900 kg 1,4-dichlorobenzene eq."/kg

$1.6 \mathrm{~kg} \mathrm{1,4-dichlorobenzene} \mathrm{eq."/kg}$

1.80E+06 kg 1,4-dichlorobenzene eq."/kg

94000 kg 1,4-dichlorobenzene eq."/kg

40 kg 1,4-dichlorobenzene eq."/kg

24 kg 1,4-dichlorobenzene eq."/kg

1300 kg 1,4-dichlorobenzene eq."/kg

300 kg 1,4-dichlorobenzene eq."/kg

30 kg 1,4-dichlorobenzene eq."/kg

24000 kg 1,4-dichlorobenzene eq."/kg

4.70E+05 kg 1,4-dichlorobenzene eq."/kg

28 kg 1,4-dichlorobenzene eq."/kg

8 kg 1,4-dichlorobenzene eq."/kg

0.35 kg 1,4-dichlorobenzene eq."/kg

36 kg 1,4-dichlorobenzene eq."/kg

2 kg 1,4-dichlorobenzene eq."/kg

2200 kg 1,4-dichlorobenzene eq."/kg

8200 kg 1,4-dichlorobenzene eq."/kg

33000 kg 1,4-dichlorobenzene eq."/kg

7600 kg 1,4-dichlorobenzene eq."/kg

8.00E+06 kg 1,4-dichlorobenzene eq."/kg

1.50E+06 kg 1,4-dichlorobenzene eq."/kg

3.60E+06 kg 1,4-dichlorobenzene eq."/kg

$1300 \mathrm{~kg} \mathrm{1,4-dichlorobenzene} \mathrm{eq."/kg}$

1.60E+05 kg 1,4-dichlorobenzene eq."/kg

1600 kg 1,4-dichlorobenzene eq." $/ \mathrm{kg}$

1.90E+05 kg 1,4-dichlorobenzene eq."/kg

36000 kg 1,4-dichlorobenzene eq."/kg

550 kg 1,4-dichlorobenzene eq."/kg

5.4 kg 1,4-dichlorobenzene eq."/kg

$15 \mathrm{~kg}$ 1,4-dichlorobenzene eq."/kg

$2800 \mathrm{~kg} \mathrm{1,4-dichlorobenzene} \mathrm{eq."} / \mathrm{kg}$

$1.7 \mathrm{~kg} 1,4$-dichlorobenzene eq." $/ \mathrm{kg}$

0.0032 kg 1,4-dichlorobenzene eq."/kg

0.12 kg 1,4-dichlorobenzene eq."/kg

2400 kg 1,4-dichlorobenzene eq." $/ \mathrm{kg}$

59000 kg 1,4-dichlorobenzene eq."/kg

$0.8 \mathrm{~kg} \mathrm{1,4-dichlorobenzene} \mathrm{eq."} / \mathrm{kg}$

$9.7 \mathrm{~kg} \mathrm{1,4-dichlorobenzene} \mathrm{eq."} / \mathrm{kg}$

19 kg 1,4-dichlorobenzene eq."/kg

16 kg 1,4-dichlorobenzene eq."/kg

3.4 kg 1,4-dichlorobenzene eq."/kg

0.052 kg 1,4-dichlorobenzene eq."/kg

13000 kg 1,4-dichlorobenzene eq."/kg

12000 kg 1,4-dichlorobenzene eq."/kg

2.5 kg 1,4-dichlorobenzene eq."/kg

1500 kg 1,4-dichlorobenzene eq." $/ \mathrm{kg}$

1500 kg 1,4-dichlorobenzene eq."/kg

$2.6 \mathrm{~kg} \mathrm{1,4-dichlorobenzene} \mathrm{eq."/kg}$

320 kg 1,4-dichlorobenzene eq."/kg

2.70E+06 kg 1,4-dichlorobenzene eq."/kg

6600 kg 1,4-dichlorobenzene eq."/kg

0.062 kg 1,4-dichlorobenzene eq."/kg

0.0026 kg 1,4-dichlorobenzene eq."/kg

5600 kg 1,4-dichlorobenzene eq."/kg

23000 kg 1,4-dichlorobenzene eq."/kg

40000 kg 1,4-dichlorobenzene eq."/kg

40000 kg 1,4-dichlorobenzene eq."/kg

40000 kg 1,4-dichlorobenzene eq."/kg

4200 kg 1,4-dichlorobenzene eq."/kg 
[E1055] folpet to sea water

[E1056] Formaldehyde (methanal) to sea water

[E1057] glyphosate to sea water

[E1058] heptachlor to sea water

[E1059] heptenophos to sea water

[E1060] hexachloro-1,3-butadiene to sea water

[E1061] hexachlorobenzene to sea water

[E1064] indeno[1,2,3-cd]pyrene to sea water

[E1065] iprodione to sea water

[E1066] isoproturon to sea water

[E1067] lead to sea water

[E1068] lindane to sea water

[E1069] linuron to sea water

[E1070] malathion to sea water

[E1072] MCPA to sea water

[E1073] mecoprop to sea water

[E1074] mercury to sea water

[E1075] metamitron to sea water

[E1076] metazachlor to sea water

[E1077] methabenzthiazuron to sea water

[E1078] methomyl to sea water

[E1079] methylbromide to sea water

[E1080] methyl-mercury to sea water

[E1081] metobromuron to sea water

[E1082] metolachlor to sea water

[E1083] mevinphos to sea water

[E1084] molybdenum to sea water

[E1085] m-xylene to sea water

[E1086] naphtalene to sea water

[E1087] nickel to sea water

[E1093] oxamyl to sea water

[E1094] oxydemethon-methyl to sea water

[E1095] o-xylene to sea water

[E1096] parathion-ethyl to sea water

[E1097] parathion-methyl to sea water

[E1098] pentachlorobenzene to sea water

[E1099] pentachloronitrobenzene to sea water

[E1100] pentachlorophenol to sea water

[E1101] permethrin to sea water

[E1102] phenanthrene to sea water

[E1103] phenol to sea water

[E1106] phoxim to sea water

[E1107] Phtalic anhydride to sea water

[E1108] pirimicarb to sea water

[E1110] propachlor to sea water

[E1111] propoxur to sea water

[E1112] propylene oxide to sea water

[E1113] p-xylene to sea water

[E1114] pyrazophos to sea water

[E1115] selenium to sea water

[E1116] simazine to sea water

[E1117] styrene (vinylbenzene) to sea water

[E1120] tetrachloroethylene (PER) to sea water

[E1121] Tetrachloromethane (carbon tetrachloride) (HC-10) to sea water

[E1122] thallium to sea water

[E1123] Thiram to sea water

[E1124] tin to sea water

[E1125] tolclophos-methyl to sea water

[E1126] toluene to sea water

[E1127] tri-allate to sea water

[E1128] triazophos to sea water

[E1129] tributyltinoxide to sea water

[E1130] trichlorfon to sea water

[E1131] Trichloroethylene (tri) to sea water

[E1132] Trichloromethane $=$ chloroform to sea water

[E1133] trifluarin to sea water

[E1134] vanadium to sea water
$21000 \mathrm{~kg}$ 1,4-dichlorobenzene eq." $/ \mathrm{kg}$ $5.6 \mathrm{~kg} \mathrm{1,4-dichlorobenzene} \mathrm{eq."} / \mathrm{kg}$

$33 \mathrm{~kg}$ 1,4-dichlorobenzene eq." $/ \mathrm{kg}$

$1100 \mathrm{~kg} \mathrm{1,4-dichlorobenzene} \mathrm{eq."} / \mathrm{kg}$ $450 \mathrm{~kg}$ 1,4-dichlorobenzene eq." $/ \mathrm{kg}$

70000 kg 1,4-dichlorobenzene eq."/kg

$2400 \mathrm{~kg} \mathrm{1,4-dichlorobenzene} \mathrm{eq."} / \mathrm{kg}$

1.10E+05 kg 1,4-dichlorobenzene eq." $/ \mathrm{kg}$

$0.72 \mathrm{~kg}$ 1,4-dichlorobenzene eq." $/ \mathrm{kg}$

$59 \mathrm{~kg}$ 1,4-dichlorobenzene eq." $/ \mathrm{kg}$

$11000 \mathrm{~kg}$ 1,4-dichlorobenzene eq." $/ \mathrm{kg}$

$230 \mathrm{~kg}$ 1,4-dichlorobenzene eq." $/ \mathrm{kg}$

$1300 \mathrm{~kg} \mathrm{1,4-dichlorobenzene} \mathrm{eq."} / \mathrm{kg}$

$5100 \mathrm{~kg} \mathrm{1,4-dichlorobenzene} \mathrm{eq."} / \mathrm{kg}$

$0.56 \mathrm{~kg} \mathrm{1,4-dichlorobenzene} \mathrm{eq."} / \mathrm{kg}$

8 kg 1,4-dichlorobenzene eq." $/ \mathrm{kg}$

$1.90 \mathrm{E}+06 \mathrm{~kg} 1,4$-dichlorobenzene eq. $" / \mathrm{kg}$

$0.49 \mathrm{~kg}$ 1,4-dichlorobenzene eq." $/ \mathrm{kg}$

$4.4 \mathrm{~kg}$ 1,4-dichlorobenzene eq." $/ \mathrm{kg}$

$48 \mathrm{~kg} \mathrm{1,4-dichlorobenzene} \mathrm{eq."} / \mathrm{kg}$

$6900 \mathrm{~kg}$ 1,4-dichlorobenzene eq." $/ \mathrm{kg}$

$2.4 \mathrm{~kg}$ 1,4-dichlorobenzene eq." $/ \mathrm{kg}$

4.30E+07 kg 1,4-dichlorobenzene eq." $/ \mathrm{kg}$

$73 \mathrm{~kg} \mathrm{1,4-dichlorobenzene} \mathrm{eq."} / \mathrm{kg}$

$1300 \mathrm{~kg}$ 1,4-dichlorobenzene eq." $/ \mathrm{kg}$

11000 kg 1,4-dichlorobenzene eq." $/ \mathrm{kg}$

$2.60 \mathrm{E}+06 \mathrm{~kg}$ 1,4-dichlorobenzene eq." $/ \mathrm{kg}$

$0.14 \mathrm{~kg} \mathrm{1,4-dichlorobenzene} \mathrm{eq."} / \mathrm{kg}$

$33 \mathrm{~kg} 1,4$-dichlorobenzene eq. "/kg

$5.80 \mathrm{E}+06 \mathrm{~kg} 1,4$-dichlorobenzene eq. "/ $/ \mathrm{kg}$

$2.8 \mathrm{~kg} \mathrm{1,4-dichlorobenzene} \mathrm{eq."} / \mathrm{kg}$

$1000 \mathrm{~kg} \mathrm{1,4-dichlorobenzene} \mathrm{eq."} / \mathrm{kg}$

$0.13 \mathrm{~kg} \mathrm{1,4-dichlorobenzene} \mathrm{eq."} / \mathrm{kg}$

$41000 \mathrm{~kg}$ 1,4-dichlorobenzene eq." $/ \mathrm{kg}$

$8100 \mathrm{~kg}$ 1,4-dichlorobenzene eq." $/ \mathrm{kg}$

$170 \mathrm{~kg}$ 1,4-dichlorobenzene eq." $/ \mathrm{kg}$

$5600 \mathrm{~kg}$ 1,4-dichlorobenzene eq." $/ \mathrm{kg}$

$78 \mathrm{~kg} \mathrm{1,4-dichlorobenzene} \mathrm{eq."} / \mathrm{kg}$

$2.80 \mathrm{E}+05 \mathrm{~kg} 1,4$-dichlorobenzene eq." $/ \mathrm{kg}$

$74 \mathrm{~kg} \mathrm{1,4-dichlorobenzene} \mathrm{eq."} / \mathrm{kg}$

$4.7 \mathrm{~kg}$ 1,4-dichlorobenzene eq." $/ \mathrm{kg}$

$300 \mathrm{~kg}$ 1,4-dichlorobenzene eq." $/ \mathrm{kg}$

$0.017 \mathrm{~kg} \mathrm{1,4-dichlorobenzene} \mathrm{eq."} / \mathrm{kg}$

$860 \mathrm{~kg}$ 1,4-dichlorobenzene eq."/kg

$27 \mathrm{~kg}$ 1,4-dichlorobenzene eq." $/ \mathrm{kg}$

$3400 \mathrm{~kg}$ 1,4-dichlorobenzene eq." $/ \mathrm{kg}$

$0.15 \mathrm{~kg}$ 1,4-dichlorobenzene eq." $/ \mathrm{kg}$

$0.13 \mathrm{~kg} 1,4$-dichlorobenzene eq." $/ \mathrm{kg}$

$1100 \mathrm{~kg}$ 1,4-dichlorobenzene eq." $/ \mathrm{kg}$

$2.90 \mathrm{E}+07 \mathrm{~kg} 1,4$-dichlorobenzene eq." $/ \mathrm{kg}$

$670 \mathrm{~kg}$ 1,4-dichlorobenzene eq." $/ \mathrm{kg}$

$0.12 \mathrm{~kg}$ 1,4-dichlorobenzene eq. $/ / \mathrm{kg}$

$0.65 \mathrm{~kg} \mathrm{1,4-dichlorobenzene} \mathrm{eq."} / \mathrm{kg}$

$1.1 \mathrm{~kg} \mathrm{1,4-dichlorobenzene} \mathrm{eq."} / \mathrm{kg}$

$3.60 \mathrm{E}+07 \mathrm{~kg}$ 1,4-dichlorobenzene eq." $/ \mathrm{kg}$

$420 \mathrm{~kg}$ 1,4-dichlorobenzene eq." $/ \mathrm{kg}$

12000 kg 1,4-dichlorobenzene eq." $/ \mathrm{kg}$

$140 \mathrm{~kg}$ 1,4-dichlorobenzene eq." $/ \mathrm{kg}$

$0.051 \mathrm{~kg} 1,4$-dichlorobenzene eq." $/ \mathrm{kg}$

$3300 \mathrm{~kg}$ 1,4-dichlorobenzene eq." $/ \mathrm{kg}$

$4900 \mathrm{~kg}$ 1,4-dichlorobenzene eq." $/ \mathrm{kg}$

$5.70 \mathrm{E}+05 \mathrm{~kg}$ 1,4-dichlorobenzene eq." $/ \mathrm{kg}$

$3600 \mathrm{~kg}$ 1,4-dichlorobenzene eq."//kg

$0.057 \mathrm{~kg} 1$ 1,-dichlorobenzene eq." $/ \mathrm{kg}$

0.056 kg 1,4-dichlorobenzene eq." $/ \mathrm{kg}$

$8300 \mathrm{~kg}$ 1,4-dichlorobenzene eq." $/ \mathrm{kg}$

1.80E+07 kg 1,4-dichlorobenzene eq." $/ \mathrm{kg}$ 
[E1135] Vinyl Chloride (chloroethene) to sea water [E1136] zinc to sea water

[E1137] zineb to sea water

[E1138] 1,1,1-trichloroethane to sea water

[E1139] 1,2,3,4-tetrachlorobenzene to sea water

[E1140] 1,2,3,5-tetrachlorobenzene to sea water

[E1141] 1,2,3-trichlorobenzene to sea water

[E1142] 1,2,4,5-tetrachlorobenzene to sea water

[E1143] 1,2,4-trichlorobenzene to sea water

[E1144] 1,2-dichlorobenzene to sea water

[E1145] 1,2-dichloroethane to sea water

[E1146] 1,3,5-trichlorobenzene to sea water

[E1147] 1,3-butadiene to sea water

[E1148] 1,3-dichlorobenzene to sea water

[E1149] 1,4-dichlorobenzene to sea water

[E1150] 1-chloro-4-nitrobenzene to sea water

[E1151] 2,3,4,6-tetrachlorophenol to sea water

[E1152] 2,3,7,8-TCDD to sea water

[E1153] 2,4,5-T to sea water

[E1154] 2,4,5-trichlorophenol to sea water

[E1155] 2,4,6-trichlorophenol to agricultural soil

[E1156] 2,4-D to agricultural soil

[E1157] 2,4-dichlorophenol to agricultural soil

[E1158] 2-chlorophenol to agricultural soil

[E1159] 3,4-dichloroaniline to agricultural soil

[E1160] 3-chloroaniline to agricultural soil

[E1161] 4-chloroaniline to agricultural soil

[E1162] acephate to agricultural soil

[E1163] Acrolein (2-propenal) to agricultural soil

[E1164] acrylonitrile to agricultural soil

[E1165] aldicarb to agricultural soil

[E1166] aldrin to agricultural soil

[E1168] anilazine to agricultural soil

[E1169] anthracene to agricultural soil

[E1170] antimony to agricultural soil

[E1171] arsenic to agricultural soil

[E1172] atrazine to agricultural soil

[E1173] azinphos-ethyl to agricultural soil

[E1174] azinphos-methyl to agricultural soil

[E1175] barium to agricultural soil

[E1176] benomyl to agricultural soil

[E1177] bentazone to agricultural soil

[E1178] benzene to agricultural soil

[E1179] benzo[a]anthracene to agricultural soil

[E1180] benzo[a]pyrene to agricultural soil

[E1181] benzo[ghi]perylene to agricultural soil

[E1182] benzo[k]fluoranthrene to agricultural soil

[E1183] benzylchloride to agricultural soil

[E1184] beryllium to agricultural soil

[E1185] bifenthrin to agricultural soil

[E1186] Butylbenzylphtalate to agricultural soil

[E1187] cadmium to agricultural soil

[E1188] captafol to agricultural soil

[E1189] captan to agricultural soil

[E1190] carbaryl to agricultural soil

[E1191] carbendazim to agricultural soil

[E1192] carbofuran to agricultural soil

[E1193] carbon disulfide to agricultural soil

[E1194] Carcinogenic PAHs to agricultural soil

[E1195] chlordane to agricultural soil

[E1196] chlorfenvinphos to agricultural soil

[E1197] chloridazon to agricultural soil

[E1198] chlorobenzene to agricultural soil

[E1199] chlorothalonil to agricultural soil

[E1200] chlorpropham to agricultural soil

[E1201] chlorpyriphos to agricultural soil

[E1202] chromium III to agricultural soil
0.02 kg 1,4-dichlorobenzene eq." $/ \mathrm{kg}$

1.10E+05 kg 1,4-dichlorobenzene eq." $/ \mathrm{kg}$

810 kg 1,4-dichlorobenzene eq." $/ \mathrm{kg}$

$0.31 \mathrm{~kg} 1,4$-dichlorobenzene eq." $/ \mathrm{kg}$

$0.39 \mathrm{~kg}$ 1,4-dichlorobenzene eq." $/ \mathrm{kg}$

$2.3 \mathrm{~kg} \mathrm{1,4-dichlorobenzene} \mathrm{eq."} / \mathrm{kg}$

0.65 kg 1,4-dichlorobenzene eq." $/ \mathrm{kg}$

0.51 kg 1,4-dichlorobenzene eq." $/ \mathrm{kg}$

$0.43 \mathrm{~kg} \mathrm{1,4-dichlorobenzene} \mathrm{eq."} / \mathrm{kg}$

$0.51 \mathrm{~kg} 1,4$-dichlorobenzene eq." $/ \mathrm{kg}$

0.059 kg 1,4-dichlorobenzene eq."/kg

$1.1 \mathrm{~kg}$ 1,4-dichlorobenzene eq."/kg

2.90E-06 kg 1,4-dichlorobenzene eq."/kg

0.37 kg 1,4-dichlorobenzene eq." $/ \mathrm{kg}$

$0.55 \mathrm{~kg}$ 1,4-dichlorobenzene eq." $/ \mathrm{kg}$

120 kg 1,4-dichlorobenzene eq."/kg

0.62 kg 1,4-dichlorobenzene eq." $/ \mathrm{kg}$

45000 kg 1,4-dichlorobenzene eq."/kg

0.0016 kg 1,4-dichlorobenzene eq."/kg

$1.3 \mathrm{~kg}$ 1,4-dichlorobenzene eq."/kg

0.0082 kg 1,4-dichlorobenzene eq."/kg

0.17 kg 1,4-dichlorobenzene eq."/kg

0.007 kg 1,4-dichlorobenzene eq."/kg

0.068 kg 1,4-dichlorobenzene eq."/kg

270 kg 1,4-dichlorobenzene eq."/kg

$0.32 \mathrm{~kg} \mathrm{1,4-dichlorobenzene} \mathrm{eq."} / \mathrm{kg}$

0.77 kg 1,4-dichlorobenzene eq." $/ \mathrm{kg}$

0.67 kg 1,4-dichlorobenzene eq." $/ \mathrm{kg}$

$250 \mathrm{~kg} \mathrm{1,4-dichlorobenzene} \mathrm{eq."/kg}$

$0.21 \mathrm{~kg} \mathrm{1,4-dichlorobenzene} \mathrm{eq."} / \mathrm{kg}$

1600 kg 1,4-dichlorobenzene eq."/kg

32 kg 1,4-dichlorobenzene eq."/kg

5.00E-05 kg 1,4-dichlorobenzene eq."/kg

$6.2 \mathrm{~kg} \mathrm{1,4-dichlorobenzene} \mathrm{eq."} / \mathrm{kg}$

14000 kg 1,4-dichlorobenzene eq."/kg

77000 kg 1,4-dichlorobenzene eq."/kg

$34 \mathrm{~kg}$ 1,4-dichlorobenzene eq."/kg

$110 \mathrm{~kg} \mathrm{1,4-dichlorobenzene} \mathrm{eq."/kg}$

0.14 kg 1,4-dichlorobenzene eq."/kg

4.20E+05 kg 1,4-dichlorobenzene eq." $/ \mathrm{kg}$

0.0058 kg 1,4-dichlorobenzene eq."/kg

0.036 kg 1,4-dichlorobenzene eq."/kg

0.0024 kg 1,4-dichlorobenzene eq."/kg

$4.5 \mathrm{~kg} \mathrm{1,4-dichlorobenzene} \mathrm{eq."} / \mathrm{kg}$

6.5 kg 1,4-dichlorobenzene eq." $/ \mathrm{kg}$

11 kg 1,4-dichlorobenzene eq."/kg

2000 kg 1,4-dichlorobenzene eq."/kg

0.082 kg 1,4-dichlorobenzene eq." $/ \mathrm{kg}$

2.70E+08 kg 1,4-dichlorobenzene eq."/kg

$0.11 \mathrm{~kg} \mathrm{1,4-dichlorobenzene} \mathrm{eq."} / \mathrm{kg}$

2.90E-05 kg 1,4-dichlorobenzene eq." $/ \mathrm{kg}$

1.10E+05 kg 1,4-dichlorobenzene eq."/kg

$4000 \mathrm{~kg}$ 1,4-dichlorobenzene eq."/kg

6.90E-05 kg 1,4-dichlorobenzene eq." $/ \mathrm{kg}$

0.0074 kg 1,4-dichlorobenzene eq."/kg

30 kg 1,4-dichlorobenzene eq."/kg

2 kg 1,4-dichlorobenzene eq." $/ \mathrm{kg}$

1.4 kg 1,4-dichlorobenzene eq."/kg

12 kg 1,4-dichlorobenzene eq."/kg

30 kg 1,4-dichlorobenzene eq."/kg

0.085 kg 1,4-dichlorobenzene eq."/kg

$0.081 \mathrm{~kg} 1,4$-dichlorobenzene eq." $/ \mathrm{kg}$

0.083 kg 1,4-dichlorobenzene eq." $/ \mathrm{kg}$

1.7 kg 1,4-dichlorobenzene eq."/kg

0.0084 kg 1,4-dichlorobenzene eq."/kg

$0.14 \mathrm{~kg}$ 1,4-dichlorobenzene eq." $/ \mathrm{kg}$

650 kg 1,4-dichlorobenzene eq."/kg 
[E1203] chromium VI to agricultural soil [E1204] chrysene to agricultural soil [E1205] cobalt to agricultural soil [E1206] copper to agricultural soil [E1207] coumaphos to agricultural soil [E1208] cyanazine to agricultural soil [E1209] cypermethrin to agricultural soil [E1210] cyromazine to agricultural soil [E1211] DDT to agricultural soil [E1212] deltamethrin to agricultural soil [E1213] demeton to agricultural soil

[E1214] desmetryn to agricultural soil

[E1215] Di(2-ethylhexyl)phtalate to agricultural soil

[E1216] diazinon to agricultural soil

[E1217] Dibutylphtalate to agricultural soil

[E1218] Dichloromethane (Methylene Chloride) to agricultural soil

[E1219] dichlorprop to agricultural soil

[E1220] dichlorvos to agricultural soil

[E1221] dieldrin to agricultural soil

[E1222] Diethylphtalate to agricultural soil

[E1223] Dihexylphtalate to agricultural soil

[E1224] Diisodecylphtalate to agricultural soil

[E1225] Diisooctylphtalate to agricultural soil

[E1226] dimethoate to agricultural soil

[E1227] Dimethylphtalate to agricultural soil

[E1228] dinoseb to agricultural soil

[E1229] dinoterb to agricultural soil

[E1230] Dioctylphtalate to agricultural soil

[E1231] disulfothon to agricultural soil

[E1232] diuron to agricultural soil

[E1233] DNOC to agricultural soil

[E1234] endosulfan to agricultural soil

[E1235] endrin to agricultural soil

[E1236] ethoprophos to agricultural soil

[E1237] ethylbenzene to agricultural soil

[E1238] ethylene to agricultural soil

[E1239] fenitrothion to agricultural soil

[E1240] fenthion to agricultural soil

[E1241] fentin acetate to agricultural soil

[E1242] fentin chloride to agricultural soil

[E1243] fentin hydroxide to agricultural soil

[E1244] fluoranthrene to agricultural soil

[E1245] folpet to agricultural soil

[E1246] Formaldehyde (methanal) to agricultural soil

[E1247] glyphosate to agricultural soil

[E1248] heptachlor to agricultural soil

[E1249] heptenophos to agricultural soil

[E1250] hexachloro-1,3-butadiene to agricultural soil

[E1251] hexachlorobenzene to agricultural soil

[E1254] indeno[1,2,3-cd]pyrene to agricultural soil

[E1255] iprodione to agricultural soil

[E1256] isoproturon to agricultural soil

[E1257] lead to agricultural soil

[E1258] lindane to agricultural soil

[E1259] linuron to agricultural soil

[E1260] malathion to agricultural soil

[E1261] MCPA to agricultural soil

[E1262] mecoprop to agricultural soil

[E1263] mercury to agricultural soil

[E1264] metamitron to agricultural soil

[E1265] metazachlor to agricultural soil

[E1266] methabenzthiazuron to agricultural soil

[E1267] methomyl to agricultural soil

[E1268] methylbromide to agricultural soil

[E1269] methyl-mercury to agricultural soil

[E1270] metobromuron to agricultural soil

[E1271] metolachlor to agricultural soil
2600 kg 1,4-dichlorobenzene eq." $/ \mathrm{kg}$

$12 \mathrm{~kg}$ 1,4-dichlorobenzene eq." $/ \mathrm{kg}$ 2.20E+06 kg 1,4-dichlorobenzene eq." $/ \mathrm{kg}$ 1.20E+05 kg 1,4-dichlorobenzene eq." $/ \mathrm{kg}$ $1.50 \mathrm{E}+05 \mathrm{~kg}$ 1,4-dichlorobenzene eq." $/ \mathrm{kg}$ $2.8 \mathrm{~kg}$ 1,4-dichlorobenzene eq." $/ \mathrm{kg}$ $300 \mathrm{~kg}$ 1,4-dichlorobenzene eq." $/ \mathrm{kg}$ $250 \mathrm{~kg}$ 1,4-dichlorobenzene eq." $/ \mathrm{kg}$

$43 \mathrm{~kg}$ 1,4-dichlorobenzene eq." $/ \mathrm{kg}$

0.06 kg 1,4-dichlorobenzene eq." $/ \mathrm{kg}$

$3.5 \mathrm{~kg}$ 1,4-dichlorobenzene eq. " $/ \mathrm{kg}$

$0.024 \mathrm{~kg} 1,4$-dichlorobenzene eq." $/ \mathrm{kg}$

1.60E-05 kg 1,4-dichlorobenzene eq." $/ \mathrm{kg}$

$7.8 \mathrm{~kg}$ 1,4-dichlorobenzene eq. " $/ \mathrm{kg}$

$0.00012 \mathrm{~kg}$ 1,4-dichlorobenzene eq." $/ \mathrm{kg}$

0.0025 kg 1,4-dichlorobenzene eq." $/ \mathrm{kg}$

3.60E-05 kg 1,4-dichlorobenzene eq." $/ \mathrm{kg}$

$0.041 \mathrm{~kg}$ 1,4-dichlorobenzene eq." $/ \mathrm{kg}$

$81 \mathrm{~kg}$ 1,4-dichlorobenzene eq." $/ \mathrm{kg}$

$0.00071 \mathrm{~kg}$ 1,4-dichlorobenzene eq." $/ \mathrm{kg}$ $0.00043 \mathrm{~kg}$ 1,4-dichlorobenzene eq." $/ \mathrm{kg}$

$0.00086 \mathrm{~kg}$ 1,4-dichlorobenzene eq." $/ \mathrm{kg}$

6.50E-05 kg 1,4-dichlorobenzene eq." $/ \mathrm{kg}$

$0.039 \mathrm{~kg}$ 1,4-dichlorobenzene eq." $/ \mathrm{kg}$

9.70E-06 kg 1,4-dichlorobenzene eq." $/ \mathrm{kg}$

$390 \mathrm{~kg}$ 1,4-dichlorobenzene eq." $/ \mathrm{kg}$

$8.7 \mathrm{~kg}$ 1,4-dichlorobenzene eq." $/ \mathrm{kg}$

1.30E-06 kg 1,4-dichlorobenzene eq." $/ \mathrm{kg}$

$0.14 \mathrm{~kg}$ 1,4-dichlorobenzene eq." $/ \mathrm{kg}$

$0.14 \mathrm{~kg}$ 1,4-dichlorobenzene eq." $/ \mathrm{kg}$

$0.0036 \mathrm{~kg} \mathrm{1,4-dichlorobenzene} \mathrm{eq."} / \mathrm{kg}$

0.0014 kg 1,4-dichlorobenzene eq." $/ \mathrm{kg}$

$10000 \mathrm{~kg}$ 1,4-dichlorobenzene eq." $/ \mathrm{kg}$

$260 \mathrm{~kg}$ 1,4-dichlorobenzene eq." $/ \mathrm{kg}$

$0.00041 \mathrm{~kg}$ 1,4-dichlorobenzene eq." $/ \mathrm{kg}$

7.80E-11 kg 1,4-dichlorobenzene eq." $/ \mathrm{kg}$

$2.3 \mathrm{~kg}$ 1,4-dichlorobenzene eq." $/ \mathrm{kg}$

$15 \mathrm{~kg}$ 1,4-dichlorobenzene eq. "/kg

$6.8 \mathrm{~kg}$ 1,4-dichlorobenzene eq." $/ \mathrm{kg}$

$95 \mathrm{~kg}$ 1,4-dichlorobenzene eq." $/ \mathrm{kg}$

$6.1 \mathrm{~kg}$ 1,4-dichlorobenzene eq. " $/ \mathrm{kg}$

$1.3 \mathrm{~kg} 1,4$-dichlorobenzene eq." $/ \mathrm{kg}$

$710 \mathrm{~kg}$ 1,4-dichlorobenzene eq." $/ \mathrm{kg}$

$0.018 \mathrm{~kg}$ 1,4-dichlorobenzene eq. "/kg

0.0028 kg 1,4-dichlorobenzene eq." $/ \mathrm{kg}$

$0.024 \mathrm{~kg}$ 1,4-dichlorobenzene eq." $/ \mathrm{kg}$

$0.026 \mathrm{~kg} \mathrm{1,4-dichlorobenzene} \mathrm{eq.} \mathrm{"/} / \mathrm{kg}$

$28000 \mathrm{~kg}$ 1,4-dichlorobenzene eq." $/ \mathrm{kg}$

$720 \mathrm{~kg}$ 1,4-dichlorobenzene eq." $/ \mathrm{kg}$

17 kg 1,4-dichlorobenzene eq." $/ \mathrm{kg}$

2.20E-05 kg 1,4-dichlorobenzene eq." $/ / \mathrm{kg}$

$1.8 \mathrm{~kg}$ 1,4-dichlorobenzene eq." $/ \mathrm{kg}$

$750 \mathrm{~kg}$ 1,4-dichlorobenzene eq." $/ \mathrm{kg}$

$1.4 \mathrm{~kg} 1,4$-dichlorobenzene eq. "/kg

$12 \mathrm{~kg}$ 1,4-dichlorobenzene eq." $/ \mathrm{kg}$

$0.66 \mathrm{~kg}$ 1,4-dichlorobenzene eq." $/ \mathrm{kg}$

$0.00062 \mathrm{~kg} 1,4$-dichlorobenzene eq." $/ \mathrm{kg}$

$0.053 \mathrm{~kg}$ 1,4-dichlorobenzene eq." $/ \mathrm{kg}$

$1.70 \mathrm{E}+05 \mathrm{~kg}$ 1,4-dichlorobenzene eq." $/ \mathrm{kg}$

$0.0011 \mathrm{~kg}$ 1,4-dichlorobenzene eq." $/ \mathrm{kg}$

$0.033 \mathrm{~kg}$ 1,4-dichlorobenzene eq." $/ \mathrm{kg}$

$1 \mathrm{~kg}$ 1,4-dichlorobenzene eq." $/ \mathrm{kg}$

$440 \mathrm{~kg}$ 1,4-dichlorobenzene eq." $/ \mathrm{kg}$

$3.1 \mathrm{~kg} \mathrm{1,4-dichlorobenzene} \mathrm{eq."} / \mathrm{kg}$

$3.80 \mathrm{E}+06 \mathrm{~kg}$ 1,4-dichlorobenzene eq." $/ \mathrm{kg}$

$14 \mathrm{~kg}$ 1,4-dichlorobenzene eq." $/ \mathrm{kg}$ $30 \mathrm{~kg} \mathrm{1,4-dichlorobenzene} \mathrm{eq."} / \mathrm{kg}$ 
[E1272] mevinphos to agricultural soil

[E1273] molybdenum to agricultural soil

[E1274] m-xylene to agricultural soil

[E1275] naphtalene to agricultural soil

[E1276] nickel to agricultural soil

[E1278] oxamyl to agricultural soil

[E1279] oxydemethon-methyl to agricultural soil

[E1280] o-xylene to agricultural soil

[E1281] parathion-ethyl to agricultural soil

[E1282] parathion-methyl to agricultural soil

[E1283] pentachlorobenzene to agricultural soil

[E1284] pentachloronitrobenzene to agricultural soil

[E1285] pentachlorophenol to agricultural soil

[E1286] permethrin to agricultural soil

[E1287] phenanthrene to agricultural soil

[E1288] phenol to agricultural soil

[E1289] phoxim to agricultural soil

[E1290] Phtalic anhydride to agricultural soil

[E1291] pirimicarb to agricultural soil

[E1293] propachlor to agricultural soil

[E1294] propoxur to agricultural soil

[E1295] propylene oxide to agricultural soil

[E1296] p-xylene to agricultural soil

[E1297] pyrazophos to agricultural soil

[E1298] selenium to agricultural soil

[E1299] simazine to agricultural soil

[E1300] styrene (vinylbenzene) to agricultural soil

[E1302] tetrachloroethylene (PER) to agricultural soil

[E1303] Tetrachloromethane (carbon tetrachloride) (HC-10) to agricultural soil

[E1304] thallium to agricultural soil

[E1305] Thiram to agricultural soil

[E1306] tin to agricultural soil

[E1307] tolclophos-methyl to agricultural soil

[E1308] toluene to agricultural soil

[E1309] tri-allate to agricultural soil

[E1310] triazophos to agricultural soil

[E1311] tributyltinoxide to agricultural soil

[E1312] trichlorfon to agricultural soil

[E1313] Trichloroethylene (tri) to agricultural soil

[E1314] Trichloromethane=chloroform to agricultural soil

[E1315] trifluarin to agricultural soil

[E1316] vanadium to agricultural soil

[E1317] Vinyl Chloride (chloroethene) to agricultural soil

[E1318] zinc to agricultural soil

[E1319] zineb to agricultural soil

[E1320] 1,1,1-trichloroethane to agricultural soil

[E1321] 1,2,3,4-tetrachlorobenzene to agricultural soil

[E1322] 1,2,3,5-tetrachlorobenzene to agricultural soil

[E1323] 1,2,3-trichlorobenzene to agricultural soil

[E1324] 1,2,4,5-tetrachlorobenzene to agricultural soil

[E1325] 1,2,4-trichlorobenzene to agricultural soil

[E1326] 1,2-dichlorobenzene to agricultural soil

[E1327] 1,2-dichloroethane to agricultural soil

[E1328] 1,3,5-trichlorobenzene to agricultural soil

[E1329] 1,3-butadiene to agricultural soil

[E1330] 1,3-dichlorobenzene to agricultural soil

[E1331] 1,4-dichlorobenzene to agricultural soil

[E1332] 1-chloro-4-nitrobenzene to agricultural soil

[E1333] 2,3,4,6-tetrachlorophenol to agricultural soil

[E1334] 2,3,7,8-TCDD to agricultural soil

[E1335] 2,4,5-T to agricultural soil

[E1336] 2,4,5-trichlorophenol to agricultural soil

[E1337] 2,4,6-trichlorophenol to industrial soil

[E1338] 2,4-D to industrial soil

[E1339] 2,4-dichlorophenol to industrial soil

[E1340] 2-chlorophenol to industrial soil

[E1341] 3,4-dichloroaniline to industrial soil
$0.34 \mathrm{~kg}$ 1,4-dichlorobenzene eq." $/ \mathrm{kg}$

1.20E+06 kg 1,4-dichlorobenzene eq."//kg

$0.00025 \mathrm{~kg}$ 1,4-dichlorobenzene eq." $/ \mathrm{kg}$

$0.057 \mathrm{~kg}$ 1,4-dichlorobenzene eq."/kg

1.20E+06 kg 1,4-dichlorobenzene eq." $/ \mathrm{kg}$

$0.0084 \mathrm{~kg}$ 1,4-dichlorobenzene eq." $/ \mathrm{kg}$

$2 \mathrm{~kg}$ 1,4-dichlorobenzene eq." $/ \mathrm{kg}$

$0.00055 \mathrm{~kg}$ 1,4-dichlorobenzene eq." $/ \mathrm{kg}$

$2.3 \mathrm{~kg}$ 1,4-dichlorobenzene eq." $/ \mathrm{kg}$

$5.9 \mathrm{~kg}$ 1,4-dichlorobenzene eq." $/ \mathrm{kg}$

28 kg 1,4-dichlorobenzene eq." $/ \mathrm{kg}$

$30 \mathrm{~kg}$ 1,4-dichlorobenzene eq." $/ \mathrm{kg}$

$0.0059 \mathrm{~kg}$ 1,4-dichlorobenzene eq." $/ \mathrm{kg}$

$5.5 \mathrm{~kg}$ 1,4-dichlorobenzene eq." $/ \mathrm{kg}$

$0.0087 \mathrm{~kg} 1,4$-dichlorobenzene eq." $/ \mathrm{kg}$

0.0017 kg 1,4-dichlorobenzene eq." $/ \mathrm{kg}$

$0.31 \mathrm{~kg}$ 1,4-dichlorobenzene eq." $/ \mathrm{kg}$

1.80E-08 kg 1,4-dichlorobenzene eq." $/ \mathrm{kg}$

$7.3 \mathrm{~kg}$ 1,4-dichlorobenzene eq." $/ \mathrm{kg}$

$0.042 \mathrm{~kg}$ 1,4-dichlorobenzene eq." $/ \mathrm{kg}$

$39 \mathrm{~kg}$ 1,4-dichlorobenzene eq." $/ \mathrm{kg}$

$0.033 \mathrm{~kg} 1,4$-dichlorobenzene eq." $/ \mathrm{kg}$

$0.00032 \mathrm{~kg}$ 1,4-dichlorobenzene eq." $/ \mathrm{kg}$

0.68 kg 1,4-dichlorobenzene eq." $/ \mathrm{kg}$

1.30E+07 kg 1,4-dichlorobenzene eq." $/ \mathrm{kg}$

$13 \mathrm{~kg}$ 1,4-dichlorobenzene eq." $/ \mathrm{kg}$

$0.00011 \mathrm{~kg}$ 1,4-dichlorobenzene eq."/kg

$0.31 \mathrm{~kg}$ 1,4-dichlorobenzene eq. "/ $/ \mathrm{kg}$

$1.1 \mathrm{~kg}$ 1,4-dichlorobenzene eq." $/ \mathrm{kg}$

1.40E+07 kg 1,4-dichlorobenzene eq."//kg

$0.7 \mathrm{~kg}$ 1,4-dichlorobenzene eq." $/ \mathrm{kg}$

$830 \mathrm{~kg}$ 1,4-dichlorobenzene eq." $/ \mathrm{kg}$

$0.13 \mathrm{~kg}$ 1,4-dichlorobenzene eq." $/ \mathrm{kg}$

$0.00045 \mathrm{~kg}$ 1,4-dichlorobenzene eq." $/ \mathrm{kg}$

0.84 kg 1,4-dichlorobenzene eq." $/ \mathrm{kg}$

$53 \mathrm{~kg}$ 1,4-dichlorobenzene eq." $/ \mathrm{kg}$

$560 \mathrm{~kg}$ 1,4-dichlorobenzene eq." $/ \mathrm{kg}$

$0.67 \mathrm{~kg}$ 1,4-dichlorobenzene eq." $/ \mathrm{kg}$

$0.0025 \mathrm{~kg}$ 1,4-dichlorobenzene eq."/kg

$0.047 \mathrm{~kg}$ 1,4-dichlorobenzene eq."/kg

$1.2 \mathrm{~kg}$ 1,4-dichlorobenzene eq." $/ \mathrm{kg}$

4.50E+06 kg 1,4-dichlorobenzene eq." $/ \mathrm{kg}$

$0.00013 \mathrm{~kg} 1,4$-dichlorobenzene eq."//kg

$7200 \mathrm{~kg}$ 1,4-dichlorobenzene eq."/kg

$3.5 \mathrm{~kg}$ 1,4-dichlorobenzene eq."/kg

$0.31 \mathrm{~kg}$ 1,4-dichlorobenzene eq." $/ \mathrm{kg}$

$1.5 \mathrm{~kg}$ 1,4-dichlorobenzene eq." $/ \mathrm{kg}$

$5.1 \mathrm{~kg}$ 1,4-dichlorobenzene eq." $/ \mathrm{kg}$

$0.86 \mathrm{~kg} 1,4$-dichlorobenzene eq." $/ \mathrm{kg}$

$1.8 \mathrm{~kg}$ 1,4-dichlorobenzene eq." $/ \mathrm{kg}$

$0.71 \mathrm{~kg}$ 1,4-dichlorobenzene eq."/kg

$0.51 \mathrm{~kg}$ 1,4-dichlorobenzene eq." $/ \mathrm{kg}$

$0.059 \mathrm{~kg}$ 1,4-dichlorobenzene eq." $/ \mathrm{kg}$

$1.3 \mathrm{~kg}$ 1,4-dichlorobenzene eq." $/ \mathrm{kg}$

2.90E-06 kg 1,4-dichlorobenzene eq." $/ \mathrm{kg}$

$0.37 \mathrm{~kg}$ 1,4-dichlorobenzene eq." $/ \mathrm{kg}$

$0.55 \mathrm{~kg}$ 1,4-dichlorobenzene eq." $/ \mathrm{kg}$

$120 \mathrm{~kg}$ 1,4-dichlorobenzene eq." $/ \mathrm{kg}$

$2.5 \mathrm{~kg}$ 1,4-dichlorobenzene eq." $/ \mathrm{kg}$

1.80E+05 kg 1,4-dichlorobenzene eq." $/ \mathrm{kg}$

$0.0055 \mathrm{~kg}$ 1,4-dichlorobenzene eq." $/ \mathrm{kg}$

$4.6 \mathrm{~kg}$ 1,4-dichlorobenzene eq." $/ \mathrm{kg}$

$0.032 \mathrm{~kg}$ 1,4-dichlorobenzene eq." $/ \mathrm{kg}$

$0.46 \mathrm{~kg} \mathrm{1,4-dichlorobenzene} \mathrm{eq."} / \mathrm{kg}$

$0.027 \mathrm{~kg}$ 1,4-dichlorobenzene eq."/kg

$0.26 \mathrm{~kg}$ 1,4-dichlorobenzene eq." $/ \mathrm{kg}$

$600 \mathrm{~kg}$ 1,4-dichlorobenzene eq." $/ \mathrm{kg}$ 
[E1342] 3-chloroaniline to industrial soil [E1343] 4-chloroaniline to industrial soil [E1344] acephate to industrial soil [E1345] Acrolein (2-propenal) to industrial soil [E1346] acrylonitrile to industrial soil [E1347] aldicarb to industrial soil [E1348] aldrin to industrial soil [E1350] anilazine to industrial soil [E1351] anthracene to industrial soil [E1352] antimony to industrial soil [E1353] arsenic to industrial soil [E1354] atrazine to industrial soil [E1355] azinphos-ethyl to industrial soil [E1356] azinphos-methyl to industrial soil [E1357] barium to industrial soil [E1358] benomyl to industrial soil [E1359] bentazone to industrial soil [E1360] benzene to industrial soil [E1361] benzo[a]anthracene to industrial soil [E1362] benzo[a]pyrene to industrial soil [E1363] benzo[ghi]perylene to industrial soil [E1364] benzo[k]fluoranthrene to industrial soil [E1365] benzylchloride to industrial soil [E1366] beryllium to industrial soil [E1367] bifenthrin to industrial soil [E1368] Butylbenzylphtalate to industrial soil [E1369] cadmium to industrial soil [E1370] captafol to industrial soil [E1371] captan to industrial soil [E1372] carbaryl to industrial soil [E1373] carbendazim to industrial soil [E1374] carbofuran to industrial soil [E1375] carbon disulfide to industrial soil [E1376] Carcinogenic PAHs to industrial soil [E1377] chlordane to industrial soil [E1378] chlorfenvinphos to industrial soil [E1379] chloridazon to industrial soil [E1380] chlorobenzene to industrial soil [E1381] chlorothalonil to industrial soil [E1382] chlorpropham to industrial soil [E1383] chlorpyriphos to industrial soil [E1384] chromium III to industrial soil [E1385] chromium VI to industrial soil [E1386] chrysene to industrial soil [E1387] cobalt to industrial soil [E1388] copper to industrial soil [E1389] coumaphos to industrial soil [E1390] cyanazine to industrial soil [E1391] cypermethrin to industrial soil [E1392] cyromazine to industrial soil [E1393] DDT to industrial soil

[E1394] deltamethrin to industrial soil [E1395] demeton to industrial soil [E1396] desmetryn to industrial soil [E1397] Di(2-ethylhexyl)phtalate to industrial soil [E1398] diazinon to industrial soil [E1399] Dibutylphtalate to industrial soil

[E1400] Dichloromethane (Methylene Chloride) to industrial soil [E1401] dichlorprop to industrial soil

[E1402] dichlorvos to industrial soil

[E1403] dieldrin to industrial soil

[E1404] Diethylphtalate to industrial soil [E1405] Dihexylphtalate to industrial soil [E1406] Diisodecylphtalate to industrial soil [E1407] Diisooctylphtalate to industrial soil [E1408] dimethoate to industrial soil

[E1409] Dimethylphtalate to industrial soil
1.2 kg 1,4-dichlorobenzene eq."/kg 2.2 kg 1,4-dichlorobenzene eq."/kg $2.1 \mathrm{~kg} \mathrm{1,4-dichlorobenzene} \mathrm{eq."/kg}$ 250 kg 1,4-dichlorobenzene eq." $/ \mathrm{kg}$ 0.27 kg 1,4-dichlorobenzene eq." $/ \mathrm{kg}$ 1600 kg 1,4-dichlorobenzene eq."/kg

33 kg 1,4-dichlorobenzene eq."/kg 0.0002 kg 1,4-dichlorobenzene eq."/kg

25 kg 1,4-dichlorobenzene eq."/kg 14000 kg 1,4-dichlorobenzene eq."/kg

77000 kg 1,4-dichlorobenzene eq."/kg

$93 \mathrm{~kg}$ 1,4-dichlorobenzene eq."/kg

14 kg 1,4-dichlorobenzene eq."/kg

$0.58 \mathrm{~kg} \mathrm{1,4-dichlorobenzene} \mathrm{eq."} / \mathrm{kg}$

4.20E+05 kg 1,4-dichlorobenzene eq."/kg

$0.023 \mathrm{~kg} \mathrm{1,4-dichlorobenzene} \mathrm{eq."} / \mathrm{kg}$

0.048 kg 1,4-dichlorobenzene eq."/kg

0.0024 kg 1,4-dichlorobenzene eq." $/ \mathrm{kg}$

$18 \mathrm{~kg} \mathrm{1,4-dichlorobenzene} \mathrm{eq."/kg}$

26 kg 1,4-dichlorobenzene eq." $/ \mathrm{kg}$

43 kg 1,4-dichlorobenzene eq."/kg

7800 kg 1,4-dichlorobenzene eq." $/ \mathrm{kg}$

$0.29 \mathrm{~kg} \mathrm{1,4-dichlorobenzene} \mathrm{eq."} / \mathrm{kg}$

2.70E+08 kg 1,4-dichlorobenzene eq."/kg

$0.45 \mathrm{~kg} \mathrm{1,4-dichlorobenzene} \mathrm{eq."} / \mathrm{kg}$

0.00012 kg 1,4-dichlorobenzene eq." $/ \mathrm{kg}$

1.10E+05 kg 1,4-dichlorobenzene eq." $/ \mathrm{kg}$

12000 kg 1,4-dichlorobenzene eq."/kg

$0.00081 \mathrm{~kg} 1,4$-dichlorobenzene eq." $/ \mathrm{kg}$

$0.04 \mathrm{~kg} \mathrm{1,4-dichlorobenzene} \mathrm{eq."} / \mathrm{kg}$

93 kg 1,4-dichlorobenzene eq." $/ \mathrm{kg}$

6.2 kg 1,4-dichlorobenzene eq." $/ \mathrm{kg}$

$1.4 \mathrm{~kg} \mathrm{1,4-dichlorobenzene} \mathrm{eq."/kg}$

48 kg 1,4-dichlorobenzene eq."/kg

120 kg 1,4-dichlorobenzene eq. "/kg

$0.31 \mathrm{~kg} \mathrm{1,4-dichlorobenzene} \mathrm{eq."} / \mathrm{kg}$

0.18 kg 1,4-dichlorobenzene eq." $/ \mathrm{kg}$

$0.083 \mathrm{~kg} \mathrm{1,4-dichlorobenzene} \mathrm{eq."} / \mathrm{kg}$

6 kg 1,4-dichlorobenzene eq."/kg

$0.03 \mathrm{~kg} \mathrm{1,4-dichlorobenzene} \mathrm{eq."} / \mathrm{kg}$

0.58 kg 1,4-dichlorobenzene eq." $/ \mathrm{kg}$

650 kg 1,4-dichlorobenzene eq."/kg

2600 kg 1,4-dichlorobenzene eq." $/ \mathrm{kg}$

47 kg 1,4-dichlorobenzene eq." $/ \mathrm{kg}$

2.20E+06 kg 1,4-dichlorobenzene eq."/kg

1.20E+05 kg 1,4-dichlorobenzene eq."/kg

4.60E+05 kg 1,4-dichlorobenzene eq." $/ \mathrm{kg}$

10 kg 1,4-dichlorobenzene eq."/kg

1000 kg 1,4-dichlorobenzene eq." $/ \mathrm{kg}$

250 kg 1,4-dichlorobenzene eq."/kg

$170 \mathrm{~kg} \mathrm{1,4-dichlorobenzene} \mathrm{eq."/kg}$

0.24 kg 1,4-dichlorobenzene eq." $/ \mathrm{kg}$

$11 \mathrm{~kg} \mathrm{1,4-dichlorobenzene} \mathrm{eq."} / \mathrm{kg}$

0.088 kg 1,4-dichlorobenzene eq." $/ \mathrm{kg}$

6.20E-05 kg 1,4-dichlorobenzene eq."/kg

27 kg 1,4-dichlorobenzene eq." $/ \mathrm{kg}$

0.00048 kg 1,4-dichlorobenzene eq." $/ \mathrm{kg}$

0.0025 kg 1,4-dichlorobenzene eq." $/ \mathrm{kg}$

0.00014 kg 1,4-dichlorobenzene eq."/kg

0.16 kg 1,4-dichlorobenzene eq." $/ \mathrm{kg}$

$310 \mathrm{~kg}$ 1,4-dichlorobenzene eq." $/ \mathrm{kg}$

0.0028 kg 1,4-dichlorobenzene eq." $/ \mathrm{kg}$

$0.0017 \mathrm{~kg} 1,4$-dichlorobenzene eq." $/ \mathrm{kg}$

0.0034 kg 1,4-dichlorobenzene eq."/kg

0.00026 kg 1,4-dichlorobenzene eq." $/ \mathrm{kg}$

$0.12 \mathrm{~kg} \mathrm{1,4-dichlorobenzene} \mathrm{eq."} / \mathrm{kg}$

3.80E-05 kg 1,4-dichlorobenzene eq."/kg 
[E1410] dinoseb to industrial soil

[E1411] dinoterb to industrial soil

[E1412] Dioctylphtalate to industrial soil

[E1413] disulfothon to industrial soil

[E1414] diuron to industrial soil

[E1415] DNOC to industrial soil

[E1416] endosulfan to industrial soil

[E1417] endrin to industrial soil

[E1418] ethoprophos to industrial soil

[E1419] ethylbenzene to industrial soil

[E1420] ethylene to industrial soil

[E1421] fenitrothion to industrial soil

[E1422] fenthion to industrial soil

[E1423] fentin acetate to industrial soil

[E1424] fentin chloride to industrial soil

[E1425] fentin hydroxide to industrial soil

[E1426] fluoranthrene to industrial soil

[E1427] folpet to industrial soil

[E1428] Formaldehyde (methanal) to industrial soil

[E1429] glyphosate to industrial soil

[E1430] heptachlor to industrial soil

[E1431] heptenophos to industrial soil

[E1432] hexachloro-1,3-butadiene to industrial soil

[E1433] hexachlorobenzene to industrial soil

[E1436] indeno[1,2,3-cd]pyrene to industrial soil

[E1437] iprodione to industrial soil

[E1438] isoproturon to industrial soil

[E1439] lead to industrial soil

[E1440] lindane to industrial soil

[E1441] linuron to industrial soil

[E1442] malathion to industrial soil

[E1443] MCPA to industrial soil

[E1444] mecoprop to industrial soil

[E1445] mercury to industrial soil

[E1446] metamitron to industrial soil

[E1447] metazachlor to industrial soil

[E1448] methabenzthiazuron to industrial soil

[E1449] methomyl to industrial soil

[E1450] methylbromide to industrial soil

[E1451] methyl-mercury to industrial soil

[E1452] metobromuron to industrial soil

[E1453] metolachlor to industrial soil

[E1454] mevinphos to industrial soil

[E1455] molybdenum to industrial soil

[E1456] m-xylene to industrial soil

[E1457] naphtalene to industrial soil

[E1458] nickel to industrial soil

[E1460] oxamyl to industrial soil

[E1461] oxydemethon-methyl to industrial soil

[E1462] o-xylene to industrial soil

[E1463] parathion-ethyl to industrial soil

[E1464] parathion-methyl to industrial soil

[E1465] pentachlorobenzene to industrial soil

[E1466] pentachloronitrobenzene to industrial soil

[E1467] pentachlorophenol to industrial soil

[E1468] permethrin to industrial soil

[E1469] phenanthrene to industrial soil

[E1470] phenol to industrial soil

[E1471] phoxim to industrial soil

[E1472] Phtalic anhydride to industrial soil

[E1473] pirimicarb to industrial soil

[E1475] propachlor to industrial soil

[E1476] propoxur to industrial soil

[E1477] propylene oxide to industrial soil

[E1478] p-xylene to industrial soil

[E1479] pyrazophos to industrial soil

[E1480] selenium to industrial soil
1100 kg 1,4-dichlorobenzene eq."/kg

36 kg 1,4-dichlorobenzene eq." $/ \mathrm{kg}$

5.20E-06 kg 1,4-dichlorobenzene eq."//kg

$0.56 \mathrm{~kg}$ 1,4-dichlorobenzene eq." $/ \mathrm{kg}$

$0.56 \mathrm{~kg}$ 1,4-dichlorobenzene eq." $/ \mathrm{kg}$

$0.014 \mathrm{~kg}$ 1,4-dichlorobenzene eq." $/ \mathrm{kg}$

$0.0055 \mathrm{~kg} \mathrm{1,4-dichlorobenzene} \mathrm{eq."} / \mathrm{kg}$

35000 kg 1,4-dichlorobenzene eq."/kg

720 kg 1,4-dichlorobenzene eq." $/ \mathrm{kg}$

$0.00041 \mathrm{~kg}$ 1,4-dichlorobenzene eq." $/ \mathrm{kg}$

7.80E-11 kg 1,4-dichlorobenzene eq." $/ \mathrm{kg}$

$8.9 \mathrm{~kg}$ 1,4-dichlorobenzene eq." $/ \mathrm{kg}$

57 kg 1,4-dichlorobenzene eq."/kg

$27 \mathrm{~kg}$ 1,4-dichlorobenzene eq." $/ \mathrm{kg}$

$370 \mathrm{~kg}$ 1,4-dichlorobenzene eq." $/ \mathrm{kg}$

24 kg 1,4-dichlorobenzene eq." $/ \mathrm{kg}$

$5.3 \mathrm{~kg}$ 1,4-dichlorobenzene eq." $/ \mathrm{kg}$

$2100 \mathrm{~kg} \mathrm{1,4-dichlorobenzene} \mathrm{eq."} / \mathrm{kg}$

$0.055 \mathrm{~kg}$ 1,4-dichlorobenzene eq." $/ \mathrm{kg}$

$0.011 \mathrm{~kg}$ 1,4-dichlorobenzene eq." $/ \mathrm{kg}$

$0.095 \mathrm{~kg}$ 1,4-dichlorobenzene eq." $/ \mathrm{kg}$

$0.1 \mathrm{~kg}$ 1,4-dichlorobenzene eq." $/ \mathrm{kg}$

34000 kg 1,4-dichlorobenzene eq."/kg

960 kg 1,4-dichlorobenzene eq." $/ \mathrm{kg}$

$68 \mathrm{~kg}$ 1,4-dichlorobenzene eq." $/ \mathrm{kg}$

$0.00018 \mathrm{~kg}$ 1,4-dichlorobenzene eq."/kg

$4.2 \mathrm{~kg}$ 1,4-dichlorobenzene eq." $/ \mathrm{kg}$

$750 \mathrm{~kg}$ 1,4-dichlorobenzene eq." $/ \mathrm{kg}$

$5.3 \mathrm{~kg}$ 1,4-dichlorobenzene eq." $/ \mathrm{kg}$

44 kg 1,4-dichlorobenzene eq." $/ \mathrm{kg}$

$2.6 \mathrm{~kg}$ 1,4-dichlorobenzene eq." $/ \mathrm{kg}$

$0.0022 \mathrm{~kg}$ 1,4-dichlorobenzene eq." $/ \mathrm{kg}$

$0.14 \mathrm{~kg}$ 1,4-dichlorobenzene eq." $/ \mathrm{kg}$

$1.70 \mathrm{E}+05 \mathrm{~kg}$ 1,4-dichlorobenzene eq." $/ \mathrm{kg}$

$0.0041 \mathrm{~kg} \mathrm{1,4-dichlorobenzene} \mathrm{eq."} / \mathrm{kg}$

$0.11 \mathrm{~kg}$ 1,4-dichlorobenzene eq." $/ \mathrm{kg}$

$3.2 \mathrm{~kg}$ 1,4-dichlorobenzene eq." $/ \mathrm{kg}$

890 kg 1,4-dichlorobenzene eq." $/ \mathrm{kg}$

$3.1 \mathrm{~kg}$ 1,4-dichlorobenzene eq." $/ \mathrm{kg}$

$3.80 \mathrm{E}+06 \mathrm{~kg}$ 1,4-dichlorobenzene eq." $/ \mathrm{kg}$

$14 \mathrm{~kg}$ 1,4-dichlorobenzene eq."//kg

$91 \mathrm{~kg}$ 1,4-dichlorobenzene eq." $/ \mathrm{kg}$

$1.4 \mathrm{~kg}$ 1,4-dichlorobenzene eq. "/kg

1.20E+06 kg 1,4-dichlorobenzene eq." "/kg

0.00025 kg 1,4-dichlorobenzene eq." $/ \mathrm{kg}$

$0.19 \mathrm{~kg}$ 1,4-dichlorobenzene eq." $/ \mathrm{kg}$

1.20E+06 kg 1,4-dichlorobenzene eq." $/ \mathrm{kg}$

$0.034 \mathrm{~kg}$ 1,4-dichlorobenzene eq." $/ \mathrm{kg}$

$7.3 \mathrm{~kg}$ 1,4-dichlorobenzene eq." $/ \mathrm{kg}$

$0.00055 \mathrm{~kg}$ 1,4-dichlorobenzene eq." $/ \mathrm{kg}$

$9.2 \mathrm{~kg}$ 1,4-dichlorobenzene eq." $/ \mathrm{kg}$

$23 \mathrm{~kg} \mathrm{1,4-dichlorobenzene} \mathrm{eq.} \mathrm{"/kg}$

$54 \mathrm{~kg}$ 1,4-dichlorobenzene eq." $/ \mathrm{kg}$

$120 \mathrm{~kg}$ 1,4-dichlorobenzene eq." $/ \mathrm{kg}$

$0.027 \mathrm{~kg}$ 1,4-dichlorobenzene eq." $/ \mathrm{kg}$

$22 \mathrm{~kg}$ 1,4-dichlorobenzene eq." $/ \mathrm{kg}$

$0.035 \mathrm{~kg}$ 1,4-dichlorobenzene eq." $/ \mathrm{kg}$

$0.0061 \mathrm{~kg} 1,4$-dichlorobenzene eq." $/ \mathrm{kg}$

$0.55 \mathrm{~kg}$ 1,4-dichlorobenzene eq." $/ \mathrm{kg}$

1.20E-08 kg 1,4-dichlorobenzene eq." $/ \mathrm{kg}$

$23 \mathrm{~kg}$ 1,4-dichlorobenzene eq." $/ \mathrm{kg}$

$0.16 \mathrm{~kg}$ 1,4-dichlorobenzene eq." $/ \mathrm{kg}$

100 kg 1,4-dichlorobenzene eq." $/ \mathrm{kg}$

$0.037 \mathrm{~kg} 1,4$-dichlorobenzene eq." $/ \mathrm{kg}$

0.00032 kg 1,4-dichlorobenzene eq." $/ \mathrm{kg}$

$2.6 \mathrm{~kg}$ 1,4-dichlorobenzene eq." $/ \mathrm{kg}$

$1.30 \mathrm{E}+07 \mathrm{~kg}$ 1,4-dichlorobenzene eq." $/ \mathrm{kg}$ 
[E1481] simazine to industrial soil

[E1482] styrene (vinylbenzene) to industrial soil

[E1484] tetrachloroethylene (PER) to industrial soil

[E1485] Tetrachloromethane (carbon tetrachloride) (HC-10) to industrial soil

[E1486] thallium to industrial soil

[E1487] Thiram to industrial soil

[E1488] tin to industrial soil

[E1489] tolclophos-methyl to industrial soil

[E1490] toluene to industrial soil

[E1491] tri-allate to industrial soil

[E1492] triazophos to industrial soil

[E1493] tributyltinoxide to industrial soil

[E1494] trichlorfon to industrial soil

[E1495] Trichloroethylene (tri) to industrial soil

[E1496] Trichloromethane=chloroform to industrial soil

[E1497] trifluarin to industrial soil

[E1498] vanadium to industrial soil

[E1499] Vinyl Chloride (chloroethene) to industrial soil

[E1500] zinc to industrial soil

[E1501] zineb to industrial soil
$31 \mathrm{~kg}$ 1,4-dichlorobenzene eq."/kg

$0.00018 \mathrm{~kg}$ 1,4-dichlorobenzene eq." $/ \mathrm{kg}$

$0.00056 \mathrm{~kg}$ 1,4-dichlorobenzene eq." $/ \mathrm{kg}$

$1.1 \mathrm{~kg}$ 1,4-dichlorobenzene eq." $/ \mathrm{kg}$

1.40E+07 kg 1,4-dichlorobenzene eq." $/ \mathrm{kg}$

$4.5 \mathrm{~kg}$ 1,4-dichlorobenzene eq. "/kg

$830 \mathrm{~kg}$ 1,4-dichlorobenzene eq." $/ \mathrm{kg}$

$0.39 \mathrm{~kg}$ 1,4-dichlorobenzene eq." $/ \mathrm{kg}$

$0.00045 \mathrm{~kg} 1,4$-dichlorobenzene eq." $/ \mathrm{kg}$

$3.4 \mathrm{~kg}$ 1,4-dichlorobenzene eq." $/ \mathrm{kg}$

$170 \mathrm{~kg}$ 1,4-dichlorobenzene eq. " $/ \mathrm{kg}$

2200 kg 1,4-dichlorobenzene eq." $/ \mathrm{kg}$

$3.7 \mathrm{~kg} 1,4$-dichlorobenzene eq." $/ \mathrm{kg}$

$0.0025 \mathrm{~kg}$ 1,4-dichlorobenzene eq." $/ \mathrm{kg}$

$0.047 \mathrm{~kg}$ 1,4-dichlorobenzene eq." $/ \mathrm{kg}$

$4.5 \mathrm{~kg} 1,4$-dichlorobenzene eq. "/kg

4.50E+06 kg 1,4-dichlorobenzene eq." $/ \mathrm{kg}$

$0.00013 \mathrm{~kg}$ 1,4-dichlorobenzene eq."/kg

$7200 \mathrm{~kg}$ 1,4-dichlorobenzene eq."//kg

$13 \mathrm{~kg}$ 1,4-dichlorobenzene eq." $/ \mathrm{kg}$

Category $=[\mathrm{C} 8]$ Sediment ecotoxicity fresh water

Description = Problem oriented approach, manual 1999; SETP

Author $=$ anonymous

Date $=1 / 18 / 00$

Environmental resources

Environmental resource

Environmental emissions

Environmental emission

[E90] 1,1,1-trichloroethane (methyl chloroform; HC-140a) to air

[E94] 1,2,3,4-tetrachlorobenzene to air

[E95] 1,2,3,5-tetrachlorobenzene to air

[E96] 1,2,3-trichlorobenzene to air

[E98] 1,2,4,5-tetrachlorobenzene to air

[E99] 1,2,4-trichlorobenzene to air

[E102] 1,2-dichlorobenzene to air

[E103] 1,2-dichloroethane to air

[E107] 1,3,5-trichlorobenzene to air

[E111] 1,3-Butadiene to air

[E112] 1,3-dichlorobenzene to air

[E116] 1,4-dichlorobenzene to air

[E128] 1-chloro-4-nitrobenzene to air

[E166] 2,3,4,6-tetrachlorophenol to air

[E168] 2,3,7,8-TCDD (tetrachloride-dibenzo-dioxin) to air

[E172] 2,4,5-T to air

[E173] 2,4,5-trichlorophenol to air

[E174] 2,4,6-trichlorophenol to air

[E175] 2,4-D to air

[E176] 2,4-dichlorophenol to air

[E185] 2-chlorophenol to air

[E208] 3,4-dichloroaniline to air

[E217] 3-chloroaniline to air

[E228] 4-chloroaniline to air

[E231] acephate to air

[E236] Acrolein (2-propenal) to air

[E237] Acrylonitrile to air

[E241] aldicarb to air

[E242] aldrin to air

[E251] anilazine to air

[E252] anthracene to air

[E253] antimony to air

[E255] arsenic to air

[E256] atrazine to air

[E257] azinphos-ethyl to air
Value

Unit

Unit

Value

$0.0001 \mathrm{~kg} \mathrm{1,4-dichlorobenzene} \mathrm{eq.} \mathrm{"/} / \mathrm{kg}$

$0.12 \mathrm{~kg}$ 1,4-dichlorobenzene eq." $/ \mathrm{kg}$

$0.081 \mathrm{~kg} \mathrm{1,4-dichlorobenzene} \mathrm{eq."} / \mathrm{kg}$

$0.0093 \mathrm{~kg}$ 1,4-dichlorobenzene eq." $/ \mathrm{kg}$

$0.085 \mathrm{~kg}$ 1,4-dichlorobenzene eq." $/ \mathrm{kg}$

$0.011 \mathrm{~kg} \mathrm{1,4-dichlorobenzene} \mathrm{eq."} / \mathrm{kg}$

0.0027 kg 1,4-dichlorobenzene eq." $/ \mathrm{kg}$

$0.0001 \mathrm{~kg} 1,4$-dichlorobenzene eq." $/ \mathrm{kg}$

$0.017 \mathrm{~kg}$ 1,4-dichlorobenzene eq." $/ \mathrm{kg}$

2.20E-07 kg 1,4-dichlorobenzene eq." $/ \mathrm{kg}$

$0.0022 \mathrm{~kg}$ 1,4-dichlorobenzene eq." $/ \mathrm{kg}$

$0.0024 \mathrm{~kg} 1,4$-dichlorobenzene eq." $/ \mathrm{kg}$

$10 \mathrm{~kg}$ 1,4-dichlorobenzene eq." $/ \mathrm{kg}$

$87 \mathrm{~kg}$ 1,4-dichlorobenzene eq." $/ \mathrm{kg}$

$6.80 \mathrm{E}+06 \mathrm{~kg}$ 1,4-dichlorobenzene eq." $/ \mathrm{kg}$

$0.61 \mathrm{~kg}$ 1,4-dichlorobenzene eq."//kg

$17 \mathrm{~kg}$ 1,4-dichlorobenzene eq." $/ \mathrm{kg}$

$5.7 \mathrm{~kg}$ 1,4-dichlorobenzene eq." $/ \mathrm{kg}$

$29 \mathrm{~kg}$ 1,4-dichlorobenzene eq." $/ \mathrm{kg}$

$0.55 \mathrm{~kg}$ 1,4-dichlorobenzene eq." $/ \mathrm{kg}$

$10 \mathrm{~kg}$ 1,4-dichlorobenzene eq."/kg

$2100 \mathrm{~kg}$ 1,4-dichlorobenzene eq." $/ \mathrm{kg}$

$93 \mathrm{~kg}$ 1,4-dichlorobenzene eq." $/ \mathrm{kg}$

$1.8 \mathrm{~kg}$ 1,4-dichlorobenzene eq." $/ \mathrm{kg}$

$40 \mathrm{~kg} 1,4$-dichlorobenzene eq. $" / \mathrm{kg}$ $390 \mathrm{~kg}$ 1,4-dichlorobenzene eq." $/ \mathrm{kg}$

$0.27 \mathrm{~kg} 1,4$-dichlorobenzene eq." $/ \mathrm{kg}$

$41000 \mathrm{~kg}$ 1,4-dichlorobenzene eq." $/ \mathrm{kg}$

$0.24 \mathrm{~kg}$ 1,4-dichlorobenzene eq."//kg

$0.88 \mathrm{~kg}$ 1,4-dichlorobenzene eq." $/ \mathrm{kg}$

$190 \mathrm{~kg}$ 1,4-dichlorobenzene eq."/kg

$9.1 \mathrm{~kg}$ 1,4-dichlorobenzene eq. "/kg

$130 \mathrm{~kg}$ 1,4-dichlorobenzene eq." $/ \mathrm{kg}$

$310 \mathrm{~kg}$ 1,4-dichlorobenzene eq." $/ \mathrm{kg}$

$210 \mathrm{~kg}$ 1,4-dichlorobenzene eq. $" / \mathrm{kg}$ 
[E258] azinphos-methyl to air

[E259] barium to air

[E260] benomyl to air

[E261] bentazone to air

[E263] Benzene to air

[E264] benzo[a]anthracene to air

[E265] benzo[a]pyrene to air

[E266] benzo[ghi]perylene to air

[E267] benzo[k]fluoranthrene to air

[E269] benzylchloride to air

[E270] beryllium to air

[E272] bifenthrin to air

[E290] Butylbenzylphtalate to air

[E385] cadmium to air

[E387] captafol to air

[E388] captan to air

[E389] carbaryl to air

[E390] carbendazim to air

[E391] carbofuran to air

[E394] carbon disulfide to air

[E404] chlordane to air

[E405] chlorfenvinphos to air

[E406] chloridazon to air

[E407] chlorobenzene to air

[E410] chlorothalonil to air

[E411] chlorpropham to air

[E412] chlorpyriphos to air

[E413] chromium (unspecified) to air

[E414] chromium III to air

[E415] chromium VI to air

[E416] chrysene to air

[E421] cobalt to air

[E422] copper to air

[E423] coumaphos to air

[E425] cyanazine to air

[E437] cypermethrin to air

[E438] cyromazine to air

[E439] DDT to air

[E441] deltamethrin to air

[E442] demeton to air

[E443] desmetryn to air

[E444] Di(2-ethylhexyl)phtalate to air

[E446] diazinon to air

[E448] Dibutylphtalate to air

[E449] Dichloromethane (Methylene Chloride) to air

[E450] dichlorprop to air

[E451] dichlorvos to air

[E452] dieldrin to air

[E456] Diethylphtalate to air

[E457] Dihexylphtalate to air

[E458] Diisodecylphtalate to ai

[E459] Diisooctylphtalate to air

[E461] dimethoate to air

[E468] Dimethylphtalate to air

[E470] dinoseb to air

[E471] dinoterb to air

[E472] Dioctylphtalate to air

[E475] disulfothon to air

[E476] diuron to air

[E478] DNOC to air

[E480] endosulfan to air

[E481] endrin to air

[E487] ethoprophos to air

[E496] Ethylbenzene to air

[E499] Ethylene (ethene) to air

[E504] fenitrothion to air

[E505] fenthion to air
$220 \mathrm{~kg}$ 1,4-dichlorobenzene eq." $/ \mathrm{kg}$

$97 \mathrm{~kg}$ 1,4-dichlorobenzene eq." $/ \mathrm{kg}$

$3.9 \mathrm{~kg}$ 1,4-dichlorobenzene eq." $/ \mathrm{kg}$

$4.5 \mathrm{~kg}$ 1,4-dichlorobenzene eq." $/ \mathrm{kg}$

6.40E-05 kg 1,4-dichlorobenzene eq."/kg

$130 \mathrm{~kg}$ 1,4-dichlorobenzene eq." $/ \mathrm{kg}$

$250 \mathrm{~kg}$ 1,4-dichlorobenzene eq." $/ \mathrm{kg}$

$140 \mathrm{~kg}$ 1,4-dichlorobenzene eq." $/ \mathrm{kg}$

13000 kg 1,4-dichlorobenzene eq." $/ \mathrm{kg}$

$0.11 \mathrm{~kg}$ 1,4-dichlorobenzene eq." $/ \mathrm{kg}$

20000 kg 1,4-dichlorobenzene eq." $/ \mathrm{kg}$

2400 kg 1,4-dichlorobenzene eq." /kg

$0.13 \mathrm{~kg}$ 1,4-dichlorobenzene eq." $/ \mathrm{kg}$

$740 \mathrm{~kg}$ 1,4-dichlorobenzene eq." $/ \mathrm{kg}$

30000 kg 1,4-dichlorobenzene eq."/kg

0.14 kg 1,4-dichlorobenzene eq." $/ \mathrm{kg}$

$32 \mathrm{~kg}$ 1,4-dichlorobenzene eq." $/ \mathrm{kg}$

$3000 \mathrm{~kg}$ 1,4-dichlorobenzene eq." $/ \mathrm{kg}$

$520 \mathrm{~kg}$ 1,4-dichlorobenzene eq."//kg

$0.027 \mathrm{~kg}$ 1,4-dichlorobenzene eq." $/ \mathrm{kg}$

27 kg 1,4-dichlorobenzene eq." $/ \mathrm{kg}$

$27 \mathrm{~kg}$ 1,4-dichlorobenzene eq." $/ \mathrm{kg}$

0.02 kg 1,4-dichlorobenzene eq." $/ \mathrm{kg}$

$0.00044 \mathrm{~kg}$ 1,4-dichlorobenzene eq." $/ \mathrm{kg}$

$1.8 \mathrm{~kg}$ 1,4-dichlorobenzene eq." $/ \mathrm{kg}$

$2 \mathrm{~kg}$ 1,4-dichlorobenzene eq." $/ \mathrm{kg}$

$330 \mathrm{~kg}$ 1,4-dichlorobenzene eq." $/ \mathrm{kg}$

$4.9 \mathrm{~kg}$ 1,4-dichlorobenzene eq." $/ \mathrm{kg}$

$4.9 \mathrm{~kg}$ 1,4-dichlorobenzene eq." $/ \mathrm{kg}$

$20 \mathrm{~kg} 1,4$-dichlorobenzene eq." $/ \mathrm{kg}$

$130 \mathrm{~kg}$ 1,4-dichlorobenzene eq." $/ \mathrm{kg}$

$1100 \mathrm{~kg} \mathrm{1,4-dichlorobenzene} \mathrm{eq."} / \mathrm{kg}$

$560 \mathrm{~kg}$ 1,4-dichlorobenzene eq." $/ \mathrm{kg}$

$3.50 \mathrm{E}+05 \mathrm{~kg} \mathrm{1,4-dichlorobenzene} \mathrm{eq."} / \mathrm{kg}$

$1500 \mathrm{~kg}$ 1,4-dichlorobenzene eq." $/ \mathrm{kg}$

$1.50 \mathrm{E}+05 \mathrm{~kg}$ 1,4-dichlorobenzene eq." $/ \mathrm{kg}$

$2800 \mathrm{~kg}$ 1,4-dichlorobenzene eq." $/ \mathrm{kg}$

$3500 \mathrm{~kg}$ 1,4-dichlorobenzene eq." $/ \mathrm{kg}$

$2700 \mathrm{~kg}$ 1,4-dichlorobenzene eq." $/ \mathrm{kg}$

$16 \mathrm{~kg}$ 1,4-dichlorobenzene eq." $/ \mathrm{kg}$

$4.1 \mathrm{~kg}$ 1,4-dichlorobenzene eq." $/ \mathrm{kg}$

$0.47 \mathrm{~kg}$ 1,4-dichlorobenzene eq." $/ \mathrm{kg}$

$160 \mathrm{~kg}$ 1,4-dichlorobenzene eq."/kg

$0.073 \mathrm{~kg}$ 1,4-dichlorobenzene eq." $/ \mathrm{kg}$

2.40E-05 kg 1,4-dichlorobenzene eq." /kg

$0.053 \mathrm{~kg}$ 1,4-dichlorobenzene eq." $/ \mathrm{kg}$

$23 \mathrm{~kg}$ 1,4-dichlorobenzene eq." $/ \mathrm{kg}$

$20 \mathrm{~kg}$ 1,4-dichlorobenzene eq." $/ \mathrm{kg}$

$0.28 \mathrm{~kg}$ 1,4-dichlorobenzene eq." $/ \mathrm{kg}$

$1.2 \mathrm{~kg}$ 1,4-dichlorobenzene eq." $/ \mathrm{kg}$

$1.2 \mathrm{~kg}$ 1,4-dichlorobenzene eq." $/ \mathrm{kg}$

$0.28 \mathrm{~kg}$ 1,4-dichlorobenzene eq." $/ \mathrm{kg}$

$9.3 \mathrm{~kg}$ 1,4-dichlorobenzene eq." $/ \mathrm{kg}$

$0.013 \mathrm{~kg}$ 1,4-dichlorobenzene eq." $/ \mathrm{kg}$

2900 kg 1,4-dichlorobenzene eq." $/ \mathrm{kg}$

1300 kg 1,4-dichlorobenzene eq." $/ \mathrm{kg}$

$0.027 \mathrm{~kg}$ 1,4-dichlorobenzene eq." $/ \mathrm{kg}$

$9.2 \mathrm{~kg}$ 1,4-dichlorobenzene eq." $/ \mathrm{kg}$

$9.2 \mathrm{~kg}$ 1,4-dichlorobenzene eq." $/ \mathrm{kg}$

$0.57 \mathrm{~kg}$ 1,4-dichlorobenzene eq." $/ \mathrm{kg}$

$9.8 \mathrm{~kg}$ 1,4-dichlorobenzene eq. "/kg

$340 \mathrm{~kg}$ 1,4-dichlorobenzene eq." $/ \mathrm{kg}$

1900 kg 1,4-dichlorobenzene eq." $/ \mathrm{kg}$

8.70E-05 kg 1,4-dichlorobenzene eq." $/ \mathrm{kg}$

9.00E-12 kg 1,4-dichlorobenzene eq." $/ \mathrm{kg}$

$1400 \mathrm{~kg}$ 1,4-dichlorobenzene eq." $/ \mathrm{kg}$

1800 kg 1,4-dichlorobenzene eq."/kg 
[E506] fentin acetate to air [E507] fentin chloride to air [E508] fentin hydroxide to air [E509] fluoranthrene to air [E510] folpet to air

[E511] Formaldehyde (methanal) to air

[E516] glyphosate to air

[E533] heptachlor to air

[E535] heptenophos to air

[E536] hexachloro-1,3-butadiene to air

[E537] hexachlorobenzene to air

[E566] indeno[1,2,3-cd]pyrene to air

[E568] iprodione to air

[E583] isoproturon to air

[E584] lead to air

[E585] lindane to air

[E586] linuron to air

[E587] malathion to air

[E589] MCPA to air

[E590] mecoprop to air

[E592] mercury to air

[E595] metamitron to air

[E596] meta-Xylene (1,3-dimethylbenzene) to air

[E597] metazachlor to air

[E598] methabenzthiazuron to air

[E603] methomyl to air

[E626] methyl-mercury to air

[E628] metobromuron to air

[E629] metolachlor to air

[E630] mevinphos to air

[E632] molybdenum to air

[E633] Naphtalene to air

[E635] nickel to air

[E650] ortho-Xylene (1,2-dimethylbenzene) to air

[E651] oxamyl to air

[E652] oxydemethon-methyl to air

[E655] parathion-ethyl to air

[E656] parathion-methyl to air

[E658] para-Xylene (1,4-dimethylbenzene) to air

[E660] pentachlorobenzene to air

[E661] pentachloronitrobenzene to air

[E662] pentachlorophenol to air

[E672] permethrin to air

[E674] phenanthrene to air

[E675] Phenol to air

[E679] phoxim to air

[E680] Phtalic anhydride to air

[E682] pirimicarb to air

[E685] Polycyclic Aromatic Hydrocarbons Carcinogenic- (carcinogenic-PAH) to air

[E686] propachlor to air

[E690] propoxur to air

[E697] Propylene Oxide to air

[E698] pyrazophos to air

[E704] selenium to air

[E705] simazine to air

[E706] styrene (vinylbenzene) to air

[E718] tetrachloroethylene (PER) (tetrachloroethene) to air

[E719] Tetrachloromethane (carbon tetrachloride) $(\mathrm{HC}-10)$ to air

[E722] thallium to air

[E723] Thiram to air

[E724] tin to air

[E725] tolclophos-methyl to air

[E727] Toluene to air

[E733] tri-allate to air

[E734] triazophos to air

[E735] tributyltinoxide to air

[E736] trichlorfon to air
$6900 \mathrm{~kg}$ 1,4-dichlorobenzene eq." $/ \mathrm{kg}$ $3000 \mathrm{~kg}$ 1,4-dichlorobenzene eq." $/ \mathrm{kg}$ $6800 \mathrm{~kg}$ 1,4-dichlorobenzene eq." $/ \mathrm{kg}$

$53 \mathrm{~kg}$ 1,4-dichlorobenzene eq." $/ \mathrm{kg}$

560 kg 1,4-dichlorobenzene eq." $/ \mathrm{kg}$

$4.5 \mathrm{~kg}$ 1,4-dichlorobenzene eq." $/ \mathrm{kg}$

$21 \mathrm{~kg} \mathrm{1,4-dichlorobenzene} \mathrm{eq."} / \mathrm{kg}$

$2 \mathrm{~kg}$ 1,4-dichlorobenzene eq." $/ \mathrm{kg}$

$15 \mathrm{~kg}$ 1,4-dichlorobenzene eq." $/ \mathrm{kg}$

$54 \mathrm{~kg}$ 1,4-dichlorobenzene eq." $/ \mathrm{kg}$

$4.3 \mathrm{~kg}$ 1,4-dichlorobenzene eq." $/ \mathrm{kg}$

$530 \mathrm{~kg}$ 1,4-dichlorobenzene eq." $/ \mathrm{kg}$

$0.23 \mathrm{~kg}$ 1,4-dichlorobenzene eq." $/ \mathrm{kg}$

$71 \mathrm{~kg}$ 1,4-dichlorobenzene eq." $/ \mathrm{kg}$

$6.2 \mathrm{~kg}$ 1,4-dichlorobenzene eq." $/ \mathrm{kg}$

$14 \mathrm{~kg}$ 1,4-dichlorobenzene eq." $/ \mathrm{kg}$

$39 \mathrm{~kg}$ 1,4-dichlorobenzene eq." $/ \mathrm{kg}$

$1100 \mathrm{~kg}$ 1,4-dichlorobenzene eq." $/ \mathrm{kg}$

$0.7 \mathrm{~kg}$ 1,4-dichlorobenzene eq." $/ \mathrm{kg}$

$25 \mathrm{~kg}$ 1,4-dichlorobenzene eq." $/ \mathrm{kg}$

$810 \mathrm{~kg}$ 1,4-dichlorobenzene eq." $/ \mathrm{kg}$

$0.49 \mathrm{~kg} 1,4$-dichlorobenzene eq." $/ \mathrm{kg}$

2.80E-05 kg 1,4-dichlorobenzene eq." $/ \mathrm{kg}$

$5.3 \mathrm{~kg}$ 1,4-dichlorobenzene eq. $/ / \mathrm{kg}$

$76 \mathrm{~kg}$ 1,4-dichlorobenzene eq. $" / \mathrm{kg}$

$10000 \mathrm{~kg}$ 1,4-dichlorobenzene eq." $/ \mathrm{kg}$

19000 kg 1,4-dichlorobenzene eq."/kg

$48 \mathrm{~kg}$ 1,4-dichlorobenzene eq." $/ \mathrm{kg}$

$1300 \mathrm{~kg}$ 1,4-dichlorobenzene eq." $/ \mathrm{kg}$

$1200 \mathrm{~kg}$ 1,4-dichlorobenzene eq." $/ \mathrm{kg}$

$210 \mathrm{~kg}$ 1,4-dichlorobenzene eq." $/ \mathrm{kg}$

$0.19 \mathrm{~kg}$ 1,4-dichlorobenzene eq." $/ \mathrm{kg}$

$1600 \mathrm{~kg}$ 1,4-dichlorobenzene eq." $/ \mathrm{kg}$

7.40E-05 kg 1,4-dichlorobenzene eq." $/ \mathrm{kg}$

$25 \mathrm{~kg}$ 1,4-dichlorobenzene eq. "/ $/ \mathrm{kg}$

530 kg 1,4-dichlorobenzene eq." $/ \mathrm{kg}$

$1900 \mathrm{~kg}$ 1,4-dichlorobenzene eq." $/ \mathrm{kg}$

$60 \mathrm{~kg}$ 1,4-dichlorobenzene eq." $/ \mathrm{kg}$

3.70E-05 kg 1,4-dichlorobenzene eq." $/ \mathrm{kg}$

$0.52 \mathrm{~kg}$ 1,4-dichlorobenzene eq. "/kg

$13 \mathrm{~kg}$ 1,4-dichlorobenzene eq." $/ \mathrm{kg}$

$24 \mathrm{~kg}$ 1,4-dichlorobenzene eq." $/ \mathrm{kg}$

21000 kg 1,4-dichlorobenzene eq."/kg

$1.4 \mathrm{~kg} 1,4$-dichlorobenzene eq. $/ / \mathrm{kg}$

$0.56 \mathrm{~kg} 1,4$-dichlorobenzene eq." $/ \mathrm{kg}$

$0.071 \mathrm{~kg} 1,4$-dichlorobenzene eq." $/ \mathrm{kg}$

1.70E-05 kg 1,4-dichlorobenzene eq." $/ \mathrm{kg}$

$2400 \mathrm{~kg} 1,4$-dichlorobenzene eq." $/ \mathrm{kg}$

560 kg 1,4-dichlorobenzene eq." $/ \mathrm{kg}$

$11 \mathrm{~kg} 1,4$-dichlorobenzene eq." $/ \mathrm{kg}$

$18000 \mathrm{~kg}$ 1,4-dichlorobenzene eq." $/ \mathrm{kg}$

$0.02 \mathrm{~kg}$ 1,4-dichlorobenzene eq." $/ \mathrm{kg}$

$170 \mathrm{~kg}$ 1,4-dichlorobenzene eq." $/ \mathrm{kg}$

$640 \mathrm{~kg}$ 1,4-dichlorobenzene eq." $/ \mathrm{kg}$

$1800 \mathrm{~kg}$ 1,4-dichlorobenzene eq." $/ \mathrm{kg}$

3.50E-05 kg 1,4-dichlorobenzene eq." $/ \mathrm{kg}$

$0.00039 \mathrm{~kg}$ 1,4-dichlorobenzene eq."/kg

$0.00014 \mathrm{~kg}$ 1,4-dichlorobenzene eq." $/ \mathrm{kg}$

$3900 \mathrm{~kg}$ 1,4-dichlorobenzene eq." $/ \mathrm{kg}$

980 kg 1,4-dichlorobenzene eq." $/ \mathrm{kg}$

$1.3 \mathrm{~kg}$ 1,4-dichlorobenzene eq." $/ \mathrm{kg}$

$0.16 \mathrm{~kg}$ 1,4-dichlorobenzene eq. $/ \mathrm{kg}$

5.00E-05 kg 1,4-dichlorobenzene eq." $/ \mathrm{kg}$

$22 \mathrm{~kg} \mathrm{1,4-dichlorobenzene} \mathrm{eq."} / \mathrm{kg}$

$3000 \mathrm{~kg}$ 1,4-dichlorobenzene eq." $/ \mathrm{kg}$

10000 kg 1,4-dichlorobenzene eq." $/ \mathrm{kg}$

$2400 \mathrm{~kg}$ 1,4-dichlorobenzene eq." $/ \mathrm{kg}$ 
[E737] Trichloroethylene (tri) to air

[E738] Trichloromethane (chloroform) to air

[E739] trifluarin to air

[E743] vanadium to air

[E745] Vinyl Chloride (chloroethene) to air

[E748] zinc to air

[E749] zineb to air

[E750] 1,1,1-trichloroethane to air

[E751] 1,2,3,4-tetrachlorobenzene to air

[E752] 1,2,3,5-tetrachlorobenzene to air

[E753] 1,2,3-trichlorobenzene to air

[E754] 1,2,4,5-tetrachlorobenzene to air

[E755] 1,2,4-trichlorobenzene to air

[E756] 1,2-dichlorobenzene to air

[E757] 1,2-dichloroethane to air

[E758] 1,3,5-trichlorobenzene to air

[E759] 1,3-butadiene to air

[E760] 1,3-dichlorobenzene to air

[E761] 1,4-dichlorobenzene to air

[E762] 1-chloro-4-nitrobenzene to air

[E763] 2,3,4,6-tetrachlorophenol to air

[E764] 2,3,7,8-TCDD to air

[E765] 2,4,5-T to air

[E766] 2,4,5-trichlorophenol to air

[E767] 2,4,6-trichlorophenol to fresh water

[E768] 2,4-D to fresh water

[E769] 2,4-dichlorophenol to fresh water

[E770] 2-chlorophenol to fresh water

[E7711] 3,4-dichloroaniline to fresh water

[E772] 3-chloroaniline to fresh water

[E773] 4-chloroaniline to fresh water

[E774] acephate to fresh water

[E775] Acrolein (2-propenal) to fresh water

[E776] acrylonitrile to fresh water

[E777] aldicarb to fresh water

[E778] aldrin to fresh water

[E780] anilazine to fresh water

[E781] anthracene to fresh water

[E782] antimony to fresh water

[E783] arsenic to fresh water

[E784] atrazine to fresh water

[E785] azinphos-ethyl to fresh water

[E786] azinphos-methyl to fresh water

[E787] barium to fresh water

[E788] benomyl to fresh water

[E789] bentazone to fresh water

[E790] benzene to fresh water

[E791] benzo[a]anthracene to fresh water

[E792] benzo[a]pyrene to fresh water

[E793] benzo[ghi]perylene to fresh water

[E794] benzo[k]fluoranthrene to fresh water

[E795] benzylchloride to fresh water

[E796] beryllium to fresh water

[E797] bifenthrin to fresh water

[E800] Butylbenzylphtalate to fresh water

[E801] cadmium to fresh water

[E802] captafol to fresh water

[E803] captan to fresh water

[E804] carbaryl to fresh water

[E805] carbendazim to fresh water

[E806] carbofuran to fresh water

[E807] carbon disulfide to fresh water

[E808] Carcinogenic PAHs to fresh water

[E810] chlordane to fresh water

[E811] chlorfenvinphos to fresh water

[E812] chloridazon to fresh water

[E814] chlorobenzene to fresh water
3.20E-05 kg 1,4-dichlorobenzene eq."/kg 4.90E-05 kg 1,4-dichlorobenzene eq."/kg

$8.1 \mathrm{~kg}$ 1,4-dichlorobenzene eq." $/ \mathrm{kg}$

$4100 \mathrm{~kg} \mathrm{1,4-dichlorobenzene} \mathrm{eq."} / \mathrm{kg}$

2.30E-06 kg 1,4-dichlorobenzene eq."/kg

$46 \mathrm{~kg}$ 1,4-dichlorobenzene eq." $/ \mathrm{kg}$

$740 \mathrm{~kg}$ 1,4-dichlorobenzene eq." $/ \mathrm{kg}$

0.09 kg 1,4-dichlorobenzene eq." $/ \mathrm{kg}$

$19 \mathrm{~kg}$ 1,4-dichlorobenzene eq." $/ \mathrm{kg}$

$16 \mathrm{~kg}$ 1,4-dichlorobenzene eq." $/ \mathrm{kg}$

$4.4 \mathrm{~kg}$ 1,4-dichlorobenzene eq." $/ \mathrm{kg}$

$15 \mathrm{~kg}$ 1,4-dichlorobenzene eq." $/ \mathrm{kg}$

$3.8 \mathrm{~kg}$ 1,4-dichlorobenzene eq." $/ \mathrm{kg}$

$0.95 \mathrm{~kg}$ 1,4-dichlorobenzene eq." $/ \mathrm{kg}$

$0.019 \mathrm{~kg}$ 1,4-dichlorobenzene eq." $/ \mathrm{kg}$

$5.2 \mathrm{~kg}$ 1,4-dichlorobenzene eq." $/ \mathrm{kg}$

$2 \mathrm{~kg}$ 1,4-dichlorobenzene eq." $/ \mathrm{kg}$

$1.2 \mathrm{~kg}$ 1,4-dichlorobenzene eq." $/ \mathrm{kg}$

$1 \mathrm{~kg}$ 1,4-dichlorobenzene eq." $/ \mathrm{kg}$

770 kg 1,4-dichlorobenzene eq."/kg

5700 kg 1,4-dichlorobenzene eq." $/ \mathrm{kg}$

5.60E+08 kg 1,4-dichlorobenzene eq." $/ \mathrm{kg}$

$12 \mathrm{~kg} \mathrm{1,4-dichlorobenzene} \mathrm{eq."} / \mathrm{kg}$

$1900 \mathrm{~kg} \mathrm{1,4-dichlorobenzene} \mathrm{eq."} / \mathrm{kg}$

$290 \mathrm{~kg}$ 1,4-dichlorobenzene eq." $/ \mathrm{kg}$

$300 \mathrm{~kg}$ 1,4-dichlorobenzene eq." $/ \mathrm{kg}$

$68 \mathrm{~kg}$ 1,4-dichlorobenzene eq." $/ \mathrm{kg}$

$1300 \mathrm{~kg}$ 1,4-dichlorobenzene eq."/kg

2400 kg 1,4-dichlorobenzene eq." $/ \mathrm{kg}$

$2300 \mathrm{~kg}$ 1,4-dichlorobenzene eq."/kg

2700 kg 1,4-dichlorobenzene eq." $/ \mathrm{kg}$

$560 \mathrm{~kg}$ 1,4-dichlorobenzene eq."/kg

1.90E+05 kg 1,4-dichlorobenzene eq." $/ \mathrm{kg}$

52 kg 1,4-dichlorobenzene eq." $/ \mathrm{kg}$

3.50E+05 kg 1,4-dichlorobenzene eq." $/ \mathrm{kg}$ $1000 \mathrm{~kg}$ 1,4-dichlorobenzene eq."/kg

$70 \mathrm{~kg} \mathrm{1,4-dichlorobenzene} \mathrm{eq."} / \mathrm{kg}$

$80000 \mathrm{~kg}$ 1,4-dichlorobenzene eq." $/ \mathrm{kg}$

$48 \mathrm{~kg}$ 1,4-dichlorobenzene eq." $/ \mathrm{kg}$ $530 \mathrm{~kg}$ 1,4-dichlorobenzene eq." $/ \mathrm{kg}$ $4300 \mathrm{~kg}$ 1,4-dichlorobenzene eq." $/ \mathrm{kg}$ $2.00 \mathrm{E}+05 \mathrm{~kg}$ 1,4-dichlorobenzene eq." $/ \mathrm{kg}$ $27000 \mathrm{~kg}$ 1,4-dichlorobenzene eq." $/ \mathrm{kg}$ $510 \mathrm{~kg}$ 1,4-dichlorobenzene eq." $/ \mathrm{kg}$ $880 \mathrm{~kg}$ 1,4-dichlorobenzene eq." $/ \mathrm{kg}$

$41 \mathrm{~kg} \mathrm{1,4-dichlorobenzene} \mathrm{eq."} / \mathrm{kg}$

$0.07 \mathrm{~kg}$ 1,4-dichlorobenzene eq."//kg $3.50 \mathrm{E}+05 \mathrm{~kg}$ 1,4-dichlorobenzene eq." $/ \mathrm{kg}$ 7.20E+05 kg 1,4-dichlorobenzene eq."/kg 1.70E+05 kg 1,4-dichlorobenzene eq." $/ \mathrm{kg}$ $3.90 \mathrm{E}+06$ kg 1,4-dichlorobenzene eq." $/ \mathrm{kg}$

$29 \mathrm{~kg}$ 1,4-dichlorobenzene eq." $/ \mathrm{kg}$ 1.10E+05 kg 1,4-dichlorobenzene eq. " $/ \mathrm{kg}$ 7.20E+05 kg 1,4-dichlorobenzene eq." $/ \mathrm{kg}$

$25 \mathrm{~kg}$ 1,4-dichlorobenzene eq." $/ \mathrm{kg}$

$3900 \mathrm{~kg}$ 1,4-dichlorobenzene eq." $/ \mathrm{kg}$

7.70E+05 kg 1,4-dichlorobenzene eq."/kg

$18 \mathrm{~kg}$ 1,4-dichlorobenzene eq." $/ \mathrm{kg}$

$1300 \mathrm{~kg}$ 1,4-dichlorobenzene eq." $/ \mathrm{kg}$

$39000 \mathrm{~kg}$ 1,4-dichlorobenzene eq." $/ \mathrm{kg}$

7600 kg 1,4-dichlorobenzene eq." $/ \mathrm{kg}$

$86 \mathrm{~kg}$ 1,4-dichlorobenzene eq." $/ \mathrm{kg}$

89000 kg 1,4-dichlorobenzene eq." $/ \mathrm{kg}$

$9100 \mathrm{~kg}$ 1,4-dichlorobenzene eq." $/ \mathrm{kg}$

$940 \mathrm{~kg}$ 1,4-dichlorobenzene eq." $/ \mathrm{kg}$

$25 \mathrm{~kg}$ 1,4-dichlorobenzene eq." $/ \mathrm{kg}$

$0.34 \mathrm{~kg}$ 1,4-dichlorobenzene eq." $/ \mathrm{kg}$ 
[E815] chlorothalonil to fresh water [E816] chlorpropham to fresh water [E817] chlorpyriphos to fresh water [E818] chromium III to fresh water [E819] chromium VI to fresh water [E820] chrysene to fresh water [E821] cobalt to fresh water [E822] copper to fresh water [E823] coumaphos to fresh water [E824] cyanazine to fresh water [E825] cypermethrin to fresh water [E826] cyromazine to fresh water [E827] DDT to fresh water

[E828] deltamethrin to fresh water [E829] demeton to fresh water [E830] desmetryn to fresh water

[E831] Di(2-ethylhexyl)phtalate to fresh water

[E832] diazinon to fresh water

[E833] Dibutylphtalate to fresh water

[E834] Dichloromethane (Methylene Chloride) to fresh water

[E835] dichlorprop to fresh water

[E836] dichlorvos to fresh water

[E837] dieldrin to fresh water

[E838] Diethylphtalate to fresh water

[E839] Dihexylphtalate to fresh water

[E840] Diisodecylphtalate to fresh water

[E841] Diisooctylphtalate to fresh water

[E842] dimethoate to fresh water

[E843] Dimethylphtalate to fresh water

[E844] dinoseb to fresh water

[E845] dinoterb to fresh water

[E846] Dioctylphtalate to fresh water

[E847] disulfothon to fresh water

[E848] diuron to fresh water

[E849] DNOC to fresh water

[E850] endosulfan to fresh water

[E851] endrin to fresh water

[E852] ethoprophos to fresh water

[E853] ethylbenzene to fresh water

[E854] ethylene to fresh water

[E855] fenitrothion to fresh water

[E856] fenthion to fresh water

[E857] fentin acetate to fresh water

[E858] fentin chloride to fresh water

[E859] fentin hydroxide to fresh water

[E860] fluoranthrene to fresh water

[E861] folpet to fresh water

[E862] Formaldehyde (methanal) to fresh water

[E863] glyphosate to fresh water

[E864] heptachlor to fresh water

[E865] heptenophos to fresh water

[E866] hexachloro-1,3-butadiene to fresh water

[E867] hexachlorobenzene to fresh water

[E870] indeno[1,2,3-cd]pyrene to fresh water

[E871] iprodione to fresh water

[E872] isoproturon to fresh water

[E873] lead to fresh water

[E874] lindane to fresh water

[E875] linuron to fresh water

[E876] malathion to fresh water

[E878] MCPA to fresh water

[E879] mecoprop to fresh water

[E880] mercury to fresh water

[E881] metamitron to fresh water

[E882] metazachlor to fresh water

[E883] methabenzthiazuron to fresh water

[E884] methomyl to fresh water
$260 \mathrm{~kg}$ 1,4-dichlorobenzene eq." $/ \mathrm{kg}$

71 kg 1,4-dichlorobenzene eq." $/ \mathrm{kg}$

4.10E+05 kg 1,4-dichlorobenzene eq." $/ \mathrm{kg}$

$18 \mathrm{~kg}$ 1,4-dichlorobenzene eq. "/ $/ \mathrm{kg}$

$71 \mathrm{~kg}$ 1,4-dichlorobenzene eq." $/ \mathrm{kg}$

$59000 \mathrm{~kg}$ 1,4-dichlorobenzene eq." $/ \mathrm{kg}$

$5600 \mathrm{~kg}$ 1,4-dichlorobenzene eq." $/ \mathrm{kg}$

$2900 \mathrm{~kg}$ 1,4-dichlorobenzene eq."//kg

2.90E+07 kg 1,4-dichlorobenzene eq." $/ \mathrm{kg}$ 43000 kg 1,4-dichlorobenzene eq." $/ \mathrm{kg}$

1.40E+07 kg 1,4-dichlorobenzene eq."/kg

21000 kg 1,4-dichlorobenzene eq." $/ \mathrm{kg}$

$31000 \mathrm{~kg}$ 1,4-dichlorobenzene eq." $/ \mathrm{kg}$

$9.80 \mathrm{E}+05 \mathrm{~kg}$ 1,4-dichlorobenzene eq." $/ \mathrm{kg}$

$16000 \mathrm{~kg}$ 1,4-dichlorobenzene eq." $/ \mathrm{kg}$

120 kg 1,4-dichlorobenzene eq." $/ \mathrm{kg}$

$100 \mathrm{~kg}$ 1,4-dichlorobenzene eq." $/ \mathrm{kg}$

77000 kg 1,4-dichlorobenzene eq."/kg

$10 \mathrm{~kg}$ 1,4-dichlorobenzene eq." $/ \mathrm{kg}$

$0.0088 \mathrm{~kg}$ 1,4-dichlorobenzene eq." $/ \mathrm{kg}$

$2.8 \mathrm{~kg}$ 1,4-dichlorobenzene eq." $/ \mathrm{kg}$

$5500 \mathrm{~kg}$ 1,4-dichlorobenzene eq." $/ \mathrm{kg}$

8200 kg 1,4-dichlorobenzene eq." $/ \mathrm{kg}$

22 kg 1,4-dichlorobenzene eq." $/ \mathrm{kg}$

$260 \mathrm{~kg}$ 1,4-dichlorobenzene eq." $/ \mathrm{kg}$

$190 \mathrm{~kg}$ 1,4-dichlorobenzene eq."//kg

$47 \mathrm{~kg}$ 1,4-dichlorobenzene eq." $/ \mathrm{kg}$

$130 \mathrm{~kg}$ 1,4-dichlorobenzene eq. "/kg

0.79 kg 1,4-dichlorobenzene eq." $/ \mathrm{kg}$

$88000 \mathrm{~kg}$ 1,4-dichlorobenzene eq."/kg

$1.00 \mathrm{E}+05 \mathrm{~kg} \mathrm{1,4-dichlorobenzene} \mathrm{eq."} / \mathrm{kg}$

$4.7 \mathrm{~kg}$ 1,4-dichlorobenzene eq." $/ \mathrm{kg}$

22000 kg 1,4-dichlorobenzene eq."/kg

22000 kg 1,4-dichlorobenzene eq." $/ \mathrm{kg}$

$19 \mathrm{~kg}$ 1,4-dichlorobenzene eq."//kg

$6000 \mathrm{~kg}$ 1,4-dichlorobenzene eq." $/ \mathrm{kg}$

2.10E+05 kg 1,4-dichlorobenzene eq." $/ \mathrm{kg}$

1.20E+05 kg 1,4-dichlorobenzene eq." $/ \mathrm{kg}$

$0.36 \mathrm{~kg}$ 1,4-dichlorobenzene eq." $/ \mathrm{kg}$

$0.014 \mathrm{~kg}$ 1,4-dichlorobenzene eq." $/ \mathrm{kg}$

$1.40 \mathrm{E}+05 \mathrm{~kg}$ 1,4-dichlorobenzene eq." $/ \mathrm{kg}$

$6.60 \mathrm{E}+05 \mathrm{~kg}$ 1,4-dichlorobenzene eq." $/ \mathrm{kg}$

4.30E+05 kg 1,4-dichlorobenzene eq." $/ \mathrm{kg}$

$2.80 \mathrm{E}+05 \mathrm{~kg}$ 1,4-dichlorobenzene eq." $/ \mathrm{kg}$

4.30E+05 kg 1,4-dichlorobenzene eq." $/ \mathrm{kg}$

39000 kg 1,4-dichlorobenzene eq." $/ \mathrm{kg}$

1.10E+05 kg 1,4-dichlorobenzene eq. " $/ \mathrm{kg}$

$150 \mathrm{~kg}$ 1,4-dichlorobenzene eq." $/ \mathrm{kg}$

$1300 \mathrm{~kg}$ 1,4-dichlorobenzene eq." $/ \mathrm{kg}$

$26000 \mathrm{~kg}$ 1,4-dichlorobenzene eq."//kg

$2800 \mathrm{~kg}$ 1,4-dichlorobenzene eq. "/kg

$52000 \mathrm{~kg} 1,4$-dichlorobenzene eq." $/ \mathrm{kg}$

$490 \mathrm{~kg}$ 1,4-dichlorobenzene eq." $/ \mathrm{kg}$

2.50E+05 kg 1,4-dichlorobenzene eq." $/ \mathrm{kg}$

$13 \mathrm{~kg}$ 1,4-dichlorobenzene eq." $/ \mathrm{kg}$

$710 \mathrm{~kg}$ 1,4-dichlorobenzene eq." $/ \mathrm{kg}$

25 kg 1,4-dichlorobenzene eq." $/ \mathrm{kg}$

$1700 \mathrm{~kg}$ 1,4-dichlorobenzene eq." $/ \mathrm{kg}$

$31000 \mathrm{~kg} \mathrm{1,4-dichlorobenzene} \mathrm{eq."} / \mathrm{kg}$

$1.20 \mathrm{E}+05 \mathrm{~kg}$ 1,4-dichlorobenzene eq." $/ \mathrm{kg}$

$18 \mathrm{~kg} \mathrm{1,4-dichlorobenzene} \mathrm{eq."} / \mathrm{kg}$

$250 \mathrm{~kg}$ 1,4-dichlorobenzene eq." $/ \mathrm{kg}$

4400 kg 1,4-dichlorobenzene eq." $/ / \mathrm{kg}$

$12 \mathrm{~kg}$ 1,4-dichlorobenzene eq." $/ \mathrm{kg}$

$110 \mathrm{~kg}$ 1,4-dichlorobenzene eq." $/ \mathrm{kg}$

1200 kg 1,4-dichlorobenzene eq." $/ \mathrm{kg}$

$1.00 \mathrm{E}+05 \mathrm{~kg}$ 1,4-dichlorobenzene eq." $/ \mathrm{kg}$ 
[E885] methylbromide to fresh water [E886] methyl-mercury to fresh water [E887] metobromuron to fresh water

[E888] metolachlor to fresh water

[E889] mevinphos to fresh water

[E890] molybdenum to fresh water

[E891] $\mathrm{m}$-xylene to fresh water

[E892] naphtalene to fresh water

[E893] nickel to fresh water

[E899] oxamyl to fresh water

[E900] oxydemethon-methyl to fresh water

[E901] o-xylene to fresh water

[E902] parathion-ethyl to fresh water

[E903] parathion-methyl to fresh water

[E904] pentachlorobenzene to fresh water

[E905] pentachloronitrobenzene to fresh water

[E906] pentachlorophenol to fresh water

[E907] permethrin to fresh water

[E908] phenanthrene to fresh water

[E909] phenol to fresh water

[E912] phoxim to fresh water

[E913] Phtalic anhydride to fresh water

[E914] pirimicarb to fresh water

[E916] propachlor to fresh water

[E917] propoxur to fresh water

[E918] propylene oxide to fresh water

[E919] p-xylene to fresh water

[E920] pyrazophos to fresh water

[E921] selenium to fresh water

[E922] simazine to fresh water

[E923] styrene (vinylbenzene) to fresh water

[E926] tetrachloroethylene (PER) to fresh water

[E927] Tetrachloromethane (carbon tetrachloride) (HC-10) to fresh water

[E928] thallium to fresh water

[E929] Thiram to fresh water

[E930] tin to fresh water

[E931] tolclophos-methyl to fresh water

[E932] toluene to fresh water

[E933] tri-allate to fresh water

[E934] triazophos to fresh water

[E935] tributyltinoxide to fresh water

[E936] trichlorfon to fresh water

[E937] Trichloroethylene (tri) to fresh water

[E938] Trichloromethane $=$ chloroform to fresh water

[E939] trifluarin to fresh water

[E940] vanadium to fresh water

[E941] Vinyl Chloride (chloroethene) to fresh water

[E942] zinc to fresh water

[E943] zineb to fresh water

[E944] 1,1,1-trichloroethane to fresh water

[E945] 1,2,3,4-tetrachlorobenzene to fresh water

[E946] 1,2,3,5-tetrachlorobenzene to fresh water

[E947] 1,2,3-trichlorobenzene to fresh water

[E948] 1,2,4,5-tetrachlorobenzene to fresh water

[E949] 1,2,4-trichlorobenzene to fresh water

[E950] 1,2-dichlorobenzene to fresh water

[E951] 1,2-dichloroethane to fresh water

[E952] 1,3,5-trichlorobenzene to fresh water

[E953] 1,3-butadiene to fresh water

[E954] 1,3-dichlorobenzene to fresh water

[E955] 1,4-dichlorobenzene to fresh water

[E956] 1-chloro-4-nitrobenzene to fresh water

[E957] 2,3,4,6-tetrachlorophenol to fresh water

[E958] 2,3,7,8-TCDD to fresh water

[E959] 2,4,5-T to fresh water

[E960] 2,4,5-trichlorophenol to fresh water

[E961] 2,4,6-trichlorophenol to sea water
$10 \mathrm{~kg}$ 1,4-dichlorobenzene eq." $/ \mathrm{kg}$

1.00E+05 kg 1,4-dichlorobenzene eq."/kg

$420 \mathrm{~kg}$ 1,4-dichlorobenzene eq." $/ \mathrm{kg}$

34000 kg 1,4-dichlorobenzene eq."/kg

74000 kg 1,4-dichlorobenzene eq."/kg

1100 kg 1,4-dichlorobenzene eq."/kg

$0.39 \mathrm{~kg}$ 1,4-dichlorobenzene eq." $/ \mathrm{kg}$

$260 \mathrm{~kg}$ 1,4-dichlorobenzene eq." $/ \mathrm{kg}$

8300 kg 1,4-dichlorobenzene eq." $/ \mathrm{kg}$

$300 \mathrm{~kg}$ 1,4-dichlorobenzene eq." $/ \mathrm{kg}$

16000 kg 1,4-dichlorobenzene eq." $/ \mathrm{kg}$

$0.45 \mathrm{~kg}$ 1,4-dichlorobenzene eq." $/ \mathrm{kg}$

8.00E+05 kg 1,4-dichlorobenzene eq." $/ \mathrm{kg}$

18000 kg 1,4-dichlorobenzene eq." $/ \mathrm{kg}$

$72 \mathrm{~kg}$ 1,4-dichlorobenzene eq." $/ \mathrm{kg}$

1100 kg 1,4-dichlorobenzene eq."/kg

$1600 \mathrm{~kg}$ 1,4-dichlorobenzene eq."//kg

6.70E+06 kg 1,4-dichlorobenzene eq." $/ \mathrm{kg}$

$560 \mathrm{~kg}$ 1,4-dichlorobenzene eq." $/ \mathrm{kg}$

$88 \mathrm{~kg}$ 1,4-dichlorobenzene eq." $/ \mathrm{kg}$

$430 \mathrm{~kg}$ 1,4-dichlorobenzene eq." $/ \mathrm{kg}$

$0.0011 \mathrm{~kg}$ 1,4-dichlorobenzene eq." $/ \mathrm{kg}$

$36000 \mathrm{~kg}$ 1,4-dichlorobenzene eq." $/ \mathrm{kg}$

$670 \mathrm{~kg}$ 1,4-dichlorobenzene eq." $/ \mathrm{kg}$

1.80E+05 kg 1,4-dichlorobenzene eq."/kg

$2.1 \mathrm{~kg}$ 1,4-dichlorobenzene eq." $/ \mathrm{kg}$

$0.33 \mathrm{~kg} \mathrm{1,4-dichlorobenzene} \mathrm{eq."} / \mathrm{kg}$

$45000 \mathrm{~kg}$ 1,4-dichlorobenzene eq." $/ \mathrm{kg}$

$3400 \mathrm{~kg}$ 1,4-dichlorobenzene eq." $/ \mathrm{kg}$

$23000 \mathrm{~kg}$ 1,4-dichlorobenzene eq." $/ \mathrm{kg}$

$0.3 \mathrm{~kg}$ 1,4-dichlorobenzene eq." $/ \mathrm{kg}$

$0.67 \mathrm{~kg}$ 1,4-dichlorobenzene eq." $/ \mathrm{kg}$

0.12 kg 1,4-dichlorobenzene eq." $/ \mathrm{kg}$

20000 kg 1,4-dichlorobenzene eq." / $/ \mathrm{kg}$

$35000 \mathrm{~kg}$ 1,4-dichlorobenzene eq." $/ \mathrm{kg}$

$5.2 \mathrm{~kg}$ 1,4-dichlorobenzene eq." $/ \mathrm{kg}$

$530 \mathrm{~kg}$ 1,4-dichlorobenzene eq." $/ \mathrm{kg}$

$0.21 \mathrm{~kg}$ 1,4-dichlorobenzene eq." $/ \mathrm{kg}$

17000 kg 1,4-dichlorobenzene eq." $/ \mathrm{kg}$

1.60E+05 kg 1,4-dichlorobenzene eq."/kg

6.10E+05 kg 1,4-dichlorobenzene eq."/kg

$76000 \mathrm{~kg}$ 1,4-dichlorobenzene eq." $/ \mathrm{kg}$

$0.082 \mathrm{~kg}$ 1,4-dichlorobenzene eq." $/ \mathrm{kg}$

$0.022 \mathrm{~kg}$ 1,4-dichlorobenzene eq." $/ \mathrm{kg}$

22000 kg 1,4-dichlorobenzene eq."//kg

$21000 \mathrm{~kg}$ 1,4-dichlorobenzene eq."/kg

$0.023 \mathrm{~kg}$ 1,4-dichlorobenzene eq." $/ \mathrm{kg}$

$240 \mathrm{~kg}$ 1,4-dichlorobenzene eq." $/ \mathrm{kg}$

22000 kg 1,4-dichlorobenzene eq." $/ \mathrm{kg}$

5.90E-05 kg 1,4-dichlorobenzene eq." $/ \mathrm{kg}$

$0.045 \mathrm{~kg}$ 1,4-dichlorobenzene eq." $/ \mathrm{kg}$

$0.033 \mathrm{~kg}$ 1,4-dichlorobenzene eq." $/ \mathrm{kg}$

$0.0043 \mathrm{~kg} 1,4$-dichlorobenzene eq." $/ \mathrm{kg}$

$0.033 \mathrm{~kg}$ 1,4-dichlorobenzene eq." $/ \mathrm{kg}$

0.0048 kg 1,4-dichlorobenzene eq." $/ \mathrm{kg}$

$0.0012 \mathrm{~kg}$ 1,4-dichlorobenzene eq." $/ \mathrm{kg}$

7.40E-05 kg 1,4-dichlorobenzene eq." $/ \mathrm{kg}$

$0.0072 \mathrm{~kg}$ 1,4-dichlorobenzene eq." $/ \mathrm{kg}$

3.80E-08 kg 1,4-dichlorobenzene eq." $/ \mathrm{kg}$

$0.001 \mathrm{~kg}$ 1,4-dichlorobenzene eq."/kg

$0.0011 \mathrm{~kg}$ 1,4-dichlorobenzene eq."/kg

$1.7 \mathrm{~kg} \mathrm{1,4-dichlorobenzene} \mathrm{eq."} / \mathrm{kg}$

$0.0014 \mathrm{~kg}$ 1,4-dichlorobenzene eq."/kg

4.30E+05 kg 1,4-dichlorobenzene eq."//kg

1.20E-10 kg 1,4-dichlorobenzene eq." $/ \mathrm{kg}$

$0.064 \mathrm{~kg}$ 1,4-dichlorobenzene eq."/kg

0.00023 kg 1,4-dichlorobenzene eq."/kg 
[E962] 2,4-D to sea water

[E963] 2,4-dichlorophenol to sea water

[E964] 2-chlorophenol to sea water

[E965] 3,4-dichloroaniline to sea water

[E966] 3-chloroaniline to sea water

[E967] 4-chloroaniline to sea water

[E968] acephate to sea water

[E969] Acrolein (2-propenal) to sea water

[E970] acrylonitrile to sea water

[E971] aldicarb to sea water

[E972] aldrin to sea water

[E974] anilazine to sea water

[E975] anthracene to sea water

[E976] antimony to sea water

[E977] arsenic to sea water

[E978] atrazine to sea water

[E979] azinphos-ethyl to sea water

[E980] azinphos-methyl to sea water

[E981] barium to sea water

[E982] benomyl to sea water

[E983] bentazone to sea water

[E984] benzene to sea water

[E985] benzo[a]anthracene to sea water

[E986] benzo[a]pyrene to sea water

[E987] benzo[ghi]perylene to sea water

[E988] benzo[k]fluoranthrene to sea water

[E989] benzylchloride to sea water

[E990] beryllium to sea water

[E991] bifenthrin to sea water

[E994] Butylbenzylphtalate to sea water

[E995] cadmium to sea water

[E996] captafol to sea water

[E997] captan to sea water

[E998] carbaryl to sea water

[E999] carbendazim to sea water

[E1000] carbofuran to sea water

[E1001] carbon disulfide to sea water

[E1002] Carcinogenic PAHs to sea water

[E1004] chlordane to sea water

[E1005] chlorfenvinphos to sea water

[E1006] chloridazon to sea water

[E1008] chlorobenzene to sea water

[E1009] chlorothalonil to sea water

[E1010] chlorpropham to sea water

[E1011] chlorpyriphos to sea water

[E1012] chromium III to sea water

[E1013] chromium VI to sea water

[E1014] chrysene to sea water

[E1015] cobalt to sea water

[E1016] copper to sea water

[E1017] coumaphos to sea water

[E1018] cyanazine to sea water

[E1019] cypermethrin to sea water

[E1020] cyromazine to sea water

[E1021] DDT to sea water

[E1022] deltamethrin to sea water

[E1023] demeton to sea water

[E1024] desmetryn to sea water

[E1025] Di(2-ethylhexyl)phtalate to sea water

[E1026] diazinon to sea water

[E1027] Dibutylphtalate to sea water

[E1028] Dichloromethane (Methylene Chloride) to sea water

[E1029] dichlorprop to sea water

[E1030] dichlorvos to sea water

[E1031] dieldrin to sea water

[E1032] Diethylphtalate to sea water

[E1033] Dihexylphtalate to sea water
8.50E-11 kg 1,4-dichlorobenzene eq." $/ \mathrm{kg}$ $0.00011 \mathrm{~kg} 1,4$-dichlorobenzene eq." $/ \mathrm{kg}$ $0.0053 \mathrm{~kg}$ 1,4-dichlorobenzene eq." $/ \mathrm{kg}$ $0.0015 \mathrm{~kg}$ 1,4-dichlorobenzene eq." $/ \mathrm{kg}$ 3.40E-06 kg 1,4-dichlorobenzene eq." $/ \mathrm{kg}$ $0.0097 \mathrm{~kg}$ 1,4-dichlorobenzene eq." $/ \mathrm{kg}$

3.10E-08 kg 1,4-dichlorobenzene eq."//kg

$3.7 \mathrm{~kg}$ 1,4-dichlorobenzene eq." $/ \mathrm{kg}$

$0.0039 \mathrm{~kg} \mathrm{1,4-dichlorobenzene} \mathrm{eq."/kg}$

$0.098 \mathrm{~kg}$ 1,4-dichlorobenzene eq."/ $/ \mathrm{kg}$

$0.11 \mathrm{~kg} \mathrm{1,4-dichlorobenzene} \mathrm{eq."} / \mathrm{kg}$

6.80E-09 kg 1,4-dichlorobenzene eq." $/ \mathrm{kg}$

$23 \mathrm{~kg}$ 1,4-dichlorobenzene eq."//kg

1.80E-20 kg 1,4-dichlorobenzene eq." $/ \mathrm{kg}$

$9.80 \mathrm{E}-20 \mathrm{~kg}$ 1,4-dichlorobenzene eq." $/ \mathrm{kg}$

$0.0072 \mathrm{~kg}$ 1,4-dichlorobenzene eq." $/ \mathrm{kg}$

$0.03 \mathrm{~kg}$ 1,4-dichlorobenzene eq." $/ \mathrm{kg}$

5.60E-05 kg 1,4-dichlorobenzene eq." $/ \mathrm{kg}$ $5.40 \mathrm{E}-19 \mathrm{~kg}$ 1,4-dichlorobenzene eq." $/ \mathrm{kg}$

1.10E-08 kg 1,4-dichlorobenzene eq." $/ \mathrm{kg}$

6.00E-09 kg 1,4-dichlorobenzene eq." $/ \mathrm{kg}$

7.00E-06 kg 1,4-dichlorobenzene eq." $/ \mathrm{kg}$

$3.2 \mathrm{~kg}$ 1,4-dichlorobenzene eq." $/ \mathrm{kg}$

$0.8 \mathrm{~kg} 1,4$-dichlorobenzene eq." $/ \mathrm{kg}$

$0.16 \mathrm{~kg}$ 1,4-dichlorobenzene eq." $/ \mathrm{kg}$

$30 \mathrm{~kg} \mathrm{1,4-dichlorobenzene} \mathrm{eq."} / \mathrm{kg}$

$0.0017 \mathrm{~kg}$ 1,4-dichlorobenzene eq." $/ \mathrm{kg}$

1.80E-16 kg 1,4-dichlorobenzene eq." $/ \mathrm{kg}$

$0.16 \mathrm{~kg}$ 1,4-dichlorobenzene eq." $/ \mathrm{kg}$

$1.00 \mathrm{E}-05 \mathrm{~kg}$ 1,4-dichlorobenzene eq." $/ \mathrm{kg}$

$6.50 \mathrm{E}-20 \mathrm{~kg} \mathrm{1,4-dichlorobenzene} \mathrm{eq."} / \mathrm{kg}$

7.30E-05 kg 1,4-dichlorobenzene eq." $/ \mathrm{kg}$

$5.70 \mathrm{E}-09 \mathrm{~kg}$ 1,4-dichlorobenzene eq." $/ \mathrm{kg}$

$5.50 \mathrm{E}-07 \mathrm{~kg}$ 1,4-dichlorobenzene eq." $/ \mathrm{kg}$

$2.40 \mathrm{E}-08 \mathrm{~kg}$ 1,4-dichlorobenzene eq." $/ \mathrm{kg}$

$0.00011 \mathrm{~kg}$ 1,4-dichlorobenzene eq." $/ \mathrm{kg}$

$0.0054 \mathrm{~kg} \mathrm{1,4-dichlorobenzene} \mathrm{eq.} / / \mathrm{kg}$

$0.38 \mathrm{~kg}$ 1,4-dichlorobenzene eq." $/ \mathrm{kg}$

$3.2 \mathrm{~kg}$ 1,4-dichlorobenzene eq. "/ $/ \mathrm{kg}$

4.80E-05 kg 1,4-dichlorobenzene eq." $/ \mathrm{kg}$

$0.0027 \mathrm{~kg} \mathrm{1,4-dichlorobenzene} \mathrm{eq."} / \mathrm{kg}$

$0.00024 \mathrm{~kg} 1,4$-dichlorobenzene eq." $/ \mathrm{kg}$

$0.095 \mathrm{~kg}$ 1,4-dichlorobenzene eq." $/ \mathrm{kg}$

2.40E-05 kg 1,4-dichlorobenzene eq." $/ \mathrm{kg}$

$0.15 \mathrm{~kg}$ 1,4-dichlorobenzene eq." $/ \mathrm{kg}$

2.30E-22 kg 1,4-dichlorobenzene eq." $/ \mathrm{kg}$

9.10E-22 kg 1,4-dichlorobenzene eq." $/ \mathrm{kg}$

$0.83 \mathrm{~kg}$ 1,4-dichlorobenzene eq." $/ \mathrm{kg}$

2.00E-18 kg 1,4-dichlorobenzene eq. "/ $/ \mathrm{kg}$

$1.00 \mathrm{E}-19 \mathrm{~kg}$ 1,4-dichlorobenzene eq." $/ \mathrm{kg}$

$150 \mathrm{~kg}$ 1,4-dichlorobenzene eq." $/ \mathrm{kg}$

1.90E-06 kg 1,4-dichlorobenzene eq." $/ \mathrm{kg}$

$4.3 \mathrm{~kg}$ 1,4-dichlorobenzene eq." $/ \mathrm{kg}$

6.50E-07 kg 1,4-dichlorobenzene eq." $/ \mathrm{kg}$

$16 \mathrm{~kg}$ 1,4-dichlorobenzene eq." $/ \mathrm{kg}$

$4.8 \mathrm{~kg} 1,4$-dichlorobenzene eq." $/ \mathrm{kg}$

$0.012 \mathrm{~kg}$ 1,4-dichlorobenzene eq." $/ \mathrm{kg}$

2.40E-06 kg 1,4-dichlorobenzene eq." $/ \mathrm{kg}$

$0.0021 \mathrm{~kg} 1,4$-dichlorobenzene eq." $/ \mathrm{kg}$

$0.046 \mathrm{~kg}$ 1,4-dichlorobenzene eq." $/ \mathrm{kg}$

3.80E-06 kg 1,4-dichlorobenzene eq." $/ \mathrm{kg}$

3.60E-06 kg 1,4-dichlorobenzene eq." "/kg

$8.30 \mathrm{E}-13 \mathrm{~kg}$ 1,4-dichlorobenzene eq." $/ \mathrm{kg}$

$0.00051 \mathrm{~kg}$ 1,4-dichlorobenzene eq." $/ \mathrm{kg}$

$1.7 \mathrm{~kg}$ 1,4-dichlorobenzene eq." $/ \mathrm{kg}$

5.20E-05 kg 1,4-dichlorobenzene eq." "/kg

$0.026 \mathrm{~kg}$ 1,4-dichlorobenzene eq." $/ \mathrm{kg}$ 
[E1034] Diisodecylphtalate to sea water [E1035] Diisooctylphtalate to sea water [E1036] dimethoate to sea water

[E1037] Dimethylphtalate to sea water

[E1038] dinoseb to sea water

[E1039] dinoterb to sea water

[E1040] Dioctylphtalate to sea water

[E1041] disulfothon to sea water

[E1042] diuron to sea water

[E1043] DNOC to sea water

[E1044] endosulfan to sea water

[E1045] endrin to sea water

[E1046] ethoprophos to sea water

[E1047] ethylbenzene to sea water

[E1048] ethylene to sea water

[E1049] fenitrothion to sea water

[E1050] fenthion to sea water

[E1051] fentin acetate to sea water

[E1052] fentin chloride to sea water

[E1053] fentin hydroxide to sea water

[E1054] fluoranthrene to sea water

[E1055] folpet to sea water

[E1056] Formaldehyde (methanal) to sea water

[E1057] glyphosate to sea water

[E1058] heptachlor to sea water

[E1059] heptenophos to sea water

[E1060] hexachloro-1,3-butadiene to sea water

[E1061] hexachlorobenzene to sea water

[E1064] indeno[1,2,3-cd]pyrene to sea water

[E1065] iprodione to sea water

[E1066] isoproturon to sea water

[E1067] lead to sea water

[E1068] lindane to sea water

[E1069] linuron to sea water

[E1070] malathion to sea water

[E1072] MCPA to sea water

[E1073] mecoprop to sea water

[E1074] mercury to sea water

[E1075] metamitron to sea water

[E1076] metazachlor to sea water

[E1077] methabenzthiazuron to sea water

[E1078] methomyl to sea water

[E1079] methylbromide to sea water

[E1080] methyl-mercury to sea water

[E1081] metobromuron to sea water

[E1082] metolachlor to sea water

[E1083] mevinphos to sea water

[E1084] molybdenum to sea water

[E1085] m-xylene to sea water

[E1086] naphtalene to sea water

[E1087] nickel to sea water

[E1093] oxamyl to sea water

[E1094] oxydemethon-methyl to sea water

[E1095] o-xylene to sea water

[E1096] parathion-ethyl to sea water

[E1097] parathion-methyl to sea water

[E1098] pentachlorobenzene to sea water

[E1099] pentachloronitrobenzene to sea water

[E1100] pentachlorophenol to sea water

[E1101] permethrin to sea water

[E1102] phenanthrene to sea water

[E1103] phenol to sea water

[E1106] phoxim to sea water

[E1107] Phtalic anhydride to sea water

[E1108] pirimicarb to sea water

[E1110] propachlor to sea water

[E1111] propoxur to sea water
0.085 kg 1,4-dichlorobenzene eq." $/ \mathrm{kg}$ $0.0087 \mathrm{~kg}$ 1,4-dichlorobenzene eq." $/ \mathrm{kg}$ 5.50E-06 kg 1,4-dichlorobenzene eq." $/ \mathrm{kg}$ 9.80E-08 kg 1,4-dichlorobenzene eq." $/ \mathrm{kg}$ 0.029 kg 1,4-dichlorobenzene eq." $/ \mathrm{kg}$

$0.019 \mathrm{~kg}$ 1,4-dichlorobenzene eq." $/ \mathrm{kg}$

0.00024 kg 1,4-dichlorobenzene eq." $/ \mathrm{kg}$

0.0046 kg 1,4-dichlorobenzene eq."/kg

$0.0046 \mathrm{~kg}$ 1,4-dichlorobenzene eq." $/ \mathrm{kg}$

3.60E-09 kg 1,4-dichlorobenzene eq."//kg

0.0045 kg 1,4-dichlorobenzene eq."//kg

$1.9 \mathrm{~kg}$ 1,4-dichlorobenzene eq." $/ \mathrm{kg}$

$0.79 \mathrm{~kg}$ 1,4-dichlorobenzene eq." $/ \mathrm{kg}$

6.30E-06 kg 1,4-dichlorobenzene eq." $/ \mathrm{kg}$

6.60E-13 kg 1,4-dichlorobenzene eq." $/ \mathrm{kg}$

$0.0055 \mathrm{~kg}$ 1,4-dichlorobenzene eq." $/ \mathrm{kg}$

$0.19 \mathrm{~kg}$ 1,4-dichlorobenzene eq." $/ \mathrm{kg}$

$0.14 \mathrm{~kg}$ 1,4-dichlorobenzene eq." $/ \mathrm{kg}$

$29 \mathrm{~kg}$ 1,4-dichlorobenzene eq." $/ \mathrm{kg}$

0.047 kg 1,4-dichlorobenzene eq." $/ \mathrm{kg}$

$2.6 \mathrm{~kg}$ 1,4-dichlorobenzene eq." $/ \mathrm{kg}$

$22 \mathrm{~kg}$ 1,4-dichlorobenzene eq." $/ \mathrm{kg}$

$0.00012 \mathrm{~kg}$ 1,4-dichlorobenzene eq."/kg

2.00E-11 kg 1,4-dichlorobenzene eq."//kg

$0.055 \mathrm{~kg}$ 1,4-dichlorobenzene eq." $/ \mathrm{kg}$

0.00017 kg 1,4-dichlorobenzene eq." $/ \mathrm{kg}$

$26 \mathrm{~kg}$ 1,4-dichlorobenzene eq." $/ \mathrm{kg}$

$3.6 \mathrm{~kg}$ 1,4-dichlorobenzene eq. "/ $/ \mathrm{kg}$

$0.0024 \mathrm{~kg}$ 1,4-dichlorobenzene eq." $/ \mathrm{kg}$

3.10E-10 kg 1,4-dichlorobenzene eq."/kg

1.10E-05 kg 1,4-dichlorobenzene eq."/kg

1.40E-22 kg 1,4-dichlorobenzene eq." $/ \mathrm{kg}$

$0.03 \mathrm{~kg}$ 1,4-dichlorobenzene eq." $/ \mathrm{kg}$

$0.06 \mathrm{~kg} \mathrm{1,4-dichlorobenzene} \mathrm{eq."} / \mathrm{kg}$

$0.011 \mathrm{~kg}$ 1,4-dichlorobenzene eq." $/ \mathrm{kg}$

3.60E-13 kg 1,4-dichlorobenzene eq." $/ \mathrm{kg}$

2.50E-10 kg 1,4-dichlorobenzene eq." $/ \mathrm{kg}$

17 kg 1,4-dichlorobenzene eq. "//kg

3.50E-10 kg 1,4-dichlorobenzene eq." $/ \mathrm{kg}$

2.20E-06 kg 1,4-dichlorobenzene eq."/kg

$0.0001 \mathrm{~kg} 1,4$-dichlorobenzene eq." $/ \mathrm{kg}$

$0.0063 \mathrm{~kg} \mathrm{1,4-dichlorobenzene} \mathrm{eq."} / \mathrm{kg}$

$0.0012 \mathrm{~kg}$ 1,4-dichlorobenzene eq."/kg

$400 \mathrm{~kg}$ 1,4-dichlorobenzene eq." $/ \mathrm{kg}$

$0.0016 \mathrm{~kg} \mathrm{1,4-dichlorobenzene} \mathrm{eq."} / \mathrm{kg}$

$0.062 \mathrm{~kg}$ 1,4-dichlorobenzene eq." $/ \mathrm{kg}$

8.80E-06 kg 1,4-dichlorobenzene eq."//kg

$1.50 \mathrm{E}-18 \mathrm{~kg}$ 1,4-dichlorobenzene eq." $/ \mathrm{kg}$

4.70E-06 kg 1,4-dichlorobenzene eq." $/ \mathrm{kg}$

$0.0045 \mathrm{~kg}$ 1,4-dichlorobenzene eq." $/ \mathrm{kg}$

$1.60 \mathrm{E}-18 \mathrm{~kg}$ 1,4-dichlorobenzene eq." $/ \mathrm{kg}$

2.10E-07 kg 1,4-dichlorobenzene eq." $/ \mathrm{kg}$

6.80E-05 kg 1,4-dichlorobenzene eq."/kg

1.20E-05 kg 1,4-dichlorobenzene eq." $/ \mathrm{kg}$

$0.14 \mathrm{~kg}$ 1,4-dichlorobenzene eq." $/ \mathrm{kg}$

$0.0074 \mathrm{~kg}$ 1,4-dichlorobenzene eq." $/ \mathrm{kg}$

$0.33 \mathrm{~kg}$ 1,4-dichlorobenzene eq." $/ \mathrm{kg}$

$3.1 \mathrm{~kg}$ 1,4-dichlorobenzene eq." $/ \mathrm{kg}$

2.70E-05 kg 1,4-dichlorobenzene eq." $/ \mathrm{kg}$

$13 \mathrm{~kg}$ 1,4-dichlorobenzene eq." $/ \mathrm{kg}$

$0.063 \mathrm{~kg}$ 1,4-dichlorobenzene eq."/kg

6.40E-06 kg 1,4-dichlorobenzene eq."/kg

$0.0054 \mathrm{~kg}$ 1,4-dichlorobenzene eq." $/ \mathrm{kg}$

9.40E-14 kg 1,4-dichlorobenzene eq."/ $/ \mathrm{kg}$

$0.0009 \mathrm{~kg}$ 1,4-dichlorobenzene eq." $/ \mathrm{kg}$

$0.00027 \mathrm{~kg}$ 1,4-dichlorobenzene eq." $/ \mathrm{kg}$

8.20E-05 kg 1,4-dichlorobenzene eq." $/ \mathrm{kg}$ 
[E1112] propylene oxide to sea water [E1113] p-xylene to sea water [E1114] pyrazophos to sea water [E1115] selenium to sea water [E1116] simazine to sea water [E1117] styrene (vinylbenzene) to sea water [E1120] tetrachloroethylene (PER) to sea water

[E1121] Tetrachloromethane (carbon tetrachloride) ( $\mathrm{HC}-10)$ to sea water [E1122] thallium to sea water

[E1123] Thiram to sea water

[E1124] tin to sea water

[E1125] tolclophos-methyl to sea water

[E1126] toluene to sea water

[E1127] tri-allate to sea water

[E1128] triazophos to sea water

[E1129] tributyltinoxide to sea water

[E1130] trichlorfon to sea water

[E1131] Trichloroethylene (tri) to sea water

[E1132] Trichloromethane $=$ chloroform to sea water

[E1133] trifluarin to sea water

[E1134] vanadium to sea water

[E1135] Vinyl Chloride (chloroethene) to sea water

[E1136] zinc to sea water

[E1137] zineb to sea water

[E1138] 1,1,1-trichloroethane to sea water

[E1139] 1,2,3,4-tetrachlorobenzene to sea water

[E1140] 1,2,3,5-tetrachlorobenzene to sea water

[E1141] 1,2,3-trichlorobenzene to sea water

[E1142] 1,2,4,5-tetrachlorobenzene to sea water

[E1143] 1,2,4-trichlorobenzene to sea water

[E1144] 1,2-dichlorobenzene to sea water

[E1145] 1,2-dichloroethane to sea water

[E1146] 1,3,5-trichlorobenzene to sea water

[E1147] 1,3-butadiene to sea water

[E1148] 1,3-dichlorobenzene to sea water

[E1149] 1,4-dichlorobenzene to sea water

[E1150] 1-chloro-4-nitrobenzene to sea water

[E1151] 2,3,4,6-tetrachlorophenol to sea water

[E1152] 2,3,7,8-TCDD to sea water

[E1153] 2,4,5-T to sea water

[E1154] 2,4,5-trichlorophenol to sea water

[E1155] 2,4,6-trichlorophenol to agricultural soil

[E1156] 2,4-D to agricultural soil

[E1157] 2,4-dichlorophenol to agricultural soil

[E1158] 2-chlorophenol to agricultural soil

[E1159] 3,4-dichloroaniline to agricultural soil

[E1160] 3-chloroaniline to agricultural soil

[E1161] 4-chloroaniline to agricultural soil

[E1162] acephate to agricultural soil

[E1163] Acrolein (2-propenal) to agricultural soil

[E1164] acrylonitrile to agricultural soil

[E1165] aldicarb to agricultural soil

[E1166] aldrin to agricultural soil

[E1168] anilazine to agricultural soil

[E1169] anthracene to agricultural soil

[E1170] antimony to agricultural soil

[E1171] arsenic to agricultural soil

[E1172] atrazine to agricultural soil

[E1173] azinphos-ethyl to agricultural soil

[E1174] azinphos-methyl to agricultural soil

[E1175] barium to agricultural soil

[E1176] benomyl to agricultural soil

[E1177] bentazone to agricultural soil

[E1178] benzene to agricultural soil

[E1179] benzo[a]anthracene to agricultural soil

[E1180] benzo[a]pyrene to agricultural soil

[E1181] benzo[ghi]perylene to agricultural soil
$0.00024 \mathrm{~kg}$ 1,4-dichlorobenzene eq. "/kg

6.10E-06 kg 1,4-dichlorobenzene eq." $/ \mathrm{kg}$

0.002 kg 1,4-dichlorobenzene eq." $/ \mathrm{kg}$

$8.60 \mathrm{E}-18 \mathrm{~kg}$ 1,4-dichlorobenzene eq." $/ \mathrm{kg}$

$0.0038 \mathrm{~kg}$ 1,4-dichlorobenzene eq." $/ \mathrm{kg}$

7.00E-06 kg 1,4-dichlorobenzene eq." $/ \mathrm{kg}$

$0.00019 \mathrm{~kg} 1,4$-dichlorobenzene eq." $/ \mathrm{kg}$

$0.00011 \mathrm{~kg}$ 1,4-dichlorobenzene eq." $/ \mathrm{kg}$

2.00E-17 kg 1,4-dichlorobenzene eq." $/ \mathrm{kg}$

$0.0095 \mathrm{~kg}$ 1,4-dichlorobenzene eq." $/ \mathrm{kg}$

4.80E-23 kg 1,4-dichlorobenzene eq." $/ \mathrm{kg}$

$0.031 \mathrm{~kg} 1,4$-dichlorobenzene eq. $/ \mathrm{kg}$

5.90E-06 kg 1,4-dichlorobenzene eq." $/ \mathrm{kg}$

$0.41 \mathrm{~kg} 1,4$-dichlorobenzene eq." $/ \mathrm{kg}$

$0.074 \mathrm{~kg} 1,4$-dichlorobenzene eq." $/ \mathrm{kg}$

$4.1 \mathrm{~kg}$ 1,4-dichlorobenzene eq." $/ \mathrm{kg}$

9.90E-07 kg 1,4-dichlorobenzene eq." $/ \mathrm{kg}$

1.30E-05 kg 1,4-dichlorobenzene eq." $/ \mathrm{kg}$

2.30E-05 kg 1,4-dichlorobenzene eq." $/ \mathrm{kg}$

$1.4 \mathrm{~kg} \mathrm{1,4-dichlorobenzene} \mathrm{eq."} / \mathrm{kg}$

5.70E-18 kg 1,4-dichlorobenzene eq." $/ \mathrm{kg}$

1.10E-06 kg 1,4-dichlorobenzene eq." $/ \mathrm{kg}$

4.50E-21 kg 1,4-dichlorobenzene eq." $/ \mathrm{kg}$

$0.0029 \mathrm{~kg}$ 1,4-dichlorobenzene eq." $/ \mathrm{kg}$

$0.00031 \mathrm{~kg}$ 1,4-dichlorobenzene eq." $/ \mathrm{kg}$

$0.032 \mathrm{~kg}$ 1,4-dichlorobenzene eq." $/ \mathrm{kg}$

$0.093 \mathrm{~kg}$ 1,4-dichlorobenzene eq." $/ \mathrm{kg}$

$0.025 \mathrm{~kg}$ 1,4-dichlorobenzene eq." $/ \mathrm{kg}$

0.029 kg 1,4-dichlorobenzene eq." $/ \mathrm{kg}$

$0.022 \mathrm{~kg}$ 1,4-dichlorobenzene eq." $/ \mathrm{kg}$

$0.018 \mathrm{~kg}$ 1,4-dichlorobenzene eq." $/ \mathrm{kg}$

$0.00063 \mathrm{~kg}$ 1,4-dichlorobenzene eq." $/ \mathrm{kg}$

$0.056 \mathrm{~kg}$ 1,4-dichlorobenzene eq." $/ \mathrm{kg}$

3.80E-05 kg 1,4-dichlorobenzene eq." $/ \mathrm{kg}$

$0.016 \mathrm{~kg}$ 1,4-dichlorobenzene eq." $/ \mathrm{kg}$

0.014 kg 1,4-dichlorobenzene eq." $/ \mathrm{kg}$

$130 \mathrm{~kg}$ 1,4-dichlorobenzene eq." $/ \mathrm{kg}$

$35 \mathrm{~kg} \mathrm{1,4-dichlorobenzene} \mathrm{eq."} / \mathrm{kg}$

4.00E+05 kg 1,4-dichlorobenzene eq." $/ \mathrm{kg}$

$0.32 \mathrm{~kg}$ 1,4-dichlorobenzene eq." $/ \mathrm{kg}$

$33 \mathrm{~kg}$ 1,4-dichlorobenzene eq. " $/ \mathrm{kg}$

$1.2 \mathrm{~kg} 1,4$-dichlorobenzene eq." $/ \mathrm{kg}$

22 kg 1,4-dichlorobenzene eq." $/ \mathrm{kg}$

$1 \mathrm{~kg} 1,4$-dichlorobenzene eq." $/ \mathrm{kg}$

$6.3 \mathrm{~kg}$ 1,4-dichlorobenzene eq." $/ \mathrm{kg}$

$2300 \mathrm{~kg}$ 1,4-dichlorobenzene eq." $/ \mathrm{kg}$

68 kg 1,4-dichlorobenzene eq." $/ \mathrm{kg}$

$150 \mathrm{~kg}$ 1,4-dichlorobenzene eq." $/ \mathrm{kg}$

26 kg 1,4-dichlorobenzene eq." $/ \mathrm{kg}$

$34000 \mathrm{~kg}$ 1,4-dichlorobenzene eq."/kg

$4.2 \mathrm{~kg} \mathrm{1,4-dichlorobenzene} \mathrm{eq."} / \mathrm{kg}$

76000 kg 1,4-dichlorobenzene eq." $/ \mathrm{kg}$

$24 \mathrm{~kg}$ 1,4-dichlorobenzene eq." $/ \mathrm{kg}$

$0.014 \mathrm{~kg}$ 1,4-dichlorobenzene eq." $/ \mathrm{kg}$

$110 \mathrm{~kg} \mathrm{1,4-dichlorobenzene} \mathrm{eq."} / \mathrm{kg}$

$24 \mathrm{~kg}$ 1,4-dichlorobenzene eq." $/ \mathrm{kg}$

$340 \mathrm{~kg}$ 1,4-dichlorobenzene eq." $/ \mathrm{kg}$

$300 \mathrm{~kg}$ 1,4-dichlorobenzene eq." $/ \mathrm{kg}$

$2000 \mathrm{~kg}$ 1,4-dichlorobenzene eq."//kg

$100 \mathrm{~kg}$ 1,4-dichlorobenzene eq." $/ \mathrm{kg}$

$260 \mathrm{~kg} 1,4$-dichlorobenzene eq." $/ \mathrm{kg}$

$0.59 \mathrm{~kg}$ 1,4-dichlorobenzene eq. " $/ \mathrm{kg}$

$6.7 \mathrm{~kg}$ 1,4-dichlorobenzene eq."//kg

$0.00054 \mathrm{~kg}$ 1,4-dichlorobenzene eq." $/ \mathrm{kg}$

$190 \mathrm{~kg}$ 1,4-dichlorobenzene eq." $/ \mathrm{kg}$

$380 \mathrm{~kg}$ 1,4-dichlorobenzene eq." $/ \mathrm{kg}$

$200 \mathrm{~kg}$ 1,4-dichlorobenzene eq." $/ \mathrm{kg}$ 
[E1182] benzo[k]fluoranthrene to agricultural soil

[E1183] benzylchloride to agricultural soil

[E1184] beryllium to agricultural soil

[E1185] bifenthrin to agricultural soil

[E1186] Butylbenzylphtalate to agricultural soil

[E1187] cadmium to agricultural soil

[E1188] captafol to agricultural soil

[E1189] captan to agricultural soil

[E1190] carbaryl to agricultural soil

[E1191] carbendazim to agricultural soil

[E1192] carbofuran to agricultural soil

[E1193] carbon disulfide to agricultural soil

[E1194] Carcinogenic PAHs to agricultural soil

[E1195] chlordane to agricultural soil

[E1196] chlorfenvinphos to agricultural soil

[E1197] chloridazon to agricultural soil

[E1198] chlorobenzene to agricultural soil

[E1199] chlorothalonil to agricultural soil

[E1200] chlorpropham to agricultural soil

[E1201] chlorpyriphos to agricultural soil

[E1202] chromium III to agricultural soil

[E1203] chromium VI to agricultural soil

[E1204] chrysene to agricultural soil

[E1205] cobalt to agricultural soil

[E1206] copper to agricultural soil

[E1207] coumaphos to agricultural soil

[E1208] cyanazine to agricultural soil

[E1209] cypermethrin to agricultural soil

[E1210] cyromazine to agricultural soil

[E1211] DDT to agricultural soil

[E1212] deltamethrin to agricultural soil

[E1213] demeton to agricultural soil

[E1214] desmetryn to agricultural soil

[E1215] Di(2-ethylhexyl)phtalate to agricultural soil

[E1216] diazinon to agricultural soil

[E1217] Dibutylphtalate to agricultural soil

[E1218] Dichloromethane (Methylene Chloride) to agricultural soil

[E1219] dichlorprop to agricultural soil

[E1220] dichlorvos to agricultural soil

[E1221] dieldrin to agricultural soil

[E1222] Diethylphtalate to agricultural soil

[E1223] Dihexylphtalate to agricultural soil

[E1224] Diisodecylphtalate to agricultural soil

[E1225] Diisooctylphtalate to agricultural soil

[E1226] dimethoate to agricultural soil

[E1227] Dimethylphtalate to agricultural soil

[E1228] dinoseb to agricultural soil

[E1229] dinoterb to agricultural soil

[E1230] Dioctylphtalate to agricultural soil

[E1231] disulfothon to agricultural soil

[E1232] diuron to agricultural soil

[E1233] DNOC to agricultural soil

[E1234] endosulfan to agricultural soil

[E1235] endrin to agricultural soil

[E1236] ethoprophos to agricultural soil

[E1237] ethylbenzene to agricultural soil

[E1238] ethylene to agricultural soil

[E1239] fenitrothion to agricultural soil

[E1240] fenthion to agricultural soil

[E1241] fentin acetate to agricultural soil

[E1242] fentin chloride to agricultural soil

[E1243] fentin hydroxide to agricultural soil

[E1244] fluoranthrene to agricultural soil

[E1245] folpet to agricultural soil

[E1246] Formaldehyde (methanal) to agricultural soil

[E1247] glyphosate to agricultural soil

[E1248] heptachlor to agricultural soil
17000 kg 1,4-dichlorobenzene eq." $/ / \mathrm{kg}$

$0.13 \mathrm{~kg}$ 1,4-dichlorobenzene eq." $/ \mathrm{kg}$

$54000 \mathrm{~kg}$ 1,4-dichlorobenzene eq."/kg

$310 \mathrm{~kg}$ 1,4-dichlorobenzene eq." $/ \mathrm{kg}$

$0.0082 \mathrm{~kg}$ 1,4-dichlorobenzene eq." $/ \mathrm{kg}$

2000 kg 1,4-dichlorobenzene eq." $/ \mathrm{kg}$

39000 kg 1,4-dichlorobenzene eq." $/ \mathrm{kg}$

$0.0035 \mathrm{~kg}$ 1,4-dichlorobenzene eq." $/ \mathrm{kg}$

$6.7 \mathrm{~kg}$ 1,4-dichlorobenzene eq." $/ \mathrm{kg}$

2000 kg 1,4-dichlorobenzene eq."/kg

$340 \mathrm{~kg}$ 1,4-dichlorobenzene eq." $/ \mathrm{kg}$

$0.28 \mathrm{~kg}$ 1,4-dichlorobenzene eq." $/ \mathrm{kg}$

$190 \mathrm{~kg} \mathrm{1,4-dichlorobenzene} \mathrm{eq."/kg}$

$9.5 \mathrm{~kg}$ 1,4-dichlorobenzene eq." $/ \mathrm{kg}$

$14 \mathrm{~kg}$ 1,4-dichlorobenzene eq." $/ \mathrm{kg}$

$1.4 \mathrm{~kg}$ 1,4-dichlorobenzene eq." $/ \mathrm{kg}$

$0.003 \mathrm{~kg}$ 1,4-dichlorobenzene eq." $/ \mathrm{kg}$

$0.73 \mathrm{~kg}$ 1,4-dichlorobenzene eq." $/ \mathrm{kg}$

$1.6 \mathrm{~kg}$ 1,4-dichlorobenzene eq."/kg

$230 \mathrm{~kg}$ 1,4-dichlorobenzene eq." $/ \mathrm{kg}$

$13 \mathrm{~kg}$ 1,4-dichlorobenzene eq."//kg

$54 \mathrm{~kg}$ 1,4-dichlorobenzene eq." $/ \mathrm{kg}$

$240 \mathrm{~kg}$ 1,4-dichlorobenzene eq." / $/ \mathrm{kg}$

$2800 \mathrm{~kg}$ 1,4-dichlorobenzene eq." $/ \mathrm{kg}$

$1500 \mathrm{~kg}$ 1,4-dichlorobenzene eq." $/ \mathrm{kg}$

$1.50 \mathrm{E}+06 \mathrm{~kg}$ 1,4-dichlorobenzene eq." $/ \mathrm{kg}$

$630 \mathrm{~kg}$ 1,4-dichlorobenzene eq." $/ \mathrm{kg}$

$3.60 \mathrm{E}+05 \mathrm{~kg}$ 1,4-dichlorobenzene eq." $/ \mathrm{kg}$

$5200 \mathrm{~kg}$ 1,4-dichlorobenzene eq."/kg

$94 \mathrm{~kg}$ 1,4-dichlorobenzene eq." $/ \mathrm{kg}$

$36 \mathrm{~kg}$ 1,4-dichlorobenzene eq." $/ \mathrm{kg}$

570 kg 1,4-dichlorobenzene eq."/kg

$1.8 \mathrm{~kg}$ 1,4-dichlorobenzene eq." $/ \mathrm{kg}$

$0.002 \mathrm{~kg}$ 1,4-dichlorobenzene eq." $/ \mathrm{kg}$

$930 \mathrm{~kg}$ 1,4-dichlorobenzene eq." $/ \mathrm{kg}$

$0.01 \mathrm{~kg}$ 1,4-dichlorobenzene eq." $/ \mathrm{kg}$

$0.00011 \mathrm{~kg}$ 1,4-dichlorobenzene eq." $/ \mathrm{kg}$

0.0069 kg 1,4-dichlorobenzene eq." $/ \mathrm{kg}$

$3.3 \mathrm{~kg}$ 1,4-dichlorobenzene eq." $/ \mathrm{kg}$

$63 \mathrm{~kg}$ 1,4-dichlorobenzene eq." $/ \mathrm{kg}$

$0.11 \mathrm{~kg}$ 1,4-dichlorobenzene eq." $/ \mathrm{kg}$

$0.044 \mathrm{~kg} 1,4$-dichlorobenzene eq." $/ \mathrm{kg}$

$0.01 \mathrm{~kg}$ 1,4-dichlorobenzene eq."/kg

$0.0014 \mathrm{~kg}$ 1,4-dichlorobenzene eq." $/ \mathrm{kg}$

$6.6 \mathrm{~kg}$ 1,4-dichlorobenzene eq." $/ \mathrm{kg}$

0.0019 kg 1,4-dichlorobenzene eq."/kg

$5600 \mathrm{~kg} 1,4$-dichlorobenzene eq." $/ \mathrm{kg}$

150 kg 1,4-dichlorobenzene eq."/kg

7.10E-05 kg 1,4-dichlorobenzene eq."/kg

$25 \mathrm{~kg}$ 1,4-dichlorobenzene eq."/kg

$25 \mathrm{~kg}$ 1,4-dichlorobenzene eq." $/ \mathrm{kg}$

$0.2 \mathrm{~kg}$ 1,4-dichlorobenzene eq." $/ \mathrm{kg}$

$0.48 \mathrm{~kg}$ 1,4-dichlorobenzene eq." $/ \mathrm{kg}$

$6400 \mathrm{~kg}$ 1,4-dichlorobenzene eq." $/ \mathrm{kg}$

$8800 \mathrm{~kg}$ 1,4-dichlorobenzene eq." $/ \mathrm{kg}$

$0.0012 \mathrm{~kg} 1,4$-dichlorobenzene eq."/kg

7.10E-10 kg 1,4-dichlorobenzene eq."/kg

$420 \mathrm{~kg}$ 1,4-dichlorobenzene eq." $/ \mathrm{kg}$

2500 kg 1,4-dichlorobenzene eq."/kg

$620 \mathrm{~kg}$ 1,4-dichlorobenzene eq." $/ \mathrm{kg}$

$410 \mathrm{~kg}$ 1,4-dichlorobenzene eq."/kg

$620 \mathrm{~kg}$ 1,4-dichlorobenzene eq." $/ \mathrm{kg}$

57 kg 1,4-dichlorobenzene eq." $/ \mathrm{kg}$ $6200 \mathrm{~kg}$ 1,4-dichlorobenzene eq." $/ \mathrm{kg}$

$7.9 \mathrm{~kg}$ 1,4-dichlorobenzene eq." $/ \mathrm{kg}$

$0.9 \mathrm{~kg}$ 1,4-dichlorobenzene eq." $/ \mathrm{kg}$

$3.2 \mathrm{~kg}$ 1,4-dichlorobenzene eq." $/ \mathrm{kg}$ 
[E1249] heptenophos to agricultural soil

[E1250] hexachloro-1,3-butadiene to agricultural soil

[E1251] hexachlorobenzene to agricultural soil

[E1254] indeno[1,2,3-cd]pyrene to agricultural soil

[E1255] iprodione to agricultural soil

[E1256] isoproturon to agricultural soil

[E1257] lead to agricultural soil

[E1258] lindane to agricultural soil

[E1259] linuron to agricultural soil

[E1260] malathion to agricultural soil

[E1261] MCPA to agricultural soil

[E1262] mecoprop to agricultural soil

[E1263] mercury to agricultural soil

[E1264] metamitron to agricultural soil

[E1265] metazachlor to agricultural soil

[E1266] methabenzthiazuron to agricultural soil

[E1267] methomyl to agricultural soil

[E1268] methylbromide to agricultural soil

[E1269] methyl-mercury to agricultural soil

[E1270] metobromuron to agricultural soil

[E1271] metolachlor to agricultural soil

[E1272] mevinphos to agricultural soil

[E1273] molybdenum to agricultural soil

[E1274] $m$-xylene to agricultural soil

[E1275] naphtalene to agricultural soil

[E1276] nickel to agricultural soil

[E1278] oxamyl to agricultural soil

[E1279] oxydemethon-methyl to agricultural soil

[E1280] o-xylene to agricultural soil

[E1281] parathion-ethyl to agricultural soil

[E1282] parathion-methyl to agricultural soil

[E1283] pentachlorobenzene to agricultural soil

[E1284] pentachloronitrobenzene to agricultural soil

[E1285] pentachlorophenol to agricultural soil

[E1286] permethrin to agricultural soil

[E1287] phenanthrene to agricultural soil

[E1288] phenol to agricultural soil

[E1289] phoxim to agricultural soil

[E1290] Phtalic anhydride to agricultural soil

[E1291] pirimicarb to agricultural soil

[E1293] propachlor to agricultural soil

[E1294] propoxur to agricultural soil

[E1295] propylene oxide to agricultural soil

[E1296] p-xylene to agricultural soil

[E1297] pyrazophos to agricultural soil

[E1298] selenium to agricultural soil

[E1299] simazine to agricultural soil

[E1300] styrene (vinylbenzene) to agricultural soi

[E1302] tetrachloroethylene (PER) to agricultural soil

[E1303] Tetrachloromethane (carbon tetrachloride) (HC-10) to agricultural soil

[E1304] thallium to agricultural soil

[E1305] Thiram to agricultural soil

[E1306] tin to agricultural soil

[E1307] tolclophos-methyl to agricultural soil

[E1308] toluene to agricultural soil

[E1309] tri-allate to agricultural soil

[E1310] triazophos to agricultural soi

[E1311] tributyltinoxide to agricultural soil

[E1312] trichlorfon to agricultural soil

[E1313] Trichloroethylene (tri) to agricultural soil

[E1314] Trichloromethane=chloroform to agricultural soil

[E1315] trifluarin to agricultural soil

[E1316] vanadium to agricultural soil

[E1317] Vinyl Chloride (chloroethene) to agricultural soil

[E1318] zinc to agricultural soil

[E1319] zineb to agricultural soil

[E1320] 1,1,1-trichloroethane to agricultural soil
$3.8 \mathrm{~kg} \mathrm{1,4-dichlorobenzene} \mathrm{eq."} / \mathrm{kg}$ 80 kg 1,4-dichlorobenzene eq."/kg $10 \mathrm{~kg} \mathrm{1,4-dichlorobenzene} \mathrm{eq."} / \mathrm{kg}$

290 kg 1,4-dichlorobenzene eq."/kg

0.019 kg 1,4-dichlorobenzene eq." $/ \mathrm{kg}$

63 kg 1,4-dichlorobenzene eq."/kg

17 kg 1,4-dichlorobenzene eq." $/ \mathrm{kg}$

25 kg 1,4-dichlorobenzene eq."/kg

$690 \mathrm{~kg} \mathrm{1,4-dichlorobenzene} \mathrm{eq."/kg}$

95 kg 1,4-dichlorobenzene eq."/kg

$0.31 \mathrm{~kg} \mathrm{1,4-dichlorobenzene} \mathrm{eq."} / \mathrm{kg}$

$20 \mathrm{~kg}$ 1,4-dichlorobenzene eq." $/ \mathrm{kg}$

2200 kg 1,4-dichlorobenzene eq." $/ \mathrm{kg}$

0.22 kg 1,4-dichlorobenzene eq." $/ \mathrm{kg}$

$2.8 \mathrm{~kg} \mathrm{1,4-dichlorobenzene} \mathrm{eq."} / \mathrm{kg}$

48 kg 1,4-dichlorobenzene eq."/kg

11000 kg 1,4-dichlorobenzene eq."/kg

0.072 kg 1,4-dichlorobenzene eq."/kg

50000 kg 1,4-dichlorobenzene eq."/kg

92 kg 1,4-dichlorobenzene eq." $/ \mathrm{kg}$

1700 kg 1,4-dichlorobenzene eq."/kg

44 kg 1,4-dichlorobenzene eq." $/ \mathrm{kg}$

580 kg 1,4-dichlorobenzene eq." $/ \mathrm{kg}$

0.0012 kg 1,4-dichlorobenzene eq." $/ \mathrm{kg}$

$1.5 \mathrm{~kg}$ 1,4-dichlorobenzene eq." $/ \mathrm{kg}$

4300 kg 1,4-dichlorobenzene eq."/kg

$13 \mathrm{~kg}$ 1,4-dichlorobenzene eq." $/ \mathrm{kg}$

220 kg 1,4-dichlorobenzene eq." $/ \mathrm{kg}$

0.002 kg 1,4-dichlorobenzene eq."/kg

340 kg 1,4-dichlorobenzene eq." $/ \mathrm{kg}$

$68 \mathrm{~kg} \mathrm{1,4-dichlorobenzene} \mathrm{eq."/kg}$

0.83 kg 1,4-dichlorobenzene eq." $/ \mathrm{kg}$

$4.3 \mathrm{~kg} \mathrm{1,4-dichlorobenzene} \mathrm{eq.} \mathrm{"/kg}$

0.74 kg 1,4-dichlorobenzene eq." $/ \mathrm{kg}$

1200 kg 1,4-dichlorobenzene eq." $/ \mathrm{kg}$

$0.32 \mathrm{~kg} \mathrm{1,4-dichlorobenzene} \mathrm{eq."} / \mathrm{kg}$

$1.3 \mathrm{~kg} \mathrm{1,4-dichlorobenzene} \mathrm{eq."} / \mathrm{kg}$

0.72 kg 1,4-dichlorobenzene eq." $/ \mathrm{kg}$

9.80E-08 kg 1,4-dichlorobenzene eq."/kg

1700 kg 1,4-dichlorobenzene eq."/kg

9.4 kg 1,4-dichlorobenzene eq." $/ \mathrm{kg}$

14000 kg 1,4-dichlorobenzene eq."/kg

0.23 kg 1,4-dichlorobenzene eq." $/ \mathrm{kg}$

0.00086 kg 1,4-dichlorobenzene eq." $/ \mathrm{kg}$

230 kg 1,4-dichlorobenzene eq."/kg

$1700 \mathrm{~kg} \mathrm{1,4-dichlorobenzene} \mathrm{eq."} / \mathrm{kg}$

2000 kg 1,4-dichlorobenzene eq." $/ \mathrm{kg}$

0.0011 kg 1,4-dichlorobenzene eq."/kg

$0.0021 \mathrm{~kg} \mathrm{1,4-dichlorobenzene} \mathrm{eq."} / \mathrm{kg}$

0.00032 kg 1,4-dichlorobenzene eq." $/ \mathrm{kg}$

11000 kg 1,4-dichlorobenzene eq."/kg

250 kg 1,4-dichlorobenzene eq." $/ \mathrm{kg}$

$3.5 \mathrm{~kg} \mathrm{1,4-dichlorobenzene} \mathrm{eq.} \mathrm{"/kg}$

$3.3 \mathrm{~kg}$ 1,4-dichlorobenzene eq." $/ \mathrm{kg}$

0.00075 kg 1,4-dichlorobenzene eq." $/ \mathrm{kg}$

18 kg 1,4-dichlorobenzene eq." $/ \mathrm{kg}$

5400 kg 1,4-dichlorobenzene eq."/kg

1500 kg 1,4-dichlorobenzene eq." $/ \mathrm{kg}$

610 kg 1,4-dichlorobenzene eq." $/ \mathrm{kg}$

0.00039 kg 1,4-dichlorobenzene eq." $/ \mathrm{kg}$

0.00024 kg 1,4-dichlorobenzene eq." $/ \mathrm{kg}$

33 kg 1,4-dichlorobenzene eq." $/ \mathrm{kg}$

11000 kg 1,4-dichlorobenzene eq." $/ \mathrm{kg}$

5.20E-05 kg 1,4-dichlorobenzene eq." $/ \mathrm{kg}$

120 kg 1,4-dichlorobenzene eq." $/ \mathrm{kg}$

300 kg 1,4-dichlorobenzene eq." $/ \mathrm{kg}$

$0.00031 \mathrm{~kg} 1,4$-dichlorobenzene eq." $/ \mathrm{kg}$ 
[E1321] 1,2,3,4-tetrachlorobenzene to agricultural soil [E1322] 1,2,3,5-tetrachlorobenzene to agricultural soil [E1323] 1,2,3-trichlorobenzene to agricultural soil

[E1324] 1,2,4,5-tetrachlorobenzene to agricultural soil

[E1325] 1,2,4-trichlorobenzene to agricultural soil

[E1326] 1,2-dichlorobenzene to agricultural soil

[E1327] 1,2-dichloroethane to agricultural soil

[E1328] 1,3,5-trichlorobenzene to agricultural soil

[E1329] 1,3-butadiene to agricultural soil

[E1330] 1,3-dichlorobenzene to agricultural soil

[E1331] 1,4-dichlorobenzene to agricultural soil

[E1332] 1-chloro-4-nitrobenzene to agricultural soil

[E1333] 2,3,4,6-tetrachlorophenol to agricultural soil

[E1334] 2,3,7,8-TCDD to agricultural soil

[E1335] 2,4,5-T to agricultural soil

[E1336] 2,4,5-trichlorophenol to agricultural soil

[E1337] 2,4,6-trichlorophenol to industrial soil

[E1338] 2,4-D to industrial soil

[E1339] 2,4-dichlorophenol to industrial soil

[E1340] 2-chlorophenol to industrial soil

[E1341] 3,4-dichloroaniline to industrial soil

[E1342] 3-chloroaniline to industrial soil

[E1343] 4-chloroaniline to industrial soil

[E1344] acephate to industrial soil

[E1345] Acrolein (2-propenal) to industrial soil

[E1346] acrylonitrile to industrial soil

[E1347] aldicarb to industrial soil

[E1348] aldrin to industrial soil

[E1350] anilazine to industrial soil

[E1351] anthracene to industrial soil

[E1352] antimony to industrial soil

[E1353] arsenic to industrial soil

[E1354] atrazine to industrial soil

[E1355] azinphos-ethyl to industrial soil

[E1356] azinphos-methyl to industrial soil

[E1357] barium to industrial soil

[E1358] benomyl to industrial soil

[E1359] bentazone to industrial soil

[E1360] benzene to industrial soil

[E1361] benzo[a]anthracene to industrial soil

[E1362] benzo[a]pyrene to industrial soil

[E1363] benzo[ghi]perylene to industrial soil

[E1364] benzo[k]fluoranthrene to industrial soil

[E1365] benzylchloride to industrial soil

[E1366] beryllium to industrial soil

[E1367] bifenthrin to industrial soil

[E1368] Butylbenzylphtalate to industrial soil

[E1369] cadmium to industrial soil

[E1370] captafol to industrial soil

[E1371] captan to industrial soil

[E1372] carbaryl to industrial soil

[E1373] carbendazim to industrial soil

[E1374] carbofuran to industrial soil

[E1375] carbon disulfide to industrial soil

[E1376] Carcinogenic PAHs to industrial soil

[E1377] chlordane to industrial soil

[E1378] chlorfenvinphos to industrial soil

[E1379] chloridazon to industrial soil

[E1380] chlorobenzene to industrial soil

[E1381] chlorothalonil to industrial soil

[E1382] chlorpropham to industrial soil

[E1383] chlorpyriphos to industrial soil

[E1384] chromium III to industrial soil

[E1385] chromium VI to industrial soil

[E1386] chrysene to industrial soil

[E1387] cobalt to industrial soil

[E1388] copper to industrial soil
$0.12 \mathrm{~kg}$ 1,4-dichlorobenzene eq." $/ \mathrm{kg}$ $0.21 \mathrm{~kg}$ 1,4-dichlorobenzene eq." $/ \mathrm{kg}$ $0.033 \mathrm{~kg}$ 1,4-dichlorobenzene eq." $/ \mathrm{kg}$

$0.1 \mathrm{~kg}$ 1,4-dichlorobenzene eq." $/ \mathrm{kg}$ $0.036 \mathrm{~kg}$ 1,4-dichlorobenzene eq." $/ \mathrm{kg}$ $0.018 \mathrm{~kg}$ 1,4-dichlorobenzene eq." $/ \mathrm{kg}$ $0.00063 \mathrm{~kg}$ 1,4-dichlorobenzene eq." $/ \mathrm{kg}$ $0.069 \mathrm{~kg}$ 1,4-dichlorobenzene eq." $/ \mathrm{kg}$ 3.80E-05 kg 1,4-dichlorobenzene eq."/kg $0.016 \mathrm{~kg}$ 1,4-dichlorobenzene eq." $/ \mathrm{kg}$ $0.014 \mathrm{~kg}$ 1,4-dichlorobenzene eq." $/ \mathrm{kg}$ $130 \mathrm{~kg}$ 1,4-dichlorobenzene eq." $/ \mathrm{kg}$ $130 \mathrm{~kg}$ 1,4-dichlorobenzene eq." $/ \mathrm{kg}$ 1.60E+06 kg 1,4-dichlorobenzene eq." $/ \mathrm{kg}$

$1.1 \mathrm{~kg}$ 1,4-dichlorobenzene eq." $/ \mathrm{kg}$

$120 \mathrm{~kg}$ 1,4-dichlorobenzene eq." $/ \mathrm{kg}$

$4.7 \mathrm{~kg}$ 1,4-dichlorobenzene eq. $" / \mathrm{kg}$

$61 \mathrm{~kg}$ 1,4-dichlorobenzene eq." $/ \mathrm{kg}$

$3.6 \mathrm{~kg}$ 1,4-dichlorobenzene eq." $/ \mathrm{kg}$

$24 \mathrm{~kg}$ 1,4-dichlorobenzene eq." $/ \mathrm{kg}$ $5000 \mathrm{~kg}$ 1,4-dichlorobenzene eq." $/ \mathrm{kg}$ $230 \mathrm{~kg}$ 1,4-dichlorobenzene eq." $/ \mathrm{kg}$ $420 \mathrm{~kg}$ 1,4-dichlorobenzene eq." $/ \mathrm{kg}$

$81 \mathrm{~kg}$ 1,4-dichlorobenzene eq." $/ \mathrm{kg}$

$34000 \mathrm{~kg}$ 1,4-dichlorobenzene eq." $/ \mathrm{kg}$

$5.3 \mathrm{~kg}$ 1,4-dichlorobenzene eq." $/ \mathrm{kg}$

$76000 \mathrm{~kg}$ 1,4-dichlorobenzene eq." $/ \mathrm{kg}$

$25 \mathrm{~kg}$ 1,4-dichlorobenzene eq." $/ \mathrm{kg}$

$0.055 \mathrm{~kg}$ 1,4-dichlorobenzene eq." $/ \mathrm{kg}$ $450 \mathrm{~kg}$ 1,4-dichlorobenzene eq." $/ \mathrm{kg}$

$24 \mathrm{~kg}$ 1,4-dichlorobenzene eq." $/ \mathrm{kg}$ $340 \mathrm{~kg}$ 1,4-dichlorobenzene eq." $/ \mathrm{kg}$ $800 \mathrm{~kg}$ 1,4-dichlorobenzene eq." $/ \mathrm{kg}$ 2700 kg 1,4-dichlorobenzene eq." $/ \mathrm{kg}$ $4100 \mathrm{~kg}$ 1,4-dichlorobenzene eq." $/ \mathrm{kg}$ $260 \mathrm{~kg}$ 1,4-dichlorobenzene eq." $/ \mathrm{kg}$ $2.4 \mathrm{~kg}$ 1,4-dichlorobenzene eq." $/ \mathrm{kg}$ $8.8 \mathrm{~kg}$ 1,4-dichlorobenzene eq." $/ \mathrm{kg}$ $0.00054 \mathrm{~kg}$ 1,4-dichlorobenzene eq." $/ \mathrm{kg}$ $740 \mathrm{~kg}$ 1,4-dichlorobenzene eq." $/ \mathrm{kg}$ $1500 \mathrm{~kg}$ 1,4-dichlorobenzene eq." $/ \mathrm{kg}$ $780 \mathrm{~kg}$ 1,4-dichlorobenzene eq." $/ \mathrm{kg}$ 68000 kg 1,4-dichlorobenzene eq." $/ \mathrm{kg}$ $0.47 \mathrm{~kg}$ 1,4-dichlorobenzene eq." $/ \mathrm{kg}$ 54000 kg 1,4-dichlorobenzene eq." $/ \mathrm{kg}$ $1200 \mathrm{~kg}$ 1,4-dichlorobenzene eq." $/ \mathrm{kg}$ $0.033 \mathrm{~kg}$ 1,4-dichlorobenzene eq." $/ \mathrm{kg}$ $2000 \mathrm{~kg}$ 1,4-dichlorobenzene eq."/kg 1.20E+05 kg 1,4-dichlorobenzene eq." $/ \mathrm{kg}$ $0.041 \mathrm{~kg} 1,4$-dichlorobenzene eq." $/ \mathrm{kg}$

$36 \mathrm{~kg}$ 1,4-dichlorobenzene eq."//kg $6200 \mathrm{~kg}$ 1,4-dichlorobenzene eq." $/ \mathrm{kg}$ $1100 \mathrm{~kg} \mathrm{1,4-dichlorobenzene} \mathrm{eq."} / \mathrm{kg}$ $0.28 \mathrm{~kg}$ 1,4-dichlorobenzene eq." $/ \mathrm{kg}$ $750 \mathrm{~kg}$ 1,4-dichlorobenzene eq." $/ \mathrm{kg}$

$38 \mathrm{~kg}$ 1,4-dichlorobenzene eq." $/ \mathrm{kg}$

50 kg 1,4-dichlorobenzene eq." $/ \mathrm{kg}$

$3.1 \mathrm{~kg}$ 1,4-dichlorobenzene eq." $/ \mathrm{kg}$ $0.003 \mathrm{~kg}$ 1,4-dichlorobenzene eq." $/ \mathrm{kg}$

$2.6 \mathrm{~kg}$ 1,4-dichlorobenzene eq." $/ \mathrm{kg}$ $5.5 \mathrm{~kg}$ 1,4-dichlorobenzene eq." $/ \mathrm{kg}$ $930 \mathrm{~kg}$ 1,4-dichlorobenzene eq." $/ \mathrm{kg}$

$13 \mathrm{~kg}$ 1,4-dichlorobenzene eq." $/ \mathrm{kg}$

$54 \mathrm{~kg}$ 1,4-dichlorobenzene eq." $/ \mathrm{kg}$ $930 \mathrm{~kg}$ 1,4-dichlorobenzene eq." $/ \mathrm{kg}$ 2800 kg 1,4-dichlorobenzene eq." $/ \mathrm{kg}$ 1500 kg 1,4-dichlorobenzene eq." $/ \mathrm{kg}$ 
[E1389] coumaphos to industrial soil

[E1390] cyanazine to industrial soil

[E1391] cypermethrin to industrial soil

[E1392] cyromazine to industrial soil

[E1393] DDT to industrial soil

[E1394] deltamethrin to industrial soil

[E1395] demeton to industrial soil

[E1396] desmetryn to industrial soil

[E1397] Di(2-ethylhexyl)phtalate to industrial soil

[E1398] diazinon to industrial soil

[E1399] Dibutylphtalate to industrial soil

[E1400] Dichloromethane (Methylene Chloride) to industrial soil

[E1401] dichlorprop to industrial soil

[E1402] dichlorvos to industrial soil

[E1403] dieldrin to industrial soil

[E1404] Diethylphtalate to industrial soil

[E1405] Dihexylphtalate to industrial soil

[E1406] Diisodecylphtalate to industrial soil

[E1407] Diisooctylphtalate to industrial soil

[E1408] dimethoate to industrial soil

[E1409] Dimethylphtalate to industrial soil

[E1410] dinoseb to industrial soil

[E1411] dinoterb to industrial soil

[E1412] Dioctylphtalate to industrial soil

[E1413] disulfothon to industrial soil

[E1414] diuron to industrial soil

[E1415] DNOC to industrial soil

[E1416] endosulfan to industrial soil

[E1417] endrin to industrial soil

[E1418] ethoprophos to industrial soil

[E1419] ethylbenzene to industrial soil

[E1420] ethylene to industrial soil

[E1421] fenitrothion to industrial soil

[E1422] fenthion to industrial soil

[E1423] fentin acetate to industrial soil

[E1424] fentin chloride to industrial soil

[E1425] fentin hydroxide to industrial soil

[E1426] fluoranthrene to industrial soil

[E1427] folpet to industrial soil

[E1428] Formaldehyde (methanal) to industrial soil

[E1429] glyphosate to industrial soil

[E1430] heptachlor to industrial soil

[E1431] heptenophos to industrial soil

[E1432] hexachloro-1,3-butadiene to industrial soil

[E1433] hexachlorobenzene to industrial soil

[E1436] indeno[1,2,3-cd]pyrene to industrial soil

[E1437] iprodione to industrial soil

[E1438] isoproturon to industrial soil

[E1439] lead to industrial soil

[E1440] lindane to industrial soil

[E1441] linuron to industrial soil

[E1442] malathion to industrial soil

[E1443] MCPA to industrial soil

[E1444] mecoprop to industrial soil

[E1445] mercury to industrial soil

[E1446] metamitron to industrial soil

[E1447] metazachlor to industrial soil

[E1448] methabenzthiazuron to industrial soil

[E1449] methomyl to industrial soil

[E1450] methylbromide to industrial soil

[E1451] methyl-mercury to industrial soil

[E1452] metobromuron to industrial soil

[E1453] metolachlor to industrial soil

[E1454] mevinphos to industrial soil

[E1455] molybdenum to industrial soil

[E1456] m-xylene to industrial soil

[E1457] naphtalene to industrial soil
4.40E+06 kg 1,4-dichlorobenzene eq."/kg 2300 kg 1,4-dichlorobenzene eq." $/ \mathrm{kg}$ 1.30E+06 kg 1,4-dichlorobenzene eq."/kg 5200 kg 1,4-dichlorobenzene eq." $/ \mathrm{kg}$ 370 kg 1,4-dichlorobenzene eq." $/ \mathrm{kg}$ 150 kg 1,4-dichlorobenzene eq."/kg 1800 kg 1,4-dichlorobenzene eq."/kg

6.6 kg 1,4-dichlorobenzene eq." $/ \mathrm{kg}$ 0.0079 kg 1,4-dichlorobenzene eq." $/ \mathrm{kg}$ 3300 kg 1,4-dichlorobenzene eq." $/ \mathrm{kg}$ 0.041 kg 1,4-dichlorobenzene eq." $/ \mathrm{kg}$ 0.00011 kg 1,4-dichlorobenzene eq." $/ \mathrm{kg}$ 0.027 kg 1,4-dichlorobenzene eq." $/ \mathrm{kg}$

$13 \mathrm{~kg} \mathrm{1,4-dichlorobenzene} \mathrm{eq."} / \mathrm{kg}$ 240 kg 1,4-dichlorobenzene eq." $/ \mathrm{kg}$ 0.41 kg 1,4-dichlorobenzene eq." $/ \mathrm{kg}$ $0.18 \mathrm{~kg} 1,4$-dichlorobenzene eq." $/ \mathrm{kg}$ 0.041 kg 1,4-dichlorobenzene eq." $/ \mathrm{kg}$ 0.0055 kg 1,4-dichlorobenzene eq."/kg

20 kg 1,4-dichlorobenzene eq." $/ \mathrm{kg}$ 0.0075 kg 1,4-dichlorobenzene eq." $/ \mathrm{kg}$ 16000 kg 1,4-dichlorobenzene eq."/kg 590 kg 1,4-dichlorobenzene eq."/kg

0.00028 kg 1,4-dichlorobenzene eq." $/ \mathrm{kg}$ 99 kg 1,4-dichlorobenzene eq."/kg

99 kg 1,4-dichlorobenzene eq." $/ \mathrm{kg}$ 0.75 kg 1,4-dichlorobenzene eq."/kg

$1.9 \mathrm{~kg}$ 1,4-dichlorobenzene eq." $/ \mathrm{kg}$ 22000 kg 1,4-dichlorobenzene eq." $/ \mathrm{kg}$ 24000 kg 1,4-dichlorobenzene eq." $/ \mathrm{kg}$ 0.0012 kg 1,4-dichlorobenzene eq." $/ \mathrm{kg}$

7.10E-10 kg 1,4-dichlorobenzene eq."/kg 1700 kg 1,4-dichlorobenzene eq."/kg 9900 kg 1,4-dichlorobenzene eq."/kg 2500 kg 1,4-dichlorobenzene eq."/kg 1600 kg 1,4-dichlorobenzene eq."/kg 2500 kg 1,4-dichlorobenzene eq." $/ \mathrm{kg}$ 230 kg 1,4-dichlorobenzene eq."/kg 18000 kg 1,4-dichlorobenzene eq."/kg $24 \mathrm{~kg}$ 1,4-dichlorobenzene eq." $/ \mathrm{kg}$ $3.6 \mathrm{~kg} \mathrm{1,4-dichlorobenzene} \mathrm{eq."} / \mathrm{kg}$ 13 kg 1,4-dichlorobenzene eq." $/ \mathrm{kg}$ 15 kg 1,4-dichlorobenzene eq." $/ \mathrm{kg}$ $97 \mathrm{~kg}$ 1,4-dichlorobenzene eq."/kg $14 \mathrm{~kg} \mathrm{1,4-dichlorobenzene} \mathrm{eq."} / \mathrm{kg}$ 1200 kg 1,4-dichlorobenzene eq."/kg 0.16 kg 1,4-dichlorobenzene eq."/kg 150 kg 1,4-dichlorobenzene eq." $/ \mathrm{kg}$

17 kg 1,4-dichlorobenzene eq." $/ \mathrm{kg}$

97 kg 1,4-dichlorobenzene eq." $/ \mathrm{kg}$ 2400 kg 1,4-dichlorobenzene eq." $/ \mathrm{kg}$ 380 kg 1,4-dichlorobenzene eq."/kg $1.1 \mathrm{~kg} \mathrm{1,4-dichlorobenzene} \mathrm{eq."/kg}$ 53 kg 1,4-dichlorobenzene eq."/kg 2200 kg 1,4-dichlorobenzene eq." $/ \mathrm{kg}$ $0.79 \mathrm{~kg}$ 1,4-dichlorobenzene eq." $/ \mathrm{kg}$ 9.8 kg 1,4-dichlorobenzene eq." $/ \mathrm{kg}$ 150 kg 1,4-dichlorobenzene eq." $/ \mathrm{kg}$ 21000 kg 1,4-dichlorobenzene eq." $/ \mathrm{kg}$ $0.073 \mathrm{~kg} \mathrm{1,4-dichlorobenzene} \mathrm{eq."} / \mathrm{kg}$ 50000 kg 1,4-dichlorobenzene eq."/kg 92 kg 1,4-dichlorobenzene eq. "/kg 5200 kg 1,4-dichlorobenzene eq." $/ \mathrm{kg}$ 180 kg 1,4-dichlorobenzene eq."/kg 580 kg 1,4-dichlorobenzene eq."/kg 0.0012 kg 1,4-dichlorobenzene eq." $/ \mathrm{kg}$ $4.9 \mathrm{~kg} 1,4$-dichlorobenzene eq. "/kg 
[E1458] nickel to industrial soil

[E1460] oxamyl to industrial soil

[E1461] oxydemethon-methyl to industrial soil

[E1462] o-xylene to industrial soil

[E1463] parathion-ethyl to industrial soil

[E1464] parathion-methyl to industrial soil

[E1465] pentachlorobenzene to industrial soil

[E1466] pentachloronitrobenzene to industrial soil

[E1467] pentachlorophenol to industrial soil

[E1468] permethrin to industrial soil

[E1469] phenanthrene to industrial soil

[E1470] phenol to industrial soil

[E1471] phoxim to industrial soil

[E1472] Phtalic anhydride to industrial soil

[E1473] pirimicarb to industrial soil

[E1475] propachlor to industrial soil

[E1476] propoxur to industrial soil

[E1477] propylene oxide to industrial soil

[E1478] p-xylene to industrial soil

[E1479] pyrazophos to industrial soil

[E1480] selenium to industrial soil

[E1481] simazine to industrial soil

[E1482] styrene (vinylbenzene) to industrial soil

[E1484] tetrachloroethylene (PER) to industrial soil

[E1485] Tetrachloromethane (carbon tetrachloride) $(\mathrm{HC}-10)$ to industrial soil

[E1486] thallium to industrial soil

[E1487] Thiram to industrial soil

[E1488] tin to industrial soil

[E1489] tolclophos-methyl to industrial soil

[E1490] toluene to industrial soil

[E1491] tri-allate to industrial soil

[E1492] triazophos to industrial soil

[E1493] tributyltinoxide to industrial soil

[E1494] trichlorfon to industrial soil

[E1495] Trichloroethylene (tri) to industrial soil

[E1496] Trichloromethane $=$ chloroform to industrial soil

[E1497] trifluarin to industrial soil

[E1498] vanadium to industrial soil

[E1499] Vinyl Chloride (chloroethene) to industrial soil

[E1500] zinc to industrial soil

[E1501] zineb to industrial soil
$4300 \mathrm{~kg}$ 1,4-dichlorobenzene eq." $/ \mathrm{kg}$

$55 \mathrm{~kg}$ 1,4-dichlorobenzene eq." $/ \mathrm{kg}$

$810 \mathrm{~kg}$ 1,4-dichlorobenzene eq." $/ \mathrm{kg}$

$0.002 \mathrm{~kg}$ 1,4-dichlorobenzene eq." $/ \mathrm{kg}$

1300 kg 1,4-dichlorobenzene eq." $/ \mathrm{kg}$

$260 \mathrm{~kg}$ 1,4-dichlorobenzene eq." $/ \mathrm{kg}$

$1.6 \mathrm{~kg} \mathrm{1,4-dichlorobenzene} \mathrm{eq."} / \mathrm{kg}$

$17 \mathrm{~kg}$ 1,4-dichlorobenzene eq." $/ \mathrm{kg}$

$3 \mathrm{~kg}$ 1,4-dichlorobenzene eq." $/ \mathrm{kg}$

$4800 \mathrm{~kg}$ 1,4-dichlorobenzene eq." $/ \mathrm{kg}$

$1.3 \mathrm{~kg}$ 1,4-dichlorobenzene eq." $/ \mathrm{kg}$

$4.7 \mathrm{~kg} \mathrm{1,4-dichlorobenzene} \mathrm{eq."} / \mathrm{kg}$

$1.3 \mathrm{~kg} 1,4$-dichlorobenzene eq." $/ \mathrm{kg}$

6.30E-08 kg 1,4-dichlorobenzene eq." $/ \mathrm{kg}$

5300 kg 1,4-dichlorobenzene eq." $/ \mathrm{kg}$

$34 \mathrm{~kg}$ 1,4-dichlorobenzene eq." $/ \mathrm{kg}$

38000 kg 1,4-dichlorobenzene eq." $/ \mathrm{kg}$

$0.25 \mathrm{~kg} 1,4$-dichlorobenzene eq." $/ \mathrm{kg}$

$0.00087 \mathrm{~kg}$ 1,4-dichlorobenzene eq."/kg

$900 \mathrm{~kg}$ 1,4-dichlorobenzene eq." $/ \mathrm{kg}$

$1700 \mathrm{~kg}$ 1,4-dichlorobenzene eq." $/ \mathrm{kg}$

$4800 \mathrm{~kg} \mathrm{1,4-dichlorobenzene} \mathrm{eq."} / \mathrm{kg}$

$0.0018 \mathrm{~kg} 1,4$-dichlorobenzene eq." $/ \mathrm{kg}$

$0.043 \mathrm{~kg} 1,4$-dichlorobenzene eq." $/ \mathrm{kg}$

0.00032 kg 1,4-dichlorobenzene eq." $/ \mathrm{kg}$

11000 kg 1,4-dichlorobenzene eq."/kg

1600 kg 1,4-dichlorobenzene eq." $/ \mathrm{kg}$

$3.5 \mathrm{~kg}$ 1,4-dichlorobenzene eq." $/ \mathrm{kg}$

$9.9 \mathrm{~kg}$ 1,4-dichlorobenzene eq." $/ \mathrm{kg}$

0.00075 kg 1,4-dichlorobenzene eq." $/ \mathrm{kg}$

$70 \mathrm{~kg} \mathrm{1,4-dichlorobenzene} \mathrm{eq."} / \mathrm{kg}$

18000 kg 1,4-dichlorobenzene eq." $/ \mathrm{kg}$

$5700 \mathrm{~kg} 1,4$-dichlorobenzene eq." $/ \mathrm{kg}$

$3400 \mathrm{~kg}$ 1,4-dichlorobenzene eq." $/ \mathrm{kg}$

$0.00039 \mathrm{~kg}$ 1,4-dichlorobenzene eq."/kg

$0.00024 \mathrm{~kg}$ 1,4-dichlorobenzene eq. "/kg

$130 \mathrm{~kg}$ 1,4-dichlorobenzene eq." $/ \mathrm{kg}$

$11000 \mathrm{~kg}$ 1,4-dichlorobenzene eq." $/ \mathrm{kg}$

5.20E-05 kg 1,4-dichlorobenzene eq."/kg

$120 \mathrm{~kg} 1,4$-dichlorobenzene eq." $/ \mathrm{kg}$

$1100 \mathrm{~kg}$ 1,4-dichlorobenzene eq."/kg

Category $=$ [C9] Sediment ecotoxicity salt water

Description = Problem oriented approach, manual 1999; SETP

Author = Huijbregts, 1999

Date $=27-09-1999$

Environmental resources

Environmental resource

Value

Environmental emissions

Environmental emission

[E90] 1,1,1-trichloroethane (methyl chloroform; HC-140a) to air

Value

Unit

[E94] 1,2,3,4-tetrachlorobenzene to air

[E95] 1,2,3,5-tetrachlorobenzene to air

[E96] 1,2,3-trichlorobenzene to air

[E98] 1,2,4,5-tetrachlorobenzene to air

[E99] 1,2,4-trichlorobenzene to air

[E102] 1,2-dichlorobenzene to air

[E103] 1,2-dichloroethane to air

[E107] 1,3,5-trichlorobenzene to air

[E111] 1,3-Butadiene to air

[E112] 1,3-dichlorobenzene to air

[E116] 1,4-dichlorobenzene to air

[E128] 1-chloro-4-nitrobenzene to air

[E166] 2,3,4,6-tetrachlorophenol to air

$$
\text { Unit }
$$

$0.11 \mathrm{~kg}$ 1,4-dichlorobenzene eq." $/ \mathrm{kg}$

$6.9 \mathrm{~kg}$ 1,4-dichlorobenzene eq." $/ \mathrm{kg}$

$7 \mathrm{~kg}$ 1,4-dichlorobenzene eq." $/ \mathrm{kg}$

$0.85 \mathrm{~kg} 1,4$-dichlorobenzene eq." $/ \mathrm{kg}$

$6.1 \mathrm{~kg}$ 1,4-dichlorobenzene eq." $/ \mathrm{kg}$

$0.84 \mathrm{~kg} \mathrm{1,4-dichlorobenzene} \mathrm{eq."} / \mathrm{kg}$

$0.28 \mathrm{~kg}$ 1,4-dichlorobenzene eq." $/ \mathrm{kg}$

$0.031 \mathrm{~kg} 1,4$-dichlorobenzene eq." $/ \mathrm{kg}$

$1.3 \mathrm{~kg}$ 1,4-dichlorobenzene eq." $/ \mathrm{kg}$

3.00E-06 kg 1,4-dichlorobenzene eq."/kg

$0.2 \mathrm{~kg} 1,4$-dichlorobenzene eq." $/ \mathrm{kg}$

$0.29 \mathrm{~kg}$ 1,4-dichlorobenzene eq." $/ \mathrm{kg}$

240 kg 1,4-dichlorobenzene eq." $/ \mathrm{kg}$

110 kg 1,4-dichlorobenzene eq."/kg 
[E168] 2,3,7,8-TCDD (tetrachloride-dibenzo-dioxin) to air [E172] 2,4,5-T to air

[E173] 2,4,5-trichlorophenol to air

[E174] 2,4,6-trichlorophenol to air

[E175] 2,4-D to air

[E176] 2,4-dichlorophenol to air

[E185] 2-chlorophenol to air

[E208] 3,4-dichloroaniline to air

[E217] 3-chloroaniline to air

[E228] 4-chloroaniline to air

[E231] acephate to air

[E236] Acrolein (2-propenal) to air

[E237] Acrylonitrile to air

[E241] aldicarb to air

[E242] aldrin to air

[E251] anilazine to air

[E252] anthracene to air

[E253] antimony to air

[E255] arsenic to air

[E256] atrazine to air

[E257] azinphos-ethyl to air

[E258] azinphos-methyl to air

[E259] barium to air

[E260] benomyl to air

[E261] bentazone to air

[E263] Benzene to air

[E264] benzo[a]anthracene to air

[E265] benzo[a]pyrene to air

[E266] benzo[ghi]perylene to air

[E267] benzo[k]fluoranthrene to air

[E269] benzylchloride to air

[E270] beryllium to air

[E272] bifenthrin to air

[E290] Butylbenzylphtalate to air

[E385] cadmium to air

[E387] captafol to air

[E388] captan to air

[E389] carbaryl to air

[E390] carbendazim to air

[E391] carbofuran to air

[E394] carbon disulfide to air

[E404] chlordane to air

[E405] chlorfenvinphos to air

[E406] chloridazon to air

[E407] chlorobenzene to air

[E410] chlorothalonil to air

[E411] chlorpropham to air

[E412] chlorpyriphos to air

[E413] chromium (unspecified) to air

[E414] chromium III to air

[E415] chromium VI to air

[E416] chrysene to air

[E421] cobalt to air

[E422] copper to air

[E423] coumaphos to air

[E425] cyanazine to air

[E437] cypermethrin to air

[E438] cyromazine to air

[E439] DDT to air

[E441] deltamethrin to air

[E442] demeton to air

[E443] desmetryn to air

[E444] Di(2-ethylhexyl)phtalate to air

[E446] diazinon to air

[E448] Dibutylphtalate to air

[E449] Dichloromethane (Methylene Chloride) to air

[E450] dichlorprop to air
8.10E+08 kg 1,4-dichlorobenzene eq." $/ \mathrm{kg}$

$0.25 \mathrm{~kg}$ 1,4-dichlorobenzene eq." $/ \mathrm{kg}$ $48 \mathrm{~kg}$ 1,4-dichlorobenzene eq." $/ \mathrm{kg}$

$4.3 \mathrm{~kg}$ 1,4-dichlorobenzene eq." $/ \mathrm{kg}$

$7.3 \mathrm{~kg} \mathrm{1,4-dichlorobenzene} \mathrm{eq."} / \mathrm{kg}$

$0.52 \mathrm{~kg}$ 1,4-dichlorobenzene eq." $/ \mathrm{kg}$

$13 \mathrm{~kg}$ 1,4-dichlorobenzene eq." $/ \mathrm{kg}$

$2100 \mathrm{~kg}$ 1,4-dichlorobenzene eq." $/ \mathrm{kg}$

$32 \mathrm{~kg}$ 1,4-dichlorobenzene eq." $/ \mathrm{kg}$

$2.3 \mathrm{~kg}$ 1,4-dichlorobenzene eq." $/ \mathrm{kg}$

$18 \mathrm{~kg}$ 1,4-dichlorobenzene eq. "/ $/ \mathrm{kg}$

$750 \mathrm{~kg}$ 1,4-dichlorobenzene eq." $/ \mathrm{kg}$

$0.77 \mathrm{~kg}$ 1,4-dichlorobenzene eq." $/ \mathrm{kg}$

$12000 \mathrm{~kg} \mathrm{1,4-dichlorobenzene} \mathrm{eq."} / \mathrm{kg}$

$5.4 \mathrm{~kg}$ 1,4-dichlorobenzene eq. " $/ \mathrm{kg}$

$0.34 \mathrm{~kg}$ 1,4-dichlorobenzene eq." $/ \mathrm{kg}$

$2100 \mathrm{~kg}$ 1,4-dichlorobenzene eq." $/ \mathrm{kg}$

$31000 \mathrm{~kg} \mathrm{1,4-dichlorobenzene} \mathrm{eq."} / \mathrm{kg}$

$2.30 \mathrm{E}+05 \mathrm{~kg}$ 1,4-dichlorobenzene eq." $/ \mathrm{kg}$

$310 \mathrm{~kg}$ 1,4-dichlorobenzene eq." $/ \mathrm{kg}$

$130 \mathrm{~kg}$ 1,4-dichlorobenzene eq." $/ \mathrm{kg}$

$57 \mathrm{~kg}$ 1,4-dichlorobenzene eq." $/ \mathrm{kg}$

$6.70 \mathrm{E}+05 \mathrm{~kg}$ 1,4-dichlorobenzene eq." $/ \mathrm{kg}$

$1.8 \mathrm{~kg}$ 1,4-dichlorobenzene eq." $/ \mathrm{kg}$

$0.94 \mathrm{~kg}$ 1,4-dichlorobenzene eq." $/ \mathrm{kg}$

$0.0013 \mathrm{~kg}$ 1,4-dichlorobenzene eq." $/ \mathrm{kg}$

$3400 \mathrm{~kg}$ 1,4-dichlorobenzene eq." $/ \mathrm{kg}$

$4100 \mathrm{~kg}$ 1,4-dichlorobenzene eq." $/ \mathrm{kg}$

$5700 \mathrm{~kg}$ 1,4-dichlorobenzene eq." $/ \mathrm{kg}$

$3.50 \mathrm{E}+05 \mathrm{~kg}$ 1,4-dichlorobenzene eq." $/ \mathrm{kg}$

$0.33 \mathrm{~kg}$ 1,4-dichlorobenzene eq." $/ \mathrm{kg}$

2.00E+08 kg 1,4-dichlorobenzene eq." $/ \mathrm{kg}$

$3700 \mathrm{~kg}$ 1,4-dichlorobenzene eq." $/ \mathrm{kg}$

$0.071 \mathrm{~kg} 1,4$-dichlorobenzene eq." $/ \mathrm{kg}$

1.10E+06 kg 1,4-dichlorobenzene eq." $/ / \mathrm{kg}$ $39000 \mathrm{~kg}$ 1,4-dichlorobenzene eq." $/ \mathrm{kg}$

$0.12 \mathrm{~kg}$ 1,4-dichlorobenzene eq." $/ \mathrm{kg}$

$1 \mathrm{~kg}$ 1,4-dichlorobenzene eq." $/ \mathrm{kg}$

$1100 \mathrm{~kg}$ 1,4-dichlorobenzene eq." $/ \mathrm{kg}$

$160 \mathrm{~kg}$ 1,4-dichlorobenzene eq." $/ \mathrm{kg}$

$0.86 \mathrm{~kg}$ 1,4-dichlorobenzene eq." $/ \mathrm{kg}$

$1600 \mathrm{~kg}$ 1,4-dichlorobenzene eq."/kg

$13 \mathrm{~kg}$ 1,4-dichlorobenzene eq." $/ \mathrm{kg}$

$0.26 \mathrm{~kg} 1,4$-dichlorobenzene eq. $/ / \mathrm{kg}$

$0.05 \mathrm{~kg}$ 1,4-dichlorobenzene eq." $/ \mathrm{kg}$

$15 \mathrm{~kg}$ 1,4-dichlorobenzene eq." $/ \mathrm{kg}$

$0.81 \mathrm{~kg}$ 1,4-dichlorobenzene eq. $/ \mathrm{kg}$

$6 \mathrm{~kg}$ 1,4-dichlorobenzene eq." $/ \mathrm{kg}$

$5300 \mathrm{~kg}$ 1,4-dichlorobenzene eq." $/ \mathrm{kg}$

$5300 \mathrm{~kg}$ 1,4-dichlorobenzene eq." $/ \mathrm{kg}$

$21000 \mathrm{~kg}$ 1,4-dichlorobenzene eq." $/ \mathrm{kg}$

$1400 \mathrm{~kg}$ 1,4-dichlorobenzene eq." //kg

$3.50 \mathrm{E}+06 \mathrm{~kg}$ 1,4-dichlorobenzene eq." $/ \mathrm{kg}$

$8.80 \mathrm{E}+05 \mathrm{~kg}$ 1,4-dichlorobenzene eq." $/ \mathrm{kg}$

4.80E+05 kg 1,4-dichlorobenzene eq." $/ \mathrm{kg}$

$810 \mathrm{~kg}$ 1,4-dichlorobenzene eq." $/ \mathrm{kg}$

$49000 \mathrm{~kg}$ 1,4-dichlorobenzene eq." $/ \mathrm{kg}$

$1300 \mathrm{~kg}$ 1,4-dichlorobenzene eq." $/ \mathrm{kg}$

25000 kg 1,4-dichlorobenzene eq."/kg

$6800 \mathrm{~kg}$ 1,4-dichlorobenzene eq." $/ \mathrm{kg}$

$11 \mathrm{~kg}$ 1,4-dichlorobenzene eq." $/ \mathrm{kg}$

$2.6 \mathrm{~kg} 1,4$-dichlorobenzene eq." $/ \mathrm{kg}$

$1.7 \mathrm{~kg}$ 1,4-dichlorobenzene eq." $/ \mathrm{kg}$

$110 \mathrm{~kg}$ 1,4-dichlorobenzene eq." $/ \mathrm{kg}$

$0.038 \mathrm{~kg}$ 1,4-dichlorobenzene eq." $/ \mathrm{kg}$

$0.0014 \mathrm{~kg}$ 1,4-dichlorobenzene eq." $/ \mathrm{kg}$

0.032 kg 1,4-dichlorobenzene eq." $/ \mathrm{kg}$ 
[E451] dichlorvos to air

[E452] dieldrin to air

[E456] Diethylphtalate to air

[E457] Dihexylphtalate to air

[E458] Diisodecylphtalate to air

[E459] Diisooctylphtalate to air

[E461] dimethoate to air

[E468] Dimethylphtalate to air

[E470] dinoseb to air

[E471] dinoterb to air

[E472] Dioctylphtalate to air

[E475] disulfothon to air

[E476] diuron to air

[E478] DNOC to air

[E480] endosulfan to air

[E481] endrin to air

[E487] ethoprophos to air

[E496] Ethylbenzene to air

[E499] Ethylene (ethene) to air

[E504] fenitrothion to air

[E505] fenthion to air

[E506] fentin acetate to air

[E507] fentin chloride to air

[E508] fentin hydroxide to air

[E509] fluoranthrene to air

[E510] folpet to air

[E511] Formaldehyde (methanal) to air

[E516] glyphosate to air

[E533] heptachlor to air

[E535] heptenophos to air

[E536] hexachloro-1,3-butadiene to air

[E537] hexachlorobenzene to air

[E566] indeno[1,2,3-cd]pyrene to air

[E568] iprodione to air

[E583] isoproturon to air

[E584] lead to air

[E585] lindane to air

[E586] linuron to air

[E587] malathion to air

[E589] MCPA to air

[E590] mecoprop to air

[E592] mercury to air

[E595] metamitron to air

[E596] meta-Xylene (1,3-dimethylbenzene) to air

[E597] metazachlor to air

[E598] methabenzthiazuron to air

[E603] methomyl to air

[E626] methyl-mercury to air

[E628] metobromuron to air

[E629] metolachlor to air

[E630] mevinphos to air

[E632] molybdenum to air

[E633] Naphtalene to air

[E635] nickel to air

[E650] ortho-Xylene (1,2-dimethylbenzene) to air

[E651] oxamyl to air

[E652] oxydemethon-methyl to air

[E655] parathion-ethyl to air

[E656] parathion-methyl to air

[E658] para-Xylene (1,4-dimethylbenzene) to air

[E660] pentachlorobenzene to air

[E661] pentachloronitrobenzene to air

[E662] pentachlorophenol to air

[E672] permethrin to air

[E674] phenanthrene to air

[E675] Phenol to air

[E679] phoxim to air
$27 \mathrm{~kg}$ 1,4-dichlorobenzene eq. " $/ \mathrm{kg}$

$170 \mathrm{~kg}$ 1,4-dichlorobenzene eq." $/ \mathrm{kg}$

$0.23 \mathrm{~kg} \mathrm{1,4-dichlorobenzene} \mathrm{eq."} / \mathrm{kg}$

$3.2 \mathrm{~kg}$ 1,4-dichlorobenzene eq." $/ \mathrm{kg}$

$7.5 \mathrm{~kg} \mathrm{1,4-dichlorobenzene} \mathrm{eq."} / \mathrm{kg}$

$5.6 \mathrm{~kg}$ 1,4-dichlorobenzene eq." $/ \mathrm{kg}$

$2 \mathrm{~kg}$ 1,4-dichlorobenzene eq." $/ \mathrm{kg}$

$0.0062 \mathrm{~kg}$ 1,4-dichlorobenzene eq."/kg

$1500 \mathrm{~kg}$ 1,4-dichlorobenzene eq." $/ \mathrm{kg}$

2100 kg 1,4-dichlorobenzene eq." $/ \mathrm{kg}$

$0.52 \mathrm{~kg}$ 1,4-dichlorobenzene eq." $/ \mathrm{kg}$

$5.7 \mathrm{~kg}$ 1,4-dichlorobenzene eq." $/ \mathrm{kg}$

$5.7 \mathrm{~kg}$ 1,4-dichlorobenzene eq." $/ \mathrm{kg}$

$0.3 \mathrm{~kg}$ 1,4-dichlorobenzene eq." $/ \mathrm{kg}$

$1.2 \mathrm{~kg}$ 1,4-dichlorobenzene eq." $/ \mathrm{kg}$

$3500 \mathrm{~kg}$ 1,4-dichlorobenzene eq." $/ \mathrm{kg}$

$930 \mathrm{~kg}$ 1,4-dichlorobenzene eq." $/ \mathrm{kg}$

$0.00061 \mathrm{~kg} 1,4$-dichlorobenzene eq. "/kg

7.10E-11 kg 1,4-dichlorobenzene eq."/kg

750 kg 1,4-dichlorobenzene eq." $/ \mathrm{kg}$

1100 kg 1,4-dichlorobenzene eq." $/ \mathrm{kg}$

53000 kg 1,4-dichlorobenzene eq." /kg

$57000 \mathrm{~kg}$ 1,4-dichlorobenzene eq." $/ \mathrm{kg}$

$51000 \mathrm{~kg}$ 1,4-dichlorobenzene eq." $/ \mathrm{kg}$

$610 \mathrm{~kg}$ 1,4-dichlorobenzene eq."/kg

$2700 \mathrm{~kg}$ 1,4-dichlorobenzene eq." $/ \mathrm{kg}$

$1.5 \mathrm{~kg}$ 1,4-dichlorobenzene eq." $/ \mathrm{kg}$

$15 \mathrm{~kg}$ 1,4-dichlorobenzene eq." $/ \mathrm{kg}$

$2.4 \mathrm{~kg}$ 1,4-dichlorobenzene eq." $/ \mathrm{kg}$

$15 \mathrm{~kg}$ 1,4-dichlorobenzene eq." $/ \mathrm{kg}$

29000 kg 1,4-dichlorobenzene eq."/kg

2800 kg 1,4-dichlorobenzene eq." $/ \mathrm{kg}$

25000 kg 1,4-dichlorobenzene eq." $/ \mathrm{kg}$

0.0052 kg 1,4-dichlorobenzene eq."/kg

20 kg 1,4-dichlorobenzene eq." $/ \mathrm{kg}$

7200 kg 1,4-dichlorobenzene eq." $/ \mathrm{kg}$

$9.2 \mathrm{~kg}$ 1,4-dichlorobenzene eq." $/ \mathrm{kg}$

$35 \mathrm{~kg}$ 1,4-dichlorobenzene eq." $/ \mathrm{kg}$

$780 \mathrm{~kg}$ 1,4-dichlorobenzene eq." $/ \mathrm{kg}$

$0.35 \mathrm{~kg}$ 1,4-dichlorobenzene eq." $/ \mathrm{kg}$

$5.3 \mathrm{~kg}$ 1,4-dichlorobenzene eq." $/ \mathrm{kg}$

1.20E+06 kg 1,4-dichlorobenzene eq." /kg

$0.19 \mathrm{~kg}$ 1,4-dichlorobenzene eq. "/ $/ \mathrm{kg}$

$0.00035 \mathrm{~kg}$ 1,4-dichlorobenzene eq."//kg

2.6 kg 1,4-dichlorobenzene eq." $/ \mathrm{kg}$

8372 kg 1,4-dichlorobenzene eq." $/ \mathrm{kg}$

$5000 \mathrm{~kg}$ 1,4-dichlorobenzene eq." $/ \mathrm{kg}$

2.80E+07 kg 1,4-dichlorobenzene eq." $/ \mathrm{kg}$

$47 \mathrm{~kg} 1,4$-dichlorobenzene eq." $/ \mathrm{kg}$ $250 \mathrm{~kg}$ 1,4-dichlorobenzene eq." $/ \mathrm{kg}$ $600 \mathrm{~kg}$ 1,4-dichlorobenzene eq." $/ \mathrm{kg}$

$1.60 \mathrm{E}+06 \mathrm{~kg}$ 1,4-dichlorobenzene eq." $/ \mathrm{kg}$

$0.32 \mathrm{~kg}$ 1,4-dichlorobenzene eq." $/ \mathrm{kg}$

$3.70 \mathrm{E}+06 \mathrm{~kg}$ 1,4-dichlorobenzene eq." $/ \mathrm{kg}$

$0.00099 \mathrm{~kg}$ 1,4-dichlorobenzene eq."/kg

$0.4 \mathrm{~kg}$ 1,4-dichlorobenzene eq." $/ \mathrm{kg}$

$210 \mathrm{~kg}$ 1,4-dichlorobenzene eq." $/ \mathrm{kg}$

$1300 \mathrm{~kg}$ 1,4-dichlorobenzene eq." $/ \mathrm{kg}$

$30 \mathrm{~kg}$ 1,4-dichlorobenzene eq."/kg

$0.00038 \mathrm{~kg}$ 1,4-dichlorobenzene eq." $/ \mathrm{kg}$

$87 \mathrm{~kg}$ 1,4-dichlorobenzene eq." $/ \mathrm{kg}$

$440 \mathrm{~kg}$ 1,4-dichlorobenzene eq." $/ \mathrm{kg}$

$69 \mathrm{~kg}$ 1,4-dichlorobenzene eq." $/ \mathrm{kg}$

$23000 \mathrm{~kg}$ 1,4-dichlorobenzene eq." //kg

$5.4 \mathrm{~kg}$ 1,4-dichlorobenzene eq." $/ \mathrm{kg}$

$0.36 \mathrm{~kg}$ 1,4-dichlorobenzene eq." $/ \mathrm{kg}$

$0.21 \mathrm{~kg}$ 1,4-dichlorobenzene eq." $/ \mathrm{kg}$ 
[E168] 2,3,7,8-TCDD (tetrachloride-dibenzo-dioxin) to air [E172] 2,4,5-T to air

[E173] 2,4,5-trichlorophenol to air

[E174] 2,4,6-trichlorophenol to air

[E175] 2,4-D to air

[E176] 2,4-dichlorophenol to air

[E185] 2-chlorophenol to air

[E208] 3,4-dichloroaniline to air

[E217] 3-chloroaniline to air

[E228] 4-chloroaniline to air

[E231] acephate to air

[E236] Acrolein (2-propenal) to air

[E237] Acrylonitrile to air

[E241] aldicarb to air

[E242] aldrin to air

[E251] anilazine to air

[E252] anthracene to air

[E253] antimony to air

[E255] arsenic to air

[E256] atrazine to air

[E257] azinphos-ethyl to air

[E258] azinphos-methyl to air

[E259] barium to air

[E260] benomyl to air

[E261] bentazone to air

[E263] Benzene to air

[E264] benzo[a]anthracene to air

[E265] benzo[a]pyrene to air

[E266] benzo[ghi]perylene to air

[E267] benzo[k]fluoranthrene to air

[E269] benzylchloride to air

[E270] beryllium to air

[E272] bifenthrin to air

[E290] Butylbenzylphtalate to air

[E385] cadmium to air

[E387] captafol to air

[E388] captan to air

[E389] carbaryl to air

[E390] carbendazim to air

[E391] carbofuran to air

[E394] carbon disulfide to air

[E404] chlordane to air

[E405] chlorfenvinphos to air

[E406] chloridazon to air

[E407] chlorobenzene to air

[E410] chlorothalonil to air

[E411] chlorpropham to air

[E412] chlorpyriphos to air

[E413] chromium (unspecified) to air

[E414] chromium III to air

[E415] chromium VI to air

[E416] chrysene to air

[E421] cobalt to air

[E422] copper to air

[E423] coumaphos to air

[E425] cyanazine to air

[E437] cypermethrin to air

[E438] cyromazine to air

[E439] DDT to air

[E441] deltamethrin to air

[E442] demeton to air

[E443] desmetryn to air

[E444] Di(2-ethylhexyl)phtalate to air

[E446] diazinon to air

[E448] Dibutylphtalate to air

[E449] Dichloromethane (Methylene Chloride) to air

[E450] dichlorprop to air
8.10E+08 kg 1,4-dichlorobenzene eq." $/ \mathrm{kg}$

$0.25 \mathrm{~kg}$ 1,4-dichlorobenzene eq." $/ \mathrm{kg}$ $48 \mathrm{~kg}$ 1,4-dichlorobenzene eq." $/ \mathrm{kg}$ $4.3 \mathrm{~kg}$ 1,4-dichlorobenzene eq." $/ \mathrm{kg}$

$7.3 \mathrm{~kg} \mathrm{1,4-dichlorobenzene} \mathrm{eq."} / \mathrm{kg}$

$0.52 \mathrm{~kg}$ 1,4-dichlorobenzene eq." $/ \mathrm{kg}$

$13 \mathrm{~kg}$ 1,4-dichlorobenzene eq." $/ \mathrm{kg}$

$2100 \mathrm{~kg}$ 1,4-dichlorobenzene eq." $/ \mathrm{kg}$

$32 \mathrm{~kg}$ 1,4-dichlorobenzene eq." $/ \mathrm{kg}$

$2.3 \mathrm{~kg}$ 1,4-dichlorobenzene eq." $/ \mathrm{kg}$

$18 \mathrm{~kg}$ 1,4-dichlorobenzene eq." $/ \mathrm{kg}$

$750 \mathrm{~kg}$ 1,4-dichlorobenzene eq." $/ \mathrm{kg}$

$0.77 \mathrm{~kg}$ 1,4-dichlorobenzene eq." $/ \mathrm{kg}$

12000 kg 1,4-dichlorobenzene eq." $/ \mathrm{kg}$

5.4 kg 1,4-dichlorobenzene eq." $/ \mathrm{kg}$

$0.34 \mathrm{~kg}$ 1,4-dichlorobenzene eq." $/ \mathrm{kg}$

$2100 \mathrm{~kg}$ 1,4-dichlorobenzene eq." $/ \mathrm{kg}$

$31000 \mathrm{~kg}$ 1,4-dichlorobenzene eq." $/ \mathrm{kg}$

$2.30 \mathrm{E}+05 \mathrm{~kg}$ 1,4-dichlorobenzene eq." $/ \mathrm{kg}$

$310 \mathrm{~kg}$ 1,4-dichlorobenzene eq." $/ \mathrm{kg}$ $130 \mathrm{~kg}$ 1,4-dichlorobenzene eq." $/ \mathrm{kg}$

$57 \mathrm{~kg}$ 1,4-dichlorobenzene eq." $/ \mathrm{kg}$

$6.70 \mathrm{E}+05 \mathrm{~kg}$ 1,4-dichlorobenzene eq." $/ \mathrm{kg}$

$1.8 \mathrm{~kg}$ 1,4-dichlorobenzene eq. "/kg $0.94 \mathrm{~kg} 1,4$-dichlorobenzene eq." $/ \mathrm{kg}$

$0.0013 \mathrm{~kg}$ 1,4-dichlorobenzene eq." $/ \mathrm{kg}$ $3400 \mathrm{~kg}$ 1,4-dichlorobenzene eq." $/ \mathrm{kg}$ $4100 \mathrm{~kg}$ 1,4-dichlorobenzene eq." $/ \mathrm{kg}$ 5700 kg 1,4-dichlorobenzene eq." $/ \mathrm{kg}$

3.50E+05 kg 1,4-dichlorobenzene eq." $/ \mathrm{kg}$ $0.33 \mathrm{~kg}$ 1,4-dichlorobenzene eq." $/ \mathrm{kg}$

2.00E+08 kg 1,4-dichlorobenzene eq." $/ \mathrm{kg}$ $3700 \mathrm{~kg}$ 1,4-dichlorobenzene eq." $/ \mathrm{kg}$ $0.071 \mathrm{~kg}$ 1,4-dichlorobenzene eq." $/ \mathrm{kg}$

1.10E+06 kg 1,4-dichlorobenzene eq." $/ \mathrm{kg}$ $39000 \mathrm{~kg}$ 1,4-dichlorobenzene eq." $/ \mathrm{kg}$

$0.12 \mathrm{~kg}$ 1,4-dichlorobenzene eq." $/ \mathrm{kg}$

$1 \mathrm{~kg}$ 1,4-dichlorobenzene eq." $/ \mathrm{kg}$ 1100 kg 1,4-dichlorobenzene eq." $/ \mathrm{kg}$ $160 \mathrm{~kg}$ 1,4-dichlorobenzene eq." $/ \mathrm{kg}$ $0.86 \mathrm{~kg}$ 1,4-dichlorobenzene eq." $/ \mathrm{kg}$ 1600 kg 1,4-dichlorobenzene eq." $/ \mathrm{kg}$

$13 \mathrm{~kg}$ 1,4-dichlorobenzene eq." $/ \mathrm{kg}$ $0.26 \mathrm{~kg}$ 1,4-dichlorobenzene eq." $/ \mathrm{kg}$ $0.05 \mathrm{~kg} 1,4$-dichlorobenzene eq." $/ \mathrm{kg}$

$15 \mathrm{~kg}$ 1,4-dichlorobenzene eq." $/ \mathrm{kg}$

$0.81 \mathrm{~kg}$ 1,4-dichlorobenzene eq." $/ \mathrm{kg}$

$6 \mathrm{~kg}$ 1,4-dichlorobenzene eq." $/ \mathrm{kg}$ $5300 \mathrm{~kg}$ 1,4-dichlorobenzene eq."/kg $5300 \mathrm{~kg}$ 1,4-dichlorobenzene eq."//kg $21000 \mathrm{~kg}$ 1,4-dichlorobenzene eq." $/ \mathrm{kg}$ 1400 kg 1,4-dichlorobenzene eq." $/ \mathrm{kg}$ 3.50E+06 kg 1,4-dichlorobenzene eq." $/ \mathrm{kg}$ $8.80 \mathrm{E}+05 \mathrm{~kg}$ 1,4-dichlorobenzene eq." $/ \mathrm{kg}$ $4.80 \mathrm{E}+05 \mathrm{~kg}$ 1,4-dichlorobenzene eq. $" / \mathrm{kg}$ $810 \mathrm{~kg}$ 1,4-dichlorobenzene eq." $/ \mathrm{kg}$ $49000 \mathrm{~kg}$ 1,4-dichlorobenzene eq." $/ \mathrm{kg}$ $1300 \mathrm{~kg}$ 1,4-dichlorobenzene eq." $/ \mathrm{kg}$ $25000 \mathrm{~kg}$ 1,4-dichlorobenzene eq." $/ \mathrm{kg}$ $6800 \mathrm{~kg}$ 1,4-dichlorobenzene eq." $/ \mathrm{kg}$

$11 \mathrm{~kg} 1,4$-dichlorobenzene eq." $/ \mathrm{kg}$

$2.6 \mathrm{~kg}$ 1,4-dichlorobenzene eq." $/ \mathrm{kg}$

$1.7 \mathrm{~kg} 1,4$-dichlorobenzene eq. $/ \mathrm{kg}$

$110 \mathrm{~kg}$ 1,4-dichlorobenzene eq." $/ \mathrm{kg}$

$0.038 \mathrm{~kg}$ 1,4-dichlorobenzene eq." $/ \mathrm{kg}$

$0.0014 \mathrm{~kg}$ 1,4-dichlorobenzene eq." $/ \mathrm{kg}$

0.032 kg 1,4-dichlorobenzene eq." $/ \mathrm{kg}$ 
[E451] dichlorvos to air

[E452] dieldrin to air

[E456] Diethylphtalate to air

[E457] Dihexylphtalate to air

[E458] Diisodecylphtalate to air

[E459] Diisooctylphtalate to air

[E461] dimethoate to air

[E468] Dimethylphtalate to air

[E470] dinoseb to air

[E471] dinoterb to air

[E472] Dioctylphtalate to air

[E475] disulfothon to air

[E476] diuron to air

[E478] DNOC to air

[E480] endosulfan to air

[E481] endrin to air

[E487] ethoprophos to air

[E496] Ethylbenzene to air

[E499] Ethylene (ethene) to air

[E504] fenitrothion to air

[E505] fenthion to air

[E506] fentin acetate to air

[E507] fentin chloride to air

[E508] fentin hydroxide to air

[E509] fluoranthrene to air

[E510] folpet to air

[E511] Formaldehyde (methanal) to air

[E516] glyphosate to air

[E533] heptachlor to air

[E535] heptenophos to air

[E536] hexachloro-1,3-butadiene to air

[E537] hexachlorobenzene to air

[E566] indeno[1,2,3-cd]pyrene to air

[E568] iprodione to air

[E583] isoproturon to air

[E584] lead to air

[E585] lindane to air

[E586] linuron to air

[E587] malathion to air

[E589] MCPA to air

[E590] mecoprop to air

[E592] mercury to air

[E595] metamitron to air

[E596] meta-Xylene (1,3-dimethylbenzene) to air

[E597] metazachlor to air

[E598] methabenzthiazuron to air

[E603] methomyl to air

[E626] methyl-mercury to air

[E628] metobromuron to air

[E629] metolachlor to air

[E630] mevinphos to air

[E632] molybdenum to air

[E633] Naphtalene to air

[E635] nickel to air

[E650] ortho-Xylene (1,2-dimethylbenzene) to air

[E651] oxamyl to air

[E652] oxydemethon-methyl to air

[E655] parathion-ethyl to air

[E656] parathion-methyl to air

[E658] para-Xylene (1,4-dimethylbenzene) to air

[E660] pentachlorobenzene to air

[E661] pentachloronitrobenzene to air

[E662] pentachlorophenol to air

[E672] permethrin to air

[E674] phenanthrene to air

[E675] Phenol to air

[E679] phoxim to air
$27 \mathrm{~kg}$ 1,4-dichlorobenzene eq." $/ \mathrm{kg}$

$170 \mathrm{~kg}$ 1,4-dichlorobenzene eq." $/ \mathrm{kg}$

$0.23 \mathrm{~kg} \mathrm{1,4-dichlorobenzene} \mathrm{eq."} / \mathrm{kg}$

$3.2 \mathrm{~kg}$ 1,4-dichlorobenzene eq." $/ \mathrm{kg}$

$7.5 \mathrm{~kg}$ 1,4-dichlorobenzene eq." $/ \mathrm{kg}$

$5.6 \mathrm{~kg}$ 1,4-dichlorobenzene eq." $/ \mathrm{kg}$

$2 \mathrm{~kg}$ 1,4-dichlorobenzene eq." $/ \mathrm{kg}$

$0.0062 \mathrm{~kg}$ 1,4-dichlorobenzene eq."/kg

$1500 \mathrm{~kg}$ 1,4-dichlorobenzene eq." $/ \mathrm{kg}$

$2100 \mathrm{~kg}$ 1,4-dichlorobenzene eq." $/ \mathrm{kg}$

$0.52 \mathrm{~kg}$ 1,4-dichlorobenzene eq." $/ \mathrm{kg}$

$5.7 \mathrm{~kg}$ 1,4-dichlorobenzene eq." $/ \mathrm{kg}$

$5.7 \mathrm{~kg}$ 1,4-dichlorobenzene eq. "/kg

$0.3 \mathrm{~kg}$ 1,4-dichlorobenzene eq." $/ \mathrm{kg}$

$1.2 \mathrm{~kg}$ 1,4-dichlorobenzene eq." $/ \mathrm{kg}$

3500 kg 1,4-dichlorobenzene eq."/kg

$930 \mathrm{~kg}$ 1,4-dichlorobenzene eq." $/ \mathrm{kg}$

$0.00061 \mathrm{~kg} 1,4$-dichlorobenzene eq." $/ \mathrm{kg}$

7.10E-11 kg 1,4-dichlorobenzene eq."/kg

$750 \mathrm{~kg}$ 1,4-dichlorobenzene eq."/kg

$1100 \mathrm{~kg}$ 1,4-dichlorobenzene eq." $/ \mathrm{kg}$

$53000 \mathrm{~kg}$ 1,4-dichlorobenzene eq." $/ \mathrm{kg}$

57000 kg 1,4-dichlorobenzene eq." $/ \mathrm{kg}$

$51000 \mathrm{~kg}$ 1,4-dichlorobenzene eq."/kg

$610 \mathrm{~kg}$ 1,4-dichlorobenzene eq."/kg

$2700 \mathrm{~kg}$ 1,4-dichlorobenzene eq." $/ \mathrm{kg}$

$1.5 \mathrm{~kg}$ 1,4-dichlorobenzene eq." $/ \mathrm{kg}$

$15 \mathrm{~kg}$ 1,4-dichlorobenzene eq." $/ \mathrm{kg}$

$2.4 \mathrm{~kg}$ 1,4-dichlorobenzene eq." $/ \mathrm{kg}$

$15 \mathrm{~kg}$ 1,4-dichlorobenzene eq." $/ \mathrm{kg}$

$29000 \mathrm{~kg}$ 1,4-dichlorobenzene eq." $/ \mathrm{kg}$

2800 kg 1,4-dichlorobenzene eq." $/ \mathrm{kg}$

25000 kg 1,4-dichlorobenzene eq." /kg

$0.0052 \mathrm{~kg}$ 1,4-dichlorobenzene eq." $/ \mathrm{kg}$

$20 \mathrm{~kg}$ 1,4-dichlorobenzene eq." $/ \mathrm{kg}$

7200 kg 1,4-dichlorobenzene eq." $/ \mathrm{kg}$

$9.2 \mathrm{~kg}$ 1,4-dichlorobenzene eq." $/ \mathrm{kg}$

$35 \mathrm{~kg}$ 1,4-dichlorobenzene eq." $/ \mathrm{kg}$

$780 \mathrm{~kg}$ 1,4-dichlorobenzene eq."/kg

$0.35 \mathrm{~kg}$ 1,4-dichlorobenzene eq." $/ \mathrm{kg}$

$5.3 \mathrm{~kg}$ 1,4-dichlorobenzene eq." $/ \mathrm{kg}$

$1.20 \mathrm{E}+06 \mathrm{~kg}$ 1,4-dichlorobenzene eq." $/ \mathrm{kg}$

$0.19 \mathrm{~kg}$ 1,4-dichlorobenzene eq." $/ \mathrm{kg}$

$0.00035 \mathrm{~kg}$ 1,4-dichlorobenzene eq." $/ \mathrm{kg}$

$2.6 \mathrm{~kg}$ 1,4-dichlorobenzene eq." $/ \mathrm{kg}$

8372 kg 1,4-dichlorobenzene eq."//kg

5000 kg 1,4-dichlorobenzene eq." $/ \mathrm{kg}$

2.80E+07 kg 1,4-dichlorobenzene eq." $/ \mathrm{kg}$

$47 \mathrm{~kg}$ 1,4-dichlorobenzene eq."/kg

$250 \mathrm{~kg}$ 1,4-dichlorobenzene eq." $/ \mathrm{kg}$

$600 \mathrm{~kg}$ 1,4-dichlorobenzene eq." $/ \mathrm{kg}$

$1.60 \mathrm{E}+06 \mathrm{~kg}$ 1,4-dichlorobenzene eq." $/ \mathrm{kg}$

$0.32 \mathrm{~kg}$ 1,4-dichlorobenzene eq." $/ \mathrm{kg}$

$3.70 \mathrm{E}+06 \mathrm{~kg}$ 1,4-dichlorobenzene eq." $/ \mathrm{kg}$

$0.00099 \mathrm{~kg}$ 1,4-dichlorobenzene eq." $/ \mathrm{kg}$

$0.4 \mathrm{~kg}$ 1,4-dichlorobenzene eq." $/ \mathrm{kg}$

$210 \mathrm{~kg}$ 1,4-dichlorobenzene eq." $/ \mathrm{kg}$

$1300 \mathrm{~kg}$ 1,4-dichlorobenzene eq." $/ \mathrm{kg}$

$30 \mathrm{~kg}$ 1,4-dichlorobenzene eq." $/ \mathrm{kg}$

$0.00038 \mathrm{~kg} 1,4$-dichlorobenzene eq." $/ \mathrm{kg}$

$87 \mathrm{~kg}$ 1,4-dichlorobenzene eq." $/ \mathrm{kg}$

$440 \mathrm{~kg}$ 1,4-dichlorobenzene eq." $/ \mathrm{kg}$

$69 \mathrm{~kg}$ 1,4-dichlorobenzene eq." $/ \mathrm{kg}$

$23000 \mathrm{~kg}$ 1,4-dichlorobenzene eq."//kg

$5.4 \mathrm{~kg}$ 1,4-dichlorobenzene eq." $/ \mathrm{kg}$

$0.36 \mathrm{~kg}$ 1,4-dichlorobenzene eq." $/ \mathrm{kg}$

$0.21 \mathrm{~kg}$ 1,4-dichlorobenzene eq." $/ \mathrm{kg}$ 
[E680] Phtalic anhydride to air

[E682] pirimicarb to air

[E685] Polycyclic Aromatic Hydrocarbons Carcinogenic- (carcinogenic-PAH) to air

[E686] propachlor to air

[E690] propoxur to air

[E697] Propylene Oxide to air

[E698] pyrazophos to air

[E704] selenium to air

[E705] simazine to air

[E706] styrene (vinylbenzene) to air

[E718] tetrachloroethylene (PER) (tetrachloroethene) to air

[E719] Tetrachloromethane (carbon tetrachloride) (HC-10) to air

[E722] thallium to air

[E723] Thiram to air

[E724] tin to air

[E725] tolclophos-methyl to air

[E727] Toluene to air

[E733] tri-allate to air

[E734] triazophos to air

[E735] tributyltinoxide to air

[E736] trichlorfon to air

[E737] Trichloroethylene (tri) to air

[E738] Trichloromethane (chloroform) to air

[E739] trifluarin to air

[E743] vanadium to air

[E745] Vinyl Chloride (chloroethene) to air

[E748] zinc to air

[E749] zineb to air

[E750] 1,1,1-trichloroethane to air

[E751] 1,2,3,4-tetrachlorobenzene to air

[E752] 1,2,3,5-tetrachlorobenzene to air

[E753] 1,2,3-trichlorobenzene to air

[E754] 1,2,4,5-tetrachlorobenzene to air

[E755] 1,2,4-trichlorobenzene to air

[E756] 1,2-dichlorobenzene to air

[E757] 1,2-dichloroethane to air

[E758] 1,3,5-trichlorobenzene to air

[E759] 1,3-butadiene to air

[E760] 1,3-dichlorobenzene to air

[E761] 1,4-dichlorobenzene to air

[E762] 1-chloro-4-nitrobenzene to air

[E763] 2,3,4,6-tetrachlorophenol to air

[E764] 2,3,7,8-TCDD to air

[E765] 2,4,5-T to air

[E766] 2,4,5-trichlorophenol to air

[E767] 2,4,6-trichlorophenol to fresh water

[E768] 2,4-D to fresh water

[E769] 2,4-dichlorophenol to fresh water

[E770] 2-chlorophenol to fresh water

[E771] 3,4-dichloroaniline to fresh water

[E772] 3-chloroaniline to fresh water

[E773] 4-chloroaniline to fresh water

[E774] acephate to fresh water

[E775] Acrolein (2-propenal) to fresh water

[E776] acrylonitrile to fresh water

[E777] aldicarb to fresh water

[E778] aldrin to fresh water

[E780] anilazine to fresh water

[E781] anthracene to fresh water

[E782] antimony to fresh water

[E783] arsenic to fresh water

[E784] atrazine to fresh water

[E785] azinphos-ethyl to fresh water

[E786] azinphos-methyl to fresh water

[E787] barium to fresh water

[E788] benomyl to fresh water

[E789] bentazone to fresh water
4.90E-05 kg 1,4-dichlorobenzene eq." $/ \mathrm{kg}$ $620 \mathrm{~kg}$ 1,4-dichlorobenzene eq." $/ \mathrm{kg}$ 14000 kg 1,4-dichlorobenzene eq." $/ \mathrm{kg}$ $6.5 \mathrm{~kg}$ 1,4-dichlorobenzene eq." $/ \mathrm{kg}$ $1800 \mathrm{~kg} \mathrm{1,4-dichlorobenzene} \mathrm{eq."} / \mathrm{kg}$ 0.066 kg 1,4-dichlorobenzene eq." $/ \mathrm{kg}$

$89 \mathrm{~kg}$ 1,4-dichlorobenzene eq." $/ \mathrm{kg}$

$9.00 \mathrm{E}+06 \mathrm{~kg}$ 1,4-dichlorobenzene eq." $/ \mathrm{kg}$ $410 \mathrm{~kg}$ 1,4-dichlorobenzene eq." $/ \mathrm{kg}$ $0.00036 \mathrm{~kg}$ 1,4-dichlorobenzene eq." $/ \mathrm{kg}$ $0.12 \mathrm{~kg}$ 1,4-dichlorobenzene eq." $/ \mathrm{kg}$ $0.31 \mathrm{~kg}$ 1,4-dichlorobenzene eq." $/ \mathrm{kg}$ $2.40 \mathrm{E}+07 \mathrm{~kg}$ 1,4-dichlorobenzene eq." $/ \mathrm{kg}$ 19 kg 1,4-dichlorobenzene eq." $/ \mathrm{kg}$ $1500 \mathrm{~kg}$ 1,4-dichlorobenzene eq." / $/ \mathrm{kg}$ $1.6 \mathrm{~kg}$ 1,4-dichlorobenzene eq." $/ \mathrm{kg}$ $0.00058 \mathrm{~kg}$ 1,4-dichlorobenzene eq." $/ \mathrm{kg}$ $39 \mathrm{~kg}$ 1,4-dichlorobenzene eq." $/ \mathrm{kg}$ $1200 \mathrm{~kg} \mathrm{1,4-dichlorobenzene} \mathrm{eq."/kg}$

$3.90 \mathrm{E}+05 \mathrm{~kg}$ 1,4-dichlorobenzene eq."/kg 270 kg 1,4-dichlorobenzene eq." $/ \mathrm{kg}$

$0.0017 \mathrm{~kg}$ 1,4-dichlorobenzene eq."/kg $0.016 \mathrm{~kg}$ 1,4-dichlorobenzene eq." $/ \mathrm{kg}$

$44 \mathrm{~kg}$ 1,4-dichlorobenzene eq." $/ \mathrm{kg}$

1.10E+07 kg 1,4-dichlorobenzene eq." $/ \mathrm{kg}$

0.00012 kg 1,4-dichlorobenzene eq." $/ \mathrm{kg}$ $68000 \mathrm{~kg}$ 1,4-dichlorobenzene eq." $/ \mathrm{kg}$ $450 \mathrm{~kg}$ 1,4-dichlorobenzene eq."/kg $0.11 \mathrm{~kg}$ 1,4-dichlorobenzene eq." $/ \mathrm{kg}$ $6.7 \mathrm{~kg}$ 1,4-dichlorobenzene eq."/kg

$7 \mathrm{~kg}$ 1,4-dichlorobenzene eq." $/ \mathrm{kg}$ $0.87 \mathrm{~kg}$ 1,4-dichlorobenzene eq." $/ \mathrm{kg}$ $5.9 \mathrm{~kg}$ 1,4-dichlorobenzene eq." $/ \mathrm{kg}$ $0.86 \mathrm{~kg}$ 1,4-dichlorobenzene eq." $/ \mathrm{kg}$ $0.28 \mathrm{~kg} 1,4$-dichlorobenzene eq." $/ \mathrm{kg}$ $0.031 \mathrm{~kg} 1,4$-dichlorobenzene eq." $/ \mathrm{kg}$ $1.3 \mathrm{~kg}$ 1,4-dichlorobenzene eq." $/ \mathrm{kg}$ 0.0099 kg 1,4-dichlorobenzene eq." $/ \mathrm{kg}$ $0.21 \mathrm{~kg}$ 1,4-dichlorobenzene eq." $/ \mathrm{kg}$ $0.29 \mathrm{~kg}$ 1,4-dichlorobenzene eq." $/ \mathrm{kg}$ 260 kg 1,4-dichlorobenzene eq."/kg $100 \mathrm{~kg}$ 1,4-dichlorobenzene eq." $/ \mathrm{kg}$ $1.50 \mathrm{E}+08 \mathrm{~kg}$ 1,4-dichlorobenzene eq."/kg $0.076 \mathrm{~kg}$ 1,4-dichlorobenzene eq." $/ \mathrm{kg}$ $81 \mathrm{~kg}$ 1,4-dichlorobenzene eq." $/ \mathrm{kg}$

$1.9 \mathrm{~kg}$ 1,4-dichlorobenzene eq." $/ \mathrm{kg}$ $3.1 \mathrm{~kg}$ 1,4-dichlorobenzene eq." $/ \mathrm{kg}$ $0.13 \mathrm{~kg} 1,4$-dichlorobenzene eq. $/ / \mathrm{kg}$ $17 \mathrm{~kg}$ 1,4-dichlorobenzene eq." $/ \mathrm{kg}$ $3500 \mathrm{~kg}$ 1,4-dichlorobenzene eq." $/ \mathrm{kg}$

$15 \mathrm{~kg}$ 1,4-dichlorobenzene eq. "/ $/ \mathrm{kg}$

$20 \mathrm{~kg}$ 1,4-dichlorobenzene eq. $/ \mathrm{kg}$

$14 \mathrm{~kg}$ 1,4-dichlorobenzene eq." $/ \mathrm{kg}$ $1600 \mathrm{~kg}$ 1,4-dichlorobenzene eq." $/ \mathrm{kg}$ $0.51 \mathrm{~kg}$ 1,4-dichlorobenzene eq." $/ \mathrm{kg}$ 11000 kg 1,4-dichlorobenzene eq."//kg

$19 \mathrm{~kg} \mathrm{1,4-dichlorobenzene} \mathrm{eq.} / \mathrm{kg}$ $0.01 \mathrm{~kg}$ 1,4-dichlorobenzene eq."/kg $4100 \mathrm{~kg}$ 1,4-dichlorobenzene eq." $/ \mathrm{kg}$ $25000 \mathrm{~kg}$ 1,4-dichlorobenzene eq." $/ \mathrm{kg}$ 1.20E+05 kg 1,4-dichlorobenzene eq." $/ / \mathrm{kg}$ $540 \mathrm{~kg}$ 1,4-dichlorobenzene eq." $/ \mathrm{kg}$ $790 \mathrm{~kg} 1,4$-dichlorobenzene eq." $/ \mathrm{kg}$

$10 \mathrm{~kg}$ 1,4-dichlorobenzene eq." $/ \mathrm{kg}$

7.10E+05 kg 1,4-dichlorobenzene eq." $/ \mathrm{kg}$ $0.75 \mathrm{~kg}$ 1,4-dichlorobenzene eq." $/ \mathrm{kg}$ $0.33 \mathrm{~kg}$ 1,4-dichlorobenzene eq."/kg 
[E790] benzene to fresh water

[E791] benzo[a]anthracene to fresh water

[E792] benzo[a]pyrene to fresh water

[E793] benzo[ghi]perylene to fresh water

[E794] benzo[k]fluoranthrene to fresh water

[E795] benzylchloride to fresh water

[E796] beryllium to fresh water

[E797] bifenthrin to fresh water

[E800] Butylbenzylphtalate to fresh water

[E801] cadmium to fresh water

[E802] captafol to fresh water

[E803] captan to fresh water

[E804] carbaryl to fresh water

[E805] carbendazim to fresh water

[E806] carbofuran to fresh water

[E807] carbon disulfide to fresh water

[E808] Carcinogenic PAHs to fresh water

[E810] chlordane to fresh water

[E811] chlorfenvinphos to fresh water

[E812] chloridazon to fresh water

[E814] chlorobenzene to fresh water

[E815] chlorothalonil to fresh water

[E816] chlorpropham to fresh water

[E817] chlorpyriphos to fresh water

[E818] chromium III to fresh water

[E819] chromium VI to fresh water

[E820] chrysene to fresh water

[E821] cobalt to fresh water

[E822] copper to fresh water

[E823] coumaphos to fresh water

[E824] cyanazine to fresh water

[E825] cypermethrin to fresh water

[E826] cyromazine to fresh water

[E827] DDT to fresh water

[E828] deltamethrin to fresh water

[E829] demeton to fresh water

[E830] desmetryn to fresh water

[E831] Di(2-ethylhexyl)phtalate to fresh water

[E832] diazinon to fresh water

[E833] Dibutylphtalate to fresh water

[E834] Dichloromethane (Methylene Chloride) to fresh water

[E835] dichlorprop to fresh water

[E836] dichlorvos to fresh water

[E837] dieldrin to fresh water

[E838] Diethylphtalate to fresh water

[E839] Dihexylphtalate to fresh water

[E840] Diisodecylphtalate to fresh water

[E841] Diisooctylphtalate to fresh water

[E842] dimethoate to fresh water

[E843] Dimethylphtalate to fresh water

[E844] dinoseb to fresh water

[E845] dinoterb to fresh water

[E846] Dioctylphtalate to fresh water

[E847] disulfothon to fresh water

[E848] diuron to fresh water

[E849] DNOC to fresh water

[E850] endosulfan to fresh water

[E851] endrin to fresh water

[E852] ethoprophos to fresh water

[E853] ethylbenzene to fresh water

[E854] ethylene to fresh water

[E855] fenitrothion to fresh water

[E856] fenthion to fresh water

[E857] fentin acetate to fresh water

[E858] fentin chloride to fresh water

[E859] fentin hydroxide to fresh water

[E860] fluoranthrene to fresh water
0.0014 kg 1,4-dichlorobenzene eq." $/ \mathrm{kg}$ $28000 \mathrm{~kg}$ 1,4-dichlorobenzene eq."/kg $36000 \mathrm{~kg}$ 1,4-dichlorobenzene eq." $/ \mathrm{kg}$ $32000 \mathrm{~kg}$ 1,4-dichlorobenzene eq."/kg 1.30E+06 kg 1,4-dichlorobenzene eq." $/ \mathrm{kg}$ $0.19 \mathrm{~kg}$ 1,4-dichlorobenzene eq. "/ $/ \mathrm{kg}$ 2.30E+08 kg 1,4-dichlorobenzene eq."/kg $810 \mathrm{~kg}$ 1,4-dichlorobenzene eq." $/ \mathrm{kg}$ $0.013 \mathrm{~kg}$ 1,4-dichlorobenzene eq." $/ \mathrm{kg}$ 2.20E+05 kg 1,4-dichlorobenzene eq." $/ \mathrm{kg}$ 1.20E+05 kg 1,4-dichlorobenzene eq."/kg $0.0013 \mathrm{~kg}$ 1,4-dichlorobenzene eq." $/ \mathrm{kg}$ $0.13 \mathrm{~kg}$ 1,4-dichlorobenzene eq." $/ \mathrm{kg}$ $860 \mathrm{~kg}$ 1,4-dichlorobenzene eq." $/ \mathrm{kg}$

$46 \mathrm{~kg}$ 1,4-dichlorobenzene eq." $/ \mathrm{kg}$

$1.4 \mathrm{~kg}$ 1,4-dichlorobenzene eq." $/ \mathrm{kg}$

$18000 \mathrm{~kg}$ 1,4-dichlorobenzene eq."/kg $270 \mathrm{~kg}$ 1,4-dichlorobenzene eq." $/ \mathrm{kg}$ $6.7 \mathrm{~kg}$ 1,4-dichlorobenzene eq." $/ \mathrm{kg}$

$1.5 \mathrm{~kg}$ 1,4-dichlorobenzene eq." $/ \mathrm{kg}$

$0.055 \mathrm{~kg}$ 1,4-dichlorobenzene eq." $/ \mathrm{kg}$

$12 \mathrm{~kg}$ 1,4-dichlorobenzene eq. "/kg

$0.45 \mathrm{~kg}$ 1,4-dichlorobenzene eq." $/ \mathrm{kg}$

$24 \mathrm{~kg}$ 1,4-dichlorobenzene eq." $/ \mathrm{kg}$

$880 \mathrm{~kg}$ 1,4-dichlorobenzene eq." $/ \mathrm{kg}$

3500 kg 1,4-dichlorobenzene eq." $/ \mathrm{kg}$

10000 kg 1,4-dichlorobenzene eq." $/ \mathrm{kg}$ $2.80 \mathrm{E}+06 \mathrm{~kg}$ 1,4-dichlorobenzene eq." $/ \mathrm{kg}$ 2.30E+05 kg 1,4-dichlorobenzene eq." $/ \mathrm{kg}$ 4.40E+06 kg 1,4-dichlorobenzene eq." $/ \mathrm{kg}$ $250 \mathrm{~kg}$ 1,4-dichlorobenzene eq." $/ \mathrm{kg}$ 27000 kg 1,4-dichlorobenzene eq." $/ \mathrm{kg}$ $1400 \mathrm{~kg}$ 1,4-dichlorobenzene eq."/kg 1600 kg 1,4-dichlorobenzene eq."/kg 2000 kg 1,4-dichlorobenzene eq." $/ \mathrm{kg}$ $120 \mathrm{~kg}$ 1,4-dichlorobenzene eq." $/ \mathrm{kg}$ $1.6 \mathrm{~kg}$ 1,4-dichlorobenzene eq." $/ \mathrm{kg}$ $0.27 \mathrm{~kg}$ 1,4-dichlorobenzene eq." $/ \mathrm{kg}$ $610 \mathrm{~kg}$ 1,4-dichlorobenzene eq." $/ \mathrm{kg}$ $0.0075 \mathrm{~kg}$ 1,4-dichlorobenzene eq." $/ \mathrm{kg}$ $0.0013 \mathrm{~kg}$ 1,4-dichlorobenzene eq." $/ \mathrm{kg}$ 0.0077 kg 1,4-dichlorobenzene eq." $/ \mathrm{kg}$ $0.91 \mathrm{~kg}$ 1,4-dichlorobenzene eq." $/ \mathrm{kg}$ $320 \mathrm{~kg}$ 1,4-dichlorobenzene eq." $/ \mathrm{kg}$ $0.094 \mathrm{~kg}$ 1,4-dichlorobenzene eq." $/ \mathrm{kg}$ $2.3 \mathrm{~kg}$ 1,4-dichlorobenzene eq." $/ \mathrm{kg}$

$3.8 \mathrm{~kg}$ 1,4-dichlorobenzene eq." $/ \mathrm{kg}$ $0.72 \mathrm{~kg}$ 1,4-dichlorobenzene eq." $/ \mathrm{kg}$ $0.91 \mathrm{~kg}$ 1,4-dichlorobenzene eq." $/ \mathrm{kg}$

$0.00043 \mathrm{~kg}$ 1,4-dichlorobenzene eq." $/ \mathrm{kg}$ 2200 kg 1,4-dichlorobenzene eq." $/ \mathrm{kg}$ $2000 \mathrm{~kg}$ 1,4-dichlorobenzene eq." $/ \mathrm{kg}$ $0.036 \mathrm{~kg}$ 1,4-dichlorobenzene eq. "/kg

$35 \mathrm{~kg}$ 1,4-dichlorobenzene eq." $/ \mathrm{kg}$

$35 \mathrm{~kg}$ 1,4-dichlorobenzene eq."/kg $0.08 \mathrm{~kg}$ 1,4-dichlorobenzene eq." $/ \mathrm{kg}$ $0.77 \mathrm{~kg}$ 1,4-dichlorobenzene eq." $/ \mathrm{kg}$ 25000 kg 1,4-dichlorobenzene eq." $/ \mathrm{kg}$ 4800 kg 1,4-dichlorobenzene eq." "/kg $0.0013 \mathrm{~kg}$ 1,4-dichlorobenzene eq." $/ \mathrm{kg}$ 3.40E-05 kg 1,4-dichlorobenzene eq." $/ \mathrm{kg}$ $340 \mathrm{~kg}$ 1,4-dichlorobenzene eq." $/ \mathrm{kg}$ 2500 kg 1,4-dichlorobenzene eq." $/ \mathrm{kg}$ 8700 kg 1,4-dichlorobenzene eq."/kg 26000 kg 1,4-dichlorobenzene eq." $/ \mathrm{kg}$ $8600 \mathrm{~kg}$ 1,4-dichlorobenzene eq." $/ \mathrm{kg}$ 2800 kg 1,4-dichlorobenzene eq."/kg 
[E861] folpet to fresh water

[E862] Formaldehyde (methanal) to fresh water

[E863] glyphosate to fresh water

[E864] heptachlor to fresh water

[E865] heptenophos to fresh water

[E866] hexachloro-1,3-butadiene to fresh water

[E867] hexachlorobenzene to fresh water

[E870] indeno[1,2,3-cd]pyrene to fresh water

[E871] iprodione to fresh water

[E872] isoproturon to fresh water

[E873] lead to fresh water

[E874] lindane to fresh water

[E875] linuron to fresh water

[E876] malathion to fresh water

[E878] MCPA to fresh water

[E879] mecoprop to fresh water

[E880] mercury to fresh water

[E881] metamitron to fresh water

[E882] metazachlor to fresh water

[E883] methabenzthiazuron to fresh water

[E884] methomyl to fresh water

[E885] methylbromide to fresh water

[E886] methyl-mercury to fresh water

[E887] metobromuron to fresh water

[E888] metolachlor to fresh water

[E889] mevinphos to fresh water

[E890] molybdenum to fresh water

[E891] $m$-xylene to fresh water

[E892] naphtalene to fresh water

[E893] nickel to fresh water

[E899] oxamyl to fresh water

[E900] oxydemethon-methyl to fresh water

[E901] o-xylene to fresh water

[E902] parathion-ethyl to fresh water

[E903] parathion-methyl to fresh water

[E904] pentachlorobenzene to fresh water

[E905] pentachloronitrobenzene to fresh water

[E906] pentachlorophenol to fresh water

[E907] permethrin to fresh water

[E908] phenanthrene to fresh water

[E909] phenol to fresh water

[E912] phoxim to fresh water

[E913] Phtalic anhydride to fresh water

[E914] pirimicarb to fresh water

[E916] propachlor to fresh water

[E917] propoxur to fresh water

[E918] propylene oxide to fresh water

[E919] p-xylene to fresh water

[E920] pyrazophos to fresh water

[E921] selenium to fresh water

[E922] simazine to fresh water

[E923] styrene (vinylbenzene) to fresh water

[E926] tetrachloroethylene (PER) to fresh water

[E927] Tetrachloromethane (carbon tetrachloride) $(\mathrm{HC}-10)$ to fresh water

[E928] thallium to fresh water

[E929] Thiram to fresh water

[E930] tin to fresh water

[E931] tolclophos-methyl to fresh water

[E932] toluene to fresh water

[E933] tri-allate to fresh water

[E934] triazophos to fresh water

[E935] tributyltinoxide to fresh water

[E936] trichlorfon to fresh water

[E937] Trichloroethylene (tri) to fresh water

[E938] Trichloromethane=chloroform to fresh water

[E939] trifluarin to fresh water

[E940] vanadium to fresh water
$16000 \mathrm{~kg}$ 1,4-dichlorobenzene eq." $/ \mathrm{kg}$

$0.2 \mathrm{~kg}$ 1,4-dichlorobenzene eq." $/ \mathrm{kg}$

$3.7 \mathrm{~kg}$ 1,4-dichlorobenzene eq." $/ \mathrm{kg}$

$10 \mathrm{~kg}$ 1,4-dichlorobenzene eq." $/ \mathrm{kg}$

$2.3 \mathrm{~kg}$ 1,4-dichlorobenzene eq." $/ \mathrm{kg}$

$28000 \mathrm{~kg}$ 1,4-dichlorobenzene eq." $/ \mathrm{kg}$

$2700 \mathrm{~kg}$ 1,4-dichlorobenzene eq." $/ \mathrm{kg}$

$50000 \mathrm{~kg} \mathrm{1,4-dichlorobenzene} \mathrm{eq."} / \mathrm{kg}$

$0.00024 \mathrm{~kg}$ 1,4-dichlorobenzene eq." $/ \mathrm{kg}$

$13 \mathrm{~kg}$ 1,4-dichlorobenzene eq." $/ \mathrm{kg}$

1100 kg 1,4-dichlorobenzene eq." $/ \mathrm{kg}$

$18 \mathrm{~kg}$ 1,4-dichlorobenzene eq." $/ \mathrm{kg}$

$730 \mathrm{~kg}$ 1,4-dichlorobenzene eq." $/ \mathrm{kg}$

$430 \mathrm{~kg}$ 1,4-dichlorobenzene eq." $/ \mathrm{kg}$

$0.044 \mathrm{~kg}$ 1,4-dichlorobenzene eq." $/ \mathrm{kg}$

$0.87 \mathrm{~kg}$ 1,4-dichlorobenzene eq." $/ \mathrm{kg}$

2.20E+05 kg 1,4-dichlorobenzene eq." $/ \mathrm{kg}$

$0.05 \mathrm{~kg}$ 1,4-dichlorobenzene eq." $/ \mathrm{kg}$

$1.5 \mathrm{~kg}$ 1,4-dichlorobenzene eq." $/ \mathrm{kg}$

$37 \mathrm{~kg}$ 1,4-dichlorobenzene eq." $/ \mathrm{kg}$

$5400 \mathrm{~kg}$ 1,4-dichlorobenzene eq." $/ \mathrm{kg}$

$0.96 \mathrm{~kg} \mathrm{1,4-dichlorobenzene} \mathrm{eq."} / \mathrm{kg}$

5.10E+06 kg 1,4-dichlorobenzene eq." $/ \mathrm{kg}$

$72 \mathrm{~kg}$ 1,4-dichlorobenzene eq." $/ \mathrm{kg}$

$810 \mathrm{~kg}$ 1,4-dichlorobenzene eq." $/ \mathrm{kg}$

$63 \mathrm{~kg}$ 1,4-dichlorobenzene eq." $/ \mathrm{kg}$

1.70E+06 kg 1,4-dichlorobenzene eq." $/ \mathrm{kg}$

$0.0021 \mathrm{~kg}$ 1,4-dichlorobenzene eq." $/ \mathrm{kg}$

$0.38 \mathrm{~kg} 1,4$-dichlorobenzene eq." $/ \mathrm{kg}$

2.20E+06 kg 1,4-dichlorobenzene eq." $/ \mathrm{kg}$

$0.053 \mathrm{~kg}$ 1,4-dichlorobenzene eq." $/ \mathrm{kg}$

$58 \mathrm{~kg}$ 1,4-dichlorobenzene eq." $/ \mathrm{kg}$

$0.0031 \mathrm{~kg}$ 1,4-dichlorobenzene eq." $/ \mathrm{kg}$

2200 kg 1,4-dichlorobenzene eq." $/ \mathrm{kg}$

$62 \mathrm{~kg}$ 1,4-dichlorobenzene eq." $/ \mathrm{kg}$

$87 \mathrm{~kg}$ 1,4-dichlorobenzene eq." $/ \mathrm{kg}$

$220 \mathrm{~kg}$ 1,4-dichlorobenzene eq." $/ \mathrm{kg}$

$22 \mathrm{~kg} \mathrm{1,4-dichlorobenzene} \mathrm{eq.} \mathrm{"} / \mathrm{kg}$

20000 kg 1,4-dichlorobenzene eq." $/ \mathrm{kg}$

$8.6 \mathrm{~kg} \mathrm{1,4-dichlorobenzene} \mathrm{eq.} / \mathrm{kg}$

0.038 kg 1,4-dichlorobenzene eq." $/ \mathrm{kg}$

$0.67 \mathrm{~kg}$ 1,4-dichlorobenzene eq." $/ \mathrm{kg}$

2.40E-08 kg 1,4-dichlorobenzene eq." $/ \mathrm{kg}$

$240 \mathrm{~kg}$ 1,4-dichlorobenzene eq." $/ \mathrm{kg}$

$2.3 \mathrm{~kg} \mathrm{1,4-dichlorobenzene} \mathrm{eq.} / \mathrm{kg}$

$520 \mathrm{~kg}$ 1,4-dichlorobenzene eq." $/ \mathrm{kg}$

$0.034 \mathrm{~kg} \mathrm{1,4-dichlorobenzene} \mathrm{eq."} / \mathrm{kg}$

$0.0016 \mathrm{~kg}$ 1,4-dichlorobenzene eq." $/ \mathrm{kg}$

$120 \mathrm{~kg}$ 1,4-dichlorobenzene eq." $/ \mathrm{kg}$

1.10E+07 kg 1,4-dichlorobenzene eq." $/ \mathrm{kg}$

$210 \mathrm{~kg}$ 1,4-dichlorobenzene eq." $/ \mathrm{kg}$

0.0016 kg 1,4-dichlorobenzene eq." $/ \mathrm{kg}$

$0.13 \mathrm{~kg}$ 1,4-dichlorobenzene eq." $/ \mathrm{kg}$

$0.31 \mathrm{~kg}$ 1,4-dichlorobenzene eq." $/ \mathrm{kg}$

$2.50 \mathrm{E}+07 \mathrm{~kg}$ 1,4-dichlorobenzene eq. " $/ \mathrm{kg}$

$6.6 \mathrm{~kg}$ 1,4-dichlorobenzene eq." $/ \mathrm{kg}$

$250 \mathrm{~kg}$ 1,4-dichlorobenzene eq." $/ \mathrm{kg}$

$5.1 \mathrm{~kg}$ 1,4-dichlorobenzene eq. $/ \mathrm{kg}$

$0.0013 \mathrm{~kg}$ 1,4-dichlorobenzene eq." $/ \mathrm{kg}$

$220 \mathrm{~kg}$ 1,4-dichlorobenzene eq." $/ \mathrm{kg}$

$2100 \mathrm{~kg}$ 1,4-dichlorobenzene eq." $/ \mathrm{kg}$

2.90E+05 kg 1,4-dichlorobenzene eq." $/ \mathrm{kg}$

$13 \mathrm{~kg}$ 1,4-dichlorobenzene eq." $/ \mathrm{kg}$

$0.0027 \mathrm{~kg}$ 1,4-dichlorobenzene eq." $/ \mathrm{kg}$

0.016 kg 1,4-dichlorobenzene eq." $/ \mathrm{kg}$

$180 \mathrm{~kg}$ 1,4-dichlorobenzene eq." $/ \mathrm{kg}$

7.90E+06 kg 1,4-dichlorobenzene eq."/kg 
[E941] Vinyl Chloride (chloroethene) to fresh water [E942] zinc to fresh water [E943] zineb to fresh water

[E944] 1,1,1-trichloroethane to fresh water [E945] 1,2,3,4-tetrachlorobenzene to fresh water [E946] 1,2,3,5-tetrachlorobenzene to fresh water [E947] 1,2,3-trichlorobenzene to fresh water [E948] 1,2,4,5-tetrachlorobenzene to fresh water [E949] 1,2,4-trichlorobenzene to fresh water [E950] 1,2-dichlorobenzene to fresh water [E951] 1,2-dichloroethane to fresh water [E952] 1,3,5-trichlorobenzene to fresh water [E953] 1,3-butadiene to fresh water [E954] 1,3-dichlorobenzene to fresh water [E955] 1,4-dichlorobenzene to fresh water [E956] 1-chloro-4-nitrobenzene to fresh water [E957] 2,3,4,6-tetrachlorophenol to fresh water [E958] 2,3,7,8-TCDD to fresh water [E959] 2,4,5-T to fresh water

[E960] 2,4,5-trichlorophenol to fresh water [E961] 2,4,6-trichlorophenol to sea water [E962] 2,4-D to sea water [E963] 2,4-dichlorophenol to sea water [E964] 2-chlorophenol to sea water [E965] 3,4-dichloroaniline to sea water [E966] 3-chloroaniline to sea water [E967] 4-chloroaniline to sea water [E968] acephate to sea water

[E969] Acrolein (2-propenal) to sea water [E970] acrylonitrile to sea water

[E971] aldicarb to sea water

[E972] aldrin to sea water

[E974] anilazine to sea water

[E975] anthracene to sea water

[E976] antimony to sea water

[E977] arsenic to sea water

[E978] atrazine to sea water

[E979] azinphos-ethyl to sea water

[E980] azinphos-methyl to sea water

[E981] barium to sea water

[E982] benomyl to sea water

[E983] bentazone to sea water

[E984] benzene to sea water

[E985] benzo[a]anthracene to sea water

[E986] benzo[a]pyrene to sea water

[E987] benzo[ghi]perylene to sea water

[E988] benzo[k]fluoranthrene to sea water

[E989] benzylchloride to sea water

[E990] beryllium to sea water

[E991] bifenthrin to sea water

[E994] Butylbenzylphtalate to sea water

[E995] cadmium to sea water

[E996] captafol to sea water

[E997] captan to sea water

[E998] carbaryl to sea water

[E999] carbendazim to sea water

[E1000] carbofuran to sea water

[E1001] carbon disulfide to sea water

[E1002] Carcinogenic PAHs to sea water

[E1004] chlordane to sea water

[E1005] chlorfenvinphos to sea water

[E1006] chloridazon to sea water

[E1008] chlorobenzene to sea water

[E1009] chlorothalonil to sea water

[E1010] chlorpropham to sea water

[E1011] chlorpyriphos to sea water

[E1012] chromium III to sea water
$0.00049 \mathrm{~kg}$ 1,4-dichlorobenzene eq. "/kg

$14000 \mathrm{~kg}$ 1,4-dichlorobenzene eq." $/ \mathrm{kg}$

$270 \mathrm{~kg}$ 1,4-dichlorobenzene eq." $/ \mathrm{kg}$

$0.19 \mathrm{~kg}$ 1,4-dichlorobenzene eq."/kg

$12 \mathrm{~kg}$ 1,4-dichlorobenzene eq. "/kg

$13 \mathrm{~kg}$ 1,4-dichlorobenzene eq."/kg

$3.5 \mathrm{~kg}$ 1,4-dichlorobenzene eq." $/ \mathrm{kg}$

$10 \mathrm{~kg}$ 1,4-dichlorobenzene eq. "/kg

$2.9 \mathrm{~kg}$ 1,4-dichlorobenzene eq." $/ \mathrm{kg}$

$1 \mathrm{~kg}$ 1,4-dichlorobenzene eq." $/ \mathrm{kg}$

0.061 kg 1,4-dichlorobenzene eq."/kg

$4.5 \mathrm{~kg}$ 1,4-dichlorobenzene eq. " $/ \mathrm{kg}$

$0.83 \mathrm{~kg}$ 1,4-dichlorobenzene eq." $/ \mathrm{kg}$

$1.2 \mathrm{~kg} \mathrm{1,4-dichlorobenzene} \mathrm{eq."} / \mathrm{kg}$

$1 \mathrm{~kg}$ 1,4-dichlorobenzene eq." $/ \mathrm{kg}$

$440 \mathrm{~kg}$ 1,4-dichlorobenzene eq." $/ \mathrm{kg}$

$250 \mathrm{~kg}$ 1,4-dichlorobenzene eq." $/ \mathrm{kg}$

1.90E+09 kg 1,4-dichlorobenzene eq." $/ \mathrm{kg}$

$0.49 \mathrm{~kg}$ 1,4-dichlorobenzene eq." $/ \mathrm{kg}$

$160 \mathrm{~kg}$ 1,4-dichlorobenzene eq." $/ \mathrm{kg}$

$8.9 \mathrm{~kg}$ 1,4-dichlorobenzene eq." $/ \mathrm{kg}$

14 kg 1,4-dichlorobenzene eq." $/ \mathrm{kg}$

$2 \mathrm{~kg} \mathrm{1,4-dichlorobenzene} \mathrm{eq."} / \mathrm{kg}$

$61 \mathrm{~kg}$ 1,4-dichlorobenzene eq." $/ \mathrm{kg}$

$4100 \mathrm{~kg}$ 1,4-dichlorobenzene eq." $/ \mathrm{kg}$

$82 \mathrm{~kg}$ 1,4-dichlorobenzene eq." $/ \mathrm{kg}$

$140 \mathrm{~kg}$ 1,4-dichlorobenzene eq." $/ \mathrm{kg}$

$35 \mathrm{~kg}$ 1,4-dichlorobenzene eq." $/ \mathrm{kg}$

$13000 \mathrm{~kg}$ 1,4-dichlorobenzene eq." $/ \mathrm{kg}$

4 kg 1,4-dichlorobenzene eq." $/ \mathrm{kg}$

$22000 \mathrm{~kg}$ 1,4-dichlorobenzene eq." $/ \mathrm{kg}$

$740 \mathrm{~kg}$ 1,4-dichlorobenzene eq." $/ \mathrm{kg}$

$0.83 \mathrm{~kg}$ 1,4-dichlorobenzene eq." $/ \mathrm{kg}$

$25000 \mathrm{~kg}$ 1,4-dichlorobenzene eq." $/ \mathrm{kg}$

$46000 \mathrm{~kg}$ 1,4-dichlorobenzene eq." $/ \mathrm{kg}$

$3.40 \mathrm{E}+05 \mathrm{~kg}$ 1,4-dichlorobenzene eq. $/ / \mathrm{kg}$

$670 \mathrm{~kg}$ 1,4-dichlorobenzene eq." $/ \mathrm{kg}$

$4700 \mathrm{~kg}$ 1,4-dichlorobenzene eq." $/ \mathrm{kg}$

$290 \mathrm{~kg}$ 1,4-dichlorobenzene eq." $/ \mathrm{kg}$

9.30E+05 kg 1,4-dichlorobenzene eq." $/ \mathrm{kg}$

$13 \mathrm{~kg}$ 1,4-dichlorobenzene eq." $/ \mathrm{kg}$

$1.8 \mathrm{~kg}$ 1,4-dichlorobenzene eq." $/ \mathrm{kg}$

$0.021 \mathrm{~kg}$ 1,4-dichlorobenzene eq." $/ \mathrm{kg}$

2.80E+05 kg 1,4-dichlorobenzene eq." $/ \mathrm{kg}$

$3.70 \mathrm{E}+05 \mathrm{~kg}$ 1,4-dichlorobenzene eq."//kg

$2.30 \mathrm{E}+05 \mathrm{~kg}$ 1,4-dichlorobenzene eq." $/ \mathrm{kg}$

4.40E+06 kg 1,4-dichlorobenzene eq." $/ \mathrm{kg}$

$1.9 \mathrm{~kg}$ 1,4-dichlorobenzene eq." $/ \mathrm{kg}$

$2.80 \mathrm{E}+08 \mathrm{~kg}$ 1,4-dichlorobenzene eq." $/ \mathrm{kg}$

$34000 \mathrm{~kg}$ 1,4-dichlorobenzene eq." $/ \mathrm{kg}$

$0.4 \mathrm{~kg}$ 1,4-dichlorobenzene eq." $/ \mathrm{kg}$

$1.90 \mathrm{E}+06 \mathrm{~kg}$ 1,4-dichlorobenzene eq." $/ \mathrm{kg}$

1.40E+05 kg 1,4-dichlorobenzene eq." $/ \mathrm{kg}$

$0.5 \mathrm{~kg}$ 1,4-dichlorobenzene eq." $/ \mathrm{kg}$

$2.1 \mathrm{~kg}$ 1,4-dichlorobenzene eq." $/ \mathrm{kg}$

$2000 \mathrm{~kg}$ 1,4-dichlorobenzene eq." $/ \mathrm{kg}$

$310 \mathrm{~kg}$ 1,4-dichlorobenzene eq." $/ \mathrm{kg}$

$45 \mathrm{~kg}$ 1,4-dichlorobenzene eq." $/ \mathrm{kg}$

80000 kg 1,4-dichlorobenzene eq." $/ \mathrm{kg}$

$15000 \mathrm{~kg}$ 1,4-dichlorobenzene eq."/kg

$33 \mathrm{~kg}$ 1,4-dichlorobenzene eq." $/ \mathrm{kg}$

$10 \mathrm{~kg}$ 1,4-dichlorobenzene eq." $/ \mathrm{kg}$

$0.45 \mathrm{~kg}$ 1,4-dichlorobenzene eq." $/ \mathrm{kg}$

$23 \mathrm{~kg}$ 1,4-dichlorobenzene eq." $/ \mathrm{kg}$

$2.5 \mathrm{~kg}$ 1,4-dichlorobenzene eq. "/kg

$220 \mathrm{~kg}$ 1,4-dichlorobenzene eq." $/ \mathrm{kg}$

$8400 \mathrm{~kg}$ 1,4-dichlorobenzene eq." $/ \mathrm{kg}$ 
[E1013] chromium VI to sea water

[E1014] chrysene to sea water

[E1015] cobalt to sea water

[E1016] copper to sea water

[E1017] coumaphos to sea water

[E1018] cyanazine to sea water

[E1019] cypermethrin to sea water

[E1020] cyromazine to sea water

[E1021] DDT to sea water

[E1022] deltamethrin to sea water

[E1023] demeton to sea water

[E1024] desmetryn to sea water

[E1025] Di(2-ethylhexyl)phtalate to sea water

[E1026] diazinon to sea water

[E1027] Dibutylphtalate to sea water

[E1028] Dichloromethane (Methylene Chloride) to sea water

[E1029] dichlorprop to sea water

[E1030] dichlorvos to sea water

[E1031] dieldrin to sea water

[E1032] Diethylphtalate to sea water

[E1033] Dihexylphtalate to sea water

[E1034] Diisodecylphtalate to sea water

[E1035] Diisooctylphtalate to sea water

[E1036] dimethoate to sea water

[E1037] Dimethylphtalate to sea water

[E1038] dinoseb to sea water

[E1039] dinoterb to sea water

[E1040] Dioctylphtalate to sea water

[E1041] disulfothon to sea water

[E1042] diuron to sea water

[E1043] DNOC to sea water

[E1044] endosulfan to sea water

[E1045] endrin to sea water

[E1046] ethoprophos to sea water

[E1047] ethylbenzene to sea water

[E1048] ethylene to sea water

[E1049] fenitrothion to sea water

[E1050] fenthion to sea water

[E1051] fentin acetate to sea water

[E1052] fentin chloride to sea water

[E1053] fentin hydroxide to sea water

[E1054] fluoranthrene to sea water

[E1055] folpet to sea water

[E1056] Formaldehyde (methanal) to sea water

[E1057] glyphosate to sea water

[E1058] heptachlor to sea water

[E1059] heptenophos to sea water

[E1060] hexachloro-1,3-butadiene to sea water

[E1061] hexachlorobenzene to sea water

[E1064] indeno[1,2,3-cd]pyrene to sea water

[E1065] iprodione to sea water

[E1066] isoproturon to sea water

[E1067] lead to sea water

[E1068] lindane to sea water

[E1069] linuron to sea water

[E1070] malathion to sea water

[E1072] MCPA to sea water

[E1073] mecoprop to sea water

[E1074] mercury to sea water

[E1075] metamitron to sea water

[E1076] metazachlor to sea water

[E1077] methabenzthiazuron to sea water

[E1078] methomyl to sea water

[E1079] methylbromide to sea water

[E1080] methyl-mercury to sea water

[E1081] metobromuron to sea water

[E1082] metolachlor to sea water
34000 kg 1,4-dichlorobenzene eq."/kg 26000 kg 1,4-dichlorobenzene eq."/kg

5.20E+06 kg 1,4-dichlorobenzene eq."/kg 1.50E+06 kg 1,4-dichlorobenzene eq."/kg

5.20E+06 kg 1,4-dichlorobenzene eq."/kg

1600 kg 1,4-dichlorobenzene eq."/kg

4.50E+05 kg 1,4-dichlorobenzene eq."/kg

2200 kg 1,4-dichlorobenzene eq."/kg

71000 kg 1,4-dichlorobenzene eq."/kg

72000 kg 1,4-dichlorobenzene eq."/kg

700 kg 1,4-dichlorobenzene eq." $/ \mathrm{kg}$

$5.5 \mathrm{~kg}$ 1,4-dichlorobenzene eq." $/ \mathrm{kg}$

11 kg 1,4-dichlorobenzene eq."/kg

2700 kg 1,4-dichlorobenzene eq."/kg

$0.16 \mathrm{~kg} \mathrm{1,4-dichlorobenzene} \mathrm{eq."/kg}$

0.0038 kg 1,4-dichlorobenzene eq." $/ \mathrm{kg}$

0.064 kg 1,4-dichlorobenzene eq." $/ \mathrm{kg}$

180 kg 1,4-dichlorobenzene eq."/kg

2100 kg 1,4-dichlorobenzene eq."/kg

0.65 kg 1,4-dichlorobenzene eq."/kg

20 kg 1,4-dichlorobenzene eq."/kg

34 kg 1,4-dichlorobenzene eq."/kg

28 kg 1,4-dichlorobenzene eq."/kg

$4.1 \mathrm{~kg} \mathrm{1,4-dichlorobenzene} \mathrm{eq."/kg}$

0.013 kg 1,4-dichlorobenzene eq." $/ \mathrm{kg}$

5000 kg 1,4-dichlorobenzene eq." $/ \mathrm{kg}$

4500 kg 1,4-dichlorobenzene eq."/kg

2.6 kg 1,4-dichlorobenzene eq." $/ \mathrm{kg}$

420 kg 1,4-dichlorobenzene eq."/kg

$420 \mathrm{~kg} \mathrm{1,4-dichlorobenzene} \mathrm{eq."/kg}$

$0.61 \mathrm{~kg}$ 1,4-dichlorobenzene eq." $/ \mathrm{kg}$

$22 \mathrm{~kg}$ 1,4-dichlorobenzene eq." $/ \mathrm{kg}$

2.00E+05 kg 1,4-dichlorobenzene eq."/kg

8900 kg 1,4-dichlorobenzene eq."/kg

0.067 kg 1,4-dichlorobenzene eq." $/ \mathrm{kg}$

0.0032 kg 1,4-dichlorobenzene eq."/kg

2900 kg 1,4-dichlorobenzene eq." $/ \mathrm{kg}$

15000 kg 1,4-dichlorobenzene eq." $/ \mathrm{kg}$

1.10E+05 kg 1,4-dichlorobenzene eq."/kg

1.10E+05 kg 1,4-dichlorobenzene eq." $/ \mathrm{kg}$

1.10E+05 kg 1,4-dichlorobenzene eq."/kg

14000 kg 1,4-dichlorobenzene eq."/kg

28000 kg 1,4-dichlorobenzene eq."/kg

6 kg 1,4-dichlorobenzene eq."/kg

30 kg 1,4-dichlorobenzene eq. "/kg

920 kg 1,4-dichlorobenzene eq."/kg

$91 \mathrm{~kg} \mathrm{1,4-dichlorobenzene} \mathrm{eq."/kg}$

47000 kg 1,4-dichlorobenzene eq." $/ \mathrm{kg}$

$3400 \mathrm{~kg} 1,4$-dichlorobenzene eq." $/ \mathrm{kg}$

3.80E+05 kg 1,4-dichlorobenzene eq." $/ \mathrm{kg}$

$0.012 \mathrm{~kg} 1,4$-dichlorobenzene eq." $/ \mathrm{kg}$

37 kg 1,4-dichlorobenzene eq. "/kg

12000 kg 1,4-dichlorobenzene eq."/kg

48 kg 1,4-dichlorobenzene eq."/kg

1700 kg 1,4-dichlorobenzene eq."/kg

2800 kg 1,4-dichlorobenzene eq."/kg

$0.69 \mathrm{~kg}$ 1,4-dichlorobenzene eq." $/ \mathrm{kg}$

$11 \mathrm{~kg} \mathrm{1,4-dichlorobenzene} \mathrm{eq."} / \mathrm{kg}$

1.90E+06 kg 1,4-dichlorobenzene eq." $/ \mathrm{kg}$

$0.38 \mathrm{~kg} \mathrm{1,4-dichlorobenzene} \mathrm{eq."} / \mathrm{kg}$

$5.2 \mathrm{~kg} \mathrm{1,4-dichlorobenzene} \mathrm{eq."/kg}$

70 kg 1,4-dichlorobenzene eq." $/ \mathrm{kg}$

8900 kg 1,4-dichlorobenzene eq."/kg

2 kg 1,4-dichlorobenzene eq." $/ \mathrm{kg}$

4.40E+07 kg 1,4-dichlorobenzene eq." $/ \mathrm{kg}$

$82 \mathrm{~kg} 1,4$-dichlorobenzene eq. $/ \mathrm{kg}$

1900 kg 1,4-dichlorobenzene eq."/kg 
[E1083] mevinphos to sea water

[E1084] molybdenum to sea water

[E1085] m-xylene to sea water

[E1086] naphtalene to sea water

[E1087] nickel to sea water

[E1093] oxamyl to sea water

[E1094] oxydemethon-methyl to sea water

[E1095] o-xylene to sea water

[E1096] parathion-ethyl to sea water

[E1097] parathion-methyl to sea water

[E1098] pentachlorobenzene to sea water

[E1099] pentachloronitrobenzene to sea water

[E1100] pentachlorophenol to sea water

[E1101] permethrin to sea water

[E1102] phenanthrene to sea water

[E1103] phenol to sea water

[E1106] phoxim to sea water

[E1107] Phtalic anhydride to sea water

[E1108] pirimicarb to sea water

[E1110] propachlor to sea water

[E1111] propoxur to sea water

[E1112] propylene oxide to sea water

[E1113] p-xylene to sea water

[E1114] pyrazophos to sea water

[E1115] selenium to sea water

[E1116] simazine to sea water

[E1117] styrene (vinylbenzene) to sea water

[E1120] tetrachloroethylene (PER) to sea water

[E1121] Tetrachloromethane (carbon tetrachloride) (HC-10) to sea water

[E1122] thallium to sea water

[E1123] Thiram to sea water

[E1124] tin to sea water

[E1125] tolclophos-methyl to sea water

[E1126] toluene to sea water

[E1127] tri-allate to sea water

[E1128] triazophos to sea water

[E1129] tributyltinoxide to sea water

[E1130] trichlorfon to sea water

[E1131] Trichloroethylene (tri) to sea water

[E1132] Trichloromethane=chloroform to sea water

[E1133] trifluarin to sea water

[E1134] vanadium to sea water

[E1135] Vinyl Chloride (chloroethene) to sea water

[E1136] zinc to sea water

[E1137] zineb to sea water

[E1138] 1,1,1-trichloroethane to sea water

[E1139] 1,2,3,4-tetrachlorobenzene to sea water

[E1140] 1,2,3,5-tetrachlorobenzene to sea water

[E1141] 1,2,3-trichlorobenzene to sea water

[E1142] 1,2,4,5-tetrachlorobenzene to sea water

[E1143] 1,2,4-trichlorobenzene to sea water

[E1144] 1,2-dichlorobenzene to sea water

[E1145] 1,2-dichloroethane to sea water

[E1146] 1,3,5-trichlorobenzene to sea water

[E1147] 1,3-butadiene to sea water

[E1148] 1,3-dichlorobenzene to sea water

[E1149] 1,4-dichlorobenzene to sea water

[E1150] 1-chloro-4-nitrobenzene to sea water

[E1151] 2,3,4,6-tetrachlorophenol to sea water

[E1152] 2,3,7,8-TCDD to sea water

[E1153] 2,4,5-T to sea water

[E1154] 2,4,5-trichlorophenol to sea water

[E1155] 2,4,6-trichlorophenol to agricultural soil

[E1156] 2,4-D to agricultural soil

[E1157] 2,4-dichlorophenol to agricultural soil

[E1158] 2-chlorophenol to agricultural soil

[E1159] 3,4-dichloroaniline to agricultural soil
$1200 \mathrm{~kg}$ 1,4-dichlorobenzene eq." $/ \mathrm{kg}$ $2.20 \mathrm{E}+06 \mathrm{~kg}$ 1,4-dichlorobenzene eq." $/ \mathrm{kg}$

$0.14 \mathrm{~kg}$ 1,4-dichlorobenzene eq." $/ \mathrm{kg}$

12 kg 1,4-dichlorobenzene eq." $/ \mathrm{kg}$

$5.70 \mathrm{E}+06 \mathrm{~kg}$ 1,4-dichlorobenzene eq." $/ \mathrm{kg}$ $0.8 \mathrm{~kg}$ 1,4-dichlorobenzene eq." $/ \mathrm{kg}$ $420 \mathrm{~kg}$ 1,4-dichlorobenzene eq." $/ \mathrm{kg}$ $0.17 \mathrm{~kg}$ 1,4-dichlorobenzene eq." $/ \mathrm{kg}$

17000 kg 1,4-dichlorobenzene eq." $/ \mathrm{kg}$

340 kg 1,4-dichlorobenzene eq. "/kg

$140 \mathrm{~kg}$ 1,4-dichlorobenzene eq." $/ \mathrm{kg}$

$550 \mathrm{~kg}$ 1,4-dichlorobenzene eq." $/ \mathrm{kg}$

$140 \mathrm{~kg}$ 1,4-dichlorobenzene eq." $/ \mathrm{kg}$

2.20E+05 kg 1,4-dichlorobenzene eq." $/ \mathrm{kg}$

$64 \mathrm{~kg}$ 1,4-dichlorobenzene eq." $/ \mathrm{kg}$

$3.2 \mathrm{~kg}$ 1,4-dichlorobenzene eq." $/ \mathrm{kg}$

$41 \mathrm{~kg}$ 1,4-dichlorobenzene eq." $/ \mathrm{kg}$

9.90E-05 kg 1,4-dichlorobenzene eq." $/ \mathrm{kg}$

$1300 \mathrm{~kg}$ 1,4-dichlorobenzene eq." $/ \mathrm{kg}$

$25 \mathrm{~kg}$ 1,4-dichlorobenzene eq." $/ \mathrm{kg}$

3600 kg 1,4-dichlorobenzene eq." $/ \mathrm{kg}$

$0.15 \mathrm{~kg}$ 1,4-dichlorobenzene eq." $/ \mathrm{kg}$

0.097 kg 1,4-dichlorobenzene eq." $/ \mathrm{kg}$

$1100 \mathrm{~kg}$ 1,4-dichlorobenzene eq." $/ \mathrm{kg}$

$1.20 \mathrm{E}+07 \mathrm{~kg}$ 1,4-dichlorobenzene eq." $/ \mathrm{kg}$

$1000 \mathrm{~kg} \mathrm{1,4-dichlorobenzene} \mathrm{eq."/kg}$

$0.093 \mathrm{~kg}$ 1,4-dichlorobenzene eq." $/ \mathrm{kg}$

$0.78 \mathrm{~kg}$ 1,4-dichlorobenzene eq." $/ \mathrm{kg}$

$0.46 \mathrm{~kg}$ 1,4-dichlorobenzene eq."/kg

3.40E+07 kg 1,4-dichlorobenzene eq." $/ \mathrm{kg}$

$37 \mathrm{~kg}$ 1,4-dichlorobenzene eq."/kg

2500 kg 1,4-dichlorobenzene eq." $/ \mathrm{kg}$

$160 \mathrm{~kg}$ 1,4-dichlorobenzene eq." $/ \mathrm{kg}$

$0.063 \mathrm{~kg}$ 1,4-dichlorobenzene eq." $/ \mathrm{kg}$

920 kg 1,4-dichlorobenzene eq." $/ \mathrm{kg}$

$6800 \mathrm{~kg}$ 1,4-dichlorobenzene eq."/kg

7.90E+05 kg 1,4-dichlorobenzene eq." $/ \mathrm{kg}$

$540 \mathrm{~kg} 1,4$-dichlorobenzene eq." $/ \mathrm{kg}$

$0.081 \mathrm{~kg}$ 1,4-dichlorobenzene eq." $/ \mathrm{kg}$

$0.033 \mathrm{~kg} 1,4$-dichlorobenzene eq." $/ \mathrm{kg}$

$3600 \mathrm{~kg} \mathrm{1,4-dichlorobenzene} \mathrm{eq."/kg}$

$1.70 \mathrm{E}+07 \mathrm{~kg}$ 1,4-dichlorobenzene eq." $/ \mathrm{kg}$

$0.029 \mathrm{~kg}$ 1,4-dichlorobenzene eq."/kg

1.10E+05 kg 1,4-dichlorobenzene eq." $/ \mathrm{kg}$

$890 \mathrm{~kg}$ 1,4-dichlorobenzene eq." $/ \mathrm{kg}$

$0.1 \mathrm{~kg}$ 1,4-dichlorobenzene eq." $/ \mathrm{kg}$

$0.16 \mathrm{~kg} 1,4$-dichlorobenzene eq." $/ \mathrm{kg}$

$0.9 \mathrm{~kg}$ 1,4-dichlorobenzene eq." $/ \mathrm{kg}$

$0.26 \mathrm{~kg}$ 1,4-dichlorobenzene eq." $/ \mathrm{kg}$

$0.21 \mathrm{~kg}$ 1,4-dichlorobenzene eq. "/kg

$0.18 \mathrm{~kg}$ 1,4-dichlorobenzene eq." $/ \mathrm{kg}$

$0.21 \mathrm{~kg}$ 1,4-dichlorobenzene eq." $/ \mathrm{kg}$

$0.022 \mathrm{~kg}$ 1,4-dichlorobenzene eq." $/ \mathrm{kg}$

$0.45 \mathrm{~kg}$ 1,4-dichlorobenzene eq." $/ \mathrm{kg}$

3.20E-06 kg 1,4-dichlorobenzene eq." $/ \mathrm{kg}$

$0.16 \mathrm{~kg}$ 1,4-dichlorobenzene eq." $/ \mathrm{kg}$

$0.21 \mathrm{~kg}$ 1,4-dichlorobenzene eq." $/ \mathrm{kg}$

$79 \mathrm{~kg}$ 1,4-dichlorobenzene eq." $/ \mathrm{kg}$

$0.68 \mathrm{~kg}$ 1,4-dichlorobenzene eq." $/ \mathrm{kg}$

$1.40 \mathrm{E}+05 \mathrm{~kg}$ 1,4-dichlorobenzene eq." $/ \mathrm{kg}$

$0.002 \mathrm{~kg}$ 1,4-dichlorobenzene eq." $/ \mathrm{kg}$

$1.6 \mathrm{~kg}$ 1,4-dichlorobenzene eq." $/ \mathrm{kg}$

$0.0095 \mathrm{~kg}$ 1,4-dichlorobenzene eq." $/ \mathrm{kg}$

$0.23 \mathrm{~kg}$ 1,4-dichlorobenzene eq." $/ \mathrm{kg}$

$0.0032 \mathrm{~kg} \mathrm{1,4-dichlorobenzene} \mathrm{eq.} \mathrm{"} / \mathrm{kg}$

$0.09 \mathrm{~kg}$ 1,4-dichlorobenzene eq." $/ \mathrm{kg}$

$330 \mathrm{~kg}$ 1,4-dichlorobenzene eq." $/ \mathrm{kg}$ 
[E1160] 3-chloroaniline to agricultural soil

[E1161] 4-chloroaniline to agricultural soil

[E1162] acephate to agricultural soil

[E1163] Acrolein (2-propenal) to agricultural soil

[E1164] acrylonitrile to agricultural soil

[E1165] aldicarb to agricultural soil

[E1166] aldrin to agricultural soil

[E1168] anilazine to agricultural soil

[E1169] anthracene to agricultural soil

[E1170] antimony to agricultural soil

[E1171] arsenic to agricultural soil

[E1172] atrazine to agricultural soil

[E1173] azinphos-ethyl to agricultural soil

[E1174] azinphos-methyl to agricultural soil

[E1175] barium to agricultural soil

[E1176] benomyl to agricultural soil

[E1177] bentazone to agricultural soil

[E1178] benzene to agricultural soil

[E1179] benzo[a]anthracene to agricultural soil

[E1180] benzo[a]pyrene to agricultural soil

[E1181] benzo[ghi]perylene to agricultural soil

[E1182] benzo[k]fluoranthrene to agricultural soil

[E1183] benzylchloride to agricultural soil

[E1184] beryllium to agricultural soil

[E1185] bifenthrin to agricultural soil

[E1186] Butylbenzylphtalate to agricultural soil

[E1187] cadmium to agricultural soil

[E1188] captafol to agricultural soil

[E1189] captan to agricultural soil

[E1190] carbaryl to agricultural soil

[E1191] carbendazim to agricultural soil

[E1192] carbofuran to agricultural soil

[E1193] carbon disulfide to agricultural soil

[E1194] Carcinogenic PAHs to agricultural soil

[E1195] chlordane to agricultural soil

[E1196] chlorfenvinphos to agricultural soil

[E1197] chloridazon to agricultural soil

[E1198] chlorobenzene to agricultural soil

[E1199] chlorothalonil to agricultural soil

[E1200] chlorpropham to agricultural soil

[E1201] chlorpyriphos to agricultural soil

[E1202] chromium III to agricultural soil

[E1203] chromium VI to agricultural soil

[E1204] chrysene to agricultural soil

[E1205] cobalt to agricultural soil

[E1206] copper to agricultural soil

[E1207] coumaphos to agricultural soil

[E1208] cyanazine to agricultural soil

[E1209] cypermethrin to agricultural soil

[E1210] cyromazine to agricultural soil

[E1211] DDT to agricultural soil

[E1212] deltamethrin to agricultural soil

[E1213] demeton to agricultural soil

[E1214] desmetryn to agricultural soil

[E1215] Di(2-ethylhexyl)phtalate to agricultural soil

[E1216] diazinon to agricultural soil

[E1217] Dibutylphtalate to agricultural soil

[E1218] Dichloromethane (Methylene Chloride) to agricultural soi

[E1219] dichlorprop to agricultural soil

[E1220] dichlorvos to agricultural soil

[E1221] dieldrin to agricultural soil

[E1222] Diethylphtalate to agricultural soil

[E1223] Dihexylphtalate to agricultural soil

[E1224] Diisodecylphtalate to agricultural soi

[E1225] Diisooctylphtalate to agricultural soil

[E1226] dimethoate to agricultural soil

[E1227] Dimethylphtalate to agricultural soil
0.45 kg 1,4-dichlorobenzene eq."/kg

$11 \mathrm{~kg}$ 1,4-dichlorobenzene eq."/kg

$0.64 \mathrm{~kg} 1,4$-dichlorobenzene eq." $/ \mathrm{kg}$

360 kg 1,4-dichlorobenzene eq." $/ \mathrm{kg}$

0.19 kg 1,4-dichlorobenzene eq."/kg

2400 kg 1,4-dichlorobenzene eq."/kg

$2.9 \mathrm{~kg} \mathrm{1,4-dichlorobenzene} \mathrm{eq."} / \mathrm{kg}$

2.10E-06 kg 1,4-dichlorobenzene eq."/kg

$8.2 \mathrm{~kg} \mathrm{1,4-dichlorobenzene} \mathrm{eq."} / \mathrm{kg}$

13000 kg 1,4-dichlorobenzene eq."/kg

77000 kg 1,4-dichlorobenzene eq."/kg

$38 \mathrm{~kg} \mathrm{1,4-dichlorobenzene} \mathrm{eq."} / \mathrm{kg}$

$8.4 \mathrm{~kg} \mathrm{1,4-dichlorobenzene} \mathrm{eq."/kg}$

$0.041 \mathrm{~kg} 1,4-$ dichlorobenzene eq." $/ \mathrm{kg}$

3.60E+05 kg 1,4-dichlorobenzene eq." $/ \mathrm{kg}$

0.0005 kg 1,4-dichlorobenzene eq." $/ \mathrm{kg}$

$0.055 \mathrm{~kg} \mathrm{1,4-dichlorobenzene} \mathrm{eq."/kg}$

0.0011 kg 1,4-dichlorobenzene eq."/kg

$15 \mathrm{~kg} \mathrm{1,4-dichlorobenzene} \mathrm{eq."/kg}$

19 kg 1,4-dichlorobenzene eq."/kg

37 kg 1,4-dichlorobenzene eq."/kg

5900 kg 1,4-dichlorobenzene eq."/kg

$0.013 \mathrm{~kg} \mathrm{1,4-dichlorobenzene} \mathrm{eq."} / \mathrm{kg}$

1.20E+08 kg 1,4-dichlorobenzene eq."/kg

$0.43 \mathrm{~kg} 1,4$-dichlorobenzene eq." $/ \mathrm{kg}$

7.10E-06 kg 1,4-dichlorobenzene eq."/kg

1.10E+05 kg 1,4-dichlorobenzene eq."/kg

5800 kg 1,4-dichlorobenzene eq."/kg

8.40E-07 kg 1,4-dichlorobenzene eq."/kg

0.00065 kg 1,4-dichlorobenzene eq."/kg

45 kg 1,4-dichlorobenzene eq."/kg

$2.1 \mathrm{~kg} \mathrm{1,4-dichlorobenzene} \mathrm{eq."} / \mathrm{kg}$

0.79 kg 1,4-dichlorobenzene eq." $/ \mathrm{kg}$

$41 \mathrm{~kg} \mathrm{1,4-dichlorobenzene} \mathrm{eq.} \mathrm{"/kg}$

$0.84 \mathrm{~kg} \mathrm{1,4-dichlorobenzene} \mathrm{eq.} \mathrm{"/kg}$

$0.1 \mathrm{~kg} \mathrm{1,4-dichlorobenzene} \mathrm{eq."} / \mathrm{kg}$

$0.1 \mathrm{~kg} \mathrm{1,4-dichlorobenzene} \mathrm{eq."} / \mathrm{kg}$

$0.037 \mathrm{~kg} \mathrm{1,4-dichlorobenzene} \mathrm{eq."/kg}$

0.47 kg 1,4-dichlorobenzene eq." $/ \mathrm{kg}$

0.011 kg 1,4-dichlorobenzene eq."/kg

0.014 kg 1,4-dichlorobenzene eq."/kg

670 kg 1,4-dichlorobenzene eq."/kg

2700 kg 1,4-dichlorobenzene eq."/kg

40 kg 1,4-dichlorobenzene eq."/kg

1.40E+06 kg 1,4-dichlorobenzene eq." $/ \mathrm{kg}$

1.20E+05 kg 1,4-dichlorobenzene eq." $/ \mathrm{kg}$

2.20E+05 kg 1,4-dichlorobenzene eq."/kg

3.7 kg 1,4-dichlorobenzene eq." $/ \mathrm{kg}$

800 kg 1,4-dichlorobenzene eq."/kg

350 kg 1,4-dichlorobenzene eq." $/ \mathrm{kg}$

$14 \mathrm{~kg}$ 1,4-dichlorobenzene eq."/kg

0.12 kg 1,4-dichlorobenzene eq." $/ \mathrm{kg}$

$4.5 \mathrm{~kg} \mathrm{1,4-dichlorobenzene} \mathrm{eq."/kg}$

0.024 kg 1,4-dichlorobenzene eq."/kg

1.10E-05 kg 1,4-dichlorobenzene eq." $/ \mathrm{kg}$

$7.5 \mathrm{~kg} \mathrm{1,4-dichlorobenzene} \mathrm{eq."} / \mathrm{kg}$

1.10E-05 kg 1,4-dichlorobenzene eq."/kg

0.00092 kg 1,4-dichlorobenzene eq."/kg

1.90E-05 kg 1,4-dichlorobenzene eq."/kg

$0.0027 \mathrm{~kg} \mathrm{1,4-dichlorobenzene} \mathrm{eq."} / \mathrm{kg}$

$2.8 \mathrm{~kg} \mathrm{1,4-dichlorobenzene} \mathrm{eq."} / \mathrm{kg}$

0.00056 kg 1,4-dichlorobenzene eq."/kg

$0.0008 \mathrm{~kg} \mathrm{1,4-dichlorobenzene} \mathrm{eq."} / \mathrm{kg}$

$0.0014 \mathrm{~kg} \mathrm{1,4-dichlorobenzene} \mathrm{eq."} / \mathrm{kg}$

$0.0001 \mathrm{~kg} \mathrm{1,4-dichlorobenzene} \mathrm{eq.} \mathrm{"/} / \mathrm{kg}$

$0.048 \mathrm{~kg} \mathrm{1,4-dichlorobenzene} \mathrm{eq."} / \mathrm{kg}$

2.30E-06 kg 1,4-dichlorobenzene eq."/kg 
[E1228] dinoseb to agricultural soil

[E1229] dinoterb to agricultural soil

[E1230] Dioctylphtalate to agricultural soil

[E1231] disulfothon to agricultural soil

[E1232] diuron to agricultural soil

[E1233] DNOC to agricultural soil

[E1234] endosulfan to agricultural soil

[E1235] endrin to agricultural soil

[E1236] ethoprophos to agricultural soil

[E1237] ethylbenzene to agricultural soil

[E1238] ethylene to agricultural soil

[E1239] fenitrothion to agricultural soil

[E1240] fenthion to agricultural soil

[E1241] fentin acetate to agricultural soil

[E1242] fentin chloride to agricultural soil

[E1243] fentin hydroxide to agricultural soil

[E1244] fluoranthrene to agricultural soil

[E1245] folpet to agricultural soil

[E1246] Formaldehyde (methanal) to agricultural soil

[E1247] glyphosate to agricultural soil

[E1248] heptachlor to agricultural soil

[E1249] heptenophos to agricultural soil

[E1250] hexachloro-1,3-butadiene to agricultural soil

[E1251] hexachlorobenzene to agricultural soil

[E1254] indeno[1,2,3-cd]pyrene to agricultural soil

[E1255] iprodione to agricultural soil

[E1256] isoproturon to agricultural soil

[E1257] lead to agricultural soil

[E1258] lindane to agricultural soil

[E1259] linuron to agricultural soil

[E1260] malathion to agricultural soil

[E1261] MCPA to agricultural soil

[E1262] mecoprop to agricultural soil

[E1263] mercury to agricultural soil

[E1264] metamitron to agricultural soil

[E1265] metazachlor to agricultural soil

[E1266] methabenzthiazuron to agricultural soil

[E1267] methomyl to agricultural soil

[E1268] methylbromide to agricultural soil

[E1269] methyl-mercury to agricultural soil

[E1270] metobromuron to agricultural soil

[E1271] metolachlor to agricultural soil

[E1272] mevinphos to agricultural soil

[E1273] molybdenum to agricultural soil

[E1274] m-xylene to agricultural soil

[E1275] naphtalene to agricultural soil

[E1276] nickel to agricultural soil

[E1278] oxamyl to agricultural soil

[E1279] oxydemethon-methyl to agricultural soil

[E1280] o-xylene to agricultural soil

[E1281] parathion-ethyl to agricultural soil

[E1282] parathion-methyl to agricultural soil

[E1283] pentachlorobenzene to agricultural soil

[E1284] pentachloronitrobenzene to agricultural soil

[E1285] pentachlorophenol to agricultural soil

[E1286] permethrin to agricultural soil

[E1287] phenanthrene to agricultural soil

[E1288] phenol to agricultural soil

[E1289] phoxim to agricultural soil

[E1290] Phtalic anhydride to agricultural soil

[E1291] pirimicarb to agricultural soil

[E1293] propachlor to agricultural soil

[E1294] propoxur to agricultural soil

[E1295] propylene oxide to agricultural soil

[E1296] p-xylene to agricultural soil

[E1297] pyrazophos to agricultural soil

[E1298] selenium to agricultural soil
150 kg 1,4-dichlorobenzene eq." $/ \mathrm{kg}$

3.1 kg 1,4-dichlorobenzene eq."/kg

1.30E-06 kg 1,4-dichlorobenzene eq."/kg

0.04 kg 1,4-dichlorobenzene eq." $/ \mathrm{kg}$

0.04 kg 1,4-dichlorobenzene eq."/kg

0.00085 kg 1,4-dichlorobenzene eq."/kg

9.00E-05 kg 1,4-dichlorobenzene eq."/kg

750 kg 1,4-dichlorobenzene eq."/kg

360 kg 1,4-dichlorobenzene eq."/kg

0.00032 kg 1,4-dichlorobenzene eq." $/ \mathrm{kg}$

7.10E-11 kg 1,4-dichlorobenzene eq."/kg

$1.1 \mathrm{~kg}$ 1,4-dichlorobenzene eq." $/ \mathrm{kg}$

9.9 kg 1,4-dichlorobenzene eq."/kg

18 kg 1,4-dichlorobenzene eq."/kg

$120 \mathrm{~kg} \mathrm{1,4-dichlorobenzene} \mathrm{eq."/kg}$

16 kg 1,4-dichlorobenzene eq."/kg

$4.3 \mathrm{~kg}$ 1,4-dichlorobenzene eq."/kg

930 kg 1,4-dichlorobenzene eq."/kg

0.018 kg 1,4-dichlorobenzene eq."/kg

0.0025 kg 1,4-dichlorobenzene eq."/kg

0.02 kg 1,4-dichlorobenzene eq." $/ \mathrm{kg}$

0.0051 kg 1,4-dichlorobenzene eq."/kg

11000 kg 1,4-dichlorobenzene eq."/kg

830 kg 1,4-dichlorobenzene eq." $/ \mathrm{kg}$

59 kg 1,4-dichlorobenzene eq."/kg

3.50E-07 kg 1,4-dichlorobenzene eq."/kg

$1.1 \mathrm{~kg} \mathrm{1,4-dichlorobenzene} \mathrm{eq."/kg}$

780 kg 1,4-dichlorobenzene eq."/kg

0.29 kg 1,4-dichlorobenzene eq." $/ \mathrm{kg}$

16 kg 1,4-dichlorobenzene eq." $/ \mathrm{kg}$

$0.37 \mathrm{~kg} 1,4$-dichlorobenzene eq." $/ \mathrm{kg}$

0.00076 kg 1,4-dichlorobenzene eq." $/ \mathrm{kg}$

0.069 kg 1,4-dichlorobenzene eq." $/ \mathrm{kg}$

1.70E+05 kg 1,4-dichlorobenzene eq."/kg

0.00089 kg 1,4-dichlorobenzene eq."/kg

0.039 kg 1,4-dichlorobenzene eq." $/ \mathrm{kg}$

$1.5 \mathrm{~kg}$ 1,4-dichlorobenzene eq." $/ \mathrm{kg}$

570 kg 1,4-dichlorobenzene eq."/kg

0.83 kg 1,4-dichlorobenzene eq." $/ \mathrm{kg}$

3.90E+06 kg 1,4-dichlorobenzene eq."/kg

$16 \mathrm{~kg}$ 1,4-dichlorobenzene eq."/kg

$41 \mathrm{~kg}$ 1,4-dichlorobenzene eq."/kg

0.038 kg 1,4-dichlorobenzene eq." $/ \mathrm{kg}$

9.60E+05 kg 1,4-dichlorobenzene eq."/kg

0.00023 kg 1,4-dichlorobenzene eq." $/ \mathrm{kg}$

$0.02 \mathrm{~kg} \mathrm{1,4-dichlorobenzene} \mathrm{eq."} / \mathrm{kg}$

1.20E+06 kg 1,4-dichlorobenzene eq."/kg

0.0024 kg 1,4-dichlorobenzene eq." $/ \mathrm{kg}$

$0.82 \mathrm{~kg} 1,4$-dichlorobenzene eq. "/kg

0.0006 kg 1,4-dichlorobenzene eq."/kg

0.96 kg 1,4-dichlorobenzene eq. "/kg

0.25 kg 1,4-dichlorobenzene eq." $/ \mathrm{kg}$

$14 \mathrm{~kg} 1,4$-dichlorobenzene eq." $/ \mathrm{kg}$

$2.3 \mathrm{~kg}$ 1,4-dichlorobenzene eq." $/ \mathrm{kg}$

$0.011 \mathrm{~kg} \mathrm{1,4-dichlorobenzene} \mathrm{eq."} / \mathrm{kg}$

4.2 kg 1,4-dichlorobenzene eq." $/ \mathrm{kg}$

0.007 kg 1,4-dichlorobenzene eq."/kg

0.0011 kg 1,4-dichlorobenzene eq."/kg

$0.041 \mathrm{~kg} \mathrm{1,4-dichlorobenzene} \mathrm{eq."} / \mathrm{kg}$

1.10E-10 kg 1,4-dichlorobenzene eq."/kg

11 kg 1,4-dichlorobenzene eq."/kg

$0.04 \mathrm{~kg} \mathrm{1,4-dichlorobenzene} \mathrm{eq."/kg}$

40 kg 1,4-dichlorobenzene eq." $/ \mathrm{kg}$

$0.016 \mathrm{~kg} \mathrm{1,4-dichlorobenzene} \mathrm{eq."} / \mathrm{kg}$

0.0002 kg 1,4-dichlorobenzene eq." $/ \mathrm{kg}$

$0.65 \mathrm{~kg} 1,4$-dichlorobenzene eq." $/ \mathrm{kg}$

5.40E+06 kg 1,4-dichlorobenzene eq."/kg 
[E1299] simazine to agricultural soil

[E1300] styrene (vinylbenzene) to agricultural soil

[E1302] tetrachloroethylene (PER) to agricultural soil

[E1303] Tetrachloromethane (carbon tetrachloride) $(\mathrm{HC}-10)$ to agricultural soil

[E1304] thallium to agricultural soil

[E1305] Thiram to agricultural soil

[E1306] tin to agricultural soil

[E1307] tolclophos-methyl to agricultural soil

[E1308] toluene to agricultural soil

[E1309] tri-allate to agricultural soil

[E1310] triazophos to agricultural soil

[E1311] tributyltinoxide to agricultural soil

[E1312] trichlorfon to agricultural soil

[E1313] Trichloroethylene (tri) to agricultural soil

[E1314] Trichloromethane=chloroform to agricultural soil

[E1315] trifluarin to agricultural soil

[E1316] vanadium to agricultural soil

[E1317] Vinyl Chloride (chloroethene) to agricultural soil

[E1318] zinc to agricultural soil

[E1319] zineb to agricultural soil

[E1320] 1,1,1-trichloroethane to agricultural soil

[E1321] 1,2,3,4-tetrachlorobenzene to agricultural soil

[E1322] 1,2,3,5-tetrachlorobenzene to agricultural soil

[E1323] 1,2,3-trichlorobenzene to agricultural soil

[E1324] 1,2,4,5-tetrachlorobenzene to agricultural soil

[E1325] 1,2,4-trichlorobenzene to agricultural soil

[E1326] 1,2-dichlorobenzene to agricultural soil

[E1327] 1,2-dichloroethane to agricultural soil

[E1328] 1,3,5-trichlorobenzene to agricultural soil

[E1329] 1,3-butadiene to agricultural soil

[E1330] 1,3-dichlorobenzene to agricultural soil

[E1331] 1,4-dichlorobenzene to agricultural soil

[E1332] 1-chloro-4-nitrobenzene to agricultural soil

[E1333] 2,3,4,6-tetrachlorophenol to agricultural soil

[E1334] 2,3,7,8-TCDD to agricultural soil

[E1335] 2,4,5-T to agricultural soil

[E1336] 2,4,5-trichlorophenol to agricultural soil

[E1337] 2,4,6-trichlorophenol to industrial soil

[E1338] 2,4-D to industrial soil

[E1339] 2,4-dichlorophenol to industrial soil

[E1340] 2-chlorophenol to industrial soil

[E1341] 3,4-dichloroaniline to industrial soil

[E1342] 3-chloroaniline to industrial soil

[E1343] 4-chloroaniline to industrial soil

[E1344] acephate to industrial soil

[E1345] Acrolein (2-propenal) to industrial soil

[E1346] acrylonitrile to industrial soil

[E1347] aldicarb to industrial soil

[E1348] aldrin to industrial soil

[E1350] anilazine to industrial soil

[E1351] anthracene to industrial soil

[E1352] antimony to industrial soil

[E1353] arsenic to industrial soil

[E1354] atrazine to industrial soil

[E1355] azinphos-ethyl to industrial soil

[E1356] azinphos-methyl to industrial soil

[E1357] barium to industrial soil

[E1358] benomyl to industrial soil

[E1359] bentazone to industrial soil

[E1360] benzene to industrial soil

[E1361] benzo[a]anthracene to industrial soil

[E1362] benzo[a]pyrene to industrial soil

[E1363] benzo[ghi]perylene to industrial soil

[E1364] benzo[k]fluoranthrene to industrial soil

[E1365] benzylchloride to industrial soil

[E1366] beryllium to industrial soil

[E1367] bifenthrin to industrial soil
19 kg 1,4-dichlorobenzene eq."/kg

7.60E-05 kg 1,4-dichlorobenzene eq." $/ \mathrm{kg}$

$0.11 \mathrm{~kg} \mathrm{1,4-dichlorobenzene} \mathrm{eq."} / \mathrm{kg}$

$0.3 \mathrm{~kg} \mathrm{1,4-dichlorobenzene} \mathrm{eq."} / \mathrm{kg}$

1.30E+07 kg 1,4-dichlorobenzene eq."/kg

0.057 kg 1,4-dichlorobenzene eq."/kg

170 kg 1,4-dichlorobenzene eq."/kg

$0.15 \mathrm{~kg} \mathrm{1,4-dichlorobenzene} \mathrm{eq."/kg}$

0.00037 kg 1,4-dichlorobenzene eq."/kg

$0.23 \mathrm{~kg} 1,4$-dichlorobenzene eq." $/ \mathrm{kg}$

$73 \mathrm{~kg} 1,4$-dichlorobenzene eq."/kg

770 kg 1,4-dichlorobenzene eq." $/ \mathrm{kg}$

$0.1 \mathrm{~kg} \mathrm{1,4-dichlorobenzene} \mathrm{eq."} / \mathrm{kg}$

$0.0015 \mathrm{~kg} \mathrm{1,4-dichlorobenzene} \mathrm{eq."} / \mathrm{kg}$

$0.013 \mathrm{~kg} \mathrm{1,4-dichlorobenzene} \mathrm{eq."/kg}$

$0.49 \mathrm{~kg} \mathrm{1,4-dichlorobenzene} \mathrm{eq."} / \mathrm{kg}$

4.10E+06 kg 1,4-dichlorobenzene eq." $/ \mathrm{kg}$

0.00012 kg 1,4-dichlorobenzene eq."/kg 7300 kg 1,4-dichlorobenzene eq."/kg

$3.8 \mathrm{~kg} \mathrm{1,4-dichlorobenzene} \mathrm{eq."} / \mathrm{kg}$

$0.1 \mathrm{~kg} \mathrm{1,4-dichlorobenzene} \mathrm{eq."} / \mathrm{kg}$

0.6 kg 1,4-dichlorobenzene eq." $/ \mathrm{kg}$

$2 \mathrm{~kg} 1,4$-dichlorobenzene eq." $/ \mathrm{kg}$

0.35 kg 1,4-dichlorobenzene eq." $/ \mathrm{kg}$

$0.74 \mathrm{~kg} 1,4$-dichlorobenzene eq." $/ \mathrm{kg}$

$0.3 \mathrm{~kg} \mathrm{1,4-dichlorobenzene} \mathrm{eq."} / \mathrm{kg}$

$0.21 \mathrm{~kg} \mathrm{1,4-dichlorobenzene} \mathrm{eq."} / \mathrm{kg}$

0.022 kg 1,4-dichlorobenzene eq."/kg

$0.55 \mathrm{~kg} 1,4$-dichlorobenzene eq. " $/ \mathrm{kg}$

3.20E-06 kg 1,4-dichlorobenzene eq."/kg

$0.16 \mathrm{~kg} 1,4$-dichlorobenzene eq." $/ \mathrm{kg}$

$0.21 \mathrm{~kg} 1,4$-dichlorobenzene eq." $/ \mathrm{kg}$

79 kg 1,4-dichlorobenzene eq."/kg

12.72 kg 1,4-dichlorobenzene eq."/kg

5.70E+05 kg 1,4-dichlorobenzene eq." $/ \mathrm{kg}$

$0.0068 \mathrm{~kg} \mathrm{1,4-dichlorobenzene} \mathrm{eq."} / \mathrm{kg}$

$5.7 \mathrm{~kg} \mathrm{1,4-dichlorobenzene} \mathrm{eq.} \mathrm{"/kg}$

0.037 kg 1,4-dichlorobenzene eq." $/ \mathrm{kg}$

0.64 kg 1,4-dichlorobenzene eq." $/ \mathrm{kg}$

$0.012 \mathrm{~kg} \mathrm{1,4-dichlorobenzene} \mathrm{eq."} / \mathrm{kg}$

$0.35 \mathrm{~kg} \mathrm{1,4-dichlorobenzene} \mathrm{eq."} / \mathrm{kg}$

740 kg 1,4-dichlorobenzene eq." $/ \mathrm{kg}$

$1.6 \mathrm{~kg} \mathrm{1,4-dichlorobenzene} \mathrm{eq.} \mathrm{"/kg}$

$3.3 \mathrm{~kg} 1,4$-dichlorobenzene eq."/kg

2 kg 1,4-dichlorobenzene eq." $/ \mathrm{kg}$

360 kg 1,4-dichlorobenzene eq."/kg

$0.23 \mathrm{~kg} \mathrm{1,4-dichlorobenzene} \mathrm{eq."} / \mathrm{kg}$

2400 kg 1,4-dichlorobenzene eq."/kg

$3 \mathrm{~kg} \mathrm{1,4-dichlorobenzene} \mathrm{eq."} / \mathrm{kg}$

8.50E-06 kg 1,4-dichlorobenzene eq."/kg

32 kg 1,4-dichlorobenzene eq."/kg

13000 kg 1,4-dichlorobenzene eq."/kg

77000 kg 1,4-dichlorobenzene eq."/kg

$100 \mathrm{~kg} \mathrm{1,4-dichlorobenzene} \mathrm{eq."} / \mathrm{kg}$

$11 \mathrm{~kg} \mathrm{1,4-dichlorobenzene} \mathrm{eq."/kg}$

$0.17 \mathrm{~kg} \mathrm{1,4-dichlorobenzene} \mathrm{eq."} / \mathrm{kg}$

3.60E+05 kg 1,4-dichlorobenzene eq." $/ \mathrm{kg}$

0.002 kg 1,4-dichlorobenzene eq."/kg

$0.072 \mathrm{~kg} \mathrm{1,4-dichlorobenzene} \mathrm{eq."/kg}$

$0.0011 \mathrm{~kg} \mathrm{1,4-dichlorobenzene} \mathrm{eq."} / \mathrm{kg}$

60 kg 1,4-dichlorobenzene eq." $/ \mathrm{kg}$

77 kg 1,4-dichlorobenzene eq." $/ \mathrm{kg}$

$150 \mathrm{~kg} \mathrm{1,4-dichlorobenzene} \mathrm{eq."} / \mathrm{kg}$

23000 kg 1,4-dichlorobenzene eq."/kg

$0.045 \mathrm{~kg} \mathrm{1,4-dichlorobenzene} \mathrm{eq."} / \mathrm{kg}$

1.20E+08 kg 1,4-dichlorobenzene eq." $/ \mathrm{kg}$

$1.7 \mathrm{~kg} \mathrm{1,4-dichlorobenzene} \mathrm{eq."} / \mathrm{kg}$ 
[E1368] Butylbenzylphtalate to industrial soil [E1369] cadmium to industrial soil [E1370] captafol to industrial soil [E1371] captan to industrial soil [E1372] carbaryl to industrial soil [E1373] carbendazim to industrial soil [E1374] carbofuran to industrial soil [E1375] carbon disulfide to industrial soil [E1376] Carcinogenic PAHs to industrial soil [E1377] chlordane to industrial soil [E1378] chlorfenvinphos to industrial soil [E1379] chloridazon to industrial soil [E1380] chlorobenzene to industrial soil [E1381] chlorothalonil to industrial soil [E1382] chlorpropham to industrial soil [E1383] chlorpyriphos to industrial soil [E1384] chromium III to industrial soil [E1385] chromium VI to industrial soil [E1386] chrysene to industrial soil [E1387] cobalt to industrial soil [E1388] copper to industrial soil [E1389] coumaphos to industrial soil [E1390] cyanazine to industrial soil [E1391] cypermethrin to industrial soil [E1392] cyromazine to industrial soil [E1393] DDT to industrial soil [E1394] deltamethrin to industrial soil [E1395] demeton to industrial soil [E1396] desmetryn to industrial soil [E1397] Di(2-ethylhexyl)phtalate to industrial soil [E1398] diazinon to industrial soil [E1399] Dibutylphtalate to industrial soil

[E1400] Dichloromethane (Methylene Chloride) to industrial soil [E1401] dichlorprop to industrial soil

[E1402] dichlorvos to industrial soil

[E1403] dieldrin to industrial soil

[E1404] Diethylphtalate to industrial soil

[E1405] Dihexylphtalate to industrial soil

[E1406] Diisodecylphtalate to industrial soil

[E1407] Diisooctylphtalate to industrial soil

[E1408] dimethoate to industrial soil

[E1409] Dimethylphtalate to industrial soil.

[E1410] dinoseb to industrial soil

[E1411] dinoterb to industrial soil

[E1412] Dioctylphtalate to industrial soil

[E1413] disulfothon to industrial soil

[E1414] diuron to industrial soil

[E1415] DNOC to industrial soil

[E1416] endosulfan to industrial soil

[E1417] endrin to industrial soil

[E1418] ethoprophos to industrial soil

[E1419] ethylbenzene to industrial soil

[E1420] ethylene to industrial soil

[E1421] fenitrothion to industrial soil

[E1422] fenthion to industrial soil

[E1423] fentin acetate to industrial soil

[E1424] fentin chloride to industrial soil

[E1425] fentin hydroxide to industrial soil

[E1426] fluoranthrene to industrial soil

[E1427] folpet to industrial soil

[E1428] Formaldehyde (methanal) to industrial soil

[E1429] glyphosate to industrial soil

[E1430] heptachlor to industrial soil

[E1431] heptenophos to industrial soil

[E1432] hexachloro-1,3-butadiene to industrial soil

[E1433] hexachlorobenzene to industrial soil

[E1436] indeno[1,2,3-cd]pyrene to industrial soil
2.80E-05 kg 1,4-dichlorobenzene eq." $/ \mathrm{kg}$ 1.10E+05 kg 1,4-dichlorobenzene eq." $/ \mathrm{kg}$ 18000 kg 1,4-dichlorobenzene eq."/kg 9.90E-06 kg 1,4-dichlorobenzene eq." $/ \mathrm{kg}$ $0.0035 \mathrm{~kg} 1,4$-dichlorobenzene eq."/kg $140 \mathrm{~kg}$ 1,4-dichlorobenzene eq." $/ \mathrm{kg}$ $6.6 \mathrm{~kg}$ 1,4-dichlorobenzene eq." $/ \mathrm{kg}$ $0.79 \mathrm{~kg}$ 1,4-dichlorobenzene eq." $/ \mathrm{kg}$ $160 \mathrm{~kg}$ 1,4-dichlorobenzene eq." $/ \mathrm{kg}$ $3.3 \mathrm{~kg}$ 1,4-dichlorobenzene eq." $/ \mathrm{kg}$

$0.37 \mathrm{~kg}$ 1,4-dichlorobenzene eq." $/ \mathrm{kg}$

$0.22 \mathrm{~kg}$ 1,4-dichlorobenzene eq." $/ \mathrm{kg}$

$0.037 \mathrm{~kg}$ 1,4-dichlorobenzene eq."/kg

$1.7 \mathrm{~kg}$ 1,4-dichlorobenzene eq." $/ \mathrm{kg}$

$0.038 \mathrm{~kg}$ 1,4-dichlorobenzene eq." $/ \mathrm{kg}$

$0.058 \mathrm{~kg}$ 1,4-dichlorobenzene eq."/kg

$670 \mathrm{~kg}$ 1,4-dichlorobenzene eq." $/ \mathrm{kg}$

$2700 \mathrm{~kg}$ 1,4-dichlorobenzene eq." $/ \mathrm{kg}$

$160 \mathrm{~kg}$ 1,4-dichlorobenzene eq." $/ \mathrm{kg}$

1.40E+06 kg 1,4-dichlorobenzene eq." $/ \mathrm{kg}$

1.20E+05 kg 1,4-dichlorobenzene eq." $/ \mathrm{kg}$

6.70E+05 kg 1,4-dichlorobenzene eq."/kg

$14 \mathrm{~kg}$ 1,4-dichlorobenzene eq." $/ \mathrm{kg}$

$2800 \mathrm{~kg}$ 1,4-dichlorobenzene eq." $/ \mathrm{kg}$

$350 \mathrm{~kg}$ 1,4-dichlorobenzene eq." $/ \mathrm{kg}$

$53 \mathrm{~kg}$ 1,4-dichlorobenzene eq." $/ \mathrm{kg}$

$0.47 \mathrm{~kg}$ 1,4-dichlorobenzene eq." $/ \mathrm{kg}$

$15 \mathrm{~kg}$ 1,4-dichlorobenzene eq." $/ \mathrm{kg}$

$0.088 \mathrm{~kg}$ 1,4-dichlorobenzene eq." $/ \mathrm{kg}$

4.40E-05 kg 1,4-dichlorobenzene eq." $/ \mathrm{kg}$

$26 \mathrm{~kg}$ 1,4-dichlorobenzene eq." $/ \mathrm{kg}$

4.50E-05 kg 1,4-dichlorobenzene eq."/kg

$0.00092 \mathrm{~kg}$ 1,4-dichlorobenzene eq." $/ \mathrm{kg}$

7.40E-05 kg 1,4-dichlorobenzene eq."//kg

$0.011 \mathrm{~kg} \mathrm{1,4-dichlorobenzene} \mathrm{eq."} / \mathrm{kg}$

$11 \mathrm{~kg}$ 1,4-dichlorobenzene eq." $/ \mathrm{kg}$

$0.0022 \mathrm{~kg}$ 1,4-dichlorobenzene eq." $/ \mathrm{kg}$

$0.0032 \mathrm{~kg}$ 1,4-dichlorobenzene eq." $/ \mathrm{kg}$

0.0054 kg 1,4-dichlorobenzene eq."/kg

$0.00041 \mathrm{~kg} 1,4$-dichlorobenzene eq." $/ \mathrm{kg}$

$0.15 \mathrm{~kg}$ 1,4-dichlorobenzene eq." $/ \mathrm{kg}$

9.10E-06 kg 1,4-dichlorobenzene eq."/kg

$430 \mathrm{~kg}$ 1,4-dichlorobenzene eq." $/ \mathrm{kg}$

$13 \mathrm{~kg}$ 1,4-dichlorobenzene eq."/kg

5.10E-06 kg 1,4-dichlorobenzene eq." $/ \mathrm{kg}$

$0.16 \mathrm{~kg} \mathrm{1,4-dichlorobenzene} \mathrm{eq."} / \mathrm{kg}$

$0.16 \mathrm{~kg}$ 1,4-dichlorobenzene eq." $/ \mathrm{kg}$

$0.0033 \mathrm{~kg}$ 1,4-dichlorobenzene eq." $/ \mathrm{kg}$

$0.00036 \mathrm{~kg}$ 1,4-dichlorobenzene eq."/kg

$2500 \mathrm{~kg}$ 1,4-dichlorobenzene eq." $/ \mathrm{kg}$

970 kg 1,4-dichlorobenzene eq."//kg

$0.00032 \mathrm{~kg}$ 1,4-dichlorobenzene eq." $/ \mathrm{kg}$

7.10E-11 kg 1,4-dichlorobenzene eq."/kg

$4.5 \mathrm{~kg}$ 1,4-dichlorobenzene eq." $/ \mathrm{kg}$

$39 \mathrm{~kg}$ 1,4-dichlorobenzene eq." $/ \mathrm{kg}$

$72 \mathrm{~kg}$ 1,4-dichlorobenzene eq." $/ \mathrm{kg}$

470 kg 1,4-dichlorobenzene eq." $/ \mathrm{kg}$

$65 \mathrm{~kg}$ 1,4-dichlorobenzene eq."/kg

17 kg 1,4-dichlorobenzene eq."/kg

2700 kg 1,4-dichlorobenzene eq." $/ \mathrm{kg}$

$0.055 \mathrm{~kg}$ 1,4-dichlorobenzene eq." $/ \mathrm{kg}$

$0.0099 \mathrm{~kg} 1,4$-dichlorobenzene eq." $/ \mathrm{kg}$

0.079 kg 1,4-dichlorobenzene eq." $/ \mathrm{kg}$

$0.02 \mathrm{~kg}$ 1,4-dichlorobenzene eq." $/ \mathrm{kg}$

$13000 \mathrm{~kg}$ 1,4-dichlorobenzene eq." $/ \mathrm{kg}$

$1100 \mathrm{~kg}$ 1,4-dichlorobenzene eq." $/ \mathrm{kg}$

240 kg 1,4-dichlorobenzene eq." $/ \mathrm{kg}$ 
[E1437] iprodione to industrial soil

[E1438] isoproturon to industrial soil

[E1439] lead to industrial soil

[E1440] lindane to industrial soil

[E1441] linuron to industrial soil

[E1442] malathion to industrial soil

[E1443] MCPA to industrial soil

[E1444] mecoprop to industrial soil

[E1445] mercury to industrial soil

[E1446] metamitron to industrial soil

[E1447] metazachlor to industrial soil

[E1448] methabenzthiazuron to industrial soil

[E1449] methomyl to industrial soil

[E1450] methylbromide to industrial soil

[E1451] methyl-mercury to industrial soil

[E1452] metobromuron to industrial soil

[E1453] metolachlor to industrial soil

[E1454] mevinphos to industrial soil

[E1455] molybdenum to industrial soil

[E1456] m-xylene to industrial soil

[E1457] naphtalene to industrial soil

[E1458] nickel to industrial soil

[E1460] oxamyl to industrial soil

[E1461] oxydemethon-methyl to industrial soil

[E1462] o-xylene to industrial soil

[E1463] parathion-ethyl to industrial soil

[E1464] parathion-methyl to industrial soil

[E1465] pentachlorobenzene to industrial soil

[E1466] pentachloronitrobenzene to industrial soil

[E1467] pentachlorophenol to industrial soil

[E1468] permethrin to industrial soil

[E1469] phenanthrene to industrial soil

[E1470] phenol to industrial soil

[E1471] phoxim to industrial soil

[E1472] Phtalic anhydride to industrial soil

[E1473] pirimicarb to industrial soil

[E1475] propachlor to industrial soil

[E1476] propoxur to industrial soil

[E1477] propylene oxide to industrial soil

[E1478] p-xylene to industrial soil

[E1479] pyrazophos to industrial soil

[E1480] selenium to industrial soil

[E1481] simazine to industrial soil

[E1482] styrene (vinylbenzene) to industrial soil

[E1484] tetrachloroethylene (PER) to industrial soil

[E1485] Tetrachloromethane (carbon tetrachloride) (HC-10) to industrial soil

[E1486] thallium to industrial soil

[E1487] Thiram to industrial soil

[E1488] tin to industrial soil

[E1489] tolclophos-methyl to industrial soil

[E1490] toluene to industrial soil

[E1491] tri-allate to industrial soil

[E1492] triazophos to industrial soil

[E1493] tributyltinoxide to industrial soil

[E1494] trichlorfon to industrial soil

[E1495] Trichloroethylene (tri) to industrial soil

[E1496] Trichloromethane=chloroform to industrial soil

[E1497] trifluarin to industrial soil

[E1498] vanadium to industrial soil

[E1499] Vinyl Chloride (chloroethene) to industrial soil

[E1500] zinc to industrial soil

[E1501] zineb to industrial soil
2.90E-06 kg 1,4-dichlorobenzene eq." $/ \mathrm{kg}$ 2.7 kg 1,4-dichlorobenzene eq."/kg 780 kg 1,4-dichlorobenzene eq." $/ \mathrm{kg}$

$1.1 \mathrm{~kg} 1,4$-dichlorobenzene eq." $/ \mathrm{kg}$

$57 \mathrm{~kg} \mathrm{1,4-dichlorobenzene} \mathrm{eq."} / \mathrm{kg}$

$1.5 \mathrm{~kg}$ 1,4-dichlorobenzene eq."/kg

0.0027 kg 1,4-dichlorobenzene eq."/kg

0.18 kg 1,4-dichlorobenzene eq." $/ \mathrm{kg}$

1.70E+05 kg 1,4-dichlorobenzene eq."/kg

0.0032 kg 1,4-dichlorobenzene eq."/kg

$0.14 \mathrm{~kg} \mathrm{1,4-dichlorobenzene} \mathrm{eq."/kg}$

4.7 kg 1,4-dichlorobenzene eq."/kg

1100 kg 1,4-dichlorobenzene eq." $/ \mathrm{kg}$

$0.83 \mathrm{~kg}$ 1,4-dichlorobenzene eq." $/ \mathrm{kg}$

3.90E+06 kg 1,4-dichlorobenzene eq."/kg

$16 \mathrm{~kg} \mathrm{1,4-dichlorobenzene} \mathrm{eq."} / \mathrm{kg}$

130 kg 1,4-dichlorobenzene eq."/kg

$0.16 \mathrm{~kg} \mathrm{1,4-dichlorobenzene} \mathrm{eq."} / \mathrm{kg}$

9.60E+05 kg 1,4-dichlorobenzene eq."/kg

0.00023 kg 1,4-dichlorobenzene eq." $/ \mathrm{kg}$

$0.067 \mathrm{~kg} \mathrm{1,4-dichlorobenzene} \mathrm{eq."/kg}$

1.20E+06 kg 1,4-dichlorobenzene eq."/kg

0.0099 kg 1,4-dichlorobenzene eq."/kg

$3 \mathrm{~kg}$ 1,4-dichlorobenzene eq." $/ \mathrm{kg}$

0.0006 kg 1,4-dichlorobenzene eq." $/ \mathrm{kg}$

$3.8 \mathrm{~kg} \mathrm{1,4-dichlorobenzene} \mathrm{eq."} / \mathrm{kg}$

0.98 kg 1,4-dichlorobenzene eq." $/ \mathrm{kg}$

27 kg 1,4-dichlorobenzene eq." $/ \mathrm{kg}$

$8.8 \mathrm{~kg} 1,4$-dichlorobenzene eq." $/ \mathrm{kg}$

0.049 kg 1,4-dichlorobenzene eq."/kg

17 kg 1,4-dichlorobenzene eq."/kg

0.028 kg 1,4-dichlorobenzene eq."/kg

0.004 kg 1,4-dichlorobenzene eq."/kg

0.072 kg 1,4-dichlorobenzene eq."/kg

6.80E-11 kg 1,4-dichlorobenzene eq."/kg

$35 \mathrm{~kg}$ 1,4-dichlorobenzene eq." $/ \mathrm{kg}$

0.15 kg 1,4-dichlorobenzene eq." $/ \mathrm{kg}$

$110 \mathrm{~kg} \mathrm{1,4-dichlorobenzene} \mathrm{eq."/kg}$

0.018 kg 1,4-dichlorobenzene eq." $/ \mathrm{kg}$

0.0002 kg 1,4-dichlorobenzene eq."/kg

2.5 kg 1,4-dichlorobenzene eq." $/ \mathrm{kg}$

$5.40 \mathrm{E}+06 \mathrm{~kg} \mathrm{1,4-dichlorobenzene} \mathrm{eq."} / \mathrm{kg}$

46 kg 1,4-dichlorobenzene eq." $/ \mathrm{kg}$

$0.00013 \mathrm{~kg} 1,4$-dichlorobenzene eq." $/ \mathrm{kg}$

0.043 kg 1,4-dichlorobenzene eq." $/ \mathrm{kg}$

$0.3 \mathrm{~kg} 1,4$-dichlorobenzene eq." $/ \mathrm{kg}$

1.30E+07 kg 1,4-dichlorobenzene eq."/kg

0.37 kg 1,4-dichlorobenzene eq."/kg

170 kg 1,4-dichlorobenzene eq."/kg

$0.44 \mathrm{~kg} \mathrm{1,4-dichlorobenzene} \mathrm{eq."} / \mathrm{kg}$

0.00037 kg 1,4-dichlorobenzene eq."/kg

$0.93 \mathrm{~kg}$ 1,4-dichlorobenzene eq." $/ \mathrm{kg}$

240 kg 1,4-dichlorobenzene eq. "/kg

3000 kg 1,4-dichlorobenzene eq." $/ \mathrm{kg}$

0.56 kg 1,4-dichlorobenzene eq." $/ \mathrm{kg}$

0.0015 kg 1,4-dichlorobenzene eq."/kg

$0.013 \mathrm{~kg} \mathrm{1,4-dichlorobenzene} \mathrm{eq."/kg}$

$1.9 \mathrm{~kg} \mathrm{1,4-dichlorobenzene} \mathrm{eq."} / \mathrm{kg}$

4.10E+06 kg 1,4-dichlorobenzene eq." $/ \mathrm{kg}$

0.00012 kg 1,4-dichlorobenzene eq." $/ \mathrm{kg}$

7300 kg 1,4-dichlorobenzene eq." $/ \mathrm{kg}$

14 kg 1,4-dichlorobenzene eq."/kg

Category $=[\mathrm{C} 10]$ Terrestrial ecotoxicity

Description $=$ Problem oriented approach, manual 1999; TETP

Author $=$ Huijbregts, 1999 
Environmental resources

Environmental resource

Environmental emissions

Environmental emission

[E90] 1,1,1-trichloroethane (methyl chloroform; HC-140a) to air [E94] 1,2,3,4-tetrachlorobenzene to air

[E95] 1,2,3,5-tetrachlorobenzene to air

[E96] 1,2,3-trichlorobenzene to air

[E98] 1,2,4,5-tetrachlorobenzene to air

[E99] 1,2,4-trichlorobenzene to air

[E102] 1,2-dichlorobenzene to air

[E103] 1,2-dichloroethane to air

[E107] 1,3,5-trichlorobenzene to air

[E111] 1,3-Butadiene to air

[E112] 1,3-dichlorobenzene to air

[E116] 1,4-dichlorobenzene to air

[E128] 1-chloro-4-nitrobenzene to air

[E166] 2,3,4,6-tetrachlorophenol to air

[E168] 2,3,7,8-TCDD (tetrachloride-dibenzo-dioxin) to air

[E172] 2,4,5-T to air

[E173] 2,4,5-trichlorophenol to air

[E174] 2,4,6-trichlorophenol to air

[E175] 2,4-D to air

[E176] 2,4-dichlorophenol to air

[E185] 2-chlorophenol to air

[E208] 3,4-dichloroaniline to air

[E217] 3-chloroaniline to air

[E228] 4-chloroaniline to air

[E231] acephate to air

[E236] Acrolein (2-propenal) to air

[E237] Acrylonitrile to air

[E241] aldicarb to air

[E242] aldrin to air

[E251] anilazine to air

[E252] anthracene to air

[E253] antimony to air

[E255] arsenic to air

[E256] atrazine to air

[E257] azinphos-ethyl to air

[E258] azinphos-methyl to air

[E259] barium to air

[E260] benomyl to air

[E261] bentazone to air

[E263] Benzene to air

[E264] benzo[a]anthracene to air

[E265] benzo[a]pyrene to air

[E266] benzo[ghi]perylene to air

[E267] benzo[k]fluoranthrene to air

[E269] benzylchloride to air

[E270] beryllium to air

[E272] bifenthrin to air

[E290] Butylbenzylphtalate to air

[E385] cadmium to air

[E387] captafol to air

[E388] captan to air

[E389] carbaryl to air

[E390] carbendazim to air

[E391] carbofuran to air

[E394] carbon disulfide to air

[E404] chlordane to air

[E405] chlorfenvinphos to air

[E406] chloridazon to air

[E407] chlorobenzene to air

[E410] chlorothalonil to air
Value

Value

0.00018 kg 1,4-dichlorobenzene eq." $/ \mathrm{kg}$

0.0099 kg 1,4-dichlorobenzene eq." $/ \mathrm{kg}$

$0.18 \mathrm{~kg}$ 1,4-dichlorobenzene eq." $/ \mathrm{kg}$

$0.075 \mathrm{~kg}$ 1,4-dichlorobenzene eq." $/ \mathrm{kg}$

$0.24 \mathrm{~kg}$ 1,4-dichlorobenzene eq."/kg

$0.0088 \mathrm{~kg}$ 1,4-dichlorobenzene eq." $/ \mathrm{kg}$

$0.00053 \mathrm{~kg}$ 1,4-dichlorobenzene eq." $/ \mathrm{kg}$

2.60E-05 kg 1,4-dichlorobenzene eq."/kg

$0.0019 \mathrm{~kg}$ 1,4-dichlorobenzene eq." $/ \mathrm{kg}$

2.30E-08 kg 1,4-dichlorobenzene eq." $/ \mathrm{kg}$

$0.00044 \mathrm{~kg}$ 1,4-dichlorobenzene eq." $/ \mathrm{kg}$

$0.012 \mathrm{~kg}$ 1,4-dichlorobenzene eq." $/ \mathrm{kg}$

$0.54 \mathrm{~kg}$ 1,4-dichlorobenzene eq." $/ \mathrm{kg}$

$0.31 \mathrm{~kg}$ 1,4-dichlorobenzene eq." $/ \mathrm{kg}$

12000 kg 1,4-dichlorobenzene eq." $/ \mathrm{kg}$

$0.32 \mathrm{~kg}$ 1,4-dichlorobenzene eq." $/ \mathrm{kg}$

$0.24 \mathrm{~kg}$ 1,4-dichlorobenzene eq." $/ \mathrm{kg}$

$0.32 \mathrm{~kg}$ 1,4-dichlorobenzene eq." $/ \mathrm{kg}$

$0.6 \mathrm{~kg}$ 1,4-dichlorobenzene eq." $/ \mathrm{kg}$

$0.03 \mathrm{~kg}$ 1,4-dichlorobenzene eq." $/ \mathrm{kg}$

$0.053 \mathrm{~kg}$ 1,4-dichlorobenzene eq."//kg

$8.7 \mathrm{~kg}$ 1,4-dichlorobenzene eq." $/ \mathrm{kg}$

$0.47 \mathrm{~kg}$ 1,4-dichlorobenzene eq." $/ \mathrm{kg}$

0.016 kg 1,4-dichlorobenzene eq." $/ \mathrm{kg}$

$0.69 \mathrm{~kg}$ 1,4-dichlorobenzene eq." $/ \mathrm{kg}$

$16 \mathrm{~kg}$ 1,4-dichlorobenzene eq." $/ \mathrm{kg}$

$0.008 \mathrm{~kg}$ 1,4-dichlorobenzene eq."//kg

2000 kg 1,4-dichlorobenzene eq." $/ \mathrm{kg}$

$0.014 \mathrm{~kg}$ 1,4-dichlorobenzene eq." $/ \mathrm{kg}$

$0.092 \mathrm{~kg} 1,4$-dichlorobenzene eq." $/ \mathrm{kg}$

$0.032 \mathrm{~kg}$ 1,4-dichlorobenzene eq." $/ \mathrm{kg}$

$0.61 \mathrm{~kg} 1,4$-dichlorobenzene eq." $/ \mathrm{kg}$

$1600 \mathrm{~kg} \mathrm{1,4-dichlorobenzene} \mathrm{eq."/kg}$

$2 \mathrm{~kg}$ 1,4-dichlorobenzene eq." $/ \mathrm{kg}$

$2.4 \mathrm{~kg}$ 1,4-dichlorobenzene eq." $/ \mathrm{kg}$

$0.19 \mathrm{~kg}$ 1,4-dichlorobenzene eq." $/ \mathrm{kg}$

$4.9 \mathrm{~kg}$ 1,4-dichlorobenzene eq." $/ \mathrm{kg}$

$0.47 \mathrm{~kg}$ 1,4-dichlorobenzene eq." $/ \mathrm{kg}$

$0.25 \mathrm{~kg}$ 1,4-dichlorobenzene eq." $/ \mathrm{kg}$

1.60E-05 kg 1,4-dichlorobenzene eq." $/ \mathrm{kg}$

$0.23 \mathrm{~kg}$ 1,4-dichlorobenzene eq." $/ \mathrm{kg}$

$0.24 \mathrm{~kg}$ 1,4-dichlorobenzene eq." $/ \mathrm{kg}$

$0.2 \mathrm{~kg}$ 1,4-dichlorobenzene eq." $/ \mathrm{kg}$

$30 \mathrm{~kg}$ 1,4-dichlorobenzene eq." $/ \mathrm{kg}$

$0.0017 \mathrm{~kg} 1,4$-dichlorobenzene eq." $/ \mathrm{kg}$

1800 kg 1,4-dichlorobenzene eq."/kg

$8.8 \mathrm{~kg}$ 1,4-dichlorobenzene eq. "/ $\mathrm{kg}$

$0.0013 \mathrm{~kg}$ 1,4-dichlorobenzene eq." $/ \mathrm{kg}$

$81 \mathrm{~kg}$ 1,4-dichlorobenzene eq."/kg

$5.9 \mathrm{~kg}$ 1,4-dichlorobenzene eq." $/ \mathrm{kg}$

$0.024 \mathrm{~kg} 1,4$-dichlorobenzene eq." $/ \mathrm{kg}$

$0.063 \mathrm{~kg}$ 1,4-dichlorobenzene eq." $/ \mathrm{kg}$

$20 \mathrm{~kg}$ 1,4-dichlorobenzene eq." $/ \mathrm{kg}$

$3 \mathrm{~kg}$ 1,4-dichlorobenzene eq." $/ \mathrm{kg}$

0.0051 kg 1,4-dichlorobenzene eq."/kg

$2.2 \mathrm{~kg}$ 1,4-dichlorobenzene eq." $/ \mathrm{kg}$

0.49 kg 1,4-dichlorobenzene eq." $/ \mathrm{kg}$

$0.00046 \mathrm{~kg}$ 1,4-dichlorobenzene eq." $/ \mathrm{kg}$

$0.00073 \mathrm{~kg}$ 1,4-dichlorobenzene eq." $/ \mathrm{kg}$

0.0071 kg 1,4-dichlorobenzene eq." $/ \mathrm{kg}$ 
[E411] chlorpropham to air [E412] chlorpyriphos to air [E413] chromium (unspecified) to air [E414] chromium III to air [E415] chromium VI to air [E416] chrysene to air

[E421] cobalt to air

[E422] copper to air

[E423] coumaphos to air

[E425] cyanazine to air

[E437] cypermethrin to air

[E438] cyromazine to air

[E439] DDT to air

[E441] deltamethrin to air

[E442] demeton to air

[E443] desmetryn to air

[E444] Di(2-ethylhexyl)phtalate to air

[E446] diazinon to air

[E448] Dibutylphtalate to air

[E449] Dichloromethane (Methylene Chloride) to air

[E450] dichlorprop to air

[E451] dichlorvos to air

[E452] dieldrin to air

[E456] Diethylphtalate to air

[E457] Dihexylphtalate to air

[E458] Diisodecylphtalate to air

[E459] Diisooctylphtalate to air

[E461] dimethoate to air

[E468] Dimethylphtalate to air

[E470] dinoseb to air

[E471] dinoterb to air

[E472] Dioctylphtalate to air

[E475] disulfothon to air

[E476] diuron to air

[E478] DNOC to air

[E480] endosulfan to air

[E481] endrin to air

[E487] ethoprophos to air

[E496] Ethylbenzene to air

[E499] Ethylene (ethene) to air

[E504] fenitrothion to air

[E505] fenthion to air

[E506] fentin acetate to air

[E507] fentin chloride to air

[E508] fentin hydroxide to air

[E509] fluoranthrene to air

[E510] folpet to air

[E511] Formaldehyde (methanal) to air

[E516] glyphosate to air

[E533] heptachlor to air

[E535] heptenophos to air

[E536] hexachloro-1,3-butadiene to air

[E537] hexachlorobenzene to air

[E566] indeno[1,2,3-cd]pyrene to air

[E568] iprodione to air

[E583] isoproturon to air

[E584] lead to air

[E585] lindane to air

[E586] linuron to air

[E587] malathion to air

[E589] MCPA to air

[E590] mecoprop to air

[E592] mercury to air

[E595] metamitron to air

[E596] meta-Xylene (1,3-dimethylbenzene) to air

[E597] metazachlor to air

[E598] methabenzthiazuron to air
$0.037 \mathrm{~kg}$ 1,4-dichlorobenzene eq." $/ \mathrm{kg}$

$0.13 \mathrm{~kg}$ 1,4-dichlorobenzene eq." $/ \mathrm{kg}$

$3000 \mathrm{~kg} \mathrm{1,4-dichlorobenzene} \mathrm{eq."} / \mathrm{kg}$

3000 kg 1,4-dichlorobenzene eq." $/ / \mathrm{kg}$

3000 kg 1,4-dichlorobenzene eq." $/ \mathrm{kg}$

$0.22 \mathrm{~kg}$ 1,4-dichlorobenzene eq." $/ \mathrm{kg}$

$110 \mathrm{~kg}$ 1,4-dichlorobenzene eq." $/ \mathrm{kg}$

$7 \mathrm{~kg}$ 1,4-dichlorobenzene eq." $/ \mathrm{kg}$

1000 kg 1,4-dichlorobenzene eq." $/ \mathrm{kg}$

$31 \mathrm{~kg}$ 1,4-dichlorobenzene eq." $/ \mathrm{kg}$

8900 kg 1,4-dichlorobenzene eq." $/ \mathrm{kg}$

$310 \mathrm{~kg}$ 1,4-dichlorobenzene eq." $/ \mathrm{kg}$

$19 \mathrm{~kg}$ 1,4-dichlorobenzene eq." $/ \mathrm{kg}$

$0.76 \mathrm{~kg}$ 1,4-dichlorobenzene eq." $/ \mathrm{kg}$

$0.3 \mathrm{~kg}$ 1,4-dichlorobenzene eq." $/ \mathrm{kg}$

$1.2 \mathrm{~kg}$ 1,4-dichlorobenzene eq." $/ \mathrm{kg}$

$0.00022 \mathrm{~kg}$ 1,4-dichlorobenzene eq." $/ \mathrm{kg}$

$0.29 \mathrm{~kg}$ 1,4-dichlorobenzene eq." $/ \mathrm{kg}$

$0.0039 \mathrm{~kg}$ 1,4-dichlorobenzene eq." $/ \mathrm{kg}$

4.30E-06 kg 1,4-dichlorobenzene eq." $/ \mathrm{kg}$

$0.00068 \mathrm{~kg}$ 1,4-dichlorobenzene eq." $/ \mathrm{kg}$

$9.8 \mathrm{~kg}$ 1,4-dichlorobenzene eq." $/ \mathrm{kg}$

$1.1 \mathrm{~kg}$ 1,4-dichlorobenzene eq." $/ \mathrm{kg}$

$0.53 \mathrm{~kg} 1,4$-dichlorobenzene eq." $/ \mathrm{kg}$

0.00078 kg 1,4-dichlorobenzene eq." $/ \mathrm{kg}$

$0.00092 \mathrm{~kg}$ 1,4-dichlorobenzene eq." $/ \mathrm{kg}$

$0.00011 \mathrm{~kg}$ 1,4-dichlorobenzene eq." $/ \mathrm{kg}$

$0.3 \mathrm{~kg}$ 1,4-dichlorobenzene eq." $/ \mathrm{kg}$

0.64 kg 1,4-dichlorobenzene eq." $/ \mathrm{kg}$

$97 \mathrm{~kg}$ 1,4-dichlorobenzene eq." $/ \mathrm{kg}$

$3.4 \mathrm{~kg}$ 1,4-dichlorobenzene eq. "/ $/ \mathrm{kg}$

9.80E-06 kg 1,4-dichlorobenzene eq."//kg

$0.043 \mathrm{~kg} 1,4$-dichlorobenzene eq." $/ \mathrm{kg}$

$0.043 \mathrm{~kg}$ 1,4-dichlorobenzene eq." $/ \mathrm{kg}$

$0.24 \mathrm{~kg} 1,4$-dichlorobenzene eq. $/ / \mathrm{kg}$

$0.036 \mathrm{~kg}$ 1,4-dichlorobenzene eq." $/ \mathrm{kg}$

$49 \mathrm{~kg}$ 1,4-dichlorobenzene eq." $/ \mathrm{kg}$

17 kg 1,4-dichlorobenzene eq."//kg

$1.40 \mathrm{E}-06 \mathrm{~kg}$ 1,4-dichlorobenzene eq."//kg

1.30E-12 kg 1,4-dichlorobenzene eq." $/ \mathrm{kg}$

$21 \mathrm{~kg}$ 1,4-dichlorobenzene eq." $/ \mathrm{kg}$

$16 \mathrm{~kg}$ 1,4-dichlorobenzene eq." $/ \mathrm{kg}$

$5.3 \mathrm{~kg}$ 1,4-dichlorobenzene eq." $/ \mathrm{kg}$

$0.26 \mathrm{~kg}$ 1,4-dichlorobenzene eq." $/ \mathrm{kg}$

$5.5 \mathrm{~kg}$ 1,4-dichlorobenzene eq." $/ \mathrm{kg}$

$0.018 \mathrm{~kg} 1,4$-dichlorobenzene eq." $/ \mathrm{kg}$

$1.7 \mathrm{~kg}$ 1,4-dichlorobenzene eq." $/ \mathrm{kg}$

$0.94 \mathrm{~kg}$ 1,4-dichlorobenzene eq." $/ \mathrm{kg}$

$0.047 \mathrm{~kg}$ 1,4-dichlorobenzene eq." $/ \mathrm{kg}$

$0.00088 \mathrm{~kg} 1,4$-dichlorobenzene eq." $/ \mathrm{kg}$

$2.2 \mathrm{~kg}$ 1,4-dichlorobenzene eq. " $/ \mathrm{kg}$

$4.2 \mathrm{~kg}$ 1,4-dichlorobenzene eq." $/ \mathrm{kg}$

$0.26 \mathrm{~kg}$ 1,4-dichlorobenzene eq." $/ \mathrm{kg}$

$0.8 \mathrm{~kg}$ 1,4-dichlorobenzene eq." $/ \mathrm{kg}$

$0.11 \mathrm{~kg}$ 1,4-dichlorobenzene eq." $/ \mathrm{kg}$

$2.5 \mathrm{~kg}$ 1,4-dichlorobenzene eq." $/ \mathrm{kg}$

$16 \mathrm{~kg}$ 1,4-dichlorobenzene eq. "/kg

$1.8 \mathrm{~kg}$ 1,4-dichlorobenzene eq. " $/ \mathrm{kg}$

$0.2 \mathrm{~kg} 1,4$-dichlorobenzene eq." $/ \mathrm{kg}$

$0.02 \mathrm{~kg}$ 1,4-dichlorobenzene eq." $/ \mathrm{kg}$

$0.043 \mathrm{~kg} 1,4$-dichlorobenzene eq." $/ \mathrm{kg}$

$1.8 \mathrm{~kg}$ 1,4-dichlorobenzene eq." $/ \mathrm{kg}$

$28000 \mathrm{~kg}$ 1,4-dichlorobenzene eq." $/ \mathrm{kg}$

$0.019 \mathrm{~kg}$ 1,4-dichlorobenzene eq." $/ \mathrm{kg}$

6.50E-07 kg 1,4-dichlorobenzene eq." $/ \mathrm{kg}$

$0.074 \mathrm{~kg}$ 1,4-dichlorobenzene eq." $/ \mathrm{kg}$

$0.45 \mathrm{~kg}$ 1,4-dichlorobenzene eq." $/ \mathrm{kg}$ 
[E603] methomyl to air

[E626] methyl-mercury to air

[E628] metobromuron to air

[E629] metolachlor to air

[E630] mevinphos to air

[E632] molybdenum to air

[E633] Naphtalene to air

[E635] nickel to air

[E650] ortho-Xylene (1,2-dimethylbenzene) to air

[E651] oxamyl to air

[E652] oxydemethon-methyl to air

[E655] parathion-ethyl to air

[E656] parathion-methyl to air

[E658] para-Xylene (1,4-dimethylbenzene) to air

[E660] pentachlorobenzene to air

[E661] pentachloronitrobenzene to air

[E662] pentachlorophenol to air

[E672] permethrin to air

[E674] phenanthrene to air

[E675] Phenol to air

[E679] phoxim to air

[E680] Phtalic anhydride to air

[E682] pirimicarb to air

[E685] Polycyclic Aromatic Hydrocarbons Carcinogenic- (carcinogenic-PAH) to air

[E686] propachlor to air

[E690] propoxur to air

[E697] Propylene Oxide to air

[E698] pyrazophos to air

[E704] selenium to air

[E705] simazine to air

[E706] styrene (vinylbenzene) to air

[E718] tetrachloroethylene (PER) (tetrachloroethene) to air

[E719] Tetrachloromethane (carbon tetrachloride) $(\mathrm{HC}-10)$ to air

[E722] thallium to air

[E723] Thiram to air

[E724] tin to air

[E725] tolclophos-methyl to air

[E727] Toluene to air

[E733] tri-allate to air

[E734] triazophos to air

[E735] tributyltinoxide to air

[E736] trichlorfon to air

[E737] Trichloroethylene (tri) to air

[E738] Trichloromethane (chloroform) to air

[E739] trifluarin to air

[E743] vanadium to air

[E745] Vinyl Chloride (chloroethene) to air

[E748] zinc to air

[E749] zineb to air

[E750] 1,1,1-trichloroethane to air

[E751] 1,2,3,4-tetrachlorobenzene to air

[E752] 1,2,3,5-tetrachlorobenzene to air

[E753] 1,2,3-trichlorobenzene to air

[E754] 1,2,4,5-tetrachlorobenzene to air

[E755] 1,2,4-trichlorobenzene to air

[E756] 1,2-dichlorobenzene to air

[E757] 1,2-dichloroethane to air

[E758] 1,3,5-trichlorobenzene to air

[E759] 1,3-butadiene to air

[E760] 1,3-dichlorobenzene to air

[E761] 1,4-dichlorobenzene to air

[E762] 1-chloro-4-nitrobenzene to air

[E763] 2,3,4,6-tetrachlorophenol to air

[E764] 2,3,7,8-TCDD to air

[E765] 2,4,5-T to air

[E766] 2,4,5-trichlorophenol to air

[E767] 2,4,6-trichlorophenol to fresh water
120 kg 1,4-dichlorobenzene eq." $/ \mathrm{kg}$ 28000 kg 1,4-dichlorobenzene eq." $/ \mathrm{kg}$

$0.99 \mathrm{~kg}$ 1,4-dichlorobenzene eq." $/ \mathrm{kg}$

$0.11 \mathrm{~kg}$ 1,4-dichlorobenzene eq." $/ \mathrm{kg}$

$43 \mathrm{~kg}$ 1,4-dichlorobenzene eq."/kg

18 kg 1,4-dichlorobenzene eq." $/ \mathrm{kg}$

$0.00083 \mathrm{~kg}$ 1,4-dichlorobenzene eq."/kg

$120 \mathrm{~kg}$ 1,4-dichlorobenzene eq." $/ \mathrm{kg}$

1.30E-06 kg 1,4-dichlorobenzene eq."/kg

$2.9 \mathrm{~kg}$ 1,4-dichlorobenzene eq. "/kg

$41 \mathrm{~kg}$ 1,4-dichlorobenzene eq." $/ \mathrm{kg}$

$1.1 \mathrm{~kg} 1,4$-dichlorobenzene eq." $/ \mathrm{kg}$

$5.7 \mathrm{~kg}$ 1,4-dichlorobenzene eq." $/ \mathrm{kg}$

$5.30 \mathrm{E}-07 \mathrm{~kg}$ 1,4-dichlorobenzene eq." $/ \mathrm{kg}$

$0.039 \mathrm{~kg}$ 1,4-dichlorobenzene eq." $/ \mathrm{kg}$

$0.12 \mathrm{~kg} 1,4$-dichlorobenzene eq." $/ \mathrm{kg}$

$2.3 \mathrm{~kg}$ 1,4-dichlorobenzene eq." $/ \mathrm{kg}$

$26 \mathrm{~kg}$ 1,4-dichlorobenzene eq."//kg

$0.00014 \mathrm{~kg}$ 1,4-dichlorobenzene eq."/kg

$0.0033 \mathrm{~kg}$ 1,4-dichlorobenzene eq." $/ \mathrm{kg}$

$0.017 \mathrm{~kg} 1,4$-dichlorobenzene eq." $/ \mathrm{kg}$

$0.0005 \mathrm{~kg}$ 1,4-dichlorobenzene eq." $/ \mathrm{kg}$

$46 \mathrm{~kg}$ 1,4-dichlorobenzene eq." $/ \mathrm{kg}$

$1 \mathrm{~kg}$ 1,4-dichlorobenzene eq." $/ \mathrm{kg}$

$0.54 \mathrm{~kg} 1,4$-dichlorobenzene eq." $/ \mathrm{kg}$

$700 \mathrm{~kg}$ 1,4-dichlorobenzene eq." $/ \mathrm{kg}$

$0.0015 \mathrm{~kg}$ 1,4-dichlorobenzene eq." $/ \mathrm{kg}$

$2.3 \mathrm{~kg}$ 1,4-dichlorobenzene eq." $/ \mathrm{kg}$

$53 \mathrm{~kg}$ 1,4-dichlorobenzene eq." $/ \mathrm{kg}$

$8.8 \mathrm{~kg}$ 1,4-dichlorobenzene eq." $/ \mathrm{kg}$

1.40E-07 kg 1,4-dichlorobenzene eq."/kg

$0.0081 \mathrm{~kg}$ 1,4-dichlorobenzene eq." $/ \mathrm{kg}$

$0.00047 \mathrm{~kg}$ 1,4-dichlorobenzene eq."/kg

$340 \mathrm{~kg}$ 1,4-dichlorobenzene eq." $/ \mathrm{kg}$

32 kg 1,4-dichlorobenzene eq."/kg

$14 \mathrm{~kg}$ 1,4-dichlorobenzene eq." $/ \mathrm{kg}$

$0.00034 \mathrm{~kg} 1,4$-dichlorobenzene eq." $/ \mathrm{kg}$

1.60E-05 kg 1,4-dichlorobenzene eq." $/ \mathrm{kg}$

$0.0069 \mathrm{~kg}$ 1,4-dichlorobenzene eq."/kg

$34 \mathrm{~kg}$ 1,4-dichlorobenzene eq." $/ \mathrm{kg}$

$17 \mathrm{~kg}$ 1,4-dichlorobenzene eq." $/ \mathrm{kg}$

1200 kg 1,4-dichlorobenzene eq." $/ \mathrm{kg}$

4.70E-06 kg 1,4-dichlorobenzene eq."/kg

4.00E-05 kg 1,4-dichlorobenzene eq."//kg

0.017 kg 1,4-dichlorobenzene eq."/kg

$670 \mathrm{~kg}$ 1,4-dichlorobenzene eq." $/ \mathrm{kg}$

2.60E-07 kg 1,4-dichlorobenzene eq."/kg

$12 \mathrm{~kg}$ 1,4-dichlorobenzene eq." $/ \mathrm{kg}$

$7.2 \mathrm{~kg}$ 1,4-dichlorobenzene eq." $/ \mathrm{kg}$

$0.00018 \mathrm{~kg}$ 1,4-dichlorobenzene eq." $/ \mathrm{kg}$

$0.0093 \mathrm{~kg}$ 1,4-dichlorobenzene eq."/kg

$0.17 \mathrm{~kg}$ 1,4-dichlorobenzene eq." $/ \mathrm{kg}$

$0.073 \mathrm{~kg} 1,4$-dichlorobenzene eq." $/ \mathrm{kg}$

$0.23 \mathrm{~kg}$ 1,4-dichlorobenzene eq." $/ \mathrm{kg}$

$0.0085 \mathrm{~kg}$ 1,4-dichlorobenzene eq." $/ \mathrm{kg}$

$0.00052 \mathrm{~kg}$ 1,4-dichlorobenzene eq."/kg

$2.60 \mathrm{E}-05 \mathrm{~kg}$ 1,4-dichlorobenzene eq."//kg

$0.0018 \mathrm{~kg}$ 1,4-dichlorobenzene eq." $/ \mathrm{kg}$

$2.10 \mathrm{E}-08 \mathrm{~kg}$ 1,4-dichlorobenzene eq."/ $/ \mathrm{kg}$

0.00042 kg 1,4-dichlorobenzene eq."/kg

$0.012 \mathrm{~kg}$ 1,4-dichlorobenzene eq." $/ \mathrm{kg}$

$0.44 \mathrm{~kg} 1,4$-dichlorobenzene eq." $/ \mathrm{kg}$

$0.0017 \mathrm{~kg}$ 1,4-dichlorobenzene eq."/kg

$590 \mathrm{~kg}$ 1,4-dichlorobenzene eq." $/ \mathrm{kg}$

3.60E-08 kg 1,4-dichlorobenzene eq."//kg

$0.061 \mathrm{~kg} 1,4$-dichlorobenzene eq." $/ \mathrm{kg}$

$0.00067 \mathrm{~kg}$ 1,4-dichlorobenzene eq." $/ \mathrm{kg}$ 
[E768] 2,4-D to fresh water

[E769] 2,4-dichlorophenol to fresh water

[E770] 2-chlorophenol to fresh water

[E771] 3,4-dichloroaniline to fresh water

[E772] 3-chloroaniline to fresh water

[E773] 4-chloroaniline to fresh water

[E774] acephate to fresh water

[E775] Acrolein (2-propenal) to fresh water

[E776] acrylonitrile to fresh water

[E777] aldicarb to fresh water

[E778] aldrin to fresh water

[E780] anilazine to fresh water

[E781] anthracene to fresh water

[E782] antimony to fresh water

[E783] arsenic to fresh water

[E784] atrazine to fresh water

[E785] azinphos-ethyl to fresh water

[E786] azinphos-methyl to fresh water

[E787] barium to fresh water

[E788] benomyl to fresh water

[E789] bentazone to fresh water

[E790] benzene to fresh water

[E791] benzo[a]anthracene to fresh water

[E792] benzo[a]pyrene to fresh water

[E793] benzo[ghi]perylene to fresh water

[E794] benzo[k]fluoranthrene to fresh water

[E795] benzylchloride to fresh water

[E796] beryllium to fresh water

[E797] bifenthrin to fresh water

[E800] Butylbenzylphtalate to fresh water

[E801] cadmium to fresh water

[E802] captafol to fresh water

[E803] captan to fresh water

[E804] carbaryl to fresh water

[E805] carbendazim to fresh water

[E806] carbofuran to fresh water

[E807] carbon disulfide to fresh water

[E808] Carcinogenic PAHs to fresh water

[E810] chlordane to fresh water

[E811] chlorfenvinphos to fresh water

[E812] chloridazon to fresh water

[E814] chlorobenzene to fresh water

[E815] chlorothalonil to fresh water

[E816] chlorpropham to fresh water

[E817] chlorpyriphos to fresh water

[E818] chromium III to fresh water

[E819] chromium VI to fresh water

[E820] chrysene to fresh water

[E821] cobalt to fresh water

[E822] copper to fresh water

[E823] coumaphos to fresh water

[E824] cyanazine to fresh water

[E825] cypermethrin to fresh water

[E826] cyromazine to fresh water

[E827] DDT to fresh water

[E828] deltamethrin to fresh water

[E829] demeton to fresh water

[E830] desmetryn to fresh water

[E831] Di(2-ethylhexyl)phtalate to fresh water

[E832] diazinon to fresh water

[E833] Dibutylphtalate to fresh water

[E834] Dichloromethane (Methylene Chloride) to fresh water

[E835] dichlorprop to fresh water

[E836] dichlorvos to fresh water

[E837] dieldrin to fresh water

[E838] Diethylphtalate to fresh water

[E839] Dihexylphtalate to fresh water
9.30E-10 kg 1,4-dichlorobenzene eq." $/ \mathrm{kg}$ 0.00096 kg 1,4-dichlorobenzene eq." $/ \mathrm{kg}$

$0.0013 \mathrm{~kg}$ 1,4-dichlorobenzene eq."/kg

$0.00076 \mathrm{~kg}$ 1,4-dichlorobenzene eq." $/ \mathrm{kg}$

9.40E-06 kg 1,4-dichlorobenzene eq." $/ \mathrm{kg}$

$0.0036 \mathrm{~kg}$ 1,4-dichlorobenzene eq." $/ \mathrm{kg}$

2.20E-08 kg 1,4-dichlorobenzene eq." $/ \mathrm{kg}$

$5.8 \mathrm{~kg}$ 1,4-dichlorobenzene eq." $/ \mathrm{kg}$

0.0039 kg 1,4-dichlorobenzene eq." $/ \mathrm{kg}$

$0.19 \mathrm{~kg}$ 1,4-dichlorobenzene eq." $/ \mathrm{kg}$

0.014 kg 1,4-dichlorobenzene eq." $/ \mathrm{kg}$

5.00E-08 kg 1,4-dichlorobenzene eq." $/ \mathrm{kg}$

$0.02 \mathrm{~kg}$ 1,4-dichlorobenzene eq." $/ \mathrm{kg}$

$1.70 \mathrm{E}-20 \mathrm{~kg}$ 1,4-dichlorobenzene eq." $/ \mathrm{kg}$

$1.00 \mathrm{E}-17 \mathrm{~kg}$ 1,4-dichlorobenzene eq." $/ \mathrm{kg}$

0.00076 kg 1,4-dichlorobenzene eq." $/ \mathrm{kg}$

$0.021 \mathrm{~kg}$ 1,4-dichlorobenzene eq." $/ \mathrm{kg}$

3.30E-06 kg 1,4-dichlorobenzene eq."/kg

$5.10 \mathrm{E}-19 \mathrm{~kg}$ 1,4-dichlorobenzene eq." $/ \mathrm{kg}$

$8.20 \mathrm{E}-08$ kg 1,4-dichlorobenzene eq." $/ \mathrm{kg}$

1.80E-07 kg 1,4-dichlorobenzene eq." $/ \mathrm{kg}$

1.40E-05 kg 1,4-dichlorobenzene eq. "/ $/ \mathrm{kg}$

$0.014 \mathrm{~kg}$ 1,4-dichlorobenzene eq." $/ \mathrm{kg}$

$0.0025 \mathrm{~kg} 1,4$-dichlorobenzene eq. $/ \mathrm{kg}$

$0.00043 \mathrm{~kg}$ 1,4-dichlorobenzene eq." $/ \mathrm{kg}$

$0.21 \mathrm{~kg}$ 1,4-dichlorobenzene eq." $/ \mathrm{kg}$

$0.00083 \mathrm{~kg}$ 1,4-dichlorobenzene eq." $/ \mathrm{kg}$

3.30E-16 kg 1,4-dichlorobenzene eq." $/ \mathrm{kg}$

$0.021 \mathrm{~kg} \mathrm{1,4-dichlorobenzene} \mathrm{eq."/kg}$

$6.60 \mathrm{E}-06$ kg 1,4-dichlorobenzene eq." $/ \mathrm{kg}$

1.40E-20 kg 1,4-dichlorobenzene eq."/kg

1.90E-07 kg 1,4-dichlorobenzene eq." $/ \mathrm{kg}$

6.20E-08 kg 1,4-dichlorobenzene eq." $/ \mathrm{kg}$

2.60E-07 kg 1,4-dichlorobenzene eq." $/ \mathrm{kg}$

6.30E-08 kg 1,4-dichlorobenzene eq." $/ \mathrm{kg}$

$3.50 \mathrm{E}-05 \mathrm{~kg} 1,4$-dichlorobenzene eq." $/ \mathrm{kg}$

$0.0048 \mathrm{~kg} 1,4$-dichlorobenzene eq. $/ / \mathrm{kg}$

$0.0021 \mathrm{~kg} \mathrm{1,4-dichlorobenzene} \mathrm{eq.} \mathrm{"/kg}$

$0.097 \mathrm{~kg}$ 1,4-dichlorobenzene eq." $/ \mathrm{kg}$

4.60E-05 kg 1,4-dichlorobenzene eq." $/ \mathrm{kg}$

$0.00038 \mathrm{~kg}$ 1,4-dichlorobenzene eq." $/ \mathrm{kg}$

0.00072 kg 1,4-dichlorobenzene eq." $/ \mathrm{kg}$

$0.0055 \mathrm{~kg}$ 1,4-dichlorobenzene eq." $/ \mathrm{kg}$

$2.50 \mathrm{E}-05 \mathrm{~kg}$ 1,4-dichlorobenzene eq." $/ \mathrm{kg}$

$0.021 \mathrm{~kg}$ 1,4-dichlorobenzene eq." $/ \mathrm{kg}$

2.30E-19 kg 1,4-dichlorobenzene eq." $/ \mathrm{kg}$

2.30E-19 kg 1,4-dichlorobenzene eq." $/ \mathrm{kg}$

$0.0084 \mathrm{~kg} 1,4$-dichlorobenzene eq. "/kg

2.70E-18 kg 1,4-dichlorobenzene eq." $/ \mathrm{kg}$

4.10E-21 kg 1,4-dichlorobenzene eq."/kg

$6 \mathrm{~kg}$ 1,4-dichlorobenzene eq." $/ \mathrm{kg}$

2.20E-06 kg 1,4-dichlorobenzene eq." $/ \mathrm{kg}$

$16 \mathrm{~kg} \mathrm{1,4-dichlorobenzene} \mathrm{eq."/kg}$

$1.90 \mathrm{E}-06 \mathrm{~kg}$ 1,4-dichlorobenzene eq." $/ \mathrm{kg}$

$0.31 \mathrm{~kg} 1,4$-dichlorobenzene eq." $/ \mathrm{kg}$

$0.032 \mathrm{~kg}$ 1,4-dichlorobenzene eq." $/ \mathrm{kg}$

$0.012 \mathrm{~kg} 1,4$-dichlorobenzene eq. "/ $/ \mathrm{kg}$

3.60E-05 kg 1,4-dichlorobenzene eq." $/ \mathrm{kg}$

6.60E-06 kg 1,4-dichlorobenzene eq." $/ \mathrm{kg}$

$0.0041 \mathrm{~kg} 1,4$-dichlorobenzene eq." $/ \mathrm{kg}$

1.30E-05 kg 1,4-dichlorobenzene eq." $/ \mathrm{kg}$

3.90E-06 kg 1,4-dichlorobenzene eq." $/ \mathrm{kg}$

6.10E-12 kg 1,4-dichlorobenzene eq." $/ \mathrm{kg}$

0.014 kg 1,4-dichlorobenzene eq." $/ \mathrm{kg}$

$0.26 \mathrm{~kg} 1,4$-dichlorobenzene eq." $/ \mathrm{kg}$

0.0056 kg 1,4-dichlorobenzene eq." $/ \mathrm{kg}$

0.00026 kg 1,4-dichlorobenzene eq." $/ \mathrm{kg}$ 
[E840] Diisodecylphtalate to fresh water [E841] Diisooctylphtalate to fresh water [E842] dimethoate to fresh water

[E843] Dimethylphtalate to fresh water

[E844] dinoseb to fresh water

[E845] dinoterb to fresh water

[E846] Dioctylphtalate to fresh water

[E847] disulfothon to fresh water

[E848] diuron to fresh water

[E849] DNOC to fresh water

[E850] endosulfan to fresh water

[E851] endrin to fresh water

[E852] ethoprophos to fresh water

[E853] ethylbenzene to fresh water

[E854] ethylene to fresh water

[E855] fenitrothion to fresh water

[E856] fenthion to fresh water

[E857] fentin acetate to fresh water

[E858] fentin chloride to fresh water

[E859] fentin hydroxide to fresh water

[E860] fluoranthrene to fresh water

[E861] folpet to fresh water

[E862] Formaldehyde (methanal) to fresh water

[E863] glyphosate to fresh water

[E864] heptachlor to fresh water

[E865] heptenophos to fresh water

[E866] hexachloro-1,3-butadiene to fresh water

[E867] hexachlorobenzene to fresh water

[E870] indeno[1,2,3-cd]pyrene to fresh water

[E871] iprodione to fresh water

[E872] isoproturon to fresh water

[E873] lead to fresh water

[E874] lindane to fresh water

[E875] linuron to fresh water

[E876] malathion to fresh water

[E878] MCPA to fresh water

[E879] mecoprop to fresh water

[E880] mercury to fresh water

[E881] metamitron to fresh water

[E882] metazachlor to fresh water

[E883] methabenzthiazuron to fresh water

[E884] methomyl to fresh water

[E885] methylbromide to fresh water

[E886] methyl-mercury to fresh water

[E887] metobromuron to fresh water

[E888] metolachlor to fresh water

[E889] mevinphos to fresh water

[E890] molybdenum to fresh water

[E891] m-xylene to fresh water

[E892] naphtalene to fresh water

[E893] nickel to fresh water

[E899] oxamyl to fresh water

[E900] oxydemethon-methyl to fresh water

[E901] o-xylene to fresh water

[E902] parathion-ethyl to fresh water

[E903] parathion-methyl to fresh water

[E904] pentachlorobenzene to fresh water

[E905] pentachloronitrobenzene to fresh water

[E906] pentachlorophenol to fresh water

[E907] permethrin to fresh water

[E908] phenanthrene to fresh water

[E909] phenol to fresh water

[E912] phoxim to fresh water

[E913] Phtalic anhydride to fresh water

[E914] pirimicarb to fresh water

[E916] propachlor to fresh water

[E917] propoxur to fresh water
$0.00038 \mathrm{~kg}$ 1,4-dichlorobenzene eq." $/ \mathrm{kg}$

6.40E-06 kg 1,4-dichlorobenzene eq."/kg

1.20E-05 kg 1,4-dichlorobenzene eq." $/ \mathrm{kg}$

0.00037 kg 1,4-dichlorobenzene eq."/kg

$0.34 \mathrm{~kg} \mathrm{1,4-dichlorobenzene} \mathrm{eq."} / \mathrm{kg}$

0.013 kg 1,4-dichlorobenzene eq."/kg

1.30E-07 kg 1,4-dichlorobenzene eq."/kg

0.0012 kg 1,4-dichlorobenzene eq."/kg

0.0012 kg 1,4-dichlorobenzene eq."/kg

8.50E-07 kg 1,4-dichlorobenzene eq."/kg

0.0018 kg 1,4-dichlorobenzene eq."/kg

0.35 kg 1,4-dichlorobenzene eq." $/ \mathrm{kg}$

$0.24 \mathrm{~kg} \mathrm{1,4-dichlorobenzene} \mathrm{eq."} / \mathrm{kg}$

1.20E-06 kg 1,4-dichlorobenzene eq."/kg

1.10E-12 kg 1,4-dichlorobenzene eq."/kg

$0.0047 \mathrm{~kg} \mathrm{1,4-dichlorobenzene} \mathrm{eq."} / \mathrm{kg}$

0.088 kg 1,4-dichlorobenzene eq."/kg

$0.0061 \mathrm{~kg} \mathrm{1,4-dichlorobenzene} \mathrm{eq."/kg}$

0.092 kg 1,4-dichlorobenzene eq."/kg

0.0021 kg 1,4-dichlorobenzene eq."/kg

0.0049 kg 1,4-dichlorobenzene eq."/kg

0.6 kg 1,4-dichlorobenzene eq."/kg

$0.0016 \mathrm{~kg} \mathrm{1,4-dichlorobenzene} \mathrm{eq."} / \mathrm{kg}$

2.20E-11 kg 1,4-dichlorobenzene eq." $/ \mathrm{kg}$

0.00053 kg 1,4-dichlorobenzene eq." $/ \mathrm{kg}$

0.0016 kg 1,4-dichlorobenzene eq."/kg

4 kg 1,4-dichlorobenzene eq."/kg

$0.26 \mathrm{~kg} \mathrm{1,4-dichlorobenzene} \mathrm{eq."} / \mathrm{kg}$

6.20E-06 kg 1,4-dichlorobenzene eq." $/ \mathrm{kg}$

4.40E-08 kg 1,4-dichlorobenzene eq." $/ \mathrm{kg}$

1.60E-05 kg 1,4-dichlorobenzene eq." $/ \mathrm{kg}$

2.90E-22 kg 1,4-dichlorobenzene eq." $/ \mathrm{kg}$

$0.16 \mathrm{~kg} \mathrm{1,4-dichlorobenzene} \mathrm{eq."} / \mathrm{kg}$

0.011 kg 1,4-dichlorobenzene eq."/kg

1.10E-05 kg 1,4-dichlorobenzene eq." $/ \mathrm{kg}$

1.40E-11 kg 1,4-dichlorobenzene eq."/kg

1.10E-08 kg 1,4-dichlorobenzene eq." $/ \mathrm{kg}$

930 kg 1,4-dichlorobenzene eq." $/ \mathrm{kg}$

8.50E-10 kg 1,4-dichlorobenzene eq."/kg

1.40E-06 kg 1,4-dichlorobenzene eq."/kg

2.00E-05 kg 1,4-dichlorobenzene eq." $/ \mathrm{kg}$

0.0022 kg 1,4-dichlorobenzene eq."/kg

0.011 kg 1,4-dichlorobenzene eq." $/ \mathrm{kg}$

$930 \mathrm{~kg} \mathrm{1,4-dichlorobenzene} \mathrm{eq."} / \mathrm{kg}$

0.00046 kg 1,4-dichlorobenzene eq." $/ \mathrm{kg}$

$0.00021 \mathrm{~kg} \mathrm{1,4-dichlorobenzene} \mathrm{eq."/kg}$

2.30E-05 kg 1,4-dichlorobenzene eq." $/ \mathrm{kg}$

2.30E-18 kg 1,4-dichlorobenzene eq."/kg

6.00E-07 kg 1,4-dichlorobenzene eq." $/ \mathrm{kg}$

$0.00049 \mathrm{~kg} \mathrm{1,4-dichlorobenzene} \mathrm{eq."} / \mathrm{kg}$

$1.00 \mathrm{E}-18 \mathrm{~kg} 1,4$-dichlorobenzene eq." $/ \mathrm{kg}$

7.10E-06 kg 1,4-dichlorobenzene eq." $/ \mathrm{kg}$

0.00046 kg 1,4-dichlorobenzene eq." $/ \mathrm{kg}$

1.20E-06 kg 1,4-dichlorobenzene eq." $/ \mathrm{kg}$

0.0031 kg 1,4-dichlorobenzene eq."/kg

$0.034 \mathrm{~kg}$ 1,4-dichlorobenzene eq." $/ \mathrm{kg}$

0.038 kg 1,4-dichlorobenzene eq." $/ \mathrm{kg}$

0.05 kg 1,4-dichlorobenzene eq." $/ \mathrm{kg}$

0.00032 kg 1,4-dichlorobenzene eq." $/ \mathrm{kg}$

0.39 kg 1,4-dichlorobenzene eq." $/ \mathrm{kg}$

6.00E-05 kg 1,4-dichlorobenzene eq." $/ \mathrm{kg}$

2.50E-06 kg 1,4-dichlorobenzene eq." $/ \mathrm{kg}$

0.015 kg 1,4-dichlorobenzene eq." $/ \mathrm{kg}$

1.20E-10 kg 1,4-dichlorobenzene eq." $/ \mathrm{kg}$

0.00093 kg 1,4-dichlorobenzene eq." $/ \mathrm{kg}$

$0.00081 \mathrm{~kg} 1,4$-dichlorobenzene eq. "/kg

$0.00031 \mathrm{~kg}$ 1,4-dichlorobenzene eq." $/ \mathrm{kg}$ 
[E918] propylene oxide to fresh water [E919] p-xylene to fresh water

[E920] pyrazophos to fresh water

[E921] selenium to fresh water

[E922] simazine to fresh water

[E923] styrene (vinylbenzene) to fresh water

[E926] tetrachloroethylene (PER) to fresh water

[E927] Tetrachloromethane (carbon tetrachloride) (HC-10) to fresh water

[E928] thallium to fresh water

[E929] Thiram to fresh water

[E930] tin to fresh water

[E931] tolclophos-methyl to fresh water

[E932] toluene to fresh water

[E933] tri-allate to fresh water

[E934] triazophos to fresh water

[E935] tributyltinoxide to fresh water

[E936] trichlorfon to fresh water

[E937] Trichloroethylene (tri) to fresh water

[E938] Trichloromethane $=$ chloroform to fresh water

[E939] trifluarin to fresh water

[E940] vanadium to fresh water

[E941] Vinyl Chloride (chloroethene) to fresh water

[E942] zinc to fresh water

[E943] zineb to fresh water

[E944] 1,1,1-trichloroethane to fresh water

[E945] 1,2,3,4-tetrachlorobenzene to fresh water

[E946] 1,2,3,5-tetrachlorobenzene to fresh water

[E947] 1,2,3-trichlorobenzene to fresh water

[E948] 1,2,4,5-tetrachlorobenzene to fresh water

[E949] 1,2,4-trichlorobenzene to fresh water

[E950] 1,2-dichlorobenzene to fresh water

[E951] 1,2-dichloroethane to fresh water

[E952] 1,3,5-trichlorobenzene to fresh water

[E953] 1,3-butadiene to fresh water

[E954] 1,3-dichlorobenzene to fresh water

[E955] 1,4-dichlorobenzene to fresh water

[E956] 1-chloro-4-nitrobenzene to fresh water

[E957] 2,3,4,6-tetrachlorophenol to fresh water

[E958] 2,3,7,8-TCDD to fresh water

[E959] 2,4,5-T to fresh water

[E960] 2,4,5-trichlorophenol to fresh water

[E961] 2,4,6-trichlorophenol to sea water

[E962] 2,4-D to sea water

[E963] 2,4-dichlorophenol to sea water

[E964] 2-chlorophenol to sea water

[E965] 3,4-dichloroaniline to sea water

[E966] 3-chloroaniline to sea water

[E967] 4-chloroaniline to sea water

[E968] acephate to sea water

[E969] Acrolein (2-propenal) to sea water

[E970] acrylonitrile to sea water

[E971] aldicarb to sea water

[E972] aldrin to sea water

[E974] anilazine to sea water

[E975] anthracene to sea water

[E976] antimony to sea water

[E977] arsenic to sea water

[E978] atrazine to sea water

[E979] azinphos-ethyl to sea water

[E980] azinphos-methyl to sea water

[E981] barium to sea water

[E982] benomyl to sea water

[E983] bentazone to sea water

[E984] benzene to sea water

[E985] benzo[a]anthracene to sea water

[E986] benzo[a]pyrene to sea water

[E987] benzo[ghi]perylene to sea water
0.00065 kg 1,4-dichlorobenzene eq."/kg 4.90E-07 kg 1,4-dichlorobenzene eq."/kg

$0.0017 \mathrm{~kg} \mathrm{1,4-dichlorobenzene} \mathrm{eq."} / \mathrm{kg}$

1.60E-17 kg 1,4-dichlorobenzene eq." $/ \mathrm{kg}$

$0.001 \mathrm{~kg} 1,4-$ dichlorobenzene eq."/kg

1.30E-07 kg 1,4-dichlorobenzene eq."/kg

0.0079 kg 1,4-dichlorobenzene eq."/kg

$0.00047 \mathrm{~kg} 1,4$-dichlorobenzene eq." $/ \mathrm{kg}$

3.10E-17 kg 1,4-dichlorobenzene eq."/kg

$0.093 \mathrm{~kg} \mathrm{1,4-dichlorobenzene} \mathrm{eq.} \mathrm{"/kg}$

7.90E-22 kg 1,4-dichlorobenzene eq."/kg

$0.00032 \mathrm{~kg} 1,4$-dichlorobenzene eq." $/ \mathrm{kg}$

1.40E-05 kg 1,4-dichlorobenzene eq."/kg

0.0027 kg 1,4-dichlorobenzene eq." $/ \mathrm{kg}$

0.039 kg 1,4-dichlorobenzene eq."/kg

$0.11 \mathrm{~kg} \mathrm{1,4-dichlorobenzene} \mathrm{eq."} / \mathrm{kg}$

7.00E-05 kg 1,4-dichlorobenzene eq."/kg

4.60E-06 kg 1,4-dichlorobenzene eq."/kg

3.90E-05 kg 1,4-dichlorobenzene eq." $/ \mathrm{kg}$

0.013 kg 1,4-dichlorobenzene eq." $/ \mathrm{kg}$

1.00E-17 kg 1,4-dichlorobenzene eq."/kg

2.60E-07 kg 1,4-dichlorobenzene eq." $/ \mathrm{kg}$

2.50E-21 kg 1,4-dichlorobenzene eq."/kg

$0.0013 \mathrm{~kg} 1,4$-dichlorobenzene eq." $/ \mathrm{kg}$

$0.00011 \mathrm{~kg} \mathrm{1,4-dichlorobenzene} \mathrm{eq."} / \mathrm{kg}$

$0.0037 \mathrm{~kg} \mathrm{1,4-dichlorobenzene} \mathrm{eq."/kg}$

0.074 kg 1,4-dichlorobenzene eq."/kg

0.035 kg 1,4-dichlorobenzene eq." $/ \mathrm{kg}$

0.095 kg 1,4-dichlorobenzene eq." $/ \mathrm{kg}$

$0.004 \mathrm{~kg} \mathrm{1,4-dichlorobenzene} \mathrm{eq.} \mathrm{"/kg}$

$0.00024 \mathrm{~kg} 1,4$-dichlorobenzene eq." $/ \mathrm{kg}$

2.00E-05 kg 1,4-dichlorobenzene eq."/kg

0.00083 kg 1,4-dichlorobenzene eq." $/ \mathrm{kg}$

4.00E-09 kg 1,4-dichlorobenzene eq."/kg

0.0002 kg 1,4-dichlorobenzene eq." $/ \mathrm{kg}$

0.0057 kg 1,4-dichlorobenzene eq." $/ \mathrm{kg}$

0.096 kg 1,4-dichlorobenzene eq."/kg

5.20E-06 kg 1,4-dichlorobenzene eq."/kg

$830 \mathrm{~kg} \mathrm{1,4-dichlorobenzene} \mathrm{eq."} / \mathrm{kg}$

6.40E-11 kg 1,4-dichlorobenzene eq."/kg

$0.00091 \mathrm{~kg} \mathrm{1,4-dichlorobenzene} \mathrm{eq.} \mathrm{"/kg}$

1.30E-05 kg 1,4-dichlorobenzene eq." /kg

1.80E-12 kg 1,4-dichlorobenzene eq." $/ \mathrm{kg}$

6.20E-06 kg 1,4-dichlorobenzene eq."/kg

2.70E-05 kg 1,4-dichlorobenzene eq."/kg

6.70E-06 kg 1,4-dichlorobenzene eq."/kg

1.70E-08 kg 1,4-dichlorobenzene eq."/kg

8.60E-05 kg 1,4-dichlorobenzene eq." $/ \mathrm{kg}$

5.30E-10 kg 1,4-dichlorobenzene eq."/kg

0.16 kg 1,4-dichlorobenzene eq." $/ \mathrm{kg}$

0.00012 kg 1,4-dichlorobenzene eq." $/ \mathrm{kg}$

0.0048 kg 1,4-dichlorobenzene eq."/kg

0.0067 kg 1,4-dichlorobenzene eq." $/ \mathrm{kg}$

7.00E-10 kg 1,4-dichlorobenzene eq." $/ \mathrm{kg}$

$0.004 \mathrm{~kg} \mathrm{1,4-dichlorobenzene} \mathrm{eq."} / \mathrm{kg}$

3.00E-20 kg 1,4-dichlorobenzene eq."/kg

3.00E-17 kg 1,4-dichlorobenzene eq."/kg

5.00E-05 kg 1,4-dichlorobenzene eq." $/ \mathrm{kg}$

0.00034 kg 1,4-dichlorobenzene eq." $/ \mathrm{kg}$

4.90E-08 kg 1,4-dichlorobenzene eq." $/ \mathrm{kg}$

6.60E-19 kg 1,4-dichlorobenzene eq." $/ \mathrm{kg}$

1.40E-09 kg 1,4-dichlorobenzene eq."/kg

3.30E-10 kg 1,4-dichlorobenzene eq." $/ \mathrm{kg}$

1.70E-06 kg 1,4-dichlorobenzene eq." $/ \mathrm{kg}$

0.0062 kg 1,4-dichlorobenzene eq." $/ \mathrm{kg}$

0.0008 kg 1,4-dichlorobenzene eq." $/ \mathrm{kg}$

0.00025 kg 1,4-dichlorobenzene eq." $/ \mathrm{kg}$ 
[E988] benzo[k]fluoranthrene to sea water [E989] benzylchloride to sea water

[E990] beryllium to sea water

[E991] bifenthrin to sea water

[E994] Butylbenzylphtalate to sea water

[E995] cadmium to sea water

[E996] captafol to sea water

[E997] captan to sea water

[E998] carbaryl to sea water

[E999] carbendazim to sea water

[E1000] carbofuran to sea water

[E1001] carbon disulfide to sea water

[E1002] Carcinogenic PAHs to sea water

[E1004] chlordane to sea water

[E1005] chlorfenvinphos to sea water

[E1006] chloridazon to sea water

[E1008] chlorobenzene to sea water

[E1009] chlorothalonil to sea water

[E1010] chlorpropham to sea water

[E1011] chlorpyriphos to sea water

[E1012] chromium III to sea water

[E1013] chromium VI to sea water

[E1014] chrysene to sea water

[E1015] cobalt to sea water

[E1016] copper to sea water

[E1017] coumaphos to sea water

[E1018] cyanazine to sea water

[E1019] cypermethrin to sea water

[E1020] cyromazine to sea water

[E1021] DDT to sea water

[E1022] deltamethrin to sea water

[E1023] demeton to sea water

[E1024] desmetryn to sea water

[E1025] Di(2-ethylhexyl)phtalate to sea water

[E1026] diazinon to sea water

[E1027] Dibutylphtalate to sea water

[E1028] Dichloromethane (Methylene Chloride) to sea water

[E1029] dichlorprop to sea water

[E1030] dichlorvos to sea water

[E1031] dieldrin to sea water

[E1032] Diethylphtalate to sea water

[E1033] Dihexylphtalate to sea water

[E1034] Diisodecylphtalate to sea water

[E1035] Diisooctylphtalate to sea water

[E1036] dimethoate to sea water

[E1037] Dimethylphtalate to sea water

[E1038] dinoseb to sea water

[E1039] dinoterb to sea water

[E1040] Dioctylphtalate to sea water

[E1041] disulfothon to sea water

[E1042] diuron to sea water

[E1043] DNOC to sea water

[E1044] endosulfan to sea water

[E1045] endrin to sea water

[E1046] ethoprophos to sea water

[E1047] ethylbenzene to sea water

[E1048] ethylene to sea water

[E1049] fenitrothion to sea water

[E1050] fenthion to sea water

[E1051] fentin acetate to sea water

[E1052] fentin chloride to sea water

[E1053] fentin hydroxide to sea water

[E1054] fluoranthrene to sea water

[E1055] folpet to sea water

[E1056] Formaldehyde (methanal) to sea water

[E1057] glyphosate to sea water

[E1058] heptachlor to sea water
$0.088 \mathrm{~kg} \mathrm{1,4-dichlorobenzene} \mathrm{eq."} / \mathrm{kg}$ 2.50E-05 kg 1,4-dichlorobenzene eq."/kg 3.90E-16 kg 1,4-dichlorobenzene eq." $/ \mathrm{kg}$ $0.00059 \mathrm{~kg}$ 1,4-dichlorobenzene eq." $/ \mathrm{kg}$ $1.00 \mathrm{E}-07 \mathrm{~kg}$ 1,4-dichlorobenzene eq." $/ \mathrm{kg}$ 1.10E-19 kg 1,4-dichlorobenzene eq."/kg $1.60 \mathrm{E}-08 \mathrm{~kg}$ 1,4-dichlorobenzene eq." $/ \mathrm{kg}$ 9.40E-10 kg 1,4-dichlorobenzene eq." $/ \mathrm{kg}$ 1.10E-09 kg 1,4-dichlorobenzene eq."/kg 1.60E-10 kg 1,4-dichlorobenzene eq."//kg

6.10E-07 kg 1,4-dichlorobenzene eq."/kg

$0.001 \mathrm{~kg}$ 1,4-dichlorobenzene eq." $/ \mathrm{kg}$

$0.00081 \mathrm{~kg}$ 1,4-dichlorobenzene eq." $/ \mathrm{kg}$

$0.28 \mathrm{~kg}$ 1,4-dichlorobenzene eq." $/ \mathrm{kg}$

8.60E-07 kg 1,4-dichlorobenzene eq."/kg

6.40E-05 kg 1,4-dichlorobenzene eq." $/ \mathrm{kg}$

$0.00041 \mathrm{~kg}$ 1,4-dichlorobenzene eq." $/ \mathrm{kg}$

$0.00038 \mathrm{~kg}$ 1,4-dichlorobenzene eq." $/ \mathrm{kg}$

4.50E-07 kg 1,4-dichlorobenzene eq." $/ \mathrm{kg}$

5.70E-05 kg 1,4-dichlorobenzene eq." $/ \mathrm{kg}$

2.00E-18 kg 1,4-dichlorobenzene eq."/kg

2.00E-18 kg 1,4-dichlorobenzene eq." $/ \mathrm{kg}$

$0.0016 \mathrm{~kg} 1,4$-dichlorobenzene eq." $/ \mathrm{kg}$

4.90E-18 kg 1,4-dichlorobenzene eq." $/ \mathrm{kg}$

2.50E-20 kg 1,4-dichlorobenzene eq."//kg

$0.5 \mathrm{~kg}$ 1,4-dichlorobenzene eq." $/ \mathrm{kg}$

4.00E-08 kg 1,4-dichlorobenzene eq." $/ \mathrm{kg}$

$0.25 \mathrm{~kg}$ 1,4-dichlorobenzene eq." $/ \mathrm{kg}$

7.30E-08 kg 1,4-dichlorobenzene eq." $/ \mathrm{kg}$

$0.96 \mathrm{~kg}$ 1,4-dichlorobenzene eq." $/ \mathrm{kg}$

$0.0014 \mathrm{~kg}$ 1,4-dichlorobenzene eq." $/ \mathrm{kg}$

$0.00023 \mathrm{~kg}$ 1,4-dichlorobenzene eq." $/ \mathrm{kg}$

7.50E-07 kg 1,4-dichlorobenzene eq." $/ \mathrm{kg}$

9.60E-07 kg 1,4-dichlorobenzene eq."/kg

8.20E-05 kg 1,4-dichlorobenzene eq." $/ \mathrm{kg}$

2.10E-07 kg 1,4-dichlorobenzene eq." $/ \mathrm{kg}$

6.50E-07 kg 1,4-dichlorobenzene eq." $/ \mathrm{kg}$

1.10E-14 kg 1,4-dichlorobenzene eq." $/ \mathrm{kg}$

$0.00022 \mathrm{~kg}$ 1,4-dichlorobenzene eq."/ $/ \mathrm{kg}$

$0.1 \mathrm{~kg}$ 1,4-dichlorobenzene eq." $/ \mathrm{kg}$

$0.0001 \mathrm{~kg}$ 1,4-dichlorobenzene eq." $/ \mathrm{kg}$

1.70E-05 kg 1,4-dichlorobenzene eq." $/ \mathrm{kg}$

6.40E-05 kg 1,4-dichlorobenzene eq." $/ \mathrm{kg}$

3.50E-06 kg 1,4-dichlorobenzene eq." $/ \mathrm{kg}$

1.80E-07 kg 1,4-dichlorobenzene eq."/kg

4.70E-06 kg 1,4-dichlorobenzene eq."/kg

$0.001 \mathrm{~kg}$ 1,4-dichlorobenzene eq." $/ \mathrm{kg}$

5.10E-05 kg 1,4-dichlorobenzene eq." $/ \mathrm{kg}$

8.80E-08 kg 1,4-dichlorobenzene eq." $/ \mathrm{kg}$

2.10E-05 kg 1,4-dichlorobenzene eq." $/ \mathrm{kg}$

2.10E-05 kg 1,4-dichlorobenzene eq." $/ \mathrm{kg}$

1.50E-09 kg 1,4-dichlorobenzene eq."/kg

1.60E-05 kg 1,4-dichlorobenzene eq." $/ \mathrm{kg}$

$0.38 \mathrm{~kg}$ 1,4-dichlorobenzene eq." $/ \mathrm{kg}$

$0.0072 \mathrm{~kg} 1,4$-dichlorobenzene eq." $/ \mathrm{kg}$

$1.00 \mathrm{E}-07 \mathrm{~kg}$ 1,4-dichlorobenzene eq."/kg

9.90E-14 kg 1,4-dichlorobenzene eq." $/ \mathrm{kg}$

$8.40 \mathrm{E}-05 \mathrm{~kg}$ 1,4-dichlorobenzene eq." $/ \mathrm{kg}$

$0.0017 \mathrm{~kg} 1,4$-dichlorobenzene eq." $/ \mathrm{kg}$

$0.00011 \mathrm{~kg}$ 1,4-dichlorobenzene eq." $/ \mathrm{kg}$

$0.0025 \mathrm{~kg}$ 1,4-dichlorobenzene eq." $/ \mathrm{kg}$

3.80E-05 kg 1,4-dichlorobenzene eq."//kg

0.00096 kg 1,4-dichlorobenzene eq." $/ \mathrm{kg}$

$0.074 \mathrm{~kg} 1,4$-dichlorobenzene eq." $/ \mathrm{kg}$

2.40E-05 kg 1,4-dichlorobenzene eq." $/ \mathrm{kg}$

4.40E-14 kg 1,4-dichlorobenzene eq."/kg

2.40E-05 kg 1,4-dichlorobenzene eq." $/ \mathrm{kg}$ 
[E1059] heptenophos to sea water

[E1060] hexachloro-1,3-butadiene to sea water

[E1061] hexachlorobenzene to sea water

[E1064] indeno[1,2,3-cd]pyrene to sea water

[E1065] iprodione to sea water

[E1066] isoproturon to sea water

[E1067] lead to sea water

[E1068] lindane to sea water

[E1069] linuron to sea water

[E1070] malathion to sea water

[E1072] MCPA to sea water

[E1073] mecoprop to sea water

[E1074] mercury to sea water

[E1075] metamitron to sea water

[E1076] metazachlor to sea water

[E1077] methabenzthiazuron to sea water

[E1078] methomyl to sea water

[E1079] methylbromide to sea water

[E1080] methyl-mercury to sea water

[E1081] metobromuron to sea water

[E1082] metolachlor to sea water

[E1083] mevinphos to sea water

[E1084] molybdenum to sea water

[E1085] m-xylene to sea water

[E1086] naphtalene to sea water

[E1087] nickel to sea water

[E1093] oxamyl to sea water

[E1094] oxydemethon-methyl to sea water

[E1095] o-xylene to sea water

[E1096] parathion-ethyl to sea water

[E1097] parathion-methyl to sea water

[E1098] pentachlorobenzene to sea water

[E1099] pentachloronitrobenzene to sea water

[E1100] pentachlorophenol to sea water

[E1101] permethrin to sea water

[E1102] phenanthrene to sea water

[E1103] phenol to sea water

[E1106] phoxim to sea water

[E1107] Phtalic anhydride to sea water

[E1108] pirimicarb to sea water

[E1110] propachlor to sea water

[E1111] propoxur to sea water

[E1112] propylene oxide to sea water

[E1113] p-xylene to sea water

[E1114] pyrazophos to sea water

[E1115] selenium to sea water

[E1116] simazine to sea water

[E1117] styrene (vinylbenzene) to sea water

[E1120] tetrachloroethylene (PER) to sea water

[E1121] Tetrachloromethane (carbon tetrachloride) $(\mathrm{HC}-10)$ to sea water

[E1122] thallium to sea water

[E1123] Thiram to sea water

[E1124] tin to sea water

[E1125] tolclophos-methyl to sea water

[E1126] toluene to sea water

[E1127] tri-allate to sea water

[E1128] triazophos to sea water

[E1129] tributyltinoxide to sea water

[E1130] trichlorfon to sea water

[E1131] Trichloroethylene (tri) to sea water

[E1132] Trichloromethane=chloroform to sea water

[E1133] trifluarin to sea water

[E1134] vanadium to sea water

[E1135] Vinyl Chloride (chloroethene) to sea water

[E1136] zinc to sea water

[E1137] zineb to sea water

[E1138] 1,1,1-trichloroethane to sea water
2.40E-05 kg 1,4-dichlorobenzene eq."/kg

$2.1 \mathrm{~kg}$ 1,4-dichlorobenzene eq." $/ \mathrm{kg}$

$0.24 \mathrm{~kg} \mathrm{1,4-dichlorobenzene} \mathrm{eq."} / \mathrm{kg}$

4.10E-06 kg 1,4-dichlorobenzene eq."/kg

1.50E-10 kg 1,4-dichlorobenzene eq."/kg

3.80E-07 kg 1,4-dichlorobenzene eq."/kg

4.60E-21 kg 1,4-dichlorobenzene eq."/kg

0.0039 kg 1,4-dichlorobenzene eq."/kg

$0.00031 \mathrm{~kg} \mathrm{1,4-dichlorobenzene} \mathrm{eq."/kg}$

2.00E-07 kg 1,4-dichlorobenzene eq."/kg

2.20E-14 kg 1,4-dichlorobenzene eq."/kg

1.80E-11 kg 1,4-dichlorobenzene eq."/kg

7600 kg 1,4-dichlorobenzene eq."/kg

1.40E-11 kg 1,4-dichlorobenzene eq."/kg

3.00E-08 kg 1,4-dichlorobenzene eq." $/ \mathrm{kg}$

6.00E-07 kg 1,4-dichlorobenzene eq."/kg

7.50E-05 kg 1,4-dichlorobenzene eq." $/ \mathrm{kg}$

$0.00091 \mathrm{~kg}$ 1,4-dichlorobenzene eq." $/ \mathrm{kg}$

$7600 \mathrm{~kg} \mathrm{1,4-dichlorobenzene} \mathrm{eq."} / \mathrm{kg}$

3.80E-05 kg 1,4-dichlorobenzene eq."/kg

5.40E-06 kg 1,4-dichlorobenzene eq."/kg

3.20E-07 kg 1,4-dichlorobenzene eq." $/ \mathrm{kg}$

2.90E-18 kg 1,4-dichlorobenzene eq."/kg

1.10E-07 kg 1,4-dichlorobenzene eq."/kg

1.90E-05 kg 1,4-dichlorobenzene eq."/kg

2.60E-18 kg 1,4-dichlorobenzene eq." $/ \mathrm{kg}$

2.30E-08 kg 1,4-dichlorobenzene eq."/kg

5.20E-06 kg 1,4-dichlorobenzene eq."/kg

2.10E-07 kg 1,4-dichlorobenzene eq."/kg

8.20E-05 kg 1,4-dichlorobenzene eq." $/ \mathrm{kg}$

$0.00071 \mathrm{~kg} 1,4$-dichlorobenzene eq. $" / \mathrm{kg}$

0.026 kg 1,4-dichlorobenzene eq." $/ \mathrm{kg}$

$0.029 \mathrm{~kg} \mathrm{1,4-dichlorobenzene} \mathrm{eq."} / \mathrm{kg}$

2.60E-06 kg 1,4-dichlorobenzene eq."/kg

0.017 kg 1,4-dichlorobenzene eq." $/ \mathrm{kg}$

6.30E-06 kg 1,4-dichlorobenzene eq." $/ \mathrm{kg}$

3.80E-08 kg 1,4-dichlorobenzene eq." $/ \mathrm{kg}$

0.0013 kg 1,4-dichlorobenzene eq." $/ \mathrm{kg}$

2.80E-12 kg 1,4-dichlorobenzene eq."/kg

1.70E-05 kg 1,4-dichlorobenzene eq." $/ \mathrm{kg}$

1.30E-05 kg 1,4-dichlorobenzene eq."/kg

3.20E-06 kg 1,4-dichlorobenzene eq."/kg

1.80E-05 kg 1,4-dichlorobenzene eq."/kg

8.90E-08 kg 1,4-dichlorobenzene eq."/kg

2.90E-05 kg 1,4-dichlorobenzene eq." $/ \mathrm{kg}$

1.80E-17 kg 1,4-dichlorobenzene eq." $/ \mathrm{kg}$

1.90E-05 kg 1,4-dichlorobenzene eq."/kg

2.70E-08 kg 1,4-dichlorobenzene eq."/kg

0.004 kg 1,4-dichlorobenzene eq." $/ \mathrm{kg}$

0.00036 kg 1,4-dichlorobenzene eq."/kg

4.20E-17 kg 1,4-dichlorobenzene eq." $/ \mathrm{kg}$

$0.00031 \mathrm{~kg} 1,4$-dichlorobenzene eq. $" / \mathrm{kg}$

7.20E-21 kg 1,4-dichlorobenzene eq."/kg

6.70E-05 kg 1,4-dichlorobenzene eq." $/ \mathrm{kg}$

1.90E-06 kg 1,4-dichlorobenzene eq."/kg

$0.00013 \mathrm{~kg} 1,4$-dichlorobenzene eq." $/ \mathrm{kg}$

0.00084 kg 1,4-dichlorobenzene eq." $/ \mathrm{kg}$

0.0069 kg 1,4-dichlorobenzene eq."/kg

4.80E-07 kg 1,4-dichlorobenzene eq. "/kg

1.90E-06 kg 1,4-dichlorobenzene eq."/kg

1.90E-05 kg 1,4-dichlorobenzene eq."/kg

0.003 kg 1,4-dichlorobenzene eq." $/ \mathrm{kg}$

2.20E-17 kg 1,4-dichlorobenzene eq."/kg

1.30E-07 kg 1,4-dichlorobenzene eq."/kg

1.90E-20 kg 1,4-dichlorobenzene eq."/kg

2.80E-05 kg 1,4-dichlorobenzene eq." $/ \mathrm{kg}$

$0.0015 \mathrm{~kg} \mathrm{1,4-dichlorobenzene} \mathrm{eq."/kg}$ 
[E1139] 1,2,3,4-tetrachlorobenzene to sea water [E1140] 1,2,3,5-tetrachlorobenzene to sea water [E1141] 1,2,3-trichlorobenzene to sea water

[E1142] 1,2,4,5-tetrachlorobenzene to sea water [E1143] 1,2,4-trichlorobenzene to sea water

[E1144] 1,2-dichlorobenzene to sea water

[E1145] 1,2-dichloroethane to sea water

[E1146] 1,3,5-trichlorobenzene to sea water

[E1147] 1,3-butadiene to sea water

[E1148] 1,3-dichlorobenzene to sea water

[E1149] 1,4-dichlorobenzene to sea water

[E1150] 1-chloro-4-nitrobenzene to sea water

[E1151] 2,3,4,6-tetrachlorophenol to sea water

[E1152] 2,3,7,8-TCDD to sea water

[E1153] 2,4,5-T to sea water

[E1154] 2,4,5-trichlorophenol to sea water

[E1155] 2,4,6-trichlorophenol to agricultural soil

[E1156] 2,4-D to agricultural soil

[E1157] 2,4-dichlorophenol to agricultural soil

[E1158] 2-chlorophenol to agricultural soil

[E1159] 3,4-dichloroaniline to agricultural soil

[E1160] 3-chloroaniline to agricultural soil

[E1161] 4-chloroaniline to agricultural soil

[E1162] acephate to agricultural soil

[E1163] Acrolein (2-propenal) to agricultural soil

[E1164] acrylonitrile to agricultural soil

[E1165] aldicarb to agricultural soil

[E1166] aldrin to agricultural soil

[E1168] anilazine to agricultural soil

[E1169] anthracene to agricultural soil

[E1170] antimony to agricultural soil

[E1171] arsenic to agricultural soil

[E1172] atrazine to agricultural soil

[E1173] azinphos-ethyl to agricultural soil

[E1174] azinphos-methyl to agricultural soil

[E1175] barium to agricultural soil

[E1176] benomyl to agricultural soil

[E1177] bentazone to agricultural soil

[E1178] benzene to agricultural soil

[E1179] benzo[a]anthracene to agricultural soil

[E1180] benzo[a]pyrene to agricultural soil

[E1181] benzo[ghi]perylene to agricultural soil

[E1182] benzo[k]fluoranthrene to agricultural soil

[E1183] benzylchloride to agricultural soil

[E1184] beryllium to agricultural soil

[E1185] bifenthrin to agricultural soil

[E1186] Butylbenzylphtalate to agricultural soil

[E1187] cadmium to agricultural soil

[E1188] captafol to agricultural soil

[E1189] captan to agricultural soil

[E1190] carbaryl to agricultural soil

[E1191] carbendazim to agricultural soil

[E1192] carbofuran to agricultural soil

[E1193] carbon disulfide to agricultural soil

[E1194] Carcinogenic PAHs to agricultural soil

[E1195] chlordane to agricultural soil

[E1196] chlorfenvinphos to agricultural soil

[E1197] chloridazon to agricultural soil

[E1198] chlorobenzene to agricultural soil

[E1199] chlorothalonil to agricultural soil

[E1200] chlorpropham to agricultural soil

[E1201] chlorpyriphos to agricultural soil

[E1202] chromium III to agricultural soil

[E1203] chromium VI to agricultural soil

[E1204] chrysene to agricultural soil

[E1205] cobalt to agricultural soil

[E1206] copper to agricultural soil
$0.83 \mathrm{~kg}$ 1,4-dichlorobenzene eq." $/ \mathrm{kg}$

15 kg 1,4-dichlorobenzene eq."//kg

$9.3 \mathrm{~kg}$ 1,4-dichlorobenzene eq." $/ \mathrm{kg}$

$19 \mathrm{~kg}$ 1,4-dichlorobenzene eq." $/ \mathrm{kg}$

$1.2 \mathrm{~kg} 1,4$-dichlorobenzene eq." $/ \mathrm{kg}$

0.054 kg 1,4-dichlorobenzene eq." $/ \mathrm{kg}$

0.0017 kg 1,4-dichlorobenzene eq." $/ \mathrm{kg}$

$0.25 \mathrm{~kg} \mathrm{1,4-dichlorobenzene} \mathrm{eq."} / \mathrm{kg}$

$0.00031 \mathrm{~kg}$ 1,4-dichlorobenzene eq." $/ \mathrm{kg}$

$0.062 \mathrm{~kg}$ 1,4-dichlorobenzene eq." $/ \mathrm{kg}$

$1 \mathrm{~kg}$ 1,4-dichlorobenzene eq." $/ \mathrm{kg}$

17 kg 1,4-dichlorobenzene eq."/kg

$1 \mathrm{~kg}$ 1,4-dichlorobenzene eq. "/kg

27000 kg 1,4-dichlorobenzene eq." $/ \mathrm{kg}$

$0.74 \mathrm{~kg} 1,4$-dichlorobenzene eq." $/ \mathrm{kg}$

$4.4 \mathrm{~kg}$ 1,4-dichlorobenzene eq." $/ \mathrm{kg}$

$0.7 \mathrm{~kg} \mathrm{1,4-dichlorobenzene} \mathrm{eq."} / \mathrm{kg}$

$1.6 \mathrm{~kg}$ 1,4-dichlorobenzene eq." $/ \mathrm{kg}$

$0.59 \mathrm{~kg}$ 1,4-dichlorobenzene eq." $/ \mathrm{kg}$

$0.38 \mathrm{~kg}$ 1,4-dichlorobenzene eq." $/ \mathrm{kg}$

26 kg 1,4-dichlorobenzene eq." $/ \mathrm{kg}$

$1.4 \mathrm{~kg}$ 1,4-dichlorobenzene eq."/kg

$16 \mathrm{~kg} \mathrm{1,4-dichlorobenzene} \mathrm{eq."} / \mathrm{kg}$

$1.7 \mathrm{~kg} \mathrm{1,4-dichlorobenzene} \mathrm{eq."} / \mathrm{kg}$

7000 kg 1,4-dichlorobenzene eq." $/ \mathrm{kg}$

$2.5 \mathrm{~kg}$ 1,4-dichlorobenzene eq." $/ \mathrm{kg}$

$4200 \mathrm{~kg}$ 1,4-dichlorobenzene eq." $/ \mathrm{kg}$

$20 \mathrm{~kg} \mathrm{1,4-dichlorobenzene} \mathrm{eq."} / \mathrm{kg}$

$0.23 \mathrm{~kg}$ 1,4-dichlorobenzene eq." $/ \mathrm{kg}$

8.9 kg 1,4-dichlorobenzene eq." $/ \mathrm{kg}$

$1.3 \mathrm{~kg}$ 1,4-dichlorobenzene eq." $/ \mathrm{kg}$

3300 kg 1,4-dichlorobenzene eq." $/ \mathrm{kg}$

$6.6 \mathrm{~kg}$ 1,4-dichlorobenzene eq." $/ \mathrm{kg}$

$220 \mathrm{~kg}$ 1,4-dichlorobenzene eq." $/ \mathrm{kg}$

$0.97 \mathrm{~kg}$ 1,4-dichlorobenzene eq." $/ \mathrm{kg}$

$10 \mathrm{~kg} 1,4$-dichlorobenzene eq." $/ \mathrm{kg}$

$3.5 \mathrm{~kg}$ 1,4-dichlorobenzene eq." $/ \mathrm{kg}$

$0.59 \mathrm{~kg}$ 1,4-dichlorobenzene eq." $/ \mathrm{kg}$

$0.0034 \mathrm{~kg}$ 1,4-dichlorobenzene eq." $/ \mathrm{kg}$

$31 \mathrm{~kg}$ 1,4-dichlorobenzene eq."/kg

$23 \mathrm{~kg}$ 1,4-dichlorobenzene eq. "/kg

$8.3 \mathrm{~kg}$ 1,4-dichlorobenzene eq."//kg

$390 \mathrm{~kg}$ 1,4-dichlorobenzene eq." $/ \mathrm{kg}$

$0.8 \mathrm{~kg}$ 1,4-dichlorobenzene eq." $/ \mathrm{kg}$

$3600 \mathrm{~kg}$ 1,4-dichlorobenzene eq. "//kg

$83 \mathrm{~kg}$ 1,4-dichlorobenzene eq." $/ \mathrm{kg}$

$0.01 \mathrm{~kg}$ 1,4-dichlorobenzene eq." $/ \mathrm{kg}$

170 kg 1,4-dichlorobenzene eq."//kg

28 kg 1,4-dichlorobenzene eq." $/ \mathrm{kg}$

$0.041 \mathrm{~kg}$ 1,4-dichlorobenzene eq." $/ \mathrm{kg}$

$0.11 \mathrm{~kg}$ 1,4-dichlorobenzene eq." $/ \mathrm{kg}$

49 kg 1,4-dichlorobenzene eq." $/ \mathrm{kg}$

$7.5 \mathrm{~kg}$ 1,4-dichlorobenzene eq." $/ \mathrm{kg}$

$1.6 \mathrm{~kg}$ 1,4-dichlorobenzene eq." $/ \mathrm{kg}$

$6.3 \mathrm{~kg}$ 1,4-dichlorobenzene eq." $/ \mathrm{kg}$

$74 \mathrm{~kg} \mathrm{1,4-dichlorobenzene} \mathrm{eq."} / \mathrm{kg}$

$1.3 \mathrm{~kg}$ 1,4-dichlorobenzene eq." $/ \mathrm{kg}$

$0.9 \mathrm{~kg}$ 1,4-dichlorobenzene eq." $/ \mathrm{kg}$

$0.12 \mathrm{~kg} 1,4$-dichlorobenzene eq." $/ \mathrm{kg}$

$0.68 \mathrm{~kg}$ 1,4-dichlorobenzene eq. "/kg

$0.13 \mathrm{~kg}$ 1,4-dichlorobenzene eq." $/ \mathrm{kg}$

17 kg 1,4-dichlorobenzene eq."/kg

$6300 \mathrm{~kg}$ 1,4-dichlorobenzene eq." $/ \mathrm{kg}$

6300 kg 1,4-dichlorobenzene eq."//kg

$4.6 \mathrm{~kg}$ 1,4-dichlorobenzene eq." $/ \mathrm{kg}$

$220 \mathrm{~kg}$ 1,4-dichlorobenzene eq " $/ \mathrm{kg}$

14 kg 1,4-dichlorobenzene eq." $/ \mathrm{kg}$ 
[E1207] coumaphos to agricultural soil [E1208] cyanazine to agricultural soil [E1209] cypermethrin to agricultural soil [E1210] cyromazine to agricultural soil [E1211] DDT to agricultural soil [E1212] deltamethrin to agricultural soil [E1213] demeton to agricultural soil

[E1214] desmetryn to agricultural soil

[E1215] Di(2-ethylhexyl)phtalate to agricultural soil

[E1216] diazinon to agricultural soil

[E1217] Dibutylphtalate to agricultural soil

[E1218] Dichloromethane (Methylene Chloride) to agricultural soil

[E1219] dichlorprop to agricultural soil

[E1220] dichlorvos to agricultural soil

[E1221] dieldrin to agricultural soil

[E1222] Diethylphtalate to agricultural soil

[E1223] Dihexylphtalate to agricultural soil

[E1224] Diisodecylphtalate to agricultural soil

[E1225] Diisooctylphtalate to agricultural soil

[E1226] dimethoate to agricultural soil

[E1227] Dimethylphtalate to agricultural soil

[E1228] dinoseb to agricultural soil

[E1229] dinoterb to agricultural soil

[E1230] Dioctylphtalate to agricultural soil

[E1231] disulfothon to agricultural soil

[E1232] diuron to agricultural soil

[E1233] DNOC to agricultural soil

[E1234] endosulfan to agricultural soil

[E1235] endrin to agricultural soil

[E1236] ethoprophos to agricultural soil

[E1237] ethylbenzene to agricultural soil

[E1238] ethylene to agricultural soil

[E1239] fenitrothion to agricultural soil

[E1240] fenthion to agricultural soil

[E1241] fentin acetate to agricultural soil

[E1242] fentin chloride to agricultural soil

[E1243] fentin hydroxide to agricultural soil

[E1244] fluoranthrene to agricultural soil

[E1245] folpet to agricultural soil

[E1246] Formaldehyde (methanal) to agricultural soil

[E1247] glyphosate to agricultural soi

[E1248] heptachlor to agricultural soi

[E1249] heptenophos to agricultural soil

[E1250] hexachloro-1,3-butadiene to agricultural soil

[E1251] hexachlorobenzene to agricultural soil

[E1254] indeno[1,2,3-cd]pyrene to agricultural soil

[E1255] iprodione to agricultural soil

[E1256] isoproturon to agricultural soil

[E1257] lead to agricultural soil

[E1258] lindane to agricultural soil

[E1259] linuron to agricultural soil

[E1260] malathion to agricultural soil

[E1261] MCPA to agricultural soil

[E1262] mecoprop to agricultural soil

[E1263] mercury to agricultural soil

[E1264] metamitron to agricultural soil

[E1265] metazachlor to agricultural soil

[E1266] methabenzthiazuron to agricultural soil

[E1267] methomyl to agricultural soil

[E1268] methylbromide to agricultural soil

[E1269] methyl-mercury to agricultural soil

[E1270] metobromuron to agricultural soil

[E1271] metolachlor to agricultural soil

[E1272] mevinphos to agricultural soil

[E1273] molybdenum to agricultural soil

[E1274] m-xylene to agricultural soil

[E1275] naphtalene to agricultural soil
16000 kg 1,4-dichlorobenzene eq."/kg

$69 \mathrm{~kg} \mathrm{1,4-dichlorobenzene} \mathrm{eq."} / \mathrm{kg}$

$90000 \mathrm{~kg}$ 1,4-dichlorobenzene eq." $/ \mathrm{kg}$

$630 \mathrm{~kg} \mathrm{1,4-dichlorobenzene} \mathrm{eq."} / \mathrm{kg}$

$60 \mathrm{~kg}$ 1,4-dichlorobenzene eq." $/ \mathrm{kg}$

$8.5 \mathrm{~kg}$ 1,4-dichlorobenzene eq." $/ \mathrm{kg}$

60 kg 1,4-dichlorobenzene eq." $/ \mathrm{kg}$

$2.9 \mathrm{~kg}$ 1,4-dichlorobenzene eq."//kg

0.0014 kg 1,4-dichlorobenzene eq." $/ \mathrm{kg}$

$12 \mathrm{~kg}$ 1,4-dichlorobenzene eq." $/ \mathrm{kg}$

$0.023 \mathrm{~kg} \mathrm{1,4-dichlorobenzene} \mathrm{eq."/kg}$

$0.00025 \mathrm{~kg}$ 1,4-dichlorobenzene eq." $/ \mathrm{kg}$

$0.0014 \mathrm{~kg}$ 1,4-dichlorobenzene eq." $/ \mathrm{kg}$

$200 \mathrm{~kg}$ 1,4-dichlorobenzene eq." $/ \mathrm{kg}$

$110 \mathrm{~kg}$ 1,4-dichlorobenzene eq." $/ \mathrm{kg}$

$2.1 \mathrm{~kg}$ 1,4-dichlorobenzene eq." $/ \mathrm{kg}$

$0.0073 \mathrm{~kg}$ 1,4-dichlorobenzene eq." $/ \mathrm{kg}$

$0.004 \mathrm{~kg} \mathrm{1,4-dichlorobenzene} \mathrm{eq."} / \mathrm{kg}$

0.00055 kg 1,4-dichlorobenzene eq." $/ \mathrm{kg}$

$0.8 \mathrm{~kg} \mathrm{1,4-dichlorobenzene} \mathrm{eq."} / \mathrm{kg}$

$1.4 \mathrm{~kg}$ 1,4-dichlorobenzene eq." $/ \mathrm{kg}$

590 kg 1,4-dichlorobenzene eq." $/ \mathrm{kg}$

$9.9 \mathrm{~kg}$ 1,4-dichlorobenzene eq." $/ \mathrm{kg}$

4.80E-05 kg 1,4-dichlorobenzene eq."/kg

$11 \mathrm{~kg}$ 1,4-dichlorobenzene eq." $/ \mathrm{kg}$

$11 \mathrm{~kg}$ 1,4-dichlorobenzene eq." $/ \mathrm{kg}$

$0.52 \mathrm{~kg}$ 1,4-dichlorobenzene eq." $/ \mathrm{kg}$

$2.7 \mathrm{~kg}$ 1,4-dichlorobenzene eq." $/ \mathrm{kg}$

$4200 \mathrm{~kg} \mathrm{1,4-dichlorobenzene} \mathrm{eq."/kg}$

$270 \mathrm{~kg}$ 1,4-dichlorobenzene eq." $/ \mathrm{kg}$

$0.0019 \mathrm{~kg}$ 1,4-dichlorobenzene eq."/kg

2.30E-09 kg 1,4-dichlorobenzene eq." $/ \mathrm{kg}$

83 kg 1,4-dichlorobenzene eq." $/ / k g$

290 kg 1,4-dichlorobenzene eq." $/ \mathrm{kg}$

$12 \mathrm{~kg}$ 1,4-dichlorobenzene eq." $/ \mathrm{kg}$

12 kg 1,4-dichlorobenzene eq." $/ / k g$

$12 \mathrm{~kg}$ 1,4-dichlorobenzene eq."/kg

$2.3 \mathrm{~kg}$ 1,4-dichlorobenzene eq." $/ \mathrm{kg}$

$110 \mathrm{~kg}$ 1,4-dichlorobenzene eq." $/ \mathrm{kg}$

$5.8 \mathrm{~kg}$ 1,4-dichlorobenzene eq." $/ \mathrm{kg}$

$0.096 \mathrm{~kg}$ 1,4-dichlorobenzene eq." $/ \mathrm{kg}$

$5.5 \mathrm{~kg}$ 1,4-dichlorobenzene eq." $/ \mathrm{kg}$

$16 \mathrm{~kg} 1,4$-dichlorobenzene eq." $/ \mathrm{kg}$

$53 \mathrm{~kg}$ 1,4-dichlorobenzene eq."/kg

$3.5 \mathrm{~kg}$ 1,4-dichlorobenzene eq."//kg

$13 \mathrm{~kg}$ 1,4-dichlorobenzene eq." $/ \mathrm{kg}$

$0.14 \mathrm{~kg}$ 1,4-dichlorobenzene eq." $/ \mathrm{kg}$

$6.4 \mathrm{~kg} \mathrm{1,4-dichlorobenzene} \mathrm{eq."} / \mathrm{kg}$

$33 \mathrm{~kg}$ 1,4-dichlorobenzene eq." $/ \mathrm{kg}$

$23 \mathrm{~kg}$ 1,4-dichlorobenzene eq. "/ $/ \mathrm{kg}$

$21 \mathrm{~kg}$ 1,4-dichlorobenzene eq." $/ \mathrm{kg}$

$0.076 \mathrm{~kg}$ 1,4-dichlorobenzene eq." $/ \mathrm{kg}$

$0.094 \mathrm{~kg}$ 1,4-dichlorobenzene eq." $/ \mathrm{kg}$

$4.7 \mathrm{~kg}$ 1,4-dichlorobenzene eq." $/ \mathrm{kg}$

$56000 \mathrm{~kg}$ 1,4-dichlorobenzene eq." $/ \mathrm{kg}$

$0.042 \mathrm{~kg}$ 1,4-dichlorobenzene eq." $/ \mathrm{kg}$

$0.17 \mathrm{~kg}$ 1,4-dichlorobenzene eq." $/ \mathrm{kg}$

$1.1 \mathrm{~kg}$ 1,4-dichlorobenzene eq." $/ \mathrm{kg}$

$300 \mathrm{~kg}$ 1,4-dichlorobenzene eq." $/ \mathrm{kg}$

$0.36 \mathrm{~kg}$ 1,4-dichlorobenzene eq." $/ \mathrm{kg}$

$56000 \mathrm{~kg}$ 1,4-dichlorobenzene eq." $/ \mathrm{kg}$

$2.2 \mathrm{~kg}$ 1,4-dichlorobenzene eq." $/ \mathrm{kg}$

$0.54 \mathrm{~kg} 1,4$-dichlorobenzene eq. "/kg

$87 \mathrm{~kg}$ 1,4-dichlorobenzene eq." $/ \mathrm{kg}$

36 kg 1,4-dichlorobenzene eq."/ $/ \mathrm{kg}$

$0.003 \mathrm{~kg}$ 1,4-dichlorobenzene eq." $/ \mathrm{kg}$

$3.1 \mathrm{~kg}$ 1,4-dichlorobenzene eq." $/ \mathrm{kg}$ 
[E1276] nickel to agricultural soil

[E1278] oxamyl to agricultural soil

[E1279] oxydemethon-methyl to agricultural soil

[E1280] o-xylene to agricultural soil

[E1281] parathion-ethyl to agricultural soil

[E1282] parathion-methyl to agricultural soil

[E1283] pentachlorobenzene to agricultural soil

[E1284] pentachloronitrobenzene to agricultural soil

[E1285] pentachlorophenol to agricultural soil

[E1286] permethrin to agricultural soil

[E1287] phenanthrene to agricultural soil

[E1288] phenol to agricultural soil

[E1289] phoxim to agricultural soil

[E1290] Phtalic anhydride to agricultural soil

[E1291] pirimicarb to agricultural soil

[E1293] propachlor to agricultural soil

[E1294] propoxur to agricultural soil

[E1295] propylene oxide to agricultural soil

[E1296] p-xylene to agricultural soil

[E1297] pyrazophos to agricultural soil

[E1298] selenium to agricultural soil

[E1299] simazine to agricultural soil

[E1300] styrene (vinylbenzene) to agricultural soil

[E1302] tetrachloroethylene (PER) to agricultural soil

[E1303] Tetrachloromethane (carbon tetrachloride) $(\mathrm{HC}-10)$ to agricultural soil

[E1304] thallium to agricultural soil

[E1305] Thiram to agricultural soil

[E1306] tin to agricultural soil

[E1307] tolclophos-methyl to agricultural soil

[E1308] toluene to agricultural soil

[E1309] tri-allate to agricultural soil

[E1310] triazophos to agricultural soil

[E1311] tributyltinoxide to agricultural soil

[E1312] trichlorfon to agricultural soil

[E1313] Trichloroethylene (tri) to agricultural soil

[E1314] Trichloromethane=chloroform to agricultural soil

[E1315] trifluarin to agricultural soil

[E1316] vanadium to agricultural soil

[E1317] Vinyl Chloride (chloroethene) to agricultural soil

[E1318] zinc to agricultural soil

[E1319] zineb to agricultural soil

[E1320] 1,1,1-trichloroethane to agricultural soil

[E1321] 1,2,3,4-tetrachlorobenzene to agricultural soil

[E1322] 1,2,3,5-tetrachlorobenzene to agricultural soil

[E1323] 1,2,3-trichlorobenzene to agricultural soil

[E1324] 1,2,4,5-tetrachlorobenzene to agricultural soil

[E1325] 1,2,4-trichlorobenzene to agricultural soil

[E1326] 1,2-dichlorobenzene to agricultural soil

[E1327] 1,2-dichloroethane to agricultural soil

[E1328] 1,3,5-trichlorobenzene to agricultural soil

[E1329] 1,3-butadiene to agricultural soil

[E1330] 1,3-dichlorobenzene to agricultural soil

[E1331] 1,4-dichlorobenzene to agricultural soil

[E1332] 1-chloro-4-nitrobenzene to agricultural soil

[E1333] 2,3,4,6-tetrachlorophenol to agricultural soil

[E1334] 2,3,7,8-TCDD to agricultural soil

[E1335] 2,4,5-T to agricultural soil

[E1336] 2,4,5-trichlorophenol to agricultural soil

[E1337] 2,4,6-trichlorophenol to industrial soil

[E1338] 2,4-D to industrial soil

[E1339] 2,4-dichlorophenol to industrial soil

[E1340] 2-chlorophenol to industrial soil

[E1341] 3,4-dichloroaniline to industrial soil

[E1342] 3-chloroaniline to industrial soil

[E1343] 4-chloroaniline to industrial soil

[E1344] acephate to industrial soil

[E1345] Acrolein (2-propenal) to industrial soil
240 kg 1,4-dichlorobenzene eq." $/ \mathrm{kg}$

5.9 kg 1,4-dichlorobenzene eq." $/ \mathrm{kg}$

92 kg 1,4-dichlorobenzene eq."/kg

0.0034 kg 1,4-dichlorobenzene eq."/kg

17 kg 1,4-dichlorobenzene eq." $/ \mathrm{kg}$

81 kg 1,4-dichlorobenzene eq."/kg

$2.1 \mathrm{~kg}$ 1,4-dichlorobenzene eq."/kg

2.7 kg 1,4-dichlorobenzene eq." $/ \mathrm{kg}$

$4.8 \mathrm{~kg} \mathrm{1,4-dichlorobenzene} \mathrm{eq."/kg}$

2250 kg 1,4-dichlorobenzene eq." $/ \mathrm{kg}$

0.037 kg 1,4-dichlorobenzene eq." $/ \mathrm{kg}$

0.045 kg 1,4-dichlorobenzene eq." $/ \mathrm{kg}$

$4.7 \mathrm{~kg} \mathrm{1,4-dichlorobenzene} \mathrm{eq."/kg}$

0.0026 kg 1,4-dichlorobenzene eq."/kg

$120 \mathrm{~kg}$ 1,4-dichlorobenzene eq." $/ \mathrm{kg}$

$2.5 \mathrm{~kg} \mathrm{1,4-dichlorobenzene} \mathrm{eq."/kg}$

1800 kg 1,4-dichlorobenzene eq." $/ \mathrm{kg}$

0.14 kg 1,4-dichlorobenzene eq." $/ \mathrm{kg}$

0.0015 kg 1,4-dichlorobenzene eq."/kg

$30 \mathrm{~kg}$ 1,4-dichlorobenzene eq."/kg

$110 \mathrm{~kg}$ 1,4-dichlorobenzene eq."/kg

29 kg 1,4-dichlorobenzene eq."/kg

0.0014 kg 1,4-dichlorobenzene eq."/kg

$0.3 \mathrm{~kg} \mathrm{1,4-dichlorobenzene} \mathrm{eq."} / \mathrm{kg}$

0.0021 kg 1,4-dichlorobenzene eq."/kg

700 kg 1,4-dichlorobenzene eq."/kg

51 kg 1,4-dichlorobenzene eq."/kg

$30 \mathrm{~kg} \mathrm{1,4-dichlorobenzene} \mathrm{eq."/kg}$

$1.8 \mathrm{~kg} 1,4$-dichlorobenzene eq."/kg

0.019 kg 1,4-dichlorobenzene eq."/kg

$1.3 \mathrm{~kg} \mathrm{1,4-dichlorobenzene} \mathrm{eq."} / \mathrm{kg}$

250 kg 1,4-dichlorobenzene eq."/kg

37 kg 1,4-dichlorobenzene eq."/kg

1900 kg 1,4-dichlorobenzene eq."/kg

0.0021 kg 1,4-dichlorobenzene eq."/kg

0.0016 kg 1,4-dichlorobenzene eq."/kg

35 kg 1,4-dichlorobenzene eq."/kg

1400 kg 1,4-dichlorobenzene eq." $/ \mathrm{kg}$

$0.00031 \mathrm{~kg}$ 1,4-dichlorobenzene eq."/kg

12 kg 1,4-dichlorobenzene eq."/kg

16 kg 1,4-dichlorobenzene eq."/kg

0.0015 kg 1,4-dichlorobenzene eq."/kg

0.77 kg 1,4-dichlorobenzene eq." $/ \mathrm{kg}$

12 kg 1,4-dichlorobenzene eq." $/ \mathrm{kg}$

$8 \mathrm{~kg}$ 1,4-dichlorobenzene eq."/kg

17 kg 1,4-dichlorobenzene eq."/kg

0.99 kg 1,4-dichlorobenzene eq."/kg

$0.054 \mathrm{~kg} \mathrm{1,4-dichlorobenzene} \mathrm{eq."/kg}$

0.0017 kg 1,4-dichlorobenzene eq."/kg

$0.22 \mathrm{~kg} \mathrm{1,4-dichlorobenzene} \mathrm{eq."} / \mathrm{kg}$

$0.00031 \mathrm{~kg} \mathrm{1,4-dichlorobenzene} \mathrm{eq."} / \mathrm{kg}$

0.062 kg 1,4-dichlorobenzene eq."/kg

$1 \mathrm{~kg}$ 1,4-dichlorobenzene eq."/kg

17 kg 1,4-dichlorobenzene eq."/kg

0.97 kg 1,4-dichlorobenzene eq." $/ \mathrm{kg}$

27000 kg 1,4-dichlorobenzene eq."/kg

0.64 kg 1,4-dichlorobenzene eq." $/ \mathrm{kg}$

$3.9 \mathrm{~kg} \mathrm{1,4-dichlorobenzene} \mathrm{eq."} / \mathrm{kg}$

0.68 kg 1,4-dichlorobenzene eq."/kg

$1.1 \mathrm{~kg}$ 1,4-dichlorobenzene eq." $/ \mathrm{kg}$

0.54 kg 1,4-dichlorobenzene eq." $/ \mathrm{kg}$

0.37 kg 1,4-dichlorobenzene eq."/kg

18 kg 1,4-dichlorobenzene eq."/kg

1.2 kg 1,4-dichlorobenzene eq."/kg

11 kg 1,4-dichlorobenzene eq."/kg

1.3 kg 1,4-dichlorobenzene eq." $/ \mathrm{kg}$

7000 kg 1,4-dichlorobenzene eq."/kg 
[E1346] acrylonitrile to industríal soil [E1347] aldicarb to industrial soil [E1348] aldrin to industrial soil [E1350] anilazine to industrial soil [E1351] anthracene to industrial soil [E1352] antimony to industrial soil [E1353] arsenic to industrial soil [E1354] atrazine to industrial soil [E1355] azinphos-ethyl to industrial soil [E1356] azinphos-methyl to industrial soil [E1357] barium to industrial soil [E1358] benomyl to industrial soil [E1359] bentazone to industrial soil [E1360] benzene to industrial soil [E1361] benzo[a]anthracene to industrial soil [E1362] benzo[a]pyrene to industrial soil

[E1363] benzo[ghi]perylene to industrial soil

$[\mathrm{E} 1364]$ benzo[k]fluoranthrene to industrial soil

[E1365] benzylchloride to industrial soil

[E1366] beryllium to industrial soil

[E1367] bifenthrin to industrial soil

[E1368] Butylbenzyiphtalate to industrial soil

[E1369] cadmium to industrial soil

[E1370] captafol to industrial soil

[E1371] captan to industrial soil

[E1372] carbaryl to industrial soil

[E1373] carbendazim to industrial soil

[E1374] carbofuran to industrial soil

[E1375] carbon disulfide to industrial soil

[E1376] Carcinogenic PAHs to industrial soil

[E1377] chlordane to industrial soil

[E1378] chlorfenvinphos to industrial soil

[E1379] chloridazon to industrial soil

[E1380] chlorobenzene to industrial soil

[E1381] chlorothalonil to industrial soil

[E1382] chlorpropham to industrial soil

[E1383] chlorpyriphos to industrial soil

[E1384] chromium III to industrial soil

[E1385] chromium VI to industrial soil

[E1386] chrysene to industrial soil

[E1387] cobalt to industrial soil

[E1388] copper to industrial soil

[E1389] coumaphos to industrial soil

[E1390] cyanazine to industrial soil

[E1391] cypermethrin to industrial soil

[E1392] cyromazine to industrial soil

[E1393] DDT to industrial soil

[E1394] deltamethrin to industrial soil

[E1395] demeton to industrial soil

[E1396] desmetryn to industrial soil

[E1397] Di(2-ethylhexyl)phtalate to industrial soil

[E1398] diazinon to industrial soil

[E1399] Dibutylphtalate to industrial soil

[E1400] Dichloromethane (Methylene Chloride) to industrial soil

[E1401] dichlorprop to industrial soil

[E1402] dichlorvos to industrial soil

[E1403] dieldrin to industrial soil

[E1404] Diethylphtalate to industrial soil

[E1405] Dihexylphtalate to industrial soil

[E1406] Diisodecylphtalate to industrial soil

[E1407] Diisooctylphtalate to industrial soil

[E1408] dimethoate to industrial soil

[E1409] Dimethylphtalate to industrial soil

[E1410] dinoseb to industrial soil

[E1411] dinoterb to industrial soi

[E1412] Dioctylphtalate to industrial soil

[E1413] disulfothon to industrial soil
$2.1 \mathrm{~kg}$ 1,4-dichlorobenzene eq." $/ \mathrm{kg}$ $4200 \mathrm{~kg}$ 1,4-dichlorobenzene eq." $/ \mathrm{kg}$

$20 \mathrm{~kg}$ 1,4-dichlorobenzene eq." $/ \mathrm{kg}$

$0.23 \mathrm{~kg} \mathrm{1,4-dichlorobenzene} \mathrm{eq."} / \mathrm{kg}$

$8.8 \mathrm{~kg}$ 1,4-dichlorobenzene eq." $/ \mathrm{kg}$

$1.3 \mathrm{~kg}$ 1,4-dichlorobenzene eq."/kg

$3300 \mathrm{~kg}$ 1,4-dichlorobenzene eq." $/ \mathrm{kg}$

$4.4 \mathrm{~kg}$ 1,4-dichlorobenzene eq." $/ \mathrm{kg}$

$72 \mathrm{~kg}$ 1,4-dichlorobenzene eq."/kg

1 kg 1,4-dichlorobenzene eq. "/kg

$10 \mathrm{~kg} 1,4$-dichlorobenzene eq." $/ \mathrm{kg}$

$3.5 \mathrm{~kg}$ 1,4-dichlorobenzene eq." $/ \mathrm{kg}$

$0.5 \mathrm{~kg}$ 1,4-dichlorobenzene eq." $/ \mathrm{kg}$

$0.0034 \mathrm{~kg} 1,4$-dichlorobenzene eq." $/ \mathrm{kg}$

$31 \mathrm{~kg}$ 1,4-dichlorobenzene eq." $/ \mathrm{kg}$

$23 \mathrm{~kg} \mathrm{1,4-dichlorobenzene} \mathrm{eq."/kg}$

$8.3 \mathrm{~kg}$ 1,4-dichlorobenzene eq. "/kg

$390 \mathrm{~kg}$ 1,4-dichlorobenzene eq."/kg

$0.71 \mathrm{~kg}$ 1,4-dichlorobenzene eq." $/ \mathrm{kg}$

$3600 \mathrm{~kg}$ 1,4-dichlorobenzene eq."//kg

$83 \mathrm{~kg}$ 1,4-dichlorobenzene eq." $/ \mathrm{kg}$

$0.01 \mathrm{~kg} 1,4$-dichlorobenzene eq. " $/ \mathrm{kg}$

170 kg 1,4-dichlorobenzene eq."/kg

$22 \mathrm{~kg}$ 1,4-dichlorobenzene eq." $/ \mathrm{kg}$

$0.12 \mathrm{~kg}$ 1,4-dichlorobenzene eq." $/ \mathrm{kg}$

0.14 kg 1,4-dichlorobenzene eq." $/ \mathrm{kg}$

$38 \mathrm{~kg}$ 1,4-dichlorobenzene eq." $/ \mathrm{kg}$

$5.9 \mathrm{~kg}$ 1,4-dichlorobenzene eq." $/ \mathrm{kg}$

$1.6 \mathrm{~kg} \mathrm{1,4-dichlorobenzene} \mathrm{eq."} / \mathrm{kg}$

$6.3 \mathrm{~kg}$ 1,4-dichlorobenzene eq." $/ \mathrm{kg}$

$73 \mathrm{~kg}$ 1,4-dichlorobenzene eq."/kg

$1.2 \mathrm{~kg} \mathrm{1,4-dichlorobenzene} \mathrm{eq."} / \mathrm{kg}$

$0.68 \mathrm{~kg}$ 1,4-dichlorobenzene eq." $/ \mathrm{kg}$

$0.12 \mathrm{~kg} \mathrm{1,4-dichlorobenzene} \mathrm{eq.} / \mathrm{kg}$

$0.61 \mathrm{~kg}$ 1,4-dichlorobenzene eq." $/ \mathrm{kg}$

$0.12 \mathrm{~kg}$ 1,4-dichlorobenzene eq." $/ \mathrm{kg}$

$17 \mathrm{~kg}$ 1,4-dichlorobenzene eq." $/ \mathrm{kg}$

$6300 \mathrm{~kg}$ 1,4-dichlorobenzene eq." $/ \mathrm{kg}$

$6300 \mathrm{~kg}$ 1,4-dichlorobenzene eq."//kg

$4.5 \mathrm{~kg}$ 1,4-dichlorobenzene eq. "/kg

220 kg 1,4-dichlorobenzene eq." $/ \mathrm{kg}$

$14 \mathrm{~kg}$ 1,4-dichlorobenzene eq." $/ \mathrm{kg}$

$12000 \mathrm{~kg}$ 1,4-dichlorobenzene eq."/kg

$63 \mathrm{~kg}$ 1,4-dichlorobenzene eq." $/ \mathrm{kg}$

$78000 \mathrm{~kg}$ 1,4-dichlorobenzene eq." $/ \mathrm{kg}$

$630 \mathrm{~kg}$ 1,4-dichlorobenzene eq."/kg

$59 \mathrm{~kg} \mathrm{1,4-dichlorobenzene} \mathrm{eq."} / \mathrm{kg}$

$8.5 \mathrm{~kg}$ 1,4-dichlorobenzene eq." $/ \mathrm{kg}$

$49 \mathrm{~kg}$ 1,4-dichlorobenzene eq."//kg

$2.6 \mathrm{~kg}$ 1,4-dichlorobenzene eq." $/ \mathrm{kg}$

$0.0014 \mathrm{~kg}$ 1,4-dichlorobenzene eq."/kg

$10 \mathrm{~kg} \mathrm{1,4-dichlorobenzene} \mathrm{eq."/kg}$

$0.023 \mathrm{~kg}$ 1,4-dichlorobenzene eq." $/ \mathrm{kg}$

$0.00025 \mathrm{~kg}$ 1,4-dichlorobenzene eq." $/ \mathrm{kg}$

0.0014 kg 1,4-dichlorobenzene eq." $/ / \mathrm{kg}$

$200 \mathrm{~kg}$ 1,4-dichlorobenzene eq." $/ \mathrm{kg}$

$100 \mathrm{~kg}$ 1,4-dichlorobenzene eq. "/kg

$2.1 \mathrm{~kg}$ 1,4-dichlorobenzene eq. $/ \mathrm{kg}$

$0.0073 \mathrm{~kg}$ 1,4-dichlorobenzene eq. "/ $/ \mathrm{kg}$

$0.004 \mathrm{~kg}$ 1,4-dichlorobenzene eq." $/ \mathrm{kg}$

$0.00055 \mathrm{~kg}$ 1,4-dichlorobenzene eq." $/ \mathrm{kg}$

$0.62 \mathrm{~kg}$ 1,4-dichlorobenzene eq. "/ $\mathrm{kg}$

$1.4 \mathrm{~kg}$ 1,4-dichlorobenzene eq." $/ \mathrm{kg}$

$420 \mathrm{~kg}$ 1,4-dichlorobenzene eq." $/ \mathrm{kg}$

$9.9 \mathrm{~kg} \mathrm{1,4-dichlorobenzene} \mathrm{eq."} / \mathrm{kg}$

4.80E-05 kg 1,4-dichlorobenzene eq."/kg

$11 \mathrm{~kg}$ 1,4-dichlorobenzene eq." $/ \mathrm{kg}$ 
[E1414] diuron to industrial soil

[E1415] DNOC to industrial soil

[E1416] endosulfan to industrial soil

[E1417] endrin to industrial soil

[E1418] ethoprophos to industrial soil

[E1419] ethylbenzene to industrial soil

[E1420] ethylene to industrial soil

[E1421] fenitrothion to industrial soil

[E1422] fenthion to industrial soil

[E1423] fentin acetate to industrial soil

[E1424] fentin chloride to industrial soil

[E1425] fentin hydroxide to industrial soil

[E1426] fluoranthrene to industrial soil

[E1427] folpet to industrial soil

[E1428] Formaldehyde (methanal) to industrial soil

[E1429] glyphosate to industrial soil

[E1430] heptachlor to industrial soil

[E1431] heptenophos to industrial soil

[E1432] hexachloro-1,3-butadiene to industrial soil

[E1433] hexachlorobenzene to industrial soil

[E1436] indeno[1,2,3-cd]pyrene to industrial soil

[E1437] iprodione to industrial soil

[E1438] isoproturon to industrial soil

[E1439] lead to industrial soil

[E1440] lindane to industrial soil

[E1441] linuron to industrial soil

[E1442] malathion to industrial soil

[E1443] MCPA to industrial soi

[E1444] mecoprop to industrial soil

[E1445] mercury to industrial soil

[E1446] metamitron to industrial soil

[E1447] metazachlor to industrial soil

[E1448] methabenzthiazuron to industrial soil

[E1449] methomyl to industrial soil

[E1450] methylbromide to industrial soil

[E1451] methyl-mercury to industrial soi

[E1452] metobromuron to industrial soil

[E1453] metolachlor to industrial soil

[E1454] mevinphos to industrial soil

[E1455] molybdenum to industrial soil

[E1456] m-xylene to industrial soil

[E1457] naphtalene to industrial soil

[E1458] nickel to industrial soil

[E1460] oxamyl to industrial soil

[E1461] oxydemethon-methyl to industrial soil

[E1462] o-xylene to industrial soil

[E1463] parathion-ethyl to industrial soil

[E1464] parathion-methyl to industrial soil

[E1465] pentachlorobenzene to industrial soil

[E1466] pentachloronitrobenzene to industrial soil

[E1467] pentachlorophenol to industrial soil

[E1468] permethrin to industrial soil

[E1469] phenanthrene to industrial soil

[E1470] phenol to industrial soil

[E1471] phoxim to industrial soil

[E1472] Phtalic anhydride to industrial soil

[E1473] pirimicarb to industrial soil

[E1475] propachlor to industrial soil

[E1476] propoxur to industrial soil

[E1477] propylene oxide to industrial soil

[E1478] p-xylene to industrial soil

[E1479] pyrazophos to industrial soil

[E1480] selenium to industrial soil

[E1481] simazine to industrial soil

[E1482] styrene (vinylbenzene) to industrial soil

[E1484] tetrachloroethylene (PER) to industrial soil

[E1485] Tetrachloromethane (carbon tetrachloride) $(\mathrm{HC}-10)$ to industrial soil
11 kg 1,4-dichlorobenzene eq."/kg

$0.49 \mathrm{~kg} 1,4$-dichlorobenzene eq." $/ \mathrm{kg}$

$2.8 \mathrm{~kg}$ 1,4-dichlorobenzene eq." $/ \mathrm{kg}$

3600 kg 1,4-dichlorobenzene eq." $/ \mathrm{kg}$

$190 \mathrm{~kg}$ 1,4-dichlorobenzene eq. "/kg

$0.0019 \mathrm{~kg} \mathrm{1,4-dichlorobenzene} \mathrm{eq."} / \mathrm{kg}$

2.30E-09 kg 1,4-dichlorobenzene eq." $/ \mathrm{kg}$

$81 \mathrm{~kg}$ 1,4-dichlorobenzene eq."/kg

$280 \mathrm{~kg}$ 1,4-dichlorobenzene eq."/kg

11 kg 1,4-dichlorobenzene eq."/kg

$11 \mathrm{~kg}$ 1,4-dichlorobenzene eq."/kg

$11 \mathrm{~kg} \mathrm{1,4-dichlorobenzene} \mathrm{eq."} / \mathrm{kg}$

$2.3 \mathrm{~kg} \mathrm{1,4-dichlorobenzene} \mathrm{eq."} / \mathrm{kg}$

78 kg 1,4-dichlorobenzene eq." $/ \mathrm{kg}$

4.4 kg 1,4-dichlorobenzene eq." $/ \mathrm{kg}$

0.096 kg 1,4-dichlorobenzene eq." $/ \mathrm{kg}$

$5.3 \mathrm{~kg}$ 1,4-dichlorobenzene eq."/kg

$16 \mathrm{~kg} \mathrm{1,4-dichlorobenzene} \mathrm{eq."/kg}$

47 kg 1,4-dichlorobenzene eq."/kg

3 kg 1,4-dichlorobenzene eq."/kg

$13 \mathrm{~kg} \mathrm{1,4-dichlorobenzene} \mathrm{eq."/kg}$

$0.3 \mathrm{~kg} \mathrm{1,4-dichlorobenzene} \mathrm{eq."} / \mathrm{kg}$

$4.6 \mathrm{~kg}$ 1,4-dichlorobenzene eq." $/ \mathrm{kg}$

33 kg 1,4-dichlorobenzene eq."/kg

22 kg 1,4-dichlorobenzene eq."/kg

18 kg 1,4-dichlorobenzene eq."/kg

0.075 kg 1,4-dichlorobenzene eq." $/ \mathrm{kg}$

0.086 kg 1,4-dichlorobenzene eq." $/ \mathrm{kg}$

$3.3 \mathrm{~kg} \mathrm{1,4-dichlorobenzene} \mathrm{eq."} / \mathrm{kg}$

56000 kg 1,4-dichlorobenzene eq."/kg

0.038 kg 1,4-dichlorobenzene eq." $/ \mathrm{kg}$

$0.15 \mathrm{~kg} \mathrm{1,4-dichlorobenzene} \mathrm{eq."} / \mathrm{kg}$

$0.88 \mathrm{~kg}$ 1,4-dichlorobenzene eq." $/ \mathrm{kg}$

220 kg 1,4-dichlorobenzene eq."/kg

$0.37 \mathrm{~kg} \mathrm{1,4-dichlorobenzene} \mathrm{eq."/kg}$

56000 kg 1,4-dichlorobenzene eq."/kg

$2.2 \mathrm{~kg}$ 1,4-dichlorobenzene eq." $/ \mathrm{kg}$

0.41 kg 1,4-dichlorobenzene eq."/kg

$90 \mathrm{~kg} \mathrm{1,4-dichlorobenzene} \mathrm{eq."/kg}$

36 kg 1,4-dichlorobenzene eq."/kg

0.003 kg 1,4-dichlorobenzene eq."/kg

$2.6 \mathrm{~kg} \mathrm{1,4-dichlorobenzene} \mathrm{eq."/kg}$

240 kg 1,4-dichlorobenzene eq." $/ \mathrm{kg}$

6 kg 1,4-dichlorobenzene eq." $/ \mathrm{kg}$

85 kg 1,4-dichlorobenzene eq."/kg

0.0034 kg 1,4-dichlorobenzene eq." $/ \mathrm{kg}$

17 kg 1,4-dichlorobenzene eq."/kg

79 kg 1,4-dichlorobenzene eq."/kg

$1.7 \mathrm{~kg}$ 1,4-dichlorobenzene eq."/kg

2.6 kg 1,4-dichlorobenzene eq." $/ \mathrm{kg}$

$4.8 \mathrm{~kg} \mathrm{1,4-dichlorobenzene} \mathrm{eq."} / \mathrm{kg}$

$250 \mathrm{~kg}$ 1,4-dichlorobenzene eq." $/ \mathrm{kg}$

0.037 kg 1,4-dichlorobenzene eq."/kg

$0.041 \mathrm{~kg} \mathrm{1,4-dichlorobenzene} \mathrm{eq."} / \mathrm{kg}$

$3.8 \mathrm{~kg} \mathrm{1,4-dichlorobenzene} \mathrm{eq."} / \mathrm{kg}$

$0.00042 \mathrm{~kg} \mathrm{1,4-dichlorobenzene} \mathrm{eq."/kg}$

94 kg 1,4-dichlorobenzene eq."/kg

2.3 kg 1,4-dichlorobenzene eq."/kg

1300 kg 1,4-dichlorobenzene eq." $/ \mathrm{kg}$

$0.12 \mathrm{~kg} \mathrm{1,4-dichlorobenzene} \mathrm{eq."} / \mathrm{kg}$

0.0015 kg 1,4-dichlorobenzene eq." $/ \mathrm{kg}$

29 kg 1,4-dichlorobenzene eq."/kg

110 kg 1,4-dichlorobenzene eq."/kg

21 kg 1,4-dichlorobenzene eq."/kg

$0.0012 \mathrm{~kg}$ 1,4-dichlorobenzene eq." $/ \mathrm{kg}$

$0.3 \mathrm{~kg} \mathrm{1,4-dichlorobenzene} \mathrm{eq.} \mathrm{"} / \mathrm{kg}$

0.0021 kg 1,4-dichlorobenzene eq."/kg 
[E1486] thallium to industrial soil

[E1487] Thiram to industrial soil

[E1488] tin to industrial soil

[E1489] tolclophos-methyl to industrial soil

[E1490] toluene to industrial soil

[E1491] tri-allate to industrial soil

[E1492] triazophos to industrial soil

[E1493] tributyltinoxide to industrial soil

[E1494] trichlorfon to industrial soil

[E1495] Trichloroethylene (tri) to industrial soil

[E1496] Trichloromethane=chloroform to industrial soil

[E1497] trifluarin to industrial soil

[E1498] vanadium to industrial soil

[E1499] Vinyl Chloride (chloroethene) to industrial soil

[E1500] zinc to industrial soil

[E1501] zineb to industrial soil
700 kg 1,4-dichlorobenzene eq." $/ \mathrm{kg}$

$81 \mathrm{~kg} \mathrm{1,4-dichlorobenzene} \mathrm{eq."/kg}$

$30 \mathrm{~kg} \mathrm{1,4-dichlorobenzene} \mathrm{eq."} / \mathrm{kg}$

1.5 kg 1,4-dichlorobenzene eq." $/ \mathrm{kg}$

0.019 kg 1,4-dichlorobenzene eq." $/ \mathrm{kg}$

$1.3 \mathrm{~kg} \mathrm{1,4-dichlorobenzene} \mathrm{eq."/kg}$

200 kg 1,4-dichlorobenzene eq."/kg

$37 \mathrm{~kg}$ 1,4-dichlorobenzene eq." $/ \mathrm{kg}$

2600 kg 1,4-dichlorobenzene eq."/kg

$0.0021 \mathrm{~kg} 1,4$-dichlorobenzene eq." $/ \mathrm{kg}$

0.0016 kg 1,4-dichlorobenzene eq." $/ \mathrm{kg}$

34 kg 1,4-dichlorobenzene eq." $/ \mathrm{kg}$

1400 kg 1,4-dichlorobenzene eq."/kg

0.00031 kg 1,4-dichlorobenzene eq."/kg

25 kg 1,4-dichlorobenzene eq."/kg

15 kg 1,4-dichlorobenzene eq."/kg

Category $=[$ C12] Acidification (Huijbregts, 1999)

Description = Problem oriented approach, manual 1999; AP; average Europe total, A\&B

Author $=$ Huijbregts, 1999

Date $=10 / 11 / 99$

Environmental resources

Environmental resource

Value

Environmental emissions

Environmental emission

Value

[E250] ammonia, ammonium to air

[E643] nitrogen oxides to air

[E709] sulphur dioxide to air

Category $=[$ C13] Eutrophication

Description = Problem oriented approach, manual 1999; EP

Author = Heijungs et al 1992

Date $=10 / 11 / 99$

Environmental resources

Environmental resource

Value

Environmental emissions

Environmental emission

Value

[E250] ammonia, ammonium to air

[E636] nitrate to air

[E640] nitrogen to air

[E641] nitrogen dioxide to air

[E642] nitrogen mono oxide to air

[E643] nitrogen oxides to air

[E676] phosphate to air

[E678] Phosphorus to air

[E779] ammonia, ammonium to fresh water

[E809] Chemical oxigen demand (COD) to fresh water

[E894] Nitrate to fresh water

[E896] Nitrogen to fresh water

[E897] nitrogen dioxide to fresh water

[E910] Phosphate to fresh water

[E911] Phosphorus to fresh water

[E973] ammonia, ammonium to sea water

[E1003] Chemical oxigen demand (COD) to sea water

[E1088] Nitrate to sea water

[E1090] Nitrogen to sea water

[E1091] nitrogen dioxide to sea water

[E1104] Phosphate to sea water

[E1105] Phosphorus to sea water

[E1167] ammonia to agricultural soil

[E1277] nitrogen dioxide to agricultural soil

$1.3 \mathrm{~kg} \mathrm{SO} 2 \mathrm{eq} . / \mathrm{kg}$

$0.41 \mathrm{~kg} \mathrm{SO} 2$ eq. $/ \mathrm{kg}$

$1 \mathrm{~kg} \mathrm{SO} 2$ eq. $/ \mathrm{kg}$

Unit

Unit

Unit

$0.35 \mathrm{~kg}$ PO4-- eq. $/ \mathrm{kg}$

$0.1 \mathrm{~kg} \mathrm{PO} 4--$ eq. $/ \mathrm{kg}$

$0.42 \mathrm{~kg}$ PO4-- eq. $/ \mathrm{kg}$

$0.13 \mathrm{~kg} \mathrm{PO} 4-\mathrm{-eq} . / \mathrm{kg}$

$0.2 \mathrm{~kg} \mathrm{PO} 4--$ eq. $/ \mathrm{kg}$

$0.13 \mathrm{~kg}$ PO4--- eq. $/ \mathrm{kg}$

$1 \mathrm{~kg} \mathrm{PO} 4--$ eq. $/ \mathrm{kg}$

$3.06 \mathrm{~kg}$ PO4-- eq. $/ \mathrm{kg}$

$0.35 \mathrm{~kg}$ PO4--- eq. $/ \mathrm{kg}$

$0.022 \mathrm{~kg} \mathrm{PO} 4---$ eq. $/ \mathrm{kg}$

$0.1 \mathrm{~kg} \mathrm{PO} 4--\mathrm{eq} . \mathrm{kg}$

$0.42 \mathrm{~kg}$ PO4--- eq. $/ \mathrm{kg}$

$0.13 \mathrm{~kg} \mathrm{PO} 4---$ eq. $/ \mathrm{kg}$

$1 \mathrm{~kg} \mathrm{PO} 4--\mathrm{eq} . / \mathrm{kg}$

$3.06 \mathrm{~kg} \mathrm{PO} 4---$ eq. $/ \mathrm{kg}$

$0.35 \mathrm{~kg}$ PO4-.- eq. $/ \mathrm{kg}$

$0.022 \mathrm{~kg}$ PO4-- eq. $/ \mathrm{kg}$

$0.1 \mathrm{~kg}$ PO4--- eq. $/ \mathrm{kg}$

$0.42 \mathrm{~kg}$ PO4--- eq. $/ \mathrm{kg}$

$0.13 \mathrm{~kg} \mathrm{PO} 4---$ eq. $/ \mathrm{kg}$

$1 \mathrm{~kg} \mathrm{PO} 4-\mathrm{eq} . / \mathrm{kg}$

$3.06 \mathrm{~kg} \mathrm{PO} 4-.-\mathrm{eq} . / \mathrm{kg}$

$0.35 \mathrm{~kg}$ PO4-.- eq. $/ \mathrm{kg}$

$0.13 \mathrm{~kg} \mathrm{PO} 4--\mathrm{eq} . / \mathrm{kg}$ 
[E1349] ammonia to industrial soil

[E1459] nitrogen dioxide to industrial soil

[E1765] nitrate to agricultural soil

[E1766] nitrate to industrial soil

[E1767] nitrogen to agricultural soil

[E1768] nitrogen to industrial soil

[E1769] nitrogen mono oxide to fresh water

[E1770] nitrogen mono oxide to sea water

[E1771] nitrogen oxides to agricultural soil

[E1772] nitrogen oxides to industrial soil

[E1773] nitrogen oxides to fresh water

[E1774] nitrogen oxides to sea water

[E1775] Phosphate to agricultural soil

[E1776] Phosphate to industrial soil

[E1777] Phosphorus to agricultural soil

[E1778] Phosphorus to industrial soil
$0.35 \mathrm{~kg}$ PO4-- eq. $/ \mathrm{kg}$

$0.13 \mathrm{~kg} P O 4--$ eq. $/ \mathrm{kg}$

$0.1 \mathrm{~kg}$ PO4--- eq. $/ \mathrm{kg}$

$0.1 \mathrm{~kg}$ PO4-- eq. $/ \mathrm{kg}$

$0.42 \mathrm{~kg}$ PO4-- eq. $/ \mathrm{kg}$

0.42 kg PO4-- eq. $/ \mathrm{kg}$

$0.2 \mathrm{~kg}$ PO $4-\mathrm{eq} . \mathrm{kg}$

$0.2 \mathrm{~kg}$ PO4-- eq. $/ \mathrm{kg}$

$0.13 \mathrm{~kg}$ PO4-- eq. $/ \mathrm{kg}$

$0.13 \mathrm{~kg}$ PO4-- eq. $/ \mathrm{kg}$

$0.13 \mathrm{~kg}$ PO4-- eq. $/ \mathrm{kg}$

$0.13 \mathrm{~kg}$ PO4-- eq. $/ \mathrm{kg}$

$1 \mathrm{~kg}$ PO4-- eq. $/ \mathrm{kg}$

$1 \mathrm{~kg}$ PO4-- eq. $/ \mathrm{kg}$

$3.06 \mathrm{~kg}$ PO4-- eq. $/ \mathrm{kg}$

3.06 kg PO4-- eq./kg

Category $=[$ C14 $]$ Odour

Description = Problem oriented approach, manual 1999; malodourous air (1/OTV)

Author $=$ Roos, 1989

Date $=27-09-1999$

Environmental resources

Environmental resource

Value

Unit

Environmental emissions

Environmental emission

[E90] 1,1,1-trichloroethane (methyl chloroform; HC-140a) to air

[E100] 1,2,4-trimethylbenzene to air

[E109] 1,3,5-trimethylbenzene (mesitylene) to air

[E120] 1-Butanol to air

[E123] 1-Butyl Acetate to air

[E126] 1-butylpropionate to air

[E189] 2-ethyl-5,5-dimethyl-1,3-dioxane to air

[E204] 2-methylpropanoic acid (isobutyric acid) to air

[E221] 3-methylbutanoic acid (isovaleric acid) to air

[E232] Acetaldehyde (ethanal) to air

[E233] Acetic acid to air

[E234] Acetone (2-propanon) to air

[E236] Acrolein (2-propenal) to air

[E250] ammonia, ammonium to air

[E288] butanoic acid (butyric acid) to air

[E289] butylacrylate to air

[E291] Butyraldehyde (butanal) to air

[E394] carbon disulfide to air

[E407] chlorobenzene to air

[E440] decaline to air

[E449] Dichloromethane (Methylene Chloride) to air

[E454] diethylamine to air

[E467] dimethylamine to air

[E484] ethanethiol (ethylmercaptan) to air

[E485] Ethanol to air

[E488] Ethyl Acetate to air

[E490] Ethyl Acrylate to air

[E497] ethylbutyrate to air

[E503] ethylthioethane (diethylsulfide) to air

[E511] Formaldehyde (methanal) to air

[E563] hydrogen sulfide to air

[E571] isobutanol (2-methylpropanol-1) to air

[E572] isobutene (2-methylpropene) to air

[E577] isopentylacetate (iso-amylacetate) to air

[E581] isopropyl benzene (cumene) to air

[E582] isopropyl propionate to air

[E593] meta-Cresol (3-methylphenol) to air

[E596] meta-Xylene (1,3-dimethylbenzene) to air

[E601] methanethiol (methylmercaptan) to air

Value

Unit

$1.89 \mathrm{E}+05 \mathrm{~m} 3 / \mathrm{kg}$

$7.14 \mathrm{E}+06 \mathrm{~m} 3 / \mathrm{kg}$

$5.56 \mathrm{E}+06 \mathrm{~m} 3 / \mathrm{kg}$

$1.30 \mathrm{E}+07 \mathrm{~m} 3 / \mathrm{kg}$

$3.23 \mathrm{E}+07 \mathrm{~m} 3 / \mathrm{kg}$

$1.16 \mathrm{E}+07 \mathrm{~m} 3 / \mathrm{kg}$

$1.79 \mathrm{E}+11 \mathrm{~m} 3 / \mathrm{kg}$

$2.00 \mathrm{E}+08 \mathrm{~m} 3 / \mathrm{kg}$

$4.55 \mathrm{E}+09 \mathrm{~m} 3 / \mathrm{kg}$

$3.70 \mathrm{E}+09 \mathrm{~m} 3 / \mathrm{kg}$

$1.64 \mathrm{E}+07 \mathrm{~m} 3 / \mathrm{kg}$

$13900 \mathrm{~m} 3 / \mathrm{kg}$

$1.45 \mathrm{E}+07 \mathrm{~m} 3 / \mathrm{kg}$

$1.00 \mathrm{E}+06 \mathrm{~m} 3 / \mathrm{kg}$

$2.86 \mathrm{E}+09 \mathrm{~m} 3 / \mathrm{kg}$

$6.67 \mathrm{E}+08 \mathrm{~m} 3 / \mathrm{kg}$

$1.19 \mathrm{E}+09 \mathrm{~m} 3 / \mathrm{kg}$

$5.56 \mathrm{E}+06 \mathrm{~m} 3 / \mathrm{kg}$

$1.00 \mathrm{E}+06 \mathrm{~m} 3 / \mathrm{kg}$

$3.57 \mathrm{E}+05 \mathrm{~m} 3 / \mathrm{kg}$

$1560 \mathrm{~m} 3 / \mathrm{kg}$

$1.11 \mathrm{E}+07 \mathrm{~m} 3 / \mathrm{kg}$

$7.14 \mathrm{E}+08 \mathrm{~m} 3 / \mathrm{kg}$

$2.27 \mathrm{E}+10 \mathrm{~m} 3 / \mathrm{kg}$

$1.56 \mathrm{E}+06 \mathrm{~m} 3 / \mathrm{kg}$

$4.76 \mathrm{E}+05 \mathrm{~m} 3 / \mathrm{kg}$

$1.22 \mathrm{E}+09 \mathrm{~m} 3 / \mathrm{kg}$

$3.33 \mathrm{E}+10 \mathrm{~m} 3 / \mathrm{kg}$

$7.14 \mathrm{E}+08 \mathrm{~m} 3 / \mathrm{kg}$

$2.04 \mathrm{E}+06 \mathrm{~m} 3 / \mathrm{kg}$

$2.33 \mathrm{E}+09 \mathrm{~m} 3 / \mathrm{kg}$

$2.86 \mathrm{E}+07 \mathrm{~m} 3 / \mathrm{kg}$

$66700 \mathrm{~m} 3 / \mathrm{kg}$

1.33E+07 m3/kg

$1.37 \mathrm{E}+07 \mathrm{~m} 3 / \mathrm{kg}$

$3.13 \mathrm{E}+06 \mathrm{~m} 3 / \mathrm{kg}$

$1.75 \mathrm{E}+09 \mathrm{~m} 3 / \mathrm{kg}$

$1.85 \mathrm{E}+06 \mathrm{~m} 3 / \mathrm{kg}$

$4.17 \mathrm{E}+09 \mathrm{~m} 3 / \mathrm{kg}$ 
[E602] Methanol to air

$13700 \mathrm{~m} 3 / \mathrm{kg}$

[E604] Methyl Acetate to air

$45500 \mathrm{~m} 3 / \mathrm{kg}$

[E606] Methyl Acrylate to air

[E608] Methyl Bromide to air

$1.00 \mathrm{E}+08 \mathrm{~m} 3 / \mathrm{kg}$

$8.33 \mathrm{E}+08 \mathrm{~m} 3 / \mathrm{kg}$

[E612] methyl dithiomethane to air

$6.67 \mathrm{E}+08 \mathrm{~m} 3 / \mathrm{kg}$

[E613] Methyl Ethyl Ketone to air

[E616] Methyl Isobutyl Ketone (4-methylpentanon-2) to air

[E618] methyl methacrylate to air

[E621] methyl propionate to air

[E623] methyl thiomethane (dimethylsulfide) to air

[E648] ortho-Cresol (2-methylphenol) to air

[E650] ortho-Xylene (1,2-dimethylbenzene) to air

[E653] para-Cresol (4-methylphenol) to air

[E658] para-Xylene (1,4-dimethylbenzene) to air

[E675] Phenol to air

[E688] Propanoic acid (propionic acid) to air

[E689] Propionaldehyde (propanal) to air

[E699] pyridine to air

[E706] styrene (vinylbenzene) to air

[E714] terephthaloyldichloride to air

[E718] tetrachloroethylene (PER) (tetrachloroethene) to air

$1.47 \mathrm{E}+06 \mathrm{~m} 3 / \mathrm{kg}$

$1.45 \mathrm{E}+06 \mathrm{~m} 3 / \mathrm{kg}$

$1.59 \mathrm{E}+06 \mathrm{~m} 3 / \mathrm{kg}$

$2.86 \mathrm{E}+05 \mathrm{~m} 3 / \mathrm{kg}$

$3.33 \mathrm{E}+09 \mathrm{~m} 3 / \mathrm{kg}$

$5.56 \mathrm{E}+08 \mathrm{~m} 3 / \mathrm{kg}$

$1.28 \mathrm{E}+06 \mathrm{~m} 3 / \mathrm{kg}$

$5.56 \mathrm{E}+09 \mathrm{~m} 3 / \mathrm{kg}$

$1.92 \mathrm{E}+06 \mathrm{~m} 3 / \mathrm{kg}$

$2.56 \mathrm{E}+07 \mathrm{~m} 3 / \mathrm{kg}$

$1.92 \mathrm{E}+08 \mathrm{~m} 3 / \mathrm{kg}$

$2.86 \mathrm{E}+08 \mathrm{~m} 3 / \mathrm{kg}$

$8.33 \mathrm{E}+06 \mathrm{~m} 3 / \mathrm{kg}$

$1.47 \mathrm{E}+07 \mathrm{~m} 3 / \mathrm{kg}$

$3.13 \mathrm{E}+08 \mathrm{~m} 3 / \mathrm{kg}$

1. $20 \mathrm{E}+05 \mathrm{~m} 3 / \mathrm{kg}$

$2.63 \mathrm{E}+05 \mathrm{~m} 3 / \mathrm{kg}$

$2.56 \mathrm{E}+05 \mathrm{~m} 3 / \mathrm{kg}$

[E737] Trichloroethylene (tri) to air

[E740] Trimethylamine to air

$3.85 \mathrm{E}+09 \mathrm{~m} 3 / \mathrm{kg}$

[E742] Valeraldehyde (pentanal) to air

4.17E+08 $\mathrm{m} 3 / \mathrm{kg}$

Category $=[\mathrm{C} 15]$ Costs of environmental impacts (Extern E)

Description = Impact pathway approach; Externalities of energy

Author $=$ European Commission, 1996

Date $=27-09-1999$

Environmental resources

Environmental resource

Value

Unit

Environmental emissions

Environmental emission

[E250] ammonia, ammonium to air

Value Unit

$4.28 \mathrm{ecu} / \mathrm{kg}$

0.004 ecu $/ \mathrm{kg}$

$0.007 \mathrm{ecu} / \mathrm{kg}$

$1.47 \mathrm{ecu} / \mathrm{kg}$

0.086 ecu $/ \mathrm{kg}$

3.69 ecu/kg

$3.69 \mathrm{ecu} / \mathrm{kg}$

7.3 ecu/kg

$5.07 \mathrm{ecu} / \mathrm{kg}$

$12.3 \mathrm{ecu} / \mathrm{kg}$

[E709] sulphur dioxide to air

[E741] TSP to air

Category $=[C 16]$ Costs of environmental impacts (EPS)

Description $=$ EPS

Author $=$ Steen, 1996

Date $=27-09-1999$

Environmental resources

Environmental resource

[E2] aluminium (AI) from earth resources

[E5] arsenic (As) from earth resources

[E8] bismuth (Bi) from earth resources

[E11] cadmium (Cd) from earth resources

[E16] cobalt (Co) from earth resources

[E17] copper $(\mathrm{Cu})$ from earth resources

[E18] chromium ( $\mathrm{Cr}$ ) from earth resources

[E26] gold $(\mathrm{Au})$ from earth resources

[E33] iron ( $\mathrm{Fe})$ from earth resources

[E38] lead $(\mathrm{Pb})$ from earth resources

[E41] manganese $(\mathrm{Mn})$ from earth resources

Value

Unit

$0.42 \mathrm{elu} / \mathrm{kg}$

$1900 \mathrm{elu} / \mathrm{kg}$

$4.00 \mathrm{E}+05$ elu $/ \mathrm{kg}$

$23000 \mathrm{elu} / \mathrm{kg}$

$136 \mathrm{elu} / \mathrm{kg}$

57 elu/kg

33 elu/kg

$8.50 \mathrm{E}+05$ elu $/ \mathrm{kg}$

$0.68 \mathrm{elu} / \mathrm{kg}$

240 elu/kg

$3.6 \mathrm{elu} / \mathrm{kg}$ 
[E42] mercury $(\mathrm{Hg})$ from earth resources [E43] molybdenum (Mo) from earth resources [E46] nickel (Ni) from earth resources [E51] platinum (Pt) from earth resources [E58] rhodium $(R h)$ from earth resources [E65] silver $(\mathrm{Ag})$ from earth resources [E75] tin (Sn) from earth resources [E76] titanium (Ti) from earth resources

[E77] tungsten (W); wolfraam from earth resources

[E78] uranium (U) from earth resources

[E79] vanadium (V) from earth resources

[E83] zinc $(\mathrm{Zn})$ from earth resources

[E84] Zirconium (Zr) from earth resources

[E85] oil crude from earth resources

[E87] coal soft from earth resources

[E88] coal hard from earth resources

[E89] coal total from earth resources

\section{Environmental emissions}

Environmental emission

[E252] anthracene to air

[E255] arsenic to air

[E264] benzo[a]anthracene to air

[E265] benzo[a]pyrene to air

[E266] benzo[ghi]perylene to air

[E267] benzo[k]fluoranthrene to air

[E385] cadmium to air

[E393] Carbon dioxide to air

[E395] Carbon Monoxide to air

[E398] CFC-11 to air

[E413] chromium (unspecified) to air

[E414] chromium III to air

[E415] chromium VI to air

[E416] chrysene to air

[E469] Dinitrogen oxide (nitrous oxide) to air

[E499] Ethylene (ethene) to air

[E509] fluoranthrene to air

[E563] hydrogen sulfide to air

[E566] indeno[1,2,3-cd]pyrene to air

[E584] lead to air

[E592] mercury to air

[E600] Methane to air

[E633] Naphtalene to air

[E641] nitrogen dioxide to air

[E643] nitrogen oxides to air

[E674] phenanthrene to air

[E683] PM10 to air

[E684] Polycyclic Aromatic Hydrocarbons (PAH) (unspecified) to air

[E685] Polycyclic Aromatic Hydrocarbons Carcinogenic- (carcinogenic-PAH) to air

[E709] sulphur dioxide to air

[E741] TSP to air

[E798] Biological Oxygen Demand (BOD) to fresh water

[E809] Chemical oxigen demand (COD) to fresh water

[E894] Nitrate to fresh water

[E896] Nitrogen to fresh water

[E910] Phosphate to fresh water

[E911] Phosphorus to fresh water

[E992] Biological Oxygen Demand (BOD) to sea water

[E1003] Chemical oxigen demand (COD) to sea water

[E1088] Nitrate to sea water

[E1090] Nitrogen to sea water

[E1104] Phosphate to sea water

[E1105] Phosphorus to sea water

\author{
40000 elu/kg \\ 2800 elu/kg \\ 40 elu/kg \\ $6.80 \mathrm{E}+05$ elu $/ \mathrm{kg}$ \\ $3.40 \mathrm{E}+06$ elu $/ \mathrm{kg}$ \\ $45000 \mathrm{elu} / \mathrm{kg}$ \\ 1480 elu/kg \\ 0.602 elu $/ \mathrm{kg}$ \\ 2720 elu/kg \\ 1260 elu/kg \\ $28.3 \mathrm{elu} / \mathrm{kg}$ \\ 49 elu/kg \\ 20.6 elu $/ \mathrm{kg}$ \\ $0.5 \mathrm{elu} / \mathrm{kg}$ \\ 0.05 elu/kg \\ 0.05 elu $/ \mathrm{kg}$ \\ 0.05 elu $/ \mathrm{kg}$
}

Value

Unit

8800 elu/kg

10 elu/kg

8800 elu/kg

8800 elu/kg

8800 elu/kg

$8800 \mathrm{elu} / \mathrm{kg}$

$21.2 \mathrm{elu} / \mathrm{kg}$

0.0636 elu/kg

$0.191 \mathrm{elu} / \mathrm{kg}$

$216 \mathrm{elu} / \mathrm{kg}$

$0.8 \mathrm{elu} / \mathrm{kg}$

$0.8 \mathrm{elu} / \mathrm{kg}$

$0.8 \mathrm{elu} / \mathrm{kg}$

8800 elu/kg

$20.3 \mathrm{elu} / \mathrm{kg}$

$3.4 \mathrm{elu} / \mathrm{kg}$

8800 elu/kg

0.142 elu $/ \mathrm{kg}$

8800 elu/kg

291 elu/kg

177 elu/kg

$1.56 \mathrm{elu} / \mathrm{kg}$

8800 elu/kg

$0.395 \mathrm{elu} / \mathrm{kg}$

0.395 elu/kg

8800 elu/kg

0.0071 elu $/ \mathrm{kg}$

$8800 \mathrm{elu} / \mathrm{kg}$

8800 elu/kg

0.0545 elu $/ \mathrm{kg}$

0.0071 elu $/ \mathrm{kg}$

0.0075 elu $/ \mathrm{kg}$

0.006 elu $/ \mathrm{kg}$

0.00226 elu $/ \mathrm{kg}$

$0.01 \mathrm{elu} / \mathrm{kg}$

0.0245 elu $/ \mathrm{kg}$

$0.075 \mathrm{elu} / \mathrm{kg}$

0.0075 elu $/ \mathrm{kg}$

$0.006 \mathrm{elu} / \mathrm{kg}$

$0.00226 \mathrm{elu} / \mathrm{kg}$

0.01 elu $/ \mathrm{kg}$

0.0245 elu $/ \mathrm{kg}$

0.075 elu/kg

Category $=[$ C17 $]$ Ecoscarcity Swiss

Description = Distance to target approach, ecoscarcity in Switzerland 
Author $=$ Ahbe et al, 1990

Date $=$ 27-09-1999

Environmental resources

Environmental resource

Value

Unit

Environmental emissions

Environmental emission

[E90] 1,1,1-trichloroethane (methyl chloroform; HC-140a) to air

[E91] 1,1,2-Trichloroethane to air

[E92] 1,1-Dichloroethane to air

[E93] 1,1-Dichloroethene to air

[E94] 1,2,3,4-tetrachlorobenzene to air

[E95] 1,2,3,5-tetrachlorobenzene to air

[E96] 1,2,3-trichlorobenzene to air

[E97] 1,2,3-Trimethyl Benzene to air

[E98] 1,2,4,5-tetrachlorobenzene to air

[E99] 1,2,4-trichlorobenzene to air

[E100] 1,2,4-trimethylbenzene to air

[E101] 1,2-Butanediol to air

[E102] 1,2-dichlorobenzene to air

[E103] 1,2-dichloroethane to air

[E104] 1,2-Dichloropropane to air

[E105] 1,2-Dihydroxy Hexane to air

[E106] 1,3 Diethyl-Cyclohexane to air

[E107] 1,3,5-trichlorobenzene to air

[E108] 1,3,5-Triethyl Cyclohex. to air

[E109] 1,3,5-trimethylbenzene (mesitylene) to air

[E110] 1,3,5-Tripropyl Cyclohex. to air

[E111] 1,3-Butadiene to air

[E112] 1,3-dichlorobenzene to air

[E113] 1,3-Dimeth. Cyclopentane to air

[E114] 1,3-Dimethyl Cyclohexane to air

[E115] 1,3-Diprop-5-Eth Cyclohex. to air

[E116] 1,4-dichlorobenzene to air

[E117] 13-Dieth.-5-Pent Cyclohex. to air

[E118] 13-Dieth-5-Me. Cyclohex. to air

[E119] 1-Butane to air

[E120] 1-Butanol to air

[E121] 1-Butene to air

[E122] 1-Butoxy Propanol to air

[E123] 1-Butyl Acetate to air

[E124] 1-Butyl Benzene to air

[E125] 1-Butyl Bromide to air

[E126] 1-butylpropionate to air

[E127] 1-C16 to air

[E128] 1-chloro-4-nitrobenzene to air

[E129] 1-Chlorobutane to air

[E130] 1-Decane to air

[E131] 1-Dodecane to air

[E132] 1-Eth.-4-Meth. Cyclohex to air

[E133] 1-Ethoxy-2-Propanol to air

[E134] 1-Heptane to air

[E135] 1-Heptanol to air

[E136] 1-Heptene to air

[E137] 1-Hexane to air

[E138] 1-Hexene to air

[E139] 1-Methoxy-2-Propanol to air

[E140] 1-Methyl-2-Pyrrolidone to air

[E141] 1-Nonane to air

[E142] 1-Nonene to air

[E143] 1-Octane to air

[E144] 1-Octanol to air

[E145] 1-Octene to air

[E146] 1-Pentadecane to air

[E147] 1-Pentane to air

[E148] 1-Pentene to air

Unit

Value

14300 Ecopoints/kg

14300 Ecopoints/kg

14300 Ecopoints/kg

14300 Ecopoints/kg

14300 Ecopoints $/ \mathrm{kg}$

14300 Ecopoints/kg

14300 Ecopoints/kg

14300 Ecopoints/kg

14300 Ecopoints/kg

14300 Ecopoints/kg

14300 Ecopoints/kg

14300 Ecopoints/kg

14300 Ecopoints/kg

14300 Ecopoints/kg

14300 Ecopoints/kg

14300 Ecopoints/kg

14300 Ecopoints/kg

14300 Ecopoints/kg

14300 Ecopoints/kg

14300 Ecopoints/kg

14300 Ecopoints/kg

14300 Ecopoints/kg

14300 Ecopoints/kg

14300 Ecopoints/kg

14300 Ecopoints/kg

14300 Ecopoints/kg

14300 Ecopoints/kg

14300 Ecopoints/kg

14300 Ecopoints/kg

14300 Ecopoints/kg

14300 Ecopoints/kg

14300 Ecopoints/kg

14300 Ecopoints $/ \mathrm{kg}$

14300 Ecopoints/kg

14300 Ecopoints/kg

14300 Ecopoints/kg

14300 Ecopoints/kg

14300 Ecopoints $/ \mathrm{kg}$

14300 Ecopoints/kg

14300 Ecopoints $/ \mathrm{kg}$

14300 Ecopoints/kg

14300 Ecopoints/kg

14300 Ecopoints/kg

14300 Ecopoints/kg

14300 Ecopoints/kg

14300 Ecopoints/kg

14300 Ecopoints/kg

14300 Ecopoints/kg

14300 Ecopoints/kg

14300 Ecopoints/kg

14300 Ecopoints/kg

14300 Ecopoints/kg

14300 Ecopoints/kg

14300 Ecopoints/kg

14300 Ecopoints $/ \mathrm{kg}$

14300 Ecopoints $/ \mathrm{kg}$

14300 Ecopoints/kg

14300 Ecopoints/kg

14300 Ecopoints/kg 
[E149] 1-Propanol to air [E150] 1-Propyl Benzene to air [E151] 1-Propyl Bromide to air [E152] 1-Tetradecane to air [E153] 1-Tridecane to air [E154] 1-Undecane to air [E155] 2- Octanol to air [E156] 2-(2-Butoxyethoxy)-Ethanol to air [E157] 2-(2-Ethoxyethoxy)-Ethanol to air [E158] 2-(Cl-methyl)-3-Cl-Propene to air [E159] 2,2,3 Trimethyl Butane to air [E160] 2,2,3,3-Tetramethyl Butane to air [E161] 2,2,4-Trimethyl Pentane to air [E162] 2,2,5-Trimethyl Hexane to air [E163] 2,2-Dimethylbutane to air [E164] 2,3 Dimethyl Pentane to air [E165] 2,3,3-Trimehyl-1-butene to air [E166] 2,3,4,6-tetrachlorophenol to air [E167] 2,3,4-Trimethyl Pentane to air [E168] 2,3,7,8-TCDD (tetrachloride-dibenzo-dioxin) to air [E169] 2,3-Dimethyl Hexane to air [E170] 2,3-Dimethyl Naptht. to air [E171] 2,3-Dimethyl-2-Butene to air [E173] 2,4,5-trichlorophenol to air [E174] 2,4,6-trichlorophenol to air [E176] 2,4-dichlorophenol to air [E177] 2,4-Dimethyl Heptane to air [E178] 2,4-Dimethyl Hexane to air [E179] 2,4-Dimethyl Pentane to air [E180] 2,5-Dimethyl Hexane to air [E181] 2,6 Diethyl Octane to air [E182] 2.3- Dimethylbutane to air [E183] 2-Butoxy-Ethanol to air [E184] 2-Butyltetrahydrofuran to air [E185] 2-chlorophenol to air [E186] 2-Ethoxy-Ethanol to air [E187] 2-Ethoxyethyl Acetate to air [E188] 2-Ethyl-1-Hexanol to air [E190] 2-Heptenes to air [E191] 2-Hexenes to air [E192] 2-Methoxy-Ethanol to air [E193] 2-Methyl-1-Butene to air [E194] 2-Methyl-1-Pentene to air [E195] 2-Methyl-2-Butene to air [E196] 2-Methyl-2-Pentene to air [E197] 2-Methylbutan-1-ol to air [E198] 2-Methylbutan-2-ol to air [E199] 2-Methylheptane to air [E200] 2-Methylhexane to air [E201] 2-Methylnonane to air [E202] 2-Methyloctane to air [E203] 2-Methylpentane to air [E204] 2-methylpropanoic acid (isobutyric acid) to air [E205] 2-Pentenes to air [E206] 3- Octanol to air [E207] 3-(Chloromethyl)-Heptane to air [E208] 3,4-dichloroaniline to air [E209] 3,4-Propyl Heptane to air [E210] 3,5 Diethyl Heptane to air [E211] 3,5-Diethyltoluene to air [E212] 3,5-Dimethylethylbenzene to air [E213] 3,7-Diethyl Nonane to air [E214] 3,8 Diethyl Decane to air [E215] 3,9-Diethyl Undecane to air [E216] 3-Carene to air

[E217] 3-chloroaniline to air

[E218] 3-Methyl-1-Butene to air
14300 Ecopoints/kg 14300 Ecopoints/kg 14300 Ecopoints/kg 14300 Ecopoints/kg 14300 Ecopoints/kg 14300 Ecopoints/kg 14300 Ecopoints/kg 14300 Ecopoints $/ \mathrm{kg}$ 14300 Ecopoints/kg 14300 Ecopoints/kg 14300 Ecopoints $/ \mathrm{kg}$ 14300 Ecopoints/kg 14300 Ecopoints $/ \mathrm{kg}$ 14300 Ecopoints $/ \mathrm{kg}$ 14300 Ecopoints/kg 14300 Ecopoints/kg 14300 Ecopoints $/ \mathrm{kg}$ 14300 Ecopoints/kg 14300 Ecopoints/kg 14300 Ecopoints/kg 14300 Ecopoints/kg 14300 Ecopoints $/ \mathrm{kg}$ 14300 Ecopoints $/ \mathrm{kg}$ 14300 Ecopoints/kg 14300 Ecopoints/kg 14300 Ecopoints $/ \mathrm{kg}$ 14300 Ecopoints/kg 14300 Ecopoints/kg 14300 Ecopoints/kg 14300 Ecopoints $/ \mathrm{kg}$ 14300 Ecopoints/kg 14300 Ecopoints/kg 14300 Ecopoints $/ \mathrm{kg}$ 14300 Ecopoints/kg 14300 Ecopoints $/ \mathrm{kg}$ 14300 Ecopoints/kg 14300 Ecopoints $/ \mathrm{kg}$ 14300 Ecopoints/kg 14300 Ecopoints/kg 14300 Ecopoints/kg 14300 Ecopoints $/ \mathrm{kg}$ 14300 Ecopoints/kg 14300 Ecopoints/kg 14300 Ecopoints $/ \mathrm{kg}$ 14300 Ecopoints $/ \mathrm{kg}$ 14300 Ecopoints $/ \mathrm{kg}$ 14300 Ecopoints/kg 14300 Ecopoints $/ \mathrm{kg}$ 14300 Ecopoints/kg 14300 Ecopoints/kg 14300 Ecopoints/kg 14300 Ecopoints $/ \mathrm{kg}$ 14300 Ecopoints/kg 14300 Ecopoints $/ \mathrm{kg}$ 14300 Ecopoints/kg 14300 Ecopoints/kg 14300 Ecopoints/kg 14300 Ecopoints/kg 14300 Ecopoints/kg 14300 Ecopoints/kg 14300 Ecopoints/kg 14300 Ecopoints/kg 14300 Ecopoints/kg 14300 Ecopoints/kg 14300 Ecopoints/kg 14300 Ecopoints/kg 14300 Ecopoints/kg 
[E219] 3-Methylbutan-1-ol to air [E220] 3-Methylbutan-2-ol to air [E221] 3-methylbutanoic acid (isovaleric acid) to air [E222] 3-Methylheptane to air [E223] 3-Methylhexane to air [E224] 3-Methylpentane to air [E225] 3-Nonenes to air [E226] 3-Octenes to air [E227] 3-Pentanol to ai [E228] 4-chloroaniline to air [E229] 4-Ethyl Heptane to air [E230] 4-Methylheptane to air [E232] Acetaldehyde (ethanal) to air

[E233] Acetic acid to air [E234] Acetone (2-propanon) to air [E235] Acetylene to air [E236] Acrolein (2-propenal) to air [E237] Acrylonitrile to air [E239] alcohols (unspec.) to air [E240] aldehydes (unspec.) to air [E243] alkanes (unspec.) to air [E244] alkenes (unspec.) to air [E245] Alkyl Phenols to air [E246] Allyl chloride to air [E247] Alpha Methyl Tetrahydrofuran to air [E248] alpha-Methyl Styrene to air [E249] alpha-Pinene to air [E252] anthracene to air

[E254] aromatics (unspecified) to air

[E262] Benzaldehyde to air

[E263] Benzene to air [E264] benzo[a]anthracene to air [E265] benzo[a]pyrene to air

[E266] benzo[ghi]perylene to air [E267] benzo[k]fluoranthrene to air [E268] Benzotrifluoride to air [E271] Beta-Pinene to air [E273] Branched C10 Alkanes to air [E274] Branched C11 Alkanes to air [E275] Branched C12 Alkanes to air [E276] Branched C13 Alkanes to air [E277] Branched C14 Alkanes to air [E278] Branched C15 Alkanes to air [E279] Branched C16 Alkanes to air [E280] Branched C17 Alkanes to air [E281] Branched C18 Alkanes to air [E282] Branched C5 Alkanes to air [E283] Branched C6 Alkanes to air [E284] Branched C7 Alkanes to air [E285] Branched C8 Alkanes to air [E286] Branched C9 Alkanes to air [E287] butane (unspec.) to air

[E288] butanoic acid (butyric acid) to air [E289] butylacrylate to air

[E290] Butylbenzylphtalate to air [E291] Butyraldehyde (butanal) to air [E292] C10 3-Alkenes to air [E293] C10 Bicycloalkanes to air [E294] C10 Cyclic Ketones to air [E295] C10 Cyclic or di-olefins to air [E296] C10 Cycloalkanes to air [E297] C10 Disub. Benzenes to air [E298] C10 Internal Alkenes to air [E299] C10 Ketones to air

[E300] C10 Monosub. Benzenes to air [E301] C10 Styrenes to air [E302] C10 Tetrasub. Benzenes to air

14300 Ecopoints/kg 14300 Ecopoints/kg 14300 Ecopoints/kg 14300 Ecopoints/kg 14300 Ecopoints/kg 14300 Ecopoints/kg 14300 Ecopoints/kg 14300 Ecopoints/kg 14300 Ecopoints/kg 14300 Ecopoints/kg 14300 Ecopoints/kg 14300 Ecopoints/kg 14300 Ecopoints/kg 14300 Ecopoints/kg 14300 Ecopoints/kg 14300 Ecopoints/kg 14300 Ecopoints/kg 14300 Ecopoints/kg 14300 Ecopoints/kg 14300 Ecopoints/kg 14300 Ecopoints $/ \mathrm{kg}$ 14300 Ecopoints/kg 14300 Ecopoints/kg 14300 Ecopoints/kg 14300 Ecopoints/kg 14300 Ecopoints/kg 14300 Ecopoints/kg 14300 Ecopoints/kg 14300 Ecopoints/kg 14300 Ecopoints/kg 14300 Ecopoints/kg 14300 Ecopoints/kg 14300 Ecopoints/kg 14300 Ecopoints/kg 14300 Ecopoints/kg 14300 Ecopoints/kg 14300 Ecopoints/kg 14300 Ecopoints/kg 14300 Ecopoints/kg 14300 Ecopoints $/ \mathrm{kg}$ 14300 Ecopoints/kg 14300 Ecopoints/kg 14300 Ecopoints/kg 14300 Ecopoints/kg 14300 Ecopoints/kg 14300 Ecopoints/kg 14300 Ecopoints/kg 14300 Ecopoints/kg 14300 Ecopoints/kg 14300 Ecopoints/kg 14300 Ecopoints/kg 14300 Ecopoints/kg 14300 Ecopoints/kg 14300 Ecopoints/kg 14300 Ecopoints/kg 14300 Ecopoints/kg 14300 Ecopoints/kg 14300 Ecopoints/kg 14300 Ecopoints/kg 14300 Ecopoints/kg 14300 Ecopoints/kg 14300 Ecopoints/kg 14300 Ecopoints/kg 14300 Ecopoints/kg 14300 Ecopoints/kg 14300 Ecopoints/kg 14300 Ecopoints/kg 
[E303] C10 Trisub. Benzenes to air [E304] C11 3-Alkenes to air [E305] C11 Bicycloalkanes to air [E306] C11 Cyclic or di-olefins to air [E307] C11 Cycloalkanes to air [E308] C11 Disub. Benzenes to air [E309] C11 Internal Alkenes to air [E310] C11 Monosub. Benzenes to air [E311] C11 Pentasub. Benzenes to air [E312] C11 Pentasub. Benzenes to air [E313] C11 Tetralin or Indane to air [E314] C11 Tetrasub. Benzenes to air [E315] C11 Trisub. Benzenes to air [E316] C12 2-Alkenes to air [E317] C12 3-Alkenes to air [E318] C12 Bicycloalkanes to air [E319] C12 Cyclic or di-olefins to air [E320] C12 Cycloalkanes to air [E321] C12 Disub. Benzenes to air [E322] C12 Disub. Naphthalenes to air [E323] C12 Hexaasub. Benzenes to air [E324] C12 Internal Alkenes to air [E325] C12 Monosub. Benzenes to air [E326] C12 Monosub. Naphth. to air [E327] C12 Tetrasub. Benzenes to air [E328] C12 Trisub. Benzenes to air [E329] C13 3-Alkenes to air [E330] C13 Bicycloalkanes to air [E331] C13 Cyclic or di-olefins to air [E332] C13 Cycloalkanes to air [E333] C13 Disub. Benzenes to air [E334] C13 Disub. Naphthalenes to air [E335] C13 Internal Alkenes to air [E336] C13 Monosub. Benzenes to air [E337] C13 Monosub. Naphth. to air [E338] C13 Trisub. Benzenes to air [E339] C13 Trisub. Naphthalenes to air [E340] C14 Bicycloalkanes to air [E341] C14 Cycloalkanes to air [E342] C15 Bicycloalkanes to air [E343] C15 Cycloalkanes to air [E344] C3 Adehydes to air [E345] C4 Aldehydes to air [E346] C4 Internal Alkenes to air [E347] C5 Adehydes to air [E348] C5 Cyclic Ketones to air [E349] C5 Internal Alkenes to air [E350] C5 Ketones to air [E351] C5 Terminal Alkenes to air [E352] C6 Adehydes to air [E353] C6 Cyclic Ketones to air [E354] C6 Cyclic or di-olefins to air [E355] C6 Cycloalkanes to air [E356] C6 internal Alkenes to air [E357] C6 Ketones to air [E358] C6 Terminal Alkenes to air [E359] C7 Adehydes to air [E360] C7 Cyclic Ketones to air [E361] C7 Cyclic or di-olefins to air [E362] C7 Cycloalkanes to air [E363] C7 Internal Alkenes to air [E364] C7 Ketones to air [E365] C7 Terminal Alkenes to air [E366] C8 Aldehydes to air [E367] C8 Cyclic Ketones to air [E368] C8 Cyclic or di-olefins to air [E369] C8 Cycloalkanes to air
14300 Ecopoints/kg 14300 Ecopoints $/ \mathrm{kg}$ 14300 Ecopoints/kg 14300 Ecopoints $/ \mathrm{kg}$ 14300 Ecopoints/kg 14300 Ecopoints/kg 14300 Ecopoints/kg 14300 Ecopoints/kg 14300 Ecopoints/kg 14300 Ecopoints/kg 14300 Ecopoints/kg 14300 Ecopoints/kg 14300 Ecopoints/kg 14300 Ecopoints/kg 14300 Ecopoints/kg 14300 Ecopoints/kg 14300 Ecopoints/kg 14300 Ecopoints/kg 14300 Ecopoints/kg 14300 Ecopoints/kg 14300 Ecopoints/kg 14300 Ecopoints/kg 14300 Ecopoints/kg 14300 Ecopoints/kg 14300 Ecopoints/kg 14300 Ecopoints/kg 14300 Ecopoints/kg 14300 Ecopoints/kg 14300 Ecopoints/kg 14300 Ecopoints/kg 14300 Ecopoints/kg 14300 Ecopoints/kg 14300 Ecopoints/kg 14300 Ecopoints/kg 14300 Ecopoints/kg 14300 Ecopoints $/ \mathrm{kg}$ 14300 Ecopoints/kg 14300 Ecopoints/kg 14300 Ecopoints/kg 14300 Ecopoints $/ \mathrm{kg}$ 14300 Ecopoints/kg 14300 Ecopoints $/ \mathrm{kg}$ 14300 Ecopoints/kg 14300 Ecopoints/kg 14300 Ecopoints/kg 14300 Ecopoints/kg 14300 Ecopoints/kg 14300 Ecopoints/kg 14300 Ecopoints/kg 14300 Ecopoints/kg 14300 Ecopoints $/ \mathrm{kg}$ 14300 Ecopoints/kg 14300 Ecopoints/kg 14300 Ecopoints/kg 14300 Ecopoints/kg 14300 Ecopoints/kg 14300 Ecopoints/kg 14300 Ecopoints/kg 14300 Ecopoints/kg 14300 Ecopoints/kg 14300 Ecopoints/kg 14300 Ecopoints/kg 14300 Ecopoints/kg 14300 Ecopoints/kg 14300 Ecopoints/kg 14300 Ecopoints/kg 14300 Ecopoints/kg 
[E370] C8 Disub. Benzenes to air [E371] C8 Internal Alkenes to air [E372] C8 Ketones to air

[E373] C8 Terminal Alkenes to air

[E374] C9 Bicycloalkanes to air

[E375] C9 Cyclic Ketones to air

[E376] C9 Cyclic or di-olefins to air

[E377] C9 Cycloalkanes to air

[E378] C9 Disub. Benzenes to air

[E379] C9 Internal Alkenes to air

[E380] C9 Ketones to air

[E381] C9 Monosub. Benzenes to air

[E382] C9 Styrenes to air

[E383] C9 Terminal Alkenes to air

[E384] C9 Trisub. Benzenes to air

[E393] Carbon dioxide to air

[E396] CFC (hard) (unspecified) to air

[E397] CFC (soft) (unspecified) to air

[E398] CFC-11 to air

[E399] CFC-113 to air

[E400] CFC-114 to air

[E401] CFC-115 to air

[E402] CFC-12 to air

[E403] CFC-13 to air

[E407] chlorobenzene to air

[E408] Chlorophenols (unspec.) to air

[E409] Chloropicrin to air

[E416] chrysene to air

[E417] cis-2-Butene to air

[E418] cis-2-Hexene to air

[E419] cis-2-Pentene to air

[E420] cis-Dichloroethene to air

[E424] Crotonaldehyde to air

[E426] Cyanides to air

[E427] cyclic alkanes (unspec.) to air

[E428] Cyclobutane to air

[E429] Cyclohexane to air

[E430] Cyclohexanol to air

[E431] Cyclohexanone to air

[E432] Cyclohexene to air

[E433] Cyclopentadiene to air

[E434] Cyclopentane to air

[E435] Cyclopentene to air

[E436] Cyclopropane to air

[E444] Di(2-ethylhexyl)phtalate to air

[E445] Diacetone alcohol to air

[E447] Dibutyl Ether to air

[E448] Dibutylphtalate to air

[E449] Dichloromethane (Methylene Chloride) to air

[E453] Diethyl Ether to air

[E454] diethylamine to air

[E455] Diethylketone to air

[E456] Diethylphtalate to air

[E457] Dihexylphtalate to air

[E458] Diisodecylphtalate to air

[E459] Diisooctylphtalate to air

[E460] Diisopropylether to air

[E462] Dimethyl Adipate to air

[E463] Dimethyl Ether to air

[E464] Dimethyl Glutarate to air

[E465] Dimethyl Naphthalenes to air

[E466] Dimethyl Succinate to air

[E467] dimethylamine to air

[E468] Dimethylphtalate to air

[E472] Dioctylphtalate to air

[E473] dioxins (unspec.) to air

[E474] diphenyl to air
14300 Ecopoints/kg

14300 Ecopoints/kg

14300 Ecopoints $/ \mathrm{kg}$

14300 Ecopoints/kg

14300 Ecopoints/kg

14300 Ecopoints/kg

14300 Ecopoints/kg

14300 Ecopoints/kg

14300 Ecopoints/kg

14300 Ecopoints $/ \mathrm{kg}$

14300 Ecopoints/kg

14300 Ecopoints/kg

14300 Ecopoints/kg

14300 Ecopoints/kg

14300 Ecopoints/kg

36 Ecopoints $/ \mathrm{kg}$

4.50E+06 Ecopoints $/ \mathrm{kg}$

$4.50 \mathrm{E}+06$ Ecopoints $/ \mathrm{kg}$

$4.50 \mathrm{E}+06$ Ecopoints $/ \mathrm{kg}$

$4.50 \mathrm{E}+06$ Ecopoints $/ \mathrm{kg}$

4.50E+06 Ecopoints $/ \mathrm{kg}$

4.50E+06 Ecopoints $/ \mathrm{kg}$

4.50E+06 Ecopoints $/ \mathrm{kg}$

4.50E+06 Ecopoints $/ \mathrm{kg}$

14300 Ecopoints/kg

14300 Ecopoints/kg

14300 Ecopoints/kg

14300 Ecopoints/kg

14300 Ecopoints/kg

14300 Ecopoints/kg

14300 Ecopoints/kg

14300 Ecopoints/kg

14300 Ecopoints/kg

14300 Ecopoints/kg

14300 Ecopoints/kg

14300 Ecopoints/kg

14300 Ecopoints $/ \mathrm{kg}$

14300 Ecopoints/kg

14300 Ecopoints/kg

14300 Ecopoints/kg

14300 Ecopoints/kg

14300 Ecopoints/kg

14300 Ecopoints/kg

14300 Ecopoints/kg

14300 Ecopoints/kg

14300 Ecopoints/kg

14300 Ecopoints/kg

14300 Ecopoints/kg

14300 Ecopoints $/ k g$

14300 Ecopoints/kg

14300 Ecopoints/kg

14300 Ecopoints/kg

14300 Ecopoints/kg

14300 Ecopoints/kg

14300 Ecopoints/kg

14300 Ecopoints/kg

14300 Ecopoints/kg

14300 Ecopoints/kg

14300 Ecopoints/kg

14300 Ecopoints/kg

14300 Ecopoints/kg

14300 Ecopoints/kg

14300 Ecopoints/kg

14300 Ecopoints/kg

14300 Ecopoints/kg

14300 Ecopoints/kg

14300 Ecopoints/kg 
14300 Ecopoints/kg

14300 Ecopoints/kg

14300 Ecopoints/kg

14300 Ecopoints/kg

14300 Ecopoints/kg

14300 Ecopoints/kg 14300 Ecopoints $/ k g$

14300 Ecopoints/kg 14300 Ecopoints/kg 14300 Ecopoints/kg 14300 Ecopoints/kg 14300 Ecopoints/kg 14300 Ecopoints/kg 14300 Ecopoints/kg 14300 Ecopoints/kg 14300 Ecopoints/kg 14300 Ecopoints/kg 14300 Ecopoints/kg 14300 Ecopoints/kg 14300 Ecopoints/kg 42300 Ecopoints/kg 14300 Ecopoints/kg 14300 Ecopoints $/ \mathrm{kg}$ 14300 Ecopoints/kg 
[E570] isobutane to air

[E571] isobutanol (2-methylpropanol-1) to air

[E572] isobutene (2-methylpropene) to air

[E573] isobutyl acetate to air

[E574] isobutyl isobutyrate to air

[E575] isobutyraldehyde to air

[E576] isopentane to air

[E577] isopentylacetate (iso-amylacetate) to air

[E578] isoprene to air

[E579] isopropanol to air

[E580] isopropyl acetate to air

[E581] isopropyl benzene (cumene) to air

[E582] isopropyl propionate to air

[E593] meta-Cresol (3-methylphenol) to air

[E594] meta-Ethyltoluene to air

[E596] meta-Xylene (1,3-dimethylbenzene) to air

[E599] Methacrolein to air

[E600] Methane to air

[E602] Methanol to air

[E604] Methyl Acetate to air

[E606] Methyl Acrylate to air

[E607] methyl amine to air

[E608] Methyl Bromide to air

[E609] Methyl Choride to air

[E610] Methyl cyclohexane to air

[E611] Methyl cyclopentane to air

[E612] methyl dithiomethane to air

[E613] Methyl Ethyl Ketone to air

[E614] Methyl Formate to air

[E615] Methyl Glyoxal to air

[E616] Methyl Isobutyl Ketone (4-methylpentanon-2) to air

[E617] Methyl iso-butyrate to air

[E618] methyl methacrylate to air

[E619] Methyl Naphthalenes to air

[E620] Methyl Nitrite to air

[E621] methyl propionate to air

[E622] Methyl propyl Ketone to air

[E623] methyl thiomethane (dimethylsulfide) to air

[E624] Methyl trans-Butyl Ether to air

[E625] Methyl-Isopropylketone to air

[E626] methyl-mercury to air

[E627] Methyl-transbutylketone to air

[E633] Naphtalene to air

[E634] Neopentane to air

[E639] Nitrobenzene to air

[E641] nitrogen dioxide to air

[E643] nitrogen oxides to air

[E645] Octyl Cyclohexane to air

[E647] organics acids (unspec.) to air

[E648] ortho-Cresol (2-methylphenol) to air

[E649] ortho-Ethyltoluene to air

[E650] ortho-Xylene (1,2-dimethylbenzene) to air

[E653] para-Cresol (4-methylphenol) to air

[E654] para-Ethyltoluene to air

[E657] para-Trifluoromethyl-Cl-Benzene to air

[E658] para-Xylene (1,4-dimethylbenzene) to air

[E660] pentachlorobenzene to air

[E661] pentachloronitrobenzene to air

[E662] pentachlorophenol to air

[E663] Pentanaldehyde to air

[E664] Pentanol to air

[E665] Perfluorobutane to air

[E666] Perfluorocyclobutane to air

[E667] Perfluoroethane (CFC-116) to air

[E668] Perfluorohexane to air

[E669] Perfluoromethane (CFC-14) to air

[E670] Perfluoropentane to air
14300 Ecopoints $/ \mathrm{kg}$

14300 Ecopoints/kg

14300 Ecopoints/kg

14300 Ecopoints/kg

14300 Ecopoints/kg

14300 Ecopoints/kg

14300 Ecopoints/kg

14300 Ecopoints/kg

14300 Ecopoints/kg

14300 Ecopoints/kg

14300 Ecopoints/kg

14300 Ecopoints/kg

14300 Ecopoints/kg

14300 Ecopoints/kg

14300 Ecopoints/kg

14300 Ecopoints/kg

14300 Ecopoints/kg

2100 Ecopoints $/ \mathrm{kg}$

14300 Ecopoints/kg

14300 Ecopoints/kg

14300 Ecopoints/kg

14300 Ecopoints/kg

14300 Ecopoints/kg

14300 Ecopoints/kg

14300 Ecopoints/kg

14300 Ecopoints/kg

14300 Ecopoints/kg

14300 Ecopoints/kg

14300 Ecopoints/kg

14300 Ecopoints/kg

14300 Ecopoints/kg

14300 Ecopoints/kg

14300 Ecopoints/kg

14300 Ecopoints/kg

14300 Ecopoints/kg

14300 Ecopoints/kg

14300 Ecopoints/kg

14300 Ecopoints/kg

14300 Ecopoints/kg

14300 Ecopoints/kg

14300 Ecopoints/kg

14300 Ecopoints/kg

14300 Ecopoints/kg

14300 Ecopoints/kg

14300 Ecopoints/kg

42300 Ecopoints/kg

42300 Ecopoints/kg

14300 Ecopoints/kg

14300 Ecopoints/kg

14300 Ecopoints/kg

14300 Ecopoints/kg

14300 Ecopoints/kg

14300 Ecopoints/kg

14300 Ecopoints/kg

14300 Ecopoints/kg

14300 Ecopoints/kg

14300 Ecopoints/kg

14300 Ecopoints/kg

14300 Ecopoints/kg

14300 Ecopoints/kg

14300 Ecopoints/kg

4.50E+06 Ecopoints/kg

4.50E+06 Ecopoints/kg

4.50E+06 Ecopoints/kg

4.50E+06 Ecopoints $/ \mathrm{kg}$

4.50E+06 Ecopoints $/ \mathrm{kg}$

4.50E+06 Ecopoints $/ \mathrm{kg}$ 
[E671] Perfluoropropane to air

[E673] petrol to air

[E674] phenanthrene to air

[E675] Phenol to air

[E680] Phtalic anhydride to air

[E681] phthalates to air

[E684] Polycyclic Aromatic Hydrocarbons (PAH) (unspecified) to air

[E685] Polycyclic Aromatic Hydrocarbons Carcinogenic- (carcinogenic-PAH) to air

[E687] Propane to air

[E688] Propanoic acid (propionic acid) to air

[E689] Propionaldehyde (propanal) to air

[E691] Propyl Acetate to air

[E692] Propyl Cyclopentane to air

[E693] Propylene to air

[E694] Propylene Carbonate to air

[E695] Propylene Glycol to air

[E696] Propylene Glycol Methyl Ether Acetate to air

[E697] Propylene Oxide to air

[E700] Sabinene to air

[E701] sec-Butanol to air

[E702] sec-Butyl Benzene to air

[E703] sec-Buytl Acetate to air

[E706] styrene (vinylbenzene) to air

[E707] Subst. C7 Ester (C12) to air

[E708] Sucts. C9 Ester (C12) to air

[E709] sulphur dioxide to air

[E713] tar to air

[E715] terpentine to air

[E716] tertiary-Butanol to air

[E717] tertiary-Butyl Acetate to air

[E718] tetrachloroethylene (PER) (tetrachloroethene) to air

[E719] Tetrachloromethane (carbon tetrachloride) (HC-10) to air

[E720] tetrafluorethylene to air

[E721] Tetralin to air

[E726] Tolualdehyde to air

[E727] Toluene to air

[E728] trans-1,2-Dichloroethene to air

[E729] trans-2-Butene to air

[E730] trans-2-Hexene to air

[E731] trans-2-Pentene to air

[E732] trans-dichloroethene to air

[E737] Trichloroethylene (tri) to air

[E738] Trichloromethane (chloroform) to air

[E742] Valeraldehyde (pentanal) to air

[E744] Vinyl Acetate to air

[E745] Vinyl Chloride (chloroethene) to air

[E746] Volatile Organic Compounds (VOC) to air

[E747] Volatile Organic Compounds non-methane- (non methane VOC) to air

[E779] ammonia, ammonium to fresh water

[E798] Biological Oxygen Demand (BOD) to fresh water

[E809] Chemical oxigen demand (COD) to fresh water

[E813] Chlorine to fresh water

[E894] Nitrate to fresh water

[E896] Nitrogen to fresh water

[E910] Phosphate to fresh water

[E911] Phosphorus to fresh water

[E924] sulphates to fresh water

[E973] ammonia, ammonium to sea water

[E992] Biological Oxygen Demand (BOD) to sea water

[E1003] Chemical oxigen demand (COD) to sea water

[E1007] Chlorine to sea water

[E1088] Nitrate to sea water

[E1090] Nitrogen to sea water

[E1104] Phosphate to sea water

[E1105] Phosphorus to sea water

[E1118] sulphates to sea water
4.50E+06 Ecopoints/kg

14300 Ecopoints $/ \mathrm{kg}$

14300 Ecopoints/kg

14300 Ecopoints/kg

14300 Ecopoints $/ \mathrm{kg}$

14300 Ecopoints $/ \mathrm{kg}$

14300 Ecopoints/kg

14300 Ecopoints/kg

14300 Ecopoints $/ \mathrm{kg}$

14300 Ecopoints/kg

14300 Ecopoints/kg

14300 Ecopoints/kg

14300 Ecopoints/kg

14300 Ecopoints/kg

14300 Ecopoints/kg

14300 Ecopoints/kg

14300 Ecopoints/kg

14300 Ecopoints/kg

14300 Ecopoints/kg

14300 Ecopoints/kg

14300 Ecopoints/kg

14300 Ecopoints/kg

14300 Ecopoints/kg

14300 Ecopoints/kg

14300 Ecopoints $/ \mathrm{kg}$

23000 Ecopoints/kg

14300 Ecopoints/kg

14300 Ecopoints/kg

14300 Ecopoints/kg

14300 Ecopoints/kg

14300 Ecopoints/kg

14300 Ecopoints/kg

14300 Ecopoints/kg

14300 Ecopoints/kg

14300 Ecopoints $/ \mathrm{kg}$

14300 Ecopoints $/ \mathrm{kg}$

14300 Ecopoints/kg

14300 Ecopoints/kg

14300 Ecopoints/kg

14300 Ecopoints/kg

14300 Ecopoints/kg

14300 Ecopoints/kg

14300 Ecopoints/kg

14300 Ecopoints/kg

14300 Ecopoints $/ \mathrm{kg}$

14300 Ecopoints/kg

14300 Ecopoints/kg

14300 Ecopoints/kg

10300 Ecopoints/kg

11500 Ecopoints/kg

3830 Ecopoints/kg

26.2 Ecopoints/kg

204 Ecopoints/kg

905 Ecopoints/kg

2.47E+05 Ecopoints/kg

$7.56 \mathrm{E}+05$ Ecopoints $/ \mathrm{kg}$

76.6 Ecopoints/kg

10300 Ecopoints $/ \mathrm{kg}$

11500 Ecopoints/kg

3830 Ecopoints $/ \mathrm{kg}$

26.2 Ecopoints/kg

204 Ecopoints/kg

905 Ecopoints/kg

2.47E+05 Ecopoints/kg

7.56E+05 Ecopoints/kg

76.6 Ecopoints/kg 
Category $=[$ C18] Ecoscarcity Norway

Description = Distance to target approach, ecoscarcity in Norway

Author = Baumann et al, 1992

Date $=27-09-1999$

Environmental resources

Environmental resource

Value

Value

emissions

Environmental emission

[E90] 1,1,1-trichloroethane (methyl chloroform; HC-140a) to air

[E91] 1,1,2-Trichloroethane to air

[E92] 1,1-Dichloroethane to air

[E93] 1,1-Dichloroethene to air

[E94] 1,2,3,4-tetrachlorobenzene to air

[E95] 1,2,3,5-tetrachlorobenzene to air

[E96] 1,2,3-trichlorobenzene to air

[E97] 1,2,3-Trimethyl Benzene to air

[E98] 1,2,4,5-tetrachlorobenzene to air

[E99] 1,2,4-trichlorobenzene to air

[E100] 1,2,4-trimethylbenzene to air

[E101] 1,2-Butanediol to air

[E102] 1,2-dichlorobenzene to air

[E103] 1,2-dichloroethane to air

[E104] 1,2-Dichloropropane to air

[E105] 1,2-Dihydroxy Hexane to air

[E106] 1,3 Diethyl-Cyclohexane to air

[E107] 1,3,5-trichlorobenzene to air

[E108] 1,3,5-Triethyl Cyclohex. to air

[E109] 1,3,5-trimethylbenzene (mesitylene) to air

[E110] 1,3,5-Tripropyl Cyclohex. to air

[E111] 1,3-Butadiene to air

[E112] 1,3-dichlorobenzene to air

[E113] 1,3-Dimeth. Cyclopentane to air

[E114] 1,3-Dimethyl Cyclohexane to air

[E115] 1,3-Diprop-5-Eth Cyclohex. to air

[E116] 1,4-dichlorobenzene to air

[E117] 13-Dieth.-5-Pent Cyclohex. to air

[E118] 13-Dieth-5-Me. Cyclohex. to air

[E119] 1-Butane to air

[E120] 1-Butanol to air

[E121] 1-Butene to air

[E122] 1-Butoxy Propanol to air

[E123] 1-Butyl Acetate to air

[E124] 1-Butyl Benzene to air

[E125] 1-Butyl Bromide to air

[E126] 1-butylpropionate to air

[E127] 1-C16 to air

[E128] 1-chloro-4-nitrobenzene to air

[E129] 1-Chlorobutane to air

[E130] 1-Decane to air

[E131] 1-Dodecane to air

[E132] 1-Eth.-4-Meth. Cyclohex to air

[E133] 1-Ethoxy-2-Propanol to air

[E134] 1-Heptane to air

[E135] 1-Heptanol to air

[E136] 1-Heptene to air

[E137] 1-Hexane to air

[E138] 1-Hexene to air

[E139] 1-Methoxy-2-Propanol to air

[E140] 1-Methyl-2-Pyrrolidone to air

[E141] 1-Nonane to air

[E142] 1-Nonene to air

[E143] 1-Octane to air

[E144] 1-Octanol to air

[E145] 1-Octene to air
Unit

Unit

9120 Ecopoints/kg

9120 Ecopoints/kg

9120 Ecopoints/kg

9120 Ecopoints/kg

9120 Ecopoints/kg

9120 Ecopoints/kg

9120 Ecopoints/kg

9120 Ecopoints/kg

9120 Ecopoints/kg

9120 Ecopoints/kg

9120 Ecopoints/kg

9120 Ecopoints/kg

9120 Ecopoints/kg

9120 Ecopoints/kg

9120 Ecopoints/kg

9120 Ecopoints/kg

9120 Ecopoints/kg

9120 Ecopoints/kg

9120 Ecopoints/kg

9120 Ecopoints/kg

9120 Ecopoints/kg

9120 Ecopoints/kg

9120 Ecopoints/kg

9120 Ecopoints/kg

9120 Ecopoints/kg

9120 Ecopoints/kg

9120 Ecopoints/kg

9120 Ecopoints $/ \mathrm{kg}$

9120 Ecopoints/kg

9120 Ecopoints/kg

9120 Ecopoints/kg

9120 Ecopoints/kg

9120 Ecopoints/kg

9120 Ecopoints/kg

9120 Ecopoints/kg

9120 Ecopoints/kg

9120 Ecopoints/kg

9120 Ecopoints/kg

9120 Ecopoints/kg

9120 Ecopoints/kg

9120 Ecopoints/kg

9120 Ecopoints/kg

9120 Ecopoints/kg

9120 Ecopoints/kg

9120 Ecopoints/kg

9120 Ecopoints/kg

9120 Ecopoints/kg

9120 Ecopoints/kg

9120 Ecopoints/kg

9120 Ecopoints/kg

9120 Ecopoints/kg

9120 Ecopoints/kg

9120 Ecopoints/kg

9120 Ecopoints/kg

9120 Ecopoints/kg

9120 Ecopoints/kg 
[E152] 1-Tetradecane to air

[E153] 1-Tridecane to air

[E154] 1-Undecane to air

[E155] 2- Octanol to air

[E156] 2-(2-Butoxyethoxy)-Ethanol to air

[E157] 2-(2-Ethoxyethoxy)-Ethanol to air

[E158] 2-(Cl-methyl)-3-Cl-Propene to air

9120 Ecopoints/kg

9120 Ecopoints/kg

9120 Ecopoints/kg

9120 Ecopoints/kg

9120 Ecopoints/kg

9120 Ecopoints/kg

9120 Ecopoints/kg

9120 Ecopoints/kg

9120 Ecopoints/kg

9120 Ecopoints/kg

9120 Ecopoints/kg

9120 Ecopoints $/ \mathrm{kg}$

9120 Ecopoints/kg

9120 Ecopoints/kg

9120 Ecopoints/kg

9120 Ecopoints/kg

9120 Ecopoints/kg

9120 Ecopoints/kg

9120 Ecopoints/kg

9120 Ecopoints/kg

9120 Ecopoints/kg

9120 Ecopoints/kg

9120 Ecopoints/kg

9120 Ecopoints/kg

9120 Ecopoints/kg

9120 Ecopoints/kg

9120 Ecopoints/kg

9120 Ecopoints/kg

9120 Ecopoints/kg

9120 Ecopoints/kg

9120 Ecopoints/kg

9120 Ecopoints/kg

9120 Ecopoints/kg

9120 Ecopoints/kg

9120 Ecopoints/kg

9120 Ecopoints/kg

9120 Ecopoints/kg

9120 Ecopoints/kg

9120 Ecopoints/kg

9120 Ecopoints/kg

9120 Ecopoints/kg

9120 Ecopoints/kg

9120 Ecopoints/kg

9120 Ecopoints/kg

9120 Ecopoints/kg

9120 Ecopoints $/ \mathrm{kg}$

9120 Ecopoints/kg

9120 Ecopoints/kg

9120 Ecopoints/kg

9120 Ecopoints/kg

9120 Ecopoints/kg

9120 Ecopoints/kg

9120 Ecopoints/kg

9120 Ecopoints/kg

9120 Ecopoints/kg

9120 Ecopoints/kg

9120 Ecopoints/kg

9120 Ecopoints/kg

9120 Ecopoints/kg

9120 Ecopoints/kg

9120 Ecopoints/kg

9120 Ecopoints/kg

[E214] 3,8 Diethyl Decane to air

[E215] 3,9-Diethyl Undecane to air 
9120 Ecopoints/kg

9120 Ecopoints/kg

9120 Ecopoints/kg

9120 Ecopoints/kg

9120 Ecopoints/kg

[E226] 3-Octenes to air

[E227] 3-Pentanol to air

[E228] 4-chloroaniline to air

[E229] 4-Ethyl Heptane to air

[E230] 4-Methylheptane to air

[E232] Acetaldehyde (ethanal) to air

[E233] Acetic acid to air

[E234] Acetone (2-propanon) to air

[E235] Acetylene to air

[E236] Acrolein (2-propenal) to air

[E237] Acrylonitrile to air

[E239] alcohols (unspec.) to air

[E240] aldehydes (unspec.) to air

[E243] alkanes (unspec.) to air

[E244] alkenes (unspec.) to air

[E245] Alkyl Phenols to air

[E246] Allyl chloride to air

[E247] Alpha Methyl Tetrahydrofuran to air

[E248] alpha-Methyl Styrene to air

[E249] alpha-Pinene to air

[E252] anthracene to air

[E254] aromatics (unspecified) to air

[E262] Benzaidehyde to air

[E263] Benzene to air

[E264] benzo[a]anthracene to air

[E265] benzo[a]pyrene to air

[E266] benzo[ghi]perylene to air

[E267] benzo[k]fluoranthrene to air

[E268] Benzotrifluoride to air

[E271] Beta-Pinene to air

[E273] Branched C10 Alkanes to air

[E274] Branched C11 Alkanes to air

[E275] Branched C12 Alkanes to air

[E276] Branched C13 Alkanes to air

[E277] Branched C14 Alkanes to air

[E278] Branched C15 Alkanes to air

[E279] Branched C16 Alkanes to air

[E280] Branched C17 Alkanes to air

[E281] Branched C18 Alkanes to air

[E282] Branched C5 Alkanes to air

[E283] Branched C6 Alkanes to air

[E284] Branched C7 Alkanes to air

[E285] Branched C8 Alkanes to air

[E286] Branched C9 Alkanes to air

[E287] butane (unspec.) to air

[E288] butanoic acid (butyric acid) to air

[E289] butylacrylate to air

[E290] Butylbenzylphtalate to air

[E291] Butyraldehyde (butanal) to air

[E292] C10 3-Alkenes to air

[E293] C10 Bicycloalkanes to air

[E294] C10 Cyclic Ketones to air

[E295] C10 Cyclic or di-olefins to air

[E296] C10 Cycloalkanes to air

[E297] C10 Disub. Benzenes to air

[E298] C10 Internal Alkenes to air

[E299] C10 Ketones to air

9120 Ecopoints/kg

9120 Ecopoints $/ \mathrm{kg}$

9120 Ecopoints/kg

9120 Ecopoints/kg

9120 Ecopoints/kg

9120 Ecopoints/kg

9120 Ecopoints/kg

9120 Ecopoints/kg

9120 Ecopoints/kg

9120 Ecopoints/kg

9120 Ecopoints/kg

9120 Ecopoints/kg

9120 Ecopoints/kg

9120 Ecopoints/kg

9120 Ecopoints/kg

9120 Ecopoints/kg

9120 Ecopoints/kg

9120 Ecopoints/kg

9120 Ecopoints $/ \mathrm{kg}$

9.80E+06 Ecopoints $/ \mathrm{kg}$

9120 Ecopoints/kg

9120 Ecopoints $/ \mathrm{kg}$

9120 Ecopoints/kg

$9.80 E+06$ Ecopoints $/ \mathrm{kg}$

$9.80 \mathrm{E}+06$ Ecopoints $/ \mathrm{kg}$

9.80E+06 Ecopoints $/ \mathrm{kg}$

$9.80 \mathrm{E}+06$ Ecopoints $/ \mathrm{kg}$

9120 Ecopoints $/ \mathrm{kg}$

9120 Ecopoints/kg

9120 Ecopoints/kg

9120 Ecopoints $/ \mathrm{kg}$

9120 Ecopoints/kg

9120 Ecopoints $/ \mathrm{kg}$

9120 Ecopoints/kg

9120 Ecopoints $/ \mathrm{kg}$

9120 Ecopoints/kg

9120 Ecopoints/kg

9120 Ecopoints/kg

9120 Ecopoints/kg

9120 Ecopoints/kg

9120 Ecopoints/kg

9120 Ecopoints/kg

9120 Ecopoints/kg

9120 Ecopoints/kg

9120 Ecopoints/kg

9120 Ecopoints/kg

9120 Ecopoints/kg

9120 Ecopoints/kg

9120 Ecopoints/kg

9120 Ecopoints/kg

9120 Ecopoints/kg

9120 Ecopoints/kg

9120 Ecopoints/kg

9120 Ecopoints/kg

9120 Ecopoints/kg

9120 Ecopoints/kg 
[E300] C10 Monosub. Benzenes to air

9120 Ecopoints/kg

[E301] C10 Styrenes to air

9120 Ecopoints/kg

9120 Ecopoints $/ \mathrm{kg}$

9120 Ecopoints $/ \mathrm{kg}$

[E303] C10 Trisub. Benzenes to air

[E304] C11 3-Alkenes to air

[E305] C11 Bicycloalkanes to air

[E306] C11 Cyclic or di-olefins to air

[E307] C11 Cycloalkanes to air

[E308] C11 Disub. Benzenes to air

[E309] C11 Internal Alkenes to air

[E310] C11 Monosub. Benzenes to air

[E311] C11 Pentasub. Benzenes to air

[E312] C11 Pentasub. Benzenes to air

[E313] C11 Tetralin or Indane to air

[E314] C11 Tetrasub. Benzenes to air

[E315] C11 Trisub. Benzenes to air

[E316] C12 2-Alkenes to air

[E317] C12 3-Alkenes to air

[E318] C12 Bicycloalkanes to air

[E319] C12 Cyclic or di-olefins to air

[E320] C12 Cycloalkanes to air

[E321] C12 Disub. Benzenes to air

[E322] C12 Disub. Naphthalenes to air

[E323] C12 Hexaasub. Benzenes to air

[E324] C12 Internal Alkenes to air

[E325] C12 Monosub. Benzenes to air

[E326] C12 Monosub. Naphth. to air

[E327] C12 Tetrasub. Benzenes to air

[E328] C12 Trisub. Benzenes to air

[E329] C13 3-Alkenes to air

[E330] C13 Bicycloalkanes to air

[E331] C13 Cyclic or di-olefins to air

[E332] C13 Cycloalkanes to air

[E333] C13 Disub. Benzenes to air

[E334] C13 Disub. Naphthalenes to air

[E335] C13 Internal Alkenes to air

[E336] C13 Monosub. Benzenes to air

[E337] C13 Monosub. Naphth. to air

[E338] C13 Trisub. Benzenes to air

[E339] C13 Trisub. Naphthalenes to air

[E340] C14 Bicycloalkanes to air

[E341] C14 Cycloalkanes to air

[E342] C15 Bicycloalkanes to air

[E343] C15 Cycloalkanes to air

[E344] C3 Adehydes to air

[E345] C4 Aldehydes to air

[E346] C4 Internal Alkenes to air

[E347] C5 Adehydes to air

[E348] C5 Cyclic Ketones to air

[E349] C5 Internal Alkenes to air

[E350] C5 Ketones to air

[E351] C5 Terminal Alkenes to air

[E352] C6 Adehydes to air

[E353] C6 Cyclic Ketones to air

[E354] C6 Cyclic or di-olefins to air

[E355] C6 Cycloalkanes to air

[E356] C6 internal Alkenes to air

[E357] C6 Ketones to air

[E358] C6 Terminal Alkenes to air

[E359] C7 Adehydes to air

[E360] C7 Cyclic Ketones to air

[E361] C7 Cyclic or di-olefins to air

[E362] C7 Cycloalkanes to air

[E363] C7 Internal Alkenes to air

[E364] C7 Ketones to air

[E365] C7 Terminal Alkenes to air

[E366] C8 Aldehydes to air

9120 Ecopoints/kg

9120 Ecopoints/kg

9120 Ecopoints/kg

9120 Ecopoints/kg

9120 Ecopoints/kg

9120 Ecopoints/kg

9120 Ecopoints $/ \mathrm{kg}$

9120 Ecopoints/kg

9120 Ecopoints $/ \mathrm{kg}$

9120 Ecopoints/kg

9120 Ecopoints/kg

9120 Ecopoints/kg

9120 Ecopoints/kg

9120 Ecopoints/kg

9120 Ecopoints/kg

9120 Ecopoints/kg

9120 Ecopoints/kg

9120 Ecopoints/kg

9120 Ecopoints/kg

9120 Ecopoints/kg

9120 Ecopoints/kg

9120 Ecopoints/kg

9120 Ecopoints/kg

9120 Ecopoints/kg

9120 Ecopoints/kg

9120 Ecopoints/kg

9120 Ecopoints/kg

9120 Ecopoints/kg

9120 Ecopoints/kg

9120 Ecopoints/kg

9120 Ecopoints/kg

9120 Ecopoints/kg

9120 Ecopoints/kg

9120 Ecopoints/kg

9120 Ecopoints/kg

9120 Ecopoints/kg

9120 Ecopoints/kg

9120 Ecopoints/kg

9120 Ecopoints/kg

9120 Ecopoints $/ \mathrm{kg}$

9120 Ecopoints/kg

9120 Ecopoints $/ \mathrm{kg}$

9120 Ecopoints/kg

9120 Ecopoints/kg

9120 Ecopoints $/ \mathrm{kg}$

9120 Ecopoints/kg

9120 Ecopoints/kg

9120 Ecopoints/kg

9120 Ecopoints $/ \mathrm{kg}$

9120 Ecopoints/kg

9120 Ecopoints $/ \mathrm{kg}$

9120 Ecopoints/kg

9120 Ecopoints $/ \mathrm{kg}$

9120 Ecopoints/kg

9120 Ecopoints $/ \mathrm{kg}$

9120 Ecopoints/kg

9120 Ecopoints/kg

9120 Ecopoints/kg

9120 Ecopoints/kg

9120 Ecopoints/kg

9120 Ecopoints/kg

9120 Ecopoints/kg

9120 Ecopoints/kg 
[E367] C8 Cyclic Ketones to air [E368] C8 Cyclic or di-olefins to air [E369] C8 Cycloalkanes to air [E370] C8 Disub. Benzenes to air [E371] C8 Internal Alkenes to air [E372] C8 Ketones to air

[E373] C8 Terminal Alkenes to air [E374] C9 Bicycloalkanes to air [E375] C9 Cyclic Ketones to air [E376] C9 Cyclic or di-olefins to air [E377] C9 Cycloalkanes to air [E378] C9 Disub. Benzenes to air [E379] C9 Internal Alkenes to air [E380] C9 Ketones to air [E381] C9 Monosub. Benzenes to air [E382] C9 Styrenes to air [E383] C9 Terminal Alkenes to air [E384] C9 Trisub. Benzenes to air [E385] cadmium to air [E393] Carbon dioxide to air [E396] CFC (hard) (unspecified) to air [E397] CFC (soft) (unspecified) to air [E398] CFC-11 to air [E399] CFC-113 to air [E400] CFC-114 to air [E401] CFC-115 to air [E402] CFC-12 to air [E403] CFC-13 to air [E407] chlorobenzene to air

[E408] Chlorophenols (unspec.) to air [E409] Chloropicrin to air

[E413] chromium (unspecified) to air

[E414] chromium III to air

[E415] chromium VI to air

[E416] chrysene to air

[E417] cis-2-Butene to air

[E418] cis-2-Hexene to air

[E419] cis-2-Pentene to air

[E420] cis-Dichloroethene to air

[E422] copper to air

[E424] Crotonaldehyde to air

[E426] Cyanides to air

[E427] cyclic alkanes (unspec.) to air

[E428] Cyclobutane to air

[E429] Cyclohexane to air

[E430] Cyclohexanol to air

[E431] Cyclohexanone to air

[E432] Cyclohexene to air

[E433] Cyclopentadiene to air

[E434] Cyclopentane to air

[E435] Cyclopentene to air

[E436] Cyclopropane to air

[E444] Di(2-ethylhexyl)phtalate to air

[E445] Diacetone alcohol to air

[E447] Dibutyl Ether to air

[E448] Dibutylphtalate to air

[E449] Dichloromethane (Methylene Chloride) to air

[E453] Diethyl Ether to air

[E454] diethylamine to air

[E455] Diethylketone to air

[E456] Diethylphtalate to air

[E457] Dihexylphtalate to air

[E458] Diisodecylphtalate to air

[E459] Diisooctylphtalate to air

[E460] Diisopropylether to air

[E462] Dimethyl Adipate to air

[E463] Dimethyl Ether to air
9120 Ecopoints/kg

9120 Ecopoints $/ \mathrm{kg}$

9120 Ecopoints/kg

9120 Ecopoints/kg

9120 Ecopoints/kg

9120 Ecopoints $/ \mathrm{kg}$

9120 Ecopoints/kg

9120 Ecopoints/kg

9120 Ecopoints/kg

9120 Ecopoints/kg

9120 Ecopoints/kg

9120 Ecopoints $/ \mathrm{kg}$

9120 Ecopoints/kg

9120 Ecopoints/kg

9120 Ecopoints/kg

9120 Ecopoints/kg

9120 Ecopoints/kg

9120 Ecopoints/kg

4.79E+07 Ecopoints $/ \mathrm{kg}$

44.6 Ecopoints $/ \mathrm{kg}$

$1.61 \mathrm{E}+06$ Ecopoints $/ \mathrm{kg}$

$1.61 E+06$ Ecopoints $/ \mathrm{kg}$

$1.61 \mathrm{E}+06$ Ecopoints $/ \mathrm{kg}$

$1.61 \mathrm{E}+06$ Ecopoints/kg

$1.61 \mathrm{E}+06$ Ecopoints $/ \mathrm{kg}$

$1.61 \mathrm{E}+06$ Ecopoints $/ \mathrm{kg}$

$1.61 \mathrm{E}+06$ Ecopoints $/ \mathrm{kg}$

$1.61 \mathrm{E}+06$ Ecopoints $/ \mathrm{kg}$

9120 Ecopoints $/ \mathrm{kg}$

9120 Ecopoints/kg

9120 Ecopoints $/ \mathrm{kg}$

$5.54 \mathrm{E}+06$ Ecopoints $/ \mathrm{kg}$

5.54E+06 Ecopoints/kg

5.54E+06 Ecopoints/kg

$9.80 \mathrm{E}+06$ Ecopoints $/ \mathrm{kg}$

9120 Ecopoints/kg

9120 Ecopoints/kg

9120 Ecopoints/kg

9120 Ecopoints/kg

6.37E+06 Ecopoints/kg

9120 Ecopoints/kg

9120 Ecopoints $/ \mathrm{kg}$

9120 Ecopoints/kg

9120 Ecopoints/kg

9120 Ecopoints/kg

9120 Ecopoints/kg

9120 Ecopoints/kg

9120 Ecopoints/kg

9120 Ecopoints/kg

9120 Ecopoints/kg

9120 Ecopoints/kg

9120 Ecopoints/kg

9120 Ecopoints/kg

9120 Ecopoints/kg

9120 Ecopoints/kg

9120 Ecopoints/kg

9120 Ecopoints/kg

9120 Ecopoints/kg

9120 Ecopoints/kg

9120 Ecopoints $/ \mathrm{kg}$

9120 Ecopoints/kg

9120 Ecopoints $/ \mathrm{kg}$

9120 Ecopoints/kg

9120 Ecopoints $/ \mathrm{kg}$

9120 Ecopoints/kg

9120 Ecopoints/kg

9120 Ecopoints $/ \mathrm{kg}$ 
[E464] Dimethyl Glutarate to air

[E465] Dimethyl Naphthalenes to air

[E466] Dimethyl Succinate to air

[E467] dimethylamine to air

[E468] Dimethylphtalate to air

[E469] Dinitrogen oxide (nitrous oxide) to air

[E472] Dioctylphtalate to air

[E473] dioxins (unspec.) to air

[E474] diphenyl to air

[E477] d-Limonene to air

[E482] esters (unspec.) to air

[E483] Ethane to air

[E484] ethanethiol (ethylmercaptan) to air

[E485] Ethanol to air

[E486] ethers (unspec.) to air

[E488] Ethyl Acetate to air

[E489] Ethyl Acetylene to air

[E490] Ethyl Acrylate to air

[E491] Ethyl Amine to air

[E492] Ethyl Chloride to air

[E493] Ethyl Cyclopentane to air

[E494] Ethyl Isopropyl Ether to air

[E495] Ethyl- trans-Butyl Ether to air

[E496] Ethylbenzene to air

[E497] ethylbutyrate to air

[E498] Ethylcyclohexane to air

[E499] Ethylene (ethene) to air

[E500] Ethylene Dibromide to air

[E501] Ethylene Dichloride to air

[E502] Ethylene Glycol to air

[E503] ethylthioethane (diethylsulfide) to air

[E509] fluoranthrene to air

[E511] Formaldehyde (methanal) to air

[E512] Formic acid to air

[E513] Furan to air

[E514] Furanes (unspec.) to air

[E515] Glyoxal to air

[E517] HALON (HBFC-2401) to air

[E518] HALON-1201 (HBFC-1201) to air

[E519] HALON-1202 to air

[E520] HALON-1211 to air

[E521] HALON-1301 to air

[E522] HALON-2311 (HBFC-2311) to air

[E523] HALON-2402 (Di-Broom-tetra-Fluor-ethane) to air

[E524] halons (unspecified) to air

[E525] HCFC-123 (1,1-Di-Chloro-222-Tri-Fluorethaan) to air

[E526] HCFC-124 (1-Chloro-1222-Tetra-Fluor-Ethane) to air

[E527] HCFC-141b (Dichlorofluorethane) to air

[E528] HCFC-142b (1-Chloro-1,1-Difluorethane) to air

[E529] HCFC-22 (Chlorodifluormethane) to air

[E530] HCFC-225ca to air

[E531] HCFC-225cb to air

[E537] hexachlorobenzene to air

[E541] Hexyl Cyclohexane to air

[E542] HFC (unspec.) to air

[E543] HFC-125 (Pentafluoroethane) to air

[E544] HFC-134 (1,1,1,2-tetra-fluor-ethane) to air

[E545] HFC-134a (1,1,1,2-tetrafluoroethane) to air

[E546] HFC-143 to air

[E547] HFC-143a (1,1,1-trifluoroethane) to air

[E548] HFC-152a (1,1-difluoroethane) to air

[E549] HFC-227ea to air

[E550] HFC-23 to air

[E551] HFC-236fa to air

[E552] HFC-245ca to air

[E553] HFC-32 to air

[E554] HFC-41 to air
9120 Ecopoints $/ \mathrm{kg}$

9120 Ecopoints/kg

9120 Ecopoints/kg

9120 Ecopoints/kg

9120 Ecopoints/kg

58800 Ecopoints/kg

9120 Ecopoints/kg

9120 Ecopoints/kg

9120 Ecopoints/kg

9120 Ecopoints $/ \mathrm{kg}$

9120 Ecopoints/kg

9120 Ecopoints/kg

9120 Ecopoints/kg

9120 Ecopoints/kg

9120 Ecopoints/kg

9120 Ecopoints/kg

9120 Ecopoints/kg

9120 Ecopoints $/ \mathrm{kg}$

9120 Ecopoints/kg

9120 Ecopoints/kg

9120 Ecopoints/kg

9120 Ecopoints/kg

9120 Ecopoints/kg

9120 Ecopoints $/ \mathrm{kg}$

9120 Ecopoints/kg

9120 Ecopoints/kg

9120 Ecopoints $/ \mathrm{kg}$

9120 Ecopoints/kg

9120 Ecopoints/kg

9120 Ecopoints/kg

9120 Ecopoints/kg

9.80E+06 Ecopoints/kg

9120 Ecopoints/kg

9120 Ecopoints/kg

9120 Ecopoints/kg

9120 Ecopoints $/ \mathrm{kg}$

9120 Ecopoints/kg

9120 Ecopoints $/ \mathrm{kg}$

9120 Ecopoints/kg

9120 Ecopoints $/ \mathrm{kg}$

9120 Ecopoints/kg

9120 Ecopoints/kg

9120 Ecopoints/kg

9120 Ecopoints/kg

9120 Ecopoints/kg

$1.61 \mathrm{E}+06$ Ecopoints/kg

$1.61 \mathrm{E}+06$ Ecopoints $/ \mathrm{kg}$

$1.61 \mathrm{E}+06$ Ecopoints $/ \mathrm{kg}$

$1.61 \mathrm{E}+06$ Ecopoints $/ \mathrm{kg}$

$1.61 \mathrm{E}+06$ Ecopoints $/ \mathrm{kg}$

$1.61 \mathrm{E}+06$ Ecopoints $/ \mathrm{kg}$

$1.61 \mathrm{E}+06$ Ecopoints/kg

9120 Ecopoints $/ \mathrm{kg}$

9120 Ecopoints/kg

9120 Ecopoints/kg

9120 Ecopoints/kg

9120 Ecopoints/kg

9120 Ecopoints/kg

9120 Ecopoints/kg

9120 Ecopoints/kg

9120 Ecopoints/kg

9120 Ecopoints/kg

9120 Ecopoints/kg

9120 Ecopoints $/ \mathrm{kg}$

9120 Ecopoints/kg

9120 Ecopoints/kg

9120 Ecopoints/kg 
[E555] HFC-43-10mee to air

[E556] Hydrocarbons to air

[E557] Hydrocarbons (excl. methane) to air

[E558] Hydrocarbons Chloro-Fluor- (HCFC) to air

[E559] Hydrocarbons haloginated to air

[E561] hydrogen chloride to air

[E562] hydrogen fluoride to air

[E565] Indan to air

[E566] indeno[1,2,3-cd]pyrene to air

[E569] isoamyl isobutyrate to air

[E570] isobutane to air

[E571] isobutanol (2-methylpropanol-1) to air

[E572] isobutene (2-methylpropene) to air

[E573] isobutyl acetate to air

[E574] isobutyl isobutyrate to air

[E575] isobutyraldehyde to air

[E576] isopentane to air

[E577] isopentylacetate (iso-amylacetate) to air

[E578] isoprene to air

[E579] isopropanol to air

[E580] isopropyl acetate to air

[E581] isopropyl benzene (cumene) to air

[E582] isopropyl propionate to air

[E584] lead to air

[E592] mercury to air

[E593] meta-Cresol (3-methylphenol) to air

[E594] meta-Ethyltoluene to air

[E596] meta-Xylene (1,3-dimethylbenzene) to air

[E599] Methacrolein to air

[E600] Methane to air

[E602] Methanol to air

[E604] Methyl Acetate to air

[E606] Methyl Acrylate to air

[E607] methyl amine to air

[E608] Methyl Bromide to air

[E609] Methyl Choride to air

[E610] Methyl cyclohexane to air

[E611] Methyl cyclopentane to air

[E612] methyl dithiomethane to air

[E613] Methyl Ethyl Ketone to air

[E614] Methyl Formate to air

[E615] Methyl Glyoxal to air

[E616] Methyl Isobutyl Ketone (4-methylpentanon-2) to air

[E617] Methyl iso-butyrate to air

[E618] methyl methacrylate to air

[E619] Methyl Naphthalenes to air

[E620] Methyl Nitrite to air

[E621] methyl propionate to air

[E622] Methyl propyl Ketone to air

[E623] methyl thiomethane (dimethylsulfide) to air

[E624] Methyl trans-Butyl Ether to air

[E625] Methyl-Isopropylketone to air

[E626] methyl-mercury to air

[E627] Methyl-transbutylketone to air

[E633] Naphtalene to air

[E634] Neopentane to air

[E635] nickel to air

[E639] Nitrobenzene to air

[E641] nitrogen dioxide to air

[E643] nitrogen oxides to air

[E645] Octyl Cyclohexane to air

[E647] organics acids (unspec.) to air

[E648] ortho-Cresol (2-methylphenol) to air

[E649] ortho-Ethyltoluene to air

[E650] ortho-Xylene (1,2-dimethylbenzene) to air

[E653] para-Cresol (4-methylphenol) to air

[E654] para-Ethyltoluene to air
9120 Ecopoints/kg

9120 Ecopoints/kg

9120 Ecopoints/kg

9120 Ecopoints/kg

9120 Ecopoints/kg

9190 Ecopoints/kg

3.30E+06 Ecopoints/kg

9120 Ecopoints/kg

9.80E+06 Ecopoints/kg

9120 Ecopoints/kg

9120 Ecopoints/kg

9120 Ecopoints/kg

9120 Ecopoints/kg

9120 Ecopoints/kg

9120 Ecopoints/kg

9120 Ecopoints/kg

9120 Ecopoints/kg

9120 Ecopoints/kg

9120 Ecopoints/kg

9120 Ecopoints/kg

9120 Ecopoints/kg

9120 Ecopoints/kg

9120 Ecopoints/kg

1.57E+06 Ecopoints/kg

8.02E+08 Ecopoints/kg

9120 Ecopoints/kg

9120 Ecopoints/kg

9120 Ecopoints/kg

9120 Ecopoints/kg

9120 Ecopoints/kg

9120 Ecopoints/kg

9120 Ecopoints/kg

9120 Ecopoints/kg

9120 Ecopoints/kg

9120 Ecopoints/kg

9120 Ecopoints/kg

9120 Ecopoints/kg

9120 Ecopoints/kg

9120 Ecopoints/kg

9120 Ecopoints/kg

9120 Ecopoints/kg

9120 Ecopoints/kg

9120 Ecopoints/kg

9120 Ecopoints/kg

9120 Ecopoints/kg

9120 Ecopoints/kg

9120 Ecopoints/kg

9120 Ecopoints/kg

9120 Ecopoints/kg

9120 Ecopoints/kg

9120 Ecopoints/kg

9120 Ecopoints/kg

9120 Ecopoints/kg

9120 Ecopoints/kg

9.80E+06 Ecopoints/kg

9120 Ecopoints/kg

2.00E+08 Ecopoints/kg

9120 Ecopoints/kg

9190 Ecopoints/kg

9190 Ecopoints/kg

9120 Ecopoints/kg

9120 Ecopoints/kg

9120 Ecopoints/kg

9120 Ecopoints/kg

9120 Ecopoints/kg

9120 Ecopoints/kg

9120 Ecopoints/kg 
[E657] para-Trifluoromethyl-Cl-Benzene to air

[E658] para-Xylene (1,4-dimethylbenzene) to air

[E660] pentachlorobenzene to air

[E661] pentachloronitrobenzene to air

[E662] pentachlorophenol to air

[E663] Pentanaldehyde to air

[E664] Pentanol to air

[E665] Perfluorobutane to air

[E666] Perfluorocyclobutane to air

[E667] Perfluoroethane (CFC-116) to air

[E668] Perfluorohexane to air

[E669] Perfluoromethane (CFC-14) to air

[E670] Perfluoropentane to air

[E671] Perfluoropropane to air

[E673] petrol to air

[E674] phenanthrene to air

[E675] Phenol to air

[E680] Phtalic anhydride to air

[E681] phthalates to air

[E684] Polycyclic Aromatic Hydrocarbons (PAH) (unspecified) to air

[E685] Polycyclic Aromatic Hydrocarbons Carcinogenic- (carcinogenic-PAH) to air

[E687] Propane to air

[E688] Propanoic acid (propionic acid) to air

[E689] Propionaldehyde (propanal) to air

[E691] Propyl Acetate to air

[E692] Propyl Cyclopentane to air

[E693] Propylene to air

[E694] Propylene Carbonate to air

[E695] Propylene Glycol to air

[E696] Propylene Glycol Methyl Ether Acetate to air

[E697] Propylene Oxide to air

[E700] Sabinene to air

[E701] sec-Butanol to air

[E702] sec-Butyl Benzene to air

[E703] sec-Buytl Acetate to air

[E706] styrene (vinylbenzene) to air

[E707] Subst. C7 Ester (C12) to air

[E708] Sucts. C9 Ester (C12) to air

[E709] sulphur dioxide to air

[E713] tar to air

[E715] terpentine to air

[E716] tertiary-Butanol to air

[E717] tertiary-Butyl Acetate to air

[E718] tetrachloroethylene (PER) (tetrachloroethene) to air

[E719] Tetrachloromethane (carbon tetrachloride) $(\mathrm{HC}-10)$ to air

[E720] tetrafluorethylene to air

[E721] Tetralin to air

[E726] Tolualdehyde to air

[E727] Toluene to air

[E728] trans-1,2-Dichloroethene to air

[E729] trans-2-Butene to air

[E730] trans-2-Hexene to air

[E731] trans-2-Pentene to air

[E732] trans-dichloroethene to air

[E737] Trichloroethylene (tri) to air

[E738] Trichloromethane (chloroform) to air

[E742] Valeraldehyde (pentanal) to air

[E744] Vinyl Acetate to air

[E745] Vinyl Chloride (chloroethene) to air

[E746] Volatile Organic Compounds (VOC) to air

[E747] Volatile Organic Compounds non-methane- (non methane VOC) to air [E748] zinc to air

[E779] ammonia, ammonium to fresh water

[E798] Biological Oxygen Demand (BOD) to fresh water

[E809] Chemical oxigen demand (COD) to fresh water

[E813] Chlorine to fresh water

[E894] Nitrate to fresh water
9120 Ecopoints/kg

9120 Ecopoints/kg

9120 Ecopoints/kg

9120 Ecopoints/kg

9120 Ecopoints $/ \mathrm{kg}$

9120 Ecopoints $/ \mathrm{kg}$

9120 Ecopoints $/ k g$

$1.61 \mathrm{E}+06$ Ecopoints $/ \mathrm{kg}$

$1.61 \mathrm{E}+06$ Ecopoints $/ \mathrm{kg}$

$1.61 \mathrm{E}+06$ Ecopoints/kg

$1.61 \mathrm{E}+06$ Ecopoints $/ \mathrm{kg}$

$1.61 \mathrm{E}+06$ Ecopoints $/ \mathrm{kg}$

$1.61 \mathrm{E}+06$ Ecopoints/kg

$1.61 \mathrm{E}+06$ Ecopoints $/ \mathrm{kg}$

9120 Ecopoints/kg

9.80E+06 Ecopoints/kg

9120 Ecopoints/kg

9120 Ecopoints $/ \mathrm{kg}$

9120 Ecopoints $/ \mathrm{kg}$

9.80E+06 Ecopoints/kg

9.80E+06 Ecopoints $/ \mathrm{kg}$

9120 Ecopoints $/ \mathrm{kg}$

9120 Ecopoints $/ \mathrm{kg}$

9120 Ecopoints/kg

9120 Ecopoints/kg

9120 Ecopoints/kg

9120 Ecopoints/kg

9120 Ecopoints $/ \mathrm{kg}$

9120 Ecopoints/kg

9120 Ecopoints/kg

9120 Ecopoints/kg

9120 Ecopoints $/ \mathrm{kg}$

9120 Ecopoints/kg

9120 Ecopoints/kg

9120 Ecopoints $/ \mathrm{kg}$

9120 Ecopoints/kg

9120 Ecopoints/kg

9120 Ecopoints $/ \mathrm{kg}$

10000 Ecopoints/kg

9120 Ecopoints/kg

9120 Ecopoints $/ \mathrm{kg}$

9120 Ecopoints $/ \mathrm{kg}$

9120 Ecopoints/kg

9120 Ecopoints/kg

9120 Ecopoints $/ \mathrm{kg}$

9120 Ecopoints/kg

9120 Ecopoints/kg

9120 Ecopoints/kg

9120 Ecopoints/kg

9120 Ecopoints $/ \mathrm{kg}$

9120 Ecopoints/kg

9120 Ecopoints/kg

9120 Ecopoints/kg

9120 Ecopoints/kg

9120 Ecopoints/kg

9120 Ecopoints/kg

9120 Ecopoints/kg

9120 Ecopoints/kg

9120 Ecopoints/kg

9120 Ecopoints/kg

9120 Ecopoints/kg

$3.92 E+05$ Ecopoints $/ \mathrm{kg}$

1.21E+05 Ecopoints $/ \mathrm{kg}$

11500 Ecopoints/kg

3830 Ecopoints/kg

26.2 Ecopoints $/ \mathrm{kg}$

27300 Ecopoints/kg 
[E896] Nitrogen to fresh water [E910] Phosphate to fresh water [E911] Phosphorus to fresh water [E924] sulphates to fresh water [E973] ammonia, ammonium to sea water

[E992] Biological Oxygen Demand (BOD) to sea water [E1003] Chemical oxigen demand (COD) to sea water [E1007] Chlorine to sea water [E1088] Nitrate to sea water [E1090] Nitrogen to sea water [E1104] Phosphate to sea water [E1105] Phosphorus to sea water [E1118] sulphates to sea water
1.21E+05 Ecopoints $/ \mathrm{kg}$ $3.03 E+05$ Ecopoints $/ \mathrm{kg}$ $9.30 \mathrm{E}+05$ Ecopoints $/ \mathrm{kg}$

76.6 Ecopoints $/ \mathrm{kg}$

1.21E+05 Ecopoints/kg

11500 Ecopoints/kg

3830 Ecopoints $/ \mathrm{kg}$

26.2 Ecopoints $/ \mathrm{kg}$

27300 Ecopoints/kg

$1.21 \mathrm{E}+05$ Ecopoints $/ \mathrm{kg}$

$3.03 E+05$ Ecopoints $/ \mathrm{kg}$

9.30E+05 Ecopoints/kg

76.6 Ecopoints/kg

Category $=[$ C19] Photochemical oxidant formation (Ecoindicator95)

Description $=$ Ecoindicator 95

Author $=$ Goedkoop, 1995

Date $=27-09-1999$

Environmental resources

Environmental resource

Value

Environmental emissions

Environmental emission

[E90] 1,1,1-trichloroethane (methyl chloroform; HC-140a) to air

[E91] 1,1,2-Trichloroethane to air

[E92] 1,1-Dichloroethane to air

[E93] 1,1-Dichloroethene to air

[E94] 1,2,3,4-tetrachlorobenzene to air

[E95] 1,2,3,5-tetrachlorobenzene to air

[E96] 1,2,3-trichlorobenzene to air

[E97] 1,2,3-Trimethyl Benzene to air

[E98] 1,2,4,5-tetrachlorobenzene to air

[E99] 1,2,4-trichlorobenzene to air

[E100] 1,2,4-trimethylbenzene to air

[E101] 1,2-Butanediol to air

[E102] 1,2-dichlorobenzene to air

[E103] 1,2-dichloroethane to air

[E104] 1,2-Dichloropropane to air

[E105] 1,2-Dihydroxy Hexane to air

[E106] 1,3 Diethyl-Cyclohexane to air

[E107] 1,3,5-trichlorobenzene to air

[E108] 1,3,5-Triethyl Cyclohex. to air

[E109] 1,3,5-trimethylbenzene (mesitylene) to air

[E110] 1,3,5-Tripropyl Cyclohex. to air

[E111] 1,3-Butadiene to air

[E112] 1,3-dichlorobenzene to air

[E113] 1,3-Dimeth. Cyclopentane to air

[E114] 1,3-Dimethyl Cyclohexane to air

[E115] 1,3-Diprop-5-Eth Cyclohex. to air

[E116] 1,4-dichlorobenzene to air

[E117] 13-Dieth.-5-Pent Cyclohex. to air

[E118] 13-Dieth-5-Me. Cyclohex. to air

[E119] 1-Butane to air

[E120] 1-Butanol to air

[E121] 1-Butene to air

[E122] 1-Butoxy Propanol to air

[E123] 1-Butyl Acetate to air

[E124] 1-Butyl Benzene to air

[E125] 1-Butyl Bromide to air

[E126] 1-butylpropionate to air

[E127] 1-C16 to air

[E128] 1-chloro-4-nitrobenzene to air

[E129] 1-Chlorobutane to air

[E130] 1-Decane to air

[E131] 1-Dodecane to air

\section{Value}

$$
\text { Unit }
$$

$0.021 \mathrm{~kg}$ ethylene eq. $/ \mathrm{kg}$

$0.021 \mathrm{~kg}$ ethylene eq. $/ \mathrm{kg}$

$0.021 \mathrm{~kg}$ ethylene eq. $/ \mathrm{kg}$

$0.021 \mathrm{~kg}$ ethylene eq. $/ \mathrm{kg}$

$0.761 \mathrm{~kg}$ ethylene eq. $/ \mathrm{kg}$

$0.761 \mathrm{~kg}$ ethylene eq. $/ \mathrm{kg}$

$0.761 \mathrm{~kg}$ ethylene eq. $/ \mathrm{kg}$

$0.761 \mathrm{~kg}$ ethylene eq. $/ \mathrm{kg}$

$0.761 \mathrm{~kg}$ ethylene eq. $/ \mathrm{kg}$

$0.761 \mathrm{~kg}$ ethylene eq. $/ \mathrm{kg}$

$0.761 \mathrm{~kg}$ ethylene eq. $/ \mathrm{kg}$

$0.196 \mathrm{~kg}$ ethylene eq. $/ \mathrm{kg}$

$0.761 \mathrm{~kg}$ ethylene eq. $/ \mathrm{kg}$

$0.021 \mathrm{~kg}$ ethylene eq. $/ \mathrm{kg}$

$0.021 \mathrm{~kg}$ ethylene eq. $/ \mathrm{kg}$

$0.196 \mathrm{~kg}$ ethylene eq. $/ \mathrm{kg}$

$0.398 \mathrm{~kg}$ ethylene eq. $/ \mathrm{kg}$

$0.761 \mathrm{~kg}$ ethylene eq. $/ \mathrm{kg}$

$0.398 \mathrm{~kg}$ ethylene eq. $/ \mathrm{kg}$

$0.761 \mathrm{~kg}$ ethylene eq. $/ \mathrm{kg}$

$0.398 \mathrm{~kg}$ ethylene eq. $/ \mathrm{kg}$

$0.398 \mathrm{~kg}$ ethylene eq. $/ \mathrm{kg}$

$0.761 \mathrm{~kg}$ ethylene eq. $/ \mathrm{kg}$

$0.398 \mathrm{~kg}$ ethylene eq. $/ \mathrm{kg}$

$0.398 \mathrm{~kg}$ ethylene eq. $/ \mathrm{kg}$

$0.398 \mathrm{~kg}$ ethylene eq. $/ \mathrm{kg}$

$0.761 \mathrm{~kg}$ ethylene eq. $/ \mathrm{kg}$

$0.398 \mathrm{~kg}$ ethylene eq. $/ \mathrm{kg}$

$0.398 \mathrm{~kg}$ ethylene eq. $/ \mathrm{kg}$

$0.398 \mathrm{~kg}$ ethylene eq. $/ \mathrm{kg}$

$0.196 \mathrm{~kg}$ ethylene eq. $/ \mathrm{kg}$

$0.398 \mathrm{~kg}$ ethylene eq. $/ \mathrm{kg}$

$0.196 \mathrm{~kg}$ ethylene eq. $/ \mathrm{kg}$

$0.398 \mathrm{~kg}$ ethylene eq. $/ \mathrm{kg}$

$0.761 \mathrm{~kg}$ ethylene eq. $/ \mathrm{kg}$

$0.021 \mathrm{~kg}$ ethylene eq. $/ \mathrm{kg}$

$0.398 \mathrm{~kg}$ ethylene eq. $/ \mathrm{kg}$

$0.398 \mathrm{~kg}$ ethylene eq. $/ \mathrm{kg}$

$0.761 \mathrm{~kg}$ ethylene eq. $/ \mathrm{kg}$

$0.021 \mathrm{~kg}$ ethylene eq. $/ \mathrm{kg}$

$0.398 \mathrm{~kg}$ ethylene eq. $/ \mathrm{kg}$

$0.398 \mathrm{~kg}$ ethylene eq. $/ \mathrm{kg}$ 
[E132] 1-Eth.-4-Meth. Cyclohex to air

[E133] 1-Ethoxy-2-Propanol to air

[E134] 1-Heptane to air

[E135] 1-Heptanol to air

[E136] 1-Heptene to air

[E137] 1-Hexane to air

[E138] 1-Hexene to air

[E139] 1-Methoxy-2-Propanol to air

[E140] 1-Methyl-2-Pyrrolidone to air

[E141] 1-Nonane to air

[E142] 1-Nonene to air

[E143] 1-Octane to air

[E144] 1-Octanol to air

[E145] 1-Octene to air

[E146] 1-Pentadecane to air

[E147] 1-Pentane to air

[E148] 1-Pentene to air

[E149] 1-Propanol to air

[E150] 1-Propyl Benzene to air

[E151] 1-Propyl Bromide to air

[E152] 1-Tetradecane to air

[E153] 1-Tridecane to air

[E154] 1-Undecane to air

[E155] 2- Octanol to air

[E156] 2-(2-Butoxyethoxy)-Ethanol to air

[E157] 2-(2-Ethoxyethoxy)-Ethanol to air

[E158] 2-(Cl-methyl)-3-Cl-Propene to air

[E159] 2,2,3 Trimethyl Butane to air

[E160] 2,2,3,3-Tetramethyl Butane to air

[E161] 2,2,4-Trimethyl Pentane to air

[E162] 2,2,5-Trimethyl Hexane to air

[E163] 2,2-Dimethylbutane to air

[E164] 2,3 Dimethyl Pentane to air

[E165] 2,3,3-Trimehyl-1-butene to air

[E166] 2,3,4,6-tetrachlorophenol to air

[E167] 2,3,4-Trimethyl Pentane to air

[E168] 2,3,7,8-TCDD (tetrachloride-dibenzo-dioxin) to air

[E169] 2,3-Dimethyl Hexane to air

[E170] 2,3-Dimethyl Naptht. to air

[E171] 2,3-Dimethyl-2-Butene to air

[E173] 2,4,5-trichlorophenol to air

[E174] 2,4,6-trichlorophenol to air

[E176] 2,4-dichlorophenol to air

[E177] 2,4-Dimethyl Heptane to air

[E178] 2,4-Dimethyl Hexane to air

[E179] 2,4-Dimethyl Pentane to air

[E180] 2,5-Dimethyl Hexane to air

[E181] 2,6 Diethyl Octane to air

[E182] 2.3- Dimethylbutane to air

[E183] 2-Butoxy-Ethanol to air

[E184] 2-Butyltetrahydrofuran to air

[E185] 2-chlorophenol to air

[E186] 2-Ethoxy-Ethanol to air

[E187] 2-Ethoxyethyl Acetate to air

[E188] 2-Ethyl-1-Hexanol to air

[E190] 2-Heptenes to air

[E191] 2-Hexenes to air

[E192] 2-Methoxy-Ethanol to air

[E193] 2-Methyl-1-Butene to air

[E194] 2-Methyl-1-Pentene to air

[E195] 2-Methyl-2-Butene to air

[E196] 2-Methyl-2-Pentene to air

[E197] 2-Methylbutan-1-ol to air

[E198] 2-Methylbutan-2-ol to air

[E199] 2-Methylheptane to air

[E200] 2-Methylhexane to air

[E201] 2-Methylnonane to air
$0.398 \mathrm{~kg}$ ethylene eq. $/ \mathrm{kg}$

$0.196 \mathrm{~kg}$ ethylene eq. $/ \mathrm{kg}$

$0.398 \mathrm{~kg}$ ethylene eq. $/ \mathrm{kg}$

$0.196 \mathrm{~kg}$ ethylene eq. $/ \mathrm{kg}$

$0.398 \mathrm{~kg}$ ethylene eq. $/ \mathrm{kg}$

$0.398 \mathrm{~kg}$ ethylene eq. $/ \mathrm{kg}$

$0.398 \mathrm{~kg}$ ethylene eq. $/ \mathrm{kg}$

$0.196 \mathrm{~kg}$ ethylene eq. $/ \mathrm{kg}$

$0.398 \mathrm{~kg}$ ethylene eq. $/ \mathrm{kg}$

$0.398 \mathrm{~kg}$ ethylene eq. $/ \mathrm{kg}$

$0.398 \mathrm{~kg}$ ethylene eq. $/ \mathrm{kg}$

$0.398 \mathrm{~kg}$ ethylene eq. $/ \mathrm{kg}$

$0.196 \mathrm{~kg}$ ethylene eq. $/ \mathrm{kg}$

$0.398 \mathrm{~kg}$ ethylene eq. $/ \mathrm{kg}$

$0.398 \mathrm{~kg}$ ethylene eq. $/ \mathrm{kg}$

$0.408 \mathrm{~kg}$ ethylene eq. $/ \mathrm{kg}$

$0.398 \mathrm{~kg}$ ethylene eq. $/ \mathrm{kg}$

$0.196 \mathrm{~kg}$ ethylene eq. $/ \mathrm{kg}$

$0.761 \mathrm{~kg}$ ethylene eq. $/ \mathrm{kg}$

$0.021 \mathrm{~kg}$ ethylene eq. $/ \mathrm{kg}$

$0.398 \mathrm{~kg}$ ethylene eq. $/ \mathrm{kg}$

$0.398 \mathrm{~kg}$ ethylene eq. $/ \mathrm{kg}$

$0.398 \mathrm{~kg}$ ethylene eq. $/ \mathrm{kg}$

$0.196 \mathrm{~kg}$ ethylene eq. $/ \mathrm{kg}$

$0.196 \mathrm{~kg}$ ethylene eq. $/ \mathrm{kg}$

$0.196 \mathrm{~kg}$ ethylene eq. $/ \mathrm{kg}$

$0.021 \mathrm{~kg}$ ethylene eq. $/ \mathrm{kg}$

$0.398 \mathrm{~kg}$ ethylene eq. $/ \mathrm{kg}$

$0.398 \mathrm{~kg}$ ethylene eq. $/ \mathrm{kg}$

$0.398 \mathrm{~kg}$ ethylene eq. $/ \mathrm{kg}$

$0.398 \mathrm{~kg}$ ethylene eq. $/ \mathrm{kg}$

$0.398 \mathrm{~kg}$ ethylene eq. $/ \mathrm{kg}$

$0.398 \mathrm{~kg}$ ethylene eq. $/ \mathrm{kg}$

$0.398 \mathrm{~kg}$ ethylene eq. $/ \mathrm{kg}$

$0.761 \mathrm{~kg}$ ethylene eq. $/ \mathrm{kg}$

$0.398 \mathrm{~kg}$ ethylene eq. $/ \mathrm{kg}$

$0.021 \mathrm{~kg}$ ethylene eq. $/ \mathrm{kg}$

$0.398 \mathrm{~kg}$ ethylene eq. $/ \mathrm{kg}$

$0.761 \mathrm{~kg}$ ethylene eq. $/ \mathrm{kg}$

$0.398 \mathrm{~kg}$ ethylene eq. $/ \mathrm{kg}$

$0.761 \mathrm{~kg}$ ethylene eq. $/ \mathrm{kg}$

$0.761 \mathrm{~kg}$ ethylene eq. $/ \mathrm{kg}$

$0.761 \mathrm{~kg}$ ethylene eq. $/ \mathrm{kg}$

$0.398 \mathrm{~kg}$ ethylene eq. $/ \mathrm{kg}$

$0.398 \mathrm{~kg}$ ethylene eq. $/ \mathrm{kg}$

$0.398 \mathrm{~kg}$ ethylene eq. $/ \mathrm{kg}$

$0.398 \mathrm{~kg}$ ethylene eq. $/ \mathrm{kg}$

$0.398 \mathrm{~kg}$ ethylene eq. $/ \mathrm{kg}$

$0.398 \mathrm{~kg}$ ethylene eq. $/ \mathrm{kg}$

$0.196 \mathrm{~kg}$ ethylene eq. $/ \mathrm{kg}$

$0.196 \mathrm{~kg}$ ethylene eq. $/ \mathrm{kg}$

$0.761 \mathrm{~kg}$ ethylene eq. $/ \mathrm{kg}$

$0.196 \mathrm{~kg}$ ethylene eq. $/ \mathrm{kg}$

$0.398 \mathrm{~kg}$ ethylene eq. $/ \mathrm{kg}$

$0.196 \mathrm{~kg}$ ethylene eq. $/ \mathrm{kg}$

$0.398 \mathrm{~kg}$ ethylene eq. $/ \mathrm{kg}$

$0.398 \mathrm{~kg}$ ethylene eq. $/ \mathrm{kg}$

$0.196 \mathrm{~kg}$ ethylene eq. $/ \mathrm{kg}$

$0.398 \mathrm{~kg}$ ethylene eq. $/ \mathrm{kg}$

$0.398 \mathrm{~kg}$ ethylene eq. $/ \mathrm{kg}$

$0.398 \mathrm{~kg}$ ethylene eq. $/ \mathrm{kg}$

$0.398 \mathrm{~kg}$ ethylene eq. $/ \mathrm{kg}$

$0.196 \mathrm{~kg}$ ethylene eq. $/ \mathrm{kg}$

$0.196 \mathrm{~kg}$ ethylene eq. $/ \mathrm{kg}$

$0.398 \mathrm{~kg}$ ethylene eq. $/ \mathrm{kg}$

$0.398 \mathrm{~kg}$ ethylene eq. $/ \mathrm{kg}$

$0.398 \mathrm{~kg}$ ethylene eq. $/ \mathrm{kg}$ 
[E202] 2-Methyloctane to air

[E203] 2-Methylpentane to air

[E204] 2-methylpropanoic acid (isobutyric acid) to air [E205] 2-Pentenes to air

[E206] 3- Octanol to air

[E207] 3-(Chloromethyl)-Heptane to air

[E208] 3,4-dichloroaniline to air

[E209] 3,4-Propyl Heptane to air

[E210] 3,5 Diethyl Heptane to air

[E211] 3,5-Diethyltoluene to air

[E212] 3,5-Dimethylethylbenzene to air

[E213] 3,7-Diethyl Nonane to air

[E214] 3,8 Diethyl Decane to air

[E215] 3,9-Diethyl Undecane to air

[E216] 3-Carene to air

[E217] 3-chloroaniline to air

[E218] 3-Methyl-1-Butene to air

[E219] 3-Methylbutan-1-ol to air

[E220] 3-Methylbutan-2-ol to air

[E221] 3-methylbutanoic acid (isovaleric acid) to air

[E222] 3-Methylheptane to air

[E223] 3-Methylhexane to air

[E224] 3-Methylpentane to air

[E225] 3-Nonenes to air

[E226] 3-Octenes to air

[E227] 3-Pentanol to air

[E228] 4-chloroaniline to air

[E229] 4-Ethyl Heptane to air

[E230] 4-Methylheptane to air

[E232] Acetaldehyde (ethanal) to air

[E233] Acetic acid to air

[E234] Acetone (2-propanon) to air

[E235] Acetylene to air

[E236] Acrolein (2-propenal) to air

[E237] Acrylonitrile to air

[E239] alcohols (unspec.) to air

[E240] aldehydes (unspec.) to air

[E243] alkanes (unspec.) to air

[E244] alkenes (unspec.) to air

[E245] Alkyl Phenols to air

[E246] Allyl chloride to air

[E247] Alpha Methyl Tetrahydrofuran to air

[E248] alpha-Methyl Styrene to air

[E249] alpha-Pinene to air

[E252] anthracene to air

[E254] aromatics (unspecified) to air

[E262] Benzaldehyde to air

[E263] Benzene to air

[E264] benzo[a]anthracene to air

[E265] benzo[a]pyrene to air

[E266] benzo[ghi]perylene to air

[E267] benzo[k]fluoranthrene to air

[E268] Benzotrifluoride to air

[E271] Beta-Pinene to air

[E273] Branched C10 Alkanes to air

[E274] Branched C11 Alkanes to air

[E275] Branched C12 Alkanes to air

[E276] Branched C13 Alkanes to air

[E277] Branched C14 Alkanes to air

[E278] Branched C15 Alkanes to air

[E279] Branched C16 Alkanes to air

[E280] Branched C17 Alkanes to air

[E281] Branched C18 Alkanes to air

[E282] Branched C5 Alkanes to air

[E283] Branched C6 Alkanes to air

[E284] Branched C7 Alkanes to air

[E285] Branched C8 Alkanes to air
$0.398 \mathrm{~kg}$ ethylene eq. $/ \mathrm{kg}$

$0.398 \mathrm{~kg}$ ethylene eq. $/ \mathrm{kg}$

$0.398 \mathrm{~kg}$ ethylene eq. $/ \mathrm{kg}$

$0.398 \mathrm{~kg}$ ethylene eq. $/ \mathrm{kg}$

$0.196 \mathrm{~kg}$ ethylene eq. $/ \mathrm{kg}$

$0.021 \mathrm{~kg}$ ethylene eq. $/ \mathrm{kg}$

$0.021 \mathrm{~kg}$ ethylene eq. $/ \mathrm{kg}$

$0.398 \mathrm{~kg}$ ethylene eq. $/ \mathrm{kg}$

$0.398 \mathrm{~kg}$ ethylene eq. $/ \mathrm{kg}$

$0.761 \mathrm{~kg}$ ethylene eq. $/ \mathrm{kg}$

$0.761 \mathrm{~kg}$ ethylene eq. $/ \mathrm{kg}$

$0.398 \mathrm{~kg}$ ethylene eq. $/ \mathrm{kg}$

$0.398 \mathrm{~kg}$ ethylene eq. $/ \mathrm{kg}$

$0.398 \mathrm{~kg}$ ethylene eq. $/ \mathrm{kg}$

$0.398 \mathrm{~kg}$ ethylene eq. $/ \mathrm{kg}$

$0.021 \mathrm{~kg}$ ethylene eq. $/ \mathrm{kg}$

$0.398 \mathrm{~kg}$ ethylene eq. $/ \mathrm{kg}$

$0.196 \mathrm{~kg}$ ethylene eq. $/ \mathrm{kg}$

$0.196 \mathrm{~kg}$ ethylene eq. $/ \mathrm{kg}$

$0.398 \mathrm{~kg}$ ethylene eq. $/ \mathrm{kg}$

$0.398 \mathrm{~kg}$ ethylene eq. $/ \mathrm{kg}$

$0.398 \mathrm{~kg}$ ethylene eq. $/ \mathrm{kg}$

$0.398 \mathrm{~kg}$ ethylene eq. $/ \mathrm{kg}$

$0.398 \mathrm{~kg}$ ethylene eq. $/ \mathrm{kg}$

$0.398 \mathrm{~kg}$ ethylene eq. $/ \mathrm{kg}$

$0.196 \mathrm{~kg}$ ethylene eq. $/ \mathrm{kg}$

$0.021 \mathrm{~kg}$ ethylene eq. $/ \mathrm{kg}$

$0.398 \mathrm{~kg}$ ethylene eq. $/ \mathrm{kg}$

$0.398 \mathrm{~kg}$ ethylene eq. $/ \mathrm{kg}$

$0.443 \mathrm{~kg}$ ethylene eq. $/ \mathrm{kg}$

$0.398 \mathrm{~kg}$ ethylene eq. $/ \mathrm{kg}$

$0.178 \mathrm{~kg}$ ethylene eq. $/ \mathrm{kg}$

$0.168 \mathrm{~kg}$ ethylene eq. $/ \mathrm{kg}$

$0.443 \mathrm{~kg}$ ethylene eq. $/ \mathrm{kg}$

$0.398 \mathrm{~kg}$ ethylene eq. $/ \mathrm{kg}$

$0.196 \mathrm{~kg}$ ethylene eq. $/ \mathrm{kg}$

$0.443 \mathrm{~kg}$ ethylene eq. $/ \mathrm{kg}$

$0.398 \mathrm{~kg}$ ethylene eq. $/ \mathrm{kg}$

$0.398 \mathrm{~kg}$ ethylene eq. $/ \mathrm{kg}$

$0.761 \mathrm{~kg}$ ethylene eq. $/ \mathrm{kg}$

$0.021 \mathrm{~kg}$ ethylene eq./kg

$0.196 \mathrm{~kg}$ ethylene eq. $/ \mathrm{kg}$

$0.761 \mathrm{~kg}$ ethylene eq. $/ \mathrm{kg}$

$0.398 \mathrm{~kg}$ ethylene eq. $/ \mathrm{kg}$

$0.761 \mathrm{~kg}$ ethylene eq. $/ \mathrm{kg}$

$0.761 \mathrm{~kg}$ ethylene eq. $/ \mathrm{kg}$

$0.443 \mathrm{~kg}$ ethylene eq. $/ \mathrm{kg}$

$0.189 \mathrm{~kg}$ ethylene eq. $/ \mathrm{kg}$

$0.761 \mathrm{~kg}$ ethylene eq. $/ \mathrm{kg}$

$0.761 \mathrm{~kg}$ ethylene eq. $/ \mathrm{kg}$

$0.761 \mathrm{~kg}$ ethylene eq. $/ \mathrm{kg}$

$0.761 \mathrm{~kg}$ ethylene eq./kg

$0.021 \mathrm{~kg}$ ethylene eq. $/ \mathrm{kg}$

$0.398 \mathrm{~kg}$ ethylene eq. $/ \mathrm{kg}$

$0.398 \mathrm{~kg}$ ethylene eq. $/ \mathrm{kg}$

$0.398 \mathrm{~kg}$ ethylene eq. $/ \mathrm{kg}$

$0.398 \mathrm{~kg}$ ethylene eq. $/ \mathrm{kg}$

$0.398 \mathrm{~kg}$ ethylene eq. $/ \mathrm{kg}$

$0.398 \mathrm{~kg}$ ethylene eq. $/ \mathrm{kg}$

$0.398 \mathrm{~kg}$ ethylene eq. $/ \mathrm{kg}$

$0.398 \mathrm{~kg}$ ethylene eq. $/ \mathrm{kg}$

$0.398 \mathrm{~kg}$ ethylene eq. $/ \mathrm{kg}$

$0.398 \mathrm{~kg}$ ethylene eq. $/ \mathrm{kg}$

$0.398 \mathrm{~kg}$ ethylene eq. $/ \mathrm{kg}$

$0.398 \mathrm{~kg}$ ethylene eq. $/ \mathrm{kg}$

$0.398 \mathrm{~kg}$ ethylene eq. $/ \mathrm{kg}$

$0.398 \mathrm{~kg}$ ethylene eq. $/ \mathrm{kg}$ 
[E286] Branched C9 Alkanes to air [E287] butane (unspec.) to air

[E288] butanoic acid (butyric acid) to air

[E289] butylacrylate to air

[E290] Butylbenzylphtalate to air

[E291] Butyraldehyde (butanal) to air

[E292] C10 3-Alkenes to air

[E293] C10 Bicycloalkanes to air

[E294] C10 Cyclic Ketones to air

[E295] C10 Cyclic or di-olefins to air

[E296] C10 Cycloalkanes to air

[E297] C10 Disub. Benzenes to air

[E298] C10 Internal Alkenes to air

[E299] C10 Ketones to air

[E300] C10 Monosub. Benzenes to air

[E301] C10 Styrenes to air

[E302] C10 Tetrasub. Benzenes to air

[E303] C10 Trisub. Benzenes to air

[E304] C11 3-Alkenes to air

[E305] C11 Bicycloalkanes to air

[E306] C11 Cyclic or di-olefins to air

[E307] C11 Cycloalkanes to air

[E308] C11 Disub. Benzenes to air

[E309] C11 internal Alkenes to air

[E310] C11 Monosub. Benzenes to air

[E311] C11 Pentasub. Benzenes to air

[E312] C11 Pentasub. Benzenes to air

[E313] C11 Tetralin or Indane to air

[E314] C11 Tetrasub. Benzenes to air

[E315] C11 Trisub. Benzenes to air

[E316] C12 2-Alkenes to air

[E317] C12 3-Alkenes to air

[E318] C12 Bicycloalkanes to air

[E319] C12 Cyclic or di-olefins to air

[E320] C12 Cycloalkanes to air

[E321] C12 Disub. Benzenes to air

[E322] C12 Disub. Naphthalenes to air

[E323] C12 Hexaasub. Benzenes to air

[E324] C12 Internal Alkenes to air

[E325] C12 Monosub. Benzenes to air

[E326] C12 Monosub. Naphth. to air

[E327] C12 Tetrasub. Benzenes to air

[E328] C12 Trisub. Benzenes to air

[E329] C13 3-Alkenes to air

[E330] C13 Bicycloalkanes to air

[E331] C13 Cyclic or di-olefins to air

[E332] C13 Cycloalkanes to air

[E333] C13 Disub. Benzenes to air

[E334] C13 Disub. Naphthalenes to air

[E335] C13 Internal Alkenes to air

[E336] C13 Monosub. Benzenes to air

[E337] C13 Monosub. Naphth. to air

[E338] C13 Trisub. Benzenes to air

[E339] C13 Trisub. Naphthalenes to air

[E340] C14 Bicycloalkanes to air

[E341] C14 Cycloalkanes to air

[E342] C15 Bicycloalkanes to air

[E343] C15 Cycloalkanes to air

[E344] C3 Adehydes to air

[E345] C4 Aldehydes to air

[E346] C4 Internal Alkenes to air

[E347] C5 Adehydes to air

[E348] C5 Cyclic Ketones to air

[E349] C5 Internal Alkenes to air

[E350] C5 Ketones to air

[E351] C5 Terminal Alkenes to air

[E352] C6 Adehydes to air
$0.398 \mathrm{~kg}$ ethylene eq. $/ \mathrm{kg}$ $0.398 \mathrm{~kg}$ ethylene eq. $/ \mathrm{kg}$ $0.398 \mathrm{~kg}$ ethylene eq. $/ \mathrm{kg}$ $0.398 \mathrm{~kg}$ ethylene eq. $/ \mathrm{kg}$ $0.761 \mathrm{~kg}$ ethylene eq. $/ \mathrm{kg}$ $0.443 \mathrm{~kg}$ ethylene eq. $/ \mathrm{kg}$ $0.398 \mathrm{~kg}$ ethylene eq. $/ \mathrm{kg}$ $0.398 \mathrm{~kg}$ ethylene eq. $/ \mathrm{kg}$ $0.326 \mathrm{~kg}$ ethylene eq. $/ \mathrm{kg}$ $0.398 \mathrm{~kg}$ ethylene eq. $/ \mathrm{kg}$ $0.398 \mathrm{~kg}$ ethylene eq. $/ \mathrm{kg}$ $0.761 \mathrm{~kg}$ ethylene eq. $/ \mathrm{kg}$ $0.398 \mathrm{~kg}$ ethylene eq. $/ \mathrm{kg}$ $0.326 \mathrm{~kg}$ ethylene eq. $/ \mathrm{kg}$ $0.761 \mathrm{~kg}$ ethylene eq. $/ \mathrm{kg}$ $0.761 \mathrm{~kg}$ ethylene eq. $/ \mathrm{kg}$ $0.761 \mathrm{~kg}$ ethylene eq. $/ \mathrm{kg}$ $0.761 \mathrm{~kg}$ ethylene eq. $/ \mathrm{kg}$ $0.398 \mathrm{~kg}$ ethylene eq. $/ \mathrm{kg}$ $0.398 \mathrm{~kg}$ ethylene eq. $/ \mathrm{kg}$ $0.398 \mathrm{~kg}$ ethylene eq. $/ \mathrm{kg}$ $0.398 \mathrm{~kg}$ ethylene eq. $/ \mathrm{kg}$ $0.761 \mathrm{~kg}$ ethylene eq. $/ \mathrm{kg}$ $0.398 \mathrm{~kg}$ ethylene eq. $/ \mathrm{kg}$ $0.761 \mathrm{~kg}$ ethylene eq. $/ \mathrm{kg}$ $0.761 \mathrm{~kg}$ ethylene eq. $/ \mathrm{kg}$ $0.761 \mathrm{~kg}$ ethylene eq. $/ \mathrm{kg}$ $0.761 \mathrm{~kg}$ ethylene eq. $/ \mathrm{kg}$ $0.761 \mathrm{~kg}$ ethylene eq. $/ \mathrm{kg}$ $0.761 \mathrm{~kg}$ ethylene eq. $/ \mathrm{kg}$ $0.398 \mathrm{~kg}$ ethylene eq. $/ \mathrm{kg}$ $0.398 \mathrm{~kg}$ ethylene eq. $/ \mathrm{kg}$ $0.398 \mathrm{~kg}$ ethylene eq. $/ \mathrm{kg}$ $0.398 \mathrm{~kg}$ ethylene eq. $/ \mathrm{kg}$ $0.398 \mathrm{~kg}$ ethylene eq. $/ \mathrm{kg}$ $0.761 \mathrm{~kg}$ ethylene eq. $/ \mathrm{kg}$ $0.761 \mathrm{~kg}$ ethylene eq. $/ \mathrm{kg}$ $0.761 \mathrm{~kg}$ ethylene eq. $/ \mathrm{kg}$ $0.398 \mathrm{~kg}$ ethylene eq. $/ \mathrm{kg}$ $0.761 \mathrm{~kg}$ ethylene eq. $/ \mathrm{kg}$ $0.761 \mathrm{~kg}$ ethylene eq. $/ \mathrm{kg}$ $0.761 \mathrm{~kg}$ ethylene eq. $/ \mathrm{kg}$ $0.761 \mathrm{~kg}$ ethylene eq. $/ \mathrm{kg}$ $0.398 \mathrm{~kg}$ ethylene eq. $/ \mathrm{kg}$ $0.398 \mathrm{~kg}$ ethylene eq. $/ \mathrm{kg}$ $0.398 \mathrm{~kg}$ ethylene eq. $/ \mathrm{kg}$ $0.398 \mathrm{~kg}$ ethylene $\mathrm{eq} . / \mathrm{kg}$ $0.761 \mathrm{~kg}$ ethylene eq. $/ \mathrm{kg}$ $0.761 \mathrm{~kg}$ ethylene eq. $/ \mathrm{kg}$ $0.398 \mathrm{~kg}$ ethylene eq. $/ \mathrm{kg}$ $0.761 \mathrm{~kg}$ ethylene eq. $/ \mathrm{kg}$ $0.761 \mathrm{~kg}$ ethylene eq. $/ \mathrm{kg}$ $0.761 \mathrm{~kg}$ ethylene eq. $/ \mathrm{kg}$ $0.761 \mathrm{~kg}$ ethylene eq. $/ \mathrm{kg}$ $0.398 \mathrm{~kg}$ ethylene eq. $/ \mathrm{kg}$ $0.398 \mathrm{~kg}$ ethylene eq. $/ \mathrm{kg}$ $0.398 \mathrm{~kg}$ ethylene eq. $/ \mathrm{kg}$ $0.398 \mathrm{~kg}$ ethylene eq. $/ \mathrm{kg}$ $0.443 \mathrm{~kg}$ ethylene eq. $/ \mathrm{kg}$ $0.443 \mathrm{~kg}$ ethylene eq. $/ \mathrm{kg}$ $0.398 \mathrm{~kg}$ ethylene eq. $/ \mathrm{kg}$ $0.443 \mathrm{~kg}$ ethylene eq. $/ \mathrm{kg}$ $0.326 \mathrm{~kg}$ ethylene eq. $/ \mathrm{kg}$ $0.398 \mathrm{~kg}$ ethylene eq. $/ \mathrm{kg}$ $0.326 \mathrm{~kg}$ ethylene eq. $/ \mathrm{kg}$ $0.398 \mathrm{~kg}$ ethylene eq. $/ \mathrm{kg}$ $0.443 \mathrm{~kg}$ ethylene eq. $/ \mathrm{kg}$ 
[E353] C6 Cyclic Ketones to air [E354] C6 Cyclic or di-olefins to air [E355] C6 Cycloalkanes to air [E356] C6 Internal Alkenes to air [E357] C6 Ketones to air [E358] C6 Terminal Alkenes to air [E359] C7 Adehydes to air [E360] C7 Cyclic Ketones to air [E361] C7 Cyclic or di-olefins to air [E362] C7 Cycloalkanes to air [E363] C7 Internal Alkenes to air [E364] C7 Ketones to air [E365] C7 Terminal Alkenes to air [E366] C8 Aldehydes to air [E367] C8 Cyclic Ketones to air [E368] C8 Cyclic or di-olefins to air [E369] C8 Cycloalkanes to air [E370] C8 Disub. Benzenes to air [E371] C8 Internal Alkenes to air [E372] C8 Ketones to air [E373] C8 Terminal Alkenes to air [E374] C9 Bicycloalkanes to air [E375] C9 Cyclic Ketones to air [E376] C9 Cyclic or di-olefins to air [E377] C9 Cycloalkanes to air [E378] C9 Disub. Benzenes to air [E379] C9 Internal Alkenes to air [E380] C9 Ketones to air [E381] C9 Monosub. Benzenes to air [E382] C9 Styrenes to air [E383] C9 Terminal Alkenes to air [E384] C9 Trisub. Benzenes to air [E386] caprolactam to air [E396] CFC (hard) (unspecified) to air [E397] CFC (soft) (unspecified) to air [E398] CFC-11 to air [E399] CFC-113 to air [E400] CFC-114 to air [E401] CFC-115 to air [E402] CFC-12 to air [E403] CFC-13 to air [E407] chlorobenzene to air [E408] Chlorophenols (unspec.) to air [E409] Chloropicrin to air [E416] chrysene to air [E417] cis-2-Butene to air [E418] cis-2-Hexene to air [E419] cis-2-Pentene to air [E420] cis-Dichloroethene to air [E424] Crotonaldehyde to air [E426] Cyanides to air [E427] cyclic alkanes (unspec.) to air [E428] Cyclobutane to air [E429] Cyclohexane to air [E430] Cyclohexanol to air [E431] Cyclohexanone to air [E432] Cyclohexene to air [E433] Cyclopentadiene to air [E434] Cyclopentane to air [E435] Cyclopentene to air [E436] Cyclopropane to air [E444] Di(2-ethylhexyl)phtalate to air [E445] Diacetone alcohol to air [E447] Dibutyl Ether to air [E448] Dibutylphtalate to air [E449] Dichloromethane (Methylene Chloride) to air [E453] Diethyl Ether to air

$0.326 \mathrm{~kg}$ ethylene eq. $/ \mathrm{kg}$ $0.398 \mathrm{~kg}$ ethylene eq. $/ \mathrm{kg}$ $0.398 \mathrm{~kg}$ ethylene eq. $/ \mathrm{kg}$ $0.398 \mathrm{~kg}$ ethylene eq. $/ \mathrm{kg}$ $0.326 \mathrm{~kg}$ ethylene eq. $/ \mathrm{kg}$ $0.398 \mathrm{~kg}$ ethylene eq. $/ \mathrm{kg}$ $0.443 \mathrm{~kg}$ ethylene eq. $/ \mathrm{kg}$ $0.326 \mathrm{~kg}$ ethylene eq. $/ \mathrm{kg}$ $0.398 \mathrm{~kg}$ ethylene eq. $/ \mathrm{kg}$ $0.398 \mathrm{~kg}$ ethylene eq. $/ \mathrm{kg}$ $0.398 \mathrm{~kg}$ ethylene eq. $/ \mathrm{kg}$ $0.326 \mathrm{~kg}$ ethylene eq. $/ \mathrm{kg}$ $0.398 \mathrm{~kg}$ ethylene eq. $/ \mathrm{kg}$ $0.443 \mathrm{~kg}$ ethylene eq. $/ \mathrm{kg}$ $0.326 \mathrm{~kg}$ ethylene eq. $/ \mathrm{kg}$ $0.398 \mathrm{~kg}$ ethylene eq. $/ \mathrm{kg}$ $0.398 \mathrm{~kg}$ ethylene eq. $/ \mathrm{kg}$ $0.761 \mathrm{~kg}$ ethylene eq. $/ \mathrm{kg}$ $0.398 \mathrm{~kg}$ ethylene eq. $/ \mathrm{kg}$ $0.326 \mathrm{~kg}$ ethylene eq. $/ \mathrm{kg}$ $0.398 \mathrm{~kg}$ ethylene eq. $/ \mathrm{kg}$ $0.398 \mathrm{~kg}$ ethylene eq. $/ \mathrm{kg}$ $0.326 \mathrm{~kg}$ ethylene eq. $/ \mathrm{kg}$ $0.398 \mathrm{~kg}$ ethylene eq. $/ \mathrm{kg}$ $0.398 \mathrm{~kg}$ ethylene eq. $/ \mathrm{kg}$ $0.761 \mathrm{~kg}$ ethylene eq. $/ \mathrm{kg}$ $0.398 \mathrm{~kg}$ ethylene eq. $/ \mathrm{kg}$ $0.326 \mathrm{~kg}$ ethylene eq. $/ \mathrm{kg}$ $0.761 \mathrm{~kg}$ ethylene eq. $/ \mathrm{kg}$ $0.761 \mathrm{~kg}$ ethylene eq. $/ \mathrm{kg}$ $0.398 \mathrm{~kg}$ ethylene eq. $/ \mathrm{kg}$ $0.761 \mathrm{~kg}$ ethylene eq. $/ \mathrm{kg}$ $0.761 \mathrm{~kg}$ ethylene eq. $/ \mathrm{kg}$ $0.021 \mathrm{~kg}$ ethylene eq. $/ \mathrm{kg}$ $0.021 \mathrm{~kg}$ ethylene eq. $/ \mathrm{kg}$ $0.021 \mathrm{~kg}$ ethylene eq. $/ \mathrm{kg}$ $0.021 \mathrm{~kg}$ ethylene eq. $/ \mathrm{kg}$ $0.021 \mathrm{~kg}$ ethylene eq. $/ \mathrm{kg}$ $0.021 \mathrm{~kg}$ ethylene eq. $/ \mathrm{kg}$ $0.021 \mathrm{~kg}$ ethylene eq. $/ \mathrm{kg}$ $0.021 \mathrm{~kg}$ ethylene eq. $/ \mathrm{kg}$ $0.021 \mathrm{~kg}$ ethylene $\mathrm{eq} . / \mathrm{kg}$ $0.761 \mathrm{~kg}$ ethylene eq. $/ \mathrm{kg}$ $0.021 \mathrm{~kg}$ ethylene eq. $/ \mathrm{kg}$ $0.761 \mathrm{~kg}$ ethylene eq. $/ \mathrm{kg}$ $0.398 \mathrm{~kg}$ ethylene eq. $/ \mathrm{kg}$ $0.398 \mathrm{~kg}$ ethylene eq. $/ \mathrm{kg}$ $0.398 \mathrm{~kg}$ ethylene eq. $/ \mathrm{kg}$ $0.021 \mathrm{~kg}$ ethylene eq. $/ \mathrm{kg}$ $0.443 \mathrm{~kg}$ ethylene eq. $/ \mathrm{kg}$ $0.398 \mathrm{~kg}$ ethylene eq. $/ \mathrm{kg}$ $0.398 \mathrm{~kg}$ ethylene eq. $/ \mathrm{kg}$ $0.398 \mathrm{~kg}$ ethylene eq. $/ \mathrm{kg}$ $0.398 \mathrm{~kg}$ ethylene eq. $/ \mathrm{kg}$ $0.398 \mathrm{~kg}$ ethylene eq. $/ \mathrm{kg}$ $0.398 \mathrm{~kg}$ ethylene eq. $/ \mathrm{kg}$ $0.398 \mathrm{~kg}$ ethylene eq. $/ \mathrm{kg}$ $0.398 \mathrm{~kg}$ ethylene eq. $/ \mathrm{kg}$ $0.398 \mathrm{~kg}$ ethylene eq. $/ \mathrm{kg}$ $0.398 \mathrm{~kg}$ ethylene eq. $/ \mathrm{kg}$ $0.398 \mathrm{~kg}$ ethylene eq. $/ \mathrm{kg}$ $0.761 \mathrm{~kg}$ ethylene eq. $/ \mathrm{kg}$ $0.196 \mathrm{~kg}$ ethylene eq. $/ \mathrm{kg}$ $0.196 \mathrm{~kg}$ ethylene eq. $/ \mathrm{kg}$ $0.761 \mathrm{~kg}$ ethylene eq. $/ \mathrm{kg}$ $0.01 \mathrm{~kg}$ ethylene eq. $/ \mathrm{kg}$ $0.398 \mathrm{~kg}$ ethylene eq. $/ \mathrm{kg}$ 
[E454] diethylamine to air [E455] Diethylketone to air [E456] Diethylphtalate to air [E457] Dihexylphtalate to air [E458] Diisodecylphtalate to air [E459] Diisooctylphtalate to air [E460] Diisopropylether to air [E462] Dimethyl Adipate to air [E463] Dimethyl Ether to air [E464] Dimethyl Glutarate to air [E465] Dimethyl Naphthalenes to air [E466] Dimethyl Succinate to air [E467] dimethylamine to air [E468] Dimethylphtalate to air [E474] diphenyl to air

[E477] d-Limonene to air [E482] esters (unspec.) to air

[E483] Ethane to air

[E484] ethanethiol (ethylmercaptan) to air [E485] Ethanol to air

[E486] ethers (unspec.) to air

[E488] Ethyl Acetate to air

[E489] Ethyl Acetylene to air

[E490] Ethyl Acrylate to air

[E491] Ethyl Amine to air

[E492] Ethyl Chloride to air

[E493] Ethyl Cyclopentane to air

[E494] Ethyl Isopropyl Ether to air

[E495] Ethyl- trans-Butyl Ether to air

[E496] Ethylbenzene to air

[E497] ethylbutyrate to air

[E498] Ethylcyclohexane to air

[E499] Ethylene (ethene) to air

[E500] Ethylene Dibromide to air

[E501] Ethylene Dichloride to air

[E502] Ethylene Glycol to air

[E503] ethylthioethane (diethylsulfide) to air

[E509] fluoranthrene to air

[E511] Formaldehyde (methanal) to air

[E512] Formic acid to air

[E513] Furan to air

[E514] Furanes (unspec.) to air

[E515] Glyoxal to air

[E517] HALON (HBFC-2401) to air

[E518] HALON-1201 (HBFC-1201) to air

[E519] HALON-1202 to air

[E520] HALON-1211 to air

[E521] HALON-1301 to air

[E522] HALON-2311 (HBFC-2311) to air

[E523] HALON-2402 (Di-Broom-tetra-Fluor-ethane) to air

[E524] halons (unspecified) to air

[E525] HCFC-123 (1,1-Di-Chloro-222-Tri-Fluorethaan) to air [E526] HCFC-124 (1-Chloro-1222-Tetra-Fluor-Ethane) to air [E527] HCFC-141b (Dichlorofluorethane) to air

[E528] HCFC-142b (1-Chloro-1,1-Difluorethane) to air

[E529] HCFC-22 (Chlorodifluormethane) to air

[E530] HCFC-225ca to air

[E531] HCFC-225cb to air

[E538] hexachlorobiphenyl to air

[E541] Hexyl Cyclohexane to air

[E542] HFC (unspec.) to air

[E543] HFC-125 (Pentafluoroethane) to air

[E544] HFC-134 (1,1,1,2-tetra-fluor-ethane) to air

[E545] HFC-134a (1,1,1,2-tetrafluoroethane) to air

[E546] HFC-143 to air

[E547] HFC-143a (1,1,1-trifluoroethane) to air

[E548] HFC-152a (1,1-difluoroethane) to air

$0.398 \mathrm{~kg}$ ethylene eq. $/ \mathrm{kg}$ $0.326 \mathrm{~kg}$ ethylene eq. $/ \mathrm{kg}$ $0.761 \mathrm{~kg}$ ethylene eq. $/ \mathrm{kg}$ $0.761 \mathrm{~kg}$ ethylene eq. $/ \mathrm{kg}$ $0.761 \mathrm{~kg}$ ethylene eq. $/ \mathrm{kg}$ $0.761 \mathrm{~kg}$ ethylene eq. $/ \mathrm{kg}$ $0.398 \mathrm{~kg}$ ethylene eq. $/ \mathrm{kg}$ $0.398 \mathrm{~kg}$ ethylene eq. $/ \mathrm{kg}$ $0.196 \mathrm{~kg}$ ethylene eq. $/ \mathrm{kg}$ $0.398 \mathrm{~kg}$ ethylene eq. $/ \mathrm{kg}$ $0.761 \mathrm{~kg}$ ethylene eq. $/ \mathrm{kg}$ $0.398 \mathrm{~kg}$ ethylene eq. $/ \mathrm{kg}$ $0.398 \mathrm{~kg}$ ethylene eq. $/ \mathrm{kg}$ $0.761 \mathrm{~kg}$ ethylene eq. $/ \mathrm{kg}$ $0.761 \mathrm{~kg}$ ethylene eq. $/ \mathrm{kg}$ $0.398 \mathrm{~kg}$ ethylene eq. $/ \mathrm{kg}$ $0.398 \mathrm{~kg}$ ethylene eq. $/ \mathrm{kg}$ $0.398 \mathrm{~kg}$ ethylene eq. $/ \mathrm{kg}$ $0.398 \mathrm{~kg}$ ethylene eq. $/ \mathrm{kg}$ $0.268 \mathrm{~kg}$ ethylene eq. $/ \mathrm{kg}$ $0.398 \mathrm{~kg}$ ethylene eq. $/ \mathrm{kg}$ $0.398 \mathrm{~kg}$ ethylene eq. $/ \mathrm{kg}$ $0.398 \mathrm{~kg}$ ethylene eq. $/ \mathrm{kg}$ $0.398 \mathrm{~kg}$ ethylene eq. $/ \mathrm{kg}$ $0.398 \mathrm{~kg}$ ethylene eq. $/ \mathrm{kg}$ $0.021 \mathrm{~kg}$ ethylene eq. $/ \mathrm{kg}$ $0.398 \mathrm{~kg}$ ethylene eq. $/ \mathrm{kg}$ $0.196 \mathrm{~kg}$ ethylene eq. $/ \mathrm{kg}$ $0.196 \mathrm{~kg}$ ethylene eq. $/ \mathrm{kg}$ $0.761 \mathrm{~kg}$ ethylene eq. $/ \mathrm{kg}$ $0.398 \mathrm{~kg}$ ethylene eq. $/ \mathrm{kg}$ $0.398 \mathrm{~kg}$ ethylene eq. $/ \mathrm{kg}$

$1 \mathrm{~kg}$ ethylene eq. $/ \mathrm{kg}$ $0.021 \mathrm{~kg}$ ethylene eq. $/ \mathrm{kg}$ $0.021 \mathrm{~kg}$ ethylene eq. $/ \mathrm{kg}$ $0.196 \mathrm{~kg}$ ethylene eq. $/ \mathrm{kg}$ $0.398 \mathrm{~kg}$ ethylene eq. $/ \mathrm{kg}$ $0.761 \mathrm{~kg}$ ethylene eq. $/ \mathrm{kg}$ $0.421 \mathrm{~kg}$ ethylene eq. $/ \mathrm{kg}$ $0.398 \mathrm{~kg}$ ethylene eq. $/ \mathrm{kg}$ $0.398 \mathrm{~kg}$ ethylene eq. $/ \mathrm{kg}$ $0.398 \mathrm{~kg}$ ethylene eq. $/ \mathrm{kg}$ $0.443 \mathrm{~kg}$ ethylene eq. $/ \mathrm{kg}$ $0.021 \mathrm{~kg}$ ethylene eq. $/ \mathrm{kg}$ $0.021 \mathrm{~kg}$ ethylene eq. $/ \mathrm{kg}$ $0.021 \mathrm{~kg}$ ethylene eq. $/ \mathrm{kg}$ $0.021 \mathrm{~kg}$ ethylene eq. $/ \mathrm{kg}$ $0.021 \mathrm{~kg}$ ethylene eq. $/ \mathrm{kg}$ $0.021 \mathrm{~kg}$ ethylene eq. $/ \mathrm{kg}$ $0.021 \mathrm{~kg}$ ethylene eq. $/ \mathrm{kg}$ $0.021 \mathrm{~kg}$ ethylene eq. $/ \mathrm{kg}$ $0.021 \mathrm{~kg}$ ethylene eq. $/ \mathrm{kg}$ $0.021 \mathrm{~kg}$ ethylene eq. $/ \mathrm{kg}$ $0.021 \mathrm{~kg}$ ethylene eq. $/ \mathrm{kg}$ $0.021 \mathrm{~kg}$ ethylene eq. $/ \mathrm{kg}$ $0.021 \mathrm{~kg}$ ethylene eq. $/ \mathrm{kg}$ $0.021 \mathrm{~kg}$ ethylene eq. $/ \mathrm{kg}$ $0.021 \mathrm{~kg}$ ethylene eq. $/ \mathrm{kg}$ $0.761 \mathrm{~kg}$ ethylene eq. $/ \mathrm{kg}$ $0.398 \mathrm{~kg}$ ethylene eq. $/ \mathrm{kg}$ $0.021 \mathrm{~kg}$ ethylene eq. $/ \mathrm{kg}$ $0.021 \mathrm{~kg}$ ethylene eq. $/ \mathrm{kg}$ $0.021 \mathrm{~kg}$ ethylene eq. $/ \mathrm{kg}$ $0.021 \mathrm{~kg}$ ethylene eq. $/ \mathrm{kg}$ $0.021 \mathrm{~kg}$ ethylene eq. $/ \mathrm{kg}$ $0.021 \mathrm{~kg}$ ethylene eq. $/ \mathrm{kg}$ $0.021 \mathrm{~kg}$ ethylene eq. $/ \mathrm{kg}$ 
[E549] HFC-227ea to air [E550] HFC-23 to air [E551] HFC-236fa to air [E552] HFC-245ca to air [E553] HFC-32 to air [E554] HFC-41 to air [E555] HFC-43-10mee to air [E556] Hydrocarbons to air [E557] Hydrocarbons (excl. methane) to air [E558] Hydrocarbons Chloro-Fluor- (HCFC) to air [E559] Hydrocarbons haloginated to air [E564] hydroxy compounds to air [E565] Indan to air [E566] indeno[1,2,3-cd]pyrene to air [E569] isoamyl isobutyrate to air [E570] isobutane to air

[E571] isobutanol (2-methylpropanol-1) to air

[E572] isobutene (2-methylpropene) to air

[E573] isobutyl acetate to air

[E574] isobutyl isobutyrate to air

[E575] isobutyraldehyde to air

[E576] isopentane to air

[E577] isopentylacetate (iso-amylacetate) to air

[E578] isoprene to air

[E579] isopropanol to air

[E580] isopropyl acetate to air

[E581] isopropyl benzene (cumene) to air

[E582] isopropyl propionate to air

[E593] meta-Cresol (3-methyiphenol) to air

[E594] meta-Ethyltoluene to air

[E596] meta-Xylene (1,3-dimethylbenzene) to air

[E599] Methacrolein to air

[E600] Methane to air

[E601] methanethiol (methylmercaptan) to air

[E602] Methanol to air

[E604] Methyl Acetate to air

[E606] Methyl Acrylate to air

[E607] methyl amine to air

[E608] Methyl Bromide to air

[E609] Methyl Choride to air

[E610] Methyl cyclohexane to air

[E611] Methyl cyclopentane to air

[E612] methyl dithiomethane to air

[E613] Methyl Ethyl Ketone to air

[E614] Methyl Formate to air

[E615] Methyl Glyoxal to air

[E616] Methyl Isobutyl Ketone (4-methylpentanon-2) to air

[E617] Methyl iso-butyrate to air

[E618] methyl methacrylate to air

[E619] Methyl Naphthalenes to air

[E620] Methyl Nitrite to air

[E621] methyl propionate to air

[E622] Methyl propyl Ketone to air

[E623] methyl thiomethane (dimethylsulfide) to air

[E624] Methyl trans-Butyl Ether to air

[E625] Methyl-Isopropylketone to air

[E626] methyl-mercury to air

[E627] Methyl-transbutylketone to air

[E631] Meythylvinyl Ketone to air

[E633] Naphtalene to air

[E634] Neopentane to air

[E639] Nitrobenzene to air

[E645] Octyl Cyclohexane to air

[E646] oil crude to air

[E647] organics acids (unspec.) to air

[E648] ortho-Cresol (2-methylphenol) to air

[E649] ortho-Ethyltoluene to air
$0.021 \mathrm{~kg}$ ethylene eq. $/ \mathrm{kg}$ $0.021 \mathrm{~kg}$ ethylene eq. $/ \mathrm{kg}$ $0.021 \mathrm{~kg}$ ethylene eq. $/ \mathrm{kg}$ $0.021 \mathrm{~kg}$ ethylene eq. $/ \mathrm{kg}$ $0.021 \mathrm{~kg}$ ethylene eq. $/ \mathrm{kg}$ $0.021 \mathrm{~kg}$ ethylene eq. $/ \mathrm{kg}$ $0.021 \mathrm{~kg}$ ethylene eq. $/ \mathrm{kg}$ $0.398 \mathrm{~kg}$ ethylene eq. $/ \mathrm{kg}$ $0.416 \mathrm{~kg}$ ethylene eq. $/ \mathrm{kg}$ $0.021 \mathrm{~kg}$ ethylene eq. $/ \mathrm{kg}$ $0.021 \mathrm{~kg}$ ethylene eq. $/ \mathrm{kg}$ $0.377 \mathrm{~kg}$ ethylene eq. $/ \mathrm{kg}$ $0.761 \mathrm{~kg}$ ethylene eq. $/ \mathrm{kg}$ $0.761 \mathrm{~kg}$ ethylene eq. $/ \mathrm{kg}$ $0.398 \mathrm{~kg}$ ethylene eq. $/ \mathrm{kg}$ $0.398 \mathrm{~kg}$ ethylene eq. $/ \mathrm{kg}$ $0.196 \mathrm{~kg}$ ethylene eq. $/ \mathrm{kg}$ $0.398 \mathrm{~kg}$ ethylene eq. $/ \mathrm{kg}$ $0.398 \mathrm{~kg}$ ethylene eq. $/ \mathrm{kg}$ $0.398 \mathrm{~kg}$ ethylene eq. $/ \mathrm{kg}$ $0.443 \mathrm{~kg}$ ethylene eq. $/ \mathrm{kg}$ $0.398 \mathrm{~kg}$ ethylene eq. $/ \mathrm{kg}$ $0.398 \mathrm{~kg}$ ethylene eq. $/ \mathrm{kg}$ $0.398 \mathrm{~kg}$ ethylene eq. $/ \mathrm{kg}$ $0.196 \mathrm{~kg}$ ethylene eq. $/ \mathrm{kg}$ $0.398 \mathrm{~kg}$ ethylene eq. $/ \mathrm{kg}$ $0.761 \mathrm{~kg}$ ethylene eq. $/ \mathrm{kg}$ $0.398 \mathrm{~kg}$ ethylene eq. $/ \mathrm{kg}$ $0.761 \mathrm{~kg}$ ethylene eq. $/ \mathrm{kg}$ $0.761 \mathrm{~kg}$ ethylene eq. $/ \mathrm{kg}$ $0.85 \mathrm{~kg}$ ethylene eq. $/ \mathrm{kg}$ $0.443 \mathrm{~kg}$ ethylene eq. $/ \mathrm{kg}$ $0.007 \mathrm{~kg}$ ethylene eq. $/ \mathrm{kg}$ $0.377 \mathrm{~kg}$ ethylene eq. $/ \mathrm{kg}$ $0.196 \mathrm{~kg}$ ethylene eq. $/ \mathrm{kg}$ $0.398 \mathrm{~kg}$ ethylene eq. $/ \mathrm{kg}$ $0.398 \mathrm{~kg}$ ethylene eq. $/ \mathrm{kg}$ $0.398 \mathrm{~kg}$ ethylene eq. $/ \mathrm{kg}$ $0.021 \mathrm{~kg}$ ethylene eq. $/ \mathrm{kg}$ $0.021 \mathrm{~kg}$ ethylene eq. $/ \mathrm{kg}$ $0.398 \mathrm{~kg}$ ethylene eq. $/ \mathrm{kg}$ $0.398 \mathrm{~kg}$ ethylene eq. $/ \mathrm{kg}$ $0.398 \mathrm{~kg}$ ethylene eq. $/ \mathrm{kg}$ $0.473 \mathrm{~kg}$ ethylene eq. $/ \mathrm{kg}$ $0.398 \mathrm{~kg}$ ethylene eq. $/ \mathrm{kg}$ $0.443 \mathrm{~kg}$ ethylene eq. $/ \mathrm{kg}$ $0.326 \mathrm{~kg}$ ethylene eq. $/ \mathrm{kg}$ $0.398 \mathrm{~kg}$ ethylene eq. $/ \mathrm{kg}$ $0.398 \mathrm{~kg}$ ethylene eq. $/ \mathrm{kg}$ $0.761 \mathrm{~kg}$ ethylene eq. $/ \mathrm{kg}$ $0.398 \mathrm{~kg}$ ethylene eq. $/ \mathrm{kg}$ $0.398 \mathrm{~kg}$ ethylene eq. $/ \mathrm{kg}$ $0.326 \mathrm{~kg}$ ethylene eq. $/ \mathrm{kg}$ $0.398 \mathrm{~kg}$ ethylene eq. $/ \mathrm{kg}$ $0.196 \mathrm{~kg}$ ethylene eq. $/ \mathrm{kg}$ $0.326 \mathrm{~kg}$ ethylene eq. $/ \mathrm{kg}$ $0.398 \mathrm{~kg}$ ethylene eq. $/ \mathrm{kg}$ $0.326 \mathrm{~kg}$ ethylene eq. $/ \mathrm{kg}$ $0.326 \mathrm{~kg}$ ethylene eq. $/ \mathrm{kg}$ $0.761 \mathrm{~kg}$ ethylene eq. $/ \mathrm{kg}$ $0.398 \mathrm{~kg}$ ethylene eq. $/ \mathrm{kg}$ $0.761 \mathrm{~kg}$ ethylene eq. $/ \mathrm{kg}$ $0.398 \mathrm{~kg}$ ethylene eq. $/ \mathrm{kg}$ $0.398 \mathrm{~kg}$ ethylene eq. $/ \mathrm{kg}$ $0.398 \mathrm{~kg}$ ethylene eq. $/ \mathrm{kg}$ $0.761 \mathrm{~kg}$ ethylene eq. $/ \mathrm{kg}$ $0.761 \mathrm{~kg}$ ethylene eq. $/ \mathrm{kg}$ 
[E650] ortho-Xylene (1,2-dimethylbenzene) to air

[E653] para-Cresol (4-methylphenol) to air

[E654] para-Ethyltoluene to air

[E657] para-Trifluoromethyl-Cl-Benzene to air

[E658] para-Xylene (1,4-dimethylbenzene) to air

[E660] pentachlorobenzene to air

[E661] pentachloronitrobenzene to air

[E662] pentachlorophenol to air

[E663] Pentanaldehyde to air

[E664] Pentanol to air

[E665] Perfluorobutane to air

[E666] Perfluorocyclobutane to air

[E667] Perfluoroethane (CFC-116) to air

[E668] Perfluorohexane to air

[E669] Perfluoromethane (CFC-14) to air

[E670] Perfluoropentane to air

[E671] Perfluoropropane to air

[E673] petrol to air

[E674] phenanthrene to air

[E675] Phenol to air

[E680] Phtalic anhydride to air

[E681] phthalates to air

[E684] Polycyclic Aromatic Hydrocarbons (PAH) (unspecified) to air

[E685] Polycyclic Aromatic Hydrocarbons Carcinogenic- (carcinogenic-PAH) to air

[E687] Propane to air

[E688] Propanoic acid (propionic acid) to air

[E689] Propionaldehyde (propanal) to air

[E691] Propyl Acetate to air

[E692] Propyl Cyclopentane to air

[E693] Propylene to air

[E694] Propylene Carbonate to air

[E695] Propylene Glycol to air

[E696] Propylene Glycol Methyl Ether Acetate to air

[E697] Propylene Oxide to air

[E700] Sabinene to air

[E701] sec-Butanol to air

[E702] sec-Butyl Benzene to air

[E703] sec-Buytl Acetate to air

[E706] styrene (vinylbenzene) to air

[E707] Subst. C7 Ester (C12) to air

[E708] Sucts. C9 Ester (C12) to air

[E715] terpentine to air

[E716] tertiary-Butanol to air

[E717] tertiary-Butyl Acetate to air

[E718] tetrachloroethylene (PER) (tetrachloroethene) to air

[E719] Tetrachloromethane (carbon tetrachloride) (HC-10) to air

[E720] tetrafluorethylene to air

[E721] Tetralin to air

[E726] Tolualdehyde to air

[E727] Toluene to air

[E728] trans-1,2-Dichloroethene to air

[E729] trans-2-Butene to air

[E730] trans-2-Hexene to air

[E731] trans-2-Pentene to air

[E732] trans-dichloroethene to air

[E737] Trichloroethylene (tri) to air

[E738] Trichloromethane (chloroform) to air

[E742] Valeraldehyde (pentanal) to air

[E744] Vinyl Acetate to air

[E745] Vinyl Chloride (chloroethene) to air

[E746] Volatile Organic Compounds (VOC) to air

[E747] Volatile Organic Compounds non-methane- (non methane VOC) to air
$0.85 \mathrm{~kg}$ ethylene eq. $/ \mathrm{kg}$

$0.761 \mathrm{~kg}$ ethylene eq. $/ \mathrm{kg}$

$0.761 \mathrm{~kg}$ ethylene eq. $/ \mathrm{kg}$

$0.021 \mathrm{~kg}$ ethylene eq. $/ \mathrm{kg}$

$0.85 \mathrm{~kg}$ ethylene eq. $/ \mathrm{kg}$

$0.761 \mathrm{~kg}$ ethylene eq. $/ \mathrm{kg}$

$0.761 \mathrm{~kg}$ ethylene eq. $/ \mathrm{kg}$

$0.761 \mathrm{~kg}$ ethylene eq. $/ \mathrm{kg}$

$0.443 \mathrm{~kg}$ ethylene eq. $/ \mathrm{kg}$

$0.196 \mathrm{~kg}$ ethylene eq. $/ \mathrm{kg}$

$0.021 \mathrm{~kg}$ ethylene eq. $/ \mathrm{kg}$

$0.021 \mathrm{~kg}$ ethylene eq. $/ \mathrm{kg}$

$0.021 \mathrm{~kg}$ ethylene eq. $/ \mathrm{kg}$

$0.021 \mathrm{~kg}$ ethylene eq. $/ \mathrm{kg}$

$0.021 \mathrm{~kg}$ ethylene eq. $/ \mathrm{kg}$

$0.021 \mathrm{~kg}$ ethylene eq. $/ \mathrm{kg}$

$0.021 \mathrm{~kg}$ ethylene eq. $/ \mathrm{kg}$

$0.398 \mathrm{~kg}$ ethylene eq. $/ \mathrm{kg}$

$0.761 \mathrm{~kg}$ ethylene eq. $/ \mathrm{kg}$

$0.761 \mathrm{~kg}$ ethylene eq. $/ \mathrm{kg}$

$0.761 \mathrm{~kg}$ ethylene eq. $/ \mathrm{kg}$

$0.761 \mathrm{~kg}$ ethylene eq. $/ \mathrm{kg}$

$0.761 \mathrm{~kg}$ ethylene eq. $/ \mathrm{kg}$

$0.761 \mathrm{~kg}$ ethylene eq. $/ \mathrm{kg}$

$0.42 \mathrm{~kg}$ ethylene eq. $/ \mathrm{kg}$

$0.398 \mathrm{~kg}$ ethylene eq. $/ \mathrm{kg}$

$0.603 \mathrm{~kg}$ ethylene eq. $/ \mathrm{kg}$

$0.398 \mathrm{~kg}$ ethylene eq. $/ \mathrm{kg}$

$0.398 \mathrm{~kg}$ ethylene eq. $/ \mathrm{kg}$

$1.03 \mathrm{~kg}$ ethylene eq. $/ \mathrm{kg}$

$0.398 \mathrm{~kg}$ ethylene eq. $/ \mathrm{kg}$

$0.196 \mathrm{~kg}$ ethylene eq. $/ \mathrm{kg}$

$0.398 \mathrm{~kg}$ ethylene eq. $/ \mathrm{kg}$

$0.398 \mathrm{~kg}$ ethylene eq. $/ \mathrm{kg}$

$0.398 \mathrm{~kg}$ ethylene eq. $/ \mathrm{kg}$

$0.196 \mathrm{~kg}$ ethylene eq. $/ \mathrm{kg}$

$0.761 \mathrm{~kg}$ ethylene eq. $/ \mathrm{kg}$

$0.398 \mathrm{~kg}$ ethylene eq. $/ \mathrm{kg}$

$0.761 \mathrm{~kg}$ ethylene eq. $/ \mathrm{kg}$

$0.398 \mathrm{~kg}$ ethylene eq. $/ \mathrm{kg}$

$0.398 \mathrm{~kg}$ ethylene eq. $/ \mathrm{kg}$

$0.377 \mathrm{~kg}$ ethylene eq. $/ \mathrm{kg}$

$0.196 \mathrm{~kg}$ ethylene eq. $/ \mathrm{kg}$

$0.398 \mathrm{~kg}$ ethylene eq. $/ \mathrm{kg}$

$0.021 \mathrm{~kg}$ ethylene eq. $/ \mathrm{kg}$

$0.021 \mathrm{~kg}$ ethylene eq. $/ \mathrm{kg}$

$0.021 \mathrm{~kg}$ ethylene eq. $/ \mathrm{kg}$

$0.761 \mathrm{~kg}$ ethylene eq. $/ \mathrm{kg}$

$0.443 \mathrm{~kg}$ ethylene $\mathrm{eq} . / \mathrm{kg}$

$0.563 \mathrm{~kg}$ ethylene eq. $/ \mathrm{kg}$

$0.021 \mathrm{~kg}$ ethylene eq. $/ \mathrm{kg}$

$0.398 \mathrm{~kg}$ ethylene eq. $/ \mathrm{kg}$

$0.398 \mathrm{~kg}$ ethylene eq. $/ \mathrm{kg}$

$0.398 \mathrm{~kg}$ ethylene eq. $/ \mathrm{kg}$

$0.021 \mathrm{~kg}$ ethylene eq. $/ \mathrm{kg}$

$0.066 \mathrm{~kg}$ ethylene eq. $/ \mathrm{kg}$

$0.398 \mathrm{~kg}$ ethylene eq. $/ \mathrm{kg}$

$0.443 \mathrm{~kg}$ ethylene eq. $/ \mathrm{kg}$

$0.223 \mathrm{~kg}$ ethylene eq. $/ \mathrm{kg}$

$0.021 \mathrm{~kg}$ ethylene eq. $/ \mathrm{kg}$

$0.398 \mathrm{~kg}$ ethylene eq. $/ \mathrm{kg}$

$0.416 \mathrm{~kg}$ ethylene eq. $/ \mathrm{kg}$

Category $=[\mathrm{C} 20]$ Depletion of the ozone layer (Ecoindicator95)

Description $=$ Ecoindicator 95

Author $=$ Goedkoop, 1995 
Environmental resources

Environmental resource

Environmental emissions

Environmental emission

[E90] 1,1,1-trichloroethane (methyl chloroform; HC-140a) to air

[E396] CFC (hard) (unspecified) to air

[E397] CFC (soft) (unspecified) to air

[E398] CFC-11 to air

[E399] CFC-113 to air

[E400] CFC-114 to air

[E401] CFC-115 to air

[E402] CFC-12 to air

[E403] CFC-13 to air

[E517] HALON (HBFC-2401) to air

[E519] HALON-1202 to air

[E520] HALON-1211 to air

[E521] HALON-1301 to air

[E522] HALON-2311 (HBFC-2311) to air

[E523] HALON-2402 (Di-Broom-tetra-Fluor-ethane) to air

[E525] HCFC-123 (1,1-Di-Chloro-222-Tri-Fluorethaan) to air

[E526] HCFC-124 (1-Chloro-1222-Tetra-Fluor-Ethane) to air

[E527] HCFC-141b (Dichlorofluorethane) to air

[E528] HCFC-142b (1-Chloro-1,1-Difluorethane) to air

[E529] HCFC-22 (Chlorodifluormethane) to air

[E530] HCFC-225ca to air

[E531] HCFC-225cb to air

[E608] Methyl Bromide to air

[E719] Tetrachloromethane (carbon tetrachloride) (HC-10) to air
[E518] HALON-1201 (HBFC-1201) to air

Category $=[\mathrm{C} 21]$ Global warming (Ecoindicator95)

Description $=$ Ecoindicator 95

Author $=$ Goedkoop, 1995

Date $=27-09-1999$

Environmental resources

Environmental resource

Environmental emissions

Environmental emission

[E90] 1,1,1-trichloroethane (methyl chloroform; HC-140a) to air

[E393] Carbon dioxide to air

[E396] CFC (hard) (unspecified) to air

[E397] CFC (soft) (unspecified) to air

[E398] CFC-11 to air

[E399] CFC-113 to air

[E400] CFC-114 to air

[E401] CFC-115 to air

[E402] CFC-12 to air

[E403] CFC-13 to air

[E449] Dichloromethane (Methylene Chloride) to air

[E469] Dinitrogen oxide (nitrous oxide) to air

[E520] HALON-1211 to air

[E521] HALON-1301 to air

[E525] HCFC-123 (1,1-Di-Chloro-222-Tri-Fluorethaan) to air [E526] HCFC-124 (1-Chloro-1222-Tetra-Fluor-Ethane) to air [E527] HCFC-141b (Dichlorofluorethane) to air [E528] HCFC-142b (1-Chloro-1,1-Difluorethane) to air [E529] HCFC-22 (Chlorodifluormethane) to air [E543] HFC-125 (Pentafluoroethane) to air [E545] HFC-134a (1,1,1,2-tetrafluoroethane) to air [E547] HFC-143a (1,1,1-trifluoroethane) to air [E548] HFC-152a (1,1-difluoroethane) to air
Value

Value

Unit

Unit

$0.12 \mathrm{~kg} \mathrm{CFC}-11$ eq. $/ \mathrm{kg}$

$1 \mathrm{~kg} \mathrm{CFC}-11$ eq. $/ \mathrm{kg}$

$0.055 \mathrm{~kg}$ CFC-11 eq. $/ \mathrm{kg}$

$1 \mathrm{~kg} \mathrm{CFC}-11$ eq. $/ \mathrm{kg}$

$1.07 \mathrm{~kg} \mathrm{CFC}-11$ eq. $/ \mathrm{kg}$

$0.8 \mathrm{~kg} \mathrm{CFC}-11$ eq. $/ \mathrm{kg}$

$0.5 \mathrm{~kg} \mathrm{CFC}-11$ eq. $/ \mathrm{kg}$

$1 \mathrm{~kg}$ CFC-11 eq. $/ \mathrm{kg}$

$1 \mathrm{~kg}$ CFC-11 eq. $/ \mathrm{kg}$

$0.25 \mathrm{~kg} \mathrm{CFC}-11$ eq. $/ \mathrm{kg}$

$1.4 \mathrm{~kg} \mathrm{CFC}-11$ eq. $/ \mathrm{kg}$

$1.25 \mathrm{~kg} \mathrm{CFC}-11$ eq. $/ \mathrm{kg}$

$4 \mathrm{~kg} \mathrm{CFC}-11$ eq. $/ \mathrm{kg}$

$16 \mathrm{~kg}$ CFC-11 eq. $/ \mathrm{kg}$

$0.14 \mathrm{~kg} \mathrm{CFC}-11$ eq. $/ \mathrm{kg}$

$7 \mathrm{~kg}$ CFC-11 eq. $/ \mathrm{kg}$

$0.02 \mathrm{~kg}$ CFC-11 eq. $/ \mathrm{kg}$

$0.022 \mathrm{~kg} \mathrm{CFC}-11$ eq. $/ \mathrm{kg}$

$0.11 \mathrm{~kg}$ CFC-11 eq. $/ \mathrm{kg}$

$0.065 \mathrm{~kg} \mathrm{CFC}-11$ eq. $/ \mathrm{kg}$

$0.055 \mathrm{~kg} \mathrm{CFC}-11$ eq. $/ \mathrm{kg}$

$0.025 \mathrm{~kg} \mathrm{CFC}-11$ eq. $/ \mathrm{kg}$

$0.033 \mathrm{~kg} \mathrm{CFC}-11$ eq. $/ \mathrm{kg}$

$0.6 \mathrm{~kg}$ CFC-11 eq. $/ \mathrm{kg}$

$1.08 \mathrm{~kg}$ CFC-11 eq. $/ \mathrm{kg}$

Value

Unit

Value

Unit

$100 \mathrm{~kg} \mathrm{CO} 2$ eq. $/ \mathrm{kg}$

$1 \mathrm{~kg} \mathrm{CO} 2$ eq. $/ \mathrm{kg}$

$7100 \mathrm{~kg} \mathrm{CO} 2 \mathrm{eq} . / \mathrm{kg}$

$1600 \mathrm{~kg} \mathrm{CO} 2 \mathrm{eq} . / \mathrm{kg}$

$3400 \mathrm{~kg} \mathrm{CO} 2 \mathrm{eq} . / \mathrm{kg}$

$4500 \mathrm{~kg} \mathrm{CO} 2 \mathrm{eq} . / \mathrm{kg}$

$7000 \mathrm{~kg} \mathrm{CO} 2$ eq. $/ \mathrm{kg}$

$7000 \mathrm{~kg} \mathrm{CO} 2 \mathrm{eq} . / \mathrm{kg}$

$7100 \mathrm{~kg} \mathrm{CO} 2 \mathrm{eq} . / \mathrm{kg}$

$13000 \mathrm{~kg} \mathrm{CO} 2 \mathrm{eq} . / \mathrm{kg}$

$15 \mathrm{~kg} \mathrm{CO} 2 \mathrm{eq} . / \mathrm{kg}$

$270 \mathrm{~kg} \mathrm{CO} 2 \mathrm{eq} . \mathrm{kg}$ $4900 \mathrm{~kg} \mathrm{CO} 2 \mathrm{eq} . / \mathrm{kg}$ $4900 \mathrm{~kg} \mathrm{CO} 2 \mathrm{eq} . / \mathrm{kg}$

$90 \mathrm{~kg} \mathrm{CO} 2 \mathrm{eq} . / \mathrm{kg}$

$440 \mathrm{~kg} \mathrm{CO} 2 \mathrm{eq} . / \mathrm{kg}$

$580 \mathrm{~kg} \mathrm{CO} 2 \mathrm{eq} . / \mathrm{kg}$

$1800 \mathrm{~kg} \mathrm{CO} 2 \mathrm{eq} . / \mathrm{kg}$

$1600 \mathrm{~kg} \mathrm{CO} 2 \mathrm{eq} . \mathrm{kg}$

$3400 \mathrm{~kg} \mathrm{CO} 2 \mathrm{eq} . / \mathrm{kg}$

$1200 \mathrm{~kg} \mathrm{CO} 2 \mathrm{eq} . / \mathrm{kg}$

$3800 \mathrm{~kg}$ CO2 eq. $/ \mathrm{kg}$

$150 \mathrm{~kg} \mathrm{CO} 2 \mathrm{eq} . / \mathrm{kg}$ 
[E600] Methane to air

[E719] Tetrachloromethane (carbon tetrachloride) $(\mathrm{HC}-10)$ to air

[E738] Trichloromethane (chloroform) to air

Category $=[\mathrm{C} 22]$ Acidification (Ecoindicator95)

Description $=$ Ecoindicator 95

Author $=$ Goedkoop, 1995

Date $=27-09-1999$

Environmental resources

Environmental resource

Environmental emissions

Environmental emission

[E250] ammonia, ammonium to air

[E561] hydrogen chloride to air

[E562] hydrogen fluoride to air

[E641] nitrogen dioxide to air

[E642] nitrogen mono oxide to air

[E643] nitrogen oxides to air

[E709] sulphur dioxide to air

Category $=[\mathrm{C} 23]$ Eutrophication (Ecoindicator95)

Description $=$ Ecoindicator 95

Author $=$ Goedkoop, 1995

Date $=27-09-1999$

Environmental resources

Environmental resource

Environmental emissions

Environmental emission

[E250] ammonia, ammonium to air

[E636] nitrate to air

[E641] nitrogen dioxide to air

[E642] nitrogen mono oxide to air

[E643] nitrogen oxides to air

[E676] phosphate to air

[E779] ammonia, ammonium to fresh water

[E809] Chemical oxigen demand (COD) to fresh water

[E896] Nitrogen to fresh water

[E910] Phosphate to fresh water

[E911] Phosphorus to fresh water

[E973] ammonia, ammonium to sea water

[E1003] Chemical oxigen demand (COD) to sea water

[E1090] Nitrogen to sea water

[E1104] Phosphate to sea water

[E1105] Phosphorus to sea water

Category $=[\mathrm{C} 24]$ heavy metals (Ecoindicator 95)

Description = Ecoindicator 95; Toxic effects by heavy metals

Author $=$ Goedkoop 1995

Date $=27-09-1999$

Environmental resources

Environmental resource

Value

Unit

Environmental emissions

Environmental emission

[E253] antimony to air

[E255] arsenic to air

[E259] barium to air

[E270] beryllium to air

[E385] cadmium to air
Value

$11 \mathrm{~kg} \mathrm{CO} 2$ eq. $/ \mathrm{kg}$

$1300 \mathrm{~kg} \mathrm{CO} 2$ eq. $/ \mathrm{kg}$

$25 \mathrm{~kg} \mathrm{CO} 2$ eq. $/ \mathrm{kg}$

Value

Value

$1.88 \mathrm{~kg} \mathrm{SO} 2 \mathrm{eq} . / \mathrm{kg}$

$0.88 \mathrm{~kg} \mathrm{SO} 2 \mathrm{eq} . / \mathrm{kg}$

$1.6 \mathrm{~kg} \mathrm{SO} 2 \mathrm{eq} . / \mathrm{kg}$

$0.7 \mathrm{~kg} \mathrm{SO} 2 \mathrm{eq} . / \mathrm{kg}$

$1.07 \mathrm{~kg} \mathrm{SO} 2 \mathrm{eq} . / \mathrm{kg}$

$0.7 \mathrm{~kg} \mathrm{SO} 2 \mathrm{eq} . / \mathrm{kg}$

$1 \mathrm{~kg} \mathrm{SO} 2 \mathrm{eq} . / \mathrm{kg}$

Value

Unit

$0.33 \mathrm{~kg}$ PO4--- eq. $/ \mathrm{kg}$

$0.42 \mathrm{~kg}$ PO4--- eq. $/ \mathrm{kg}$

$0.13 \mathrm{~kg} \mathrm{PO} 4---$ eq. $/ \mathrm{kg}$

$0.2 \mathrm{~kg}$ PO4--- eq. $/ \mathrm{kg}$

$0.13 \mathrm{~kg} \mathrm{PO} 4---$ eq. $/ \mathrm{kg}$

$1 \mathrm{~kg}$ PO4--- eq. $/ \mathrm{kg}$

$0.33 \mathrm{~kg}$ PO4--- eq. $/ \mathrm{kg}$

0.022 kg PO4--- eq. $/ \mathrm{kg}$

$0.42 \mathrm{~kg}$ PO4--- eq. $/ \mathrm{kg}$

$1 \mathrm{~kg} \mathrm{PO} 4--$ eq. $/ \mathrm{kg}$

$3.06 \mathrm{~kg}$ PO4--- eq. $/ \mathrm{kg}$

$0.33 \mathrm{~kg}$ PO4--- eq. $/ \mathrm{kg}$

$0.022 \mathrm{~kg} \mathrm{PO} 4--$ eq. $/ \mathrm{kg}$

$0.42 \mathrm{~kg}$ PO4--- eq. $/ \mathrm{kg}$

$1 \mathrm{~kg} \mathrm{PO} 4---\mathrm{eq} . / \mathrm{kg}$

3.06 kg PO4--- eq. $/ \mathrm{kg}$ 
[E414] chromium III to air

[E415] chromium VI to air

[E421] cobalt to air

[E422] copper to air

[E532] Heavy metals to air

[E584] lead to air

[E588] Mangane to air

[E592] mercury to air

[E626] methyl-mercury to air

[E632] molybdenum to air

[E635] nickel to air

[E704] selenium to air

[E722] thallium to air

[E724] tin to air

[E743] vanadium to air

[E748] zinc to air

[E782] antimony to fresh water

[E783] arsenic to fresh water

[E787] barium to fresh water

[E799] Borium to fresh water

[E801] cadmium to fresh water

[E818] chromium III to fresh water

[E819] chromium VI to fresh water

[E822] copper to fresh water

[E873] lead to fresh water

[E877] mangane to fresh water

[E880] mercury to fresh water

[E890] molybdenum to fresh water

[E976] antimony to sea water

[E977] arsenic to sea water

[E981] barium to sea water

[E993] Borium to sea water

[E995] cadmium to sea water

[E1012] chromium III to sea water

[E1013] chromium VI to sea water

[E1016] copper to sea water

[E1067] lead to sea water

[E1071] mangane to sea water

[E1074] mercury to sea water

[E1084] molybdenum to sea water

[E1087] nickel to sea water

Category $=[\mathrm{C} 25]$ carcinogenic (Ecoindicator95)

Description $=$ Ecoindicator 95; carcinogenic effects

Author $=$ Goedkoop, 1995

Date $=27-09-1999$

Environmental resources

Environmental resource

Value

Unit

Environmental emissions

Environmental emission

[E254] aromatics (unspecified) to air

[E255] arsenic to air

[E263] Benzene to air

[E265] benzo[a]pyrene to air

[E415] chromium VI to air

[E496] Ethylbenzene to air

[E509] fluoranthrene to air

[E635] nickel to air

[E684] Polycyclic Aromatic Hydrocarbons (PAH) (unspecified) to air

[E685] Polycyclic Aromatic Hydrocarbons Carcinogenic- (carcinogenic-PAH) to air

[E713] tar to air

Value

Unit

$1.10 \mathrm{E}-05-/ \mathrm{kg}$

$0.044-/ \mathrm{kg}$

$1.10 \mathrm{E}-05-/ \mathrm{kg}$

$1-/ \mathrm{kg}$

$0.44-/ \mathrm{kg}$

$1.10 \mathrm{E}-05-/ \mathrm{kg}$

$1-/ \mathrm{kg}$

$0.44-/ \mathrm{kg}$

$1-/ \mathrm{kg}$

$1-/ \mathrm{kg}$

$1.10 \mathrm{E}-05-/ \mathrm{kg}$

Category $=[\mathrm{C} 26]$ winter smog (Ecoindicator95) 
Description $=$ Ecoindicator 95

Author $=$ Goedkoop, 1995

Date $=27-09-1999$

Environmental resources

Environmental resource

Value

Unit

Environmental emissions

Environmental emission

[E392] Carbon black to air

[E479] dust to air

[E659] Particulates (diesel) (black smoke)(soot) to air

[E683] PM10 to air

[E741] TSP to air

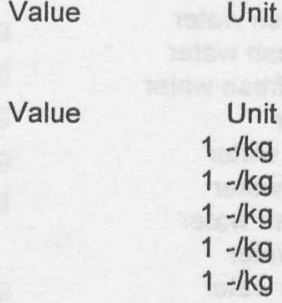

Category $=[\mathrm{C} 27]$ pesticides (ecoindicator95)

Description = Ecoindicator 95; Toxic effects of pesticides

Author $=$ Goedkoop, 1995

Date $=27-09-1999$

Environmental resources

Environmental resource

Environmental emissions

Environmental emission

[E765] 2,4,5-T to air

[E768] 2,4-D to fresh water

[E774] acephate to fresh water

[E777] aldicarb to fresh water

[E778] aldrin to fresh water

[E780] anilazine to fresh water

[E784] atrazine to fresh water

[E785] azinphos-ethyl to fresh water

[E786] azinphos-methyl to fresh water

[E788] benomyl to fresh water

[E789] bentazone to fresh water

[E797] bifenthrin to fresh water

[E802] captafol to fresh water

[E803] captan to fresh water

[E804] carbaryl to fresh water

[E805] carbendazim to fresh water

[E806] carbofuran to fresh water

[E810] chlordane to fresh water

[E811] chlorfenvinphos to fresh water

[E812] chloridazon to fresh water

[E815] chlorothalonil to fresh water

[E816] chlorpropham to fresh water

[E817] chlorpyriphos to fresh water

[E823] coumaphos to fresh water

[E824] cyanazine to fresh water

[E825] cypermethrin to fresh water

[E826] cyromazine to fresh water

[E827] DDT to fresh water

[E828] deltamethrin to fresh water

[E829] demeton to fresh water

[E830] desmetryn to fresh water

[E832] diazinon to fresh water

[E835] dichlorprop to fresh water

[E836] dichlorvos to fresh water

[E837] dieldrin to fresh water

[E842] dimethoate to fresh water

[E844] dinoseb to fresh water

[E845] dinoterb to fresh water

[E847] disulfothon to fresh water

[E848] diuron to fresh water

[E849] DNOC to fresh water

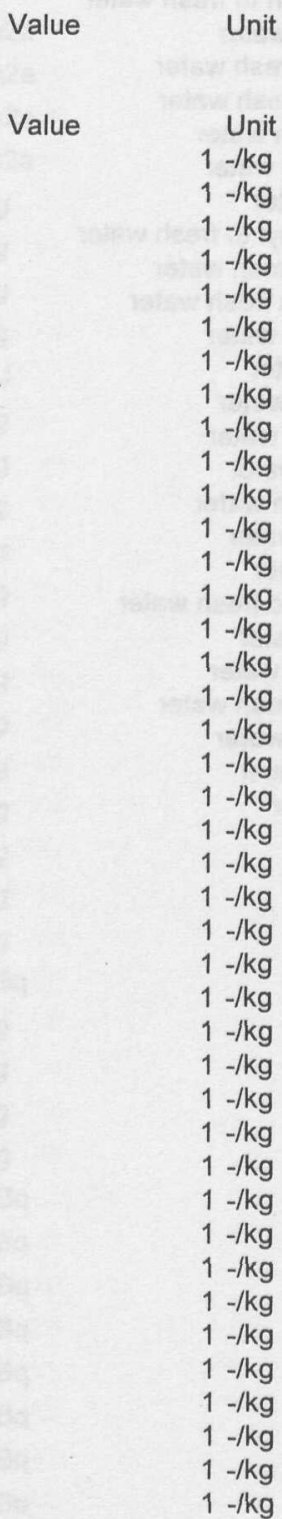


[E850] endosulfan to fresh water

[E851] endrin to fresh water

[E852] ethoprophos to fresh water

[E855] fenitrothion to fresh water

[E856] fenthion to fresh water

[E857] fentin acetate to fresh water

[E858] fentin chloride to fresh water

[E859] fentin hydroxide to fresh water

[E861] folpet to fresh water

[E863] glyphosate to fresh water

[E864] heptachlor to fresh water

[E865] heptenophos to fresh water

[E871] iprodione to fresh water

[E872] isoproturon to fresh water

[E874] lindane to fresh water

[E875] linuron to fresh water

[E876] malathion to fresh water

[E878] MCPA to fresh water

[E879] mecoprop to fresh water

[E881] metamitron to fresh water

[E882] metazachlor to fresh water

[E883] methabenzthiazuron to fresh water

[E884] methomyl to fresh water

[E885] methylbromide to fresh water

[E887] metobromuron to fresh water

[E888] metolachlor to fresh water

[E889] mevinphos to fresh water

[E899] oxamyl to fresh water

[E900] oxydemethon-methyl to fresh water

[E902] parathion-ethyl to fresh water

[E903] parathion-methyl to fresh water

[E907] permethrin to fresh water

[E912] phoxim to fresh water

[E914] pirimicarb to fresh water

[E916] propachlor to fresh water

[E917] propoxur to fresh water

[E920] pyrazophos to fresh water

[E922] simazine to fresh water

[E929] Thiram to fresh water

[E931] tolclophos-methyl to fresh water

[E933] tri-allate to fresh water

[E934] triazophos to fresh water

[E935] tributyltinoxide to fresh water

[E936] trichlorfon to fresh water

[E939] trifluarin to fresh water

[E943] zineb to fresh water 
Appendix D. Inputs and outputs not assigned to an impact category

\begin{tabular}{|c|c|c|}
\hline Name & Compartment & Unit \\
\hline Barit ab Erz & from earth resources & $\mathrm{kg}$ \\
\hline Bauxit & from earth resources & $\mathrm{kg}$ \\
\hline Bentonit ab Erz & from earth resources & $\mathrm{kg}$ \\
\hline dolomite & from earth resources & $\mathrm{kg}$ \\
\hline flussspat & from earth resources & $\mathrm{kg}$ \\
\hline Grubengas (Methan) & from earth resources & $\mathrm{kg}$ \\
\hline Holz & from earth resources & $t$ \\
\hline Kalkstein vor Abbau & from earth resources & $\mathrm{kg}$ \\
\hline $\mathrm{KCl}$ & from earth resources & $\mathrm{kg}$ \\
\hline Kies vor Abbau & from earth resources & $\mathrm{kg}$ \\
\hline quarzsand & from earth resources & $\mathrm{kg}$ \\
\hline Sand vor Abbau & from earth resources & $\mathrm{kg}$ \\
\hline Space Benthos II-III & from earth resources & $\mathrm{m} 2 \mathrm{a}$ \\
\hline Space Benthos II-IV & from earth resources & $\mathrm{m} 2 \mathrm{a}$ \\
\hline Space II-III & from earth resources & $\mathrm{m} 2 \mathrm{a}$ \\
\hline Space III-IV & from earth resources & $\mathrm{m} 2 \mathrm{a}$ \\
\hline Space II-IV & from earth resources & $\mathrm{m} 2 \mathrm{a}$ \\
\hline Space IV-IV & from earth resources & $\mathrm{m} 2 \mathrm{a}$ \\
\hline Steinsalz vor Abbau & from earth resources & $\mathrm{kg}$ \\
\hline titanium ore & from earth resources & $\mathrm{kg}$ \\
\hline Ton & from earth resources & $\mathrm{kg}$ \\
\hline Wasser & from earth resources & $\mathrm{kg}$ \\
\hline heat & to air & TJ \\
\hline Aluminium & to air & $\mathrm{kg}$ \\
\hline B & to air & $\mathrm{kg}$ \\
\hline $\mathrm{Br}$ & to air & $\mathrm{kg}$ \\
\hline Butene & to air & $\mathrm{kg}$ \\
\hline $\mathrm{Ca}$ & to air & $\mathrm{kg}$ \\
\hline Dichloromonofluormethane & to air & $\mathrm{kg}$ \\
\hline Iron & to air & $\mathrm{kg}$ \\
\hline $\mathrm{He}$ & to air & $\mathrm{kg}$ \\
\hline Hexane & to air & $\mathrm{kg}$ \\
\hline I (lodium) & to air - & $\mathrm{kg}$ \\
\hline K (kalium) & to air & $\mathrm{kg}$ \\
\hline La (Lanthane) & to air & $\mathrm{kg}$ \\
\hline$M g$ & to air & $\mathrm{kg}$ \\
\hline LT Radio. Rn222 & to air & $\mathrm{kBq}$ \\
\hline $\mathrm{Na}$ & to air & $\mathrm{kg}$ \\
\hline NMVOC & to air & $\mathrm{kg}$ \\
\hline Pentane & air & $\mathrm{kg}$ \\
\hline Pt (Platina) & air & $\mathrm{kg}$ \\
\hline Radio. Aerosole & air & $\mathrm{kBq}$ \\
\hline Radio. Ag110m & air & $\mathrm{kBq}$ \\
\hline Radio. Am241 & air & $\mathrm{kBq}$ \\
\hline Radio. other beta & air & $\mathrm{kBq}$ \\
\hline Radio. Ar4 & air & $\mathrm{kBq}$ \\
\hline Radio. Ba140 & air & $\mathrm{kBq}$ \\
\hline Radio. C14 & air & $\mathrm{kBq}$ \\
\hline Radio. Ce141 & air & $\mathrm{kBq}$ \\
\hline
\end{tabular}




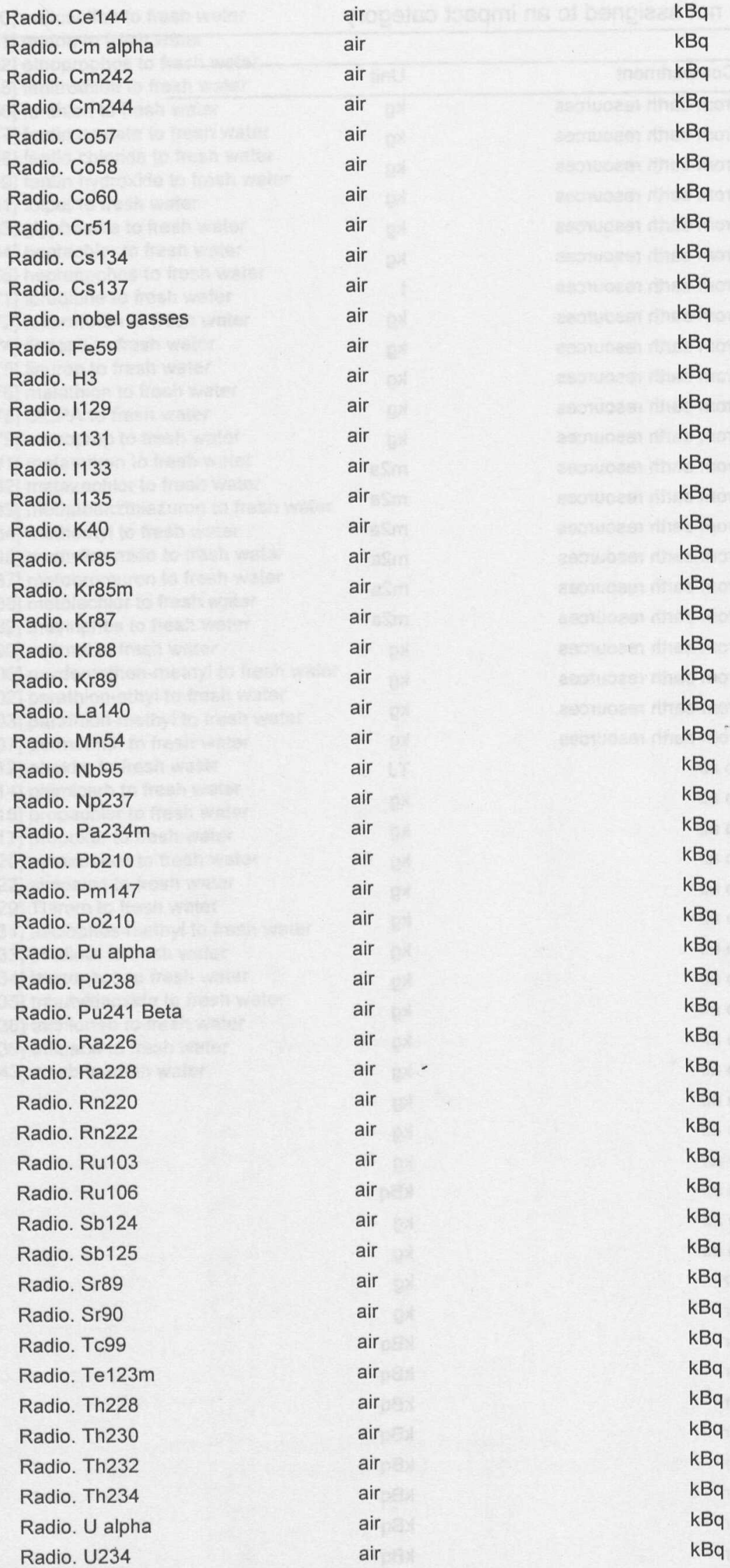




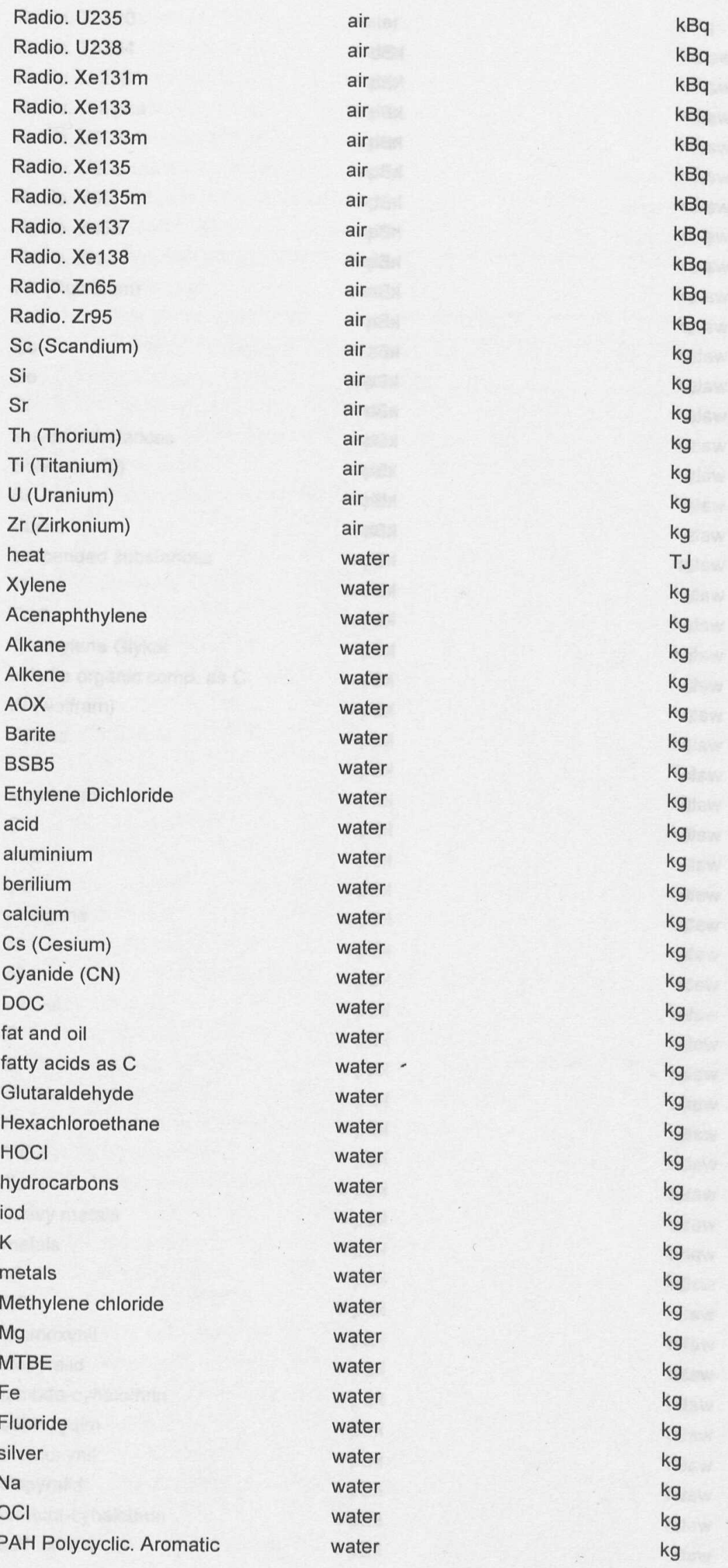


Hydrocarbons

Radio. Ag110m

Radio. Alpha-radiator

water

$\mathrm{kBq}$

Radio. Am241

water

$\mathrm{kBq}$

Radio. Ba140

water

$\mathrm{kBq}$

Radio. C14

Radio. Cd109

Radio. Ce141

water

$\mathrm{kBq}$

water

$\mathrm{kBq}$

water

$\mathrm{kBq}$

Radio. Ce144

water

$\mathrm{kBq}$

Radio. $\mathrm{Cm}$ alpha

water

$\mathrm{kBq}$

Radio. Co57

water

$\mathrm{kBq}$

water

$\mathrm{kBq}$

Radio. Co58

water

$\mathrm{kBq}$

Radio. Co60

Radio. Cr51

Radio. Cs134

Radio. Cs136

Radio. Cs137

Radio. Fe59

water

$\mathrm{kBq}$

water

$\mathrm{kBq}$

water

$\mathrm{kBq}$

water

$\mathrm{kBq}$

water

$\mathrm{kBq}$

water

$\mathrm{kBq}$

Radio. H3

water

$\mathrm{kBq}$

Radio. 1129

water

$\mathrm{kBq}$

Radio. 1131

Radio. 1133

Radio. K 40

water

$\mathrm{kBq}$

water

$\mathrm{kBq}$

Radio. La140

water

$\mathrm{kBq}$

water

$\mathrm{kBq}$

Radio. Mn54

water

Radio. Mo99

water

$\mathrm{kBq}$

Radio. Na24

water

$\mathrm{kBq}$

$\mathrm{kBq}$

Radio. Nb95

water

$\mathrm{kBq}$

Radio. Np237

Radio. Nucl. mixed

Radio. Pa234m

Radio. $\mathrm{Pb} 210$

Radio. Po 210

Radio. Pu alpha

Radio. Pu241 beta

water

$\mathrm{kBq}$

water

$\mathrm{kBq}$

water

$\mathrm{kBq}$

water

$\mathrm{kBq}$

water

$\mathrm{kBq}$

water $\mathrm{kBq}$

water $\mathrm{kBq}$

water - $\mathrm{kBq}$

water $\mathrm{kBq}$

water $\quad \mathrm{kBq}$

water $\quad \mathrm{kBq}$

water $\quad \mathrm{kBq}$

water $\quad \mathrm{kBq}$

water $\quad \mathrm{kBq}$

water $\mathrm{kBq}$

water $\quad \mathrm{kBq}$

water $\mathrm{kBq}$

water $\quad \mathrm{kBq}$

water $\mathrm{kBq}$

water $\mathrm{kBq}$

water $\mathrm{kBq}$

water $\quad \mathrm{kBq}$

water $\quad \mathrm{kBq}$

water $\mathrm{kBq}$

Radio. Th 232 


\begin{tabular}{|c|c|c|}
\hline Radio. Th230 & water & $\mathrm{kBq}$ \\
\hline Radio. Th234 & water & $\mathrm{kBq}$ \\
\hline Radio. U 238 & water & $\mathrm{kBq}$ \\
\hline Radio. $U$ alpha & water & $\mathrm{kBq}$ \\
\hline Radio. U234 & water & $\mathrm{kBq}$ \\
\hline Radio. U235 & water & $\mathrm{kBq}$ \\
\hline Radio. Y90 & water & $\mathrm{kBq}$ \\
\hline Radio. Zn65 & water & $\mathrm{kBq}$ \\
\hline Radio. Zr95 & water & $\mathrm{kBq}$ \\
\hline Rb (Rubidium) & water & $\mathrm{kg}$ \\
\hline salt & water & $\mathrm{kg}$ \\
\hline $\mathrm{Sb}$ & water & $\mathrm{kg}$ \\
\hline $\mathrm{Se}$ & water & $\mathrm{kg}$ \\
\hline $\mathrm{Si}$ & water & $\mathrm{kg}$ \\
\hline solved substances & water & $\mathrm{kg}$ \\
\hline Solvents $(\mathrm{Cl})$ & water & $\mathrm{kg}$ \\
\hline $\mathrm{Sr}$ & water & $\mathrm{kg}$ \\
\hline Sulfite & water & $\mathrm{kg}$ \\
\hline suspended substances & water & $\mathrm{kg}$ \\
\hline $\mathrm{Ti}$ & water & $\mathrm{kg}$ \\
\hline TOC & water & $\mathrm{kg}$ \\
\hline Triethylene Glykol & water & $\mathrm{kg}$ \\
\hline volatile organic comp. as C & water & $\mathrm{kg}$ \\
\hline W (wolfram) & water & $\mathrm{kg}$ \\
\hline Xylene & water & $\mathrm{kg}$ \\
\hline heat & soil & TJ \\
\hline aluminium & soil & $\mathrm{kg}$ \\
\hline C & soil & $\mathrm{kg}$ \\
\hline calcium & soil & $\mathrm{kg}$ \\
\hline iron & soil & $\mathrm{kg}$ \\
\hline mangane & soil & $\mathrm{kg}$ \\
\hline N & soil & $\mathrm{kg}$ \\
\hline oil & soil & $\mathrm{kg}$ \\
\hline oil biol. & soil & $\mathrm{kg}$ \\
\hline$P$ & soil & $\mathrm{kg}$ \\
\hline S & soil - & $\mathrm{kg}$ \\
\hline chlorine & to air & $\mathrm{kg}$ \\
\hline fluor & to air & $\mathrm{kg}$ \\
\hline $\mathrm{TiO} 2$ & water & $\mathrm{kg}$ \\
\hline $\mathrm{Te}$ & air & $\mathrm{kg}$ \\
\hline heavy metals & air & $\mathrm{kg}$ \\
\hline metals & air & $\mathrm{kg}$ \\
\hline oil & water & $\mathrm{kg}$ \\
\hline $\mathrm{HC}$ & water & $\mathrm{kg}$ \\
\hline bromoxynil & air & $\mathrm{kg}$ \\
\hline clopyralid & air & $\mathrm{kg}$ \\
\hline lambda-cyhalothrin & air & $\mathrm{kg}$ \\
\hline sethoxydim & air & $\mathrm{kg}$ \\
\hline bromoxynil & soil & $\mathrm{kg}$ \\
\hline clopyralid & soil & $\mathrm{kg}$ \\
\hline lambda-cyhalothrin & soil & $\mathrm{kg}$ \\
\hline sethoxidim & soil & $\mathrm{kg}$ \\
\hline
\end{tabular}




\begin{tabular}{lll} 
bromoxynil & water & $\mathrm{kg}$ \\
clopyralid & water & $\mathrm{kg}$ \\
lambda-cyhalothrin & water & $\mathrm{kg}$ \\
sethoxidim & water & $\mathrm{kg}$ \\
heptane & to air & $\mathrm{kg}$ \\
hydrogen sulphide & to fresh water & $\mathrm{kg}$ \\
Nitrites & to fresh water & $\mathrm{kg}$ \\
sulphur dioxide & to fresh water & $\mathrm{kg}$ \\
\hline
\end{tabular}

. Intervention tables

\begin{tabular}{|c|c|c|c|}
\hline Environmental flow & $\begin{array}{l}\text { BAseline } \\
\text { (Dutch) }\end{array}$ & Swedish & Cork \\
\hline [E16] cobalt (Co) from earth resources & $-3.16 \mathrm{E}-06$ & $-2.67 \mathrm{E}-06$ & $-3.06 \mathrm{E}-06 \mathrm{~kg}$ \\
\hline [E17] copper $(\mathrm{Cu})$ from earth resources & -0.7435 & -0.74809 & $-0.66445 \mathrm{~kg}$ \\
\hline [E18] chromium $(\mathrm{Cr})$ from earth resources & -0.26267 & -0.35548 & $-0.23928 \mathrm{~kg}$ \\
\hline$[\mathrm{E} 33]$ iron $(\mathrm{Fe})$ from earth resources & -150.33 & -149.98 & $-129.96 \mathrm{~kg}$ \\
\hline [E38] lead $(\mathrm{Pb})$ from earth resources & -0.8873 & -1.3594 & $-0.83331 \mathrm{~kg}$ \\
\hline [E41] manganese $(\mathrm{Mn})$ from earth resources & -0.092737 & -0.12201 & $-0.083536 \mathrm{~kg}$ \\
\hline [E43] molybdenum (Mo) from earth resources & $-2.90 E-06$ & $-1.95 E-06$ & $-2.85 \mathrm{E}-06 \mathrm{~kg}$ \\
\hline [E46] nickel ( $\mathrm{Ni})$ from earth resources & -0.15328 & -0.20956 & $-0.14015 \mathrm{~kg}$ \\
\hline [E49] palladium $(\mathrm{Pd})$ from earth resources & $-5.53 E-07$ & $-7.91 \mathrm{E}-07$ & $-5.19 \mathrm{E}-07 \mathrm{~kg}$ \\
\hline [E50] phosphorus $(P)$ from earth resources & -16.445 & -12.495 & $-11.692 \mathrm{~kg}$ \\
\hline [E51] platinum (Pt) from earth resources & $-6.59 \mathrm{E}-07$ & $-9.49 \mathrm{E}-07$ & $-6.20 \mathrm{E}-07 \mathrm{~kg}$ \\
\hline [E57] rhenium $(\mathrm{Re})$ from earth resources & $-4.93 E-07$ & $-6.90 \mathrm{E}-07$ & $-4.63 \mathrm{E}-07 \mathrm{~kg}$ \\
\hline [E58] rhodium (Rh) from earth resources & $-5.94 \mathrm{E}-07$ & $-8.52 E-07$ & $-5.59 \mathrm{E}-07 \mathrm{~kg}$ \\
\hline [E65] silver (Ag) from earth resources & -0.0046019 & -0.0040948 & $-0.0043543 \mathrm{~kg}$ \\
\hline [E68] sulfur (S) from earth resources & -27.031 & -9.5491 & $-27.031 \mathrm{~kg}$ \\
\hline [E75] tin (Sn) from earth resources & -0.0025542 & -0.002271 & $-0.0024167 \mathrm{~kg}$ \\
\hline$[E 78]$ uranium $(U)$ from earth resources & -0.051527 & -0.038311 & $-0.0394 \mathrm{~kg}$ \\
\hline [E83] zinc $(\mathrm{Zn})$ from earth resources & -0.023439 & -0.03648 & $-0.021899 \mathrm{~kg}$ \\
\hline [E85] oil crude from earth resources & -1602 & -1427.7 & $-1610.1 \mathrm{~kg}$ \\
\hline [E86] natural gas from earth resources & -1174.8 & -784.1 & $-2040.2 \mathrm{~m} 3$ \\
\hline [E87] coal soft from earth resources & -1148.5 & -713.99 & $-1181.9 \mathrm{~kg}$ \\
\hline [E88] coal hard from earth resources & -529.61 & -391.07 & $-371.26 \mathrm{~kg}$ \\
\hline [E90] 1,1,1-trichloroethane (methyl chloroform; $\mathrm{HC}-140 \mathrm{a}$ ) to air & 0.11022 & 0.085923 & $0.11954 \mathrm{~kg}$ \\
\hline [E97] 1,2,3-Trimethyl Benzene to air & 0.19434 & 0.18674 & $0.18891 \mathrm{~kg}$ \\
\hline [E100] 1,2,4-trimethylbenzene to air & 0.23057 & 0.21499 & $0.22821 \mathrm{~kg}$ \\
\hline [E109] 1,3,5-trimethylbenzene (mesitylene) to air & 0.20222 & 0.19288 & $0.19746 \mathrm{~kg}$ \\
\hline [E123] 1-Butyl Acetate to air & 0.025271 & 0.0197 & $0.027408 \mathrm{~kg}$ \\
\hline [E130] 1-Decane to air & 0.069229 & 0.053968 & $0.075081 \mathrm{~kg}$ \\
\hline [E131] 1-Dodecane to air & 0.086402 & 0.067355 & $0.093707 \mathrm{~kg}$ \\
\hline [E134] 1-Heptane to air & 0.037392 & 0.029149 & $0.040553 \mathrm{~kg}$ \\
\hline [E137] 1-Hexane to air & 0.057614 & 0.044913 & $0.062485 \mathrm{~kg}$ \\
\hline [E141] 1-Nonane to air & 0.080836 & 0.063016 & $0.08767 \mathrm{~kg}$ \\
\hline [E143] 1-Octane to air & 0.028805 & 0.022455 & $0.031241 \mathrm{~kg}$ \\
\hline [E147] 1-Pentane to air & 0.014401 & 0.011226 & $0.015618 \mathrm{~kg}$ \\
\hline [E150] 1-Propyl Benzene to air & 0.19025 & 0.18339 & $0.18458 \mathrm{~kg}$ \\
\hline [E154] 1-Undecane to air & 0.086402 & 0.067355 & $0.093707 \mathrm{~kg}$ \\
\hline [E168] 2,3,7,8-TCDD (tetrachloride-dibenzo-dioxin) to air & $7.49 \mathrm{E}-10$ & 7.09E-10 & $6.12 \mathrm{E}-10 \mathrm{~kg}$ \\
\hline [E182] 2.3- Dimethylbutane to air & 0.020225 & 0.015767 & $0.021935 \mathrm{~kg}$ \\
\hline [E199] 2-Methylheptane to air & 0.063408 & 0.04943 & $0.068768 \mathrm{~kg}$ \\
\hline
\end{tabular}


[E200] 2-Methylhexane to air [E201] 2-Methylnonane to air [E202] 2-Methyloctane to air [E203] 2-Methylpentane to air [E223] 3-Methylhexane to air [E224] 3-Methylpentane to air [E232] Acetaldehyde (ethanal) to air [E233] Acetic acid to air [E234] Acetone (2-propanon) to air [E235] Acetylene to air [E236] Acrolein (2-propenal) to air [E239] aldehydes (unspec.) to air [E242] alkanes (unspec.) to air [E243] alkenes (unspec.) to air [E249] ammonia, ammonium to air [E252] antimony to air [E253] aromatics (unspecified) to air [E254] arsenic to air [E258] barium to air [E261] Benzaldehyde to air [E262] Benzene to air [E264] benzo[a]pyrene to air [E269] beryllium to air [E286] butane (unspec.) to air [E384] cadmium to air [E392] Carbon dioxide to air [E394] Carbon Monoxide to air [E397] CFC-11 to air [E399] CFC-114 to air [E401] CFC-12 to air [E402] CFC-13 to air [E411] chlorpyriphos to air [E412] chromium (unspecified) to air [E420] cobalt to air [E421] copper to air [E425] Cyanides to air [E440] deltamethrin to air

[E448] Dichloromethane (Methylene Chloride) to air [E460] dimethoate to air

[E468] Dinitrogen oxide (nitrous oxide) to air [E482] Ethane to air [E484] Ethanol to air [E487] Ethyl Acetate to air [E495] Ethylbenzene to air [E498] Ethylene (ethene) to air [E500] Ethylene Dichloride to air [E510] Formaldehyde (methanal) to air [E515] glyphosate to air [E520] HALON-1301 to air [E528] HCFC-22 (Chlorodifluormethane) to air [E533] heptane to air [E536] hexachlorobenzene to air

\begin{tabular}{|c|c|c|}
\hline 1 & 09 & \\
\hline 040416 & .031507 & 0.043833 \\
\hline 026012 & 020278 & 28211 \\
\hline 051797 & .040378 & $.056175 \mathrm{k}$ \\
\hline 014408 & 0.011232 & $.015626 \mathrm{k}$ \\
\hline 037392 & .029149 & $553 \mathrm{k}$ \\
\hline .001233 & .0009408 & $588 \mathrm{k}$ \\
\hline .0096433 & 0.0069185 & $0.012838 \mathrm{k}$ \\
\hline 0.11139 & .086818 & 0.1 \\
\hline 0.72736 & 0.70768 & $0.70128 \mathrm{k}$ \\
\hline $1.06 \mathrm{E}-06$ & 1.05E-06 & 9.63 \\
\hline 4.00E-05 & 2.96E-05 & $5 \mathrm{k}$ \\
\hline 0.047882 & 0.039719 & 0.04 \\
\hline 0022927 & 0017444 & kr \\
\hline 21.408 & 16.266 & 5.2 \\
\hline 0.0029855 & 0.002347 & 0.002 \\
\hline 0.0015145 & 0.0010898 & 0.001 \\
\hline 0.0078946 & 0.0064217 & 0.00 \\
\hline 0.00 & 0.000 & 0.0 \\
\hline 5.53E-07 & $5.50 \mathrm{E}-07$ & $7 \mathrm{k}$ \\
\hline 3 & & \\
\hline 3.54E-05 & $2.64 \mathrm{E}-05$ & 3.3 \\
\hline 1.11E-05 & $8.22 E-06$ & 8.2 \\
\hline 0.1652 & 13447 & $27 \mathrm{kc}$ \\
\hline .0059176 & .0047136 & $0.0059012 \mathrm{~kg}$ \\
\hline 7254 & & \\
\hline 57.156 & 47.533 & 90 \\
\hline $1.25 \mathrm{E}$ & 9.0 & \\
\hline 0.000423 & 0.0003144 & 0.000 \\
\hline 2.69E-06 & 1.96E-06 & $1.76 \mathrm{E}-06 \mathrm{~kg}$ \\
\hline $1.68 \mathrm{E}-06$ & $1.22 \mathrm{E}-06$ & \\
\hline 0.013647 & 0.010369 & $6 \mathrm{~kg}$ \\
\hline 0.0033873 & 0.00 & $\mathrm{~kg}$ \\
\hline 0.001 & 0.0009 & 0.0 \\
\hline 0.040295 & 905 & $2 \mathrm{~kg}$ \\
\hline $8.48 \mathrm{E}-$ & $6.68 \mathrm{E}-$ & \\
\hline 3.70E-05 & 2.81E-05 & 2.63E-05 kg \\
\hline 0.087648 & 0.068326 & 0.09 \\
\hline 0.0056904 & .0043235 & $.0040457 \mathrm{~kg}$ \\
\hline 30.384 & 23.159 & $21.622 \mathrm{~kg}$ \\
\hline 3.225 & 3. & 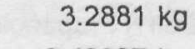 \\
\hline 0.40033 & 0.31205 & $.43337 \mathrm{~kg}$ \\
\hline 0.043323 & 0.033773 & $0.046986 \mathrm{k}$ \\
\hline 0.22002 & 0.20704 & $0.21555 \mathrm{~kg}$ \\
\hline 3.3703 & 5362 & $3.24 \mathrm{~kg}$ \\
\hline 0001221 & 8.44E-05 & .0001518 \\
\hline 3.6656 & 3.5582 & $3.5377 \mathrm{~kg}$ \\
\hline 0.0017776 & 0.0013506 & $0.0012638 k$ \\
\hline 0.0005758 & 0.0005186 & $0.0005448 \mathrm{k}$ \\
\hline $2.98 \mathrm{E}-06$ & 2.17E-06 & $.99 \mathrm{E}-06$ \\
\hline 0.028313 & 0.024539 & 0.02686 \\
\hline 4.56E-09 & 5.10E-09 & 4.13E-0 \\
\hline
\end{tabular}


[E544] HFC-134a (1,1,1,2-tetrafluoroethane) to air

[E559] hydrogen chloride to air

[E560] hydrogen fluoride to air

[E561] hydrogen sulfide to air

[E570] isobutyl acetate to air

[E573] isopentane to air

[E577] isopropyl acetate to air

[E578] isopropyl benzene (cumene) to air

[E581] lead to air

[E584] malathion to air

[E585] Mangane to air

[E586] MCPA to air

[E588] mercury to air

[E590] meta-Ethyltoluene to air

[E592] meta-Xylene (1,3-dimethylbenzene) to air

[E596] Methane to air

[E598] Methanol to air

[E599] methomyl to air

[E600] Methyl Acetate to air

[E608] Methyl Ethyl Ketone to air

[E611] Methyl Isobutyl Ketone (4-methylpentanon-2) to air

[E619] Methyl trans-Butyl Ether to air

[E627] molybdenum to air

[E630] nickel to air

[E634] nitrogen to air

[E637] nitrogen oxides to air

[E642] ortho-Ethyltoluene to air

[E643] ortho-Xylene (1,2-dimethylbenzene) to air

[E647] para-Ethyltoluene to air

[E651] para-Xylene (1,4-dimethylbenzene) to air

[E653] pentachlorobenzene to air

[E655] pentachlorophenol to air

[E660] Perfluoroethane (CFC-116) to air

[E662] Perfluoromethane (CFC-14) to air

[E665] permethrin to air

[E668] Phenol to air

[E671] Phosphorus to air

[E676] PM10 to air

[E677] Polycyclic Aromatic Hydrocarbons (PAH) (unspecified) to air

[E680] Propane to air

[E681] Propanoic acid (propionic acid) to air

[E682] Propionaldehyde (propanal) to air

[E686] Propylene to air

[E697] selenium to air

[E702] suiphur dioxide to air

[E703] Sulphur hexafluoride to air

[E711] tetrachloroethylene (PER) (tetrachloroethene) to air

[E712] Tetrachloromethane (carbon tetrachloride) $(\mathrm{HC}-10)$ to air

[E715] thallium to air

[E717] tin to air

[E720] Toluene to air

[E729] trichlorfon to air
$-7.32 \mathrm{E}-1$

1.3237

0.15508

0.049989

$-4.97 \mathrm{E}-17$

1.0317

0.12061

0.034578

0.024531

0.031468

0.0085869

0.019481

0.18729

0.15153

0.018935

0.045489

0.0037265

0.0031749

0.19987

0.24148

24.552

0.062396

0.0065717

0.011298

0.19977

0.068888

$7.41 \mathrm{E}-05$

0.0002665

0.010368

0.33587

37.382

0.20072

0.22227

0.19988

0.24061

1.22E-08

1.97E-09

5.71E-05

0.0005136

0.0005539

$5.29 \mathrm{E}-06$

0.0006957

6.8834

0.0005312

0.20094

0.0006523

5.53E- 07

0.73645

0.0015874

41.229

3.38E-06

0.095065

3.03E-05

4.17E-06

0.017938

0.67142

0.0088882
0.0066939

0.015187

0.18142

0.12166

0.014386

0.037225

0.0028313

0.0024938

0.19105

0.22349

17.966

0.048629

0.0049931

0.0088071

0.15573

0.053702

0.0001159

0.0002209

0.0091329

0.22525

37.059

0.19171

0.20852

0.19106

0.22264

1.36E-08

2.20E-09

8.26E-05

0.0007435

0.0004209

2.78E-06

0.0005796

5.9896

0.0004486

0.15927

0.0004381

5.50E-07

0.71683

0.0013327

34.083

2.56E-06

0.074108

2.10E-05

3.07E-06

0.014104

0.59426

0.0067531
$-1.33 \mathrm{E}-16 \mathrm{~kg}$

$1.3006 \mathrm{~kg}$

$0.14523 \mathrm{~kg}$

$0.06183 \mathrm{~kg}$

$0.034128 \mathrm{~kg}$

$0.0093128 \mathrm{~kg}$

$0.021128 \mathrm{~kg}$

$0.18117 \mathrm{~kg}$

$0.15189 \mathrm{~kg}$

$0.013462 \mathrm{~kg}$

$0.044606 \mathrm{~kg}$

$0.0026494 \mathrm{~kg}$

$0.0031691 \mathrm{~kg}$

$0.19492 \mathrm{~kg}$

$0.24004 \mathrm{~kg}$

$26.609 \mathrm{~kg}$

$0.066711 \mathrm{~kg}$

$0.0046723 \mathrm{~kg}$

$0.012253 \mathrm{~kg}$

$0.21666 \mathrm{~kg}$

$0.074711 \mathrm{~kg}$

$6.96 \mathrm{E}-05 \mathrm{~kg}$

$0.0002134 \mathrm{~kg}$

$0.0090703 \mathrm{~kg}$

$0.57431 \mathrm{~kg}$

$39.359 \mathrm{~kg}$

$0.19583 \mathrm{~kg}$

$0.21921 \mathrm{~kg}$

$0.19492 \mathrm{~kg}$

$0.2392 \mathrm{~kg}$

$1.10 \mathrm{E}-08 \mathrm{~kg}$

$1.78 \mathrm{E}-09 \mathrm{~kg}$

$5.02 \mathrm{E}-05 \mathrm{~kg}$

$0.0004517 \mathrm{~kg}$

$0.0003938 \mathrm{~kg}$

$4.19 \mathrm{E}-06 \mathrm{~kg}$

$0.0005622 \mathrm{~kg}$

$7.8811 \mathrm{~kg}$

$0.0007896 \mathrm{~kg}$

$0.24263 \mathrm{~kg}$

$0.0012385 \mathrm{~kg}$

$5.03 \mathrm{E}-07 \mathrm{~kg}$

$0.70988 \mathrm{~kg}$

$0.0014367 \mathrm{~kg}$

$46.494 \mathrm{~kg}$

$2.40 \mathrm{E}-06 \mathrm{~kg}$

$0.1031 \mathrm{~kg}$

$3.71 \mathrm{E}-05 \mathrm{~kg}$

$3.17 \mathrm{E}-06 \mathrm{~kg}$

$0.018044 \mathrm{~kg}$

$0.68648 \mathrm{~kg}$

$0.0063192 \mathrm{~kg}$ 
[E730] Trichloroethylene (tri) to air

[E731] Trichloromethane (chloroform) to air

[E736] vanadium to air

[E738] Vinyl Chloride (chloroethene) to air

[E741] zinc to air

[E743] 1,1,1-trichloroethane to air

[E772] ammonia, ammonium to fresh water

[E775] antimony to fresh water

[E776] arsenic to fresh water

[E780] barium to fresh water

[E783] benzene to fresh water

[E791] Biological Oxygen Demand (BOD) to fresh water

[E792] Borium to fresh water

[E794] cadmium to fresh water

[E802] Chemical oxigen demand (COD) to fresh water

[E806] Chlorine to fresh water

[E807] chlorobenzene to fresh water

[E810] chlorpyriphos to fresh water

[E811] chromium III to fresh water

[E812] chromium VI to fresh water

[E814] cobalt to fresh water

[E815] copper to fresh water

[E821] deltamethrin to fresh water

[E824] Di(2-ethylhexyl)phtalate to fresh water

[E826] Dibutylphtalate to fresh water

[E835] dimethoate to fresh water

[E836] Dimethylphtalate to fresh water

[E846] ethylbenzene to fresh water

[E855] Formaldehyde (methanal) to fresh water

[E856] glyphosate to fresh water

[E861] hydrogen sulfide to fresh water

[E865] lead to fresh water

[E868] malathion to fresh water

[E869] mangane to fresh water

[E870] MCPA to fresh water

[E872] mercury to fresh water

[E876] methomyl to fresh water

[E882] molybdenum to fresh water

[E885] nickel to fresh water

[E886] Nitrate to fresh water

[E887] Nitrites to fresh water

[E888] Nitrogen to fresh water

[E898] permethrin to fresh water

[E900] phenol to fresh water

[E901] Phosphate to fresh water

[E902] Phosphorus to fresh water

[E914] sulphates to fresh water

[E915] sulphur dioxide to fresh water

[E916] tetrachloroethylene (PER) to fresh water

[E917] Tetrachloromethane (carbon tetrachloride) to fresh water

[E920] tin to fresh water

[E922] toluene to fresh water

\begin{tabular}{|c|c|c|}
\hline 0.10667 & 0.083157 & . \\
\hline 22E-06 & 2.23E-06 & $6 \mathrm{k}$ \\
\hline 028781 & 0.02507 & 761 \\
\hline $99 \mathrm{E}-05$ & 1.37E-05 & 2.47E-05 k \\
\hline 0.049842 & 0.063005 & $87 \mathrm{k}$ \\
\hline $9.90 \mathrm{E}-07$ & $1.55 \mathrm{E}-06$ & $9.31 \mathrm{E}$ \\
\hline 0.12186 & 0.10656 & 0.11 \\
\hline 0.045258 & 0.040636 & 0.044 \\
\hline 0.00292 & 0.0016916 & $0.0023658 \mathrm{~kg}$ \\
\hline 0.29769 & 75 & 0.2 \\
\hline 0098211 & 0.008 & 0.009 \\
\hline 0020506 & 0.0016212 & 003 \\
\hline 0019806 & 0.0017887 & 0.00 \\
\hline 054054 & 0.0048521 & 0.00 \\
\hline 1.3879 & 39 & 12 \\
\hline 127.88 & 3 & \\
\hline 3.84 & 6.07 & 3.63 \\
\hline $6.50 \mathrm{E}-05$ & 4.94E-05 & $4.62 \mathrm{E}$ \\
\hline 0.015471 & 0.0 & 0.01 \\
\hline 6 & & \\
\hline 391 & 195 & .00 \\
\hline .008216 & 571 & 0.006 \\
\hline $9.64 \mathrm{E}-07$ & 7 & 6.86 \\
\hline $1.31 \mathrm{E}-08$ & 1.5 & \\
\hline 1.72 & 9 & 7 \\
\hline $2.69 E-05$ & & 1.9 \\
\hline 1.08E-07 & -08 & 2.37 \\
\hline ? & 8 & . \\
\hline 4 & & \\
\hline 0001516 & 0.0001152 & $0.0001078 \mathrm{k}$ \\
\hline & 0.0 & $0.0010335 \mathrm{k}$ \\
\hline 0.011849 & 0.00 & \\
\hline 7.77E-05 & $5.90 \mathrm{E}-05$ & $.52 \mathrm{E}$ \\
\hline 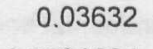 & & \\
\hline 0001624 & 0.000 & 0 \\
\hline 23 & 5 & 1.35E-05 \\
\hline & & \\
\hline 046464 & 0028464 & $.0036547 \mathrm{k}$ \\
\hline 0075558 & 5 & man \\
\hline 0.17294 & & \\
\hline 0023119 & 0017155 & 0016 \\
\hline & & \\
\hline $1.45 \mathrm{E}-05$ & 1.10E-05 & 1.03E-05 \\
\hline 0.010866 & 00098 & 01007 \\
\hline 0.090854 & 0.053482 & $0.073737 \mathrm{k}$ \\
\hline 0.054532 & 0.041434 & 0.038792 \\
\hline | & 1 & 5.938 \\
\hline 0.003465 & 0.0030096 & .0002144 \\
\hline 1.66E-07 & $1.14 \mathrm{E}-07$ & 2.06E-07 \\
\hline 2.53E-07 & 1.75E-07 & $3.14 \mathrm{E}-07 \mathrm{k}$ \\
\hline 0.000594 & 0.0004506 & 0.0004186 \\
\hline 0081126 & 0073558 & 0078040 \\
\hline
\end{tabular}


[E925] tributyltinoxide to fresh water [E926] trichlorfon to fresh water [E927] Trichloroethylene (tri) to fresh water [E928] Trichloromethane=chloroform to fresh water [E930] vanadium to fresh water

[E931] Vinyl Chloride (chloroethene) to fresh water [E932] zinc to fresh water

[E1185] chlorpyriphos to agricultural soil [E1196] deltamethrin to agricultural soil [E1210] dimethoate to agricultural soil [E1231] glyphosate to agricultural soil [E1242] malathion to agricultural soil [E1243] MCPA to agricultural soil [E1249] methomyl to agricultural soil [E1268] permethrin to agricultural soil [E1292] trichlorfon to agricultural soil [E1333] arsenic to agricultural soil [E1349] cadmium to industrial soil [E1364] chromium III to industrial soil [E1367] cobalt to industrial soil [E1368] copper to industrial soil [E1417] lead to industrial soil [E1423] mercury to industrial soil [E1436] nickel to industrial soil [E1476] zinc to industrial soil [E1478] Barit ab Erz to industrial soil [E1479] Bauxit to industrial soil [E1480] Bentonit ab Erz to industrial soil [E1481] dolomite to industrial soil

[E1482] Erdoelgas to industrial soil

[E1483] flussspat to industrial soil

[E1484] Grubengas (Methan) to industrial soil [E1485] Holz to industrial soil

[E1486] Kalkstein vor Abbau to industrial soil [E1488] Kies vor Abbau to industrial soil [E1489] quarzsand to industrial soil

[E1490] Sand vor Abbau to industrial soil

[E1491] Space Benthos II-III to industrial soil

[E1492] Space Benthos II-IV to industrial soil

[E1493] Space II-III to industrial soil

[E1494] Space III-IV to industrial soil

[E1495] Space II-IV to industrial soil

[E1496] Space IV-IV to industrial soil

[E1497] Steinsalz vor Abbau to industrial soil

[E1498] titanium ore to industrial soil

[E1499] Ton to industrial soil

[E1500] Wasser to industrial soil

[E1501] heat to industrial soil

[E1502] Aluminium from earth resources

[E1503] B from earth resources

[E1504] Br from earth resources

[E1505] Butene from earth resources

\begin{tabular}{|c|c|c|}
\hline 3989 & 0.0003822 & $0.0003806 \mathrm{~kg}$ \\
\hline 0.0001163 & 8.84E-05 & $8.27 \mathrm{E}-05 \mathrm{~kg}$ \\
\hline $1.05 \mathrm{E}-05$ & 7.23E-06 & $1.30 \mathrm{E}-05 \mathrm{~kg}$ \\
\hline $3.84 \mathrm{E}-05$ & 2.65E-05 & $4.77 \mathrm{E}-05 \mathrm{~kg}$ \\
\hline 0.0075229 & 0.004374 & $0.0060531 \mathrm{~kg}$ \\
\hline 4.70E-08 & $3.25 E-08$ & $5.84 \mathrm{E}-08 \mathrm{~kg}$ \\
\hline 0.028126 & 0.020642 & $0.023242 \mathrm{~kg}$ \\
\hline 0.011104 & 0.0084366 & $0.0078945 \mathrm{~kg}$ \\
\hline 0.0003298 & 0.0002506 & $0.0002345 \mathrm{~kg}$ \\
\hline 0.0046329 & 0.0035201 & $0.0032939 \mathrm{~kg}$ \\
\hline 0.027697 & 0.021044 & $0.019692 \mathrm{~kg}$ \\
\hline 0.01541 & 0.011708 & $0.010956 \mathrm{~kg}$ \\
\hline 0.058163 & 0.044192 & $0.041352 \mathrm{~kg}$ \\
\hline 0.0053631 & 0.0040748 & $0.003813 \mathrm{~kg}$ \\
\hline 0.0049602 & 0.0037688 & $0.0035266 \mathrm{~kg}$ \\
\hline 0.035502 & 0.026974 & $0.025241 \mathrm{~kg}$ \\
\hline 4.53E-05 & $3.82 E-05$ & $5.01 \mathrm{E}-05 \mathrm{~kg}$ \\
\hline 1.38E-05 & $1.20 \mathrm{E}-05$ & $1.12 \mathrm{E}-05 \mathrm{~kg}$ \\
\hline 0.0005669 & 0.0004785 & $0.0006268 \mathrm{~kg}$ \\
\hline 1.95E-06 & $1.75 E-06$ & $1.84 \mathrm{E}-06 \mathrm{~kg}$ \\
\hline $9.74 E-06$ & 8.73E-06 & $9.21 \mathrm{E}-06 \mathrm{~kg}$ \\
\hline $4.64 \mathrm{E}-05$ & 4.14E-05 & $4.35 \mathrm{E}-05 \mathrm{~kg}$ \\
\hline 3.23E-07 & 2.71E-07 & $3.06 \mathrm{E}-07 \mathrm{~kg}$ \\
\hline $1.46 \mathrm{E}-05$ & 1.31E-05 & $1.38 \mathrm{E}-05 \mathrm{~kg}$ \\
\hline 0.001797 & 0.0015212 & $0.0019713 \mathrm{~kg}$ \\
\hline-8.5448 & -7.245 & $-9.5006 \mathrm{~kg}$ \\
\hline-11.922 & -12.876 & $-16.103 \mathrm{~kg}$ \\
\hline-694.45 & -528.02 & $-494.17 \mathrm{~kg}$ \\
\hline-157 & -119.29 & $-111.62 \mathrm{~kg}$ \\
\hline-101.24 & -91.078 & $-95.779 \mathrm{Nm} 3$ \\
\hline-0.11539 & -0.091303 & $-0.19857 \mathrm{~kg}$ \\
\hline-9.8096 & -6.0786 & $-8.609 \mathrm{~kg}$ \\
\hline-0.29977 & -0.23283 & $-0.27084 t$ \\
\hline-355.43 & -317.28 & $-408.28 \mathrm{~kg}$ \\
\hline-2400.7 & -3171.6 & $-2146.6 \mathrm{~kg}$ \\
\hline-10.706 & -8.4714 & $-18.424 \mathrm{~kg}$ \\
\hline-699.21 & -529.11 & $-529.33 \mathrm{~kg}$ \\
\hline-133.97 & -114.23 & $-149.93 \mathrm{~m} 2 \mathrm{a}$ \\
\hline-0.45914 & -0.34793 & $-0.32646 \mathrm{~m} 2 a$ \\
\hline-216.86 & -168.9 & $-162 m 2 a$ \\
\hline-92.468 & -136.87 & $-88.634 \mathrm{~m} 2 a$ \\
\hline-59.787 & -78.078 & $-54.539 \mathrm{~m} 2 \mathrm{a}$ \\
\hline-1.5156 & -0.92748 & $-1.4722 \mathrm{~m} 2 \mathrm{a}$ \\
\hline-158.54 & -125.49 & $-229.84 \mathrm{~kg}$ \\
\hline-318.24 & -251.81 & $-547.64 \mathrm{~kg}$ \\
\hline-34.314 & -34.404 & $-27.649 \mathrm{~kg}$ \\
\hline$-2.84 E+05$ & -55362 & $-2.67 E+05 \mathrm{~kg}$ \\
\hline 0.11261 & 0.08963 & $0.13779 \mathrm{TJ}$ \\
\hline 0.036198 & 0.025927 & $0.027314 \mathrm{~kg}$ \\
\hline 0.020541 & 0.015183 & $0.014308 \mathrm{~kg}$ \\
\hline 0.0017622 & 0.0013008 & $0.0011227 \mathrm{~kg}$ \\
\hline 0.016229 & 0.023664 & $0.015316 \mathrm{~kg}$ \\
\hline
\end{tabular}


[E1506] Ca from earth resources

[E1507] Dichloromonofluormethane from earth resources

[E1508] Iron from earth resources

[E1509] He from earth resources

[E1510] Hexane from earth resources

[E1511] I (lodium) from earth resources

[E1512] K (kalium) from earth resources

[E1513] La (Lanthane) from earth resources

[E1514] Mg from earth resources

[E1515] LT Radio. Rn222 from earth resources

[E1516] Na from earth resources

[E1517] NMVOC from earth resources

[E1518] Pentane from earth resources

[E1519] Pt (Platina) from earth resources

[E1520] Radio. Aerosole from earth resources

[E1521] Radio. Ag110m from earth resources

[E1522] Radio. Am241 from earth resources

[E1523] Radio. other beta from earth resources

[E1524] Radio. Ar4 from earth resources

[E1525] Radio. Ba140 to air

[E1526] Radio. C14 to air

[E1527] Radio. Ce141 to air

[E1528] Radio. Ce144 to air

[E1529] Radio. Cm alpha to air

[E1530] Radio. Cm242 to air

[E1531] Radio. Cm244 to air

[E1532] Radio. Co57 to air

[E1533] Radio. Co58 to air

[E1534] Radio. Co60 to air

[E1535] Radio. Cr51 to air

[E1536] Radio. Cs134 to air

[E1537] Radio. Cs137 to air

[E1538] Radio. nobel gasses to air

[E1539] Radio. Fe59 to air

[E1540] Radio. H3 to air

[E1541] Radio. I129 to air

[E1542] Radio. 1131 air

[E1543] Radio. 1133 air

[E1544] Radio. 1135 air

[E1545] Radio. K40 air

[E1546] Radio. Kr85 air

[E1547] Radio. Kr85m air

[E1548] Radio. Kr87 air

[E1549] Radio. Kr88 air

[E1550] Radio. Kr89 air

[E1551] Radio. La140 air

[E1552] Radio. Mn54 air

[E1553] Radio. Nb95 air

[E1554] Radio. Np237 air

[E1555] Radio. Pa234m air

[E1556] Radio. Pb210 air

[E1557] Radio. Pm147 air

\begin{tabular}{|c|c|c|}
\hline 793 & 0.029386 & $0.027535 \mathrm{~kg}$ \\
\hline 0.0037605 & 0.0059415 & $0.0035442 \mathrm{~kg}$ \\
\hline 0.032599 & 0.026385 & $0.025416 \mathrm{~kg}$ \\
\hline 0.10207 & 0.091883 & $0.09656 \mathrm{~kg}$ \\
\hline 0.059254 & 0.051494 & $0.056212 \mathrm{~kg}$ \\
\hline 0.0008399 & 0.0006165 & $0.0005532 \mathrm{~kg}$ \\
\hline 0.03008 & 0.028755 & $0.025389 \mathrm{~kg}$ \\
\hline 1.83E-05 & 1.30E-05 & $1.43 \mathrm{E}-05 \mathrm{~kg}$ \\
\hline 0.012889 & 0.0092554 & $0.0096966 \mathrm{~kg}$ \\
\hline $2.65 E+06$ & 1.97E+06 & 1.89E+06 kBq \\
\hline 0.011814 & 0.0098601 & $0.0096186 \mathrm{~kg}$ \\
\hline 17.312 & 16.389 & $17.008 \mathrm{~kg}$ \\
\hline 0.19573 & 0.16048 & $0.22067 \mathrm{~kg}$ \\
\hline 4.20E-06 & $6.66 \mathrm{E}-06$ & $3.95 \mathrm{E}-06 \mathrm{~kg}$ \\
\hline $9.92 E+05$ & $7.54 E+05$ & 7.06E+05 kBq \\
\hline $9.33 \mathrm{E}-06$ & $6.49 \mathrm{E}-06$ & 4.68E-06 kBq \\
\hline 0.0003799 & 0.0002815 & $0.0002709 \mathrm{kBq}$ \\
\hline $1.48 \mathrm{E}-05$ & 1.10E-05 & 1.09E-05 kBq \\
\hline 19.056 & 13.184 & $9.2345 \mathrm{kBq}$ \\
\hline 0.0001509 & 0.0001116 & $0.0001078 \mathrm{kBq}$ \\
\hline 34.305 & 25.412 & $24.493 \mathrm{kBq}$ \\
\hline 0.0010366 & 0.0007875 & $0.0007369 \mathrm{kBq}$ \\
\hline 0.0040299 & 0.0029868 & $0.0028739 \mathrm{kBq}$ \\
\hline 0.000602 & 0.0004462 & $0.0004292 \mathrm{kBq}$ \\
\hline 1.22E-09 & $8.89 \mathrm{E}-10$ & 7.84E-10 kBq \\
\hline $1.11 \mathrm{E}-08$ & 8.03E-09 & 7.08E-09 kBq \\
\hline $1.47 \mathrm{E}-08$ & 1.02E-08 & 7.05E-09 kBq \\
\hline 0.0004868 & 0.0003639 & $0.0003585 \mathrm{kBq}$ \\
\hline 0.000794 & 0.0005895 & $0.0005691 \mathrm{kBq}$ \\
\hline $5.74 \mathrm{E}-05$ & 4.19E-05 & 3.81E-05 kBq \\
\hline 0.014332 & 0.010623 & $0.010232 \mathrm{kBq}$ \\
\hline 0.027794 & 0.020604 & $0.019827 \mathrm{kBq}$ \\
\hline 19.928 & 14.821 & $14.761 \mathrm{kBq}$ \\
\hline 4.60E-07 & 3.33E-07 & 2.86E-07 kBq \\
\hline 273.09 & 202.91 & $196.13 \mathrm{kBq}$ \\
\hline 0.10834 & 0.080301 & $0.077249 \mathrm{kBq}$ \\
\hline 0.038408 & 0.028519 & $0.028123 \mathrm{kBq}$ \\
\hline 0.0059792 & 0.0044723 & $0.004417 \mathrm{kBq}$ \\
\hline 0.0085463 & 0.0063941 & $0.0063138 \mathrm{kBq}$ \\
\hline 0.040251 & 0.029833 & $0.026833 \mathrm{kBq}$ \\
\hline $1.86 \mathrm{E}+06$ & $1.38 E+06$ & $1.33 E+06 \mathrm{kBq}$ \\
\hline 10.868 & 8.0681 & $7.9729 \mathrm{kBq}$ \\
\hline 3.4243 & 2.5372 & $2.4874 \mathrm{kBq}$ \\
\hline 77.319 & 57.827 & $57.065 \mathrm{kBq}$ \\
\hline 3.4543 & 2.5649 & $2.5374 \mathrm{kBq}$ \\
\hline 4.85E-05 & $3.56 \mathrm{E}-05$ & $3.31 \mathrm{E}-05 \mathrm{kBq}$ \\
\hline 1.43E-05 & $1.04 \mathrm{E}-05$ & $9.37 \mathrm{E}-06 \mathrm{kBq}$ \\
\hline 2.17E-06 & $1.55 E-06$ & 1.26E-06 kBq \\
\hline $1.99 \mathrm{E}-08$ & $1.47 \mathrm{E}-08$ & $1.42 \mathrm{E}-08 \mathrm{kBq}$ \\
\hline 0.011927 & 0.00884 & $0.0084895 \mathrm{kBq}$ \\
\hline 0.27468 & 0.20356 & $0.18929 \mathrm{kBq}$ \\
\hline 0.010237 & 0.0075874 & $0.0072982 \mathrm{kBq}$ \\
\hline
\end{tabular}


[E1558] Radio. Po210 air [E1559] Radio. Pu alpha air [E1560] Radio. Pu238 air [E1561] Radio. Pu241 Beta air [E1562] Radio. Ra226 air [E1563] Radio. Ra228 air [E1564] Radio. Rn220 air [E1565] Radio. Rn222 air [E1566] Radio. Ru103 air [E1567] Radio. Ru106 air [E1568] Radio. Sb124 air [E1569] Radio. Sb125 air [E1570] Radio. Sr89 air [E1571] Radio. Sr90 air [E1572] Radio. Tc99 air [E1573] Radio. Te123m air [E1574] Radio. Th228 air [E1575] Radio. Th230 air [E1576] Radio. Th232 air [E1577] Radio. Th234 air [E1578] Radio. U alpha air [E1579] Radio. U234 air [E1580] Radio. U235 air [E1581] Radio. U238 air [E1582] Radio. Xe131m air [E1583] Radio. Xe133 air [E1584] Radio. Xe133m air [E1585] Radio. Xe135 air [E1586] Radio. Xe135m air [E1587] Radio. Xe137 air [E1588] Radio. Xe138 air [E1589] Radio. Zn65 air [E1590] Radio. Zr95 air [E1591] Sc (Scandium) air [E1592] Si air [E1593] Sr air [E1594] Th (Thorium) air [E1595] Ti (Titanium) air [E1596] U (Uranium) air [E1597] Zr (Zirkonium) air [E1598] heat air [E1599] Xylene air [E1600] Acenaphthylene air [E1601] Alkane air [E1602] Alkene air [E1603] AOX air [E1604] Barite air [E1605] BSB5 air [E1606] Ethylene Dichloride air [E1607] acid air [E1608] aluminium air [E1609] berilium air

\begin{tabular}{|c|c|c|}
\hline 9136 & 0.29006 & $0.26702 \mathrm{kBq}$ \\
\hline 0.001203 & 0.000892 & $0.0008578 \mathrm{kBq}$ \\
\hline 2.67E-08 & 1.93E-08 & $1.67 \mathrm{E}-08 \mathrm{kBq}$ \\
\hline 0.033142 & 0.02456 & $0.023628 \mathrm{kBq}$ \\
\hline 0.41101 & 0.30445 & $0.29084 \mathrm{kBq}$ \\
\hline 0.0198 & 0.014681 & $0.01322 \mathrm{kBq}$ \\
\hline 0.28035 & 0.10169 & $-0.32118 \mathrm{kBq}$ \\
\hline 28817 & 21346 & $20519 \mathrm{kBq}$ \\
\hline 2.46E-07 & 1.79E-07 & $1.62 \mathrm{E}-07 \mathrm{kBq}$ \\
\hline 0.1203 & 0.089198 & $0.08578 \mathrm{kBq}$ \\
\hline $3.20 \mathrm{E}-06$ & 2.27E-06 & 1.80E-06 kBq \\
\hline 2.76E-06 & 2.05E-06 & $2.01 \mathrm{E}-06 \mathrm{kBq}$ \\
\hline $2.60 \mathrm{E}-05$ & $1.90 \mathrm{E}-05$ & 1.72E-05 kBq \\
\hline 0.019893 & 0.014742 & $0.014185 \mathrm{kBq}$ \\
\hline 8.43E-07 & $6.25 \mathrm{E}-07$ & $6.01 \mathrm{E}-07 \mathrm{kBq}$ \\
\hline 3.86E-05 & 2.67E-05 & 1.85E-05 kBq \\
\hline 0.01681 & 0.012463 & $0.011222 \mathrm{kBq}$ \\
\hline 0.13255 & 0.098175 & $0.09432 \mathrm{kBq}$ \\
\hline 0.010646 & 0.0078946 & $0.0071069 \mathrm{kBq}$ \\
\hline 0.011927 & 0.00884 & $0.0084895 \mathrm{kBq}$ \\
\hline 0.42686 & 0.31621 & $0.30379 \mathrm{kBq}$ \\
\hline 0.14307 & 0.10604 & $0.10186 \mathrm{kBq}$ \\
\hline 0.0069344 & 0.0051375 & $0.0049379 \mathrm{kBq}$ \\
\hline 0.17194 & 0.12735 & $0.12103 \mathrm{kBq}$ \\
\hline 15.69 & 11.623 & $11.382 \mathrm{kBq}$ \\
\hline 1382.9 & 1031.4 & $1014.6 \mathrm{kBq}$ \\
\hline 0.31314 & 0.2196 & $0.16467 \mathrm{kBq}$ \\
\hline 376.59 & 280.39 & $276.54 \mathrm{kBq}$ \\
\hline 103.03 & 76.487 & $75.55 \mathrm{kBq}$ \\
\hline 2.1382 & 1.5852 & $1.5583 \mathrm{kBq}$ \\
\hline 28.555 & 21.2 & $20.95 \mathrm{kBq}$ \\
\hline 0.0001052 & 7.74E-05 & 7.30E-05 kBq \\
\hline $6.14 \mathrm{E}-07$ & 4.30E-07 & 3.22E-07 kBq \\
\hline $5.83 E-06$ & 4.04E-06 & 4.47E-06 kg \\
\hline 0.11084 & 0.080571 & $0.080231 \mathrm{~kg}$ \\
\hline 0.000635 & 0.0004643 & $0.0004897 \mathrm{~kg}$ \\
\hline $1.04 \mathrm{E}-05$ & 7.44E-06 & $7.74 \mathrm{E}-06 \mathrm{~kg}$ \\
\hline 0.0014074 & 0.0009991 & $0.0010181 \mathrm{~kg}$ \\
\hline 1.12E-05 & 8.26E-06 & $8.33 \mathrm{E}-06 \mathrm{~kg}$ \\
\hline 1.96E-05 & 1.09E-05 & $1.90 \mathrm{E}-05 \mathrm{~kg}$ \\
\hline-0.0016004 & -0.0009161 & -0.0013749 TJ \\
\hline 0.026771 & 0.031167 & $0.022328 \mathrm{~kg}$ \\
\hline 0.0001703 & 7.64E-05 & $0.0003741 \mathrm{~kg}$ \\
\hline 0.009647 & 0.0087046 & $0.0092686 \mathrm{~kg}$ \\
\hline 0.0008874 & 0.0008009 & $0.0008524 \mathrm{~kg}$ \\
\hline 0.0007773 & 0.0006419 & $0.0007137 \mathrm{~kg}$ \\
\hline 1.6686 & 1.4225 & $1.8669 \mathrm{~kg}$ \\
\hline 0.15508 & 0.12573 & $0.14062 \mathrm{~kg}$ \\
\hline $6.27 \mathrm{E}-05$ & 4.33E-05 & $7.80 \mathrm{E}-05 \mathrm{~kg}$ \\
\hline 0.011356 & 0.0078327 & $0.015792 \mathrm{~kg}$ \\
\hline 1.4566 & 0.83807 & $1.1772 \mathrm{~kg}$ \\
\hline 1.57E-06 & 1.16E-06 & 1.10E-06 kg \\
\hline
\end{tabular}


[E1610] calcium air

[E1611] Cs (Cesium) air

[E1612] Cyanide (CN) air

[E1613] DOC air

[E1614] fat and oil air

[E1615] fatty acids as C air

[E1616] Glutaraldehyde air

[E1617] Hexachloroethane air

[E1618] HOCl air

[E1619] hydrocarbons air

[E1620] iod air

[E1621] K air

[E1622] metals water

[E1623] Methylene chloride water

[E1624] Mg water

[E1625] MTBE water

[E1626] Fe water

[E1627] Fluoride water

[E1628] silver water

[E1629] Na water

[E1630] OCl water

[E1631] PAH Polycyclic. Aromatic Hydrocarbons water

[E1632] Radio. Ag110m water

[E1633] Radio. Alpha-radiator water

[E1634] Radio. Am241 water

[E1635] Radio. Ba140 water

[E1636] Radio. C14 water

[E1637] Radio. Cd109 water

[E1638] Radio. Ce141 water

[E1639] Radio. Ce144 water

[E1640] Radio. Cm alpha water

[E1641] Radio. Co57 water

[E1642] Radio. Co58 water

[E1643] Radio. Co60 water

[E1644] Radio. Cr51 water

[E1645] Radio. Cs134 water

[E1646] Radio. Cs136 water

[E1647] Radio. Cs137 water

[E1648] Radio. Fe59 water

[E1649] Radio. H3 water

[E1650] Radio. 1129 water

[E1651] Radio. 1131 water

[E1652] Radio. 1133 water

[E1653] Radio. K 40 water

[E1654] Radio. La140 water

[E1655] Radio. Mn54 water

[E1656] Radio. Mo99 water

[E1657] Radio. Na24 water

[E1658] Radio. Nb95 water

[E1659] Radio. Np237 water

[E1660] Radio. Nucl. mixed water

[E1661] Radio. Pa234m water

\begin{tabular}{|c|c|c|}
\hline 3.8549 & 3.1608 & $3.4971 \mathrm{~kg}$ \\
\hline 7.25E-05 & $6.58 \mathrm{E}-05$ & $6.85 \mathrm{E}-05 \mathrm{~kg}$ \\
\hline 0.0017808 & 0.0013246 & $0.0016646 \mathrm{~kg}$ \\
\hline 0.016669 & 0.011046 & $0.02897 \mathrm{~kg}$ \\
\hline 1.4064 & 1.2613 & $1.3607 \mathrm{~kg}$ \\
\hline 0.37108 & 0.33606 & $0.35378 \mathrm{~kg}$ \\
\hline 0.0002058 & 0.0001755 & $0.0002302 \mathrm{~kg}$ \\
\hline 1.40E-09 & $9.64 \mathrm{E}-10$ & $1.73 \mathrm{E}-09 \mathrm{~kg}$ \\
\hline 0.0026837 & 0.0017771 & $0.0009119 \mathrm{~kg}$ \\
\hline 0.057618 & 0.045586 & $0.098622 \mathrm{~kg}$ \\
\hline 0.0072367 & 0.006564 & $0.0068406 \mathrm{~kg}$ \\
\hline 0.78508 & 0.56897 & $0.68592 \mathrm{~kg}$ \\
\hline 0.21239 & 0.16805 & $0.36548 \mathrm{~kg}$ \\
\hline 0.0007879 & 0.0006643 & $0.0008714 \mathrm{~kg}$ \\
\hline 1.2327 & 0.73724 & $1.0021 \mathrm{~kg}$ \\
\hline $6.14 \mathrm{E}-06$ & $9.54 \mathrm{E}-06$ & $5.76 \mathrm{E}-06 \mathrm{~kg}$ \\
\hline 1.583 & 1.116 & $1.4166 \mathrm{~kg}$ \\
\hline 0.041717 & 0.038489 & $0.040781 \mathrm{~kg}$ \\
\hline 5.34E-05 & 4.93E-05 & $4.95 \mathrm{E}-05 \mathrm{~kg}$ \\
\hline 33.808 & 36.107 & $31.754 \mathrm{~kg}$ \\
\hline 0.0026838 & 0.0017772 & $0.000912 \mathrm{~kg}$ \\
\hline 0.0009813 & 0.0009035 & $0.0009259 \mathrm{~kg}$ \\
\hline 0.13338 & 0.10046 & $0.10174 \mathrm{kBq}$ \\
\hline $9.95 \mathrm{E}-06$ & 7.22E-06 & $6.33 \mathrm{E}-06 \mathrm{kBq}$ \\
\hline 0.04997 & 0.037035 & $0.035631 \mathrm{kBq}$ \\
\hline 0.0018854 & 0.0014023 & $0.0013965 \mathrm{kBq}$ \\
\hline 2.5249 & 1.8719 & $1.8003 \mathrm{kBq}$ \\
\hline 1.09E-05 & $8.10 \mathrm{E}-06$ & 8.06E-06 kBq \\
\hline 0.000281 & 0.000209 & $0.0002082 \mathrm{kBq}$ \\
\hline 1.1441 & 0.84783 & $0.81592 \mathrm{kBq}$ \\
\hline 0.066147 & 0.049024 & $0.047166 \mathrm{kBq}$ \\
\hline 0.0019274 & 0.0014334 & $0.0014279 \mathrm{kBq}$ \\
\hline 0.80412 & 0.59925 & $0.59832 \mathrm{kBq}$ \\
\hline 12.005 & 8.9017 & $8.5967 \mathrm{kBq}$ \\
\hline 0.041348 & 0.030751 & $0.030629 \mathrm{kBq}$ \\
\hline 2.5684 & 1.9046 & $1.833 \mathrm{kBq}$ \\
\hline $1.01 \mathrm{E}-05$ & 7.51E-06 & 7.48E-06 kBq \\
\hline 23.629 & 17.522 & $16.862 \mathrm{kBq}$ \\
\hline 3.33E-05 & 2.48E-05 & 2.47E-05 kBq \\
\hline 74827 & 55454 & $53362 \mathrm{kBq}$ \\
\hline 7.2324 & 5.3602 & $5.1569 \mathrm{kBq}$ \\
\hline 0.011341 & 0.0084699 & $0.0084802 \mathrm{kBq}$ \\
\hline 0.0086151 & 0.0064071 & $0.0063817 \mathrm{kBq}$ \\
\hline-0.10853 & -0.095951 & $-0.14957 \mathrm{kBq}$ \\
\hline 0.0003905 & 0.0002904 & $0.0002892 \mathrm{kBq}$ \\
\hline 1.7279 & 1.2809 & $1.2334 \mathrm{kBq}$ \\
\hline 0.0001317 & $9.79 \mathrm{E}-05$ & 9.75E-05 kBq \\
\hline 0.057934 & 0.043087 & $0.042918 \mathrm{kBq}$ \\
\hline 0.0010686 & 0.0007947 & $0.0007915 \mathrm{kBq}$ \\
\hline 0.0031877 & 0.0023631 & $0.0022732 \mathrm{kBq}$ \\
\hline 8663.6 & 6582.5 & $6159.6 \mathrm{kBq}$ \\
\hline 0.22079 & 0.16361 & $0.15715 \mathrm{kBq}$ \\
\hline
\end{tabular}


[E1662] Radio. Pb 210 water [E1663] Radio. Po 210 water [E1664] Radio. Pu alpha water [E1665] Radio. Pu241 beta water [E1666] Radio. Ra 224 water [E1667] Radio. Ra 226 water [E1668] Radio. Ra 228 water [E1669] Radio. Ru103 water [E1670] Radio. Ru106 water [E1671] Radio. Sb122 water [E1672] Radio. Sb124 water [E1673] Radio. Sb125 water [E1674] Radio. Spalt- u. Aktiv. prod. water [E1675] Radio. Sr89 water [E1676] Radio. Sr90 water [E1677] Radio. Tc99 water [E1678] Radio. Tc99m water [E1679] Radio. Te123m water [E1680] Radio. Te132 water [E1681] Radio. Th 228 water [E1682] Radio. Th 232 water [E1683] Radio. Th230 water [E1684] Radio. Th234 water [E1685] Radio. U 238 water [E1686] Radio. U alpha water [E1687] Radio. U234 water [E1688] Radio. U235 water [E1689] Radio. Y90 water [E1690] Radio. Zn65 water [E1691] Radio. Zr95 water [E1692] Rb (Rubidium) water [E1693] salt water [E1694] Sb water [E1695] Se water [E1696] Si water [E1697] solved substances water [E1698] Solvents (CI) water [E1699] Sr water [E1700] Sulfite water

[E1701] suspended substanses water [E1702] Ti water [E1703] TOC water [E1704] Triethylene Glykol water [E1705] volatile organic comp. as $C$ water [E1706] W (wolfram) water [E1707] Xylene water [E1708] heat water [E1709] aluminium water [E1710] C water [E1711] calcium water [E1712] iron water [E1713] mangane water

\begin{tabular}{|c|c|c|}
\hline-0.08698 & -0.076886 & $-0.11975 \mathrm{kBq}$ \\
\hline-0.08698 & -0.076886 & $-0.11975 \mathrm{kBq}$ \\
\hline 0.19893 & 0.14742 & $0.14185 \mathrm{kBq}$ \\
\hline 4.9382 & 3.6605 & $3.5212 \mathrm{kBq}$ \\
\hline 3.6161 & 3.2814 & $3.4178 \mathrm{kBq}$ \\
\hline 917.09 & 680.65 & $654.35 \mathrm{kBq}$ \\
\hline 7.2369 & 6.5642 & $6.8408 \mathrm{kBq}$ \\
\hline 0.0006312 & 0.0004695 & $0.0004676 \mathrm{kBq}$ \\
\hline 12.03 & 8.9198 & $8.578 \mathrm{kBq}$ \\
\hline 0.0018854 & 0.0014023 & $0.0013965 \mathrm{kBq}$ \\
\hline 0.056806 & 0.042554 & $0.042771 \mathrm{kBq}$ \\
\hline 0.015382 & 0.011441 & $0.011394 \mathrm{kBq}$ \\
\hline 0.066298 & 0.046154 & $0.033236 \mathrm{kBq}$ \\
\hline 0.0042549 & 0.0031644 & $0.0031522 \mathrm{kBq}$ \\
\hline 2.4117 & 1.7873 & $1.7198 \mathrm{kBq}$ \\
\hline 1.2649 & 0.93755 & $0.90198 \mathrm{kBq}$ \\
\hline 0.0008871 & 0.0006598 & $0.0006571 \mathrm{kBq}$ \\
\hline 7.95E-05 & 5.91E-05 & $5.89 \mathrm{E}-05 \mathrm{kBq}$ \\
\hline 3.25E-05 & 2.42E-05 & $2.41 \mathrm{E}-05 \mathrm{kBq}$ \\
\hline 14.472 & 13.14 & $13.679 \mathrm{kBq}$ \\
\hline-0.020261 & -0.017916 & $-0.027917 \mathrm{kBq}$ \\
\hline 34.544 & 25.593 & $24.585 \mathrm{kBq}$ \\
\hline 0.22203 & 0.16448 & $0.15794 \mathrm{kBq}$ \\
\hline 0.63372 & 0.46337 & $0.42257 \mathrm{kBq}$ \\
\hline 14.416 & 10.674 & $10.253 \mathrm{kBq}$ \\
\hline 0.2948 & 0.21842 & $0.20985 \mathrm{kBq}$ \\
\hline 0.43957 & 0.32561 & $0.31281 \mathrm{kBq}$ \\
\hline 0.0002179 & 0.0001621 & $0.0001614 \mathrm{kBq}$ \\
\hline 0.12238 & 0.091018 & $0.090654 \mathrm{kBq}$ \\
\hline 0.1026 & 0.076042 & $0.073147 \mathrm{kBq}$ \\
\hline 0.0007254 & 0.0006583 & $0.0006854 \mathrm{~kg}$ \\
\hline 2.1405 & 1.5307 & $1.5652 \mathrm{~kg}$ \\
\hline $6.13 E-06$ & 5.89E-06 & 1.74E-06 kg \\
\hline 0.0073071 & 0.0042149 & $0.0059036 \mathrm{~kg}$ \\
\hline 0.0010827 & 0.0008914 & $0.0011027 \mathrm{~kg}$ \\
\hline 0.82706 & 0.62933 & $0.70293 \mathrm{~kg}$ \\
\hline 1.65E-05 & 1.67E-05 & $1.42 \mathrm{E}-05 \mathrm{~kg}$ \\
\hline 0.45548 & 0.40803 & $0.42822 \mathrm{~kg}$ \\
\hline 0.0027271 & 0.0040448 & $0.0024617 \mathrm{~kg}$ \\
\hline 5.3479 & 4.5812 & $5.916 \mathrm{~kg}$ \\
\hline 0.085895 & 0.049627 & $0.069535 \mathrm{~kg}$ \\
\hline 30.201 & 26.114 & $24.335 \mathrm{~kg}$ \\
\hline 0.016669 & 0.011046 & $0.02897 \mathrm{~kg}$ \\
\hline 0.025337 & 0.023 & $0.023946 \mathrm{~kg}$ \\
\hline 3.10E-05 & $2.30 E-05$ & 2.08E-05 kg \\
\hline 0.007042 & 0.0063974 & $0.0067654 \mathrm{~kg}$ \\
\hline-0.0006908 & -0.0005762 & $-0.0006933 \mathrm{TJ}$ \\
\hline 0.11333 & 0.095586 & $0.12529 \mathrm{~kg}$ \\
\hline 0.38271 & 0.32062 & $0.40813 \mathrm{~kg}$ \\
\hline 0.45319 & 0.38233 & $0.501 \mathrm{~kg}$ \\
\hline 0.22665 & 0.19118 & $0.25068 \mathrm{~kg}$ \\
\hline 0.0045319 & 0.0038233 & $0.00501 \mathrm{~kg}$ \\
\hline
\end{tabular}


[E1714] N water

[E1715] oil water

[E1716] oil biol. water

[E1717] P water

[E1718] S water

[E1719] chlorine water

[E1720] fluor water

[E1722] Te water

[E1723] heavy metals water

[E1724] metals water

[E1725] oil water

[E1726] HC water

[E1727] bromoxynil water

[E1728] clopyralid water

[E1729] lambda-cyhalothrin water

[E1730] sethoxydim water

[E1731] bromoxynil water

[E1732] clopyralid water

[E1733] lambda-cyhalothrin water

[E1734] sethoxidim soil

[E1735] bromoxynil soil

[E1736] clopyralid soil

[E1737] lambda-cyhalothrin soil

[E1738] sethoxidim soil

\begin{tabular}{rrr}
55.395 & 42.088 & $39.384 \mathrm{~kg}$ \\
0.070075 & 0.062012 & $0.065932 \mathrm{~kg}$ \\
0.0048416 & 0.0037557 & $0.0043744 \mathrm{~kg}$ \\
0.0065833 & 0.0054251 & $0.0070187 \mathrm{~kg}$ \\
0.068008 & 0.057367 & $0.075192 \mathrm{~kg}$ \\
0.029266 & 0.023157 & $0.050361 \mathrm{~kg}$ \\
0.01087 & 0.008601 & $0.018706 \mathrm{~kg}$ \\
0.0001157 & $9.09 \mathrm{E}-05$ & $0.0001164 \mathrm{~kg}$ \\
$8.79 \mathrm{E}-19$ & $6.68 \mathrm{E}-19$ & $6.25 \mathrm{E}-19 \mathrm{~kg}$ \\
$7.35 \mathrm{E}-07$ & $5.59 \mathrm{E}-07$ & $5.23 \mathrm{E}-07 \mathrm{~kg}$ \\
0.03575 & 0.027162 & $0.025417 \mathrm{~kg}$ \\
$1.77 \mathrm{E}-05$ & $1.34 \mathrm{E}-05$ & $1.26 \mathrm{E}-05 \mathrm{~kg}$ \\
0.0042804 & 0.0032522 & $0.0030432 \mathrm{~kg}$ \\
0.0043056 & 0.0032714 & $0.0030611 \mathrm{~kg}$ \\
$2.67 \mathrm{E}-05$ & $2.03 \mathrm{E}-05$ & $1.90 \mathrm{E}-05 \mathrm{~kg}$ \\
0.0029208 & 0.0022192 & $0.0020766 \mathrm{~kg}$ \\
0.016971 & 0.012894 & $0.012066 \mathrm{~kg}$ \\
0.0034747 & 0.00264 & $0.0024704 \mathrm{~kg}$ \\
0.0004155 & 0.0003157 & $0.0002954 \mathrm{~kg}$ \\
0.026186 & 0.019896 & $0.018618 \mathrm{~kg}$ \\
0.0001093 & $8.30 \mathrm{E}-05$ & $7.77 \mathrm{E}-05 \mathrm{~kg}$ \\
\hline $4.00 \mathrm{E}-05$ & $3.04 \mathrm{E}-05$ & $2.85 \mathrm{E}-05 \mathrm{~kg}$ \\
$1.16 \mathrm{E}-06$ & $8.80 \mathrm{E}-07$ & $8.23 \mathrm{E}-07 \mathrm{~kg}$ \\
0.0001498 & 0.0001138 & $0.0001065 \mathrm{~kg}$ \\
\hline & & \\
\hline
\end{tabular}




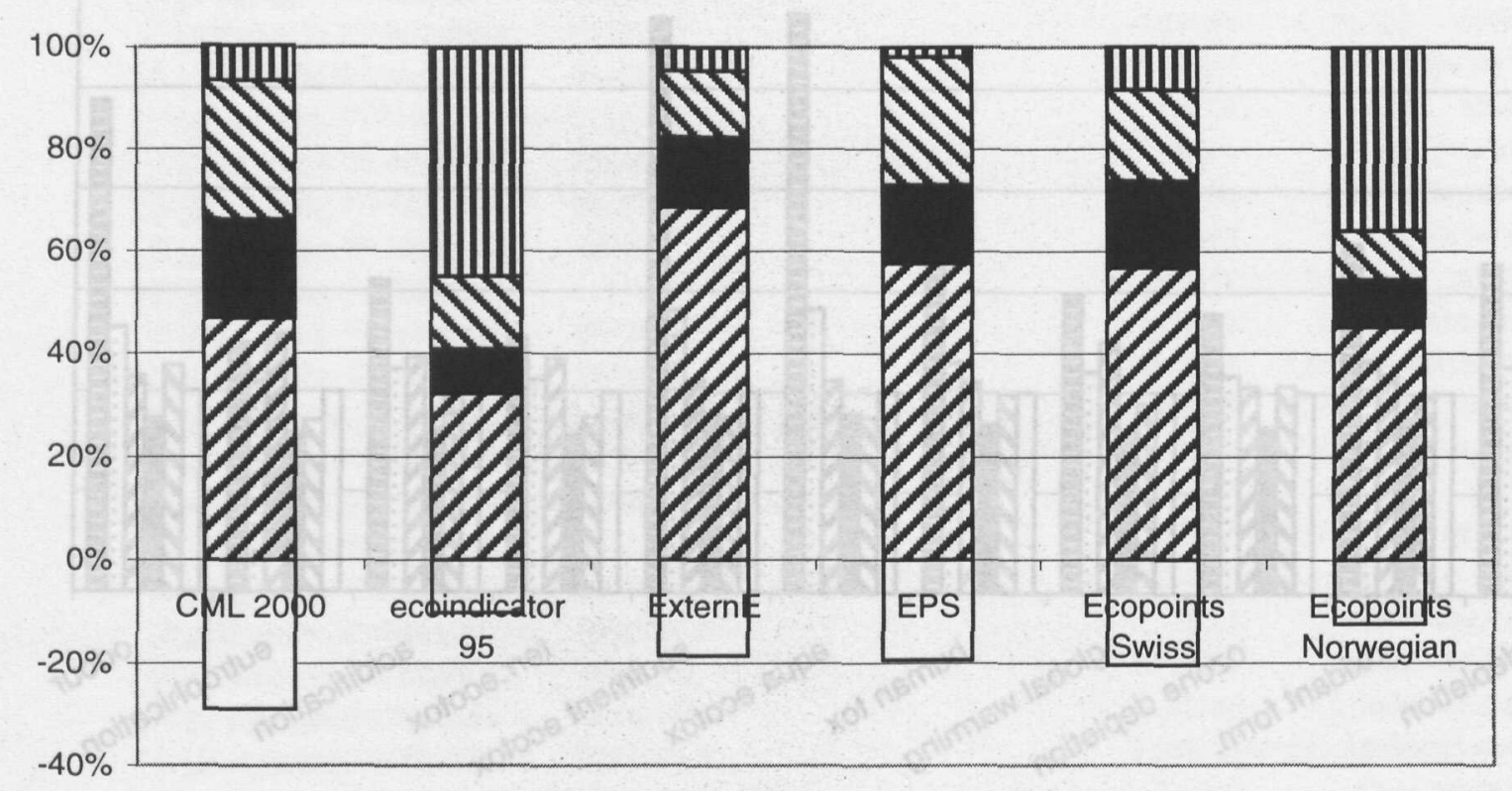

$\square$ raw materials $\mathbf{\square}$ linoleum production $\mathbf{\nabla}$ laying and use $\mathbf{\square}$ discarding $\square$ avoided electr. use

Figure 7. Environmental profile of the baseline system calculated with the Ecoindicator 95, split-up into life cycle stages

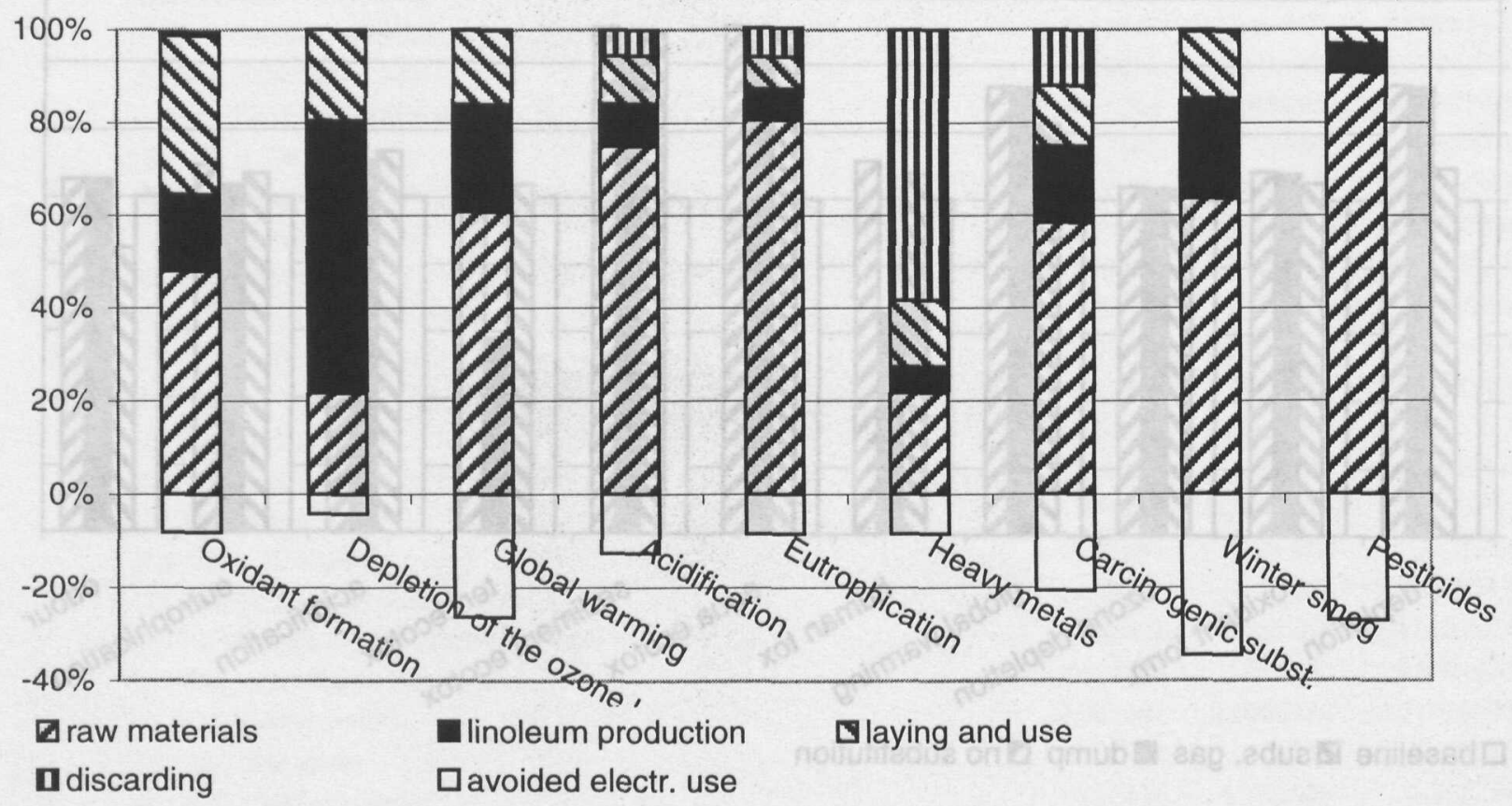

Figure 8. Total weighted results for the baseline system calculated with different methods 


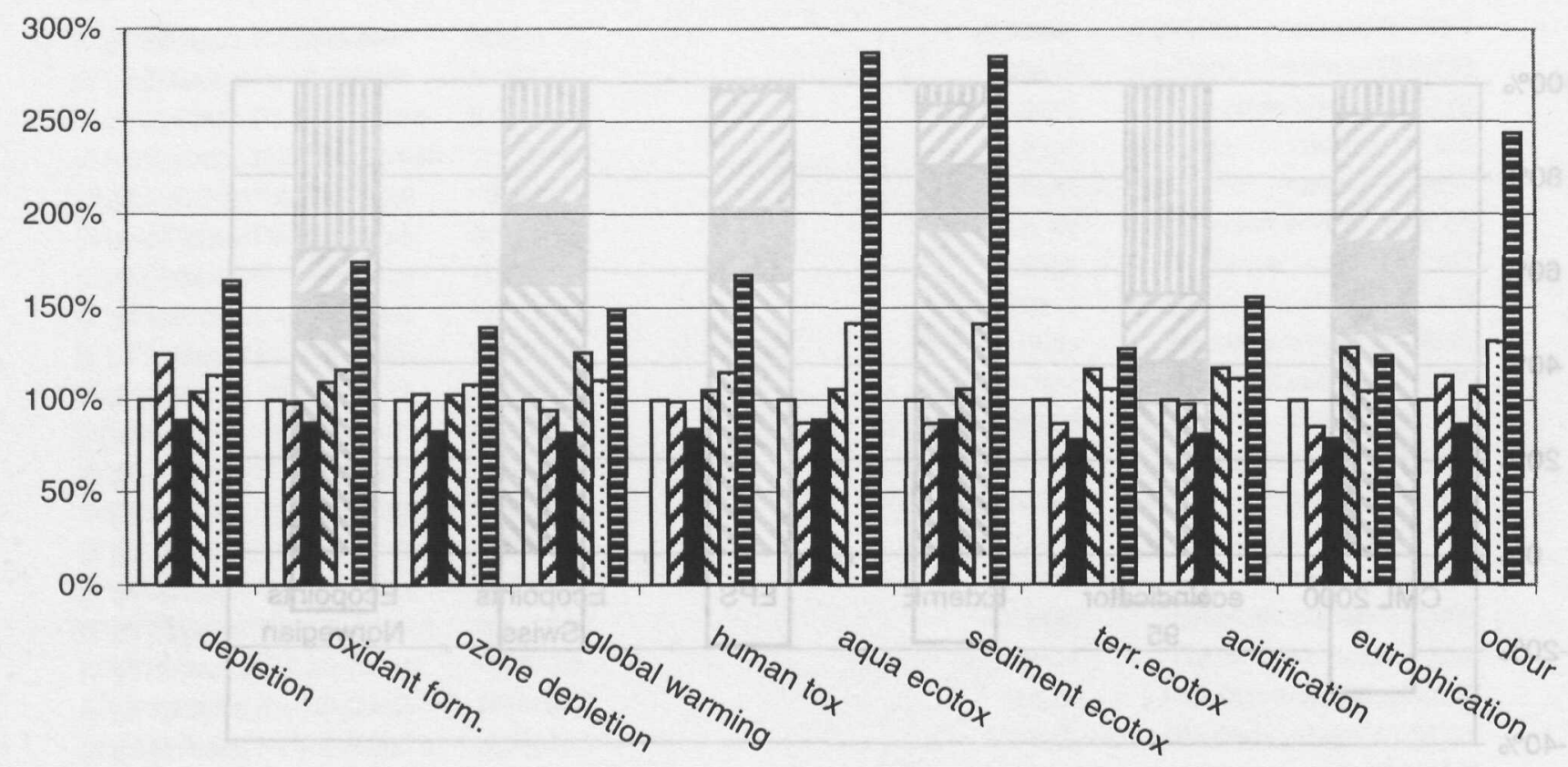

口Baseline $\square$ Cork $\square 2,0 \mathrm{~mm}$ \No Talloil $\square 10 \%$ yellow $\mathbf{\square} 10 \%$ red

Figure 5. Environmental profile for the baseline system and five alternative scenarios related to differences in ingredients

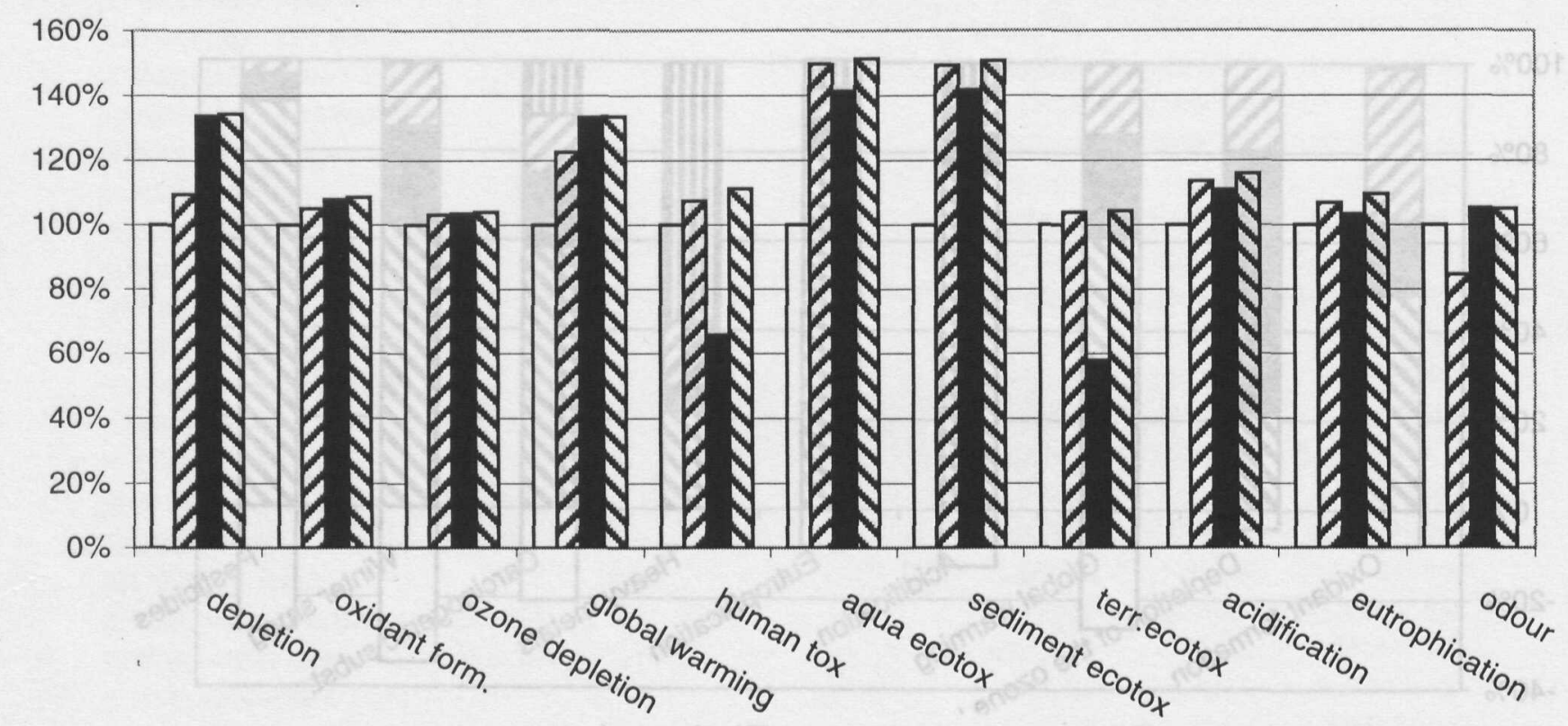

$\square$ baseline $\square$ subs. gas $\square$ dump $\nabla$ no substitution

Figure 6. Environmental profile for the baseline system and three different scenarios related to handling linoleum waste 


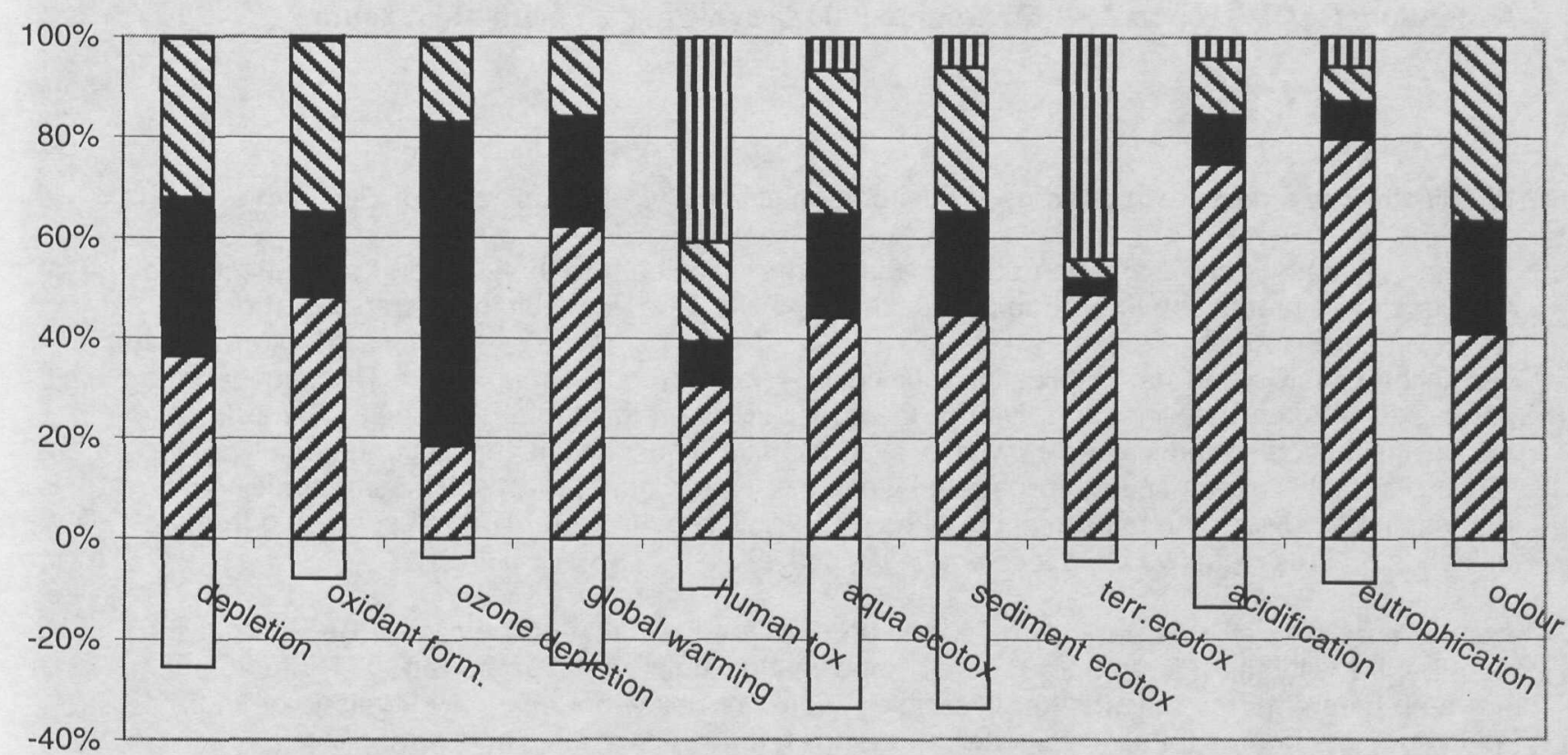

$\square$ raw materials $\mathbf{\square}$ linoleum production $\mathbf{\nabla}$ laying and use $\mathbf{\square}$ discarding $\square$ avoided electr. use

Figure 3. The contribution of life cycle stages to the category results

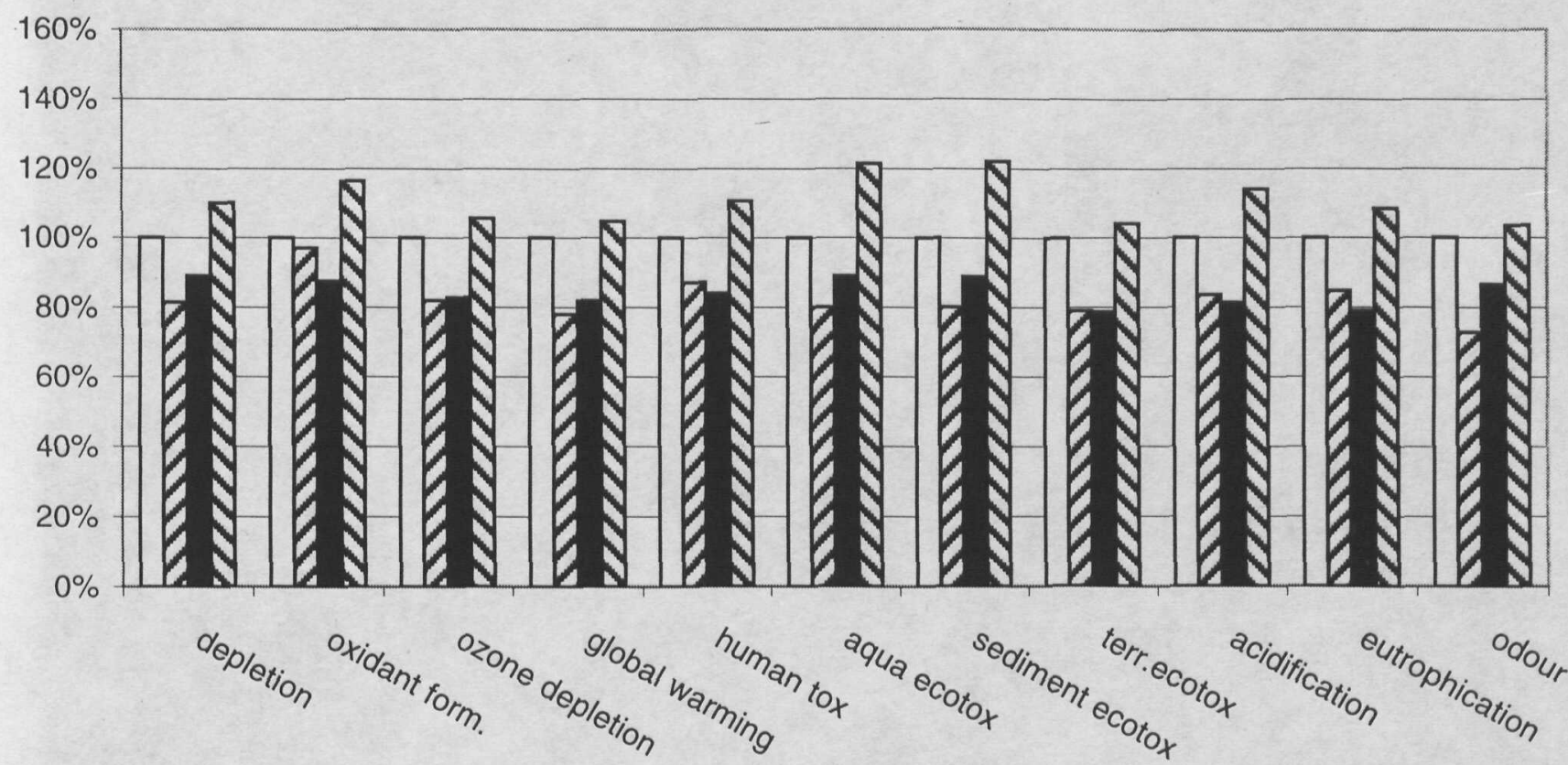

口Baseline $\square$ Swedish $\mathbf{\square} 2,0 \mathrm{~mm}$ \USA

Figure 4. Environmental profile for the baseline system and three alternative scenarios related to the country of use 
Addendum to CML report 151: Environmental life cycle assessment of linoleum

L.S.

We discovered a mistake in our report "Environmental Life Cycle Assessment of Linoleum".

This fault concerns the estimation of the transport from linoleum to the USA and only affects the USA scenario, production in Europe plus sales in the US. Due to a fault in the translation from $\mathrm{m}^{2}$ to $\mathrm{kg}$, the transport by freighter was a factor 3 to low $(7.5 \mathrm{t} / \mathrm{km}$ instead of $21.5 \mathrm{t} / \mathrm{km})$. The correction of this value has implications for figure 4 in the report, in which the USA scenario is compared with the baseline, and also for the conclusions on this topic. Transport to (and in) the USA with the corrected values now has a substantial influence on the environmental profile, ranging from $4 \%$ higher scores for terrestrial ecotoxicity and odour to $21-22 \%$ percent for aquatic and sediment toxicity. As the transport takes place mainly by freighter it is not surprising that the influence on those last two impact categories is highest.

Furthermore, figure 7 did not fully reflect the correct data and text of the report. Because of the poor print quality the figures 3 to 8 are all somewhat difficult to read in the report. The difference between different gray tones is hardly visible. Therefore below not only new versions of figure 4 and 7 , but the figures for all five scenarios are given for easier reading and comparison .

The Authors

30 November 2000

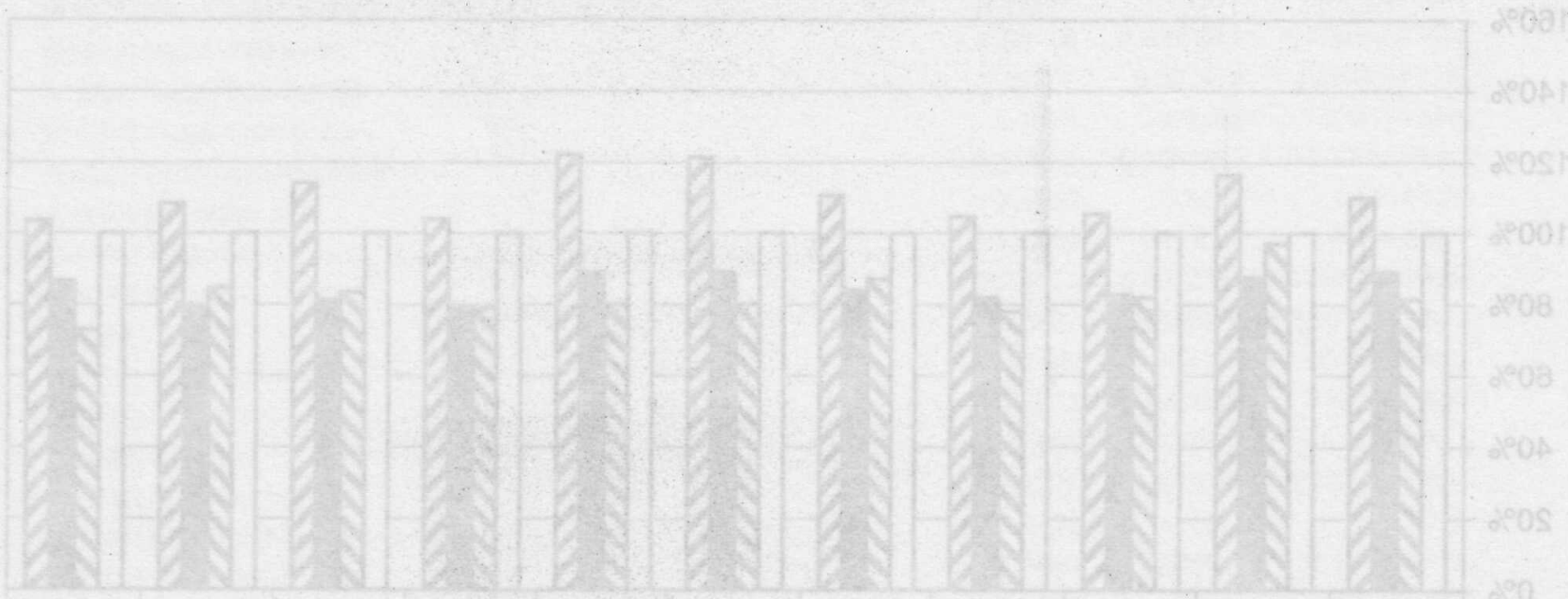

